\title{
Convincing Catalytic Performance of Oxo-tethered Ruthenium Complexes for Asymmetric Transfer Hydrogenation of Cyclic $\alpha$-Halogenated Ketones through Dynamic Kinetic Resolution
}

Taichiro Touge,${ }^{* \dagger}$ Hideki Nara,${ }^{\dagger}$ Michio Kida,${ }^{\dagger}$ Kazuhiko Matsumura, ${ }^{\dagger}$ Yoshihito Kayaki, ${ }^{* \dagger}$

\begin{abstract}
$\dagger$ Corporate Research and Development Division, Takasago International Corporation, 1-4-11 Nishi-yawata, Hiratsuka City, Kanagawa 254-0073, Japan

$\$$ Department of Chemical Science and Engineering, School of Materials and Chemical Technology, Tokyo Institute of Technology, 2-12-1-E4-1 O-okayama, Meguro-ku, Tokyo 152-8552, Japan
\end{abstract}

taichiro touge@takasago.com

ykayaki@,o.cc.titech.ac.jp 


\section{Contents}

General Information $\quad$ S2

A. Ancillary Data for Asymmtric Transfer Hydrogenation of 7a-7c. S3

B. Synthesis of Ketones. S6

C. Asymmetric Transfer Hydrogenation of $\alpha$-Substituted Ketones Using $(R, R)-2 \mathrm{a}, 2 \mathrm{~b}$ and 2c.

D. Characterization Data for Reduction Products.

E. Synthesis of (+)-PHNO.

F. Determination of Absolute Configuration of Products.

G. References.

H. NMR Spectra for Ketones and Reduction Products and Derivatized Products.

I. Chiral HPLC Charts for Reduction Products.

J. HR-MS Spectra for Ketones, Reduction Products and Derivatized Products.

\section{General Information}

All reactions and manipulations were conducted under a nitrogen atmosphere unless otherwise noted. Synthesis of ruthenium catalysts was performed with solvents purchased as an anhydrous grade. NMR Spectra were obtained on Agilent 400-MR DD2 and Bruker BioSpin Avance III 500 Systems. NMR chemical shifts are listed in ppm relative to $\mathrm{CHCl}_{3}$ (7.26 ppm for ${ }^{1} \mathrm{H}$, and $77.0 \mathrm{ppm}$ for ${ }^{13} \mathrm{C}$ ) or $\mathrm{CH}_{3} \mathrm{OH}\left(3.30 \mathrm{ppm}\right.$ for ${ }^{1} \mathrm{H}$, and $49.0 \mathrm{ppm}$ for ${ }^{13} \mathrm{C}$ ). The following abbreviations are used to designate peak splitting patterns: $\mathrm{s}=$ singlet, $\mathrm{d}=$ doublet, $\mathrm{t}=$ triplet, $\mathrm{q}=$ quartet, $\mathrm{dd}=\mathrm{a}$ doublet of doublets, $\mathrm{ddd}=$ a doublet of doublets of doublets, $\mathrm{m}=$ multiplet, $\mathrm{br}=$ broad. Optical rotations were obtained on a JASCO P-1020 Polarimeter. Mass spectra were recorded on Bruker Impact II, Thermo Fisher Scientific TRACE1310, EXACTIV GC, Orbitrap GC-MS, and JEOL JMS-T100GCV instruments. High performance liquid chromatography (HPLC) analysis was performed using a GL-Science GL-7400 system comprised of a column oven (GL-7430), a gradient unit with a pump and a degasser (GL-7430), a UV detector (GL-7450), and an auto sampler (GL-7420), and a HITACHI Chromaster system equipped with a column oven (5310), a gradient unit with a pump, degasser (5110), a UV detector (5410) and an auto sampler (5210). Ketones $(\mathbf{3 k}, \mathbf{3 l})$ were purchased from Sigma-Aldrich. 
A. Ancillary Data for Asymmetric Transfer Hydrogenation of 7a-7c.

(1) Asymmetric Transfer Hydrogenation of 6-Chloro-3-methylcyclohex-2-en-1-one (7a)

Table S1. Asymmetric Transfer Hydrogenation of 6-Chloro-3-methylcyclohex-2-en-1-one (7a) ${ }^{\text {[a] }}$

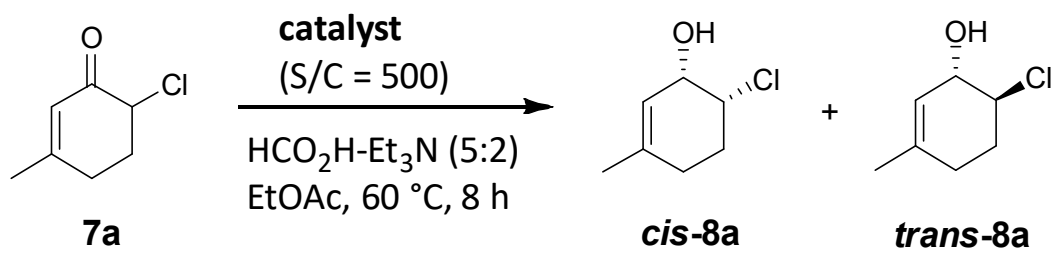

\begin{tabular}{|c|c|c|c|c|}
\hline Entry & Catalyst & Yield (\%) & cis/trans $^{[\mathrm{d}]}$ & Ee (\%) $)^{[\mathrm{e}]}$ \\
\hline 1 & Ts-DENEB & $>95^{[\mathrm{b}]}$ & $96 / 4$ & 68 \\
2 & Ms-DENEB & $>95^{[\mathrm{b}]}$ & $95 / 5$ & 72 \\
3 & TIPPs-DENEB & $\mathbf{9 0}^{[\mathrm{c}]}$ & $>\mathbf{9 9 / 1}$ & $\mathbf{9 3}$ \\
\hline
\end{tabular}

[a] Typical reaction conditions: substrate $(1.0 \mathrm{mmol})$, catalyst $(0.002 \mathrm{mmol}), \mathrm{HCO}_{2} \mathrm{H}-\mathrm{Et}_{3} \mathrm{~N}$ (5:2) azeotropic mixture $(0.5 \mathrm{~mL})$, and AcOEt $(1.0 \mathrm{~mL})$. [b] Estimated by TLC analysis. [c] Isolated yield. [d] Determined by ${ }^{1} \mathrm{H}-\mathrm{NMR}$ analysis of the crude products. [e] Determined by HPLC analysis. 
(2) Asymmetric Transfer Hydrogenation of Ethyl 2-oxocyclohex-3-ene-1-carboxylate (7b)

Table S2. Asymmetric Transfer Hydrogenation of Ethyl 2-oxocyclohex-3-ene-1-carboxylate (7b) [a]

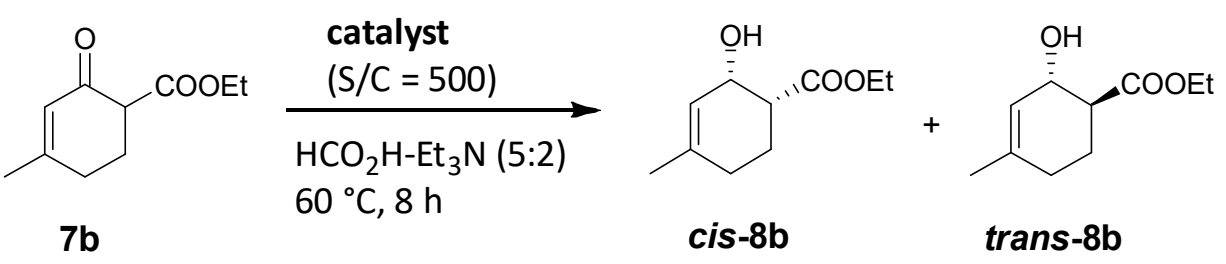

\begin{tabular}{|c|c|c|c|c|}
\hline Entry & Catalyst & Yield (\%) & cis/trans $^{[\mathrm{d}]}$ & Ee (\%) $)^{[\mathrm{e}]}$ \\
\hline 1 & Ts-DENEB & $>95^{[\mathrm{b}]}$ & $96 / 4$ & 83 \\
2 & Ms-DENEB & $>95^{[\mathrm{b}]}$ & $94 / 6$ & 76 \\
3 & TIPPs-DENEB & $\mathbf{9 0}^{[\mathrm{c}]}$ & $>\mathbf{9 9 / 1}$ & $\mathbf{9 1}$ \\
\hline
\end{tabular}

[a] Typical reaction conditions: substrate $(1.0 \mathrm{mmol})$, catalyst $(0.002 \mathrm{mmol})$ and $\mathrm{HCO}_{2} \mathrm{H}_{-} \mathrm{Et}_{3} \mathrm{~N}(5: 2)$ azeotropic mixture $(0.5 \mathrm{~mL})$. [b] Estimated by TLC analysis. [c] Isolated yield. [d] Determined by ${ }^{1} \mathrm{H}-\mathrm{NMR}$ analysis of the crude products. [e] Determined by HPLC analysis. 
(3) Asymmetric Transfer Hydrogenation of $N$-(tert-Butyl)-4-methyl-2-oxocyclohex-3-ene-1-carboxamide (7c)

Table S3. Asymmetric Transfer Hydrogenation of $N$-(tert-Butyl)-4-methyl-2-oxocyclohex-3-ene-1-carboxamide (7c) ${ }^{[a]}$

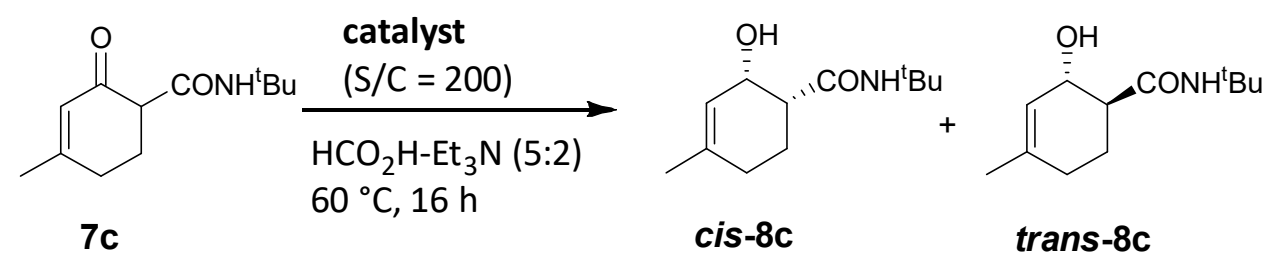

\begin{tabular}{|c|c|c|c|c|}
\hline Entry & Catalyst & Yield (\%) & cis/trans $^{[\mathrm{d}]}$ & Ee (\%) ${ }^{[\mathrm{e}]}$ \\
\hline 1 & Ts-DENEB & $>95^{[\mathrm{b}]}$ & $>99 / 1$ & 88 \\
2 & Ms-DENEB & $>95^{[\mathrm{b}]}$ & $>99 / 1$ & 87 \\
3 & TIPPs-DENEB & $\mathbf{9 4}^{[\mathrm{c}]}$ & $>99 / 1$ & 96 \\
\hline
\end{tabular}

[a] Typical reaction conditions: substrate $(1.0 \mathrm{mmol})$, catalyst $(0.005 \mathrm{mmol})$ and $\mathrm{HCO}_{2} \mathrm{H}_{-}-\mathrm{Et}_{3} \mathrm{~N}(5: 2)$ azeotropic mixture $(0.5 \mathrm{~mL})$. [b] Estimated by TLC analysis. [c] Isolated yield. [d] Determined by ${ }^{1} \mathrm{H}-\mathrm{NMR}$ analysis of the crude products. [e] Determined by HPLC analysis. 


\section{B. Synthesis of Ketones.}

(a) Synthesis of 2-chloro-3,4-dihydronaphthalen-1(2H)-one (3a) ${ }^{[1]}$

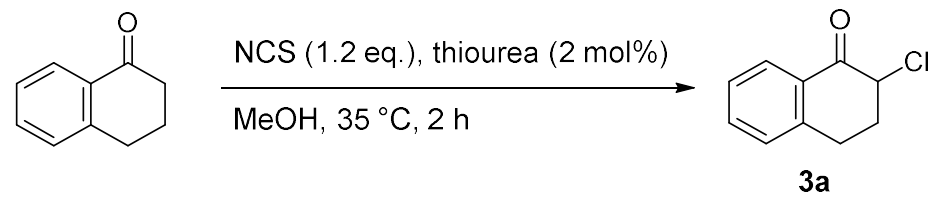

A MeOH solution $(100 \mathrm{~mL})$ containing $\alpha$-tetralone $(10.00 \mathrm{~g}, 68.4 \mathrm{mmol})$, thiourea $(104.0$ $\mathrm{mg}, 1.37 \mathrm{mmol}$ ), and $\mathrm{N}$-chlorosuccinimide (10.96 g, $82.08 \mathrm{mmol}, 1.20$ eq.) was heated by oil bath under $\mathrm{N}_{2}$ atmosphere at $35{ }^{\circ} \mathrm{C}$, for $2 \mathrm{~h}$. The solution was concentrated under a reduced pressure, then AcOEt $(50 \mathrm{~mL})$ and $\mathrm{H}_{2} \mathrm{O}(20 \mathrm{~mL})$ were added and stirred for 10 min. The organic layer was separated, and the aqueous phase was extracted with AcOEt (2 $\times 50 \mathrm{~mL}$ ). The combined organic portions were dried over $\mathrm{MgSO}_{4}$ and concentrated to give a crude product. Purification by silica gel column chromatography (eluent: Hexane/AcOEt $=5 / 1$ ) gave the product (3a) as a light brown solid ( $7.6 \mathrm{~g}, 62 \%$ yield).

${ }^{1} \mathrm{H}$ NMR $\left(400 \mathrm{MHz}, \mathrm{CDCl}_{3}\right) \delta 8.08(\mathrm{dd}, J=7.8,1.6 \mathrm{~Hz}, 1 \mathrm{H}), 7.54-7.50(\mathrm{~m}, 1 \mathrm{H})$, $7.37-7.33(\mathrm{~m}, 1 \mathrm{H}), 7.27(\mathrm{~d}, J=7.6 \mathrm{~Hz}, 1 \mathrm{H}), 4.63$ (dd, $J=7.8,3.6 \mathrm{~Hz}, 1 \mathrm{H}), 3.29$ (ddd, $J=$ 14.6, 9.8, $4.7 \mathrm{~Hz}, 1 \mathrm{H}$ ), 3.00 (ddd, $J=14.6,9.8,4.7 \mathrm{~Hz}, 1 \mathrm{H}), 2.62-2.55$ (m, 1H), 2.50-2.42 $(\mathrm{m}, 1 \mathrm{H}) ;{ }^{13} \mathrm{C}\left\{{ }^{1} \mathrm{H}\right\}$ NMR $\left(125 \mathrm{MHz}, \mathrm{CDCl}_{3}\right) \delta 190.8,143.1,134.1,130.4,128.7,128.5$, $127.1,59.8,32.4,26.3$.

HRMS (APCI) $m / z:[\mathrm{M}+\mathrm{H}]^{+}$calcd for $\mathrm{C}_{10} \mathrm{H}_{10} \mathrm{ClO}: 181.0415$; found: 181.0399 .

Following this method, ketones $\mathbf{3 b}, \mathbf{3 c}, \mathbf{3 d}, \mathbf{3 e}, \mathbf{3 f}, \mathbf{3 g}, \mathbf{3 h}, \mathbf{3} \mathbf{i}$, and $\mathbf{3} \mathbf{j}$ were synthesized.

\section{2-Chloro-7-methoxy-3,4-dihydronaphthalen-1(2H)-one (3b)}<smiles>COc1ccc2c(c1)C(=O)C(Cl)CC2</smiles>

The chlorination was carried out on a $19.9 \mathrm{mmol}$ scale. A crude product was purified by silica gel column chromatography (eluent: Hexane/AcOEt $=5 / 1$ ). A light brown solid of 3b (4.1 g) was obtained in $98 \%$ yield.

${ }^{1} \mathrm{H}$ NMR $\left(500 \mathrm{MHz}, \mathrm{CDCl}_{3}\right) \delta 7.54(\mathrm{~d}, J=2.8 \mathrm{~Hz}, 1 \mathrm{H}), 7.17(\mathrm{~d}, J=8.4 \mathrm{~Hz}, 1 \mathrm{H}), 7.11(\mathrm{dd}$, $J=8.4,2.8 \mathrm{~Hz}, 1 \mathrm{H}), 4.62(\mathrm{dd}, J=7.6,3.8 \mathrm{~Hz}, 1 \mathrm{H}), 3.84(\mathrm{~s}, 3 \mathrm{H}), 3.22(\mathrm{ddd}, J=14.5,10.2$, $4.6 \mathrm{~Hz}, 1 \mathrm{H}$ ), 2.93 (ddd, $J=14.5,9.4,4.6 \mathrm{~Hz}, 1 \mathrm{H}), 2.58-2.53$ (m, 1H), 2.47-2.41 (m, 1H); ${ }^{13} \mathrm{C}\left\{{ }^{1} \mathrm{H}\right\} \mathrm{NMR}\left(125 \mathrm{MHz}, \mathrm{CDCl}_{3}\right) \delta 190.8,158.6,135.7,131.2,130.0,122.7,110.1,59.7$, 55.5, 32.6, 25.5.

HRMS (APCI) $m / z:[\mathrm{M}+\mathrm{H}]^{+}$calcd for $\mathrm{C}_{11} \mathrm{H}_{11} \mathrm{ClO}_{2}: 211.0520$; found: 211.0520. 


\section{2-Chloro-7-bromo-3,4-dihydronaphthalen-1(2H)-one (3c)}<smiles>O=C1c2cc(Br)ccc2CCC1Cl</smiles>

The chlorination was carried out on a $13.3 \mathrm{mmol}$ scale. A crude product was purified by silica gel column chromatography (eluent: Hexane/AcOEt $=15 / 1$ to 8/1). A white solid of $3 \mathrm{c}(2.6 \mathrm{~g})$ was obtained in $75 \%$ yield.

${ }^{1} \mathrm{H}$ NMR $\left(500 \mathrm{MHz}, \mathrm{CDCl}_{3}\right) \delta 8.19(\mathrm{~d}, J=2.3 \mathrm{~Hz}, 1 \mathrm{H}), 7.62(\mathrm{dd}, J=8.2,2.3 \mathrm{~Hz}, 1 \mathrm{H}), 7.17$ $(\mathrm{d}, J=8.2 \mathrm{~Hz}, 1 \mathrm{H}), 4.61(\mathrm{dd}, J=7.4,3.8 \mathrm{~Hz}, 1 \mathrm{H}), 3.23(\mathrm{ddd}, J=15.1,11.0,5.2 \mathrm{~Hz}, 1 \mathrm{H})$, $2.95(\mathrm{dt}, J=11.0,5.2 \mathrm{~Hz}, 1 \mathrm{H}), 2.60-2.53(\mathrm{~m}, 1 \mathrm{H}), 2.48-2.43(\mathrm{~m}, 1 \mathrm{H}) ;{ }^{13} \mathrm{C}\left\{{ }^{1} \mathrm{H}\right\} \mathrm{NMR}(125$ $\left.\mathrm{MHz}, \mathrm{CDCl}_{3}\right) \delta 189.5,141.7,136.9,131.9,131.2,130.5,121.1,59.0,31.9,25.6$.

HRMS (APCI) $m / z$ : [M+H] $]^{+}$calcd for $\mathrm{C}_{10} \mathrm{H}_{8} \mathrm{BrClO}$ : 258.9520; found: 258.9515 .

2-Chloro-2,3-dihydro-1 $H$-inden-1-one (3d)<smiles>O=C1c2ccccc2CC1Cl</smiles>

The chlorination was carried out on a $51.3 \mathrm{mmol}$ scale. A crude product was purified by silica gel column chromatography (eluent: Hexane/AcOEt $=10 / 1$ to $5 / 1$ ). A pale yellow solid of $\mathbf{3 d}(6.3 \mathrm{~g})$ was obtained in $73 \%$ yield.

${ }^{1} \mathrm{H}$ NMR $\left(400 \mathrm{MHz}, \mathrm{CDCl}_{3}\right) \delta$ 7.83-7.81 (m, 1H), 7.69-7.65 (m, 1H), 7.47-7.42 (m, 2H), 4.57 (dd, $J=7.9,3.8 \mathrm{~Hz}, 1 \mathrm{H}), 3.79$ (dd, $J=17.6,7.9 \mathrm{~Hz}, 1 \mathrm{H}), 3.30$ (dd, $J=17.6,3.8 \mathrm{~Hz}$, $1 \mathrm{H}) ;{ }^{13} \mathrm{C}\left\{{ }^{1} \mathrm{H}\right\} \mathrm{NMR}\left(125 \mathrm{MHz}, \mathrm{CDCl}_{3}\right) \delta 199.1,150.7,136.0,133.9,128.3,126.4,125.0$, 55.7, 37.5.

HRMS (APCI) $m / z:[\mathrm{M}+\mathrm{H}]^{+}$calcd for $\mathrm{C}_{9} \mathrm{H}_{8} \mathrm{ClO}_{3}$ : 167.0258; found: 167.0245.

\section{2-Chloro-5-methoxy-2,3-dihydro-1H-inden-1-one (3e)}<smiles>COc1ccc2c(c1)CC(Cl)C2=O</smiles>

The chlorination was carried out on a $18.5 \mathrm{mmol}$ scale. A crude product was purified by silica gel column chromatography (eluent: Hexane/AcOEt $=5 / 1$ to 3/1). A white solid of 3e (3.3 g) was obtained in $92 \%$ yield.

${ }^{1} \mathrm{H}$ NMR $\left(500 \mathrm{MHz}, \mathrm{CDCl}_{3}\right) \delta 7.75(\mathrm{~d}, J=8.6 \mathrm{~Hz}, 1 \mathrm{H}), 6.95(\mathrm{dd}, J=8.6,1.8 \mathrm{~Hz}, 1 \mathrm{H})$, 6.88-6.87 (m, 1H), $4.54(\mathrm{dd}, J=7.7,3.8 \mathrm{~Hz}, 1 \mathrm{H}), 3.90(\mathrm{~s}, 3 \mathrm{H}), 3.72(\mathrm{dd}, J=17.3,7.7 \mathrm{~Hz}$, 1H), $3.25(\mathrm{dd}, J=17.3,3.8 \mathrm{~Hz}, 1 \mathrm{H}) ;{ }^{13} \mathrm{C}\left\{{ }^{1} \mathrm{H}\right\} \mathrm{NMR}\left(125 \mathrm{MHz}, \mathrm{CDCl}_{3}\right) \delta$ 197.3, 166.4, 


\section{2,5-Dichloro-2,3-dihydro-1H-inden-1-one (3f)}<smiles>O=C1c2ccc(Cl)cc2CC1Cl</smiles>

The chlorination was carried out on a $18.0 \mathrm{mmol}$ scale. A crude product was purified by silica gel column chromatography (eluent: Hexane/AcOEt $=10 / 1$ to $5 / 1$ ). A white solid of 3f $(3.2 \mathrm{~g})$ was obtained in $88 \%$ yield.

${ }^{1} \mathrm{H}$ NMR $\left(500 \mathrm{MHz}, \mathrm{CDCl}_{3}\right) \delta 7.75(\mathrm{~d}, J=8.0 \mathrm{~Hz}, 1 \mathrm{H}), 7.46-7.27(\mathrm{~m}, 2 \mathrm{H}), 4.56(\mathrm{dd}, J=$ $7.5,4.0 \mathrm{~Hz}, 1 \mathrm{H}), 3.76(\mathrm{dd}, J=18.0,7.5 \mathrm{~Hz}, 1 \mathrm{H}), 3.28(\mathrm{dd}, J=18.0,4.0 \mathrm{~Hz}, 1 \mathrm{H}) ;{ }^{13} \mathrm{C}\left\{{ }^{1} \mathrm{H}\right\}$ NMR $\left(125 \mathrm{MHz}, \mathrm{CDCl}_{3}\right) \delta 197.8,152.1,142.7,132.2,129.2,126.6,126.1,55.4,37.2$. HRMS (APCI) $m / z:[\mathrm{M}+\mathrm{H}]^{+}$calcd for $\mathrm{C}_{9} \mathrm{H}_{6} \mathrm{Cl}_{2} \mathrm{O}: 200.9868$; found: 200.9868 .

\section{2-Chloro-7-hydroxy-2,3-dihydro-1H-inden-1-one (3g)}<smiles>O=C1c2c(O)cccc2CC1Cl</smiles>

The chlorination was carried out on a $15.7 \mathrm{mmol}$ scale. A crude product was purified by silica gel column chromatography (eluent: Hexane/AcOEt $=10 / 1$ to $5 / 1$ ). A light yellow solid of $\mathbf{3 g}(2.8 \mathrm{~g})$ was obtained in $96 \%$ yield.

${ }^{1} \mathrm{H}$ NMR $\left(500 \mathrm{MHz}, \mathrm{CDCl}_{3}\right) \delta 7.56(\mathrm{~d}, J=7.8 \mathrm{~Hz}, 1 \mathrm{H}), 6.92(\mathrm{~d}, J=7.8 \mathrm{~Hz}, 1 \mathrm{H}), 6.82(\mathrm{~d}, J$ $=7.8 \mathrm{~Hz}, 1 \mathrm{H}), 4.59(\mathrm{dd}, J=7.7,3.7 \mathrm{~Hz}, 1 \mathrm{H}), 3.75(\mathrm{dd}, J=17.8,7.7 \mathrm{~Hz}, 1 \mathrm{H}), 3.25(\mathrm{dd}, J=$ 17.8, 3.7 Hz, $1 \mathrm{H}) ;{ }^{13} \mathrm{C}\left\{{ }^{1} \mathrm{H}\right\}$ NMR $\left(125 \mathrm{MHz}, \mathrm{CDCl}_{3}\right) \delta 201.8,158.0,150.6,139.2,119.6$, 117.2, 114.6, 55.1, 37.6.

HRMS (APCI) $m / z:[\mathrm{M}+\mathrm{H}]^{+}$calcd for $\mathrm{C}_{9} \mathrm{H}_{7} \mathrm{ClO}_{2}: 183.0207$; found: 183.0205 .

\section{2-Chloro-3,3-dimethyl-2,3-dihydro-1H-inden-1-one (3h)}<smiles>CC1(C)c2ccccc2C(=O)C1Cl</smiles>

The chlorination was carried out on a $18.7 \mathrm{mmol}$ scale. A crude product was purified by silica gel column chromatography (eluent: Hexane/AcOEt $=20 / 1$ ). A light brown oil of $\mathbf{3 h}$ 
$(2.4 \mathrm{~g})$ was obtained in $67 \%$ yield.

${ }^{1} \mathrm{H}$ NMR $\left(500 \mathrm{MHz}, \mathrm{CDCl}_{3}\right) \delta 7.77(\mathrm{~d}, J=7.7 \mathrm{~Hz}, 1 \mathrm{H}), 7.70-7.67(\mathrm{~m}, 1 \mathrm{H}), 7.52(\mathrm{~d}, J=7.7$ $\mathrm{Hz}, 1 \mathrm{H}), 7.43(\mathrm{t}, J=7.7 \mathrm{~Hz}, 1 \mathrm{H}), 4.44(\mathrm{~s}, 1 \mathrm{H}), 1.56(\mathrm{~s}, 3 \mathrm{H}), 1.29(\mathrm{~s}, 3 \mathrm{H}) ;{ }^{13} \mathrm{C}$ NMR $(125$ $\left.\mathrm{MHz}, \mathrm{CDCl}_{3}\right) \delta 201.8,158.0,150.6,139.2,119.6,117.2,114.6,55.1,37.6$.

${ }^{13} \mathrm{C}\left\{{ }^{1} \mathrm{H}\right\}$ NMR $\left(125 \mathrm{MHz}, \mathrm{CDCl}_{3}\right) \delta 197.7,159.9,136.0,131.9,128.2,124.3,123.5,70.6$, 43.7, 28.1, 26.1.

HRMS (APCI) $m / z$ : [M+H] $]^{+}$calcd for $\mathrm{C}_{11} \mathrm{H}_{11} \mathrm{ClO}$ : 195.0571; found: 195.0575.

\section{6-Chloro-6,7,8,9-tetrahydro-5H-benzo[7]annulen-5-one (3i)}<smiles>O=C1c2ccccc2CCCC1Cl</smiles>

The chlorination was carried out on a $31.2 \mathrm{mmol}$ scale. A crude product was purified by silica gel column chromatography (eluent: Toluene/Hexane $=3 / 1$ to $1 / 1$ ). A yellow liquid of $3 \mathbf{i}(0.9 \mathrm{~g})$ was obtained in $15 \%$ yield.

${ }^{1} \mathrm{H}$ NMR $\left(400 \mathrm{MHz}, \mathrm{CDCl}_{3}\right) \delta 7.64(\mathrm{dd}, J=7.8,1.6 \mathrm{~Hz}, 1 \mathrm{H}), 7.44-7.18(\mathrm{~m}, 3 \mathrm{H}), 4.80(\mathrm{dd}$, $J=8.8,4.8 \mathrm{~Hz}, 1 \mathrm{H}), 3.05-2.90(\mathrm{~m}, 2 \mathrm{H}), 2.44-2.35(\mathrm{~m}, 1 \mathrm{H}), 2.25-2.18(\mathrm{~m}, 1 \mathrm{H}), 2.07-1.96$ $(\mathrm{m}, 2 \mathrm{H}) ;{ }^{13} \mathrm{C}\left\{{ }^{1} \mathrm{H}\right\}$ NMR $\left(125 \mathrm{MHz}, \mathrm{CDCl}_{3}\right) \delta 199.5,140.3,137.4,132.1,129.8,129.4$, 126.7, 63.4, 33.9, 33.7, 23.6.

HRMS (APCI) $m / z:[\mathrm{M}+\mathrm{H}]^{+}$calcd for $\mathrm{C}_{11} \mathrm{H}_{12} \mathrm{ClO}$ : 195.0571; found: 195.0554 .

\section{2-Chloroacenaphthylen-1(2H)-one (3j)}<smiles>O=C1c2cccc3cccc(c23)C1Cl</smiles>

The chlorination was carried out on a $17.8 \mathrm{mmol}$ scale. A crude product was purified by silica gel column chromatography (eluent: Toluene only). A pale brown powder of $\mathbf{3 j}$ (3.0 g) was obtained in $84 \%$ yield.

${ }^{1} \mathrm{H}$ NMR $\left(400 \mathrm{MHz}, \mathrm{CDCl}_{3}\right) \delta 8.17(\mathrm{~d}, J=8.0 \mathrm{~Hz}, 1 \mathrm{H}), 8.04(\mathrm{~d}, J=6.8 \mathrm{~Hz}, 1 \mathrm{H}), 7.95-7.93$ $(\mathrm{m}, 1 \mathrm{H}), 7.79-7.71(\mathrm{~m}, 3 \mathrm{H}), 5.50(\mathrm{~s}, 1 \mathrm{H}) ;{ }^{13} \mathrm{C}\left\{{ }^{1} \mathrm{H}\right\} \mathrm{NMR}\left(125 \mathrm{MHz}, \mathrm{CDCl}_{3}\right) \delta 196.9,141.6$, 135.3, 132.3, 130.9, 130.6, 128.8, 128.6, 126.0, 123.1, 122.4, 56.3.

HRMS (ESI) $m / z:[\mathrm{M}+\mathrm{H}]^{+}$calcd for $\mathrm{C}_{12} \mathrm{H}_{8} \mathrm{ClO}: 203.0258$; found: 203.0259 . 
(b) Synthesis of Methyl 1-oxo-2,3-dihydro- $1 H$-indene-2-carboxylate (5a) ${ }^{[2]}$
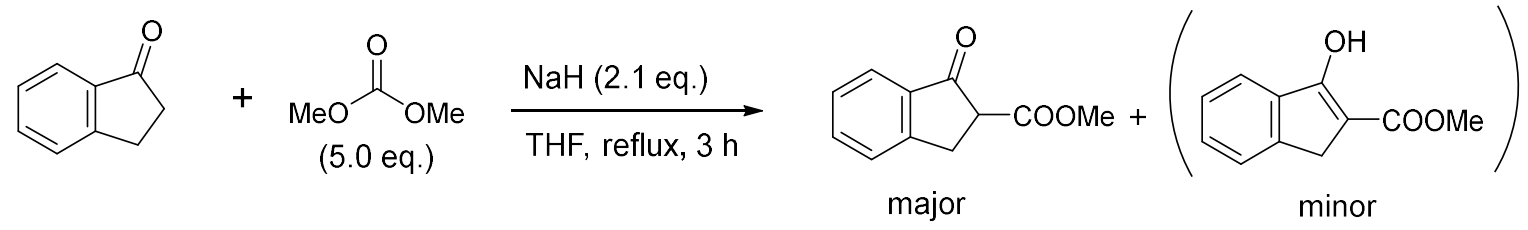

$5 a$

A THF solution $(120 \mathrm{~mL})$ containing 1-indanone $(5.00 \mathrm{~g}, 37.8 \mathrm{mmol})$ and $\mathrm{NaH}(60 \%)$ (3.18 g, $79.4 \mathrm{mmol})$ was added dimethyl carbonate $(16.0 \mathrm{~g}, 172.4 \mathrm{mmol})$ slowly, then heated by oil bath under $\mathrm{N}_{2}$ atmosphere at $65^{\circ} \mathrm{C}$ for $3 \mathrm{~h}$. After cooling the reaction mixture, a $1 \mathrm{~N} \mathrm{HCl}$ solution was added to neutralize the reaction mixture, then $\mathrm{CHCl}_{3}(100 \mathrm{~mL})$ was added and the product was extracted with $\mathrm{CHCl}_{3}(2 \times 50 \mathrm{~mL})$ and the combined organic layers were washed with brine $(100 \mathrm{~mL})$, dried over anhydrous $\mathrm{MgSO}_{4}$, and concentrated under reduced pressure to afford the crude product. Purification by silica gel column chromatography (eluent: Hexane/AcOEt $=20 / 1)$ gave the product $(\mathbf{5 l})$ as a pale yellow oil (6.5 g, 90\% yield).

${ }^{1} \mathrm{H}$ NMR $\left(400 \mathrm{MHz}, \mathrm{CDCl}_{3}\right) \delta 7.79-7.77(\mathrm{~m}, 1 \mathrm{H}), 7.65-7.61(\mathrm{~m}, 1 \mathrm{H}), 7.52-7.50(\mathrm{~m}, 1 \mathrm{H})$, $7.42-7.38(\mathrm{~m}, 1 \mathrm{H}), 3.80(\mathrm{~s}, 3 \mathrm{H}), 3.74(\mathrm{dd}, J=8.3,4.0 \mathrm{~Hz}, 1 \mathrm{H}), 3.60-3.50(\mathrm{~m}, 1 \mathrm{H}), 3.78$ $(\mathrm{dd}, J=17.4,8.3 \mathrm{~Hz}, 1 \mathrm{H}) ;{ }^{13} \mathrm{C}\left\{{ }^{1} \mathrm{H}\right\} \mathrm{NMR}\left(125 \mathrm{MHz}, \mathrm{CDCl}_{3}\right) \delta 199.4,169.5,153.6,135.4$, $135.2,127.8,126.5,124.7,53.1,52.8,30.2$.

HRMS (ESI) $m / z:[\mathrm{M}+\mathrm{H}]^{+}$calcd for $\mathrm{C}_{11} \mathrm{H}_{10} \mathrm{O}_{3}: 191.0703$; found: 191.0699 .

Following this method, ketones $\mathbf{5 b}$ and $\mathbf{5 i}$ were synthesized.

Methyl 5-oxo-6,7,8,9-tetrahydro-5H-benzo[7]annulene-6-carboxylate (5b)

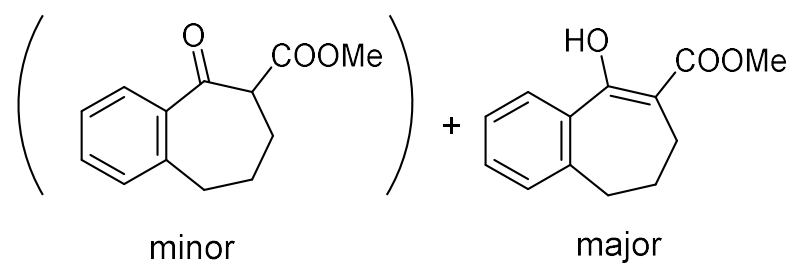

The reaction was carried out on a $31.2 \mathrm{mmol}$ scale. A crude product was purified by silica gel column chromatography (eluent: Hexane/AcOEt $=20 / 1)$. A colorless oil of $\mathbf{5 b}(6.5 \mathrm{~g})$ was obtained in $96 \%$ yield.

${ }^{1} \mathrm{H}$ NMR (400 MHz, $\left.\mathrm{CDCl}_{3}\right) \delta 12.6(\mathrm{~s}, 1 \mathrm{H}), 7.63-7.61(\mathrm{~m}, 1 \mathrm{H}), 7.34-7.29(\mathrm{~m}, 2 \mathrm{H})$, $7.22-7.20(\mathrm{~m}, 1 \mathrm{H}), 3.82(\mathrm{~s}, 3 \mathrm{H}), 2.63(\mathrm{t}, J=6.8 \mathrm{~Hz}, 2 \mathrm{H}), 2.20-2.00(\mathrm{~m}, 4 \mathrm{H}) ;{ }^{13} \mathrm{C}\left\{{ }^{1} \mathrm{H}\right\}$ NMR $\left(125 \mathrm{MHz}, \mathrm{CDCl}_{3}\right) \delta 173.3,170.4,141.0,135.6,130.1,129.1,127.1,126.3,100.1$, 51.6, 33.4, 31.7, 21.7.

HRMS (ESI) $m / z:[\mathrm{M}+\mathrm{H}]^{+}$calcd for $\mathrm{C}_{13} \mathrm{H}_{15} \mathrm{O}_{3}: 219.1016$; found: 219.1020 . 
Methyl 2-oxo-1,2-dihydroacenaphthylene-1-carboxylate (5i)<smiles>CC(=O)C1=C(O)C2=CC=Cc3cccc(c31)C2C(C)=O</smiles>

The reaction was carried out on a $17.8 \mathrm{mmol}$ scale. A crude product was purified by silica gel column chromatography (eluent: Hexane/AcOEt $=10 / 1$ to 5/1). An orange powder of 5i $(3.6 \mathrm{~g})$ was obtained in $89 \%$ yield.

${ }^{1} \mathrm{H}$ NMR $\left(400 \mathrm{MHz}, \mathrm{CDCl}_{3}\right) \delta 7.90(\mathrm{t}, J=8.4 \mathrm{~Hz}, 1 \mathrm{H}), 7.77-7.75(\mathrm{~m}, 2 \mathrm{H}), 7.69-7.58(\mathrm{~m}$, $3 \mathrm{H}), 4.00(\mathrm{~s}, 3 \mathrm{H}) ;{ }^{13} \mathrm{C}\left\{{ }^{1} \mathrm{H}\right\}$ NMR $\left(125 \mathrm{MHz}, \mathrm{CDCl}_{3}\right) \delta 168.1,142.7,133.6,132.0,130.8$, $128.5,128.3,127.3,125.2,124.8,122.7,121.8,102.7,52.9$.

HRMS (ESI) $m / z:[\mathrm{M}+\mathrm{H}]^{+}$calcd for $\mathrm{C}_{14} \mathrm{H}_{11} \mathrm{O}_{3}: 227.0703$; found: 227.0706 .

(c) Synthesis of $N$-(tert-Butyl)-1-oxo-1,2,3,4-tetrahydronaphthalene-2-carboxamide $(5 d)^{[3]}$<smiles>O=C1CCCc2ccccc21</smiles><smiles>[CH2+]C(C)(C)N=C=O</smiles>

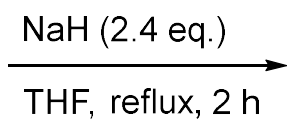<smiles>CCCCCCCCC(=O)C1CCc2ccccc2C1=O</smiles>

$5 d$

A THF solution $(340 \mathrm{~mL})$ containing $\alpha$-tetralone $(10.00 \mathrm{~g}, 68.4 \mathrm{mmol})$ and $\mathrm{NaH}(60 \%)$ $(6.57 \mathrm{~g}, 164.2 \mathrm{mmol})$ under $\mathrm{N}_{2}$ atmosphere was stirred $15 \mathrm{~min}$ at $30{ }^{\circ} \mathrm{C}$, then tert-butyl isocyanate $(8.14 \mathrm{~g}, 82.1 \mathrm{mmol})$ was added to the mixture slowly. The reaction mixture was heated by oil bath at $65{ }^{\circ} \mathrm{C}$ for $2 \mathrm{~h}$. After cooling the reaction mixture, a $1 \mathrm{~N} \mathrm{HCl}$ solution was added to neutralize the reaction mixture, then AcOEt $(200 \mathrm{~mL})$ and $\mathrm{H}_{2} \mathrm{O}(100 \mathrm{~mL})$ were added and the product was extracted with AcOEt $(2 \times 100 \mathrm{~mL})$ and the combined organic layers were washed with brine $(100 \mathrm{~mL})$, dried over anhydrous $\mathrm{MgSO}_{4}$, and concentrated under reduced pressure to afford the crude product. The crude product was dissolved in $\mathrm{CHCl}_{3}(70 \mathrm{~mL})$ then heptane $(300 \mathrm{~mL})$ was added slowly at room temperature. The slurry was cooled to $0{ }^{\circ} \mathrm{C}$ and stirred for $1 \mathrm{~h}$, the insoluble components were separated and the resulting cake was washed by a heptane $/ \mathrm{CHCl}_{3}(10 / 1)$ solution $(50 \mathrm{~mL})$. The obtained solid was dried under reduced pressure to give the product $(\mathbf{5 d})$ as a white powder (12.5 g, 74\% yield).

${ }^{1} \mathrm{H}$ NMR $\left(400 \mathrm{MHz}, \mathrm{CDCl}_{3}\right) \delta 8.01(\mathrm{dd}, J=8.0,1.2 \mathrm{~Hz}, 1 \mathrm{H}), 7.51-7.47(\mathrm{~m}, 1 \mathrm{H})$, 7.33-7.23 (m, 2H), $6.90(\mathrm{br}, 1 \mathrm{H}), 3.29$ (t, $J=6.8 \mathrm{~Hz}, 1 \mathrm{H}), 3.13-3.10(\mathrm{~m}, 1 \mathrm{H}), 2.93-2.87$ (m, 1H), 2.45-2.41 (m, 2H), $1.36(\mathrm{~s}, 9 \mathrm{H}) ;{ }^{13} \mathrm{C}\left\{{ }^{1} \mathrm{H}\right\} \mathrm{NMR}\left(125 \mathrm{MHz}, \mathrm{CDCl}_{3}\right) \delta 197.1,166.4$, $144.9,134.1,131.9,128.8,127.7,126.7,53.2,51.3,28.7,27.8,25.2$. 
HRMS (ESI) $m / z:[\mathrm{M}+\mathrm{H}]^{+}$calcd for $\mathrm{C}_{15} \mathrm{H}_{20} \mathrm{NO}_{2}$ : 246.1489; found: 246.1489.

Following this method, ketones $\mathbf{5 c}, \mathbf{5 e}$, and $\mathbf{5 h}$ were synthesized.

\section{$N$-(tert-Butyl)-1-oxo-2,3-dihydro-1H-indene-2-carboxamide (5c)}<smiles>CCCCCC(=O)C1Cc2ccccc2C1=O</smiles>

The reaction was carried out on a $75.7 \mathrm{mmol}$ scale. A white powder of 5c $(6.5 \mathrm{~g})$ was obtained in $75 \%$ yield.

${ }^{1} \mathrm{H}$ NMR $\left(400 \mathrm{MHz}, \mathrm{CDCl}_{3}\right) \delta 7.74(\mathrm{~d}, J=7.6 \mathrm{~Hz}, 1 \mathrm{H}), 7.65-7.61(\mathrm{~m}, 1 \mathrm{H}), 7.53-7.51(\mathrm{~m}$, $1 \mathrm{H}), 7.40-7.36(\mathrm{~m}, 1 \mathrm{H}), 7.02$ (br, $1 \mathrm{H}), 3.77$ (dd, $J=17.8,3.8 \mathrm{~Hz}, 1 \mathrm{H}), 3.50(\mathrm{dd}, J=8.2$, $3.8 \mathrm{~Hz}, 1 \mathrm{H}), 3.30(\mathrm{dd}, J=17.8,8.2 \mathrm{~Hz}, 1 \mathrm{H}), 1.39(\mathrm{~s}, 9 \mathrm{H}) ;{ }^{13} \mathrm{C}\left\{{ }^{1} \mathrm{H}\right\} \mathrm{NMR}(125 \mathrm{MHz}$, $\left.\mathrm{CDCl}_{3}\right) \delta 203.8,165.2,154.4,135.6,135.4,127.5,126.7,124.3,53.5,51.4,28.7,28.5$. HRMS (APCI) $m / z$ : [M+H] $]^{+}$calcd for $\mathrm{C}_{14} \mathrm{H}_{18} \mathrm{NO}_{2}: 232.1332$; found: 232.1309 .

\section{$\mathrm{N}$-(tert-butyl)-5-oxo-6,7,8,9-tetrahydro-5H-benzo[7]annulene-6-carboxamide (5e)}<smiles>CCCCCCCCC(=O)C1CCCc2ccccc2C1=O</smiles>

The reaction was carried out on a $62.4 \mathrm{mmol}$ scale. A pale orange powder of $5 \mathbf{e}(1.9 \mathrm{~g})$ was obtained in $12 \%$ yield.

${ }^{1} \mathrm{H}$ NMR $\left(400 \mathrm{MHz}, \mathrm{CDCl}_{3}\right) \delta 7.63(\mathrm{dd}, J=7.6,1.2 \mathrm{~Hz}, 1 \mathrm{H}), 7.45-7.41(\mathrm{~m}, 1 \mathrm{H})$, $7.32-7.28(\mathrm{~m}, 1 \mathrm{H}), 7.21(\mathrm{~d}, J=7.6 \mathrm{~Hz}, 1 \mathrm{H}), 7.09(\mathrm{br}, 1 \mathrm{H}), 3.55(\mathrm{dd}, J=9.6,4.8 \mathrm{~Hz}, 1 \mathrm{H})$, 2.92-2.89 (m, 2H), 2.20-1.90 (m, 4H), $1.38(\mathrm{~s}, 9 \mathrm{H}) ;{ }^{13} \mathrm{C}\left\{{ }^{1} \mathrm{H}\right\} \mathrm{NMR}\left(125 \mathrm{MHz}, \mathrm{CDCl}_{3}\right) \delta$ 206.1, 167.4, 141.4, 139.0, 132.3, 129.7, 128.4, 126.6, 56.2, 51.2, 32.5, 28.7, 26.8, 24.3. HRMS (ESI) $m / z$ : [M+H] $]^{+}$calcd for $\mathrm{C}_{16} \mathrm{H}_{22} \mathrm{NO}_{2}$ : 260.1645; found: 260.1647 .

$N$-(tert-Butyl)-2-oxo-1,2-dihydroacenaphthylene-1-carboxamide (5h)

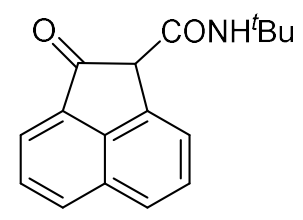

The reaction was carried out on a $17.8 \mathrm{mmol}$ scale. An off-white powder of $\mathbf{5 h}(3.1 \mathrm{~g})$ was obtained in $65 \%$ yield. 
${ }^{1} \mathrm{H}$ NMR $\left(400 \mathrm{MHz}, \mathrm{CDCl}_{3}\right) \delta 8.15-8.13(\mathrm{~m}, 1 \mathrm{H}), 7.99(\mathrm{~d}, J=6.8 \mathrm{~Hz}, 1 \mathrm{H}), 7.93(\mathrm{~d}, J=6.8$ $\mathrm{Hz}, 1 \mathrm{H}), 7.88$ (d, $J=8.4 \mathrm{~Hz}, 1 \mathrm{H}), 7.74-7.68(\mathrm{~m}, 2 \mathrm{H}), 7.10$ (br, 1H), 4.52 (s, 1H), 1.41 (s, $9 \mathrm{H}) ;{ }^{13} \mathrm{C}\left\{{ }^{1} \mathrm{H}\right\}$ NMR $\left(125 \mathrm{MHz}, \mathrm{CDCl}_{3}\right) \delta 201.3,163.7,142.3,133.5,132.6,132.6,130.7$, $128.9,127.8,124.5,124.4,122.3,57.4,51.6,28.8$.

HRMS (ESI) $m / z:[\mathrm{M}+\mathrm{H}]^{+}$calcd for $\mathrm{C}_{17} \mathrm{H}_{18} \mathrm{NO}_{2}$ : 268.1332; found: 268.1334 .

(d) Synthesis of 2-(Phenylsulfonyl)-3,4-dihydronaphthalen-1(2H)-one (5g) ${ }^{[4]}$

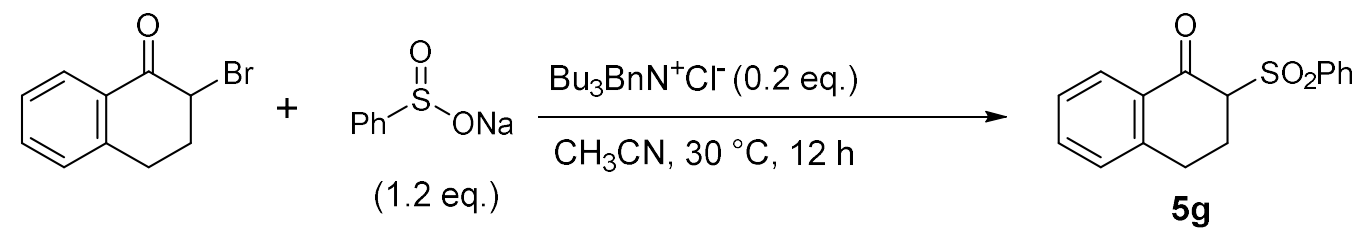

An $\mathrm{CH}_{3} \mathrm{CN}$ solution (50 mL) containing 2-bromo-1-tetralone (5.00 g, $22.2 \mathrm{mmol}$ ), sodium benzenesulfinate $(4.38 \mathrm{~g}, 26.65 \mathrm{mmol})$ and tri- $n$-butylbenzylammonium chloride $(1.01 \mathrm{~g}$, $4.44 \mathrm{mmol}$ ) was stirred under $\mathrm{N}_{2}$ atmosphere at $30^{\circ} \mathrm{C}$ for $12 \mathrm{~h}$. After cooling the reaction mixture, water $(25 \mathrm{~mL})$ and $\mathrm{CHCl}_{3}(50 \mathrm{~mL})$ were added and the product was extracted with $\mathrm{CHCl}_{3}(2 \times 30 \mathrm{~mL})$. The combined organic layers were washed with brine $(50 \mathrm{~mL})$, dried over anhydrous $\mathrm{MgSO}_{4}$, and concentrated under reduced pressure to afford the crude product. Purification by silica gel column chromatography (eluent: Hexane/AcOEt $=3 / 1$ to 1/1) gave the product $(\mathbf{5 g})$ as a pale brown powder (3.7 $\mathrm{g}, 59 \%$ yield).

${ }^{1} \mathrm{H}$ NMR $\left(400 \mathrm{MHz}, \mathrm{CDCl}_{3}\right) \delta$ 7.99-7.91 (m, 3H), 7.69-7.65 (m, 1H), 7.59-7.50 (m, 3H), $7.33-7.26(\mathrm{~m}, 2 \mathrm{H}), 4.12(\mathrm{t}, J=6.0 \mathrm{~Hz}, 1 \mathrm{H}), 3.50(\mathrm{ddd}, J=15.7,11.1,4.8 \mathrm{~Hz}, 1 \mathrm{H}), 3.00$ (ddd, $J=11.4,11.4,5.6 \mathrm{~Hz}, 1 \mathrm{H}), 2.90-2.82(\mathrm{~m}, 1 \mathrm{H}), 2.70-2.62(\mathrm{~m}, 1 \mathrm{H}) ;{ }^{13} \mathrm{C}\left\{{ }^{1} \mathrm{H}\right\} \mathrm{NMR}$ $\left(125 \mathrm{MHz}, \mathrm{CDCl}_{3}\right) \delta 188.6,143.5,138.9,134.5,134.0,131.7,129.1,129.0,128.9,127.9$, 127.0, 69.6, 26.5, 23.6 .

HRMS (ESI) $m / z:[\mathrm{M}+\mathrm{H}]^{+}$calcd for $\mathrm{C}_{16} \mathrm{H}_{15} \mathrm{O}_{3} \mathrm{~S}: 287.0736$; found: 287.0740 .

Following this method, ketone $\mathbf{5 f}$ was synthesized.

2-(Phenylsulfonyl)-2,3-dihydro-1 $H$-inden-1-one (5f)<smiles>O=C1c2ccccc2CC1S(=O)(=O)c1ccccc1</smiles>

The reaction was carried out on a $23.7 \mathrm{mmol}$ scale. A crude product was purified by silica gel column chromatography (eluent: Hexane/AcOEt $=3 / 1$ to $1 / 1$ ). A pale orange powder of $\mathbf{5 f}(3.3 \mathrm{~g})$ was obtained in $52 \%$ yield.

${ }^{1} \mathrm{H}$ NMR (400 MHz, $\left.\mathrm{CDCl}_{3}\right) \delta$ 7.94-7.92 (m, 2H), 7.72-7.50 (m, 6H), 7.40-7.36 (m, 1H), $4.29(\mathrm{dd}, J=8.6,3.4 \mathrm{~Hz}, 1 \mathrm{H}), 3.82$ (dd, $J=18.3,3.4 \mathrm{~Hz}, 1 \mathrm{H}), 3.54$ (dd, $J=18.3,8.6 \mathrm{~Hz}$, $1 \mathrm{H}) ;{ }^{13} \mathrm{C}\left\{{ }^{1} \mathrm{H}\right\} \mathrm{NMR}\left(125 \mathrm{MHz}, \mathrm{CDCl}_{3}\right) \delta 194.4,151.8,137.5,135.9,135.7,134.2,129.2$, 
129.0, 128.2, 126.4, 124.8, 68.6, 28.1.

HRMS (ESI) $m / z$ : $[\mathrm{M}+\mathrm{H}]^{+}$calcd for $\mathrm{C}_{15} \mathrm{H}_{13} \mathrm{O}_{3} \mathrm{~S}: 273.0580$; found: 273.0580 .

(e) Synthesis of 6-Chloro-3-methylcyclohex-2-en-1-one (7a) ${ }^{[5]}$

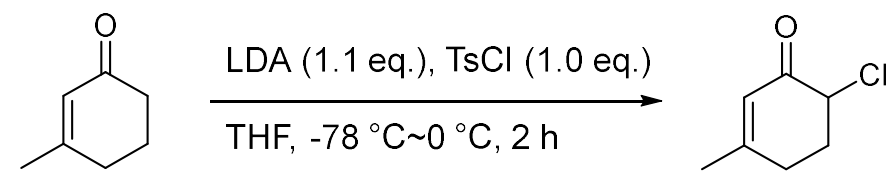

$7 a$

A THF solution $(150 \mathrm{~mL})$ containing $N, N$-diisopropylamine $(4.79 \mathrm{~g}, 55.0 \mathrm{mmol})$ was cooled to $-78{ }^{\circ} \mathrm{C}$ under $\mathrm{N}_{2}$ atmosphere, then $n$-BuLi $(1.59 \mathrm{M})(34.6 \mathrm{~mL}, 55.0 \mathrm{mmol})$ was added slowly and stirred for $1 \mathrm{~h}$. To the resulting solution, 3-methylcyclohex-2-en-1-one $(5.50 \mathrm{~g}, 50.0 \mathrm{mmol})$ was added and stirred for $1 \mathrm{~h}$ at $0{ }^{\circ} \mathrm{C}$. Then $p$-toluenesulfonyl chloride $(9.53 \mathrm{~g}, 50.0 \mathrm{mmol})$ in THF $(100 \mathrm{~mL})$ were added to the reaction mixture and stirred at $0{ }^{\circ} \mathrm{C}$. After stirring for $5 \mathrm{~h}$, water $(150 \mathrm{~mL})$ was added and the product was extracted with $\mathrm{CHCl}_{3}(3 \times 10 \mathrm{~mL})$. The combined organic layers were washed with brine $(100 \mathrm{~mL})$, dried over anhydrous $\mathrm{MgSO}_{4}$, and concentrated under reduced pressure to afford the crude product. Purification by silica gel column chromatography (eluent: Hexane/AcOEt $=10 / 1$ to $5 / 1)$ gave the product (7a) as a colorless oil ( $5.8 \mathrm{~g}, 80 \%$ yield).

${ }^{1} \mathrm{H}$ NMR $\left(500 \mathrm{MHz}, \mathrm{CDCl}_{3}\right) \delta 5.93(\mathrm{~d}, J=1.0 \mathrm{~Hz}, 1 \mathrm{H}), 4.37-4.33(\mathrm{~m}, 1 \mathrm{H}), 2.63-2.57(\mathrm{~m}$, $1 \mathrm{H}), 2.45-2.27(\mathrm{~m}, 3 \mathrm{H}), 2.00(\mathrm{~s}, 3 \mathrm{H}) ;{ }^{13} \mathrm{C}\left\{{ }^{1} \mathrm{H}\right\} \mathrm{NMR}\left(125 \mathrm{MHz}, \mathrm{CDCl}_{3}\right) \delta 191.5,163.0$, $124.3,58.1,31.7,28.5,24.3$.

HRMS (APCI) $m / z:[\mathrm{M}+\mathrm{H}]^{+}$calcd for $\mathrm{C}_{7} \mathrm{H}_{9} \mathrm{ClO}: 145.0415$; found: 145.0413 .

(f) Synthesis of Ethyl 4-methyl-2-oxocyclohex-3-ene-1-carboxylate (7b) ${ }^{[6]}$

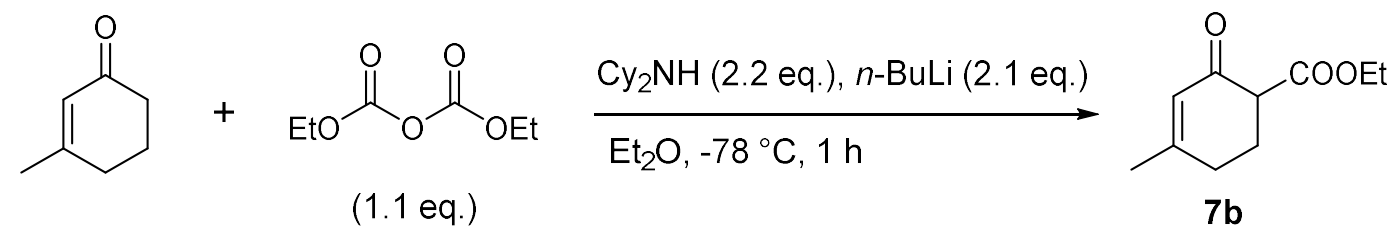

An $\mathrm{Et}_{2} \mathrm{O}$ solution $(150 \mathrm{~mL})$ containing $N, N$-dicyclohexylamine $(19.95 \mathrm{~g}, 110.0 \mathrm{mmol})$ was cooled to $-78{ }^{\circ} \mathrm{C}$ under $\mathrm{N}_{2}$ atmosphere, then $n$-BuLi $(1.59 \mathrm{M})(66.0 \mathrm{~mL}, 105.0 \mathrm{mmol})$ was added slowly and stirred for $1 \mathrm{~h}$. To the resulting solution, 3-methylcyclohex-2-en-1-one $(5.50 \mathrm{~g}, 50.0 \mathrm{mmol})$ in $\mathrm{Et}_{2} \mathrm{O}(100 \mathrm{~mL})$ was added and stirred for $1 \mathrm{~h}$ at $-78{ }^{\circ} \mathrm{C}$. Then diethyl dicarbonate $(8.92 \mathrm{~g}, 55 \mathrm{mmol})$ in $\mathrm{Et}_{2} \mathrm{O}(20 \mathrm{~mL})$ was added to reaction mixture and stirred at $-78^{\circ} \mathrm{C}$. After stirring for $1 \mathrm{~h}$, an aqueous solution of citric acid (a mixture of citric acid $10 \mathrm{~g}$ and water $90 \mathrm{~mL})$ was added and the product was extracted with tert-BuOMe $(3 \times 100$ $\mathrm{mL})$. The combined organic layers were washed with brine $(100 \mathrm{~mL})$, dried over anhydrous $\mathrm{MgSO}_{4}$, and concentrated under reduced pressure to afford the crude product. Purification by silica gel column chromatography (eluent: hexane/AcOEt $=7 / 1$ to $3 / 1$ ) gave the product $(7 \mathbf{b})$ as a pale yellow oil $(7.7 \mathrm{~g}, 85 \%$ yield $)$. 
${ }^{1} \mathrm{H}$ NMR $\left(500 \mathrm{MHz}, \mathrm{CDCl}_{3}\right) \delta 5.93(\mathrm{q}, J=1.1 \mathrm{~Hz}, 1 \mathrm{H}), 4.25-4.17(\mathrm{~m}, 2 \mathrm{H}), 3.33-3.30(\mathrm{~m}$, $1 \mathrm{H}), 2.45-2.27(\mathrm{~m}, 3 \mathrm{H}), 2.21-2.17(\mathrm{~m}, 1 \mathrm{H}), 1.98(\mathrm{~s}, 3 \mathrm{H}), 1.28(\mathrm{t}, J=7.1 \mathrm{~Hz}, 3 \mathrm{H}) ;{ }^{13} \mathrm{C}\left\{{ }^{1} \mathrm{H}\right\}$ NMR $\left(125 \mathrm{MHz}, \mathrm{CDCl}_{3}\right) \delta 193.8,170.3,162.8,125.8,61.1,52.4,19.4,25.4,24.3,14.1$. HRMS (FI) $m / z:[\mathrm{M}]^{+}$calcd for $\mathrm{C}_{10} \mathrm{H}_{14} \mathrm{O}_{3}: 182.0943$; found: 182.0952 .

(g) Synthesis of $N$-(tert-Butyl)-4-methyl-2-oxocyclohex-3-ene-1-carboxamide (7c)

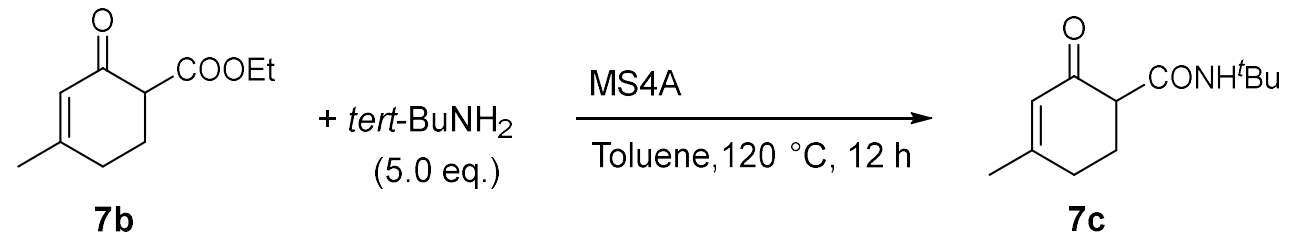

A toluene solution $(30 \mathrm{~mL})$ containing ethyl 4-methyl-2-oxocyclohex-3-ene-1-carboxylate (7b) $(2.50 \mathrm{~g}, 13.72 \mathrm{mmol})$, tert-butylamine $(5.01 \mathrm{~g}, 68.6 \mathrm{mmol})$ and molecular sieves $4 \AA$ $(2.0 \mathrm{~g})$ in a $50 \mathrm{~mL}$ stainless steel autoclave was stirred under $\mathrm{N}_{2}$ atmosphere at $120{ }^{\circ} \mathrm{C}$ for $12 \mathrm{~h}$. After cooling the reaction mixture, molecular sieves were removed by filtration. Then the filtrate was concentrated under reduced pressure to afford the crude product. Purification by silica gel column chromatography (eluent: hexane/AcOEt $=5 / 1$ to $2 / 1$ ) gave the product (7c) as a light brown solid (2.2 g, $75 \%$ yield).

${ }^{1} \mathrm{H}$ NMR $\left(400 \mathrm{MHz}, \mathrm{CDCl}_{3}\right) \delta 6.80(\mathrm{~s}, 1 \mathrm{H}), 5.89(\mathrm{~d}, J=0.5 \mathrm{~Hz}, 1 \mathrm{H}), 3.03-2.98(\mathrm{~m}, 1 \mathrm{H})$, 2.60-2.50 (m, 1H), 2.34-2.22 (m, 3H), $1.97(\mathrm{~s}, 3 \mathrm{H}), 1.34(\mathrm{~s}, 9 \mathrm{H}) ;{ }^{13} \mathrm{C}\left\{{ }^{1} \mathrm{H}\right\}$ NMR $(125$ $\mathrm{MHz}$

$\left.\mathrm{CDCl}_{3}\right) \delta 197.7,166.6,165.5,125.9,51.2,50.9,29.7,28.7,24.5,24.3$.

HRMS (FI) $m / z$ : [M] $]^{+}$calcd for $\mathrm{C}_{12} \mathrm{H}_{19} \mathrm{NO}_{2}$ : 209.1416; found: 209.1426 . 


\section{Asymmetric Transfer Hydrogenation of $\alpha$-Substituted Ketones Using $(R, R)-2 a, 2 b$, and $2 \mathrm{c}$.}

General procedures under the conditions of $\mathrm{S} / \mathrm{C}=500,60^{\circ} \mathrm{C}$, and $8 \mathrm{~h}$.

Under $\mathrm{N}_{2}$ atmosphere, a mixture of ketone $(1.0 \mathrm{mmol})$ and the $\mathrm{Ru}$ catalyst $(0.02 \mathrm{mmol})$ in an azeotrope of formic acid and triethylamine (a 5:2 molar ratio, $0.5 \mathrm{~mL}$ ) and solvent (1 $\mathrm{mL}$, if nessesary) was heated by aluminum block heater at $60{ }^{\circ} \mathrm{C}$ for $8 \mathrm{~h}$. After the reaction completion, water $(3 \mathrm{~mL})$ and AcOEt $(5 \mathrm{~mL})$ was added. The biphasic layers were separated, the aqueous layer was extracted with AcOEt $(3 \times 5 \mathrm{~mL})$, and the combined organic portions were washed with brine $(3 \mathrm{~mL})$. After drying over $\mathrm{MgSO}_{4}$, filtration, and solvent removal under reduced pressure, the crude residue was purified by silica gel column chromatography to afford the desired product. The optical purity of product was determined by chiral HPLC analysis using a Daicel Chiralcel OD-H, OJ-H, OD-3, OJ-3, OZ-3 or Chiralpak AD-H, AS-H, AD-3. AS-3, IA, IB, QN-AX column $(4.6 \mathrm{~mm} \times 25 \mathrm{~cm}$ or $4.6 \mathrm{~mm} \times 15 \mathrm{~cm}$ ) column with hexane/2-propanol as eluent where a clear base-line separation was obtained.

Specific Example: Asymmetric Transfer Hydrogenation of 2-chloro-3,4-dihydronaphthalen-1(2H)-one (3a) under the conditions of $\mathrm{S} / \mathrm{C}=1,000,60{ }^{\circ} \mathrm{C}$, and $8 \mathrm{~h}$.

Under $\mathrm{N}_{2}$ atmosphere, 2-chloro-3,4-dihydronaphthalen-1(2H)-one $(0.18 \mathrm{~g}, 1.0 \mathrm{mmol})$ and $(\boldsymbol{R}, \boldsymbol{R})-\mathbf{2 a}(0.65 \mathrm{mg}, 0.001 \mathrm{mmol})$ in an azeotrope of formic acid and triethylamine (a 5:2 molar ratio, $0.5 \mathrm{~mL})$ and AcOEt $(1.0 \mathrm{~mL})$ heated by aluminum block heater at $60{ }^{\circ} \mathrm{C}$ for $8 \mathrm{~h}$. After the reaction completion, water $(3 \mathrm{~mL})$ and AcOEt $(5 \mathrm{~mL})$ was added. The biphasic layers were separated, the aqueous layer was extracted with AcOEt $(3 \times 5 \mathrm{~mL})$, and the combined organic portions were washed with brine $(3 \mathrm{~mL})$. After drying over $\mathrm{MgSO}_{4}$, filtration, and solvent removal under reduced pressure, the crude residue was purified by silica gel column chromatography (eluent: Hexane/AcOEt $=5 / 1$ ) to afford the desired product $(0.175 \mathrm{~g}, 96 \%$ yield). The optical purity of product was determined by chiral HPLC analysis using a Daicel Chiralcel OJ-H $(4.6 \mathrm{~mm} \times 25 \mathrm{~cm})$ column with hexane/2-propanol (97/3) as eluent where a clear base-line separation was obtained. 


\section{Characterization Data for Reduction Products.}

\section{(1S,2R)-2-Chloro-1,2,3,4-tetrahydronaphthalen-1-ol (cis-4a)}<smiles>OC1c2ccccc2CC[C@@H]1Cl</smiles>

According to the general procedure (ketone: $0.360 \mathrm{~g}(2.0 \mathrm{mmol})$, cat. $(\boldsymbol{R}, \boldsymbol{R})-\mathbf{3}$, eluent of silica gel column chromatography: Hexane/AcOEt $=5 / 1), 0.351 \mathrm{~g}$ of cis-4a was obtained as a white solid ( $96 \%$ yield).

${ }^{1} \mathrm{H}$ NMR $\left(400 \mathrm{MHz}, \mathrm{CDCl}_{3}\right) \delta 7.50-7.48(\mathrm{~m}, 1 \mathrm{H}), 7.27-7.22(\mathrm{~m}, 2 \mathrm{H}), 7.23-7.11(\mathrm{~m}, 1 \mathrm{H})$, 4.87-4.84 (m, 1H), 4.57-4.54 (m, 1H), $3.09(\mathrm{dt}, J=17.0,7.0 \mathrm{~Hz}, 1 \mathrm{H}), 2.82(\mathrm{dt}, J=17.5$, $7.0 \mathrm{~Hz}, 1 \mathrm{H}), 2.45-2.40(\mathrm{~m}, 2 \mathrm{H}), 2.24-2.17(\mathrm{~m}, 1 \mathrm{H}) ;{ }^{13} \mathrm{C}\left\{{ }^{1} \mathrm{H}\right\} \mathrm{NMR}\left(125 \mathrm{MHz}, \mathrm{CDCl}_{3}\right) \delta$ $135.8,134.9,128.8,128.5,128.2,126.5,70.1,63.2,27.6,26.6$. All characterization data are in agreement with those reported. ${ }^{[7]}$

HRMS (FI) $m / z$ : [M] $]^{+}$calcd for $\mathrm{C}_{10} \mathrm{H}_{11} \mathrm{ClO}$ : 182.0498; found: 182.0506 .

The enantiomeric excess was determined by HPLC analysis (Chiralcel OJ-H, $250 \times 4.6$ $\mathrm{mm}$ column, hexane/2-propanol 97:3, $1.0 \mathrm{~mL} / \mathrm{min}, 220 \mathrm{~nm}, 35^{\circ} \mathrm{C},(1 R, 2 S)$ isomer 14.9 min (minor), $(1 S, 2 R)$ isomer 21.8 min (major)); $[\alpha]_{\mathrm{D}}^{20}-9.1$ (c 2.28 in $\left.\mathrm{CHCl}_{3}\right)$ cis/trans $=$ $>99 / 1,>99.9 \%$ ee $(1 S, 2 R)\left(\right.$ lit. $^{[7]}[\alpha]_{\mathrm{D}}{ }^{20}-8.2\left(\mathrm{c} 0.8\right.$ in $\left.\mathrm{CHCl}_{3}\right) 99 \%$ ee $(1 S, 2 R),>98 \%$ de).

\section{(1S,2R)-2-Chloro-7-methoxy-1,2,3,4-tetrahydronaphthalen-1-ol (cis-4b)}<smiles>COc1ccc2c(c1)[C@H](O)[C@H](Cl)CC2</smiles>

According to the general procedure (ketone: $0.211 \mathrm{~g}(1.0 \mathrm{mmol})$, cat. $(\boldsymbol{R}, \boldsymbol{R})-\mathbf{3}$, eluent of silica gel column chromatography: Hexane/AcOEt $=5 / 1), 0.212 \mathrm{~g}$ of $\boldsymbol{c i s}-\mathbf{4 b}$ was obtained as a white solid ( $>99 \%$ yield).

${ }^{1} \mathrm{H}$ NMR $\left(500 \mathrm{MHz}, \mathrm{CDCl}_{3}\right) \delta 7.05-7.02(\mathrm{~m}, 1 \mathrm{H}), 6.82(\mathrm{dd}, J=8.2,2.7 \mathrm{~Hz}, 1 \mathrm{H}), 4.82(\mathrm{dd}$, $J=8.2,3.2 \mathrm{~Hz}, 1 \mathrm{H}), 4.70(\mathrm{dt}, J=5.6,3.2 \mathrm{~Hz}, 1 \mathrm{H}), 3.80(\mathrm{~s}, 3 \mathrm{H}), 3.04(\mathrm{dt}, J=12.0,6.6 \mathrm{~Hz}$, $1 \mathrm{H}), 2.76(\mathrm{dt}, J=12.0,6.6, \mathrm{~Hz}, 1 \mathrm{H}), 2.45(\mathrm{~d}, J=8.0 \mathrm{~Hz}, 1 \mathrm{H}), 2.44-2.36(\mathrm{~m}, 1 \mathrm{H})$, 2.22-2.15 (m, $1 \mathrm{H}) ;{ }^{13} \mathrm{C}\left\{{ }^{1} \mathrm{H}\right\}$ NMR $\left(125 \mathrm{MHz}, \mathrm{CDCl}_{3}\right) \delta 158.3,136.9,129.6,126.8,115.0$, 112.6, 70.3, 63.3, 55.3, 27.9, 25.5.

HRMS (APCI) $m / z$ : [M-Cl] $]^{+}$calcd for $\mathrm{C}_{11} \mathrm{H}_{13} \mathrm{O}_{2}: 177.0910$; found: 177.0908 .

The enantiomeric excess was determined by HPLC analysis (Chiralpak AS-3, $250 \times 4.6$ $\mathrm{mm}$ column, hexane/2-propanol 95:5, $1.0 \mathrm{~mL} / \mathrm{min}, 220 \mathrm{~nm}, 35^{\circ} \mathrm{C},(1 R, 2 S)$ isomer 17.2 $\min$ (minor), $(1 S, 2 R)$ isomer 23.7 min (major)); $[\alpha]_{\mathrm{D}^{20}}-38.7$ (c 1.90 in $\left.\mathrm{CHCl}_{3}\right)$ cis/trans $=$ $>99 / 1,>99.9 \%$ ee $(1 S, 2 R)$. 


\section{(1S,2R)-2-Chloro-7-bromo-1,2,3,4-tetrahydronaphthalen-1-ol (cis-4c)}<smiles>OC1c2cc(Br)ccc2CC[C@@H]1Cl</smiles>

According to the general procedure (ketone: $0.170 \mathrm{~g}(0.66 \mathrm{mmol})$, cat. $(\boldsymbol{R}, \boldsymbol{R})-3$, eluent of silica gel column chromatography: Hexane/AcOEt $=5 / 1), 0.171 \mathrm{~g}$ of cis-4c was obtained as a white solid ( $>99 \%$ yield).

${ }^{1} \mathrm{H}$ NMR $\left(500 \mathrm{MHz}, \mathrm{CDCl}_{3}\right) \delta 7.68(\mathrm{~d}, J=1.9 \mathrm{~Hz}, 1 \mathrm{H}), 7.35(\mathrm{dd}, J=8.2,1.9, \mathrm{~Hz}, 1 \mathrm{H})$, $7.00(\mathrm{~d}, J=8.2 \mathrm{~Hz}, 1 \mathrm{H}), 4.81(\mathrm{dd}, J=8.4,3.2, \mathrm{~Hz}, 1 \mathrm{H}), 4.56(\mathrm{dt}, J=5.3,3.2, \mathrm{~Hz}, 1 \mathrm{H})$, $3.05(\mathrm{dt}, J=12.0,6.5, \mathrm{~Hz}, 1 \mathrm{H}), 2.76(\mathrm{dt}, J=12.0,6.5, \mathrm{~Hz}, 1 \mathrm{H}), 2.48(\mathrm{~d}, J=8.4 \mathrm{~Hz}, 1 \mathrm{H})$, 2.43-2.37 (m, 1H), 2.24-2.17 (m, 1H); ${ }^{13} \mathrm{C}\left\{{ }^{1} \mathrm{H}\right\}$ NMR (125 MHz, $\left.\mathrm{CDCl}_{3}\right) \delta 138.1,133.8$, $131.3,131.1,130.2,120.1,69.7,62.7,27.6,25.6$.

HRMS (CI) $m / z$ : [M] $]^{+}$calcd for $\mathrm{C}_{10} \mathrm{H}_{10} \mathrm{BrClO}$ : 259.9598; found: 259.9599 .

The enantiomeric excess was determined by HPLC analysis (Chiralcel OD-3, $150 \times 4.6$ $\mathrm{mm}$ column, hexane/2-propanol 98:2, $0.8 \mathrm{~mL} / \mathrm{min}, 220 \mathrm{~nm}, 35^{\circ} \mathrm{C},(1 R, 2 S)$ isomer $9.0 \mathrm{~min}$ (minor), $(1 S, 2 R)$ isomer 9.9 min (major)); $[\alpha]_{\mathrm{D}}^{20}-72.5$ (c 1.42 in $\left.\mathrm{CHCl}_{3}\right)$ cis/trans $=>99 / 1$, $>99.9 \%$ ee $(1 S, 2 R)$.

\section{(1S,2R)-2-Chloro-2,3-dihydro-1H-inden-1-ol (cis-4d)}<smiles>O[C@H]1c2ccccc2CC1Cl</smiles>

According to the general procedure (ketone: $0.166 \mathrm{~g}(1.0 \mathrm{mmol})$, cat. $(\boldsymbol{R}, \boldsymbol{R})-\mathbf{3}$, eluent of silica gel column chromatography: Hexane/AcOEt $=4 / 1), 0.168 \mathrm{~g}$ of $\boldsymbol{c i s}-\mathbf{4 d}$ was obtained as a white solid ( $>99 \%$ yield).

${ }^{1} \mathrm{H}$ NMR (400 MHz, $\left.\mathrm{CDCl}_{3}\right) \delta$ 7.47-7.45 (m,1H), 7.33-7.25 (m, 3H), 5.18-5.15 (m, 1H), $4.79(\mathrm{dt}, J=5.5,3.5 \mathrm{~Hz}, 1 \mathrm{H}), 3.34(\mathrm{dd}, J=17.0,5.5 \mathrm{~Hz}, 1 \mathrm{H}), 3.26(\mathrm{dd}, J=17.0,3.5 \mathrm{~Hz}$, $1 \mathrm{H}), 2.51(\mathrm{~d}, J=9.5 \mathrm{~Hz}, 1 \mathrm{H}) ;{ }^{13} \mathrm{C}\left\{{ }^{1} \mathrm{H}\right\} \mathrm{NMR}\left(125 \mathrm{MHz}, \mathrm{CDCl}_{3}\right) \delta 141.4,138.9,128.8$, $127.5,125.0,124.7,76.4,66.0,39.7$. All characterization data are in agreement with those reported. ${ }^{[7]}$

HRMS (FI) $m / z:[\mathrm{M}]^{+}$calcd for $\mathrm{C}_{9} \mathrm{H}_{9} \mathrm{ClO}: 168.0342$; found: 168.0324 .

The enantiomeric excess was determined by HPLC analysis (Chiralcel OJ-H, $250 \times 4.6$ $\mathrm{mm}$ column, hexane/2-propanol 97:3, $1.0 \mathrm{~mL} / \mathrm{min}, 220 \mathrm{~nm}, 30^{\circ} \mathrm{C},(1 R, 2 S)$ isomer 15.7 min (minor), $(1 S, 2 R)$ isomer 26.7 min (major)); $[\alpha]_{\mathrm{D}}^{20}-58.6$ (c 0.72 in $\left.\mathrm{CHCl}_{3}\right)$ cis/trans $=$

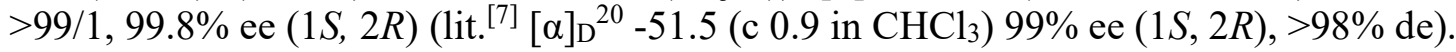




\section{(1S,2R)-2-Chloro-5-methoxy-2,3-dihydro-1H-inden-1-ol (cis-4e)}<smiles>COc1ccc2c(c1)C[C@H](Cl)[C@H]2O</smiles>

According to the general procedure (ketone: $0.167 \mathrm{~g}(1.0 \mathrm{mmol})$, cat. $(\boldsymbol{R}, \boldsymbol{R})-3$, eluent of silica gel column chromatography: Hexane/AcOEt $=4 / 1$ to 3/1), $0.198 \mathrm{~g}$ of cis-4e was obtained as a white solid ( $>99 \%$ yield).

${ }^{1} \mathrm{H}$ NMR $\left(500 \mathrm{MHz}, \mathrm{CDCl}_{3}\right) \delta 7.36(\mathrm{~d}, J=8.3 \mathrm{~Hz}, 1 \mathrm{H}), 6.83(\mathrm{dd}, J=8.3,2.2 \mathrm{~Hz}, 1 \mathrm{H})$, 6.79-6.78 (m, 1H), $5.09(\mathrm{dd}, J=8.3,4.7 \mathrm{~Hz}, 1 \mathrm{H}), 4.77-4.74(\mathrm{~m}, 1 \mathrm{H}), 3.80(\mathrm{~s}, 3 \mathrm{H})$, 3.33-3.21 (m, 2H), $2.42(\mathrm{~d}, J=8.5 \mathrm{~Hz}, 1 \mathrm{H}) ;{ }^{13} \mathrm{C}\left\{{ }^{1} \mathrm{H}\right\}$ NMR $\left(125 \mathrm{MHz}, \mathrm{CDCl}_{3}\right) \delta 160.6$, $140.8,133.5,125.7,113.5,110.3,65.5,55.4,39.8$.

HRMS (CI) $m / z:[\mathrm{M}]^{+}$calcd for $\mathrm{C}_{10} \mathrm{H}_{11} \mathrm{ClO}_{2}$ : 198.0442; found: 198.0442 .

The enantiomeric excess was determined by HPLC analysis (Chiralcel OJ-3, $250 \times 4.6 \mathrm{~mm}$ column, hexane/2-propanol 90:10, $1.0 \mathrm{~mL} / \mathrm{min}, 220 \mathrm{~nm}, 35^{\circ} \mathrm{C},(1 R, 2 S)$ isomer $13.9 \mathrm{~min}$ (minor), $\left(1 S, 2 R\right.$ ) isomer 20.8 min (major)); $[\alpha]_{\mathrm{D}}{ }^{20}-39.0$ (c 1.77 in $\mathrm{CHCl}_{3}$ ) cis/trans $=$ $>99 / 1,>99.9 \%$ ee $(1 S, 2 R)$.

\section{(1S,2R)-2,5-Dichloro-2,3-dihydro-1H-inden-1-ol (cis-4f)}<smiles>O[C@H]1c2ccc(Cl)cc2CC1Cl</smiles>

According to the general procedure (ketone: $0.201 \mathrm{~g}(1.0 \mathrm{mmol})$, cat. $(\boldsymbol{R}, \boldsymbol{R})-3$, eluent of silica gel column chromatography: Hexane/AcOEt $=5 / 1), 0.202 \mathrm{~g}$ of cis-4f was obtained as a white solid ( $>99 \%$ yield).

${ }^{1} \mathrm{H}$ NMR $\left(500 \mathrm{MHz}, \mathrm{CDCl}_{3}\right) \delta 7.38(\mathrm{~d}, J=8.0 \mathrm{~Hz}, 1 \mathrm{H}), 7.30-7.24(\mathrm{~m}, 2 \mathrm{H}), 5.13(\mathrm{dd}, J=$ 9.4, $4.8 \mathrm{~Hz}, 1 \mathrm{H}), 4.81(\mathrm{dt}, J=4.8,3.1 \mathrm{~Hz}, 1 \mathrm{H}), 3.35-3.22(\mathrm{~m}, 2 \mathrm{H}), 2.50(\mathrm{~d}, J=9.4 \mathrm{~Hz}$, $1 \mathrm{H}) ;{ }^{13} \mathrm{C}\left\{{ }^{1} \mathrm{H}\right\}$ NMR $\left(125 \mathrm{MHz}, \mathrm{CDCl}_{3}\right) \delta 140.7,140.0,134.6,127.8,125.9,125.4,75.8$, 65.8, 39.5.

HRMS (CI) $m / z:[\mathrm{M}]^{+}$calcd for $\mathrm{C}_{9} \mathrm{H}_{8} \mathrm{Cl}_{2} \mathrm{O}$ : 201.9947; found: 201.9946.

The enantiomeric excess was determined by HPLC analysis (Chiralcel OZ-3, $250 \times 4.6$ $\mathrm{mm}$ column, hexane/2-propanol 97:3, $1.0 \mathrm{~mL} / \mathrm{min}, 220 \mathrm{~nm}, 35^{\circ} \mathrm{C},(1 R, 2 S)$ isomer 11.3 $\min$ (minor), $(1 S, 2 R)$ isomer 16.1 min (major)); $[\alpha]_{\mathrm{D}}{ }^{20}-29.7$ (c 1.80 in $\left.\mathrm{CHCl}_{3}\right)$ cis/trans $=$ $>99 / 1,>99.9 \%$ ee $(1 S, 2 R)$. 


\section{(1S,2R)-2-Chloro-3,3-dimethyl-2,3-dihydro-1H-inden-1-ol (cis-4g)}<smiles>Oc1cccc2c1[C@@H](O)[C@H](Cl)C2</smiles>

According to the general procedure (ketone: $0.182 \mathrm{~g}(1.0 \mathrm{mmol})$, cat. $(\boldsymbol{R}, \boldsymbol{R})-\mathbf{3}$, eluent of silica gel column chromatography: Hexane/AcOEt $=5 / 1$ to $4 / 1), 0.177 \mathrm{~g}$ of cis-4g was obtained as a white solid ( $96 \%$ yield).

${ }^{1} \mathrm{H}$ NMR $\left(500 \mathrm{MHz}, \mathrm{CDCl}_{3}\right) \delta 7.20-7.15(\mathrm{~m}, 1 \mathrm{H}), 6.81-6.75(\mathrm{~m}, 2 \mathrm{H}), 6.72(\mathrm{br}, 1 \mathrm{H})$, 5.45-5.41 (m, 1H), 4.89-4.87 (m, 1H), 3.37-3.22 (m, 2H), $3.00(\mathrm{br}, 1 \mathrm{H}) ;{ }^{13} \mathrm{C}\left\{{ }^{1} \mathrm{H}\right\} \mathrm{NMR}$ $\left(125 \mathrm{MHz}, \mathrm{CDCl}_{3}\right) \delta 154.9,139.4,130.7,124.5,117.1,114.6,76.2,66.4,40.1$.

HRMS (FI) calcd for $m / z$ : [M] ${ }^{+} \mathrm{C}_{9} \mathrm{H}_{9} \mathrm{ClO}_{2}$ : 184.0291; found: 184.0286 .

The enantiomeric excess was determined by HPLC analysis (Chiralcel OJ-3, $250 \times 4.6 \mathrm{~mm}$ column, hexane/2-propanol 90:10, $1.0 \mathrm{~mL} / \mathrm{min}, 220 \mathrm{~nm}, 35^{\circ} \mathrm{C},(1 R, 2 S)$ isomer $17.3 \mathrm{~min}$ (minor), $\left(1 S, 2 R\right.$ ) isomer 19.9 min (major)); $[\alpha]_{\mathrm{D}}{ }^{20}-94.4$ (c 0.76 in $\mathrm{CHCl}_{3}$ ) cis/trans $=$ $>99 / 1,99.8 \%$ ee $(1 S, 2 R)$.

\section{(1S,2R)-2-Chloro-2,3-dihydro-1H-indene-1,7-diol (cis-4h)}<smiles>CC1(C)c2ccccc2[C@H](O)[C@H]1Cl</smiles>

According to the general procedure (ketone: $0.195 \mathrm{~g}(1.0 \mathrm{mmol})$, cat. $(\boldsymbol{R}, \boldsymbol{R})-\mathbf{5}$, eluent of silica gel column chromatography: Hexane/AcOEt $=15 / 1$ to $10 / 1), 0.143 \mathrm{~g}$ of $\boldsymbol{c i s}-\mathbf{4 h}$ was obtained as a pale pink oil ( $73 \%$ yield).

${ }^{1} \mathrm{H}$ NMR $\left(500 \mathrm{MHz}, \mathrm{CDCl}_{3}\right) \delta$ 7.46-7.44 $(\mathrm{m}, 1 \mathrm{H}), 7.37-7.34(\mathrm{~m}, 1 \mathrm{H}), 7.31-7.27(\mathrm{~m}, 1 \mathrm{H})$, $7.24-7.20(\mathrm{~m}, 1 \mathrm{H}), 5.13(\mathrm{t}, J=5.3 \mathrm{~Hz}, 1 \mathrm{H}), 4.33(\mathrm{~d}, J=5.3 \mathrm{~Hz}, 1 \mathrm{H}), 2.48(\mathrm{~d}, J=5.3 \mathrm{~Hz}$, $1 \mathrm{H}), 1.40(\mathrm{~s}, 3 \mathrm{H}), 1.35(\mathrm{~s}, 3 \mathrm{H}) ;{ }^{13} \mathrm{C}\left\{{ }^{1} \mathrm{H}\right\}$ NMR $\left(125 \mathrm{MHz}, \mathrm{CDCl}_{3}\right) \delta 149.1,139.3,129.5$, 127.5, 125.3, 122.6, 74.5, 74.4, 46.6, 26.7, 26.6.

HRMS (FI) calcd for $m / z$ : [M] ${ }^{+} \mathrm{C}_{11} \mathrm{H}_{13} \mathrm{ClO}$ : 196.0655; found: 196.0662 .

The enantiomeric excess was determined by HPLC analysis (Chiralcel OZ-3, $250 \times 4.6$ $\mathrm{mm}$ column, hexane/2-propanol 97:3, $1.0 \mathrm{~mL} / \mathrm{min}, 220 \mathrm{~nm}, 35^{\circ} \mathrm{C},(1 R, 2 S)$ isomer $7.5 \mathrm{~min}$ (minor), $(1 S, 2 R)$ isomer 8.0 min (major)); $[\alpha]_{\mathrm{D}}{ }^{20}+8.24$ (c 0.57 in $\left.\mathrm{CHCl}_{3}\right)$ cis/trans $=95 / 5$, $97 \%$ ee $(1 S, 2 R)$. 


\section{(5S,6R)-6-Chloro-6,7,8,9-tetrahydro-5H-benzo[7]annulen-5-ol (cis-4i)}<smiles>OC1c2ccccc2CCC[C@H]1Cl</smiles>

According to the general procedure (ketone: $0.194 \mathrm{~g}(1.0 \mathrm{mmol})$, cat. $(\boldsymbol{R}, \boldsymbol{R})-\mathbf{4}$, eluent of silica gel column chromatography: Hexane/AcOEt $=5 / 1$ to 3/1), $0.196 \mathrm{~g}$ of cis-4i was obtained as a white solid ( $>99 \%$ yield).

${ }^{1} \mathrm{H}$ NMR $\left(400 \mathrm{MHz}, \mathrm{CDCl}_{3}\right) \delta 7.30-7.28(\mathrm{~m}, 1 \mathrm{H}), 7.23-7.18(\mathrm{~m}, 2 \mathrm{H}), 7.10-7.08(\mathrm{~m}, 1 \mathrm{H})$, $5.07(\mathrm{~d}, J=4.4 \mathrm{~Hz}, 1 \mathrm{H}), 4.40-4.37(\mathrm{~m}, 1 \mathrm{H}), 3.14-3.08(\mathrm{~m}, 1 \mathrm{H}), 2.66-2.60(\mathrm{~m}, 1 \mathrm{H})$, $2.53-2.45(\mathrm{~m}, 2 \mathrm{H}), 2.26-2.20(\mathrm{~m}, 1 \mathrm{H}), 1.90-1.88(\mathrm{~m}, 1 \mathrm{H}), 1.64-1.59(\mathrm{~m}, 1 \mathrm{H}) ;{ }^{13} \mathrm{C}\left\{{ }^{1} \mathrm{H}\right\}$ NMR $\left(125 \mathrm{MHz}, \mathrm{CDCl}_{3}\right) \delta 141.6,138.0,129.9,128.6,128.4,126.2,79.2,65.6,36.1,34.7$, 25.6 .

HRMS (FI) $m / z:[\mathrm{M}]^{+}$calcd for $\mathrm{C}_{11} \mathrm{H}_{13} \mathrm{ClO}$ : 196.0655; found: 196.0663 .

The enantiomeric excess was determined by HPLC analysis (Chiralcel OJ-H, $250 \times 4.6$ $\mathrm{mm}$ column, hexane/2-propanol 97:3, $1.0 \mathrm{~mL} / \mathrm{min}, 220 \mathrm{~nm}, 30{ }^{\circ} \mathrm{C},(5 R, 6 S)$ isomer 20.4 $\min$ (minor), $(5 S, 6 R)$ isomer $25.9 \mathrm{~min}$ (major), $(5 S, 6 S)$ and $(5 R, 6 R)$ isomers $16.1 \mathrm{~min}$ and $16.9 \mathrm{~min}) ;[\alpha]_{\mathrm{D}}^{20}-23.3\left(\mathrm{c} 0.70\right.$ in $\left.\mathrm{CHCl}_{3}\right)$ cis/trans $=>99 / 1,97 \%$ ee $(5 S, 6 R)$.

\section{(1S,2R)-2-Chloro-1,2-dihydroacenaphthylen-1-ol (cis-4j)}<smiles>OC1c2cccc3cccc(c23)[C@H]1Cl</smiles>

According to the general procedure (ketone: $0.202 \mathrm{~g}(1.0 \mathrm{mmol})$, cat. $(\boldsymbol{R}, \boldsymbol{R})-\mathbf{5}$, eluent of silica gel column chromatography: Hexane/AcOEt $=5 / 1), 0.194 \mathrm{~g}$ of $\boldsymbol{c i s}-\mathbf{4 j}$ was obtained as a white solid ( $95 \%$ yield).

${ }^{1} \mathrm{H}$ NMR $\left(500 \mathrm{MHz}, \mathrm{CDCl}_{3}\right) \delta 7.81-7.77(\mathrm{~m}, 2 \mathrm{H}), 7.62-7.56(\mathrm{~m}, 4 \mathrm{H}), 5.88(\mathrm{~d}, J=6.0 \mathrm{~Hz}$, $1 \mathrm{H}), 5.68(\mathrm{~m}, 1 \mathrm{H}), 2.80(\mathrm{~d}, J=9.5 \mathrm{~Hz}, 1 \mathrm{H}) ;{ }^{13} \mathrm{C}\left\{{ }^{1} \mathrm{H}\right\} \mathrm{NMR}\left(125 \mathrm{MHz}, \mathrm{CDCl}_{3}\right) \delta 141.6$, $140.2,134.7,130.9,128.6,128.4,125.8,125.1,121.8,121.3,74.6,64.1$.

HRMS (FI) $m / z:[\mathrm{M}]^{+}$calcd for $\mathrm{C}_{12} \mathrm{H}_{9} \mathrm{CO}: 204.0342$; found: 204.0361 .

The enantiomeric excess was determined by HPLC analysis (Chiralpak AS-3, $250 \times 4.6$ $\mathrm{mm}$ column, hexane/2-propanol 95:5, $1.0 \mathrm{~mL} / \mathrm{min}, 220 \mathrm{~nm}, 35^{\circ} \mathrm{C},(1 R, 2 S)$ isomer 11.4 $\min$ (minor), $(1 S, 2 R)$ isomer 12.9 min (major); $[\alpha]_{\mathrm{D}}{ }^{20}-41.4$ (c 0.61 in $\left.\mathrm{CHCl}_{3}\right)$ cis/trans $=$ $>99 / 1,98 \%$ ee $(1 S, 2 R)$. 


\section{(1S,2R)-2-Bromo-2,3-dihydro-1H-inden-1-ol (cis-4k)}<smiles>O[C@H]1c2ccccc2CC1Br</smiles>

According to the general procedure (ketone: $0.211 \mathrm{~g}(1.0 \mathrm{mmol})$, cat. $(\boldsymbol{R}, \boldsymbol{R})-\mathbf{4}$, eluent of silica gel column chromatography: Hexane/AcOEt $=20 / 1$ to $15 / 1), 0.198 \mathrm{~g}$ of cis-4k was obtained as a white solid (93\% yield).

${ }^{1} \mathrm{H}$ NMR $\left(500 \mathrm{MHz}, \mathrm{CDCl}_{3}\right) \delta 7.47-7.44(\mathrm{~m}, 1 \mathrm{H}), 7.32-7.28(\mathrm{~m}, 2 \mathrm{H}), 7.28-7.24(\mathrm{~m}, 1 \mathrm{H})$, $4.97(\mathrm{dd}, J=9.3,4.6 \mathrm{~Hz}, 1 \mathrm{H}), 4.94-4.91(\mathrm{~m}, 1 \mathrm{H}), 3.40(\mathrm{~m}, 2 \mathrm{H}), 2.42(\mathrm{~d}, J=9.3 \mathrm{~Hz}, 1 \mathrm{H})$; ${ }^{13} \mathrm{C}\left\{{ }^{1} \mathrm{H}\right\}$ NMR $\left(125 \mathrm{MHz}, \mathrm{CDCl}_{3}\right) \delta 141.7,139.2,128.7,127.5,125.0,124.6,76.3,60.7$, 40.2 .

All characterization data are in agreement with those reported ${ }^{[7]}$

HRMS (FI) calcd for $m / z$ : [M] ${ }^{+} \mathrm{C}_{9} \mathrm{H}_{9} \mathrm{BrO}: 211.9837$; found: 211.9851 .

The enantiomeric excess was determined by HPLC analysis (Chiralpak AS-3, $250 \times 4.6$ $\mathrm{mm}$ column, hexane/2-propanol 97:3, $1.0 \mathrm{~mL} / \mathrm{min}, 220 \mathrm{~nm}, 35^{\circ} \mathrm{C},(1 R, 2 S)$ isomer 12.1 $\min$ (minor), $(1 S, 2 R)$ isomer 13.5 min (major)); $[\alpha]_{\mathrm{D}}{ }^{20}-32.0$ (c 1.07 in $\left.\mathrm{CHCl}_{3}\right)$ cis/trans $=$ $>99 / 1,99.5 \%$ ee $(1 S, 2 R)\left(\right.$ lit. $^{[7]}[\alpha]_{\mathrm{D}}{ }^{20}+59.4\left(\mathrm{c} 0.8\right.$ in $\left.\mathrm{CHCl}_{3}\right) 99 \%$ ee $(1 R, 2 S),>98 \%$ de).

\section{(1S,2R)-2-Bromo-1,2,3,4-tetrahydronaphthalen-1-ol (cis-4l)}<smiles>OC1c2ccccc2CC[C@@H]1Br</smiles>

According to the general procedure (ketone: $0.225 \mathrm{~g}(1.0 \mathrm{mmol})$, cat. $(\boldsymbol{R}, \boldsymbol{R})-\mathbf{4}$, eluent of silica gel column chromatography: Hexane/AcOEt $=20 / 1$ to $10 / 1), 0.218$ g of cis-4l was obtained as a white solid (96\% yield).

${ }^{1} \mathrm{H}$ NMR $\left(500 \mathrm{MHz}, \mathrm{CDCl}_{3}\right) \delta 7.48-7.46(\mathrm{~m}, 1 \mathrm{H}), 7.27-7.22(\mathrm{~m}, 2 \mathrm{H}), 7.13-7.10(\mathrm{~m}, 1 \mathrm{H})$, $4.81-4.77(\mathrm{~m}, 1 \mathrm{H}), 4.71(\mathrm{dt}, J=5.8,3.0 \mathrm{~Hz}, 1 \mathrm{H}), 3.10(\mathrm{dt}, J=12.0,6.6 \mathrm{~Hz}, 1 \mathrm{H}), 2.87(\mathrm{dt}$, $J=12.0,6.6 \mathrm{~Hz}, 1 \mathrm{H}), 2.55-2.48(\mathrm{~m}, 1 \mathrm{H}), 2.40(\mathrm{~d}, J=7.5 \mathrm{~Hz}, 1 \mathrm{H}), 2.33-2.26(\mathrm{~m}, 1 \mathrm{H})$; ${ }^{13} \mathrm{C}\left\{{ }^{1} \mathrm{H}\right\}$ NMR $\left(125 \mathrm{MHz}, \mathrm{CDCl}_{3}\right) \delta 136.1,134.6,128.6,128.5,128.2,126.5,70.2,58.5$, 28.3, 27.6. All characterization data are in agreement with those reported. ${ }^{[7]}$

HRMS (FI) calcd for $m / z$ : [M] ${ }^{+} \mathrm{C}_{10} \mathrm{H}_{11} \mathrm{BrO}$ : 225.9993; found: 226.0007 .

The enantiomeric excess was determined by HPLC analysis (Chiralpak AS-3, $250 \times 4.6$ $\mathrm{mm}$ column, hexane/2-propanol 97:3, $1.0 \mathrm{~mL} / \mathrm{min}, 220 \mathrm{~nm}, 35^{\circ} \mathrm{C},(1 R, 2 S)$ isomer 10.8 min (minor), $(1 S, 2 R)$ isomer 12.5 min (major)); $[\alpha]_{\mathrm{D}^{20}}-8.05$ (c 0.59 in $\left.\mathrm{CHCl}_{3}\right)$ cis/trans $=$ $>99 / 1,>99.9 \%$ ee $(1 S, 2 R)\left(\right.$ lit. $^{[7]}[\alpha]_{\mathrm{D}}{ }^{20}+7.25\left(\right.$ c 0.8 in $\left.\mathrm{CHCl}_{3}\right) 96 \%$ ee $(1 R, 2 S),>98 \%$ de). 


\section{Methyl (1R,2R)-1-hydroxy-2,3-dihydro-1 H-indene-2-carboxylate (cis-6a)}<smiles>CC(=O)C1Cc2ccccc2[C@H]1O</smiles>

According to the general procedure (ketone: $0.190 \mathrm{~g}(1.0 \mathrm{mmol})$, cat. $(\boldsymbol{R}, \boldsymbol{R})-\mathbf{3}$, eluent of silica gel column chromatography: Hexane/AcOEt $=3 / 1), 0.192 \mathrm{~g}$ of cis-6a was obtained as a white solid ( $>99 \%$ ee).

${ }^{1} \mathrm{H}$ NMR $\left(400 \mathrm{MHz}, \mathrm{CDCl}_{3}\right) \delta$ 7.43-7.41 (m, 1H), 7.29-7.25 (m, 3H), $5.32(\mathrm{~m}, 1 \mathrm{H}), 3.77$ (s, 3H), 3.47-3.36 (m, 2H), $3.08(\mathrm{dd}, J=14.4,6.4 \mathrm{~Hz}, 1 \mathrm{H}), 2.89(\mathrm{~d}, J=6.4 \mathrm{~Hz}, 1 \mathrm{H})$; ${ }^{13} \mathrm{C}\left\{{ }^{1} \mathrm{H}\right\}$ NMR $\left(125 \mathrm{MHz}, \mathrm{CDCl}_{3}\right) \delta 173.4,142.5,141.7,129.0,127.2,125.0,124.8,75.8$, $51.9,49.4,32.7$.

HRMS (FI) calcd for $m / z$ : [M] $]^{+} \mathrm{C}_{11} \mathrm{H}_{12} \mathrm{O}_{3}$ : 192.0786; found: 192.0795 .

The enantiomeric excess was determined by HPLC analysis (Chiralcel OJ-H, $250 \times 4.6$ $\mathrm{mm}$ column, hexane/2-propanol 97:3, $1.0 \mathrm{~mL} / \mathrm{min}, 220 \mathrm{~nm}, 35^{\circ} \mathrm{C},(1 \mathrm{~S}, 2 S)$ isomer 25.5 $\min$ (minor), $(1 R, 2 R)$ isomer 31.6 min (major)); $[\alpha]_{\mathrm{D}}^{20}-8.4\left(\right.$ c 1.0 in $\left.\mathrm{CHCl}_{3}\right)$ cis/trans $=$ $>99 / 1,99.6 \%$ ee $(1 R, 2 R)$.

\section{Methyl (5R,6R)-5-hydroxy-6,7,8,9-tetrahydro-5H-benzo[7]annulene-6-carboxylate (cis-6b)}<smiles>CC(=O)[C@H]1CCCc2ccccc2[C@@H]1O</smiles>

According to the general procedure (ketone: $0.218 \mathrm{~g}(1.0 \mathrm{mmol})$, cat. $(\boldsymbol{R}, \boldsymbol{R})-4$, eluent of silica gel column chromatography: Hexane/AcOEt $=5 / 1$ to $3 / 1), 0.218$ g of cis-6b was obtained as white solid ( $>99 \%$ yield).

${ }^{1} \mathrm{H}$ NMR $\left(400 \mathrm{MHz}, \mathrm{CDCl}_{3}\right) \delta$ 7.26-7.07 (m, 4H), $5.06(\mathrm{~m}, 1 \mathrm{H}), 3.68(\mathrm{~s}, 1 \mathrm{H}), 3.24-3.17$ (m, 2H), 2.84-2.80 (m, 1H), 2.68-2.35 (m, 1H), 2.03-1.94 (m, 2H) , 1.58-1.55 (m, 1H); ${ }^{13} \mathrm{C}\left\{{ }^{1} \mathrm{H}\right\} \mathrm{NMR}\left(125 \mathrm{MHz}, \mathrm{CDCl}_{3}\right) \delta 176.0,142.2,139.9,130.0,128.4,128.0,126.0,75.6$, 51.8, 49.0, 53.0, 28.5, 26.7.

HRMS (FI) calcd for $m / z$ : [M] $]^{+} \mathrm{C}_{13} \mathrm{H}_{16} \mathrm{O}_{3}$ : 220.1099; found: 220.1107 .

The enantiomeric excess was determined by HPLC analysis (Chiralcel OD-H, $250 \times 4.6$ $\mathrm{mm}$ column, hexane/2-propanol 98:2, $1.0 \mathrm{~mL} / \mathrm{min}, 220 \mathrm{~nm}, 40{ }^{\circ} \mathrm{C},(5 R, 6 R)$ isomer 18.6 $\min$ (major), $(5 S, 6 S)$ isomer $20.0 \mathrm{~min}$ (minor)); $[\alpha]_{\mathrm{D}}{ }^{20}-30.3$ (c 1.0 in $\left.\mathrm{CHCl}_{3}\right)$ cis/trans $=$ $>99 / 1,98.9 \%$ ee $(5 R, 6 R)$. 
<smiles>CCCCC(=O)[C@H]1Cc2ccccc2[C@H]1O</smiles>

According to the general procedure (ketone: $0.230 \mathrm{~g}(1.0 \mathrm{mmol})$, cat. $(\boldsymbol{R}, \boldsymbol{R})-\mathbf{3}$, eluent of silica gel column chromatography: Hexane/AcOEt $=3 / 1), 0.231 \mathrm{~g}$ of cis-6c was obtained as a white solid ( $99 \%$ yield).

${ }^{1} \mathrm{H}$ NMR $\left(400 \mathrm{MHz}, \mathrm{CDCl}_{3}\right) \delta 7.43-7.41(\mathrm{~m}, 1 \mathrm{H}), 7.28-7.22(\mathrm{~m}, 3 \mathrm{H}), 5.76(\mathrm{br}, 1 \mathrm{H}), 5.25$ (m, $1 \mathrm{H}), 4.18(\mathrm{~d}, J=5.2 \mathrm{~Hz}, 1 \mathrm{H}), 3.31(\mathrm{dd}, J=14.8,7.2 \mathrm{~Hz}, 1 \mathrm{H}), 3.09-2.98(\mathrm{~m}, 2 \mathrm{H}), 1.38$ $(\mathrm{s}, 9 \mathrm{H}) ;{ }^{13} \mathrm{C}\left\{{ }^{1} \mathrm{H}\right\}$ NMR $\left(125 \mathrm{MHz}, \mathrm{CDCl}_{3}\right) \delta 172.9,143.5,141.7,128.7,127.1,124.8$, 124.7, 75.9, 51.6, 49.8, 34.4, 28.8 .

HRMS (FI) calcd for $m / z$ : [M] ${ }^{+} \mathrm{C}_{14} \mathrm{H}_{19} \mathrm{NO}_{2}$ : 233.1416; found: 233.1415 .

The enantiomeric excess was determined by HPLC analysis (Chiralcel OJ-H, $250 \times 4.6$ $\mathrm{mm}$ column, hexane/2-propanol 95:5, $1.0 \mathrm{~mL} / \mathrm{min}, 220 \mathrm{~nm}, 30^{\circ} \mathrm{C},(1 S, 2 S)$ isomer $9.9 \mathrm{~min}$ (minor), $(1 R, 2 R)$ isomer 14.0 min (major)); $[\alpha]_{\mathrm{D}}{ }^{20}-50.2$ (c 1.53 in $\left.\mathrm{CHCl}_{3}\right)$ cis/trans $=$ $>99 / 1,99.9 \%$ ee $(1 R, 2 R)$.

(1R,2R)- $N$-(tert-Butyl)-1-hydroxy-1,2,3,4-tetrahydronaphthalene-2-carboxamide (cis-6d)

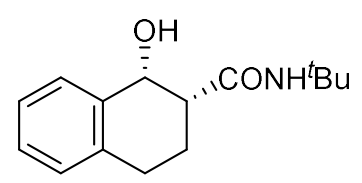

According to the general procedure (ketone: $0.245 \mathrm{~g}(1.0 \mathrm{mmol})$, cat. $(\boldsymbol{R}, \boldsymbol{R})-\mathbf{3}$, eluent of silica gel column chromatography: Hexane/AcOEt $=3 / 1), 0.247 \mathrm{~g}$ of cis-6d was obtained as a white solid ( $>99 \%$ yield).

${ }^{1} \mathrm{H}$ NMR (400 MHz, $\left.\mathrm{CDCl}_{3}\right) \delta$ 7.34-7.32 (m, 1H), 7.26-7.17 (m, 2H), 7.11-7.09 (m, 1H), $5.85(\mathrm{br}, 1 \mathrm{H}), 4.91(\mathrm{~m}, 1 \mathrm{H}), 4.39(\mathrm{~d}, J=2.8 \mathrm{~Hz}, 1 \mathrm{H}), 2.97-2.91(\mathrm{~m}, 1 \mathrm{H}), 2.84-2.77(\mathrm{~m}$, 1H), 2.41-2.29 (m, 1H), 1.90-1.84 (m, 1H), $1.38(\mathrm{~s}, 9 \mathrm{H}) ;{ }^{13} \mathrm{C}\left\{{ }^{1} \mathrm{H}\right\}$ NMR $(125 \mathrm{MHz}$, $\left.\mathrm{CDCl}_{3}\right) \delta 175.0,136.8,135.7,129.8,128.9,128.1,126.2,68.1,51.4,46.3,28.8,28.5,21.5$. HRMS (FI) calcd for $m / z$ : [M] ${ }^{+} \mathrm{C}_{15} \mathrm{H}_{21} \mathrm{NO}_{2}$ : 247.1572; found: 247.1565 .

The enantiomeric excess was determined by HPLC analysis (Chiralcel OJ-H, $250 \times 4.6$ $\mathrm{mm}$ column, hexane/2-propanol 95:5, $1.0 \mathrm{~mL} / \mathrm{min}, 220 \mathrm{~nm}, 30^{\circ} \mathrm{C},(1 S, 2 S)$ isomer 13.9 min (minor), $\left(1 R, 2 R\right.$ ) isomer 26.9 min (major)); $[\alpha]_{\mathrm{D}}{ }^{20}+4.1$ (c 1.6 in $\left.\mathrm{CHCl}_{3}\right)$ cis/trans $=$ $>99 / 1,99.9 \%$ ee $(1 R, 2 R)$. 
(5R,6R)- $N$-(tert-Butyl)-5-hydroxy-6,7,8,9-tetrahydro-5H-benzo[7]annulene-6-carboxa mide (cis-6e)<smiles>CCCCCCCCC1CCCc2ccccc2[C@H]1O</smiles>

According to the general procedure (ketone: $0.182 \mathrm{~g}(0.70 \mathrm{mmol})$, cat. $(\boldsymbol{R}, \boldsymbol{R})-\mathbf{4}$, eluent of silica gel column chromatography: Hexane/AcOEt $=5 / 1), 0.183 \mathrm{~g}$ of cis-6e was obtained as a colorless oil ( $>99 \%$ yield).

${ }^{1} \mathrm{H}$ NMR $\left(400 \mathrm{MHz}, \mathrm{CDCl}_{3}\right) \delta 7.18-7.04(\mathrm{~m}, 4 \mathrm{H}), 6.02(\mathrm{br}, 1 \mathrm{H}), 4.90(\mathrm{~m}, 1 \mathrm{H}), 4.09(\mathrm{~d}, J=$ $1.6 \mathrm{~Hz}, 1 \mathrm{H}), 3.26-3.20(\mathrm{~m}, 1 \mathrm{H}), 2.58(\mathrm{dd}, J=14.0,7.6 \mathrm{~Hz}, 1 \mathrm{H}), 2.42-2.35(\mathrm{~m}, 2 \mathrm{H})$, $1.97-1.89(\mathrm{~m}, 1 \mathrm{H}), 1.85-1.79(\mathrm{~m}, 1 \mathrm{H}), 1.50-1.42(\mathrm{~m}, 1 \mathrm{H}), 1.29(\mathrm{~s}, 9 \mathrm{H}) ;{ }^{13} \mathrm{C}\left\{{ }^{1} \mathrm{H}\right\} \mathrm{NMR}$ $\left(125 \mathrm{MHz}, \mathrm{CDCl}_{3}\right) \delta 175.0,136.8,135.7,129.8,128.9,128.1,126.2,68.1,51.4,46.3,28.8$, 28.5, 21.5.

HRMS (FI) $m / z:[\mathrm{M}]^{+}$calcd for $\mathrm{C}_{16} \mathrm{H}_{23} \mathrm{NO}_{2}: 261.1729$; found: 261.1727 .

The enantiomeric excess was determined by HPLC analysis (Chiralpak AS-H, $250 \times 4.6$ $\mathrm{mm}$ column, hexane/2-propanol 95:5, $1.0 \mathrm{~mL} / \mathrm{min}, 220 \mathrm{~nm}, 30{ }^{\circ} \mathrm{C},(5 R, 6 R)$ isomer 11.4 min (major), $(5 S, 6 S)$ isomer 18.4 min (minor)); $[\alpha]_{\mathrm{D}}{ }^{20}-17.5$ (c 0.96 in $\mathrm{CHCl}_{3}$ ) cis/trans $=$ $>99 / 1,99.9 \%$ ee $(5 R, 6 R)$.

\section{(1S,2R)-2-(Phenylsulfonyl)-2,3-dihydro-1H-inden-1-ol (cis-6f)}<smiles>O[C@H]1c2ccccc2CC1[Se]c1ccccc1</smiles>

According to the general procedure (ketone: $0.136 \mathrm{~g}(0.50 \mathrm{mmol})$, cat. $(\boldsymbol{R}, \boldsymbol{R})-3$, eluent of silica gel column chromatography: Hexane/AcOEt $=5 / 1$ to $3 / 1), 0.136 \mathrm{~g}$ of cis-6f was obtained as an off-white solid ( $99 \%$ yield).

${ }^{1} \mathrm{H}$ NMR $\left(400 \mathrm{MHz}, \mathrm{CDCl}_{3}\right) \delta 8.04-8.02(\mathrm{~m}, 2 \mathrm{H}), 7.70-7.65(\mathrm{~m}, 1 \mathrm{H}), 7.60-7.57(\mathrm{~m}, 2 \mathrm{H})$, 7.39-7.37 (m, 1H), 7.31-7.22 (m, 3H), $5.30(\mathrm{~m}, 1 \mathrm{H}), 3.98-3.93(\mathrm{~m}, 1 \mathrm{H}), 3.69$ (d, $J=16.0$, $8.8 \mathrm{~Hz}, 1 \mathrm{H}), 3.22(\mathrm{~m}, 1 \mathrm{H}), 3.18(\mathrm{dd}, J=16.0,8.0 \mathrm{~Hz}, 1 \mathrm{H}) ;{ }^{13} \mathrm{C}\left\{{ }^{1} \mathrm{H}\right\}$ NMR $(125 \mathrm{MHz}$, $\left.\mathrm{CDCl}_{3}\right) \delta 141.7,139.6,139.2,133.9,129.5,129.2,128.5,127.8,125.0,124.9,74.6,66.9$, 31.3 .

All characterization data are in agreement with those reported. ${ }^{[4]}$ HRMS (FI) $m / z$ : [M] $]^{+}$calcd for $\mathrm{C}_{15} \mathrm{H}_{14} \mathrm{O}_{3} \mathrm{~S}$ : 274.0664; found: 274.0676 .

The enantiomeric excess was determined by HPLC analysis (Chiralpak AD-H, $250 \times 4.6$ $\mathrm{mm}$ column, hexane/2-propanol 80:20, $1.0 \mathrm{~mL} / \mathrm{min}, 220 \mathrm{~nm}, 30^{\circ} \mathrm{C},(1 \mathrm{~S}, 2 R)$ isomer 14.6 $\min$ (major), $(1 R, 2 S)$ isomer $18.0 \mathrm{~min}$ (minor)); $[\alpha]_{\mathrm{D}}{ }^{20}+10.7$ (c 0.37 in acetone) cis/trans $=$ $>99 / 1,>99.9 \%$ ee $(1 S, 2 R)\left(\right.$ lit. $^{[5]}[\alpha]_{\mathrm{D}}{ }^{24}-8.46$ (c 0.65 in acetone) $99 \%$ ee $(1 R, 2 S)$, dr $>99: 1)$. 


\section{(1S,2R)-2-(Phenylsulfonyl)-1,2,3,4-tetrahydronaphthalen-1-ol (cis-6g)}<smiles>O[C@H]1c2ccccc2CC[C@H]1Oc1ccccc1</smiles>

According to the general procedure (ketone: $0.143 \mathrm{~g}(0.50 \mathrm{mmol})$, cat. $(\boldsymbol{R}, \boldsymbol{R})-3$, eluent of silica gel column chromatography: Hexane/AcOEt $=5 / 1$ to $3 / 1), 0.144$ g of cis-6g was obtained as a white solid ( $>99 \%$ yield).

${ }^{1} \mathrm{H}$ NMR (400 MHz, $\left.\mathrm{CDCl}_{3}\right) \delta 8.00-7.98(\mathrm{~m}, 2 \mathrm{H}), 7.72-7.68(\mathrm{~m}, 1 \mathrm{H}), 7.63-7.59(\mathrm{~m}, 2 \mathrm{H})$, 7.26-7.16 (m, 3H), 7.13-7.11 (m, 1H), $5.06(\mathrm{~m}, 1 \mathrm{H}), 3.31-3.26(\mathrm{~m}, 2 \mathrm{H}), 3.12-3.06(\mathrm{~m}$, $1 \mathrm{H}), 2.87-2.78(\mathrm{~m}, 1 \mathrm{H}), 2.51-2.40(\mathrm{~m}, 1 \mathrm{H}), 2.27-2.22(\mathrm{~m}, 1 \mathrm{H}) ;{ }^{13} \mathrm{C}\left\{{ }^{1} \mathrm{H}\right\}$ NMR $(125 \mathrm{MHz}$, $\left.\mathrm{CDCl}_{3}\right) \delta 137.9,135.5,135.0,134.0,129.9,129.3,129.0,128.9,128.8,126.5,65.8,65.0$, $28.1,16.4$. All characterization data are in agreement with those reported. ${ }^{[4]}$

HRMS (ESI) $m / z$ : [M+Na] $]^{+}$calcd for $\mathrm{C}_{16} \mathrm{H}_{16} \mathrm{O}_{3} \mathrm{SNa}$ : 311.0712; found: 311.0716 .

The enantiomeric excess was determined by HPLC analysis (Chiralpak AD-H, $250 \times 4.6$ $\mathrm{mm}$ column, hexane/2-propanol 80:20, $1.0 \mathrm{~mL} / \mathrm{min}, 220 \mathrm{~nm}, 30^{\circ} \mathrm{C},(1 S, 2 R)$ isomer 20.6 min (major), $(1 R, 2 S)$ isomer 29.0 min (minor)); $[\alpha]_{\mathrm{D}}{ }^{20}+82.7$ (c 0.68 in Acetone) cis/trans $=>99 / 1,>99.9 \%$ ee $(1 S, 2 R)\left(\right.$ lit. $^{[5]}[\alpha]_{\mathrm{D}}{ }^{24}-67.33$ (c 0.30 in acetone) $>99 \%$ ee $(1 R, 2 S), \mathrm{dr}$ $>99: 1)$.

$(1 R, 2 R)-N$-(tert-Butyl)-2-hydroxy-1,2-dihydroacenaphthylene-1-carboxamide (cis-6h)

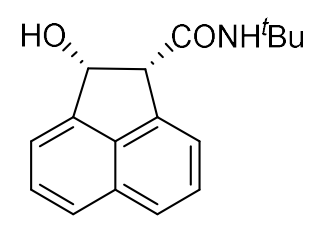

According to the general procedure (ketone: $0.267 \mathrm{~g}(0.50 \mathrm{mmol})$, cat. $(\boldsymbol{R}, \boldsymbol{R})-3$, eluent of silica gel column chromatography: Hexane/AcOEt $=1 / 1), 0.215 \mathrm{~g}$ of cis-6h was obtained as an off-white powder $(80 \%$ yield $)$.

${ }^{1} \mathrm{H}$ NMR $\left(400 \mathrm{MHz}, \mathrm{CDCl}_{3}\right) \delta$ 7.76-7.70 (m, 2H), 7.59-7.58 (m, 2H), 7.52-7.49 (m, $\left.1 \mathrm{H}\right)$, $7.33-7.31(\mathrm{~m}, 1 \mathrm{H}), 5.99(\mathrm{br}, 1 \mathrm{H}), 5.86(\mathrm{t}, J=7.2 \mathrm{~Hz}, 1 \mathrm{H}), 4.42(\mathrm{~d}, J=7.2 \mathrm{~Hz}, 1 \mathrm{H}), 4.30(\mathrm{~d}$, $J=7.2 \mathrm{~Hz}, 1 \mathrm{H}), 1.34(\mathrm{~s}, 9 \mathrm{H}) ;{ }^{13} \mathrm{C}\left\{{ }^{1} \mathrm{H}\right\}$ NMR $\left(125 \mathrm{MHz}, \mathrm{CDCl}_{3}\right) \delta 170.3,144.1,140.1$, 137.5, 131.4, 128.6, 127.9, 124.7, 124.3, 121.0, 120.0, 75.2, 56.4, 51.8, 28.6.

HRMS (FI) $m / z:[\mathrm{M}]^{+}$calcd for $\mathrm{C}_{17} \mathrm{H}_{19} \mathrm{NO}_{2}: 269.1416$; found: 269.1422 .

The enantiomeric excess was determined by HPLC analysis (Chiralpak AS-H, $250 \times 4.6$ $\mathrm{mm}$ column, hexane/2-propanol 95:5, $1.0 \mathrm{~mL} / \mathrm{min}, 220 \mathrm{~nm}, 30{ }^{\circ} \mathrm{C},(1 S, 2 S)$ isomer 14.7 $\min$ (minor), $(1 R, 2 R)$ isomer 22.5 min (major)); $[\alpha]_{\mathrm{D}^{20}}+45.6$ (c 0.89 in $\left.\mathrm{CHCl}_{3}\right)$ cis/trans $=$ $>99 / 1,>99.9 \%$ ee. 
Methyl (1R,2R)-2-hydroxy-1,2-dihydroacenaphthylene-1-carboxylate (cis-6i)<smiles>COC(=O)[C@H]1c2cccc3cccc(c23)[C@H]1O</smiles>

According to the general procedure (ketone: $0.113 \mathrm{~g}(0.50 \mathrm{mmol})$, cat. $(\boldsymbol{R}, \boldsymbol{R})-\mathbf{5}$, eluent of silica gel column chromatography: Hexane/AcOEt $=3 / 1), 0.110 \mathrm{~g}$ of cis-6i was obtained as a light brown oil ( $96 \%$ yield).

${ }^{1} \mathrm{H}$ NMR $\left(500 \mathrm{MHz}, \mathrm{CDCl}_{3}\right) \delta 7.77-7.71(\mathrm{~m}, 2 \mathrm{H}), 7.58-7.47(\mathrm{~m}, 4 \mathrm{H}), 6.06(\mathrm{~d}, J=7.2,3.1$ $\mathrm{Hz}, 1 \mathrm{H}), 4.39(\mathrm{~m}, 1 \mathrm{H}), 3.81(\mathrm{~s}, 3 \mathrm{H}), 2.77(\mathrm{~d}, J=7.0 \mathrm{~Hz}, 1 \mathrm{H}) ;{ }^{13} \mathrm{C}\left\{{ }^{1} \mathrm{H}\right\} \mathrm{NMR}(125 \mathrm{MHz}$, $\left.\mathrm{CDCl}_{3}\right) \delta 172.3,143.1,138.1,136.2,131.1,128.3,128.2,125.2,124.3,120.8,120.6,77.7$, $59.5,52.5$.

HRMS (FI) calcd for $m / z$ : [M] $]^{+} \mathrm{C}_{14} \mathrm{H}_{12} \mathrm{O}_{3}$ : 228.0786; found: 228.0799 .

The enantiomeric excess was determined by HPLC analysis (Chiralcel AD-3, $150 \times 4.6$ $\mathrm{mm}$ column, hexane/2-propanol 93:7, $1.0 \mathrm{~mL} / \mathrm{min}, 220 \mathrm{~nm}, 30{ }^{\circ} \mathrm{C},(1 R, 2 R)$ isomer 11.3 $\min$ (major), $(1 S, 2 S)$ isomer $14.3 \mathrm{~min}$ (minor); $[\alpha]_{\mathrm{D}}{ }^{20}+58.9\left(\right.$ c 0.41 in $\left.\mathrm{CHCl}_{3}\right)$ cis/trans $=$ 91/9, $96 \%$ ee.

\section{(1S,6R)-6-Chloro-3-methylcyclohex-2-en-1-ol (cis-8a)}<smiles>CC1=CC(O)[C@H](Cl)CC1</smiles>

According to the general procedure (ketone: $0.434 \mathrm{~g}(3.0 \mathrm{mmol})$, cat. $(\boldsymbol{R}, \boldsymbol{R})-\mathbf{5}$, eluent of silica gel column chromatography: Hexane/AcOEt $=10 / 1), 0.396 \mathrm{~g}$ of cis-8a was obtained as a pale yellow oil ( $90 \%$ yield).

${ }^{1} \mathrm{H}$ NMR $\left(500 \mathrm{MHz}, \mathrm{CDCl}_{3}\right) \delta 5.47-5.43(\mathrm{~m}, 1 \mathrm{H}), 4.31-4.26(\mathrm{~m}, 1 \mathrm{H}), 4.23-4.20(\mathrm{~m}, 1 \mathrm{H})$, 2.25 (br, $1 \mathrm{H}), 2.23-2.16(\mathrm{~m}, 1 \mathrm{H}), 2.07-1.93(\mathrm{~m}, 1 \mathrm{H}), 1.71(\mathrm{~s}, 3 \mathrm{H}) ;{ }^{13} \mathrm{C}\left\{{ }^{1} \mathrm{H}\right\}$ NMR $(125$ $\left.\mathrm{MHz}, \mathrm{CDCl}_{3}\right) \delta 138.6,121.5,66.9,62.9,29.0,27.3,23.1$.

HRMS (CI) $m / z$ : [M] $]^{+}$calcd for $\mathrm{C}_{7} \mathrm{H}_{11} \mathrm{ClO}$ : 146.0493; found: 146.0492 .

The enantiomeric excess was determined by HPLC analysis (Chiralpak AD-3, $150 \times 4.6$ $\mathrm{mm}$ column, hexane/2-propanol 98:2, $1.0 \mathrm{~mL} / \mathrm{min}, 210 \mathrm{~nm}, 35^{\circ} \mathrm{C},(1 R, 6 S)$ isomer 11.1 min (minor), $(1 S, 6 R)$ isomer 12.1 min (major)); $[\alpha]_{\mathrm{D}}^{20}+1.1$ (c 2.24 in $\left.\mathrm{CHCl}_{3}\right)$ cis/trans $=$ $>99 / 1,93 \%$ ee $(1 S, 6 R)$. 
Ethyl (1R,2S)-2-hydroxy-4-methylcyclohex-3-ene-1-carboxylate (cis-8b)<smiles>CCOC(=O)[C@H]1CCC(C)=C[C@H]1O</smiles>

According to the general procedure (ketone: $0.364 \mathrm{~g}(2.0 \mathrm{mmol})$, cat. $(\boldsymbol{R}, \boldsymbol{R})-\mathbf{5}$, eluent of silica gel column chromatography: Toluene/AcOEt $=10 / 1), 0.331 \mathrm{~g}$ of $\mathbf{c i s}-\mathbf{8 b}$ was obtained as a light brown oil (90\% yield).

${ }^{1} \mathrm{H}$ NMR $\left(500 \mathrm{MHz}, \mathrm{CDCl}_{3}\right) \delta 5.63-5.60(\mathrm{~m}, 1 \mathrm{H}), 4.45-4.40(\mathrm{~m}, 1 \mathrm{H}), 4.20(\mathrm{q}, J=7.1 \mathrm{~Hz}$, $2 \mathrm{H}), 2.68(\mathrm{~d}, J=5.6 \mathrm{~Hz}, 1 \mathrm{H}), 2.53-2.48(\mathrm{~m}, 1 \mathrm{H}), 2.08-1.90(\mathrm{~m}, 4 \mathrm{H}), 1.72(\mathrm{~s}, 3 \mathrm{H}), 1.29(\mathrm{t}$, $J=7.1 \mathrm{~Hz}, 3 \mathrm{H}) ;{ }^{13} \mathrm{C}\left\{{ }^{1} \mathrm{H}\right\} \mathrm{NMR}\left(125 \mathrm{MHz}, \mathrm{CDCl}_{3}\right) \delta 174.6,139.5,122.2,64.3,60.6,45.0$, 30.0, 23.4, 19.7, 14.2.

HRMS (FI) $m / z$ : [M] $]^{+}$calcd for $\mathrm{C}_{10} \mathrm{H}_{16} \mathrm{O}_{3}$ : 184.1099; found: 184.1092 .

The enantiomeric excess was determined by HPLC analysis (Chiralcel OZ-3, $250 \times 4.6$ $\mathrm{mm}$ column, hexane/2-propanol 95:5, $1.0 \mathrm{~mL} / \mathrm{min}, 210 \mathrm{~nm}, 30{ }^{\circ} \mathrm{C},(1 R, 2 S)$ isomer $8.7 \mathrm{~min}$ (major), $(1 S, 2 R)$ isomer 9.8 min (minor)); $[\alpha]_{\mathrm{D}}^{20}+143.4$ (c 1.45 in $\mathrm{CHCl}_{3}$ ) cis/trans $=$ $>99 / 1,91 \%$ ee $(1 R, 2 S)$.

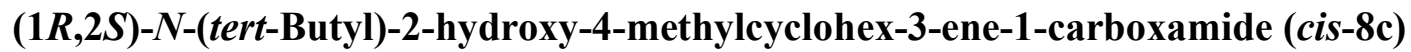

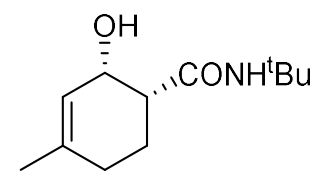

According to the general procedure (ketone: $0.105 \mathrm{~g}(0.50 \mathrm{mmol})$, cat. $(\boldsymbol{R}, \boldsymbol{R})-\mathbf{5}$, eluent of silica gel column chromatography: Hexane/AcOEt $=4 / 1$ to $3 / 1), 0.099 \mathrm{~g}$ of cis-8c was obtained as a light brown oil ( $94 \%$ yield).

${ }^{1} \mathrm{H}$ NMR $\left(500 \mathrm{MHz}, \mathrm{CDCl}_{3}\right) \delta 5.81(\mathrm{~s}, 1 \mathrm{H}), 5.63(\mathrm{~d}, J=4.0 \mathrm{~Hz}, 1 \mathrm{H}), 4.35(\mathrm{~m}, 1 \mathrm{H}), 3.80(\mathrm{~d}$, $J=6.4 \mathrm{~Hz}, 1 \mathrm{H}), 2.21-2.18(\mathrm{~m}, 1 \mathrm{H}), 2.10-2.00(\mathrm{~m}, 3 \mathrm{H}), 1.76-1.70(\mathrm{~m}, 1 \mathrm{H}), 1.70(\mathrm{~s}, 3 \mathrm{H})$, $1.36(\mathrm{~s}, 9 \mathrm{H}) ;{ }^{13} \mathrm{C}\left\{{ }^{1} \mathrm{H}\right\}$ NMR $\left(125 \mathrm{MHz}, \mathrm{CDCl}_{3}\right) \delta 175.0,138.9,122.5,64.3,51.2,45.9$, 30.4, 28.8, 23.4, 21.4 .

HRMS (FI) $m / z:[\mathrm{M}]^{+}$calcd for $\mathrm{C}_{12} \mathrm{H}_{21} \mathrm{NO}_{2}$ : 211.1572; found: 211.1581 .

The enantiomeric excess was determined by HPLC analysis (Chiralpak QN-AX, $150 \times 4.6$ $\mathrm{mm}$ column, hexane/2-propanol 96:4, $1.0 \mathrm{~mL} / \mathrm{min}, 210 \mathrm{~nm}, 30^{\circ} \mathrm{C},(1 R, 2 S)$ isomer $9.2 \mathrm{~min}$ (major), $(1 S, 2 R)$ isomer 10.5 min (minor)); $[\alpha]_{\mathrm{D}}^{20}+89.5$ (c 0.44 in $\left.\mathrm{CHCl}_{3}\right)$ cis/trans $=$ $>99 / 1,96 \%$ ee $(1 R, 2 S)$. 


\section{E. Synthesis of (+)-PHNO.}

(a) Synthesis of 7-(benzyloxy)-2-chloro-3,4-dihydronaphthalen-1(2H)-one (10) ${ }^{[1]}$

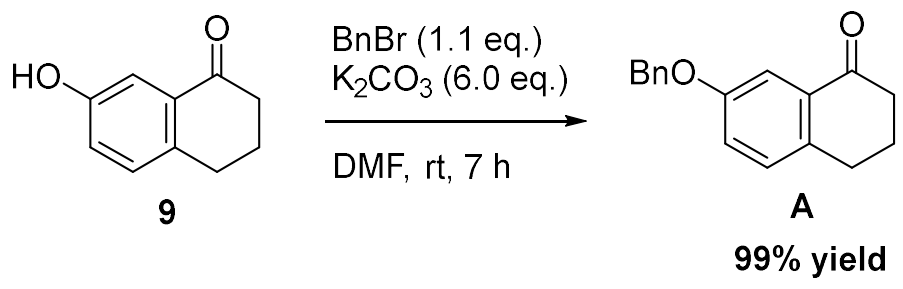

A suspension of 7-hydroxy-3,4-dihydronaphthalen-1(2H)-one (9) $(4.21 \mathrm{~g}, 26.0 \mathrm{mmol})$ and $\mathrm{K}_{2} \mathrm{CO}_{3}(21.56 \mathrm{~g}, 156.0 \mathrm{mmol})$ in DMF $(85 \mathrm{~mL})$ was added to benzyl bromide $4.88 \mathrm{~g}, 3.39$ $\mathrm{mL}, 28.6 \mathrm{mmol}$ ) at room temperature. The reaction mixture was stirred for $7 \mathrm{~h}$. The reaction mixture was poured to cooled water $(300 \mathrm{~mL})$, then AcOEt $(300 \mathrm{~mL})$ was added and stirred for $10 \mathrm{~min}$. The organic layers were separated, the aqueous phase was extracted with AcOEt $(2 \times 300 \mathrm{~mL})$, and the combined organic portions were dried over $\mathrm{MgSO}_{4}$, and concentrated to give a crude product. Purification by silica gel column chromatography (eluent: Hexane/AcOEt $=10 / 1$ to 5/1) gave the product,

7-(benzyloxy)-3,4-dihydronaphthalen-1(2H)-one (A), as a white solid (6.48 g, 99\% yield).

\section{Characterization data for $\mathbf{A}$}

${ }^{1} \mathrm{H}$ NMR $\left(500 \mathrm{MHz}, \mathrm{CDCl}_{3}\right) \delta 7.61(\mathrm{~d}, J=2.7 \mathrm{~Hz}, 1 \mathrm{H}), 7.46-7.43(\mathrm{~m}, 2 \mathrm{H}), 7.38(\mathrm{t}, J=7.2$ $\mathrm{Hz}, 2 \mathrm{H}), 7.34-7.30(\mathrm{~m}, 1 \mathrm{H}), 7.18-7.17(\mathrm{~m}, 1 \mathrm{H}), 7.12$ (dd, $J=8.4,2.7 \mathrm{~Hz}, 1 \mathrm{H}), 5.09$ (s, 2H), $2.90(\mathrm{t}, J=5.3 \mathrm{~Hz}, 2 \mathrm{H}), 2.63(\mathrm{t}, J=6.3 \mathrm{~Hz}, 2 \mathrm{H}), 2.14-2.09(\mathrm{~m}, 2 \mathrm{H}) ;{ }^{13} \mathrm{C}\left\{{ }^{1} \mathrm{H}\right\} \mathrm{NMR}$ $\left(125 \mathrm{MHz}, \mathrm{CDCl}_{3}\right) \delta 198.3,157.5,137.4,136.7,133.4,130.0,128.6,128.0,127.5,122.3$, $110.3,70.1,39.0,28.9,23.4$.

HRMS (APCI) $m / z$ : [M+H] $]^{+}$calcd for $\mathrm{C}_{17} \mathrm{H}_{10} \mathrm{O}_{2}: 253.1223$; found: 253.1223 .<smiles>O=C1CCCc2ccc(OBr)cc21</smiles>

A

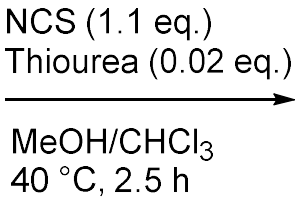

$40^{\circ} \mathrm{C}, 2.5 \mathrm{~h}$<smiles>O=C1c2cc(OCBr)ccc2CCC1Cl</smiles>

$93 \%$ yield

Subsequently, a mixed solution of $\mathrm{MeOH}(55 \mathrm{~mL})$ and chloroform $(11 \mathrm{~mL})$ containing 7-(benzyloxy)-3,4-dihydronaphthalen-1(2H)-one (A) $(5.50 \mathrm{~g}, 19.8 \mathrm{mmol})$, thiourea $(33.0$ $\mathrm{mg}, 0.40 \mathrm{mmol})$ and $N$-chlorosuccinimide $(3.20 \mathrm{~g}, 21.8 \mathrm{mmol}, 1.10 \mathrm{eq}$.$) was heated under$ $\mathrm{N}_{2}$ atmosphere at $40{ }^{\circ} \mathrm{C}$, for $2.5 \mathrm{~h}$. The solution was concentrated under pressure, then AcOEt $(100 \mathrm{~mL})$ and $\mathrm{H}_{2} \mathrm{O}(80 \mathrm{~mL})$ were added and stirred $10 \mathrm{~min}$. The organic layers were separated, the aqueous phase was extracted with AcOEt $(2 \times 100 \mathrm{~mL})$, and the combined organic portions were dried over $\mathrm{MgSO}_{4}$, and concentrated to give a crude product. Purification by silica gel column chromatography (eluent: Hexane/AcOEt $=10 / 1$ to $5 / 1$ ) gave the product (10) as a white solid (5.8 g, 93\% yield). 
Characterization data for $\mathbf{1 0}$

${ }^{1} \mathrm{H}$ NMR $\left(500 \mathrm{MHz}, \mathrm{CDCl}_{3}\right) \delta 7.64(\mathrm{~d}, J=2.0 \mathrm{~Hz}, 1 \mathrm{H}), 7.45-7.43(\mathrm{~m}, 2 \mathrm{H}), 7.39(\mathrm{t}, J=7.0$ $\mathrm{Hz}, 2 \mathrm{H}), 7.35-7.31(\mathrm{~m}, 1 \mathrm{H}), 7.19-7.18(\mathrm{~m}, 2 \mathrm{H}), 4.62(\mathrm{dd}, J=8.0,3.5 \mathrm{~Hz}, 1 \mathrm{H}), 3.22$ (ddd, $J=14.5,9.8,4.6 \mathrm{~Hz}, 1 \mathrm{H}), 2.93(\mathrm{ddd}, J=14.5,9.8,4.6 \mathrm{~Hz}, 1 \mathrm{H}), 2.59-2.53(\mathrm{~m}, 1 \mathrm{H})$, $2.48-2.41(\mathrm{~m}, 1 \mathrm{H}) ;{ }^{13} \mathrm{C}\left\{{ }^{1} \mathrm{H}\right\} \mathrm{NMR}\left(125 \mathrm{MHz}, \mathrm{CDCl}_{3}\right) \delta 190.7,157.8,136.4,136.0,131.2$, 130.0, 128.6, 128.1, 127.6, 123.3, 11.4, 70.2, 59.7, 32.6, 25.5.

HRMS (APCI) $m / z:[\mathrm{M}+\mathrm{H}]^{+}$calcd for $\mathrm{C}_{17} \mathrm{H}_{15} \mathrm{ClO}_{2}: 287.0833$; found: 287.0837 .

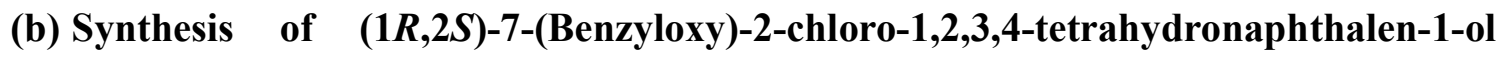
(11)<smiles>O=C1c2cc(OCc3ccccc3)ccc2CCC1Cl</smiles>

10

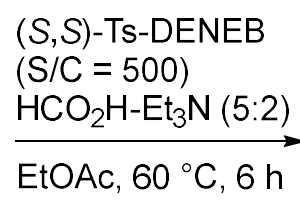

EtOAc $60^{\circ} \mathrm{C}, 6 \mathrm{~h}$<smiles>O[C@H]1c2cc(O[Bi])ccc2CC[C@@H]1Cl</smiles>

$98 \%$ yield

cis/trans $=>99 / 1$

$>99.9 \%$ ee

Under $\quad \mathrm{N}_{2}$ atmosphere, a mixture of 7-(benzyloxy)-2-chloro-3,4-dihydronaphthalen-1(2H)-one (10) $(4.00 \mathrm{~g}, 14.0 \mathrm{mmol})$ and $(S, S)$-Ts-DENEB $(22.7 \mathrm{mg}, 0.035 \mathrm{mmol}, \mathrm{S} / \mathrm{C}=500)$ in an azeotrope of formic acid and triethylamine $(5: 2$ molar ratios, $7.0 \mathrm{~mL})$ and AcOEt $(28.0 \mathrm{~mL})$ was stirred at $60{ }^{\circ} \mathrm{C}$ for $5 \mathrm{~h}$. After the reaction completion, water $(50 \mathrm{~mL})$ and AcOEt $(50 \mathrm{~mL})$ was added. The biphasic layers were separated, the aqueous layer was extracted with AcOEt $(4 \times 50 \mathrm{~mL})$, and the combined organic portions were washed with brine $(20 \mathrm{~mL})$. After drying over $\mathrm{MgSO}_{4}$, filtration, and solvent removal under reduced pressure, the crude residue was purified by silica gel column chromatography (eluent: Hexane/AcOEt $=7 / 1$ to $4 / 1$ ) to afford the product (11) as a white solid (3.95 g, 98\% yield, cis/trans $=>99 / 1,>99.9 \%$ ee).

${ }^{1} \mathrm{H}$ NMR $\left(500 \mathrm{MHz}, \mathrm{CDCl}_{3}\right) \delta$ 7.44-7.41 (m, 2H), 7.40-7.37 (m, 2H), 7.33-7.30 (m, 1H), $7.13(\mathrm{~d}, J=2.6 \mathrm{~Hz}, 1 \mathrm{H}), 7.04-7.72(\mathrm{~m}, 1 \mathrm{H}), 6.89(\mathrm{dd}, J=8.4,2.6 \mathrm{~Hz}, 1 \mathrm{H}), 5.06(\mathrm{~d}, J=3.2$, $2 \mathrm{H}), 4.81(\mathrm{dd}, J=8.0,3.2 \mathrm{~Hz}, 1 \mathrm{H}), 4.55(\mathrm{dt}, J=5.6,3.2 \mathrm{~Hz}, 1 \mathrm{H}), 3.04(\mathrm{dt}, J=11.8,6.5 \mathrm{~Hz}$, $1 \mathrm{H}), 2.76(\mathrm{dt}, J=11.8,6.5 \mathrm{~Hz}, 1 \mathrm{H}), 2.43(\mathrm{~d}, J=8.0 \mathrm{~Hz}, 1 \mathrm{H}), 2.42-2.35(\mathrm{~m}, 1 \mathrm{H}), 2.22-2.15$ $(\mathrm{m}, 1 \mathrm{H}) ;{ }^{13} \mathrm{C}\left\{{ }^{1} \mathrm{H}\right\}$ NMR $\left(125 \mathrm{MHz}, \mathrm{CDCl}_{3}\right) \delta 157.5,137.0,136.9,129.6,128.6,127.9$, 127.5, 127.1, 115.8, 113.8, 70.2, 70.1, 63.3, 27.9, 25.5.

HRMS (CI) $m / z$ : [M] $]^{+}$calcd for $\mathrm{C}_{17} \mathrm{H}_{17} \mathrm{ClO}_{2}$ : 288.0912; found: 288.0911 .

The enantiomeric excess was determined by HPLC analysis (Chiralcel OZ-3, $250 \times 4.6$ $\mathrm{mm}$ column, hexane/2-propanol 95:5, $1.0 \mathrm{~mL} / \mathrm{min}, 220 \mathrm{~nm}, 35^{\circ} \mathrm{C},(1 R, 2 S)$ isomer 10.0 $\min$ (major), $(1 S, 2 R)$ isomer $11.5 \mathrm{~min}$ (minor))

$[\alpha]_{\mathrm{D}}^{20}+44.7\left(\mathrm{c} 0.53\right.$ in $\left.\mathrm{CHCl}_{3}\right)$ cis/trans $=>99 / 1,>99.9 \%$ ee $(1 R, 2 S)$. 
(c) Synthesis of (1R,2R)-2-Amino-7-(benzyloxy)-1,2,3,4-tetrahydronaphthalen-1-ol (12)<smiles>OC1c2cc(OCc3ccccc3)ccc2CCC1Cl</smiles>

11
$\underset{\mathrm{DMF}, 95^{\circ} \mathrm{C}, 8 \mathrm{~h}}{\stackrel{\mathrm{NaN}_{3}(4.0 \text { eq. })}{\longrightarrow}}$<smiles>N[C@H]1CCc2ccc(OCc3ccccc3)cc2[C@H]1O</smiles>

B

$75 \%$ yield

$(1 R, 2 S)$-7-(benzyloxy)-2-chloro-1,2,3,4-tetrahydronaphthalen-1-ol $\quad(\mathbf{1 1}) \quad(3.00 \mathrm{~g}, \quad 10.4$ mmol) was dissolved in DMF $(156 \mathrm{~mL})$ and $\mathrm{NaN}_{3}(2.70 \mathrm{~g}, 41.6 \mathrm{mmol})$ was added. ${ }^{[8]}$ The reaction mixture was heated at $95{ }^{\circ} \mathrm{C}$ for $8 \mathrm{~h}$, cooled to ambient temperature, quenched with water $(400 \mathrm{~mL})$ and extracted with AcOEt $/$ hexane $=7 / 3$ solution $(4 \times 300 \mathrm{~mL})$. Then the combined organic portions were dried over $\mathrm{MgSO}_{4}$ and concentrated to give a crude product. Purification by silica gel column chromatography (eluent: Hexane/AcOEt $=15 / 1$ to $10 / 1)$ gave the product,

$(1 R, 2 R)$-2-azido-7-(benzyloxy)-1,2,3,4-tetrahydronaphthalen-1-ol (B), as a white solid (2.3 g, $75 \%$ yield).

\section{Characterization data for $\mathbf{B}$}

${ }^{1} \mathrm{H}$ NMR $\left(500 \mathrm{MHz}, \mathrm{CDCl}_{3}\right) \delta$ 7.44-7.41 (m, 2H), 7.40-7.37 (m, 2H), 7.34-7.30 (m, 1H), $7.16(\mathrm{~d}, J=2.5 \mathrm{~Hz}, 1 \mathrm{H}), 7.01(\mathrm{~d}, J=8.4 \mathrm{~Hz}, 1 \mathrm{H}), 6.86(\mathrm{dd}, J=8.4,2.5 \mathrm{~Hz}, 1 \mathrm{H}), 5.06(\mathrm{~d}, J$ $=2.2 \mathrm{~Hz}, 2 \mathrm{H}), 4.59(\mathrm{dd}, J=7.7,5.5 \mathrm{~Hz}, 1 \mathrm{H}), 3.71-3.66(\mathrm{~m}, 1 \mathrm{H}), 2.85(\mathrm{dd}, J=7.7,5.5 \mathrm{~Hz}$, $2 \mathrm{H}), 2.31(\mathrm{~d}, J=5.5 \mathrm{~Hz}, 1 \mathrm{H}), 2.24-2.18(\mathrm{~m}, 1 \mathrm{H}), 1.92-1.84(\mathrm{~m}, 1 \mathrm{H}) ;{ }^{13} \mathrm{C}\left\{{ }^{1} \mathrm{H}\right\} \operatorname{NMR}(125$ $\left.\mathrm{MHz}, \mathrm{CDCl}_{3}\right) \delta 157.6,137.4,137.0,139.5,128.6,128.0,127.5,127.4,115.4,112.7,72.7$, 70.1, 64.5, 26.5, 25.8.

HRMS (FI) $m / z:[\mathrm{M}]^{+}$calcd for $\mathrm{C}_{17} \mathrm{H}_{17} \mathrm{~N}_{3} \mathrm{O}_{2}: 295.1321$; found: 295.1320 .

$[\alpha]_{\mathrm{D}}^{20}+52.9$ (c 3.58 in $\left.\mathrm{CHCl}_{3}\right)>99 \%$ ee $(1 R, 2 R)$.<smiles>N[C@H]1CCc2ccc(OCc3ccccc3)cc2[C@H]1O</smiles>

B

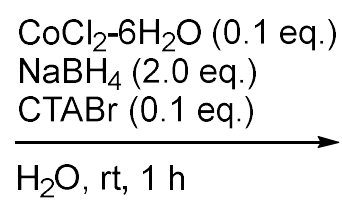

$\mathrm{H}_{2} \mathrm{O}, \mathrm{rt}, 1 \mathrm{~h}$<smiles>N[C@H]1CCc2ccc(OCc3ccccc3)cc2[C@H]1O</smiles>

12

$87 \%$ yield

Then, a solution of $\mathrm{NaBH}_{4}\left(454 \mathrm{mg}, 6.0 \mathrm{mmol}, 2.0\right.$ eq.) in $\mathrm{H}_{2} \mathrm{O}(12 \mathrm{~mL})$ was added dropwise to a mixture of (1R,2R)-2-azido-7-(benzyloxy)-1,2,3,4-tetrahydronaphthalen-1-ol (B) $(1.77 \mathrm{~g}, 6.00 \mathrm{mmol}), \mathrm{CoCl}_{2} \cdot 6 \mathrm{H}_{2} \mathrm{O}$ (71.4 mg, $\left.0.6 \mathrm{mmol}, 0.1 \mathrm{eq}.\right)$, and $\mathrm{CTABr}$ (cetyltrimethyammonium bromide) $(218.6 \mathrm{mg}, 0.6 \mathrm{mmol})$ under stirring at $25{ }^{\circ} \mathrm{C}$. ${ }^{[9]}$ Formation of a black precipitate indicated in situ generation of cobalt boride species. The mixute was stirred at $25{ }^{\circ} \mathrm{C}$ for $30 \mathrm{~min}$. At the end of the reaction, the mixture was extracted with $\mathrm{Et}_{2} \mathrm{O}(5 \times 70 \mathrm{~mL})$. The combined organic portions were dried over $\mathrm{MgSO}_{4}$ and concentrated to give a crude product. Purification by silica gel column chromatography (eluent: $\mathrm{CHCl}_{3} / \mathrm{MeOH}=20 / 1$ to 3/1) gave the product (12) as a light brown solid (1.4 g, 87\% yield). 
Characterization data for $\mathbf{1 2}$

${ }^{1} \mathrm{H}$ NMR $\left(500 \mathrm{MHz}, \mathrm{CDCl}_{3}\right) \delta$ 7.44-7.41 (m, 2H), 7.39-7.35 (m, 2H), 7.33-7.29 (m, 1H), $7.22-7.18(\mathrm{~m}, 1 \mathrm{H}), 6.99(\mathrm{~d}, J=8.4 \mathrm{~Hz}, 1 \mathrm{H}), 6.83(\mathrm{dd}, J=8.4,2.7 \mathrm{~Hz}, 1 \mathrm{H}), 5.06(\mathrm{~d}, J=2.6$ $\mathrm{Hz}, 2 \mathrm{H}), 4.32(\mathrm{~d}, J=8.2 \mathrm{~Hz}, 1 \mathrm{H}), 2.89-2.77(\mathrm{~m}, 3 \mathrm{H}), 2.10-1.85(\mathrm{~m}, 4 \mathrm{H}), 1.40-1.35(\mathrm{~m}$, $1 \mathrm{H}) ;{ }^{13} \mathrm{C}\left\{{ }^{1} \mathrm{H}\right\}$ NMR $\left(125 \mathrm{MHz}, \mathrm{CDCl}_{3}\right) \delta 157.5,139.2,137.2,129.4,128.5,128.1,127.9$, 127.5, 114.8, 112.3, 75.6, 70.1, 54.7, 30.6, 27.4.

HRMS (ESI) $m / z:[\mathrm{M}+\mathrm{H}]^{+}$calcd for $\mathrm{C}_{17} \mathrm{H}_{19} \mathrm{NO}_{2}: 270.1489$; found: 270.1487 .

$[\alpha]_{\mathrm{D}}^{20}+52.2\left(\mathrm{c} 0.15\right.$ in $\left.\mathrm{CHCl}_{3}\right)>99 \%$ ee $(1 R, 2 R)$.

\section{(d) Synthesis of}

(4aR,10bR)-9-(Benzyloxy)-4a,5,6,10b-tetrahydro-2H-naphtho[1,2-b][1,4]oxazin-3( 4H)-one (13) ${ }^{[10]}$

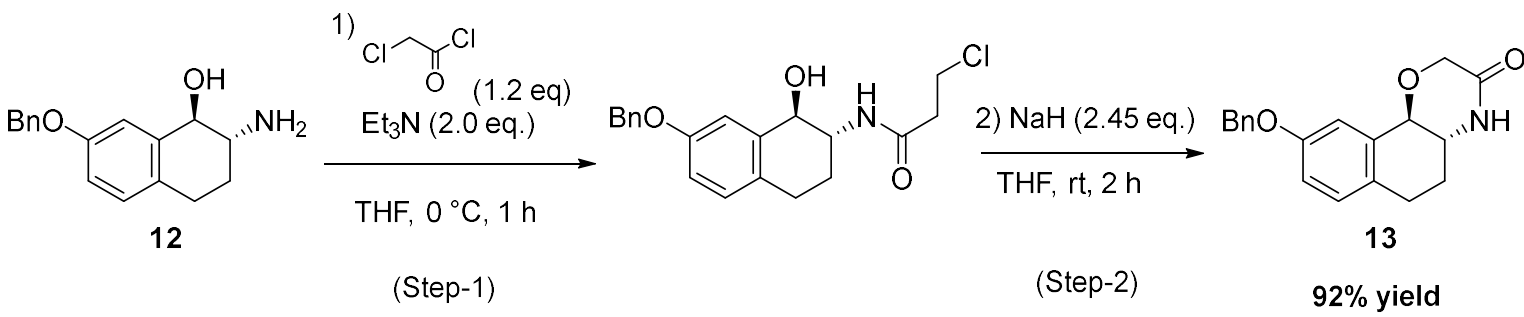

To a solution of $(1 R, 2 R)$-2-amino-7-(benzyloxy)-1,2,3,4-tetrahydronaphthalen-1-ol (12) $(600 \mathrm{mg}, 2.23 \mathrm{mmol})$ and triethylamine $(337.9 \mathrm{mg}, 462.8 \mu \mathrm{L}, 3.34 \mathrm{mmol}, 1.5 \mathrm{eq}$.$) in THF$ $(12 \mathrm{~mL})$ at $0{ }^{\circ} \mathrm{C}$ was added chloroacetyl chloride $(301.68 \mathrm{mg}, 212.5 \mu \mathrm{L}, 2.67 \mathrm{mmol}, 1.2$ eq.). The reaction mixture was stirred at $0{ }^{\circ} \mathrm{C}$ for $1 \mathrm{~h}$. After this time, a saturated aqueous $\mathrm{NH}_{4} \mathrm{Cl}$ solution $(12 \mathrm{~mL})$ and AcOEt $(30 \mathrm{~mL})$ were added. The mixture was extracted with $\operatorname{AcOEt}(3 \times 30 \mathrm{~mL})$. The combined organic portions were washed with water $(2 \times 10 \mathrm{~mL})$, dried over $\mathrm{MgSO}_{4}$, filered and the filtrate was concentrated under vacuum to give a crude product containing $N$-((1R,2R)-7-(benzyloxy)-1-hydroxy-1,2,3,4-tetrahydronaphthalen-2yl)-3-chloropropanamide. Subsequently, the product was dissolved in THF (12 mL) and treated with several portions of sodium hydride (60\% dispersion in mineral oil, $217.8 \mathrm{mg}$, 2.45 eq.) over a period of $5 \mathrm{~min}$. The mixture was stirred at room temperature for $2 \mathrm{~h}$. After this time a saturated aqueous $\mathrm{NH}_{4} \mathrm{Cl}$ solution $(12 \mathrm{~mL})$ and AcOEt $(30 \mathrm{~mL})$ were added. The mixture was extracted with AcOEt $(3 \times 30 \mathrm{~mL})$. The combined organic portions were washed with water $(2 \times 10 \mathrm{~mL})$, dried over $\mathrm{MgSO}_{4}$, filered and the filtrate was concentrated under vacuum to give a crude product. Purification by silica gel column chromatography (eluent: Hexane/AcOEt $=2 / 1$ to $1 / 1$ ) gave the product (13) as a white solid (634 mg, 92\% yield).

${ }^{1} \mathrm{H}$ NMR (500 MHz, $\left.\mathrm{CDCl}_{3}\right) \delta$ 7.44-7.42 (m, 2H), 7.40-7.37 (m, 2H), 7.34-7.30 (m, 1H), $7.18(\mathrm{~d}, J=2.5 \mathrm{~Hz}, 1 \mathrm{H}), 7.04-7.01(\mathrm{~m}, 1 \mathrm{H}), 6.87(\mathrm{dd}, J=8.4,2.5 \mathrm{~Hz}, 1 \mathrm{H}), 6.23(\mathrm{br}, 1 \mathrm{H})$, $5.06(\mathrm{~d}, J=3.5 \mathrm{~Hz}, 2 \mathrm{H}), 4.52-4.40(\mathrm{~m}, 3 \mathrm{H}), 3.67-3.62(\mathrm{~m}, 1 \mathrm{H}), 2.95-2.90(\mathrm{~m}, 2 \mathrm{H})$, 2.09-2.02 (m, 1H), 1.90-1.83 (m, 1H); ${ }^{13} \mathrm{C}\left\{{ }^{1} \mathrm{H}\right\}$ NMR (125 MHz, $\left.\mathrm{CDCl}_{3}\right) \delta 169.2,157.6$, 137.0, 134.8, 129.6, 128.6, 127.9, 127.5, 126.7, 115.6, 110.4, 77.1, 70.1, 68.6, 53.5, 27.5, 26.4.

HRMS (APCI) $m / z:[\mathrm{M}+\mathrm{H}]^{+}$calcd for $\mathrm{C}_{19} \mathrm{H}_{19} \mathrm{NO}_{3}: 310.1438$; found: 310.1437 .

$[\alpha]_{\mathrm{D}}^{20}-4.46\left(\mathrm{c} 0.19\right.$ in $\left.\mathrm{CHCl}_{3}\right)>99 \%$ ee $(4 \mathrm{a} R, 10 \mathrm{~b} R)$. 
(e) Synthesis of

(4aR,10bR)-9-(Benzyloxy)-4-propionyl-4a,5,6,10b-tetrahydro-2H-naphtho[1,2-b][1 ,4]oxazin-3(4H)-one (14)<smiles>O=C1CO[C@H]2c3cc(OCc4ccccc4)ccc3CC[C@H]2N1</smiles>

13

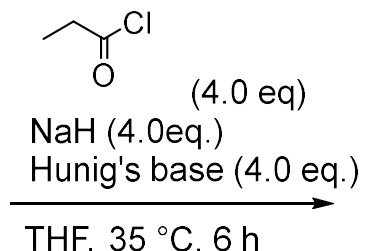

THF, $35^{\circ} \mathrm{C}, 6 \mathrm{~h}$<smiles>CCC(=O)N1C(=O)CO[C@H]2c3cc(OCc4ccccc4)ccc3CC[C@H]21</smiles>

14

$86 \%$ yield

(4a $R, 10 \mathrm{~b} R$ )-9-(benzyloxy)-4a,5,6,10b-tetrahydro-2H-naphtho[1,2-b][1,4]oxazin-3(4H)-one (13) $(155.0 \mathrm{mg}, 0.5 \mathrm{mmol})$ was dissolved in THF $(30 \mathrm{~mL})$ at room temperature. Hunig's base $(258.5 \mathrm{mg}, 4.0 \mathrm{mmol})$ and sodium hydride (60\% dispersion in mineral oil, $40.0 \mathrm{mg}$, 4.0 eq.) was then added to the reaction solution and allowed to stir for $10 \mathrm{~min}$. Propionyl chloride $(50.9 \mathrm{mg}, 48.0 \mu \mathrm{L}, 0.55 \mathrm{mmol}, 1.1$ eq.) was then added at the same temperature. The reaction was allowed to stir at $35^{\circ} \mathrm{C}$ for $6 \mathrm{~h}$. After this time, water $(30 \mathrm{~mL})$ and AcOEt $(50 \mathrm{~mL})$ were added. The mixture was extracted with AcOEt $(3 \times 50 \mathrm{~mL})$. The combined organic portions were washed with water $(2 \times 10 \mathrm{~mL})$, dried over $\mathrm{MgSO}_{4}$, filered and the filtrate was concentrated under vacuum to give a crude product. Purification by silica gel column chromatography (eluent: Hexane/AcOEt $=20 / 1$ to $10 / 1$ ) gave the product (14) as a white solid (157 mg, 86\% yield).

${ }^{1} \mathrm{H}$ NMR $\left(500 \mathrm{MHz}, \mathrm{CDCl}_{3}\right) \delta$ 7.45-7.42 (m, 2H), 7.39-7.37 (m, 2H), 7.33-7.30 (m, 1H), $7.15(\mathrm{~d}, J=2.4 \mathrm{~Hz}, 1 \mathrm{H}), 7.06-7.04(\mathrm{~m}, 1 \mathrm{H}), 6.88(\mathrm{dd}, J=8.4,2.6, \mathrm{~Hz}, 1 \mathrm{H}), 5.06(\mathrm{~d}, J=$ $2.6 \mathrm{~Hz}, 2 \mathrm{H}), 4.65-4.59(\mathrm{~m}, 1 \mathrm{H}), 4.47-4.42(\mathrm{~m}, 1 \mathrm{H}), 4.09(\mathrm{dt}, J=10.7,2.6, \mathrm{~Hz}, 1 \mathrm{H})$, $3.06-2.83(\mathrm{~m}, 4 \mathrm{H}), 2.69-2.63(\mathrm{~m}, 1 \mathrm{H}), 1.57-1.43(\mathrm{~m}, 2 \mathrm{H}), 1.17(\mathrm{t}, J=7.3 \mathrm{~Hz}, 3 \mathrm{H})$; ${ }^{13} \mathrm{C}\left\{{ }^{1} \mathrm{H}\right\}$ NMR $\left(125 \mathrm{MHz}, \mathrm{CDCl}_{3}\right) \delta 177.5,170.6,157.5,137.0,135.5,129.5,128.6,128.0$, 127.5, 127.0, 115.4, 110.1, 77.4, 70.5, 70.1, 57.2, 33.8, 27.0, 26.1, 8.9.

HRMS (APCI) $m / z$ : [M+H] calcd for $\mathrm{C}_{22} \mathrm{H}_{23} \mathrm{NO}_{4}: 366.1670$; found: 366.1698 .

$[\alpha]_{\mathrm{D}}{ }^{20}-192.0\left(\mathrm{c} 0.83\right.$ in $\left.\mathrm{CHCl}_{3}\right)>99 \%$ ee $(4 \mathrm{a} R, 10 \mathrm{~b} R)$.

(f) Synthesis of (+)-PHNO:

$(4 \mathrm{a} R, 10 \mathrm{~b} R)$-4-Propyl-3,4,4a,5,6,10b-hexahydro-2 $H$-naphtho[1,2-b][1,4]oxazin-9-ol<smiles>[B]Oc1ccc2c(c1)[C@@H]1OCC(=O)N(C(=O)CC)[C@@H]1CC2</smiles>

14

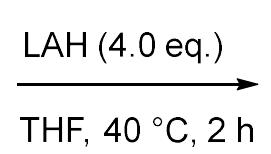

THF, $40^{\circ} \mathrm{C}, 2 \mathrm{~h}$<smiles>CCCN1CCO[C@@H]2c3cc(OCc4ccccc4)ccc3CC[C@H]21</smiles>

C

$85 \%$ yield

A suspension of $(4 \mathrm{a} R, 10 \mathrm{~b} R)-9$-(benzyloxy)-4-propionyl-4a,5,6,10b-tetrahydro- $2 \mathrm{H}$ naphtho[1,2-b][1,4]oxazin-3(4H)-one (14) $(100 \mathrm{mg}, 0.238 \mathrm{mmol})$ in THF $(5 \mathrm{~mL})$ was added to a $1 \mathrm{M}$ solution of $\mathrm{LiAlH}_{4}$ in $\mathrm{Et}_{2} \mathrm{O}(1.09 \mathrm{~mL}, 2.74 \mathrm{mmol}, 4.0$ eq. $)$ at $5{ }^{\circ} \mathrm{C}$. The 
reaction mixture was allowed to warm to $40{ }^{\circ} \mathrm{C}$ then stirred for $2 \mathrm{~h}$. After cooling $\left(5^{\circ} \mathrm{C}\right)$, a saturated aqueous solution of Rochell salt $(5 \mathrm{~mL})$ and tert-BuOMe $(20 \mathrm{~mL})$ were added. The mixture was extracted with tert-BuOMe $(2 \times 20 \mathrm{~mL})$ and AcOEt $(2 \times 20 \mathrm{~mL})$. The combined organic portions were dried over $\mathrm{MgSO}_{4}$, filered and the filtrate was concentrated under vacuum to give a crude product. Purification by silica gel column chromatography (eluent: Hexane/AcOEt $=5 / 1$ to $3 / 1$ ) gave the product, $(4 \mathrm{a} R, 10 \mathrm{~b} R)-9-($ benzyloxy)-4-propyl-3,4,4a,5,6,10b-

hexahydro-2H-naphtho[1,2-b][1,4]oxazine (C), as a white solid (78.5 mg, 85\% yield).

Characterization data for $\mathbf{C}$

${ }^{1} \mathrm{H}$ NMR $\left(500 \mathrm{MHz}, \mathrm{CDCl}_{3}\right) \delta 7.45-7.41(\mathrm{~m}, 2 \mathrm{H}), 7.40-7.36(\mathrm{~m}, 2 \mathrm{H}), 7.33-7.29(\mathrm{~m}, 1 \mathrm{H})$, $7.19(\mathrm{~d}, J=2.3 \mathrm{~Hz}, 1 \mathrm{H}), 7.00-6.97(\mathrm{~m}, 1 \mathrm{H}), 6.82(\mathrm{dd}, J=8.6,2.3, \mathrm{~Hz}, 1 \mathrm{H}), 5.05(\mathrm{~d}, J=$ $5.9 \mathrm{~Hz}, 2 \mathrm{H}), 4.31(\mathrm{~d}, J=8.6 \mathrm{~Hz}, 1 \mathrm{H}), 4.08(\mathrm{dd}, J=11.5,2.4 \mathrm{~Hz}, 1 \mathrm{H}), 3.92$ (dt, $J=11.5$, $2.4 \mathrm{~Hz}, 1 \mathrm{H}), 2.92-2.86(\mathrm{~m}, 1 \mathrm{H}), 2.86-2.70(\mathrm{~m}, 3 \mathrm{H}), 2.48(\mathrm{dt}, J=11.5,3.4 \mathrm{~Hz}, 1 \mathrm{H})$, 2.30-2.23 (m, 3H), 1.60-1.48 (m, 3H), $0.92(\mathrm{t}, J=7.4 \mathrm{~Hz}, 3 \mathrm{H}) ;{ }^{13} \mathrm{C}\left\{{ }^{1} \mathrm{H}\right\}$ NMR $(125 \mathrm{MHz}$, $\left.\mathrm{CDCl}_{3}\right) \delta 157.5,137.6,137.3,129.0,128.5,127.8,127.5,127.4,114.8,110.5,79.0,70.0$, 67.4, 62.6, 55.2, 52.4, 27.3, 24.3, 18.8, 12.0.

HRMS (ESI) $m / z$ : $[\mathrm{M}+\mathrm{H}]^{+}$calcd for $\mathrm{C}_{22} \mathrm{H}_{27} \mathrm{NO}_{2}$ : 338.2115; found: 338.2119 .<smiles>CCCN1CCO[C@@H]2c3cc(O[Hg])ccc3CC[C@H]21</smiles>

C

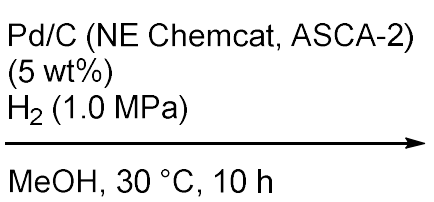

$\mathrm{MeOH}, 30^{\circ} \mathrm{C}, 10 \mathrm{~h}$<smiles>CCCN1CCO[C@@H]2c3cc(O)ccc3CC[C@H]21</smiles>

(+)-PHNO

$>99 \%$ yield cis/trans $=>99 / 1,99.8 \%$ ee

Subsequently, in a cylinder type $50 \mathrm{~mL}$ stainless steel autoclave, a mixture of (4a $R, 10 \mathrm{~b} R$ )-9-(benzyloxy)-4-propyl-3,4,4a,5,6,10b-hexahydro-2H-naphtho[1,2-b][1,4] oxaz ine $(\mathbf{C})(50 \mathrm{mg}, 0.148 \mathrm{mmol})$ and $5 \%$ palladium on carbon (wet, NE Chemcat, type ASCA-2, $2.5 \mathrm{mg}, 5 \mathrm{wt} \%)$ in $\mathrm{MeOH}(10 \mathrm{~mL})$ was stirred at $30{ }^{\circ} \mathrm{C}$ for $10 \mathrm{~h}$ under hydrogen atmosphere (1.0 MPa). The mixture was filtered through a pad of Celite, and the solvent was removed under reduced pressure to give the product (+)-PHNO as a white solid. (36.6 $\mathrm{mg},>99 \%$ yield. cis/trans $=>99 / 1,99.8 \%$ ee)

\section{Characterization data for (+)-PHNO}

${ }^{1} \mathrm{H}$ NMR $\left(500 \mathrm{MHz}, \mathrm{CD}_{3} \mathrm{OD}\right) \delta 6.96(\mathrm{~d}, J=8.3 \mathrm{~Hz}, 1 \mathrm{H}), 6.92(\mathrm{~d}, J=2.4 \mathrm{~Hz}, 1 \mathrm{H}), 6.69(\mathrm{dd}$, $J=8.3,2.4 \mathrm{~Hz}, 1 \mathrm{H}), 4.70(\mathrm{~d}, J=9.5 \mathrm{~Hz}, 1 \mathrm{H}), 4.16-4.11(\mathrm{~m}, 1 \mathrm{H}), 4.14(\mathrm{t}, J=12.4 \mathrm{~Hz}, 1 \mathrm{H})$, $3.64(\mathrm{~d}, J=12.4 \mathrm{~Hz}, 1 \mathrm{H}), 3.47(\mathrm{dt}, J=12.4,5.0 \mathrm{~Hz}, 1 \mathrm{H}), 3.34-3.23(\mathrm{~m}, 2 \mathrm{H}), 3.09(\mathrm{dt}, J=$ $12.4,5.0 \mathrm{~Hz}, 1 \mathrm{H}), 2.94-2.90(\mathrm{~m}, 2 \mathrm{H}), 2.58-2.52(\mathrm{~m}, 1 \mathrm{H}), 1.93-1.85(\mathrm{~m}, 2 \mathrm{H}), 1.80-1.72(\mathrm{~m}$, $1 \mathrm{H}), 1.07(\mathrm{t}, J=7.4 \mathrm{~Hz}, 3 \mathrm{H}) ;{ }^{13} \mathrm{C}\left\{{ }^{1} \mathrm{H}\right\}$ NMR $\left(125 \mathrm{MHz}, \mathrm{CD}_{3} \mathrm{OD}\right) \delta 157.2,135.7,130.3$, $125.8,116.7,112.8,77.8,65.5,65.4,55.6,52.5,27.5,23.2,18.0,11.2$.

HRMS (ESI) $m / z:[\mathrm{M}+\mathrm{H}]^{+}$calcd for $\mathrm{C}_{15} \mathrm{H}_{21} \mathrm{NO}_{2}: 248.1645$; found: 248.1643 .

The enantiomeric excess was determined by HPLC analysis (Chiralcel OZ-3, $250 \times 4.6$ $\mathrm{mm}$ column, hexane/2-propanol 90:10, $1.0 \mathrm{~mL} / \mathrm{min}, 220 \mathrm{~nm}, 30{ }^{\circ} \mathrm{C}$, (-)-PHNO $8.2 \mathrm{~min}$ (minor), (+)-PHNO 9.7 min (major))

$[\alpha]_{\mathrm{D}}{ }^{20}+58.2(\mathrm{c} 0.06$ in EtOH $)$ cis/trans $=>99 / 1,99.8 \%$ ee $(4 \mathrm{a} R, 10 \mathrm{~b} R) .\left(\right.$ lit. $^{[11]}[\alpha]_{\mathrm{D}}{ }^{24}+59.54$ (c 0.0964 in $\mathrm{EtOH})(4 \mathrm{a} R, 10 \mathrm{~b} R))$. 


\section{F. Determination of Absolute Configuration of Products.}

a) Synthesis of Methyl

$(1 R, 2 R)-1-((4,5-d i c h l o r o-2-((3 a R, 6 R, 7 \mathrm{a} R)-8,8$-dimethyl-2,2-dioxidohexahydro-3H-3 a,6-methanobenzo[c] isothiazole-1-carbonyl)benzoyl)oxy)-2,3-dihydro-1 $H$-indene-2carboxylate $^{[12]}$

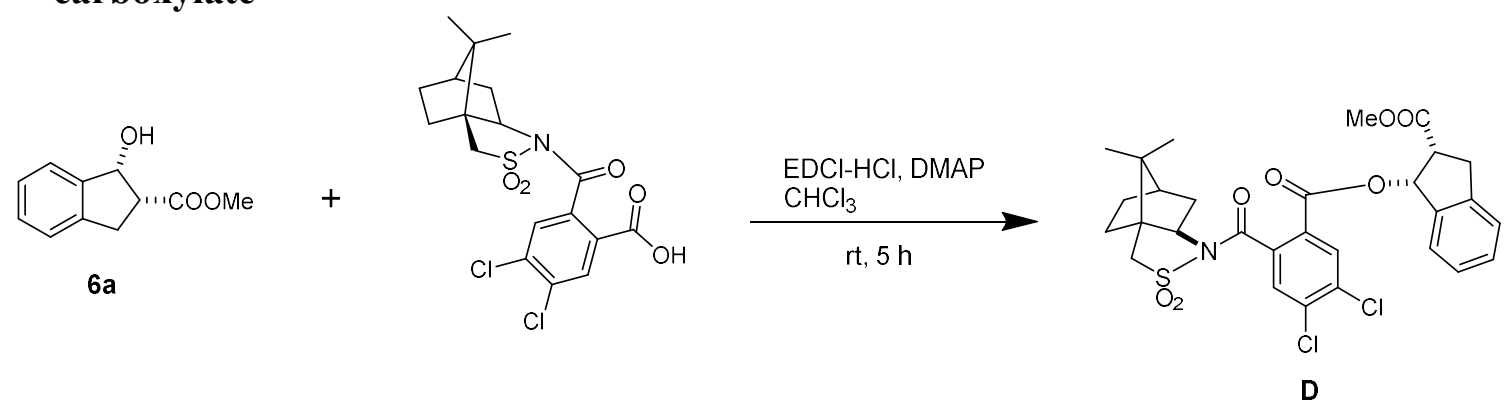

To a mixture of $N$-(2-carboxy-4,5-dichlorobenzoyl)-(-)-10,2-camphorsultam (344.3 mg, $0.80 \mathrm{mmol})$, 1-ethyl-3-(3-dimethylaminopropyl) carbodiimide hydrochloride $(152.7 \mathrm{mg}$, $0.80 \mathrm{mmol})$, and $N, N$-dimethyl-4-aminopyridine $(97.3 \mathrm{mg}, 0.80 \mathrm{mmol})$ in $\mathrm{CHCl}_{3}(2 \mathrm{~mL})$ was added methyl $(1 R, 2 R)$-1-hydroxy-2,3-dihydro-1 $H$-indene-2-carboxylate 6a $(102.0 \mathrm{mg}$, $0.53 \mathrm{mmol})$ in $\mathrm{CHCl}_{3}(2 \mathrm{~mL})$. The reaction mixture was stirred for $5 \mathrm{~h}$ at room temperature and then quenched by a saturated aqueous $\mathrm{NH}_{4} \mathrm{Cl}$ solution. The aqueous layer was extracted with $\mathrm{CHCl}_{3}(3 \mathrm{~mL} \times 3)$. The combined organic portions were dried over $\mathrm{Mg}_{2} \mathrm{SO}_{4}$ and evaporated in vacuo. The residue was purified by column chromatography (eluent: Hexane/AcOEt $=4 / 1$ to $2 / 1)$ to give ester $\mathbf{D}(290 \mathrm{mg})$ in $90 \%$ yield as a white solid. Single crystals were obtained by recrystallization from a 2-propanol solution.

${ }^{1} \mathrm{H}$ NMR $\left(500 \mathrm{MHz}, \mathrm{CDCl}_{3}\right) \delta 7.92(\mathrm{~s}, 1 \mathrm{H}), 7.47-7.44(\mathrm{~m}, 1 \mathrm{H}), 7.36-7.32(\mathrm{~m}, 2 \mathrm{H})$, $7.25-7.21(\mathrm{~m}, 1 \mathrm{H}), 6.62(\mathrm{~d}, J=6.5 \mathrm{~Hz}, 1 \mathrm{H}), 3.99-3.97(\mathrm{~m}, 1 \mathrm{H}), 3.74-3.69(\mathrm{~m}, 1 \mathrm{H})$, $3.66-3.60(\mathrm{~m}, 1 \mathrm{H}), 3.64(\mathrm{~s}, 3 \mathrm{H}), 3.40(\mathrm{dd}, J=33.0,14.0 \mathrm{~Hz}, 2 \mathrm{H}), 3.18-3.11(\mathrm{~m}, 1 \mathrm{H})$, 2.55-2.51 (m, 1H), 2.21-2.15 (m, 1H), 1.98-1.90 (m, 3H), 1.47-1.38 (m, 2H), $1.29(\mathrm{~s}, 3 \mathrm{H})$, $1.00(\mathrm{~s}, 3 \mathrm{H}) ;{ }^{13} \mathrm{C}\left\{{ }^{1} \mathrm{H}\right\} \mathrm{NMR}\left(125 \mathrm{MHz}, \mathrm{CDCl}_{3}\right) \delta 171.1,165.1,162.5,143.2,138.4,137.0$, 135.0, 134.8, 131.7, 131.0, 130.0, 127.9, 127.2, 126.5, 124.8, 78.2, 65.6, 53.0, 52.2, 48.5, 48.4, 47.8, 44.8, 37.7, 33.0, 32.9, 26.5, 20.9, 20.1. HRMS (ESI) $m / z:[\mathrm{M}+\mathrm{H}]^{+}$calcd for $\mathrm{C}_{29} \mathrm{H}_{29} \mathrm{NO}_{7} \mathrm{SCl}_{2}$ : 606.1115; found: 606.1120. 


\section{b) X-ray Structure Determination for $\mathbf{D}$ derived from $\mathbf{6 a}$.}

Measurements were made on a Rigaku Saturn CCD area detector equipped with graphite-monochromated Mo- $K \alpha$ radiation $(\lambda=0.71075 \AA)$ under nitrogen stream at $93 \mathrm{~K}$. The single crystal was mounted on a glass fiber. Indexing was performed from eighteen images. The crystal-to-detector distance was $75.21 \mathrm{~mm}$. The data were collected to a maximum $2 \theta$ value of $55.0^{\circ}$. A total of 1440 oscillation images were collected. A sweep of data was carried out using $\omega$ scans from -120.0 to $60.0^{\circ}$ in $0.5^{\circ}$ steps, at $\chi=45.0^{\circ}$ and $\phi=$ $0.0^{\circ}$. A second sweep was performed using $\omega$ scans from -120.0 to $60.0^{\circ}$ in $0.5^{\circ}$ steps, at $\chi$ $=45.0^{\circ}$ and $\phi=90.0^{\circ}$. A third sweep was performed using $\omega$ scans from -120.0 to $60.0^{\circ}$ in $0.5^{\circ}$ steps, at $\chi=45.0^{\circ}$ and $\phi=180.0^{\circ}$. A last sweep was performed using $\omega$ scans from -120.0 to $60.0^{\circ}$ in $0.5^{\circ}$ steps, at $\chi=45.0^{\circ}$ and $\phi=270.0^{\circ}$. The exposure rate was $16.0 \mathrm{sec} /{ }^{\circ}$. The detector swing angle in each sweep was $-29.95^{\circ}$. Intensity data were collected for Lorentz-polarization effects as well as absorption. Structure solution and refinements were performed with the CrystalStructure program package. The heavy atom positions were determined by direct methods (SIR97) ${ }^{13}$, and the remaining non-hydrogen atoms were found by subsequent Fourier techniques. An empirical absorption correction based on equivalent reflections was applied to all data. All non-hydrogen atoms other than solvent molecules were refined anisotropically by full-matrix least-square techniques based on $F^{2}$ using CRYSTALS ${ }^{14}$ program. All hydrogen atoms were constrained to ride on their parent atom. Relevant crystallographic data are compiled in Table S1.

Table S4. Crystallographic Data for D

\begin{tabular}{|c|c|}
\hline & $\begin{array}{l}(1 R, 2 R)-1-((4,5 \text {-dichloro-2- }((3 \mathrm{a} R, 6 R, 7 \mathrm{a} R)-8,8 \text {-dimethyl-2,2- } \\
\text { dioxidohexahydro-3H-3a,6-methanobenzo[c]isothiazole-1- } \\
\text { carbonyl)benzoyl)oxy)-2,3-dihydro- } 1 H \text {-indene-2-carboxylate }\end{array}$ \\
\hline empirical formula & $\mathrm{C}_{29} \mathrm{H}_{29} \mathrm{Cl}_{2} \mathrm{NO}_{7} \mathrm{~S}$ \\
\hline formula weight & 606.52 \\
\hline crystal color & Colorless \\
\hline crystal system & Orthorhombic \\
\hline space group & $P 2{ }_{1} 2_{1} 2_{1}(\# 19)$ \\
\hline$a, \AA$ & $12.021(3)$ \\
\hline$b, \AA$ & $12.312(3)$ \\
\hline$c, \AA$ & $19.081(5)$ \\
\hline$V, \AA^{3}$ & $2824.0(13)$ \\
\hline$Z$ & 4 \\
\hline$D_{\text {calcd }}, \mathrm{g} \mathrm{cm}^{-3}$ & 1.426 \\
\hline $\mathrm{F}_{000}$ & 1264.00 \\
\hline$\mu, \mathrm{cm}^{-1}(\operatorname{Mo} K \alpha)$ & 3.518 \\
\hline Exposure rate & $16.0 \mathrm{sec} /{ }^{\circ}$ \\
\hline no. of reflections measured & 31725 \\
\hline no. of unique reflections & 6464 \\
\hline no. of variables & 391 \\
\hline$R 1(I>2.00 \sigma(I))$ & 0.0465 \\
\hline wR2 (All reflections) & 0.1056 \\
\hline GOF on $F^{2}$ & 1.000 \\
\hline Flack parameter & $0.04(6)$ \\
\hline
\end{tabular}




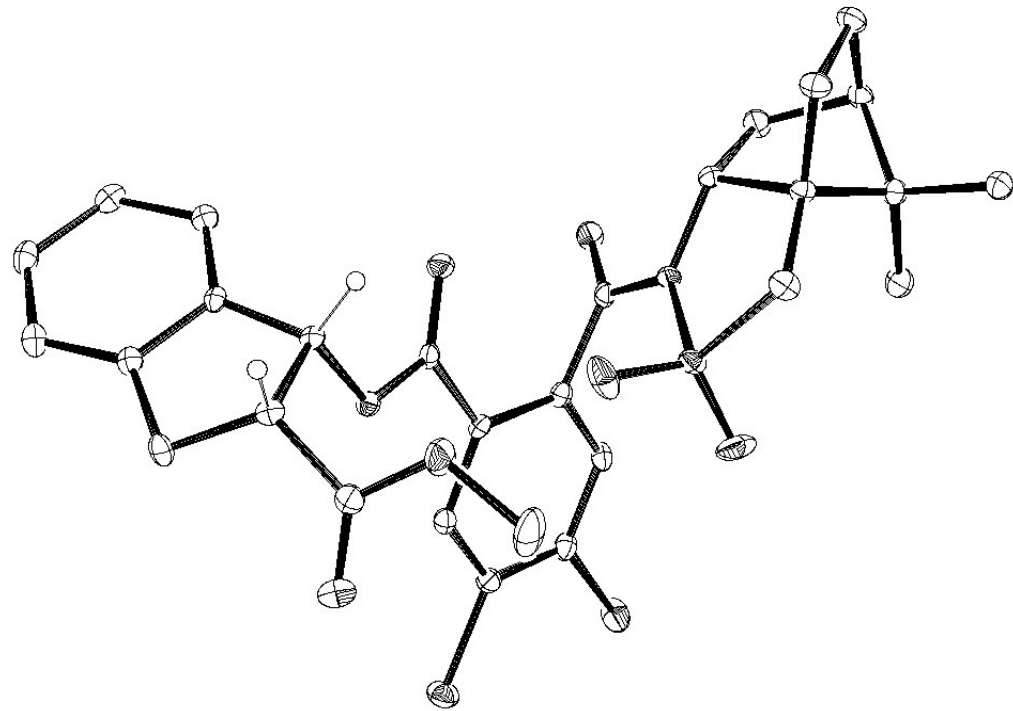

Figure S1. X-ray crystallographic structure of $\mathbf{D}$. All hydrogens except those attached to chiral carbons are omitted for clarity, and the ellipsoids represent $30 \%$ probability. 


\section{G. References.}

(1) Mei, Y.; Bentley, P. A; Du, J. Thiourea Catalysis of NCS in the Synthesis of $\alpha$-Chloroketones. Tetrahedron Lett. 2008, 49, 3802-3804.

(2) Pan, C.; Zeng, X.; Guan, Y.; Jiang, X.; Li, L.; Zhang, H. Design and Synthesis of Brazilin-Like Compounds. Synlett 2011, 3, 425-429.

(3) Lian, X.; Lin, L.; Fu, K.; Ma, B.; Liu, X.; Fen, X. A New Approach to the Asymmetric Mannich Reaction Catalyzed by Chiral $N, N^{\prime}$-Dioside-metal Complexes. Chem. Sci. 2017, 8, 1238-1242.

(4) Ding, Z.; Yang, J.; Wang, T.; Shen, Z.; Zhang, Y. Dynamic Kinetic Resolution of $\beta$-Keto Sulfones via Asymmetric Transfer Hydrogenation. Chem. Commun. 2009, 571-573.

(5) Brummond, K. M., Gesenberg, K. D. $\alpha$-Chlorination of Ketones Using p-Toluenesulfonyl Chloride. Tetrahedron Lett. 1999, 40, 2231-2234.

(6) Hellou, J.; Kingston, J. F.; Fallis, A. G. Diethyl Dicarbonate: A Convenient Reagent for the Preparation of $\beta$-Ketoesters. Synthesis 1984, 12, 1014-1017.

(7) Ros, A.; Magriz, A.; Dietrich, H.; Fernández, R.; Alvarez, E.; Lassaletta, J. M. Enantioselective Synthesis of Vicinal Halohydrins via Dynamic Kinetic Resolution. Org. Lett. 2006, 8, 127-130.

(8) Marigo, M.; Bachmann, S.; Halland, N.; Braunton, A.; Jørgensen, K. A. Highly Enantioselective Direct Organocatalytic $\alpha$-Chlorination of Ketones. Angew. Chem., Int. Ed. 2004, 18, 5507-5510.

(9) Fringuelli, F.; Pizzo, F.; Vaccaro, L. Cobalt(II) Chloride-Catalyzed Chemoselective Sodium Borohydride Reduction of Azides in Water. Synthesis 2000, 5, 646-650.

(10) Gonzalez, A. Z.: Eksterowicz, J. Bartberger, M. D.; Beck, H. P.; Canon, J.; Chen, A.; Chow, D.; Duquett, J.; Fox, B. M.; Fu, J.; Huang, X.; Houze, J. B.; Jin, L.; Li, Y.; Li, Z.; Ling, Y.; Lo, M.-C.; Long, A. M.; McGee, L. R.; McIntosh, J.; McMinn, D. L.; Oliner, J. D.; Osgood, T.; Rew, Y.; Saiki, A. Y.; Shaffer, P.; Wortman, S.; Yakowec, P.; Yan, X.; Ye, Q.; Yu, D.; Zhao, X.; Zhou, J.; Olson, S. H.; Medina, J. C.; Sun, D. Selective and Potent Morpholinone Inhibitors of the MDM2-p53 Protein-Protein Interaction. J. Med. Chem. 2014, 57, 2472-2488.

(11) Jones, J. H.; Anderson, P. S.; Baldwin, J. J.; Clineschmidt, B. V.; McClure, D. E.; Lundell, G. F.; Randall, W. C.; Martin, G. E.; Williams, M.; Hirshfield, J. M.; Smith, G.; Lumma, P. K. Synthesis of 4-Substituted $2 H$-Naphth[1,2-b]-1,4-oxazines, a New Class of Dopamine Agonists. J. Med. Chem. 1984, 27. 1607-1613.

(12) Terada, M.; Moriya, K.; Kanomata, K.; Sorimachi, K. Chiral Brønsted Acid Catalyzed Stereoselective Addition of Azlactones to 3-Vinylindoles for Facile Access to Enantioenriched Tryptophan Derivatives. Angew. Chem., Int. Ed. 2011, 50, 12586-12590.

(13) Altomare, A.; Buria, M. C.; Camalli, M.; Cascarano, G.; Giacovazzo, C.; Guagliardi, A.; Moliterni, A. G. G.; Polidori, G., Spagna, R., SIR97: a New Tool for Crystal Structure Determination and Refinement. J. Appl. Crystallogr. 1999, 32, 115-119.

(14) CRYSTALS Issue 11; Carruthers, J. R.; Rollett, J. S.; Betteridge, P. W.; Kinna, D.; Pearce, L.; Larsen, A.; Gabe, E. Chemical Crystallography Laboratory, Oxford, UK, 1999. 
H. NMR Spectra for Ketones and Reduction Products.

2-Chloro-3,4-dihydronaphthalen-1(2H)-one (3a)

${ }^{1} \mathrm{H}-\mathrm{NMR}\left(400 \mathrm{MHz}, \mathrm{CDCl}_{3}\right)$

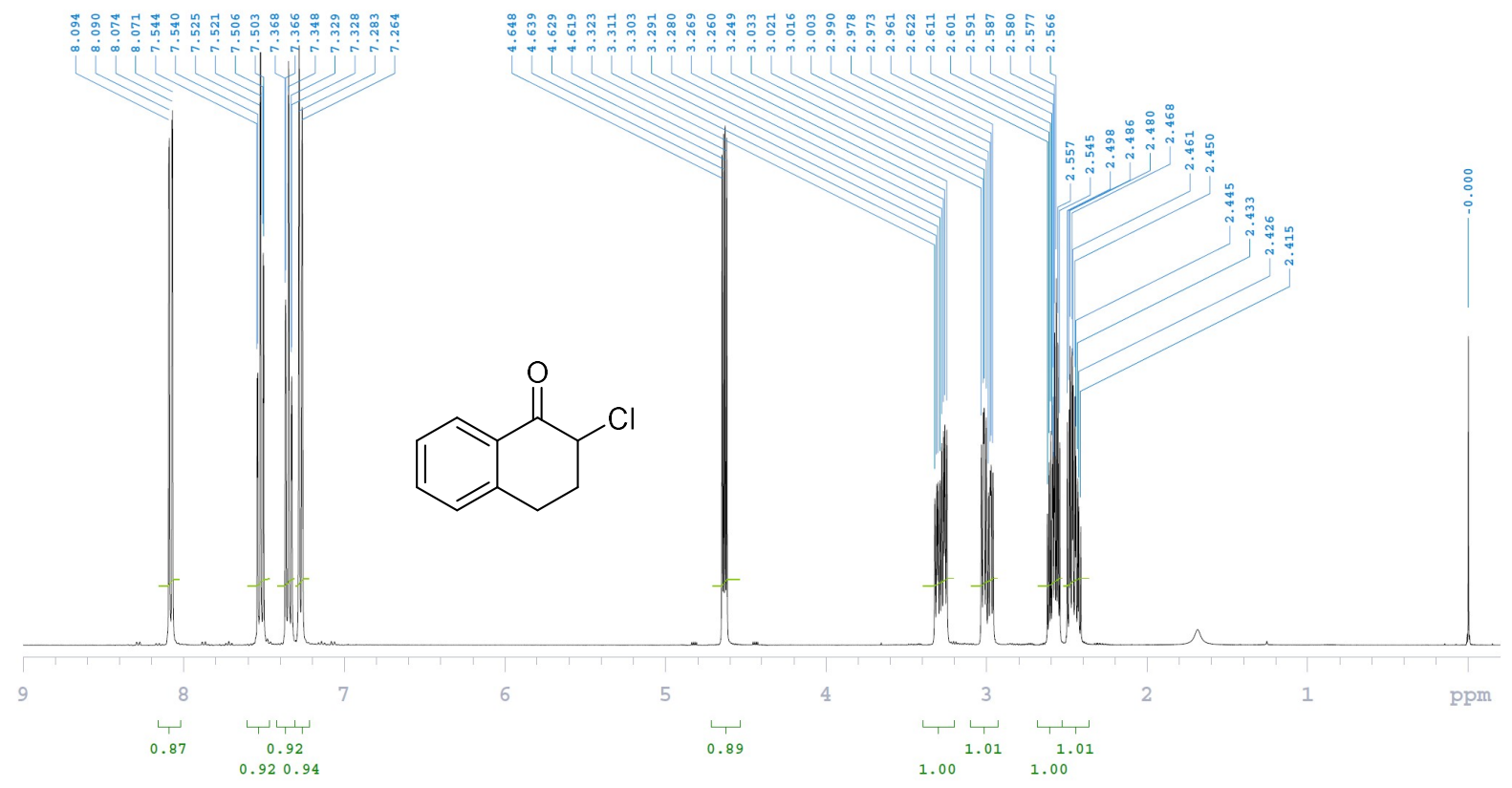

${ }^{13} \mathrm{C}$ NMR (125 MHz, $\left.\mathrm{CDCl}_{3}\right)$
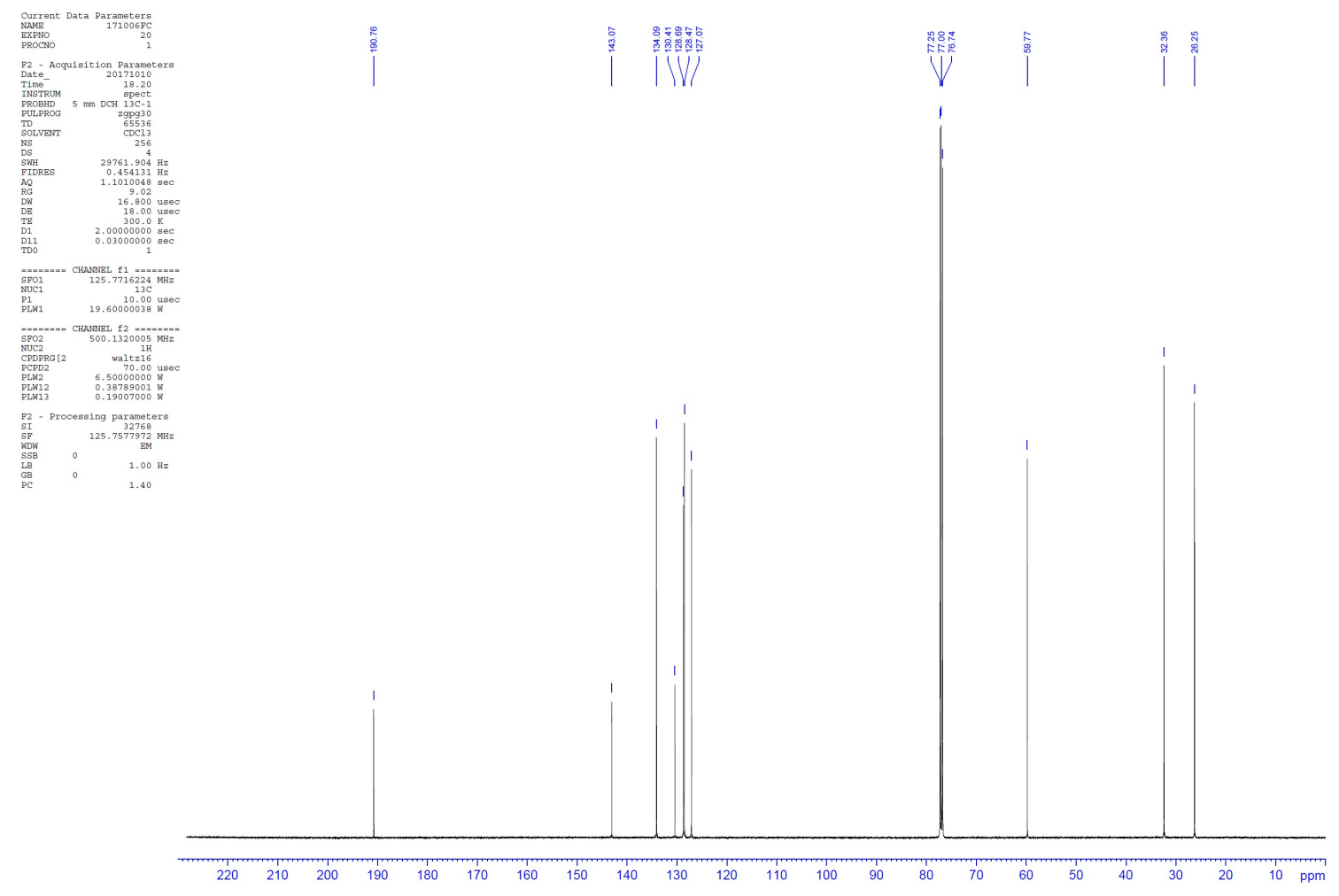
2-Chloro-7-methoxy-3,4-dihydronaphthalen-1(2H)-one (3b)

${ }^{1} \mathrm{H}-\mathrm{NMR}\left(500 \mathrm{MHz}, \mathrm{CDCl}_{3}\right.$ )
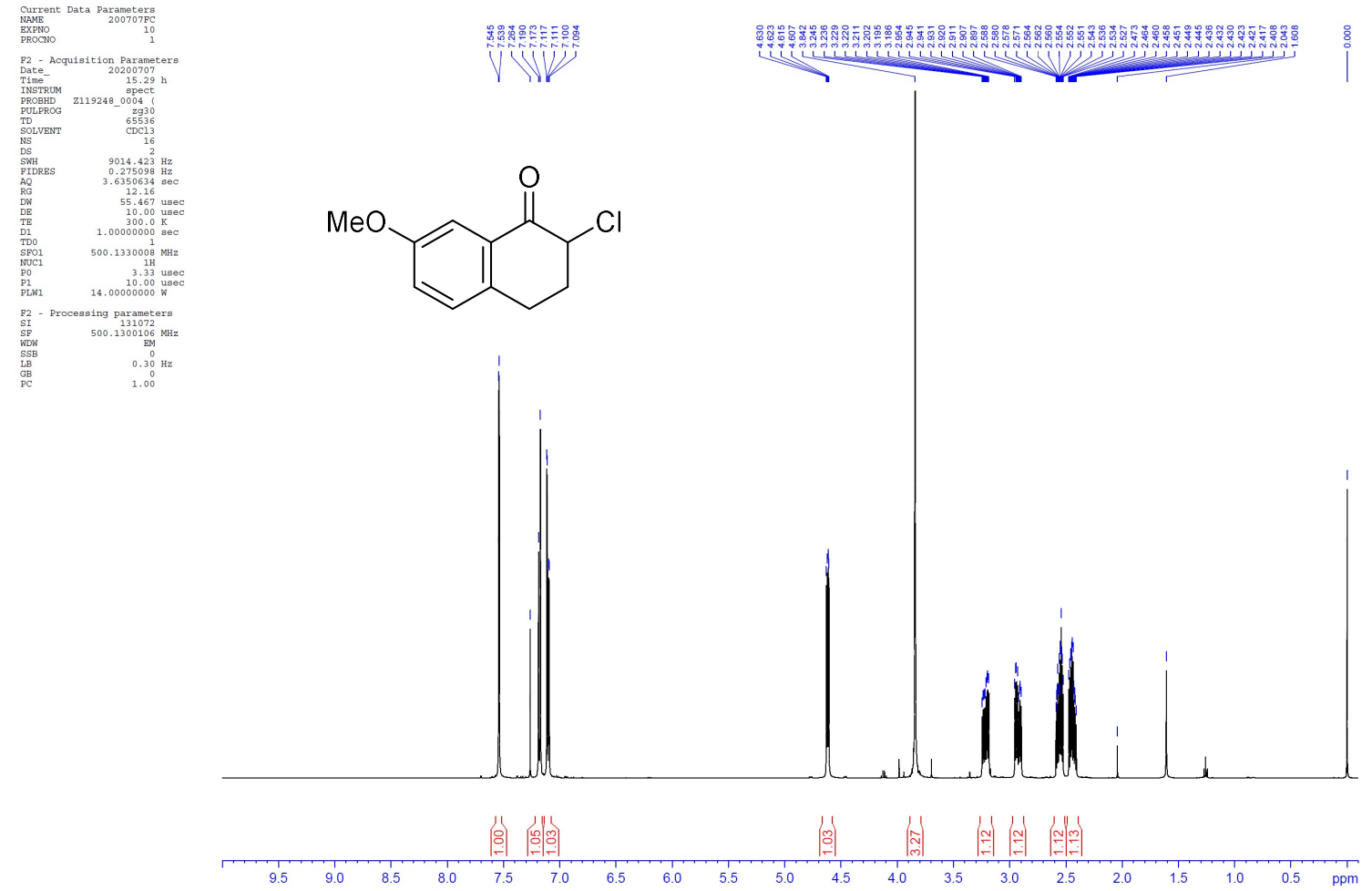

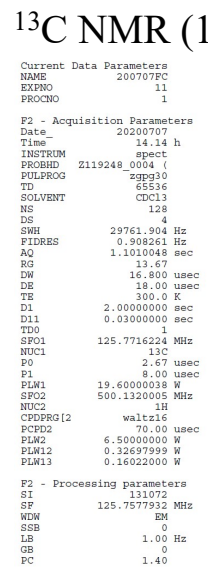
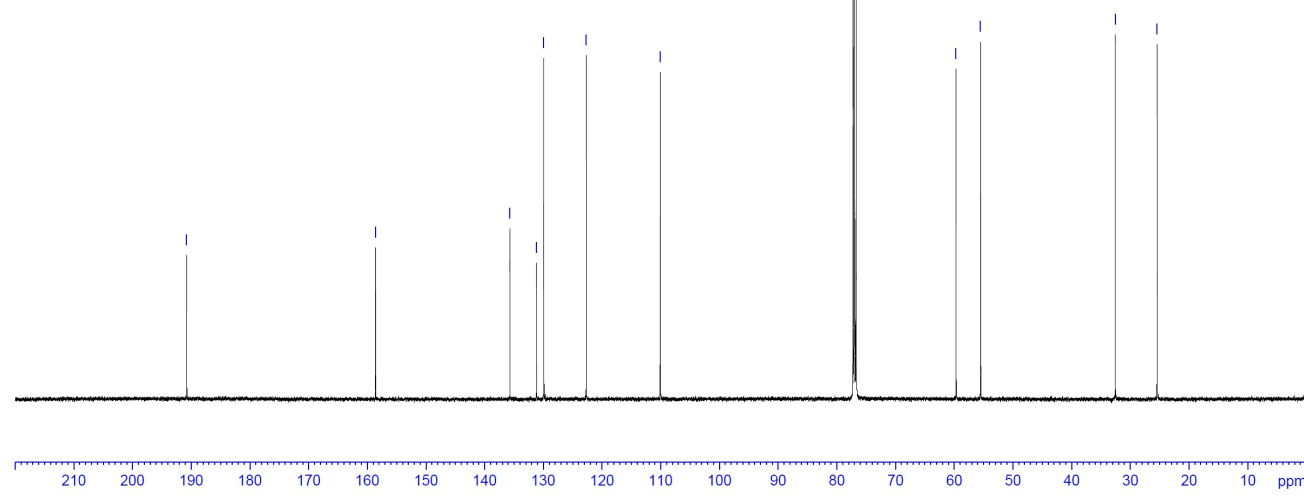
2-Chloro-7-bromo-3,4-dihydronaphthalen-1(2H)-one (3c)

${ }^{1} \mathrm{H}-\mathrm{NMR}\left(500 \mathrm{MHz}, \mathrm{CDCl}_{3}\right)$

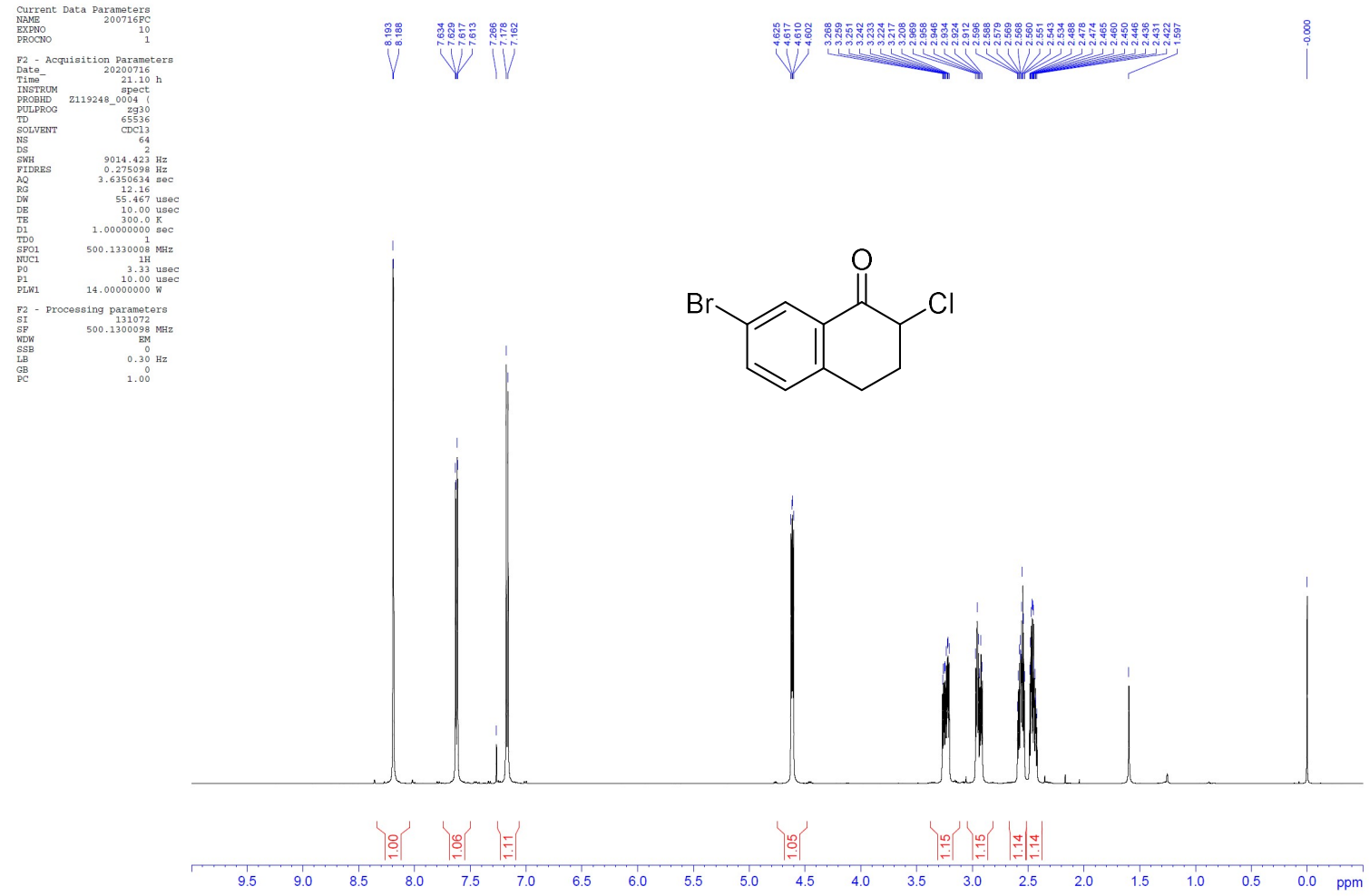

${ }^{13} \mathrm{C} \mathrm{NMR}\left(125 \mathrm{MHz}, \mathrm{CDCl}_{3}\right)$
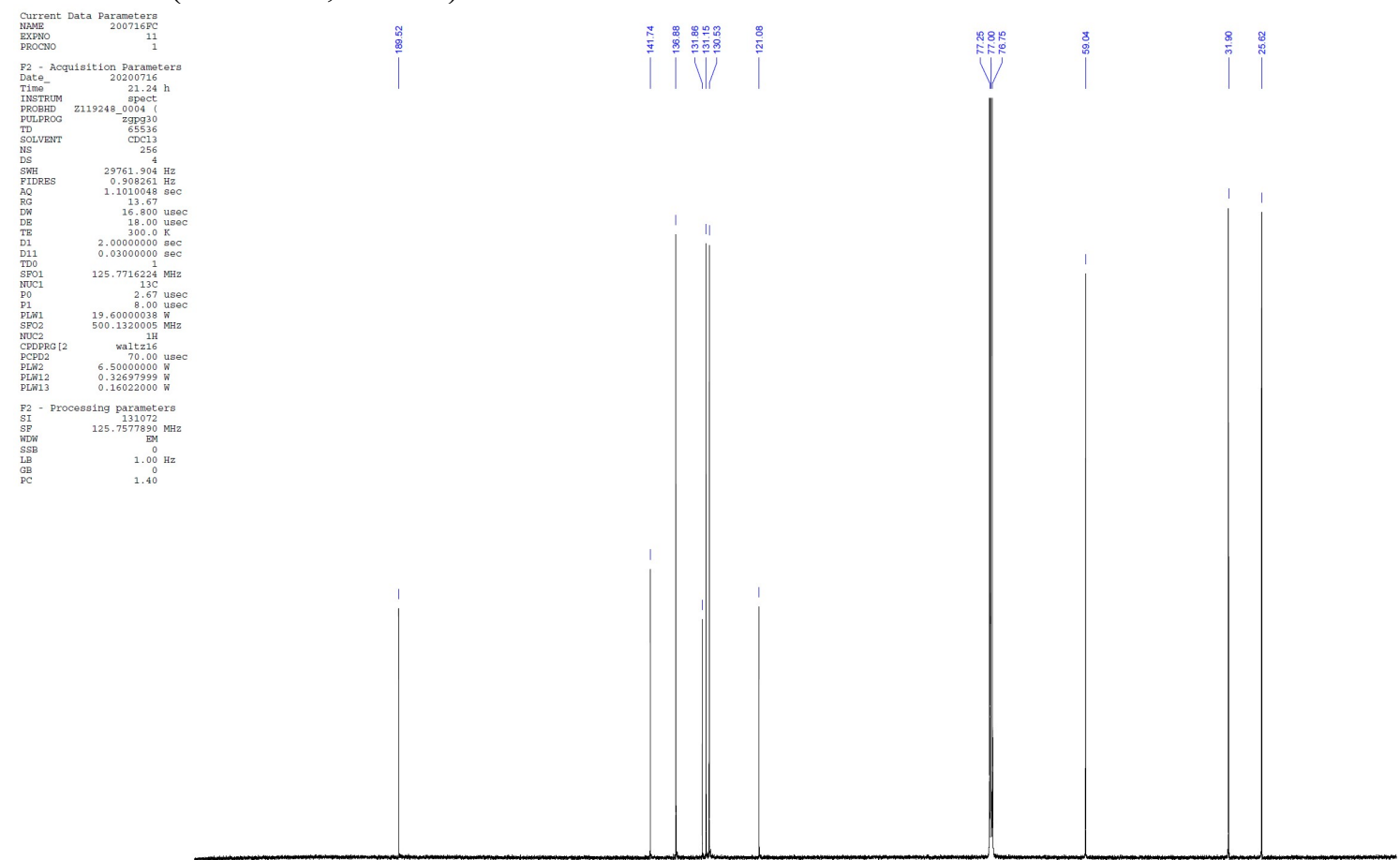

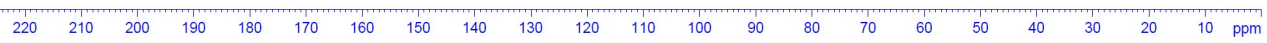


2-Chloro-2,3-dihydro-1 $H$-inden-1-one (3d)

${ }^{1} \mathrm{H}-\mathrm{NMR}\left(400 \mathrm{MHz}, \mathrm{CDCl}_{3}\right)$

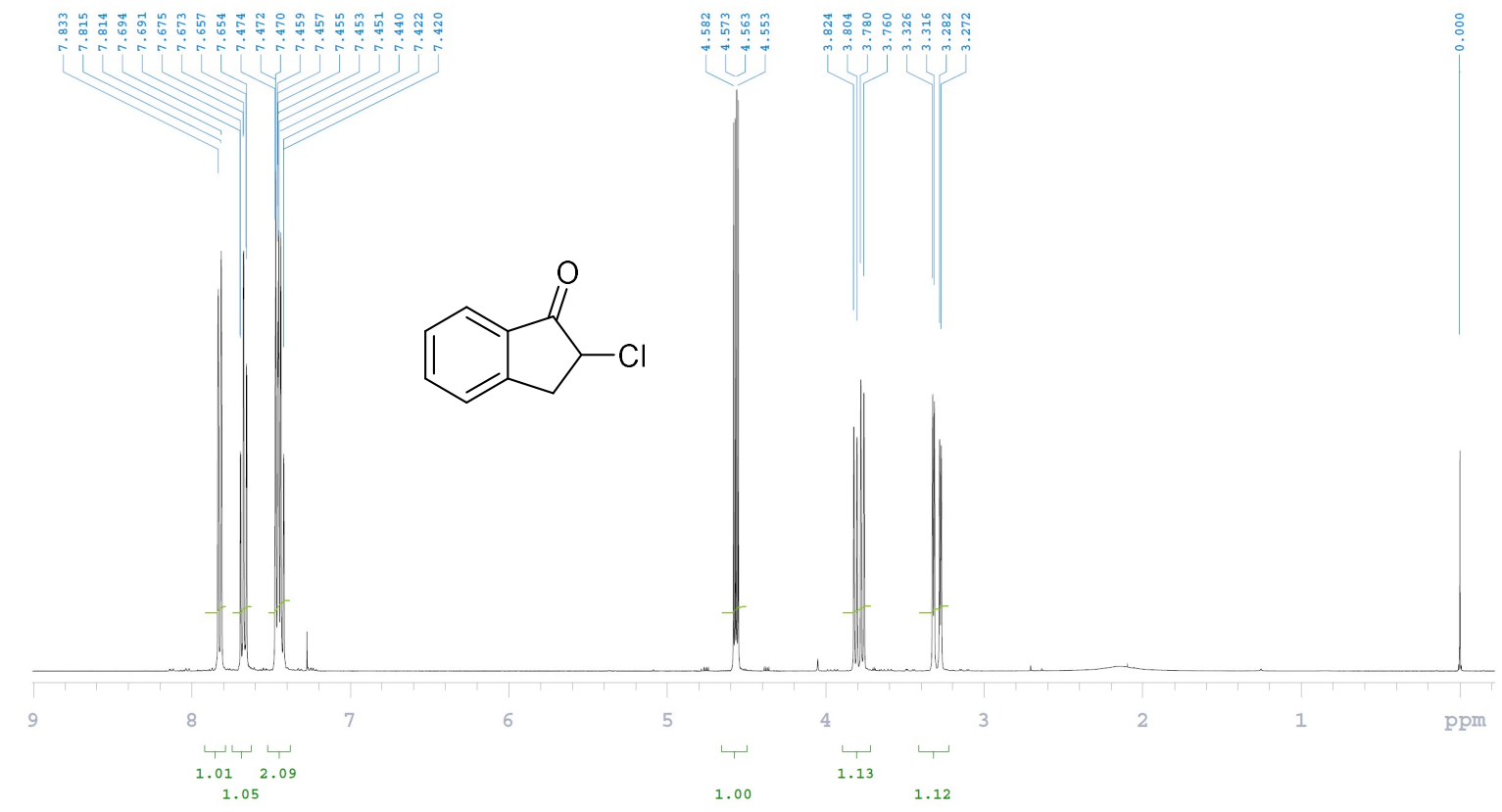

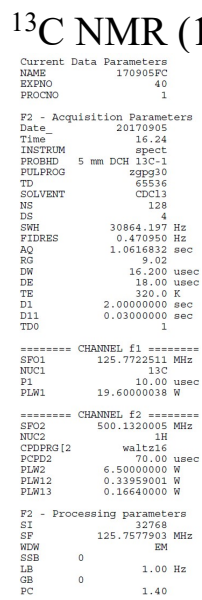

$\left.25 \mathrm{MHz}, \mathrm{CDCl}_{3}\right)$

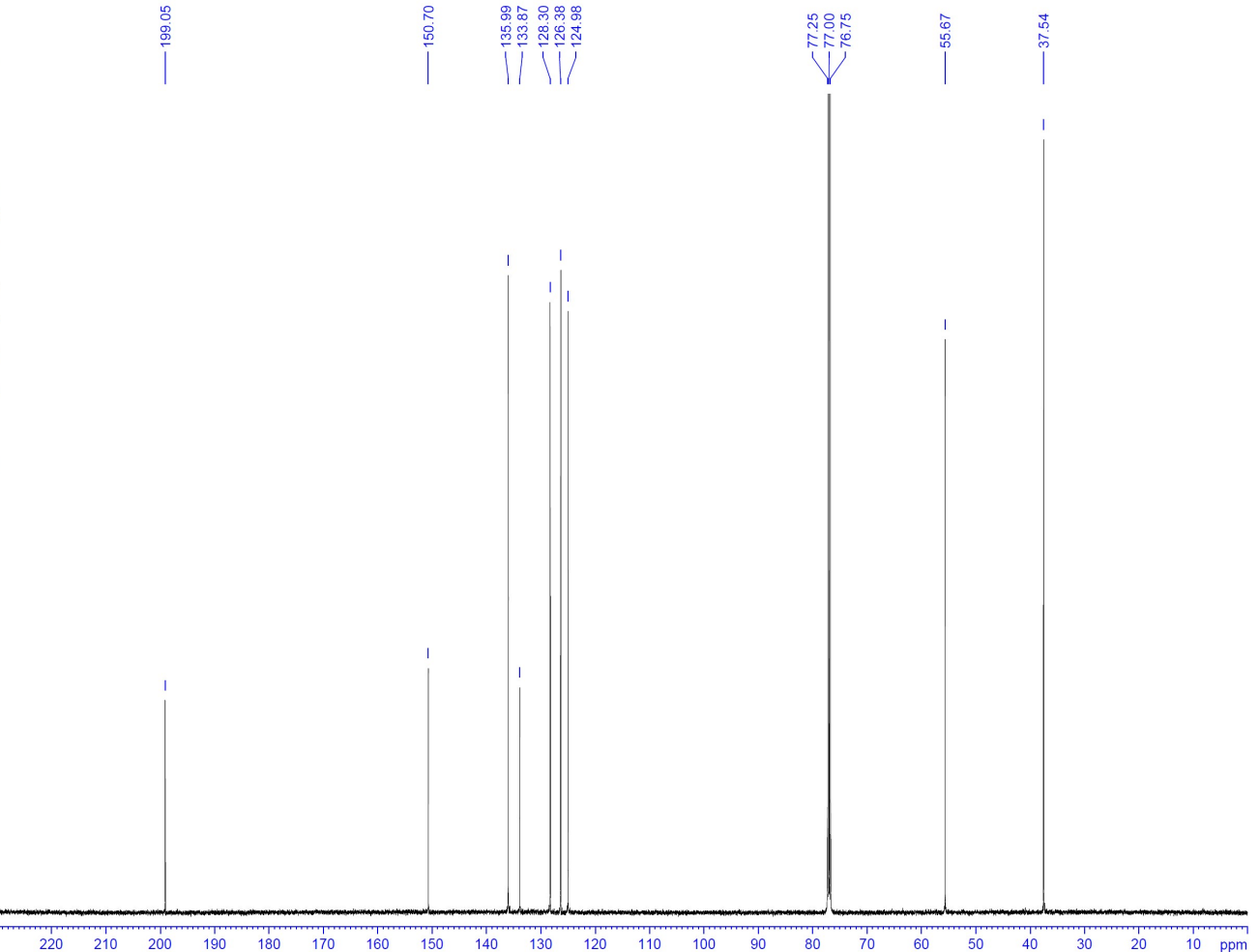


2-Chloro-5-methoxy-2,3-dihydro-1H-inden-1-one (3e)

${ }^{1} \mathrm{H}-\mathrm{NMR}\left(500 \mathrm{MHz}, \mathrm{CDCl}_{3}\right)$
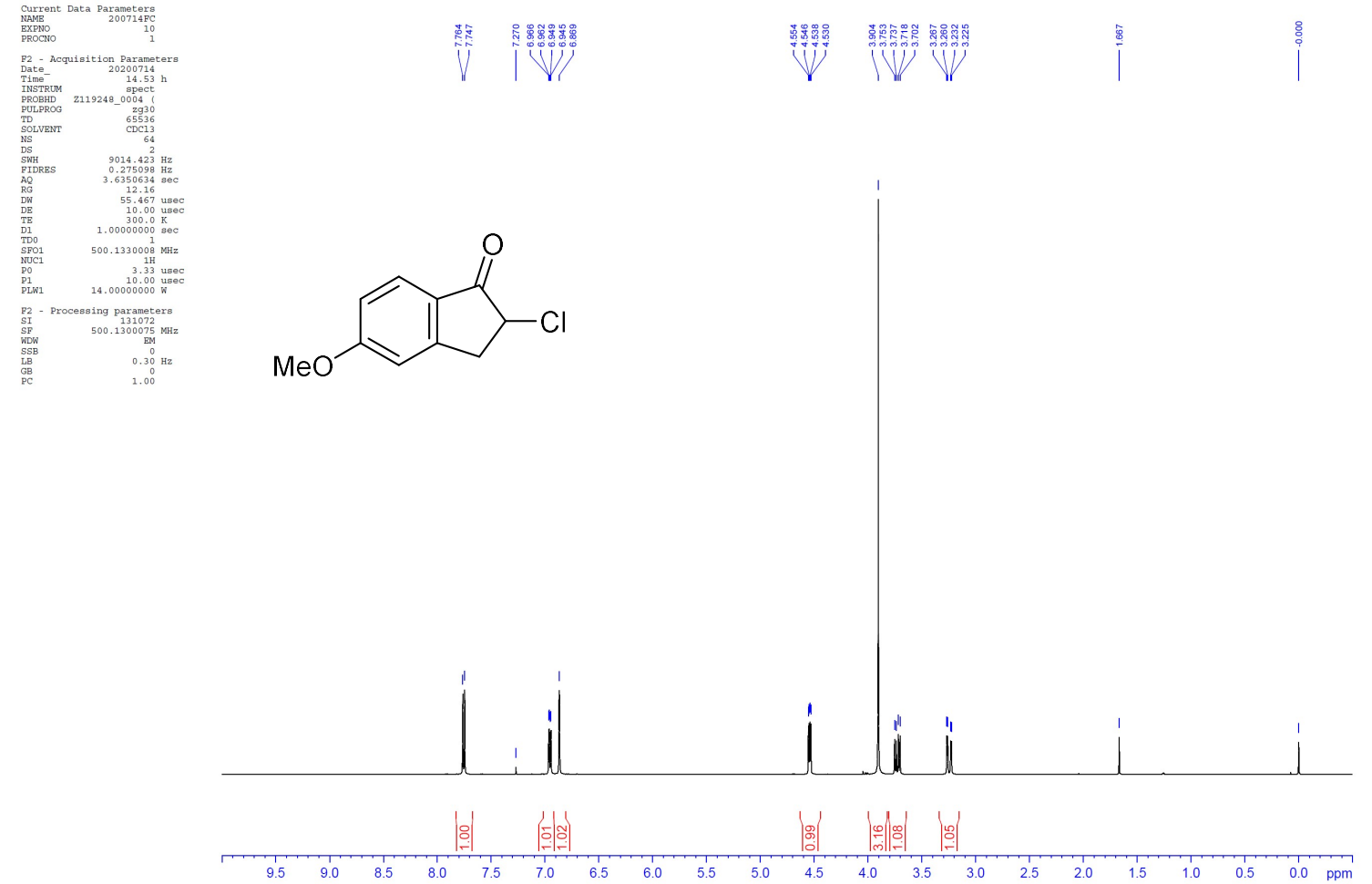

${ }^{13} \mathrm{C} \mathrm{NMR}\left(125 \mathrm{MHz}, \mathrm{CDCl}_{3}\right)$
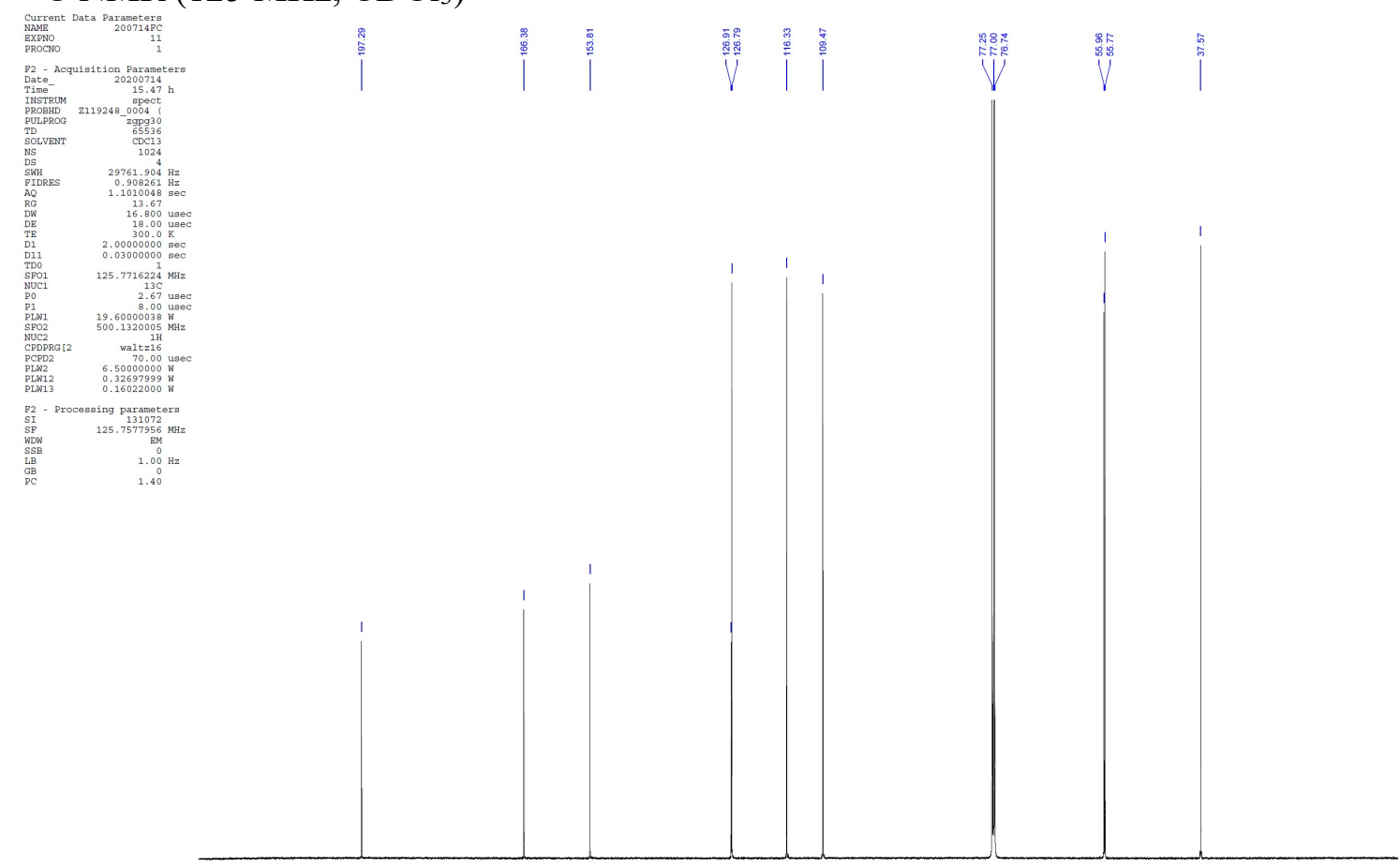

$\begin{array}{lllllllllllllllllllllll}220 & 210 & 200 & 190 & 180 & 170 & 160 & 150 & 140 & 130 & 120 & 110 & 100 & 90 & 80 & 70 & 60 & 50 & 40 & 30 & 20 & 10 & p p m\end{array}$ 
2,5-Dichloro-2,3-dihydro-1 $H$-inden-1-one (3f)

${ }^{1} \mathrm{H}-\mathrm{NMR}\left(500 \mathrm{MHz}, \mathrm{CDCl}_{3}\right.$ )
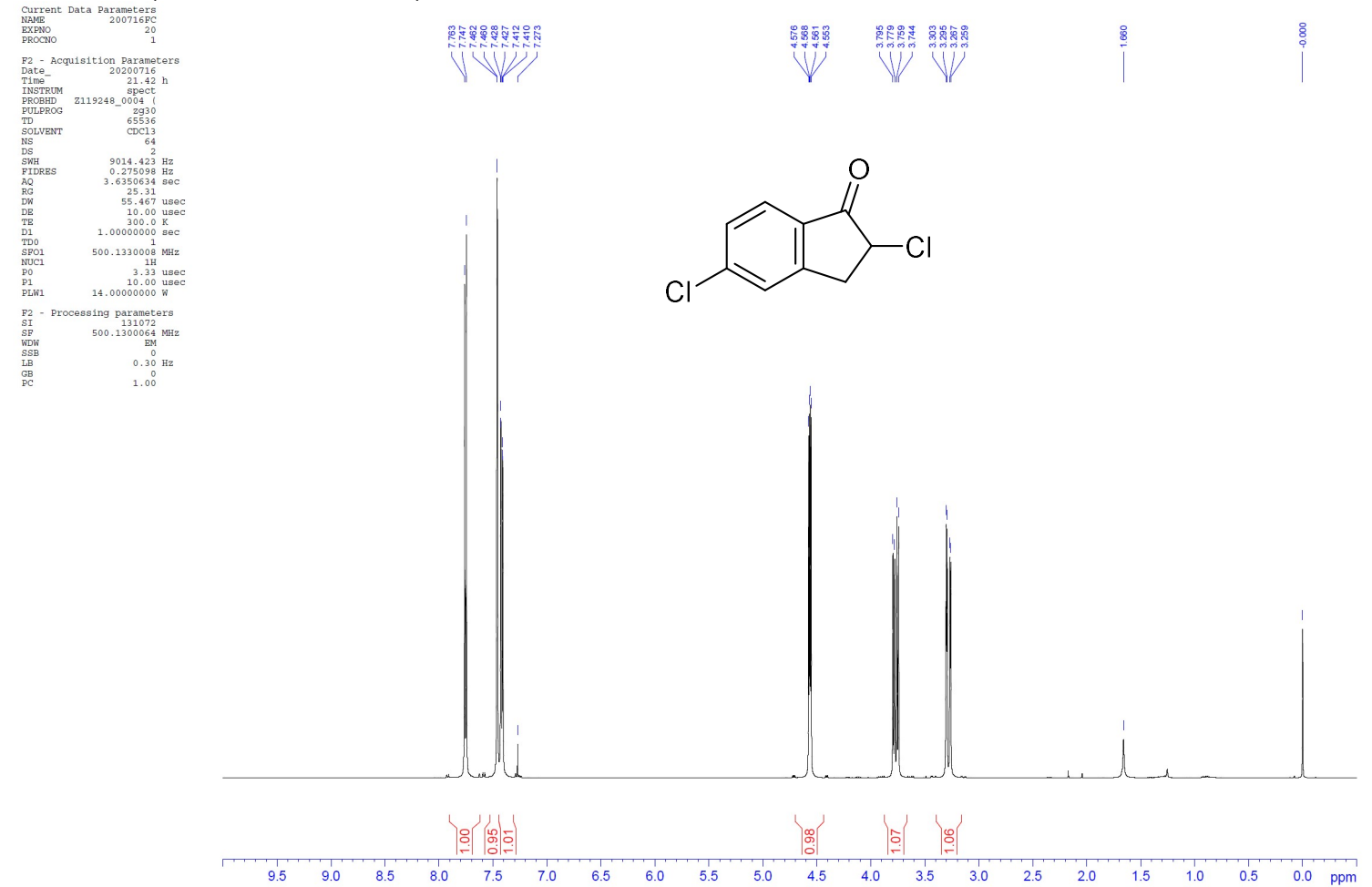

${ }^{13} \mathrm{C} \mathrm{NMR}\left(125 \mathrm{MHz}, \mathrm{CDCl}_{3}\right)$
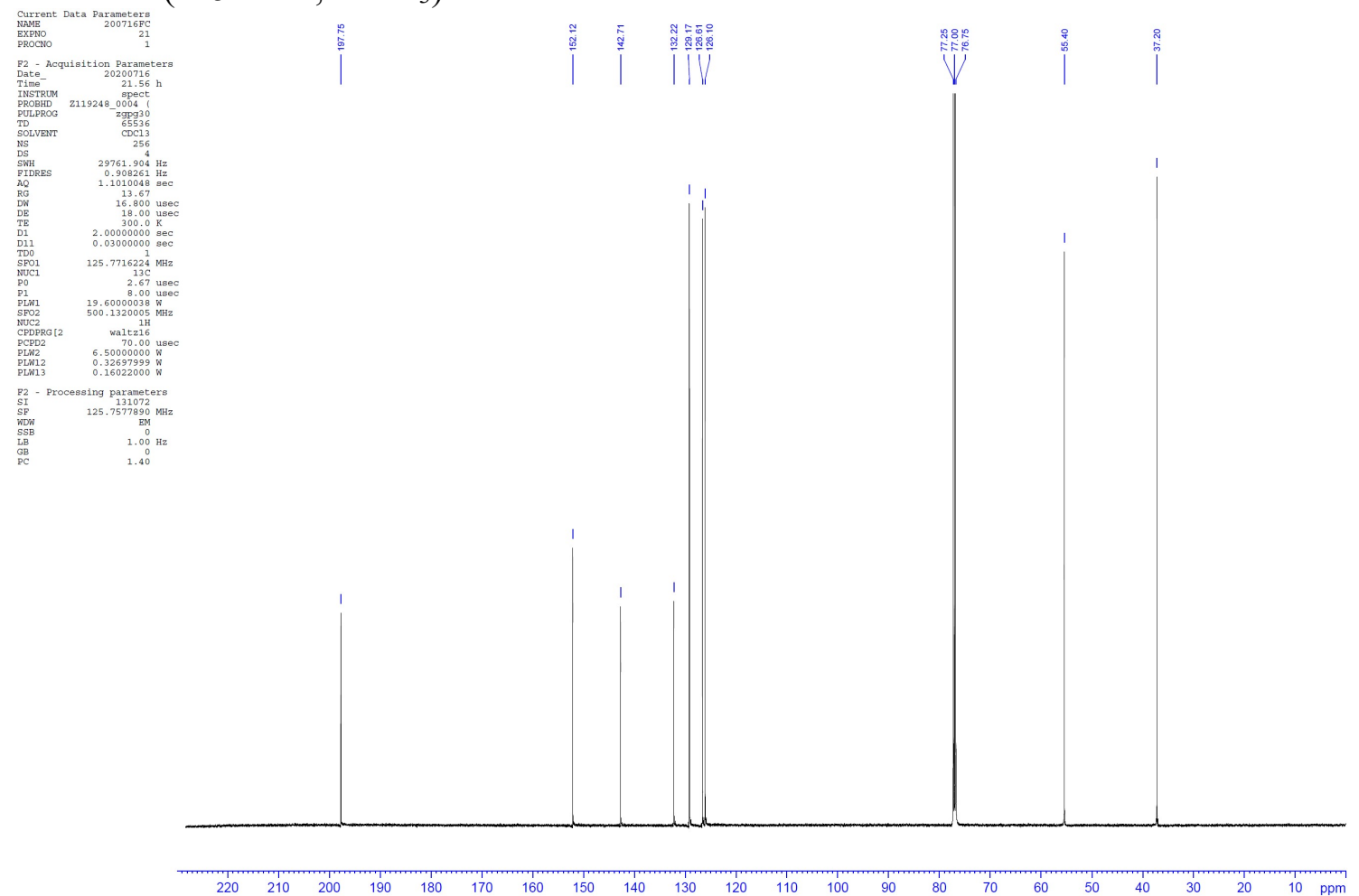
2-Chloro-7-hydroxy-2,3-dihydro-1H-inden-1-one (3g)

${ }^{1} \mathrm{H}-\mathrm{NMR}\left(500 \mathrm{MHz}, \mathrm{CDCl}_{3}\right.$ )

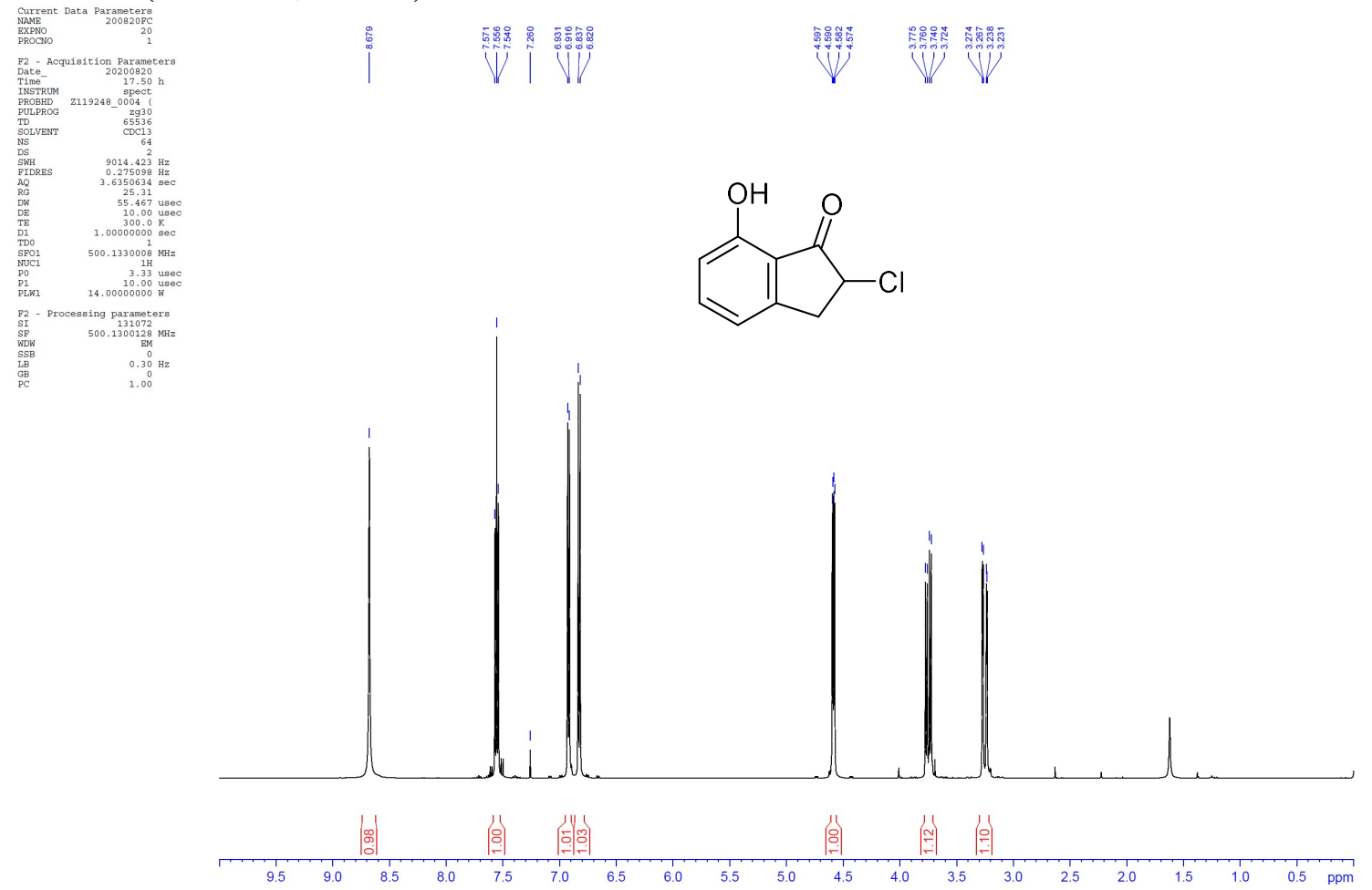

${ }^{13} \mathrm{C} \mathrm{NMR}\left(125 \mathrm{MHz}, \mathrm{CDCl}_{3}\right)$

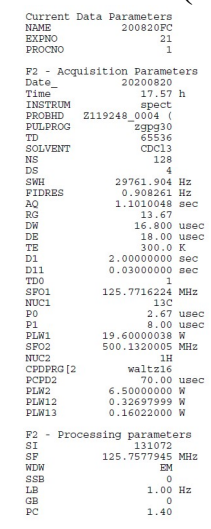


2-Chloro-3,3-dimethyl-2,3-dihydro-1H-inden-1-one (3h)

${ }^{1} \mathrm{H}-\mathrm{NMR}\left(500 \mathrm{MHz}, \mathrm{CDCl}_{3}\right)$

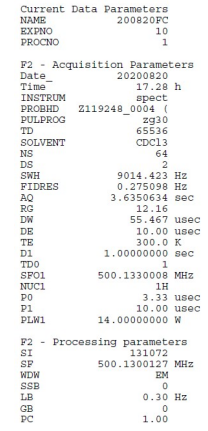

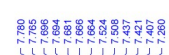
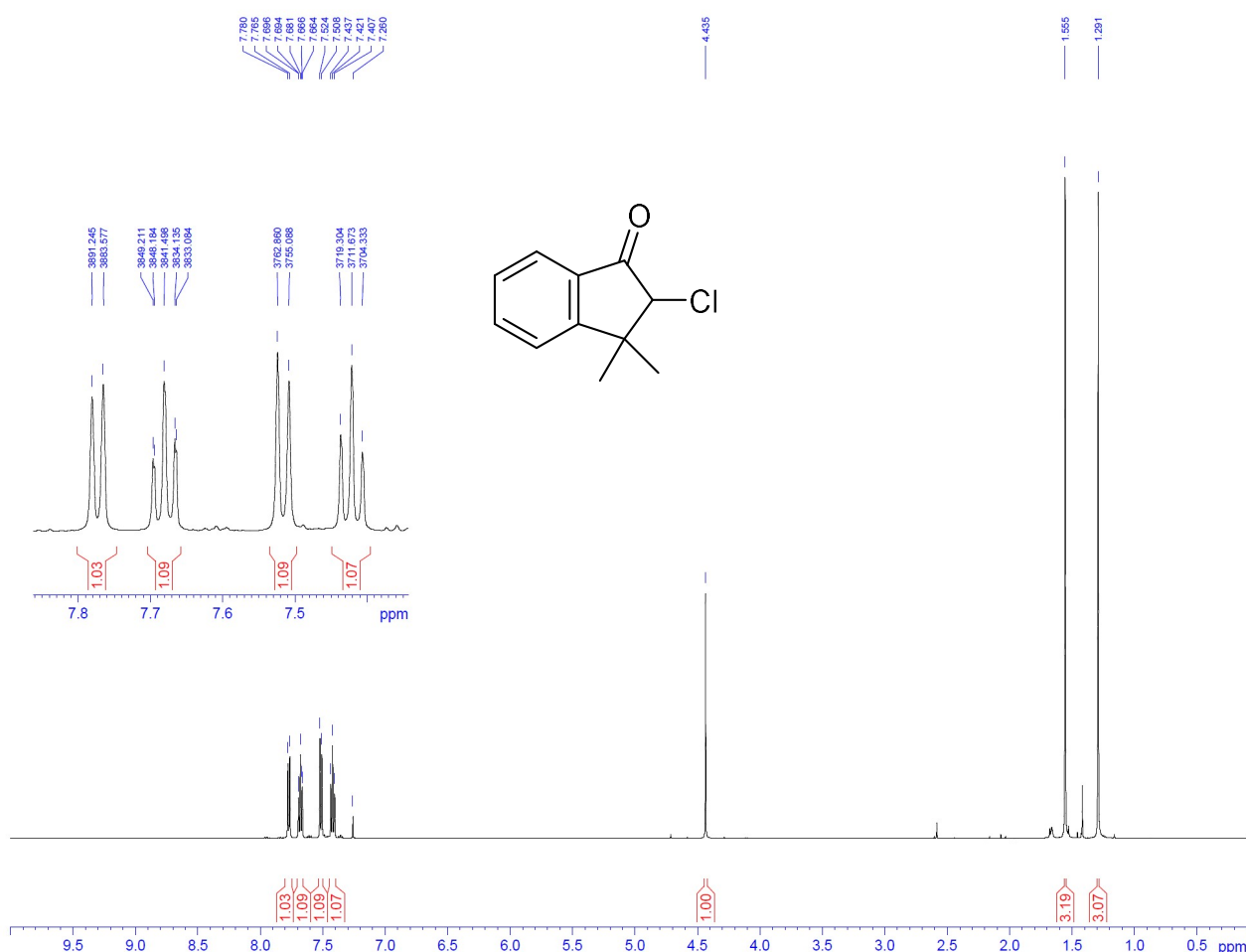

${ }^{13} \mathrm{C} \mathrm{NMR}\left(125 \mathrm{MHz}, \mathrm{CDCl}_{3}\right)$
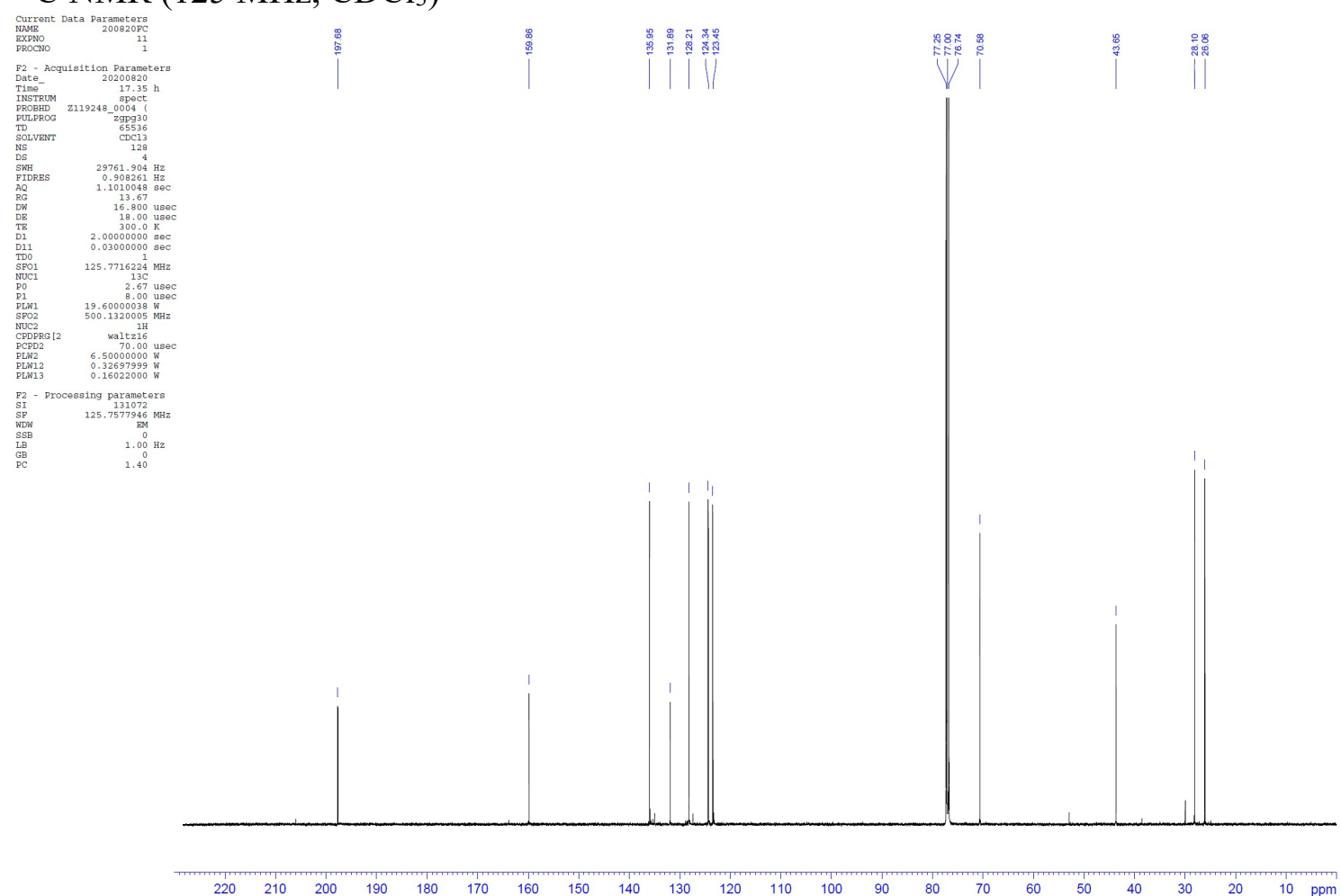
6-Chloro-6,7,8,9-tetrahydro-5H-benzo[7]annulen-5-one (3i)

${ }^{1} \mathrm{H}-\mathrm{NMR}\left(400 \mathrm{MHz}, \mathrm{CDCl}_{3}\right)$

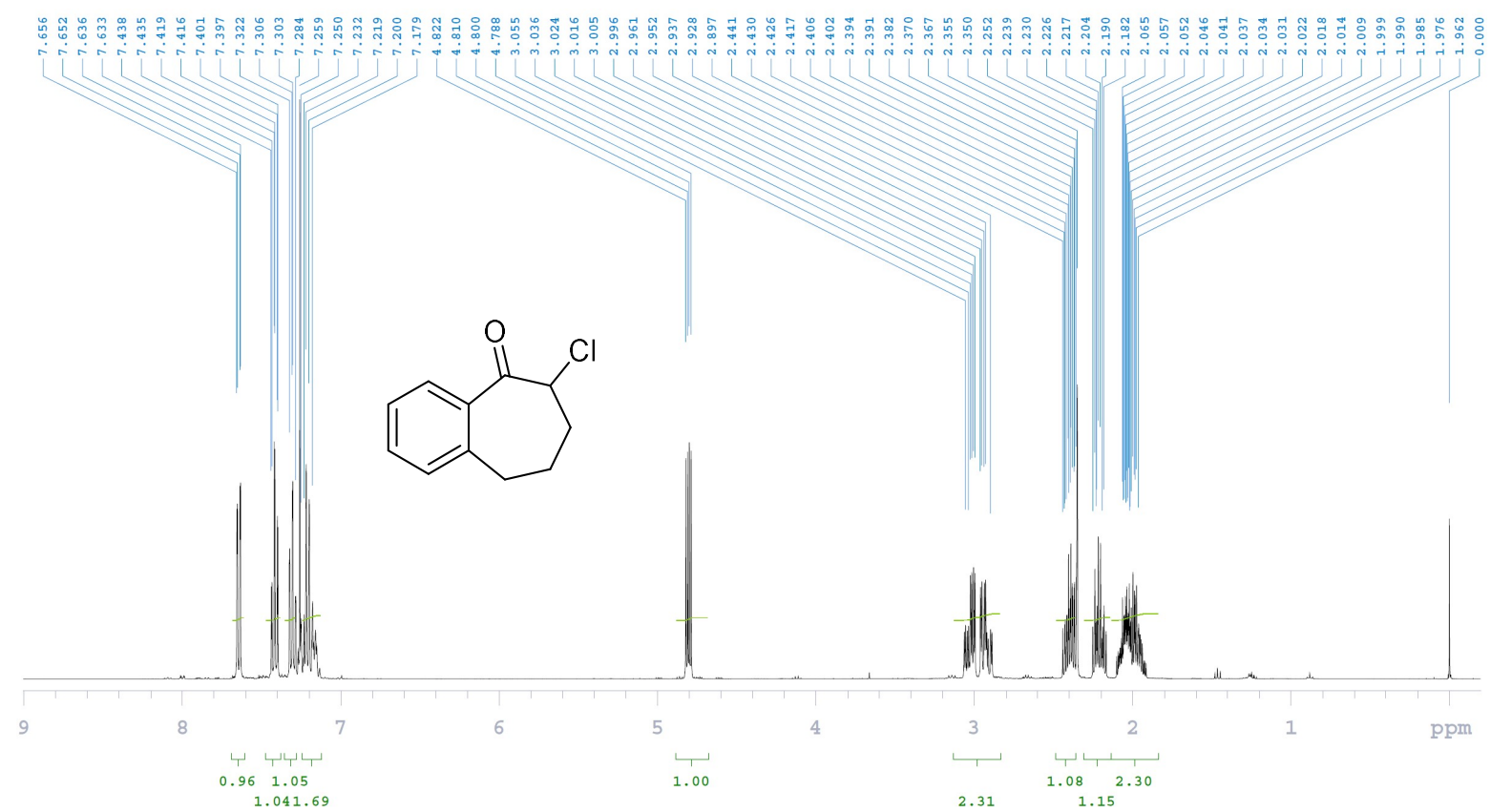

${ }^{13} \mathrm{C}$ NMR (125 MHz, $\left.\mathrm{CDCl}_{3}\right)$
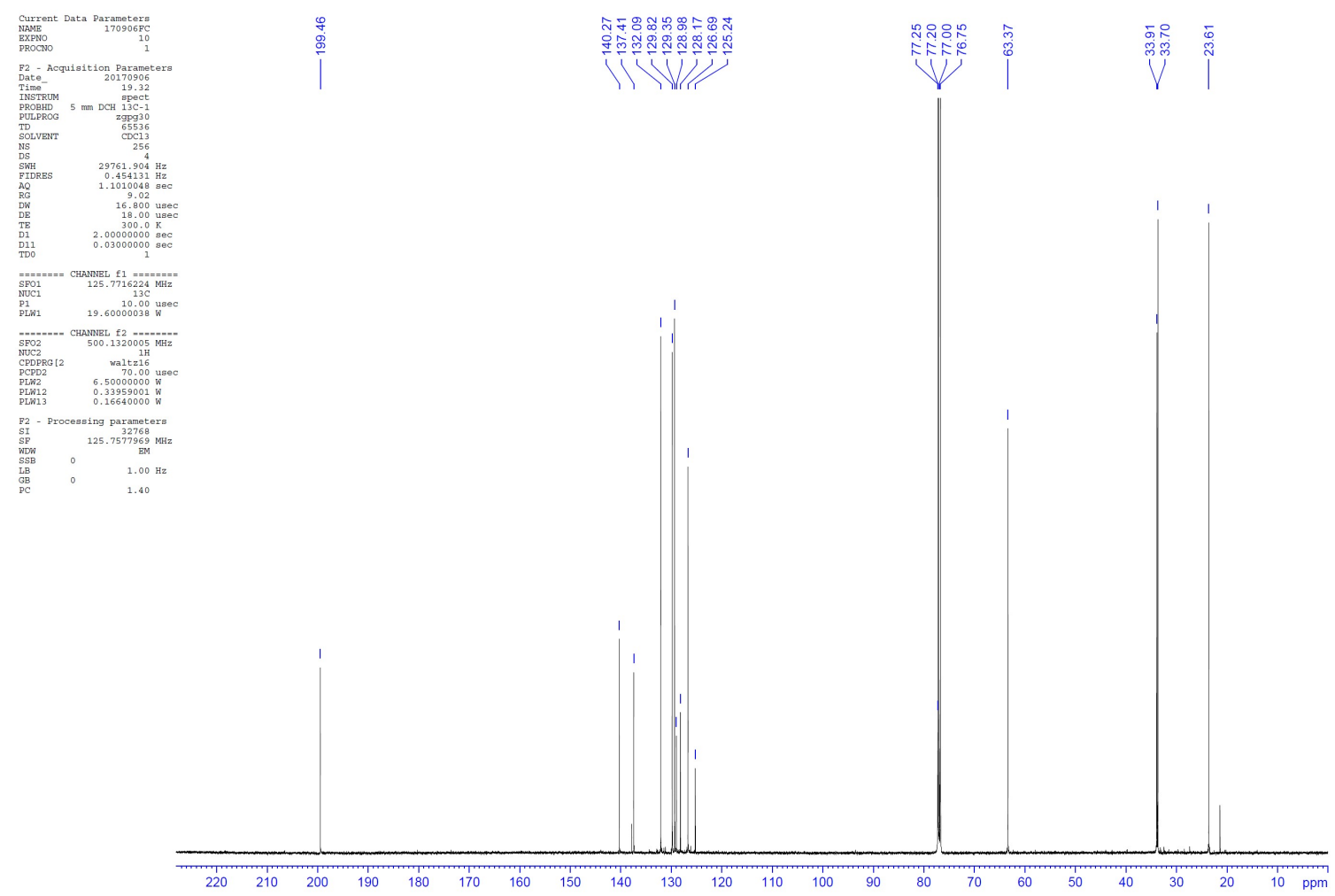
2-Chloroacenaphthylen-1(2H)-one (3j)

${ }^{1} \mathrm{H}-\mathrm{NMR}\left(400 \mathrm{MHz}, \mathrm{CDCl}_{3}\right)$

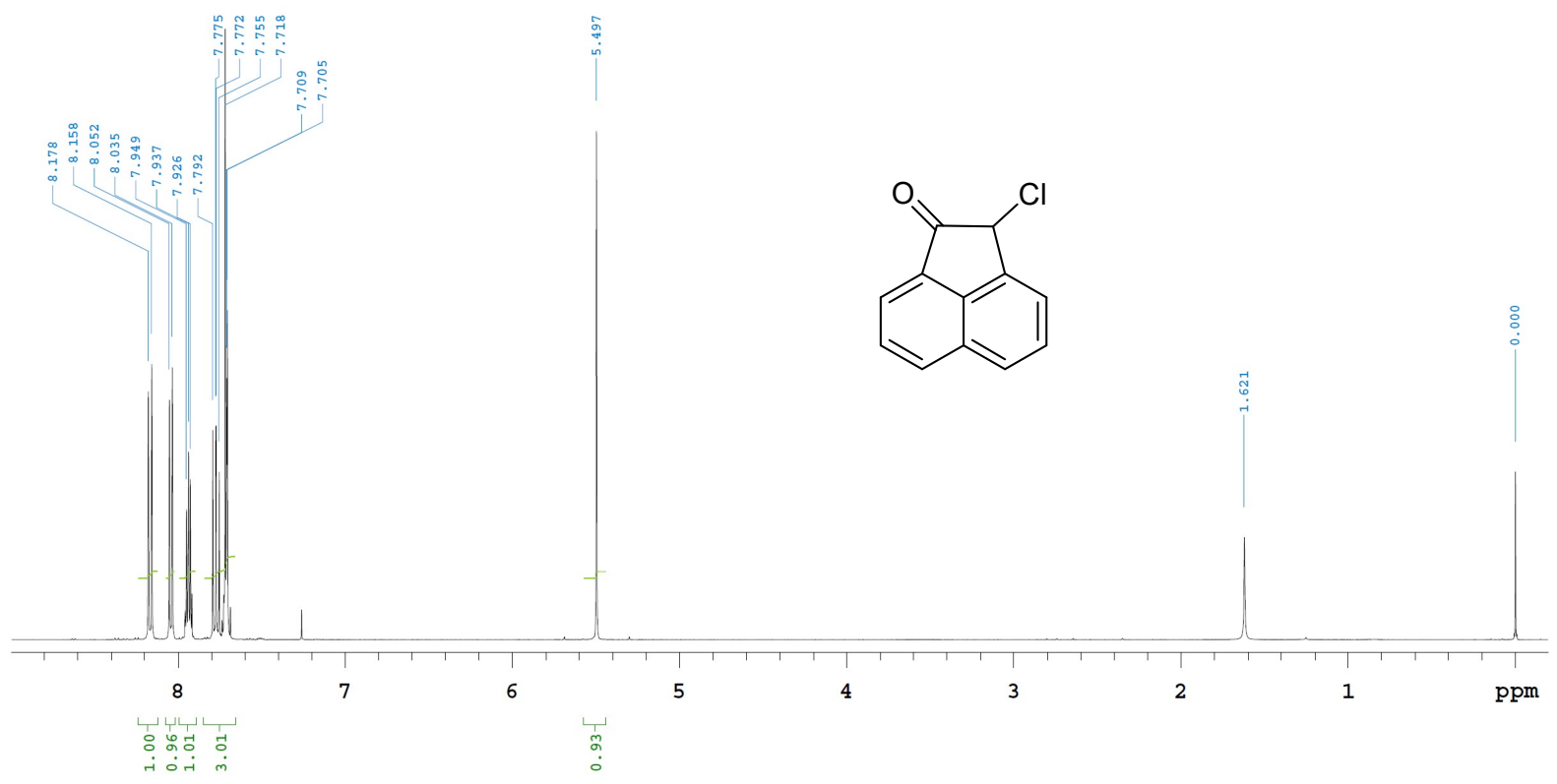

${ }^{13} \mathrm{C} \mathrm{NMR}\left(125 \mathrm{MHz}, \mathrm{CDCl}_{3}\right)$

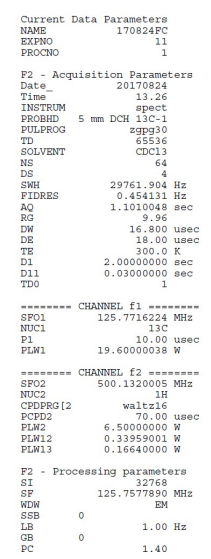

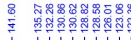

|lvil|
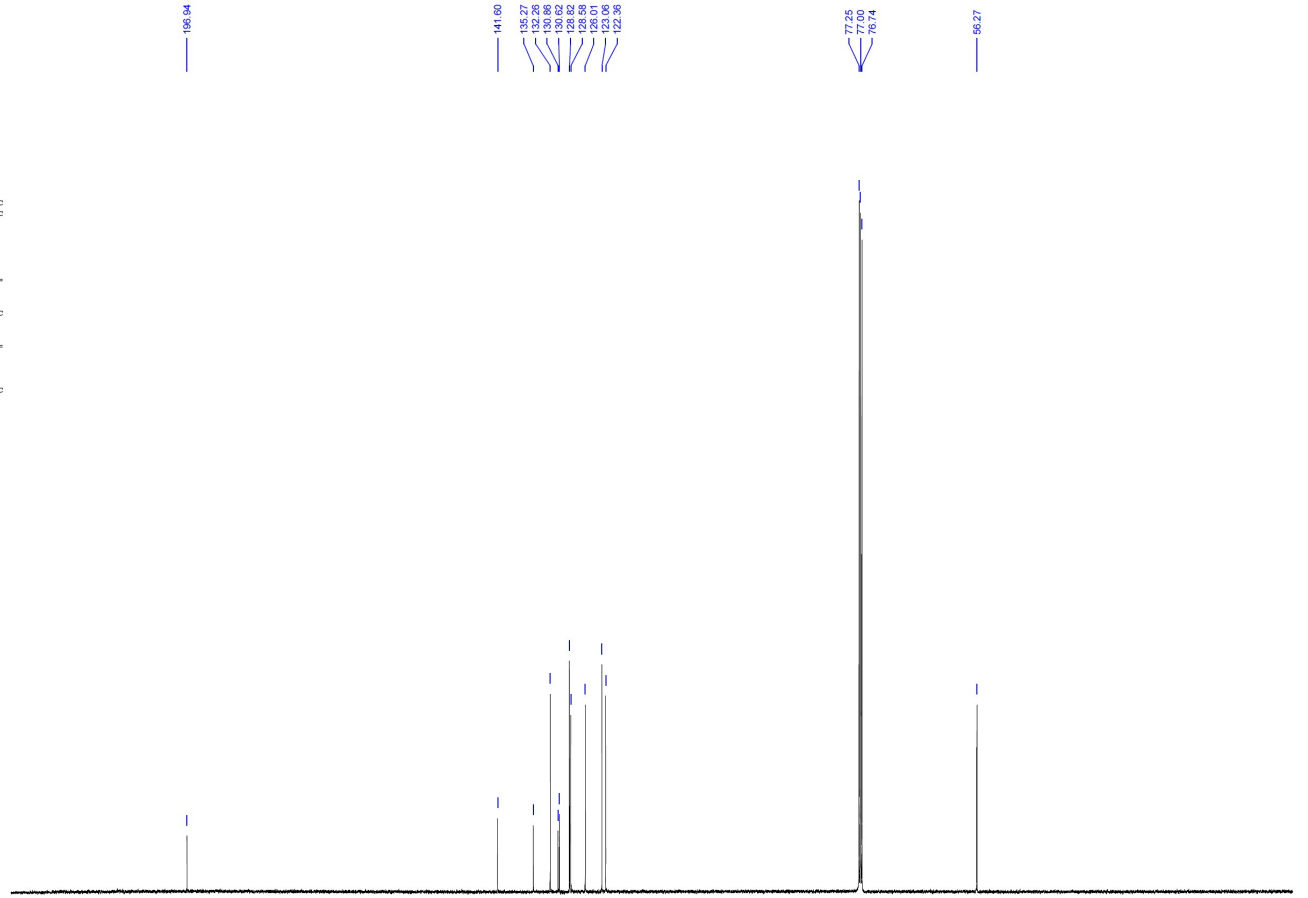
Methyl 1-oxo-2,3-dihydro- $1 H$-indene-2-carboxylate (5a)

${ }^{1} \mathrm{H}-\mathrm{NMR}\left(400 \mathrm{MHz}, \mathrm{CDCl}_{3}\right)$

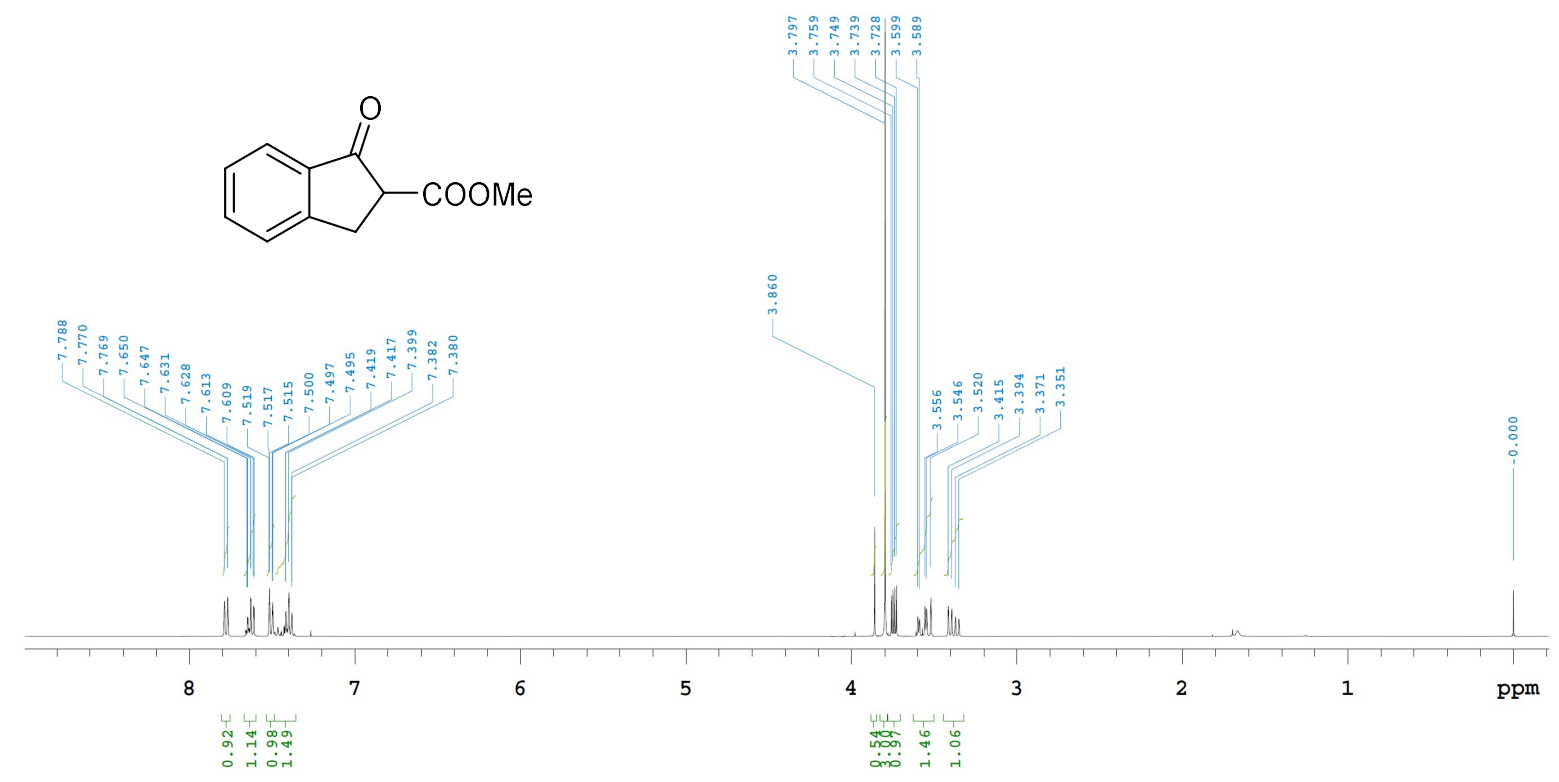

${ }^{13} \mathrm{C} \mathrm{NMR}\left(125 \mathrm{MHz}, \mathrm{CDCl}_{3}\right)$
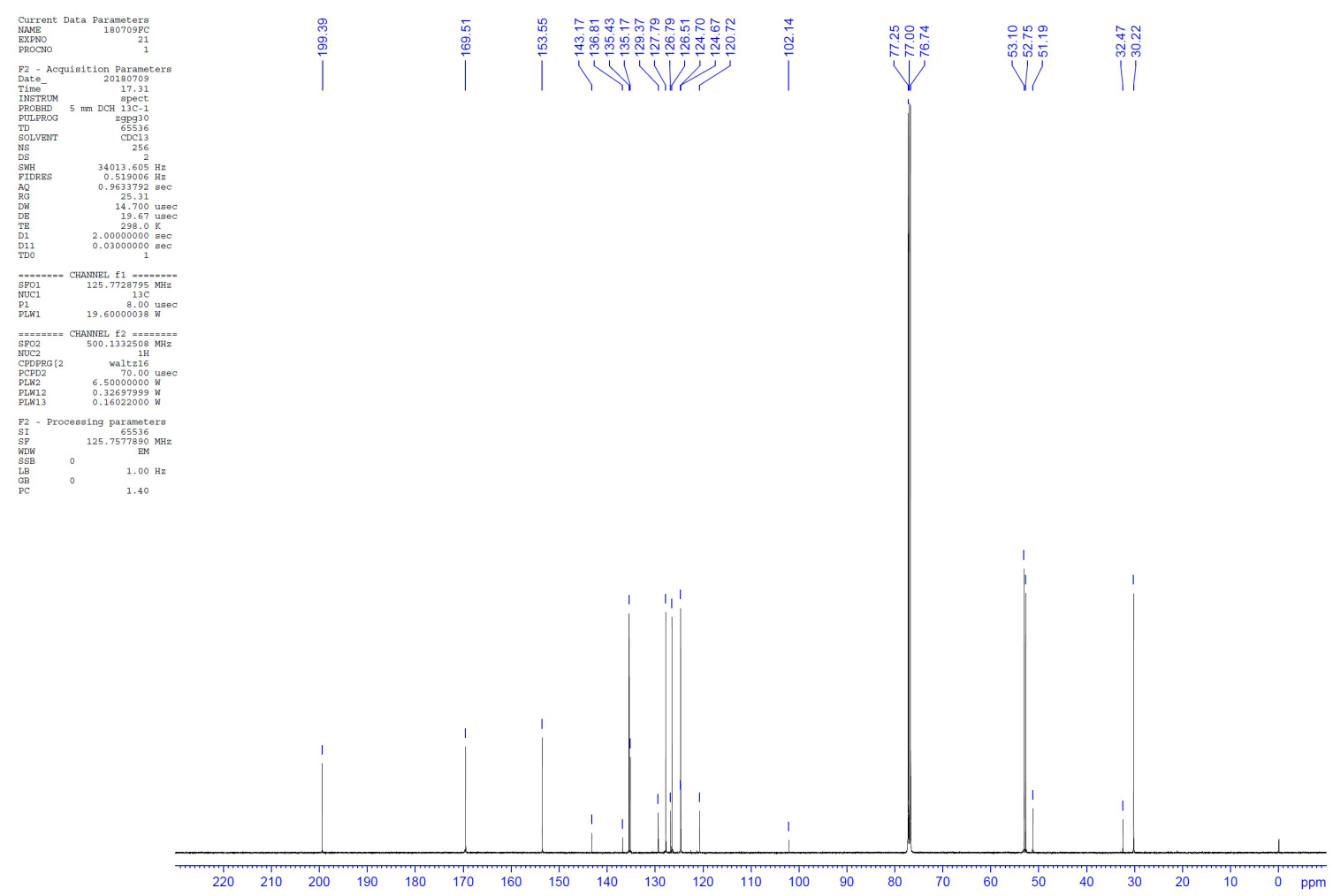
Methyl 5-oxo-6,7,8,9-tetrahydro-5H-benzo[7] annulene-6-carboxylate (5b)

${ }^{1} \mathrm{H}-\mathrm{NMR}\left(400 \mathrm{MHz}, \mathrm{CDCl}_{3}\right)$

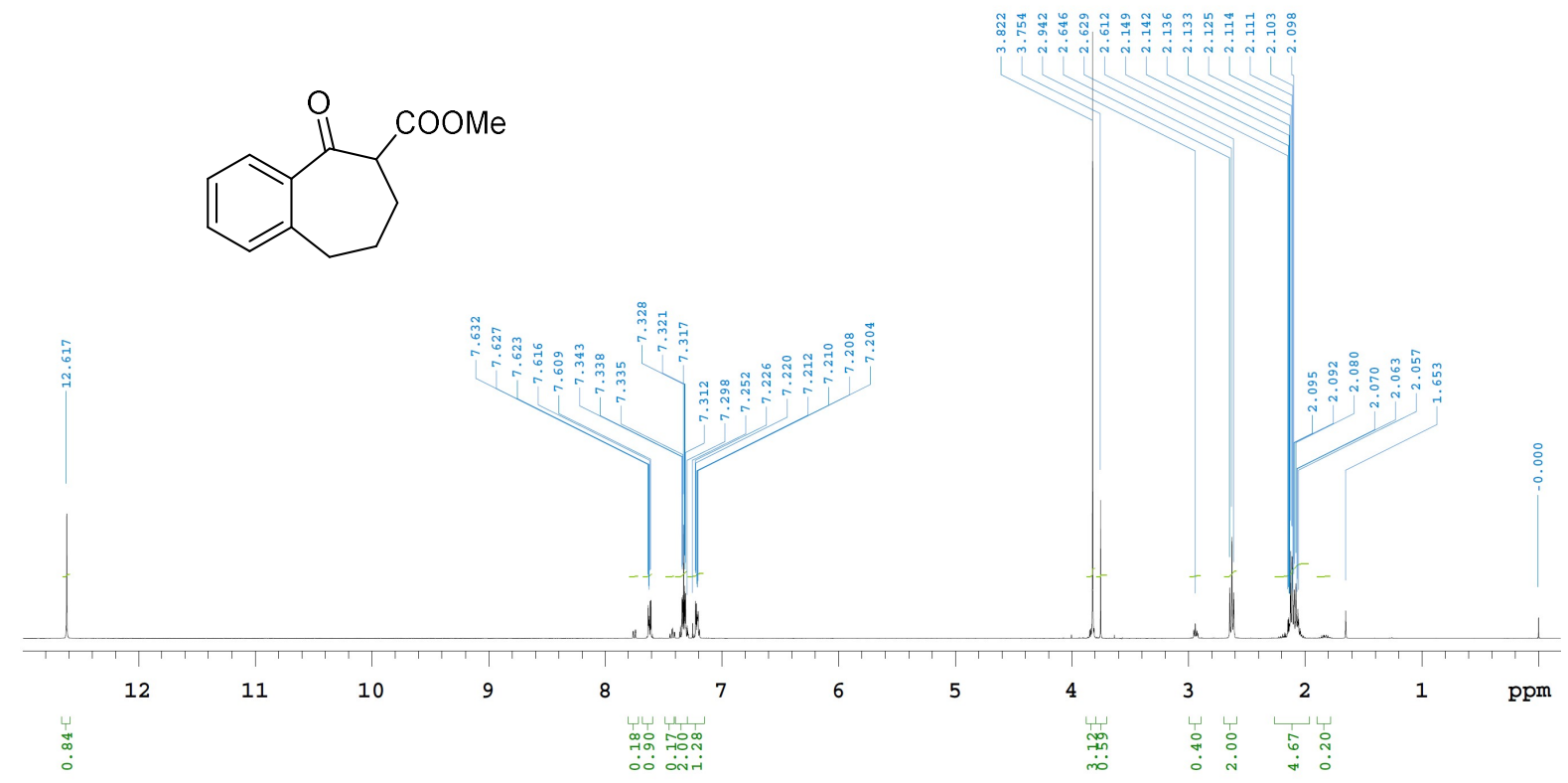

${ }^{13} \mathrm{C} \mathrm{NMR}\left(125 \mathrm{MHz}, \mathrm{CDCl}_{3}\right)$
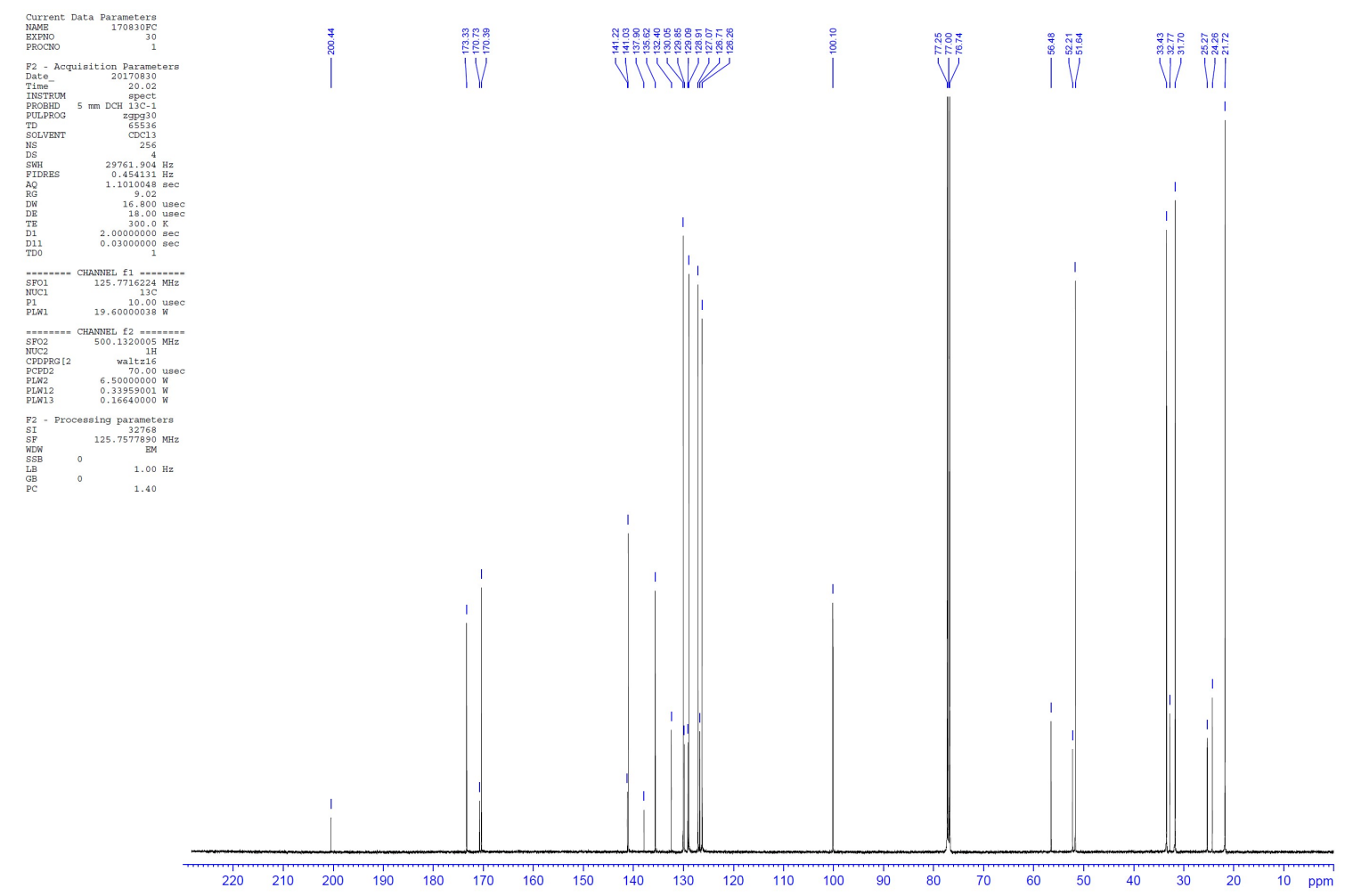
$N$-(tert-Butyl)-1-oxo-2,3-dihydro-1H-indene-2-carboxamide (5c)

${ }^{1} \mathrm{H}-\mathrm{NMR}\left(400 \mathrm{MHz}, \mathrm{CDCl}_{3}\right)$

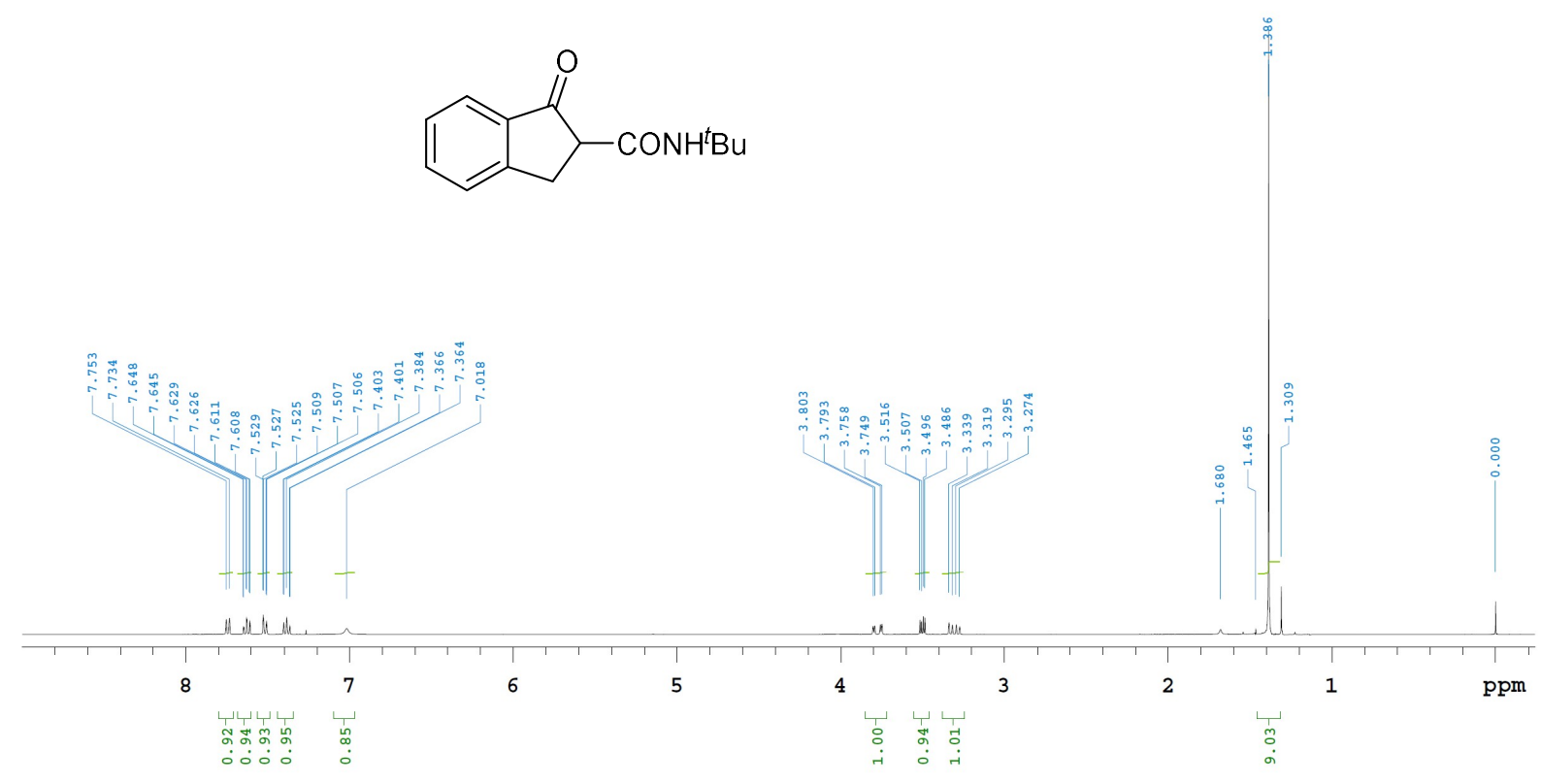

${ }^{13} \mathrm{C} \mathrm{NMR}\left(125 \mathrm{MHz}, \mathrm{CDCl}_{3}\right)$
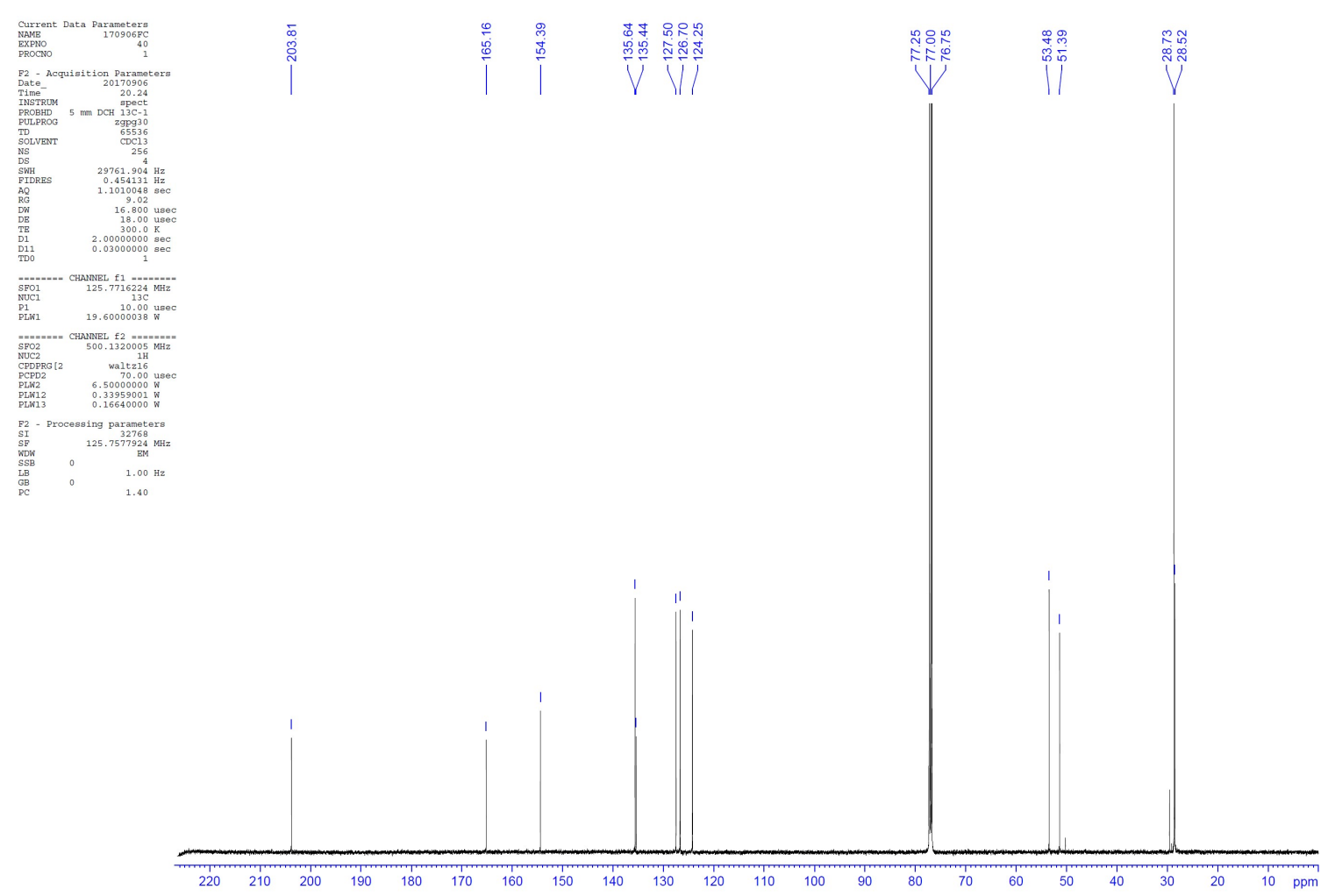
$N$-(tert-Butyl)-1-oxo-1,2,3,4-tetrahydronaphthalene-2-carboxamide (5d)

${ }^{1} \mathrm{H}-\mathrm{NMR}\left(400 \mathrm{MHz}, \mathrm{CDCl}_{3}\right)$

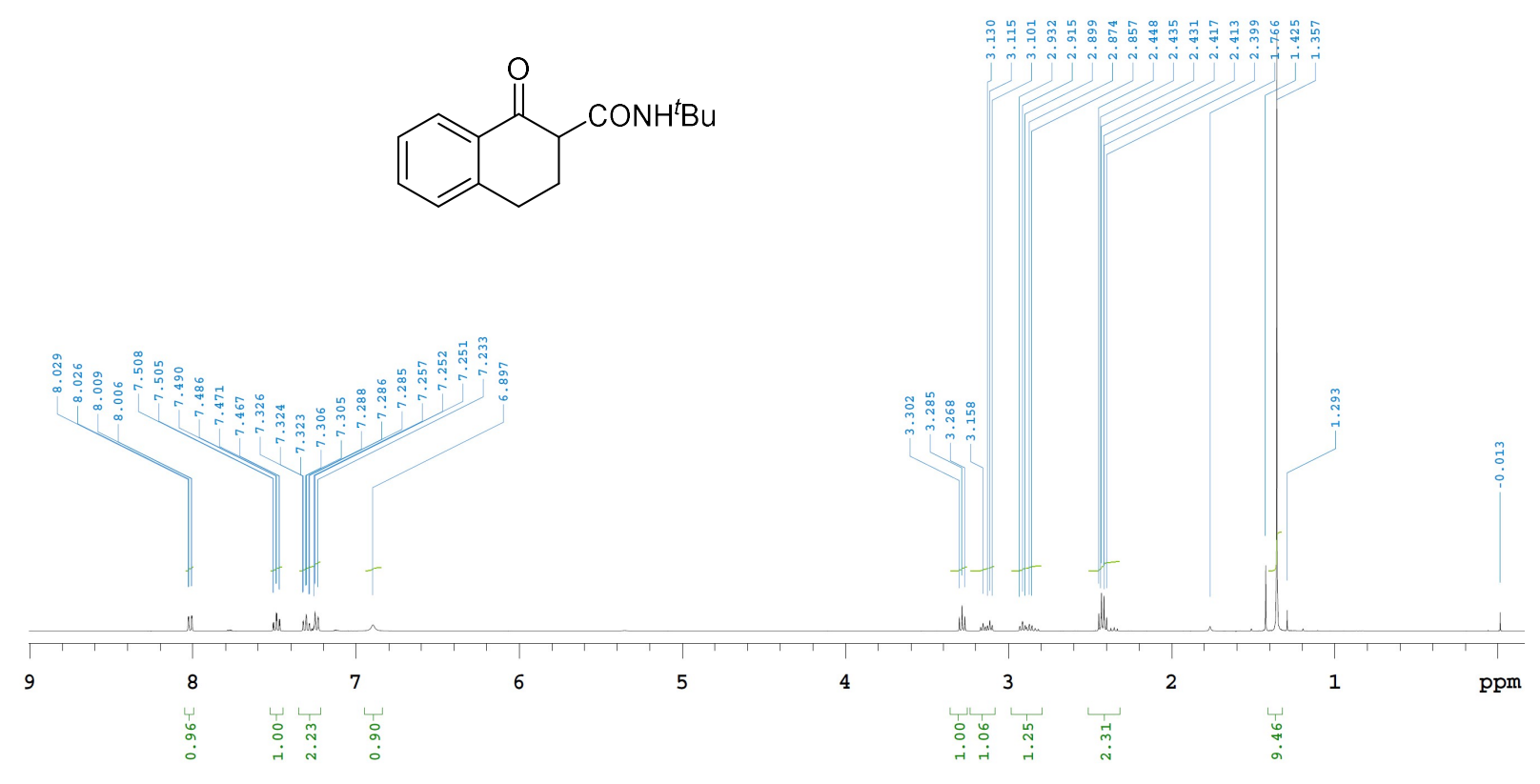

${ }^{13} \mathrm{C} \mathrm{NMR}\left(125 \mathrm{MHz}, \mathrm{CDCl}_{3}\right)$

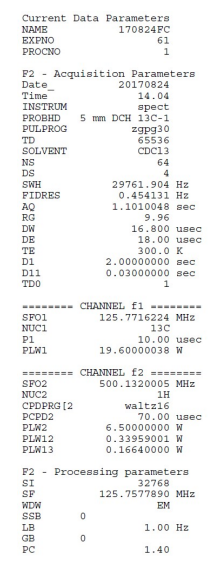

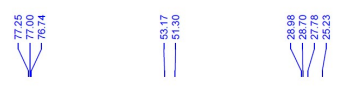

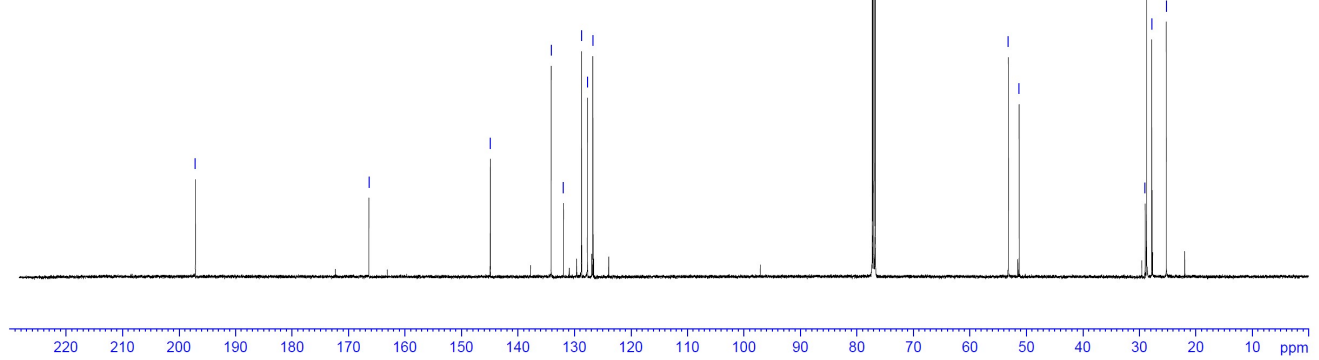


$\mathrm{N}$-(tert-Butyl)-5-oxo-6,7,8,9-tetrahydro-5H-benzo[7]annulene-6-carboxamide (5e)

${ }^{1} \mathrm{H}-\mathrm{NMR}\left(400 \mathrm{MHz}, \mathrm{CDCl}_{3}\right)$

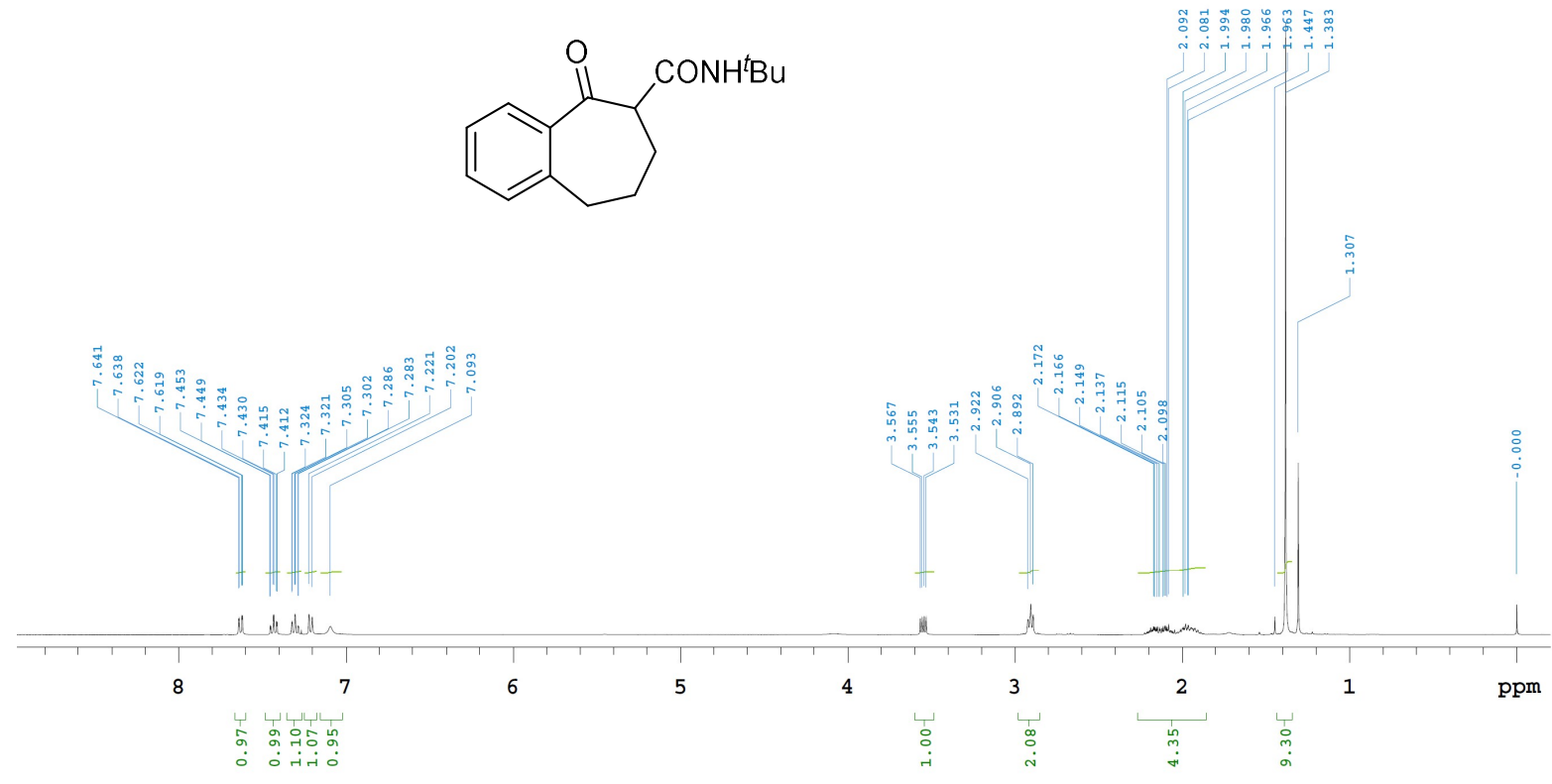

${ }^{13} \mathrm{C} \mathrm{NMR}\left(125 \mathrm{MHz}, \mathrm{CDCl}_{3}\right)$

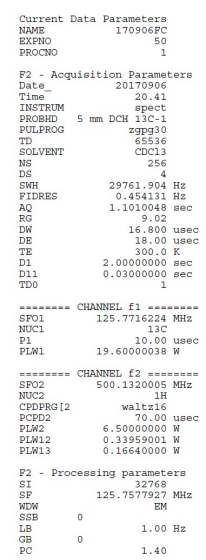

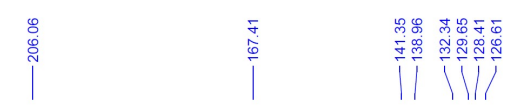

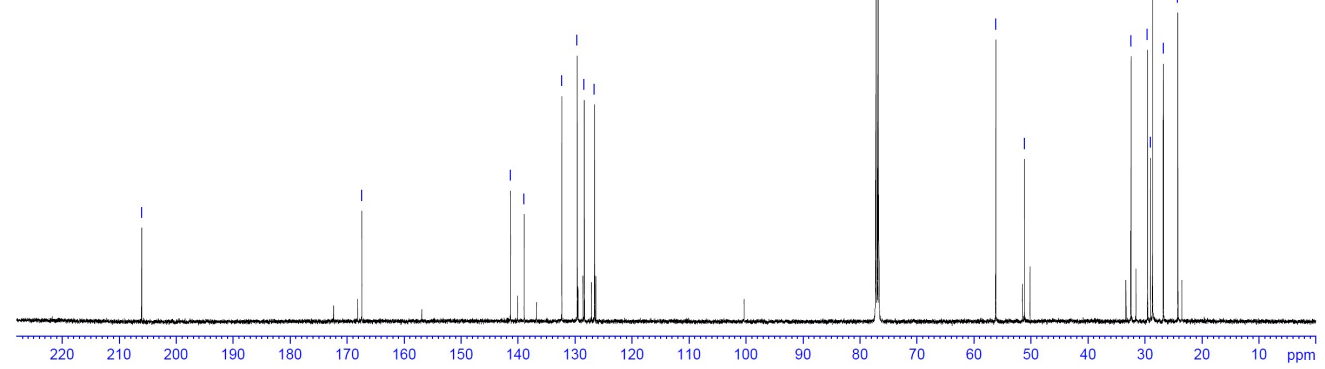


2-(Phenylsulfonyl)-2,3-dihydro- $1 H$-inden-1-one (5f)

${ }^{1} \mathrm{H}-\mathrm{NMR}\left(400 \mathrm{MHz}, \mathrm{CDCl}_{3}\right.$ )

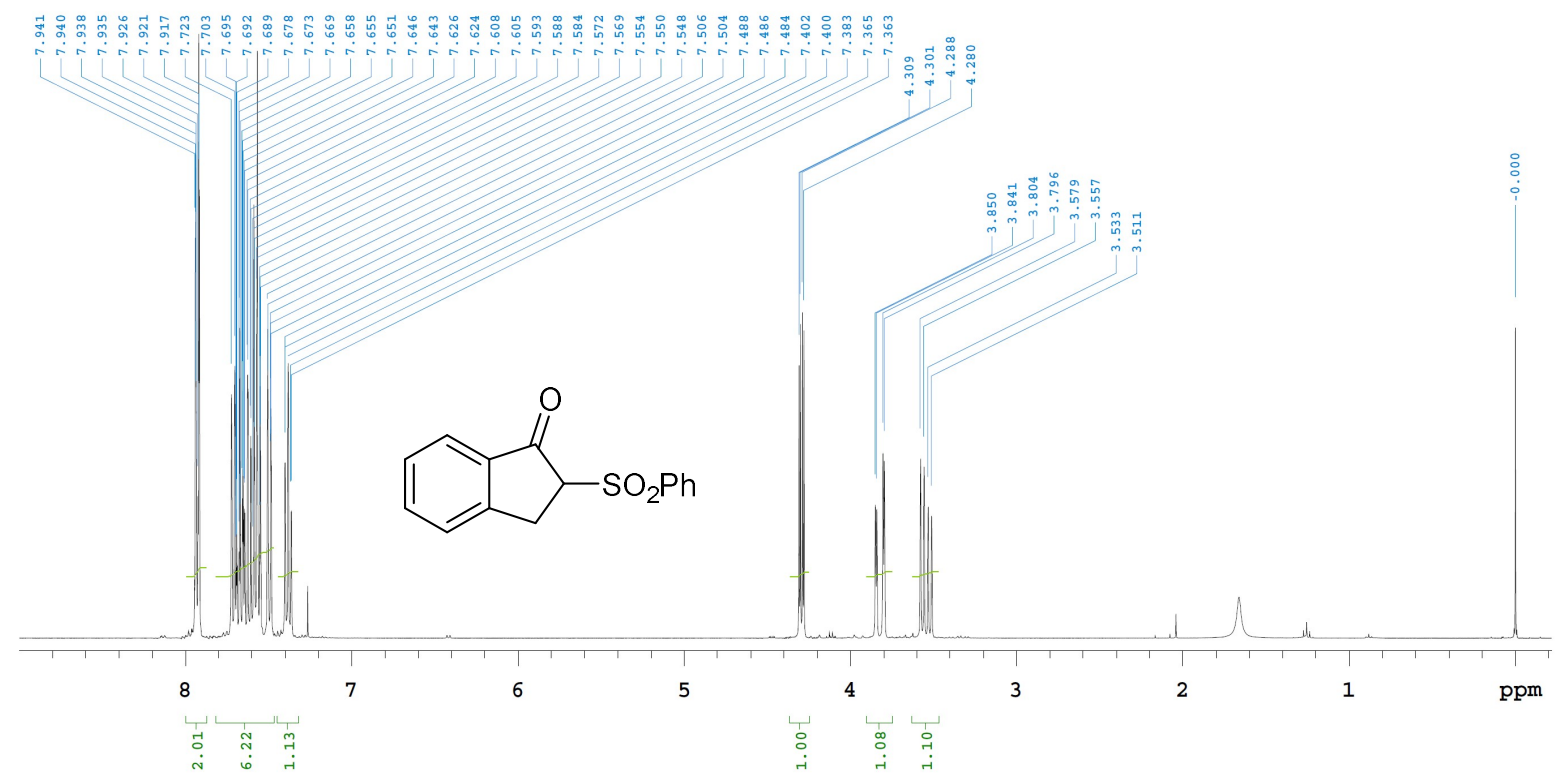

${ }^{13} \mathrm{C} \mathrm{NMR}\left(125 \mathrm{MHz}, \mathrm{CDCl}_{3}\right)$
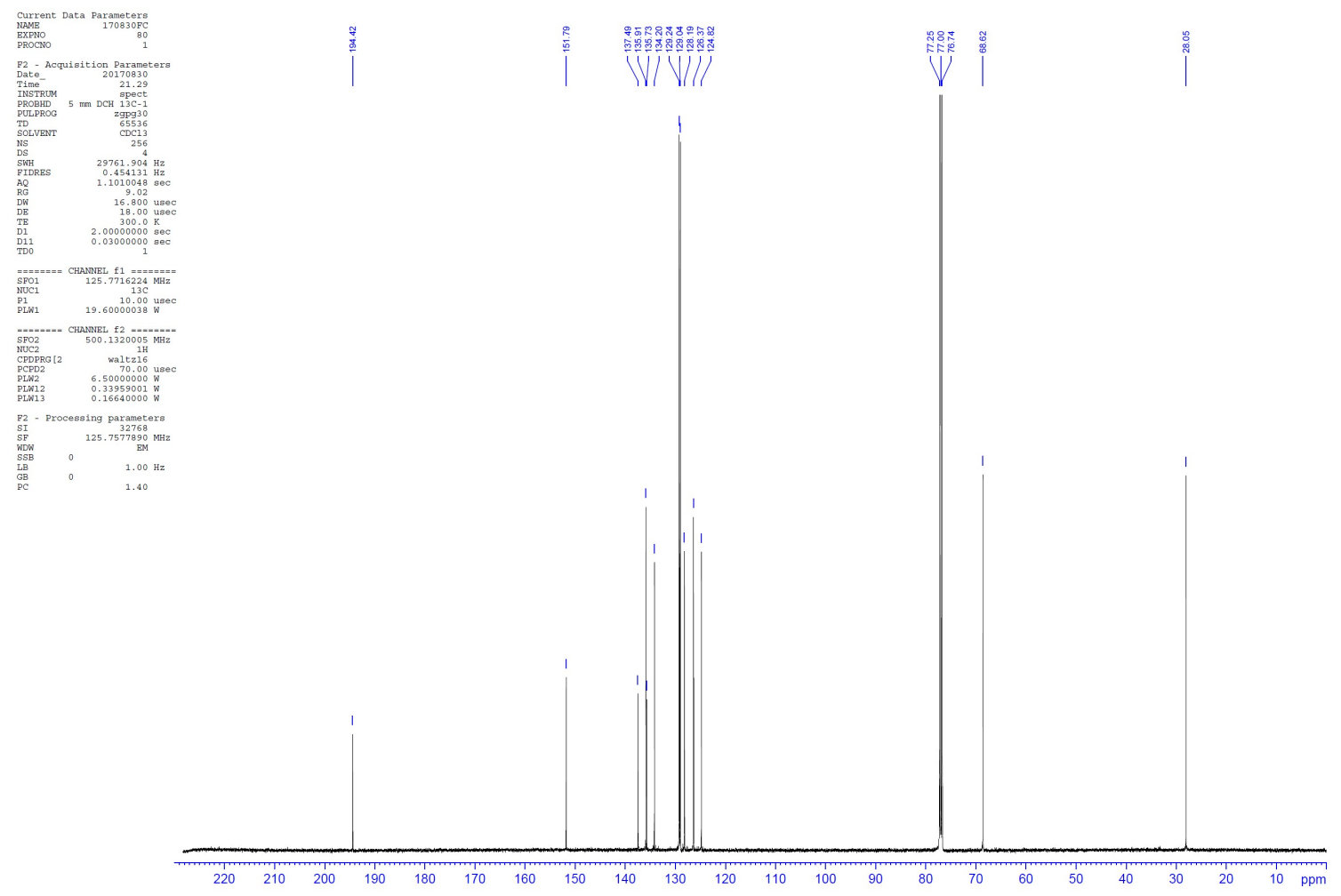
2-(Phenylsulfonyl)-3,4-dihydronaphthalen-1(2H)-one (5g)

${ }^{1} \mathrm{H}-\mathrm{NMR}\left(400 \mathrm{MHz}, \mathrm{CDCl}_{3}\right.$ )

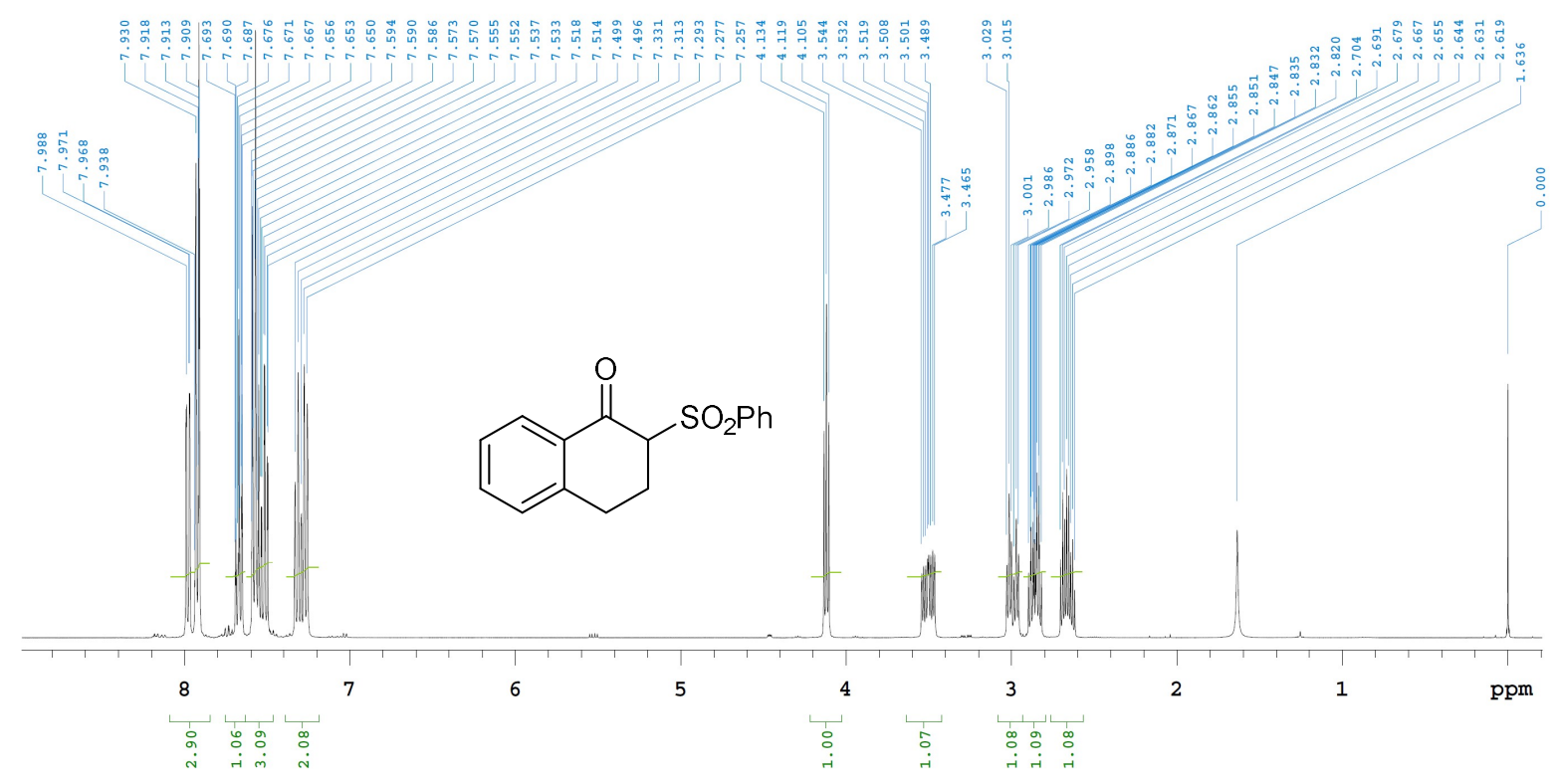

${ }^{13} \mathrm{C} \mathrm{NMR}\left(125 \mathrm{MHz}, \mathrm{CDCl}_{3}\right)$
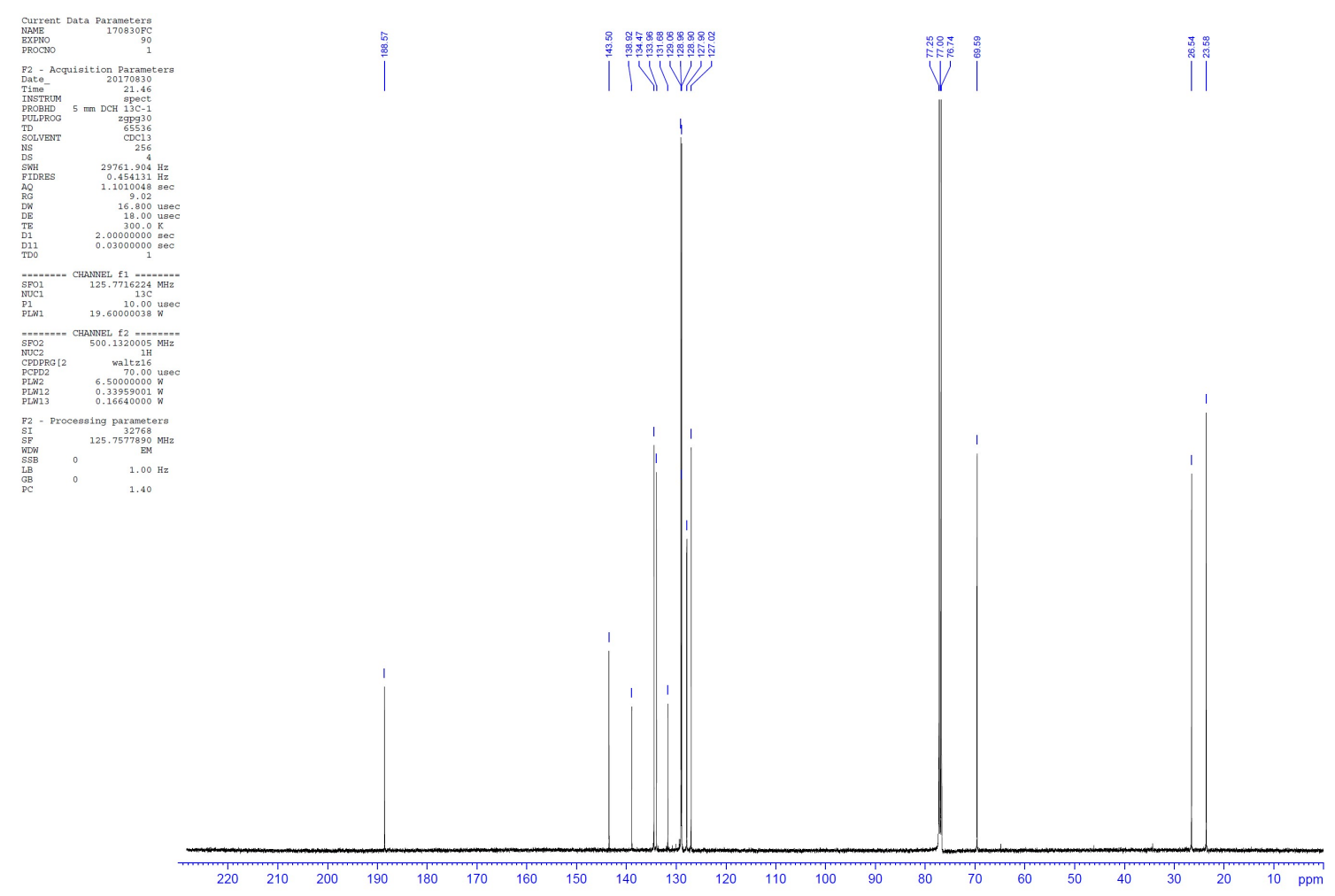
$N$-(tert-Butyl)-2-oxo-1,2-dihydroacenaphthylene-1-carboxamide (5h)

${ }^{1} \mathrm{H}-\mathrm{NMR}\left(400 \mathrm{MHz}, \mathrm{CDCl}_{3}\right)$

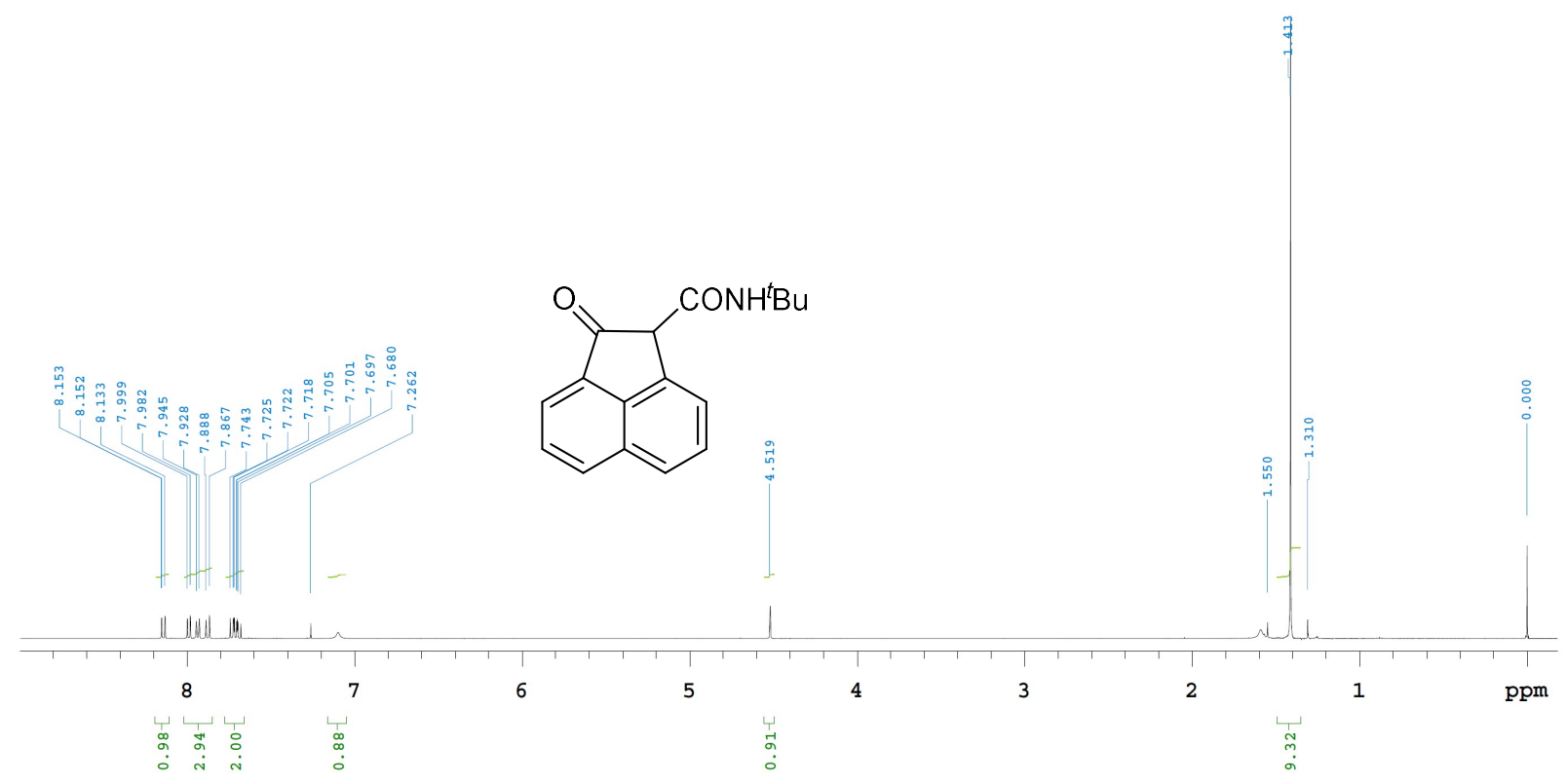

${ }^{13} \mathrm{C}$ NMR $\left(125 \mathrm{MHz}, \mathrm{CDCl}_{3}\right)$
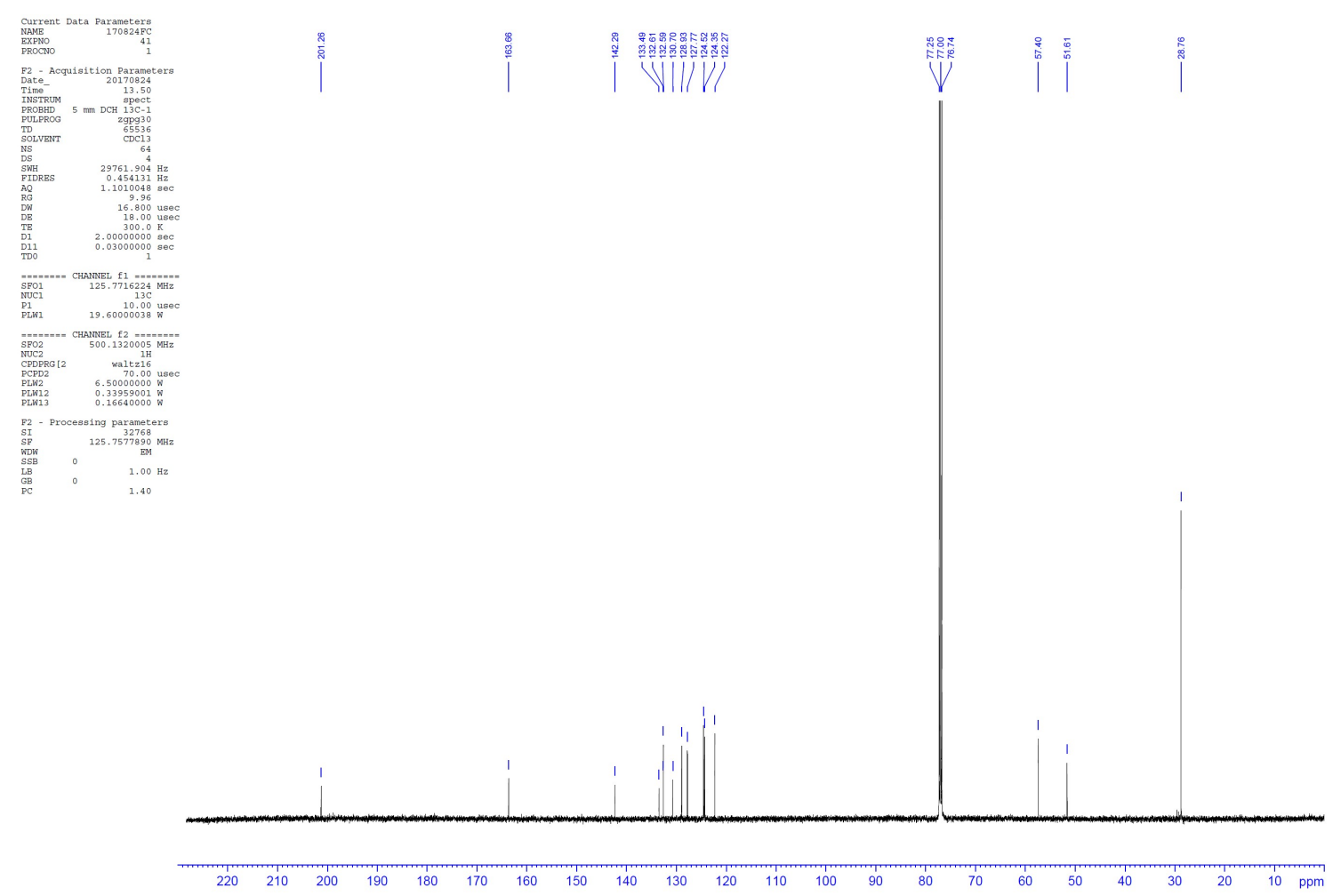
Methyl 2-oxo-1,2-dihydroacenaphthylene-1-carboxylate (5i)

${ }^{1} \mathrm{H}-\mathrm{NMR}\left(400 \mathrm{MHz}, \mathrm{CDCl}_{3}\right.$ )

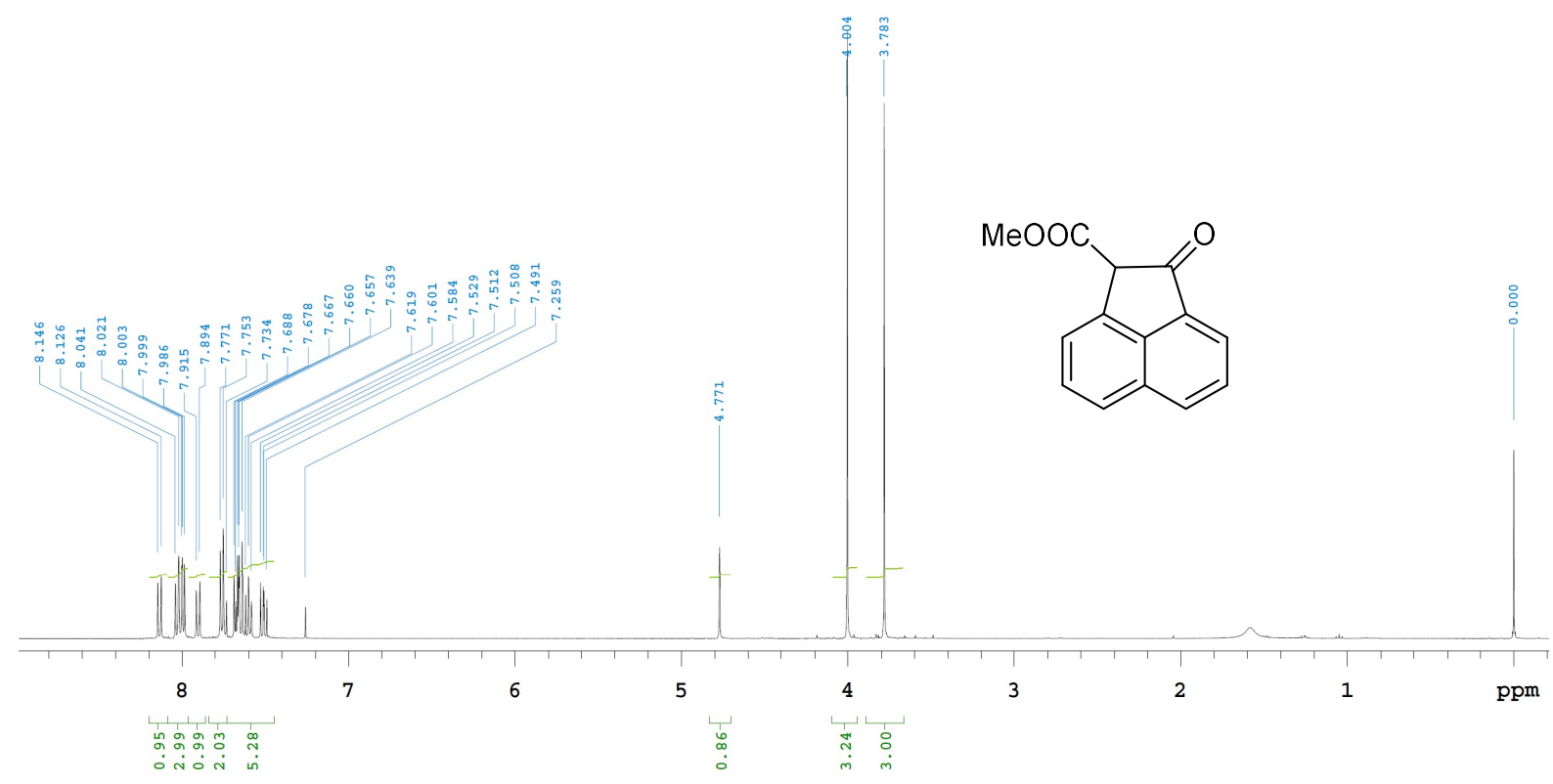

${ }^{13} \mathrm{C}$ NMR $\left(125 \mathrm{MHz}, \mathrm{CDCl}_{3}\right)$
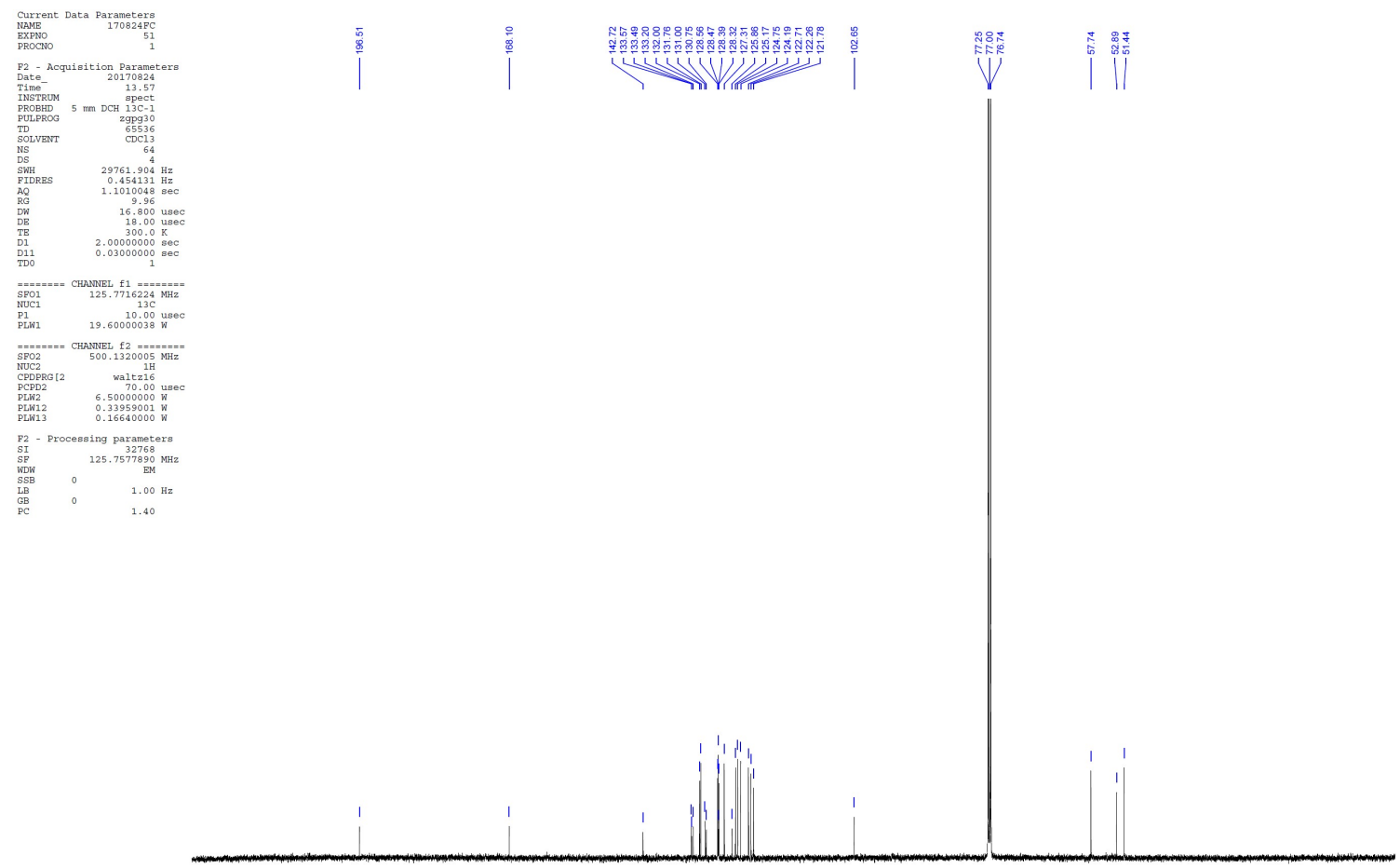

$\begin{array}{lllllllllllllllllllllll}220 & 210 & 200 & 190 & 180 & 170 & 160 & 150 & 140 & 130 & 120 & 110 & 100 & 90 & 80 & 70 & 60 & 50 & 40 & 30 & 20 & 10 & \mathrm{ppm}\end{array}$ 
6-Chloro-3-methylcyclohex-2-en-1-one (7a)

${ }^{1} \mathrm{H}-\mathrm{NMR}\left(500 \mathrm{MHz}, \mathrm{CDCl}_{3}\right)$

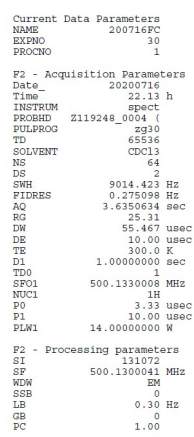

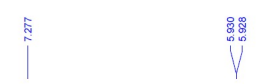

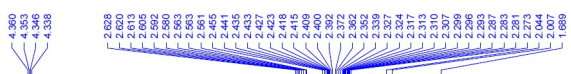

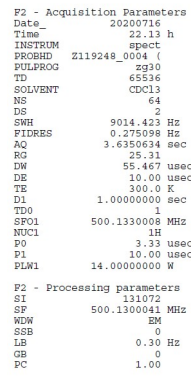

W

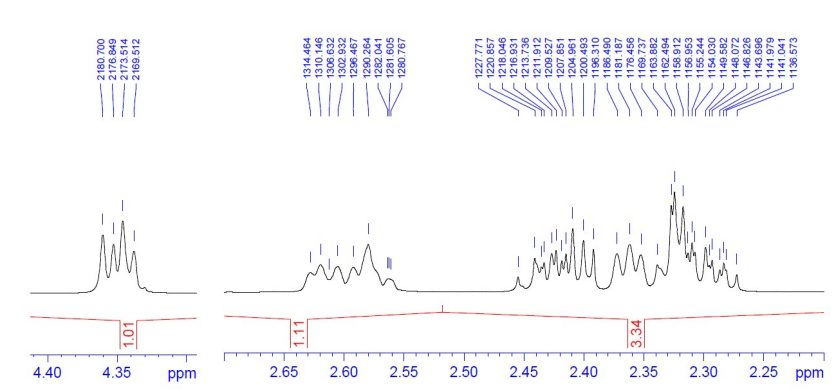<smiles>CC1=CC(=O)C(Cl)CC1</smiles>

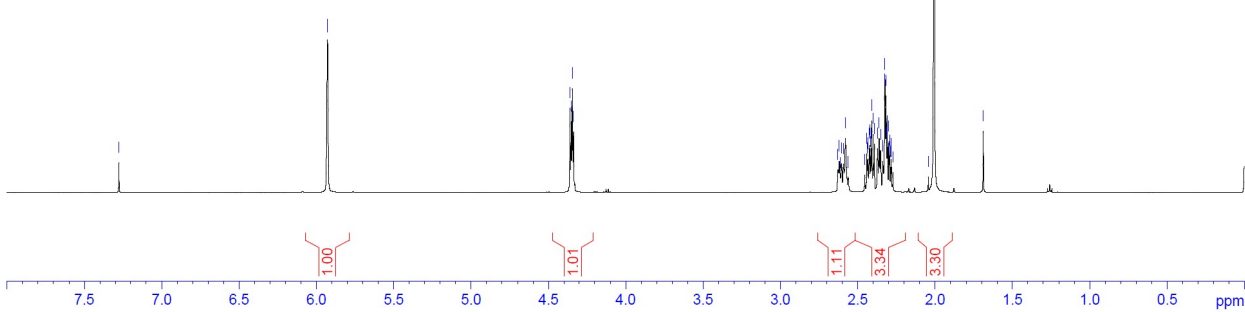

${ }^{13} \mathrm{C} \mathrm{NMR}\left(125 \mathrm{MHz}, \mathrm{CDCl}_{3}\right)$
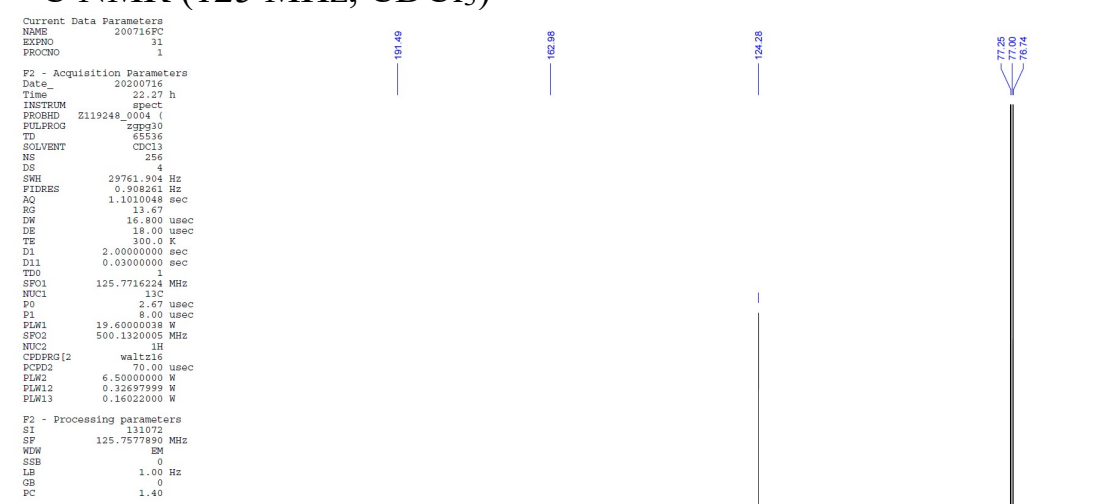

|

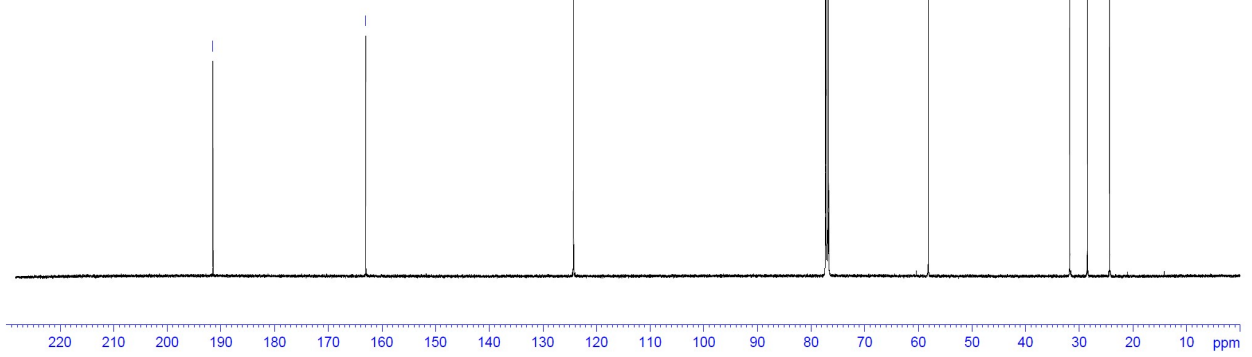


Ethyl 4-methyl-2-oxocyclohex-3-ene-1-carboxylate (7b)

${ }^{1} \mathrm{H}-\mathrm{NMR}\left(500 \mathrm{MHz}, \mathrm{CDCl}_{3}\right)$
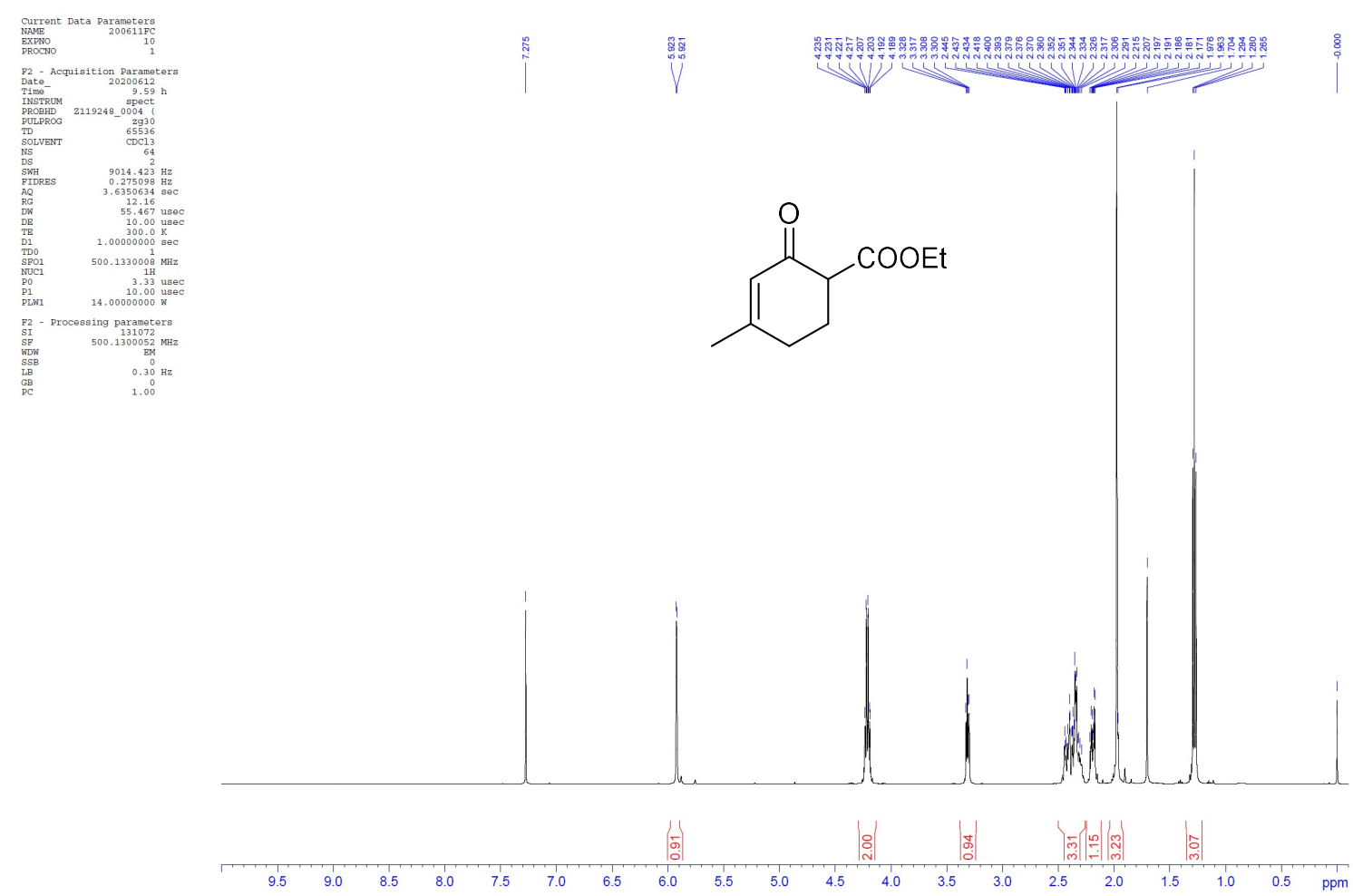

${ }^{13} \mathrm{C} \mathrm{NMR}\left(125 \mathrm{MHz}, \mathrm{CDCl}_{3}\right)$

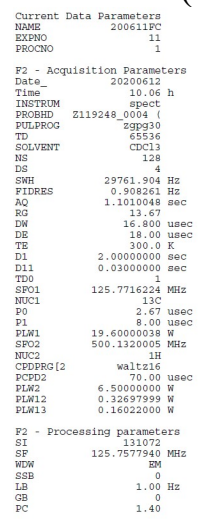

造
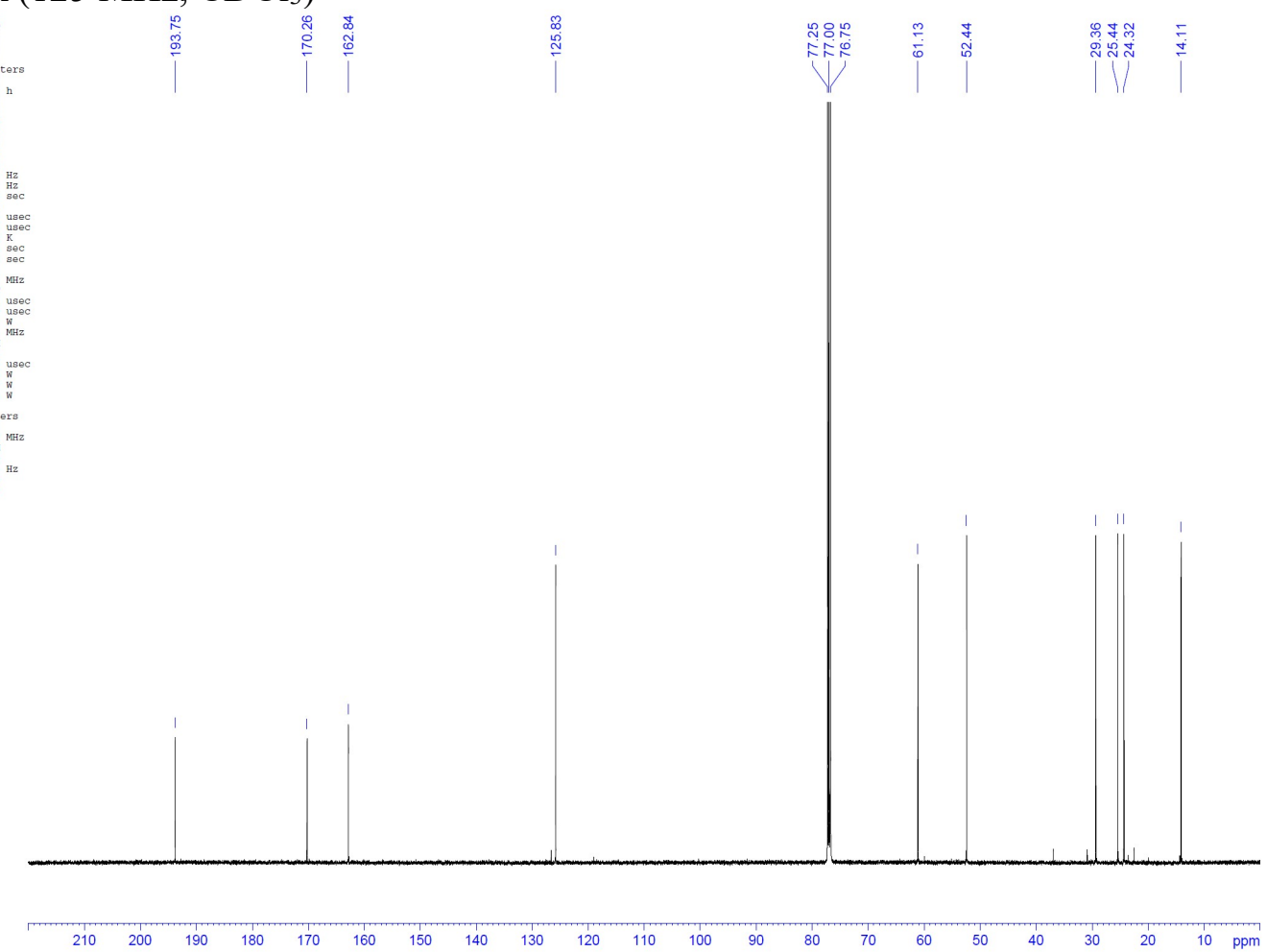
$N$-(tert-Butyl)-4-methyl-2-oxocyclohex-3-ene-1-carboxamide (7c)

${ }^{1} \mathrm{H}-\mathrm{NMR}\left(500 \mathrm{MHz}, \mathrm{CDCl}_{3}\right)$
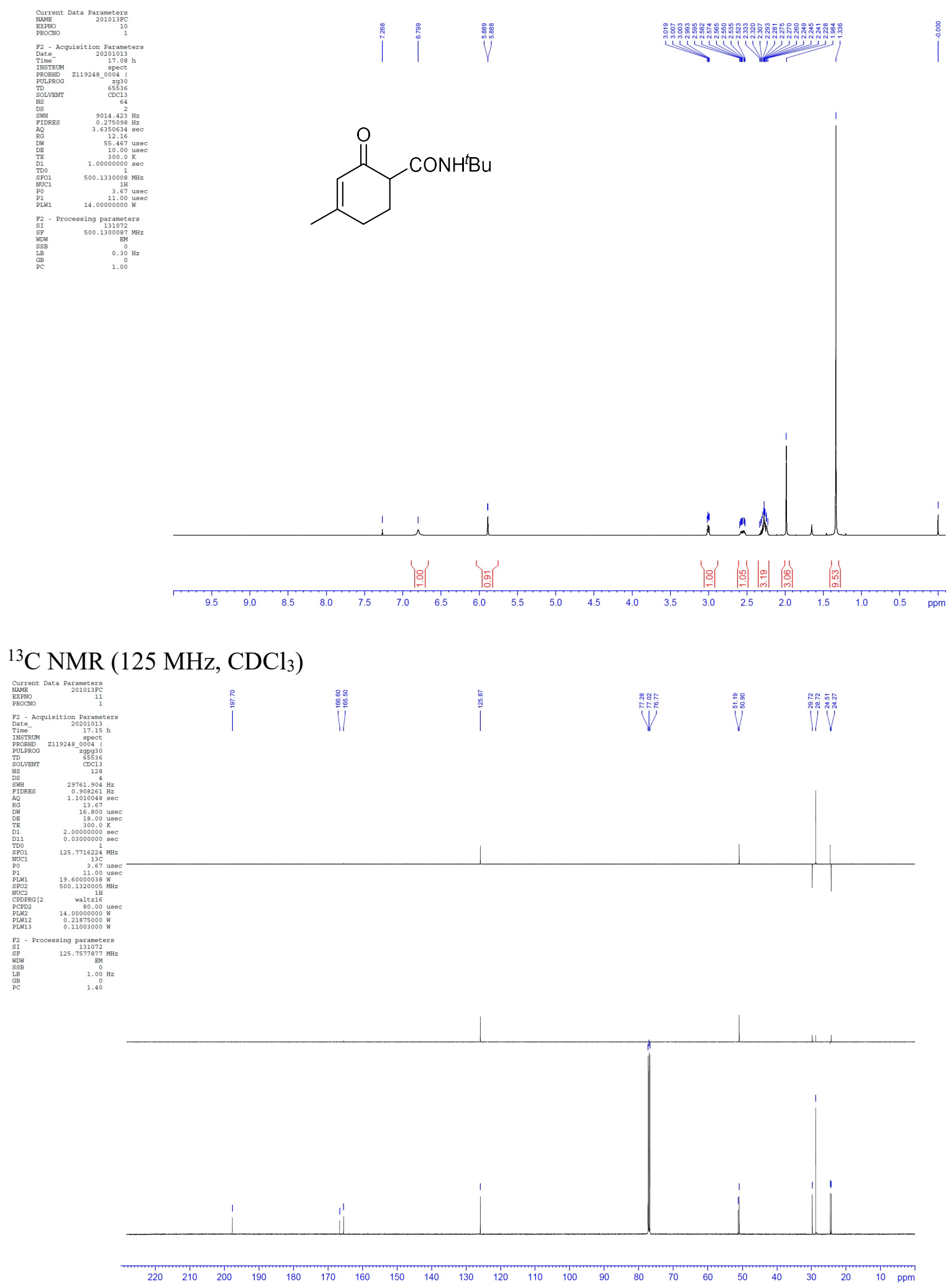
(1S,2R)-2-Chloro-1,2,3,4-tetrahydronaphthalen-1-ol (cis-4a)

${ }^{1} \mathrm{H}-\mathrm{NMR}\left(400 \mathrm{MHz}, \mathrm{CDCl}_{3}\right)$

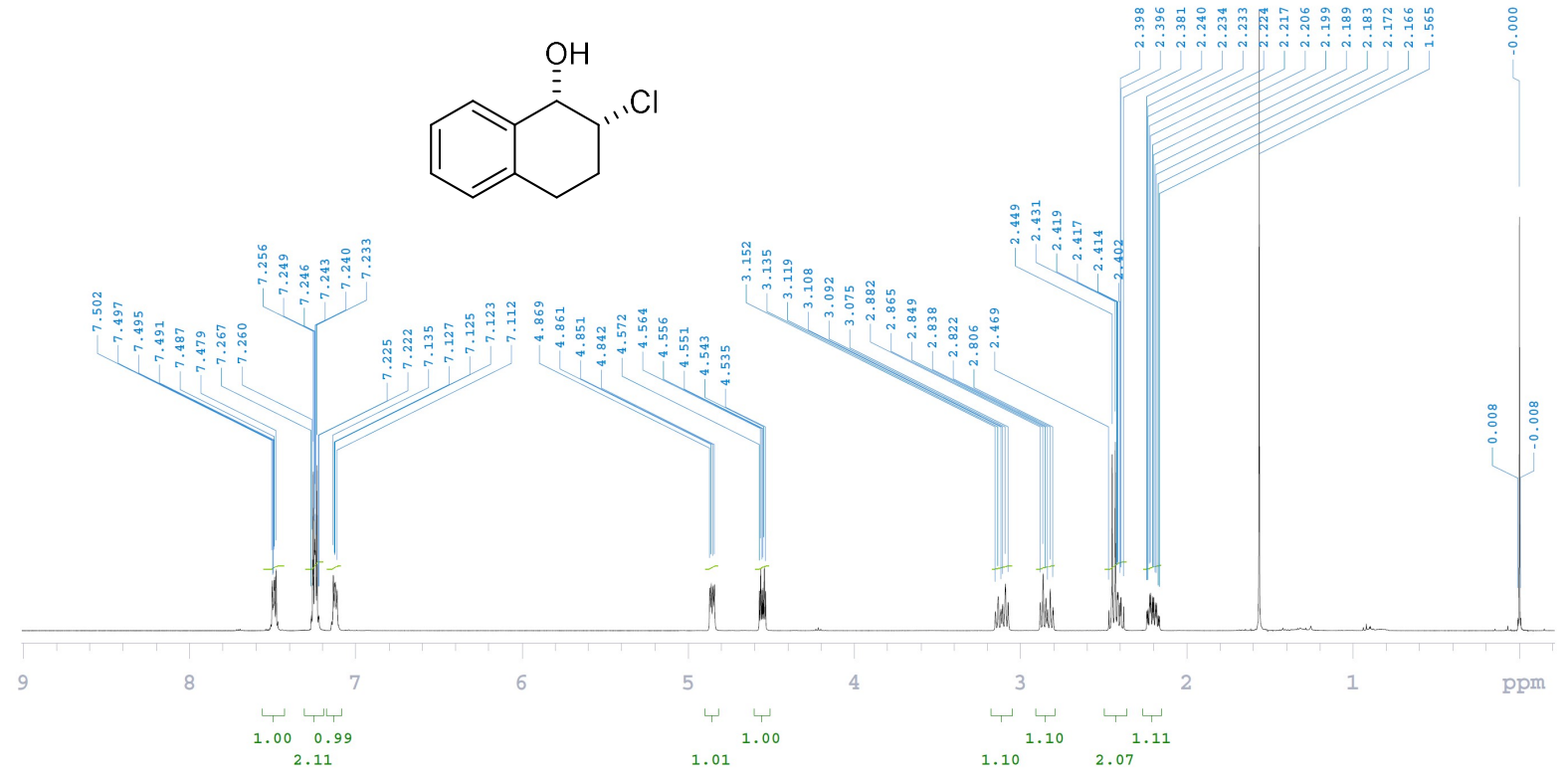

${ }^{13} \mathrm{C} \mathrm{NMR}\left(125 \mathrm{MHz}, \mathrm{CDCl}_{3}\right)$
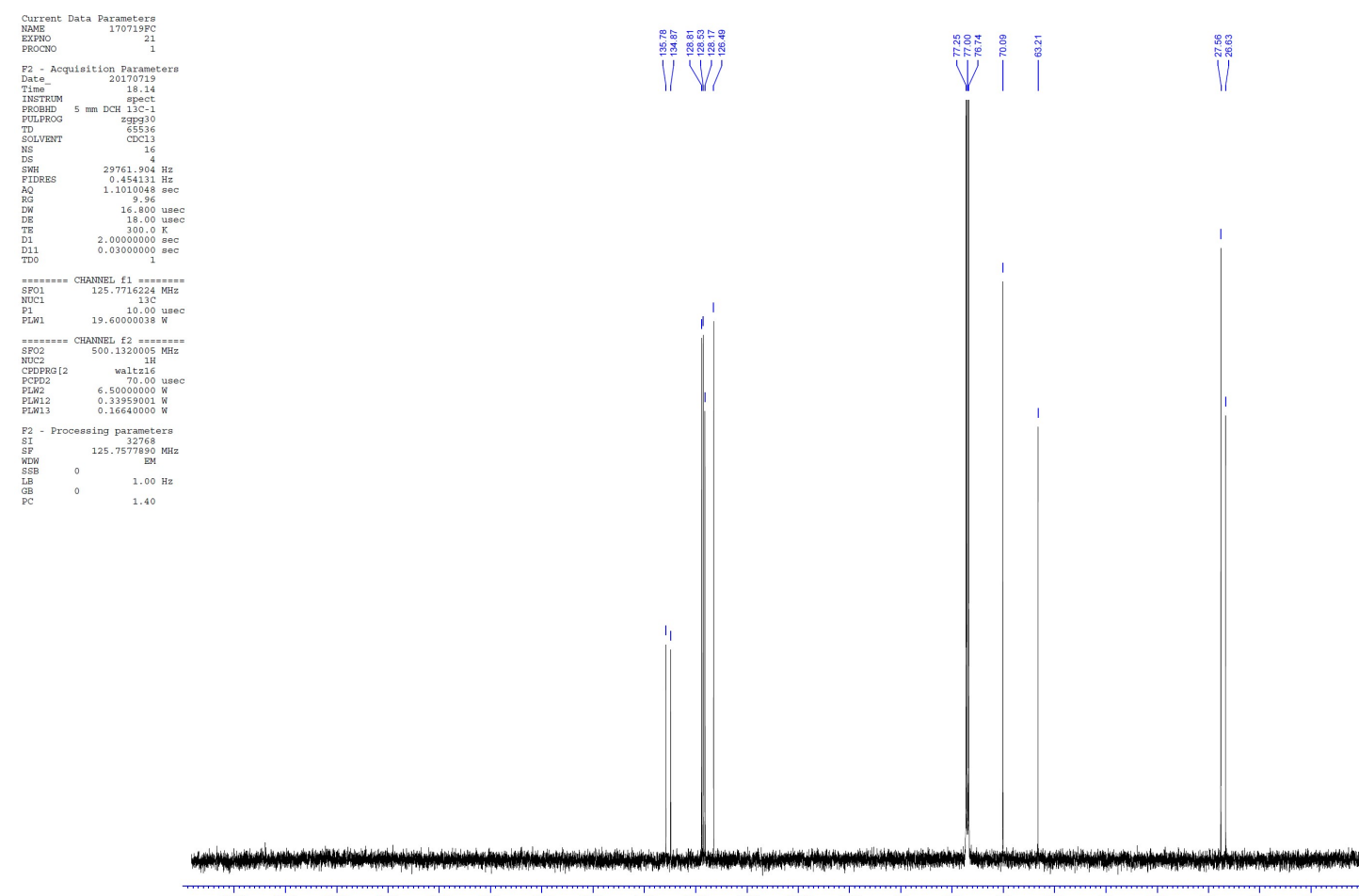
(1S,2R)-2-Chloro-7-methoxy-1,2,3,4-tetrahydronaphthalen-1-ol (cis-4b)

${ }^{1} \mathrm{H}-\mathrm{NMR}\left(500 \mathrm{MHz}, \mathrm{CDCl}_{3}\right)$
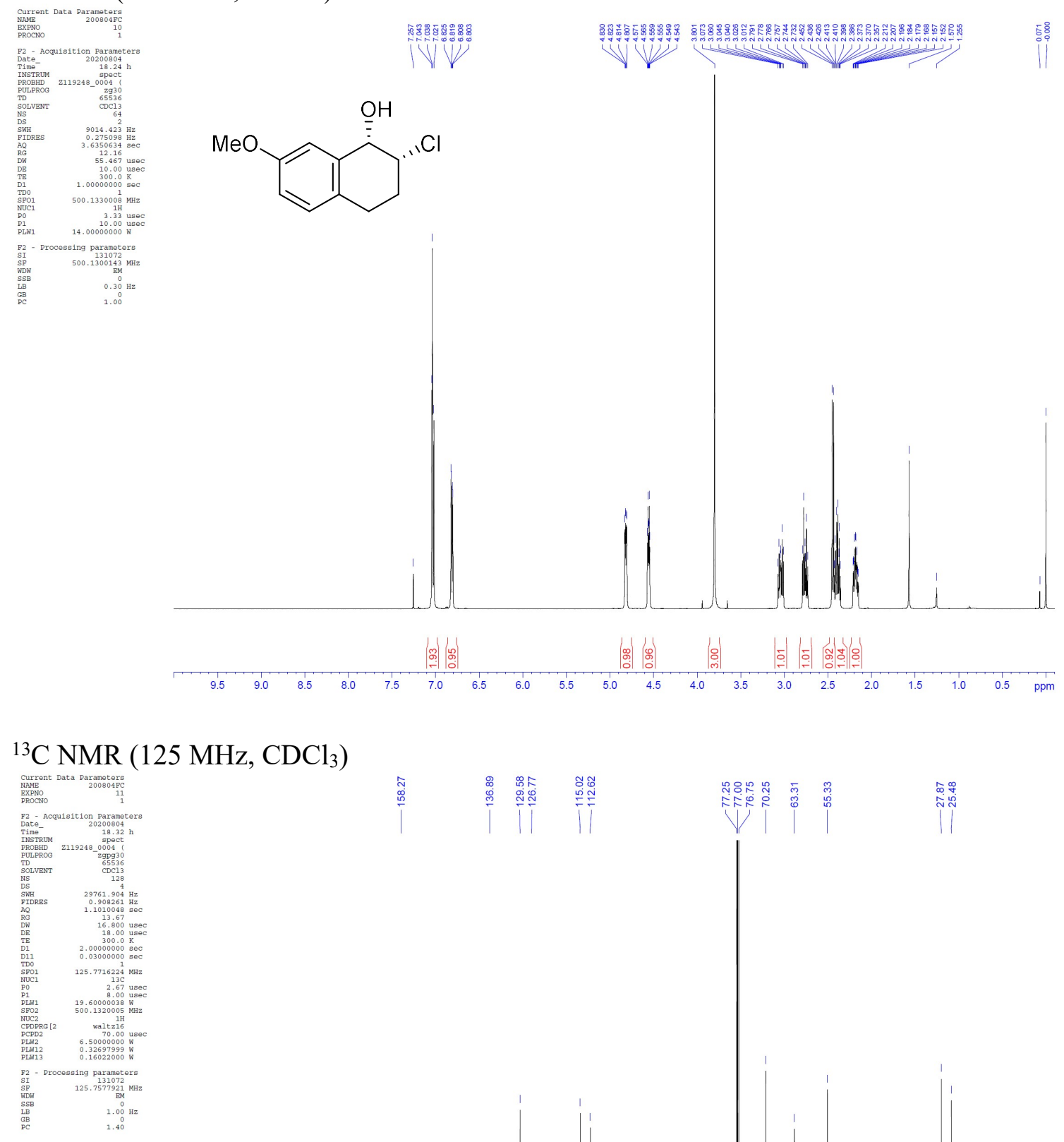
(1S,2R)-2-Chloro-7-bromo-1,2,3,4-tetrahydronaphthalen-1-ol (cis-4c)

${ }^{1} \mathrm{H}-\mathrm{NMR}\left(500 \mathrm{MHz}, \mathrm{CDCl}_{3}\right.$ )
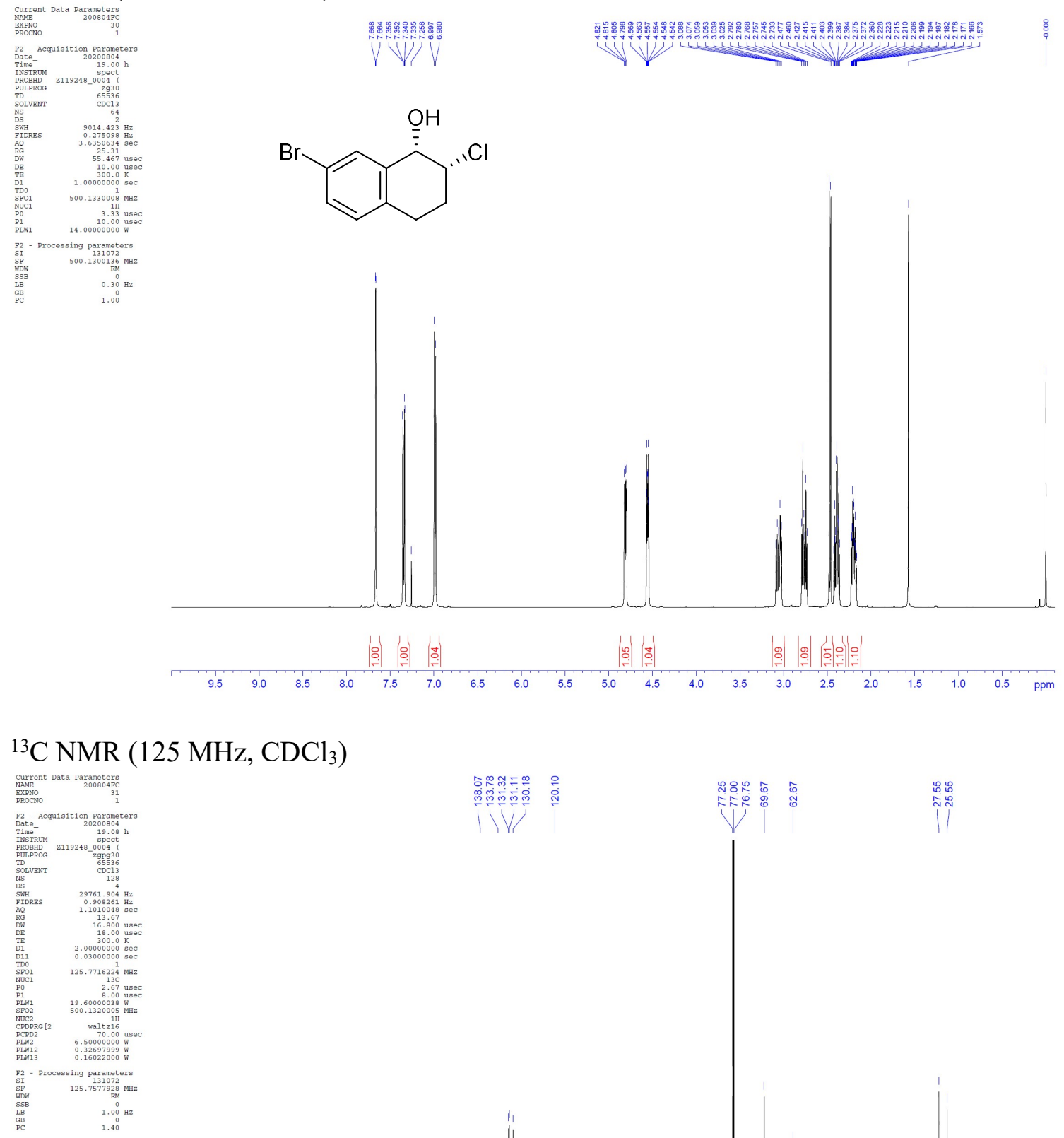
(1S,2R)-2-Chloro-2,3-dihydro-1H-inden-1-ol ((cis-4d)

${ }^{1} \mathrm{H}-\mathrm{NMR}\left(400 \mathrm{MHz}, \mathrm{CDCl}_{3}\right)$

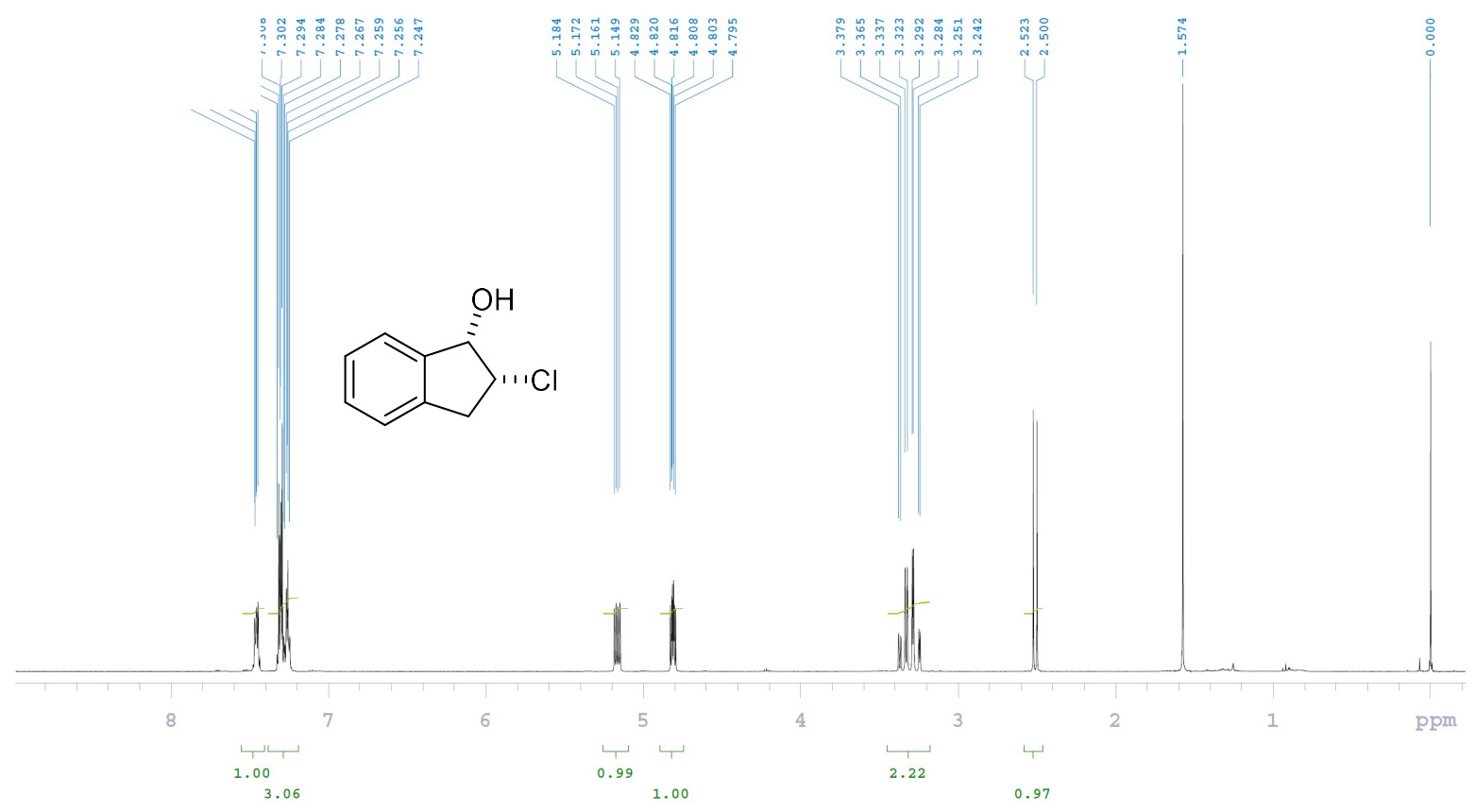

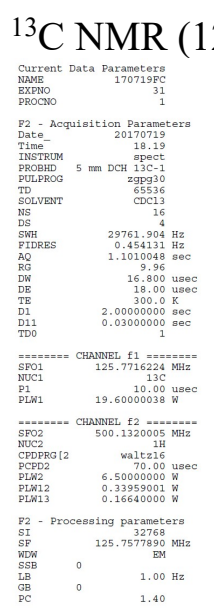

$\left.125 \mathrm{MHz}, \mathrm{CDCl}_{3}\right)$
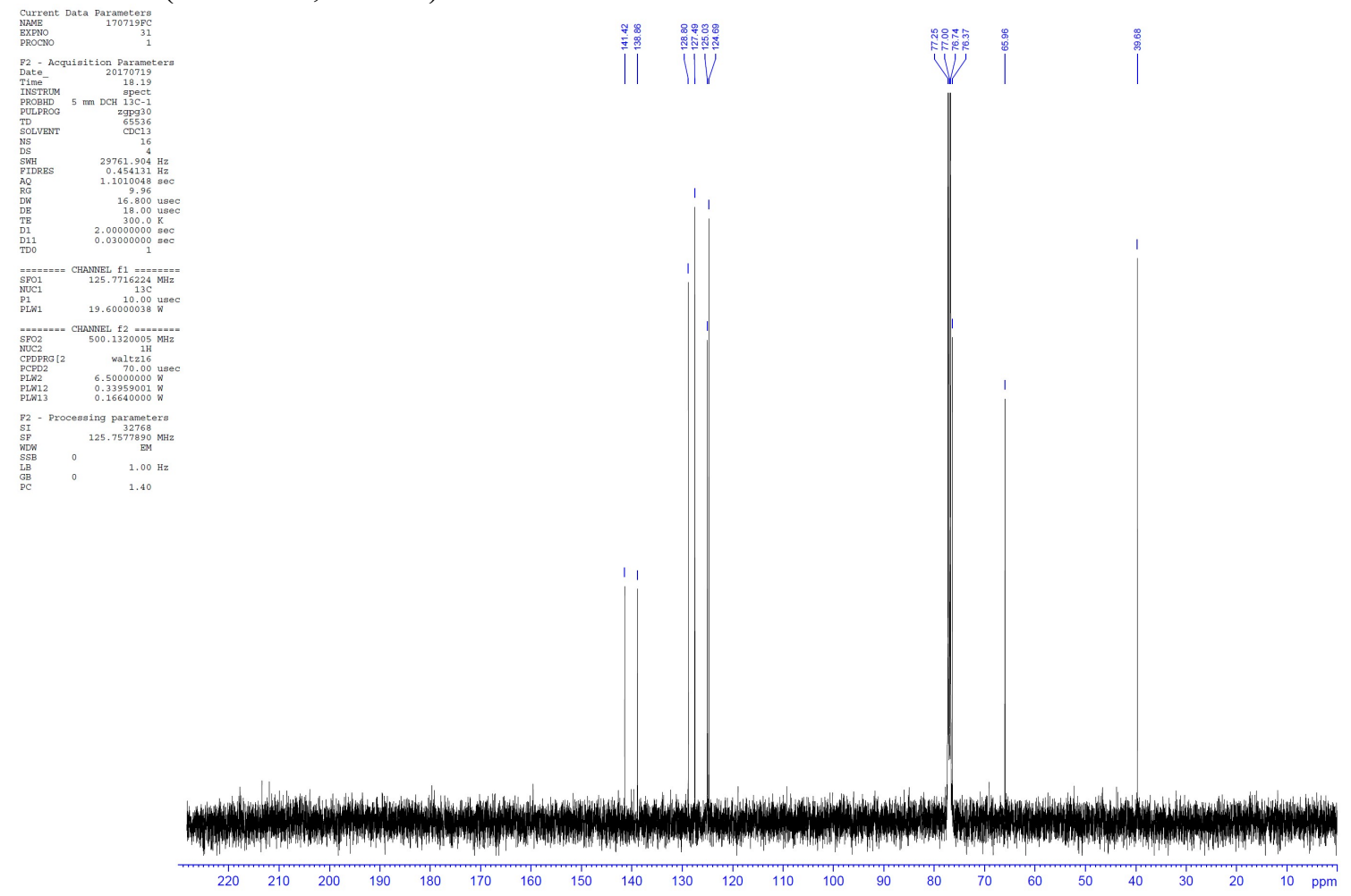
(1S,2R)-2-Chloro-5-methoxy-2,3-dihydro-1H-inden-1-ol (cis-4e)

${ }^{1} \mathrm{H}-\mathrm{NMR}\left(500 \mathrm{MHz}, \mathrm{CDCl}_{3}\right)$
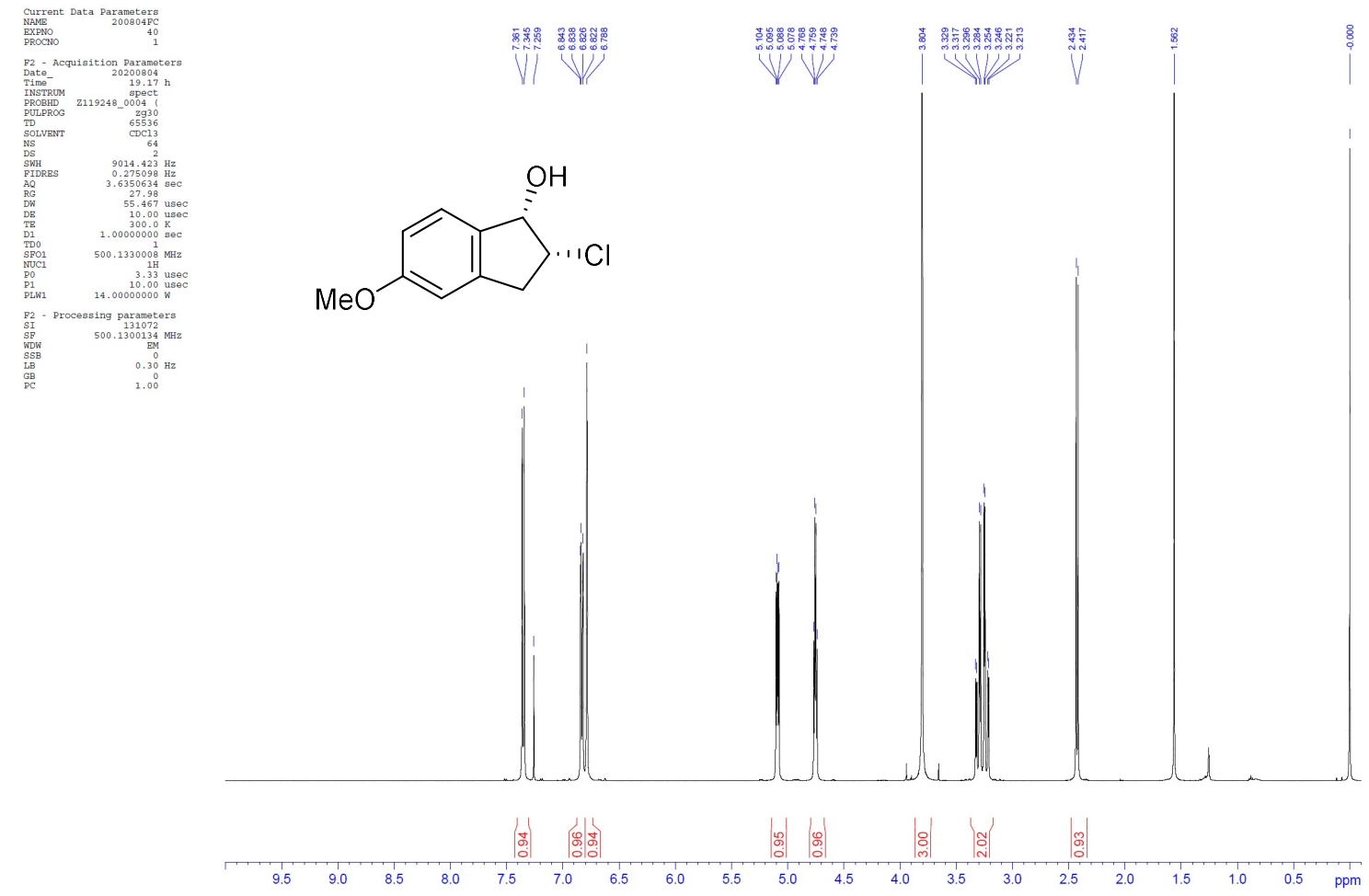

${ }^{13} \mathrm{C} \mathrm{NMR}\left(125 \mathrm{MHz}, \mathrm{CDCl}_{3}\right)$
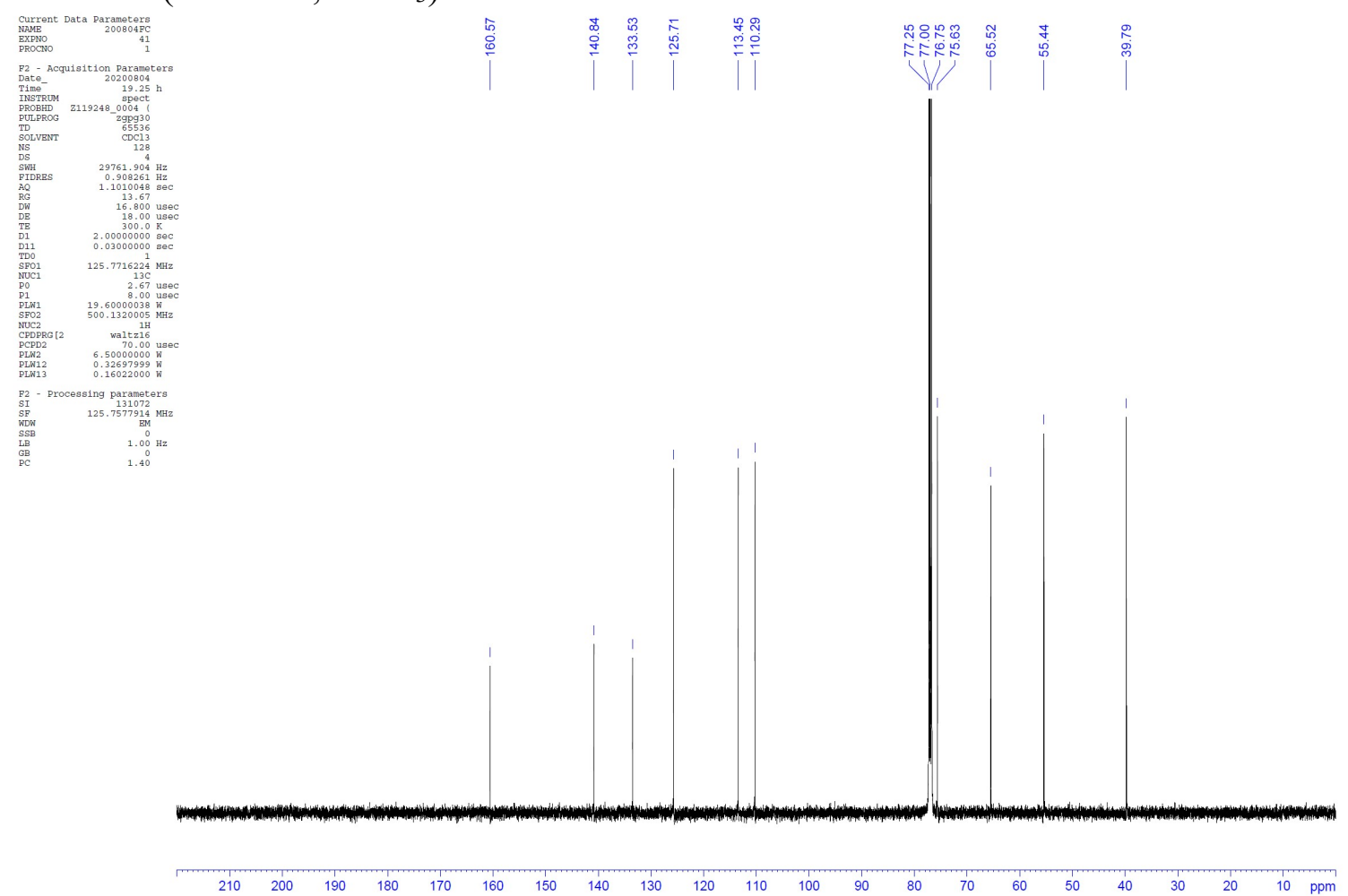
(1S,2R)-2,5-Dichloro-2,3-dihydro-1H-inden-1-ol (cis-4f)

${ }^{1} \mathrm{H}-\mathrm{NMR}\left(500 \mathrm{MHz}, \mathrm{CDCl}_{3}\right)$
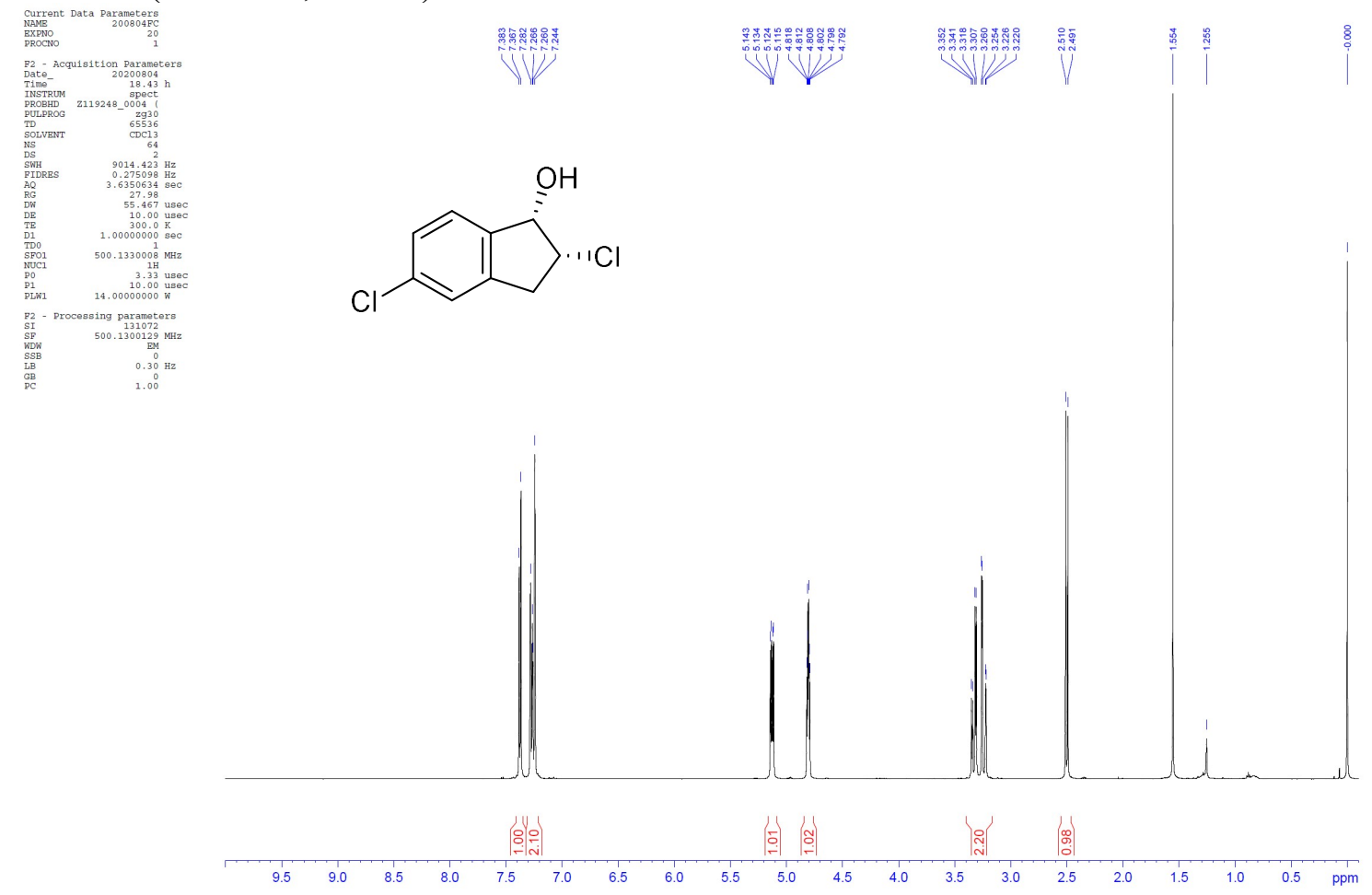

${ }^{13} \mathrm{C} \mathrm{NMR}\left(125 \mathrm{MHz}, \mathrm{CDCl}_{3}\right)$

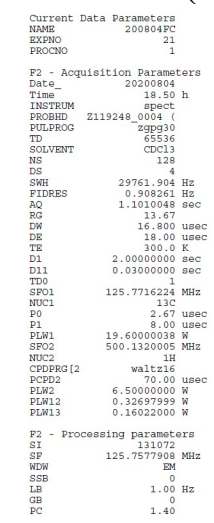


(1S,2R)-2-Chloro-2,3-dihydro-1H-indene-1,7-diol (cis-4g)

${ }^{1} \mathrm{H}-\mathrm{NMR}\left(500 \mathrm{MHz}, \mathrm{CDCl}_{3}\right)$

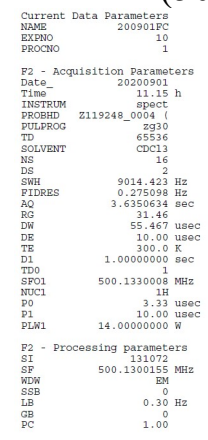<smiles>Oc1cccc2c1[C@@H](O)[C@H](Cl)C2</smiles>

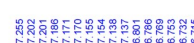

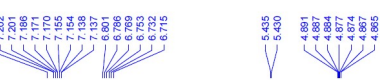

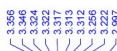
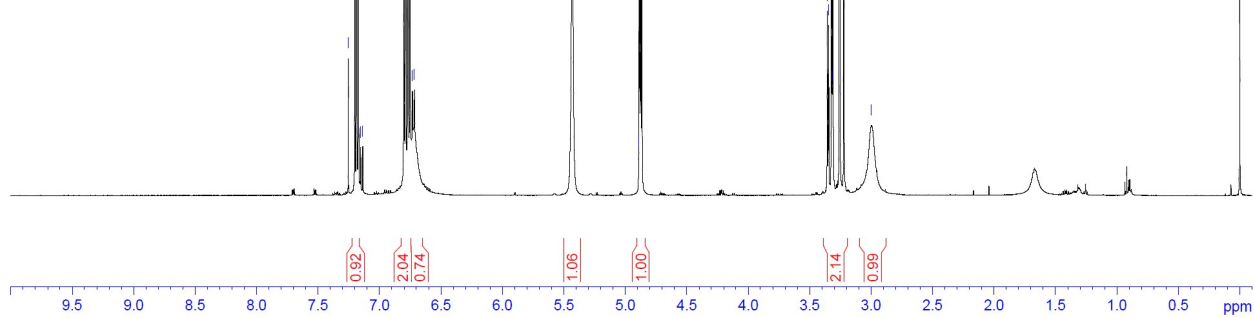

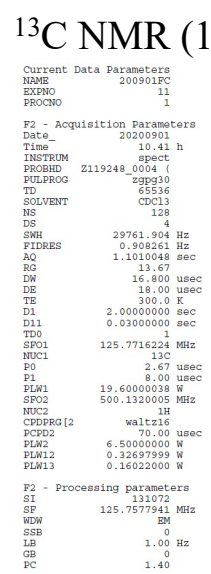

$\left(125 \mathrm{MHz}, \mathrm{CDCl}_{3}\right)$

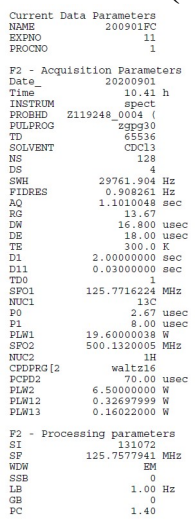


(1S,2R)-2-Chloro-3,3-dimethyl-2,3-dihydro-1H-inden-1-ol (cis-4h)

${ }^{1} \mathrm{H}-\mathrm{NMR}\left(500 \mathrm{MHz}, \mathrm{CDCl}_{3}\right)$
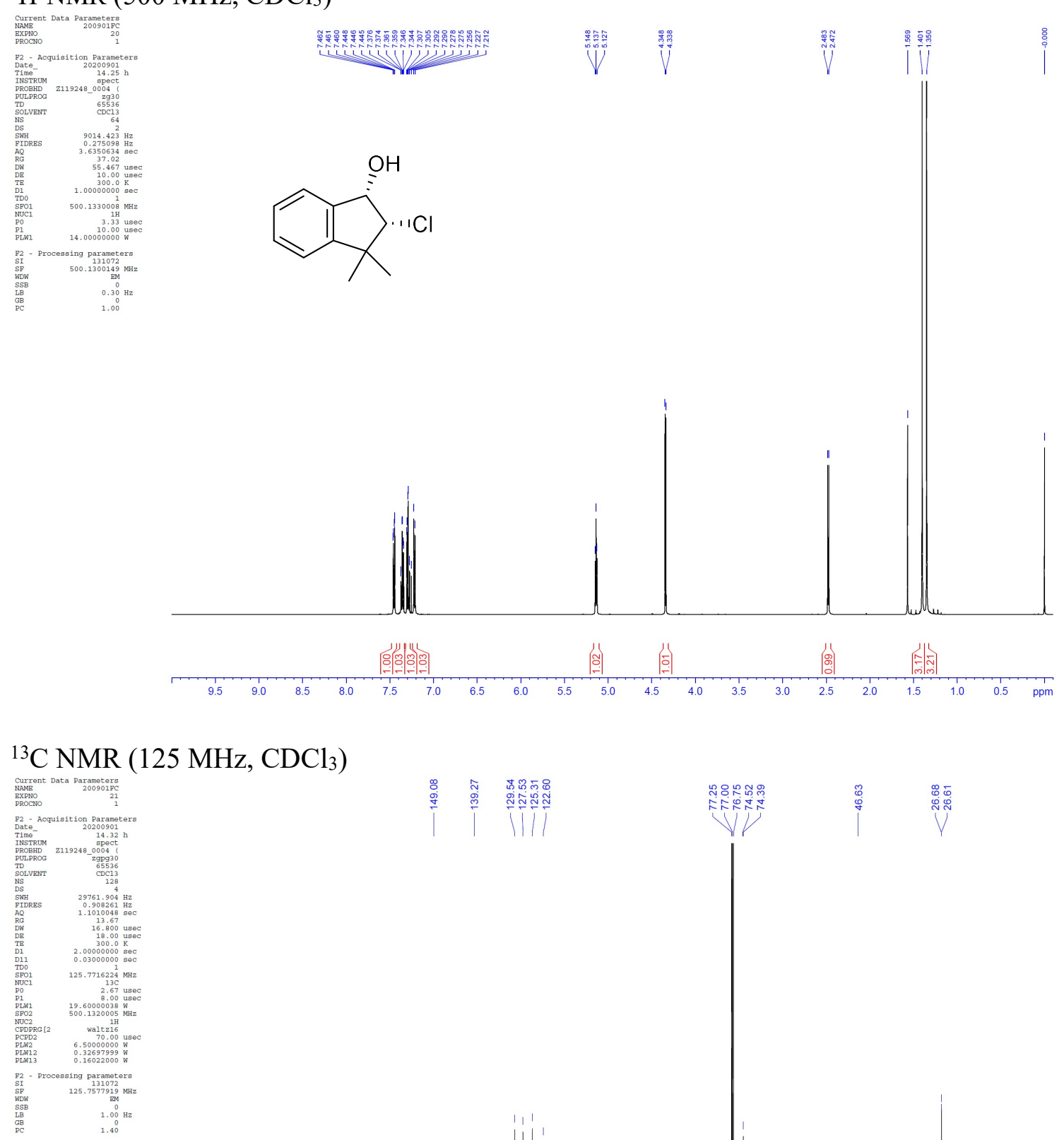
(5S,6R)-6-Chloro-6,7,8,9-tetrahydro-5H-benzo[7]annulen-5-ol (cis-4i)

${ }^{1} \mathrm{H}-\mathrm{NMR}\left(400 \mathrm{MHz}, \mathrm{CDCl}_{3}\right)$

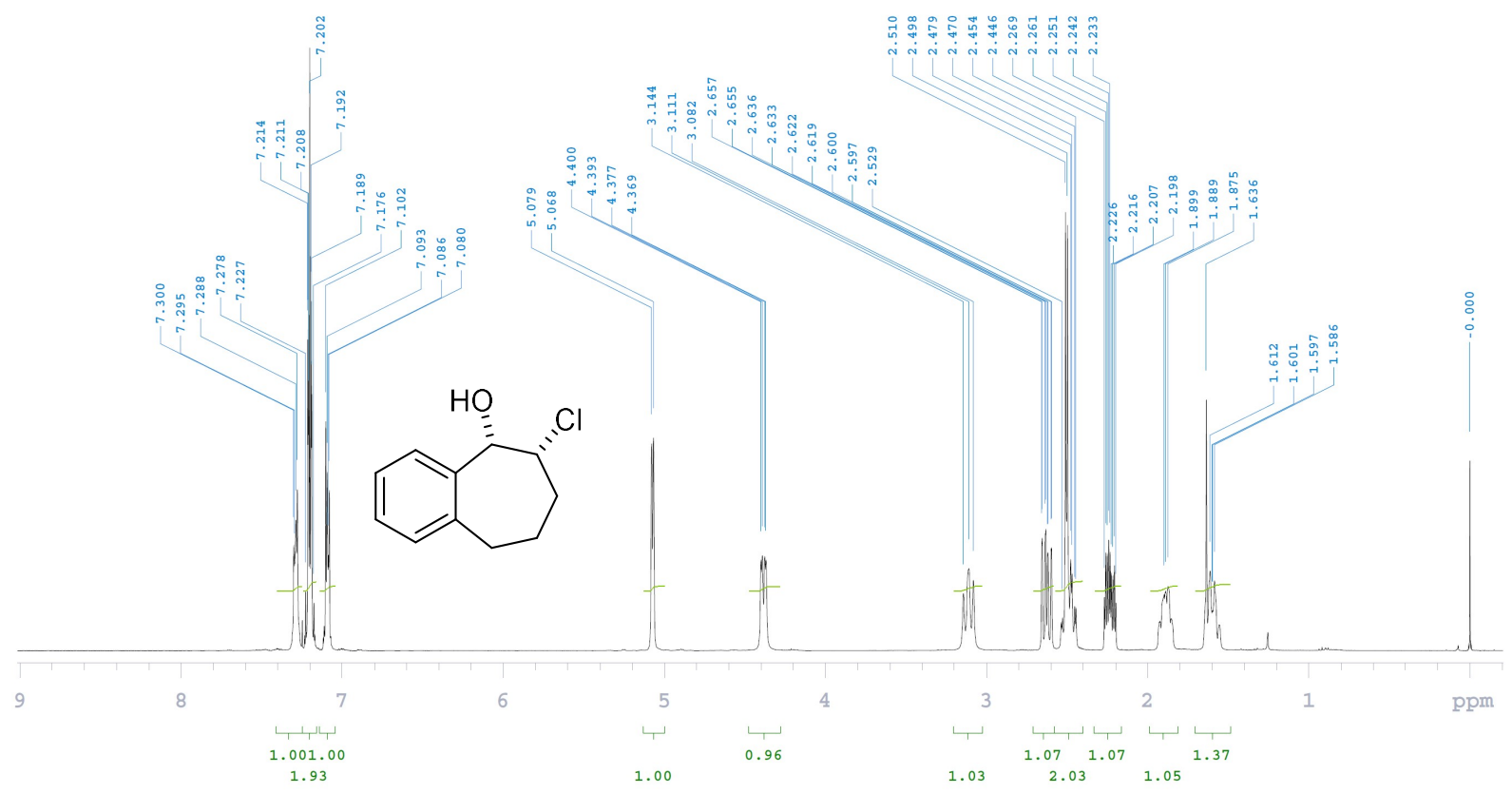

${ }^{13} \mathrm{C} \mathrm{NMR}\left(125 \mathrm{MHz}, \mathrm{CDCl}_{3}\right)$

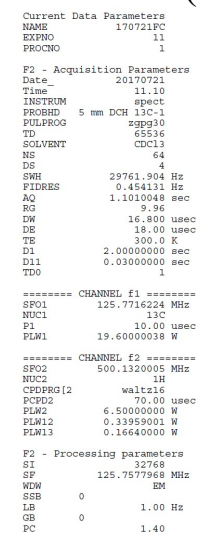


(1S,2R)-2-Chloro-1,2-dihydroacenaphthylen-1-ol (cis-4j)

${ }^{1} \mathrm{H}-\mathrm{NMR}\left(500 \mathrm{MHz}, \mathrm{CDCl}_{3}\right)$
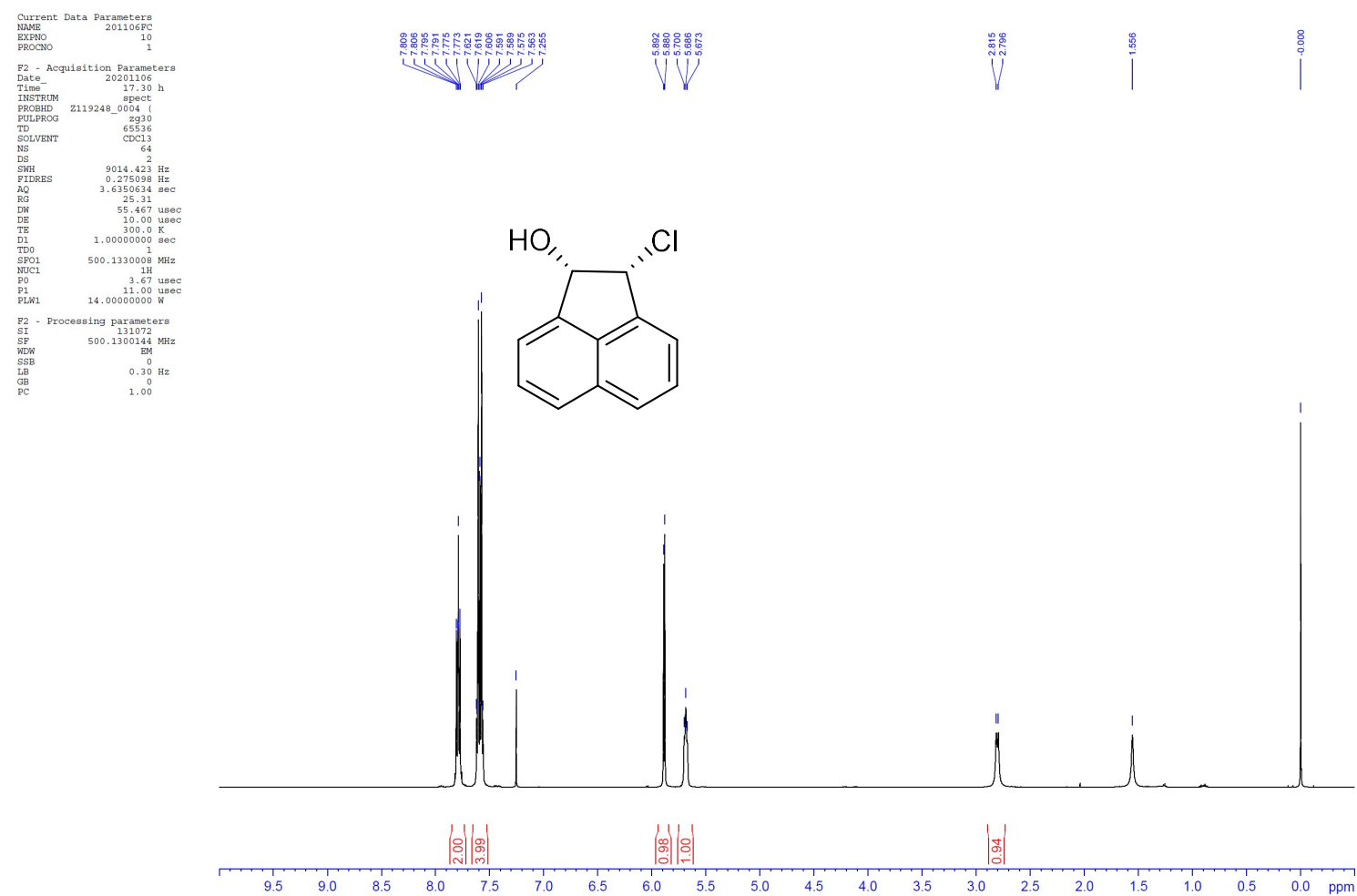

${ }^{13} \mathrm{C} \mathrm{NMR}\left(125 \mathrm{MHz}, \mathrm{CDCl}_{3}\right)$

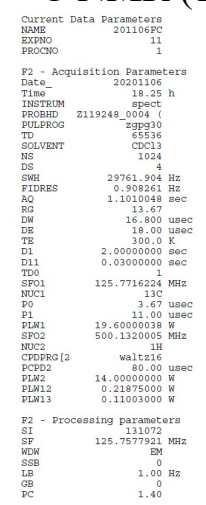

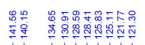

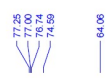

II IVI/

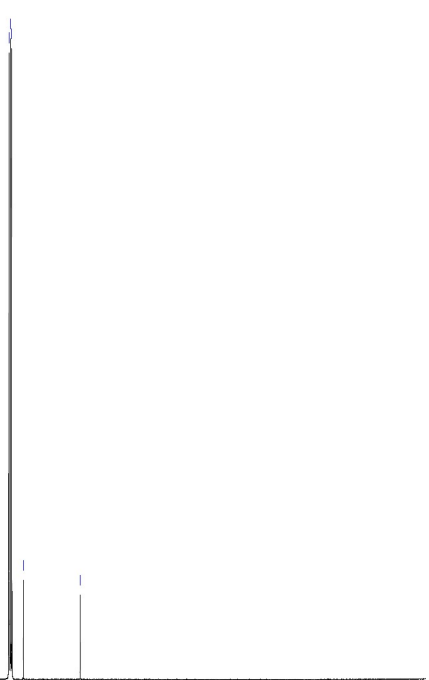

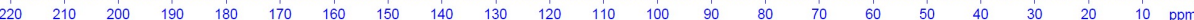


(1S,2R)-2-Bromo-2,3-dihydro-1 H-inden-1-ol (cis-4k)

${ }^{1} \mathrm{H}-\mathrm{NMR}\left(500 \mathrm{MHz}, \mathrm{CDCl}_{3}\right)$

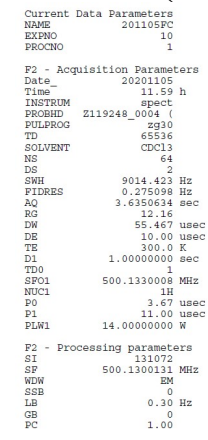

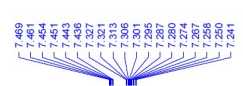
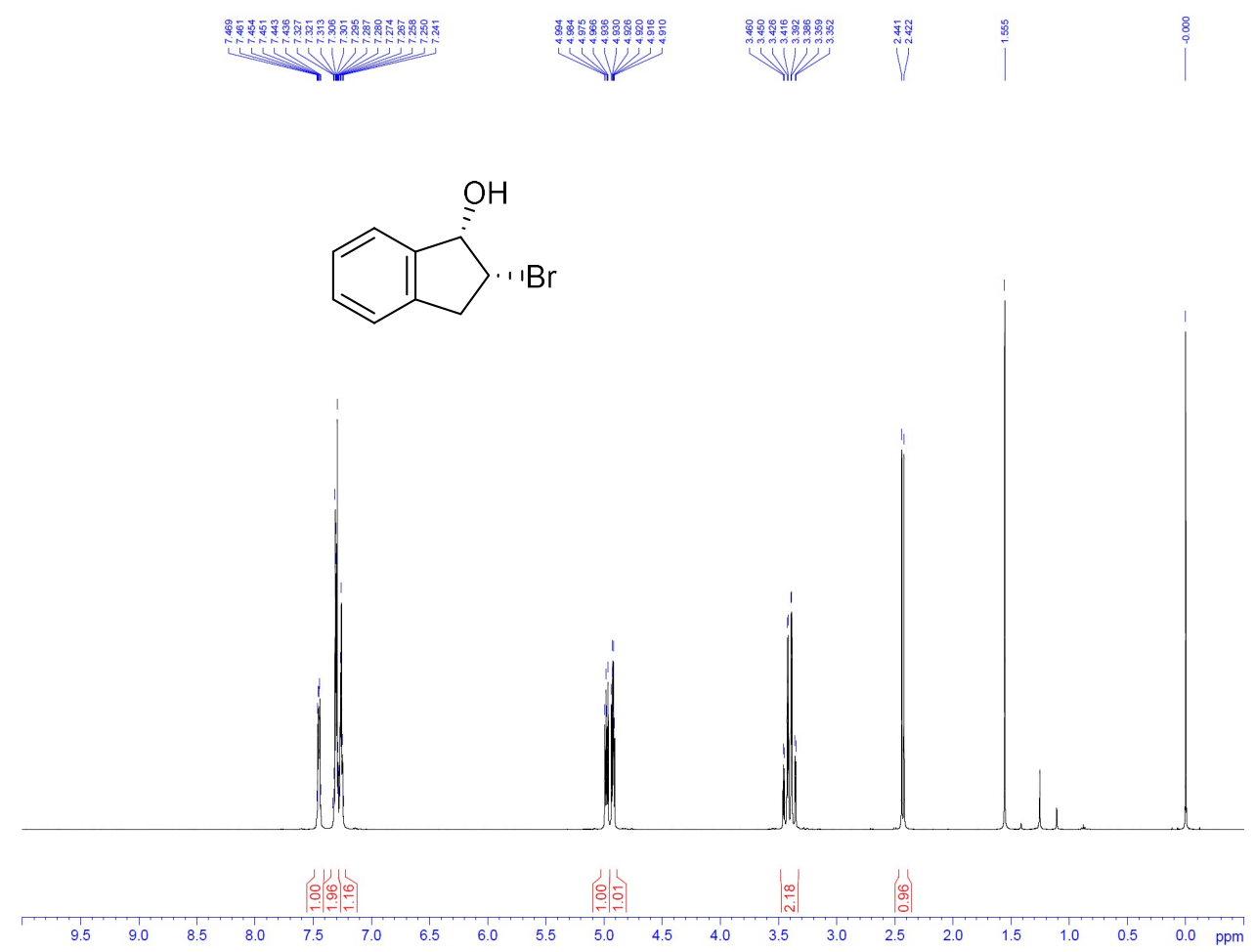

${ }^{13} \mathrm{C}$ NMR (125 MHz, $\left.\mathrm{CDCl}_{3}\right)$
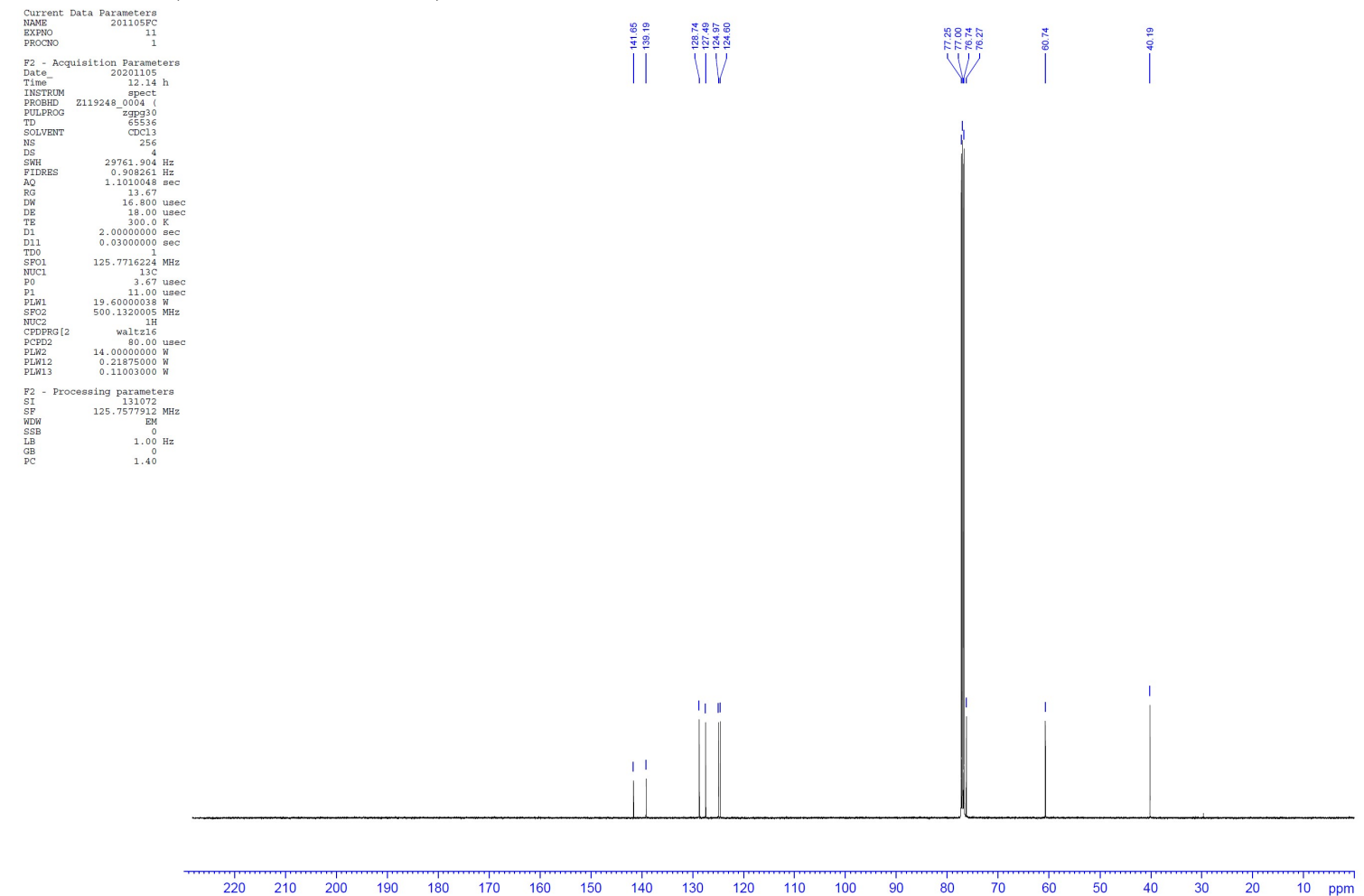
(1S,2R)-2-Bromo-1,2,3,4-tetrahydronaphthalen-1-ol (cis-4l)
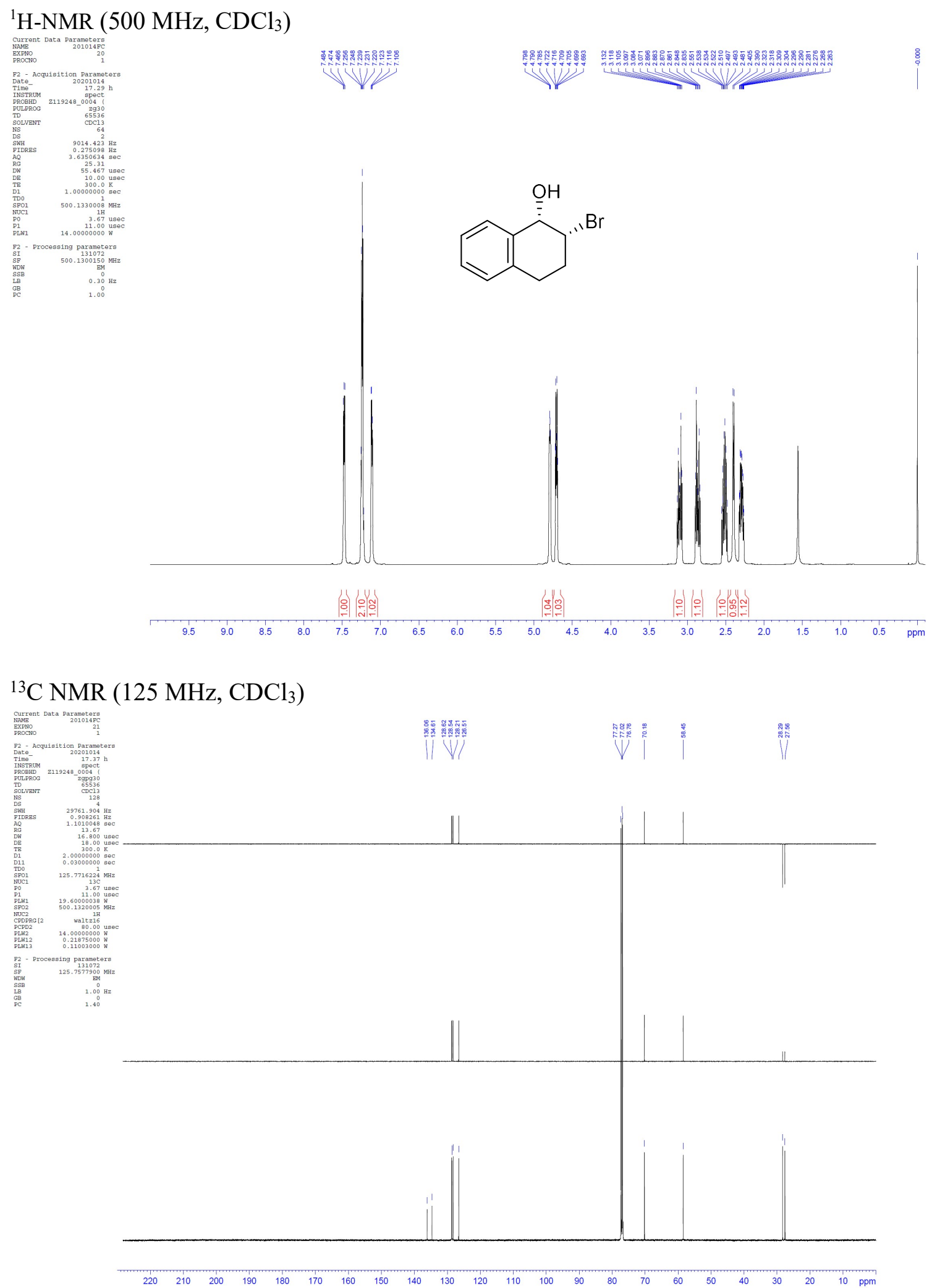
Methyl (1R,2R)-1-hydroxy-2,3-dihydro-1H-indene-2-carboxylate (cis-6a)

${ }^{1} \mathrm{H}-\mathrm{NMR}\left(400 \mathrm{MHz}, \mathrm{CDCl}_{3}\right)$

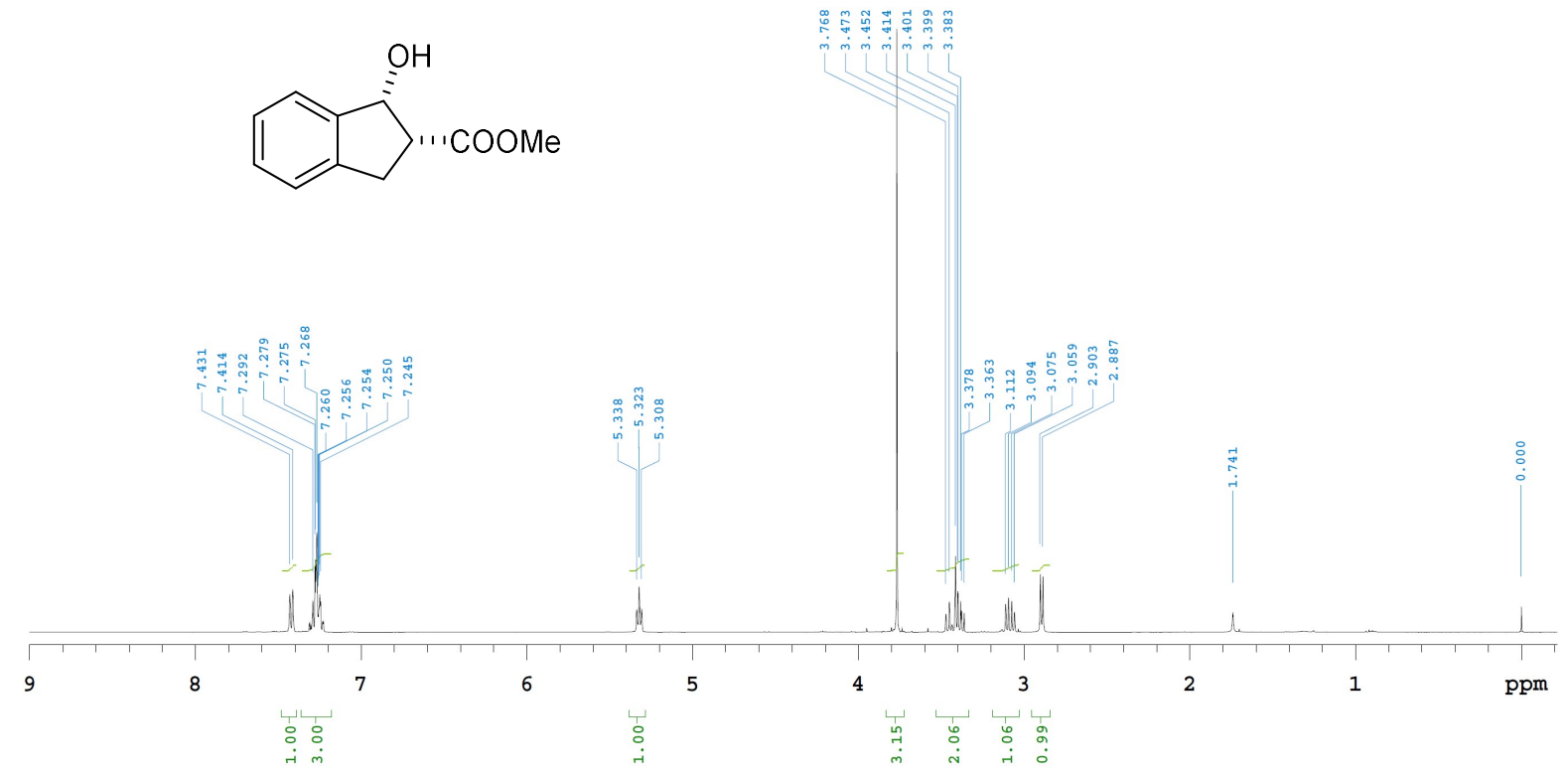

${ }^{13} \mathrm{C}$ NMR (125 MHz, $\left.\mathrm{CDCl}_{3}\right)$
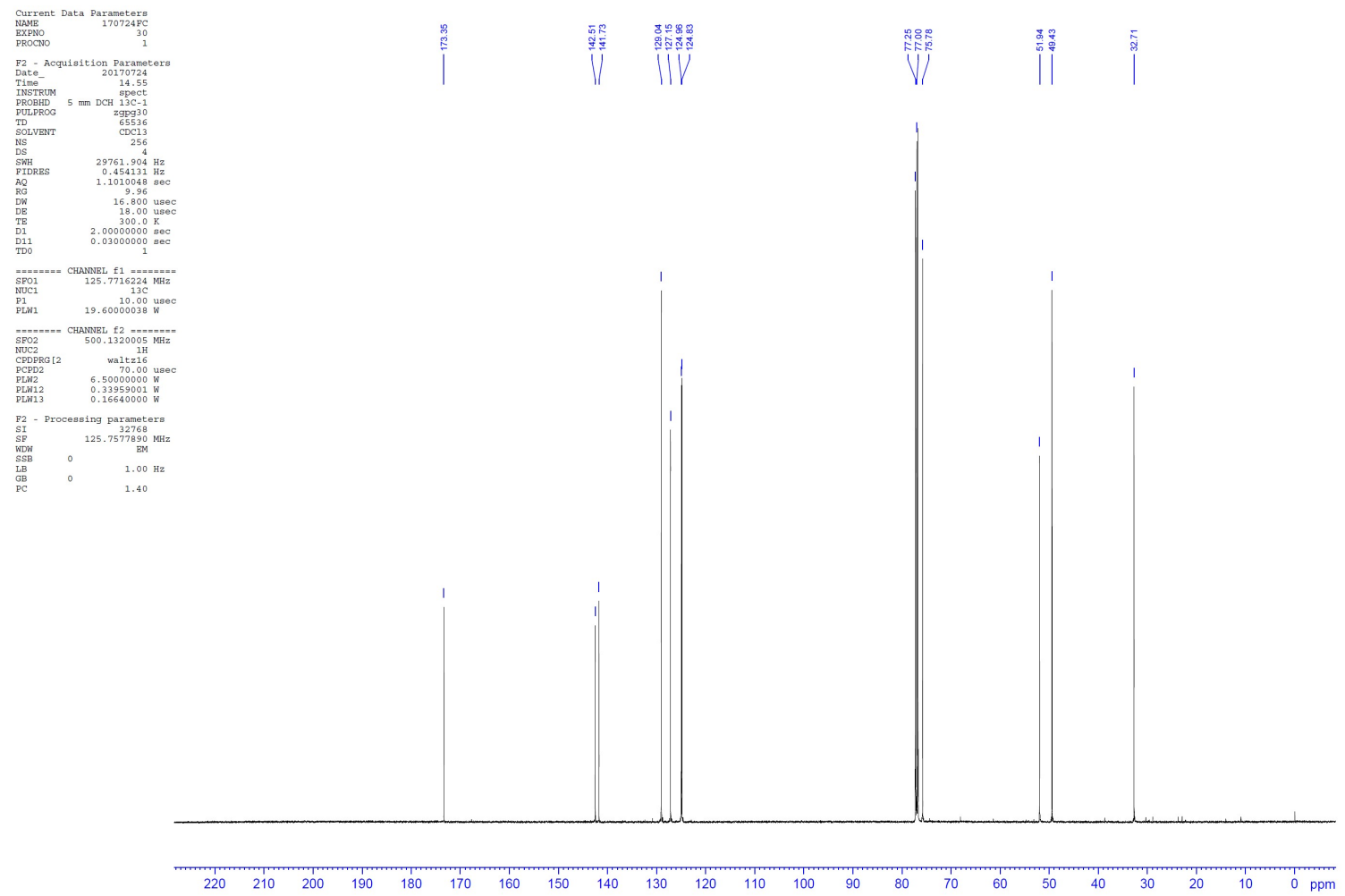
Methyl $(5 R, 6 R)-5$-hydroxy-6,7,8,9-tetrahydro-5H-benzo[7] annulene-6-carboxylate (cis-6b)

${ }^{1} \mathrm{H}-\mathrm{NMR}\left(400 \mathrm{MHz}, \mathrm{CDCl}_{3}\right)$

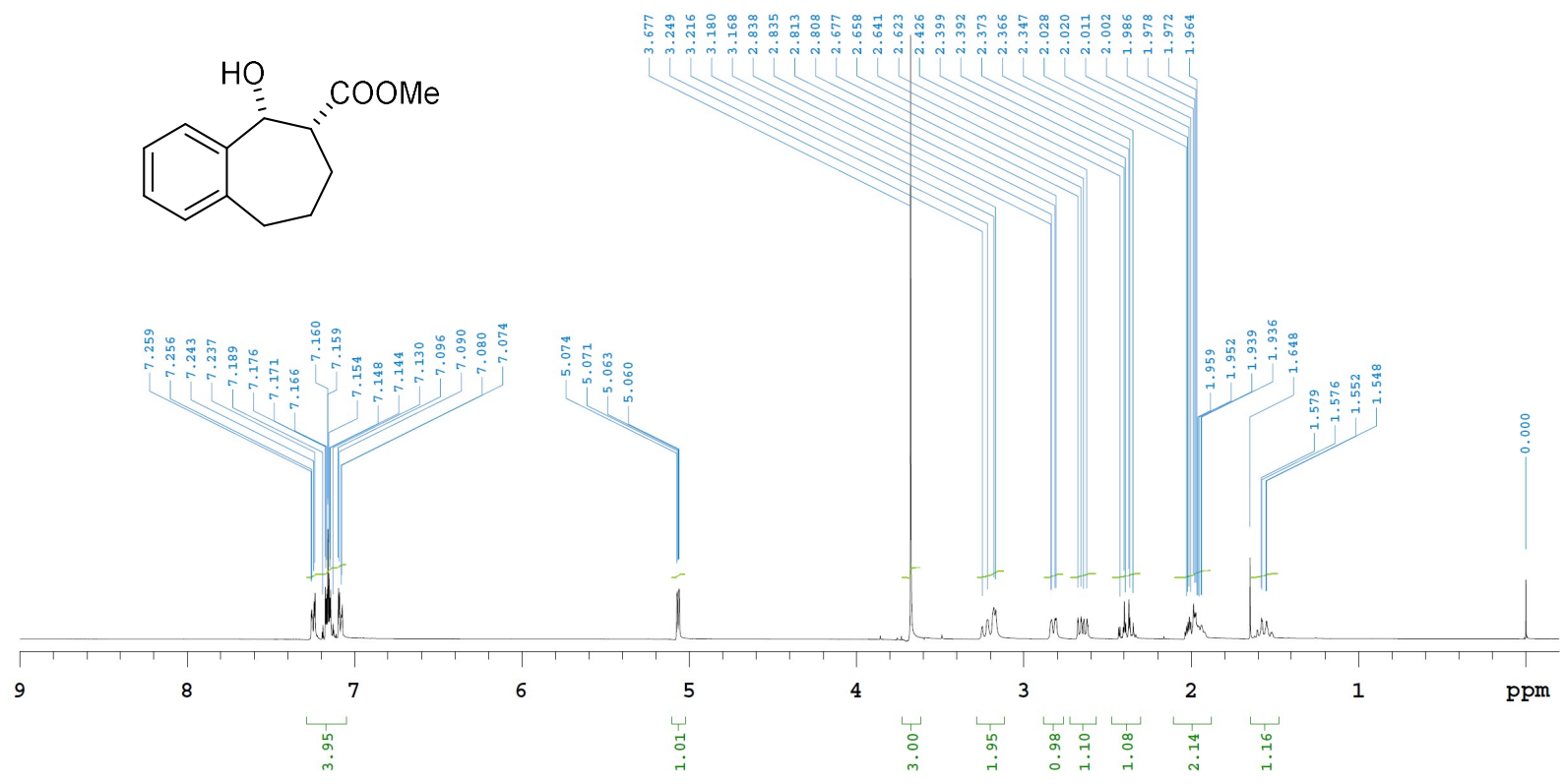

${ }^{13} \mathrm{C} \mathrm{NMR}\left(125 \mathrm{MHz}, \mathrm{CDCl}_{3}\right)$
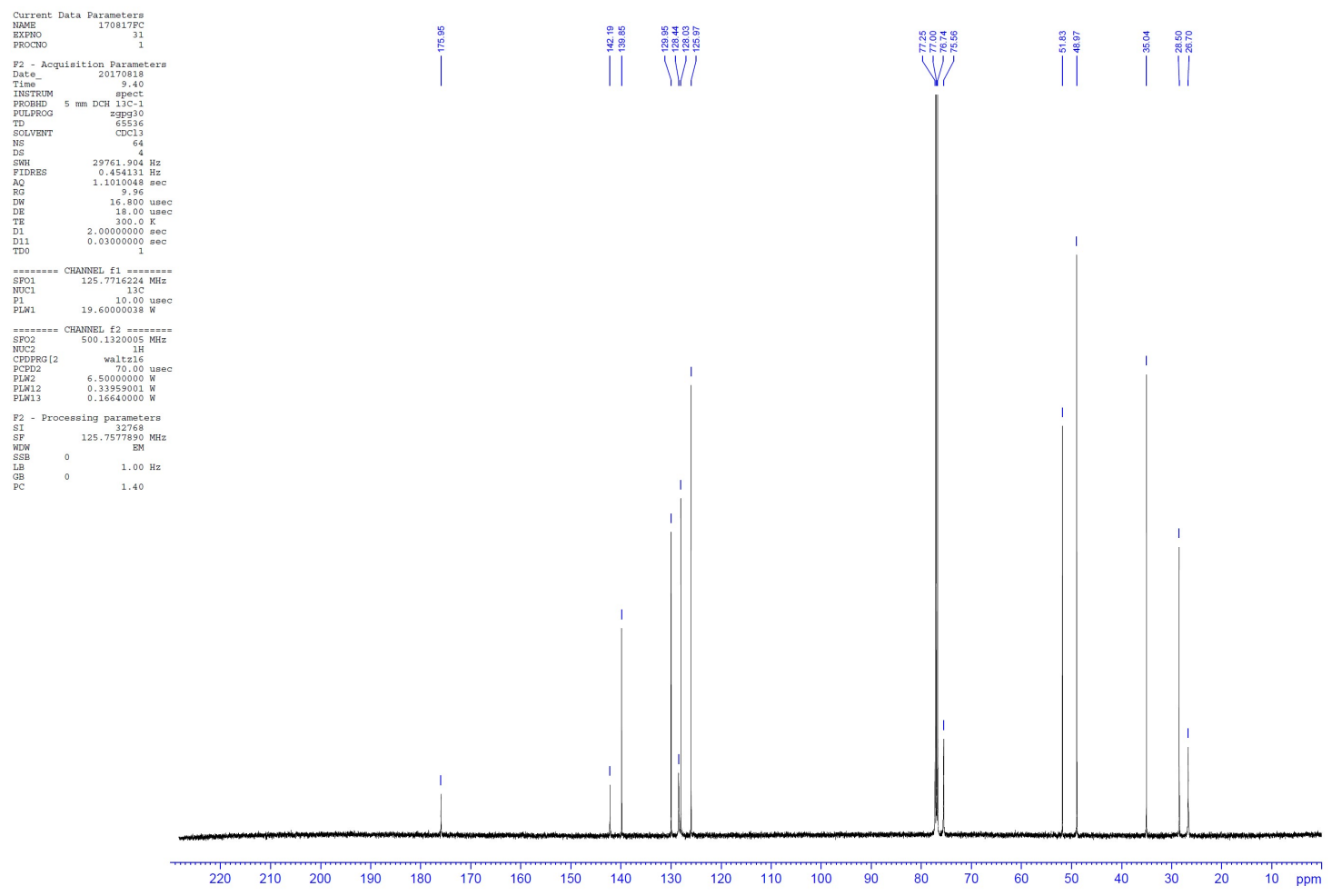
$(1 R, 2 R)-N$-(tert-Butyl)-1-hydroxy-2,3-dihydro-1H-indene-2-carboxamide (cis-6c)

${ }^{1} \mathrm{H}-\mathrm{NMR}\left(400 \mathrm{MHz}, \mathrm{CDCl}_{3}\right)$

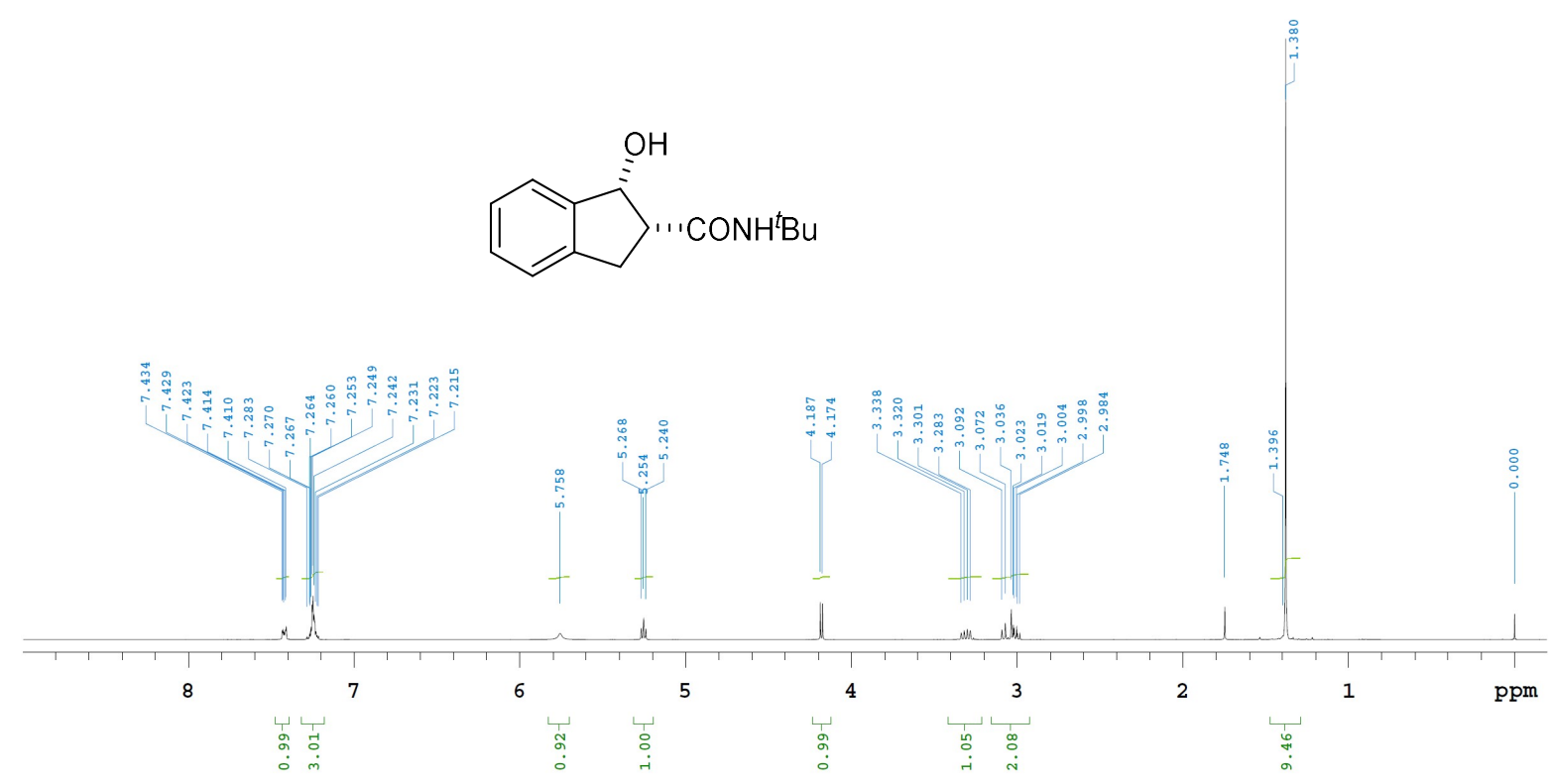

${ }^{13} \mathrm{C}$ NMR $\left(125 \mathrm{MHz}, \mathrm{CDCl}_{3}\right)$

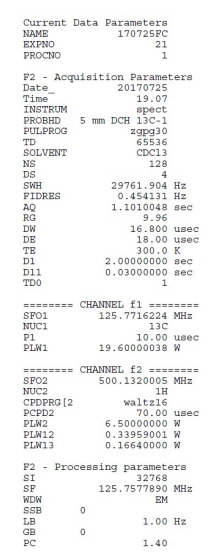

11
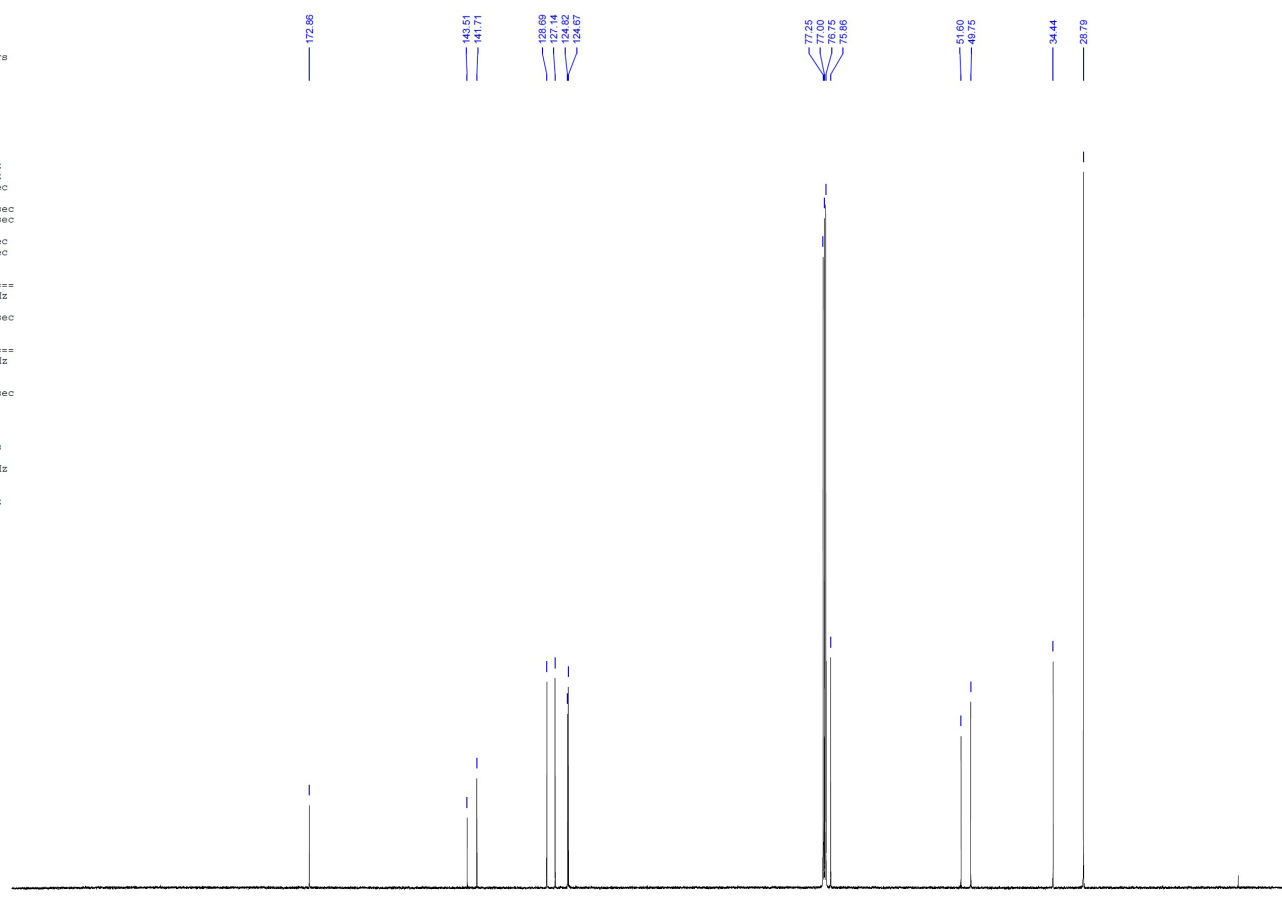

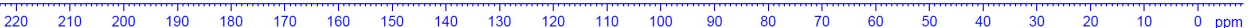


$(1 R, 2 R)-N$-(tert-Butyl)-1-hydroxy-1,2,3,4-tetrahydronaphthalene-2-carboxamide (cis-6d)

${ }^{1} \mathrm{H}-\mathrm{NMR}\left(400 \mathrm{MHz}, \mathrm{CDCl}_{3}\right)$

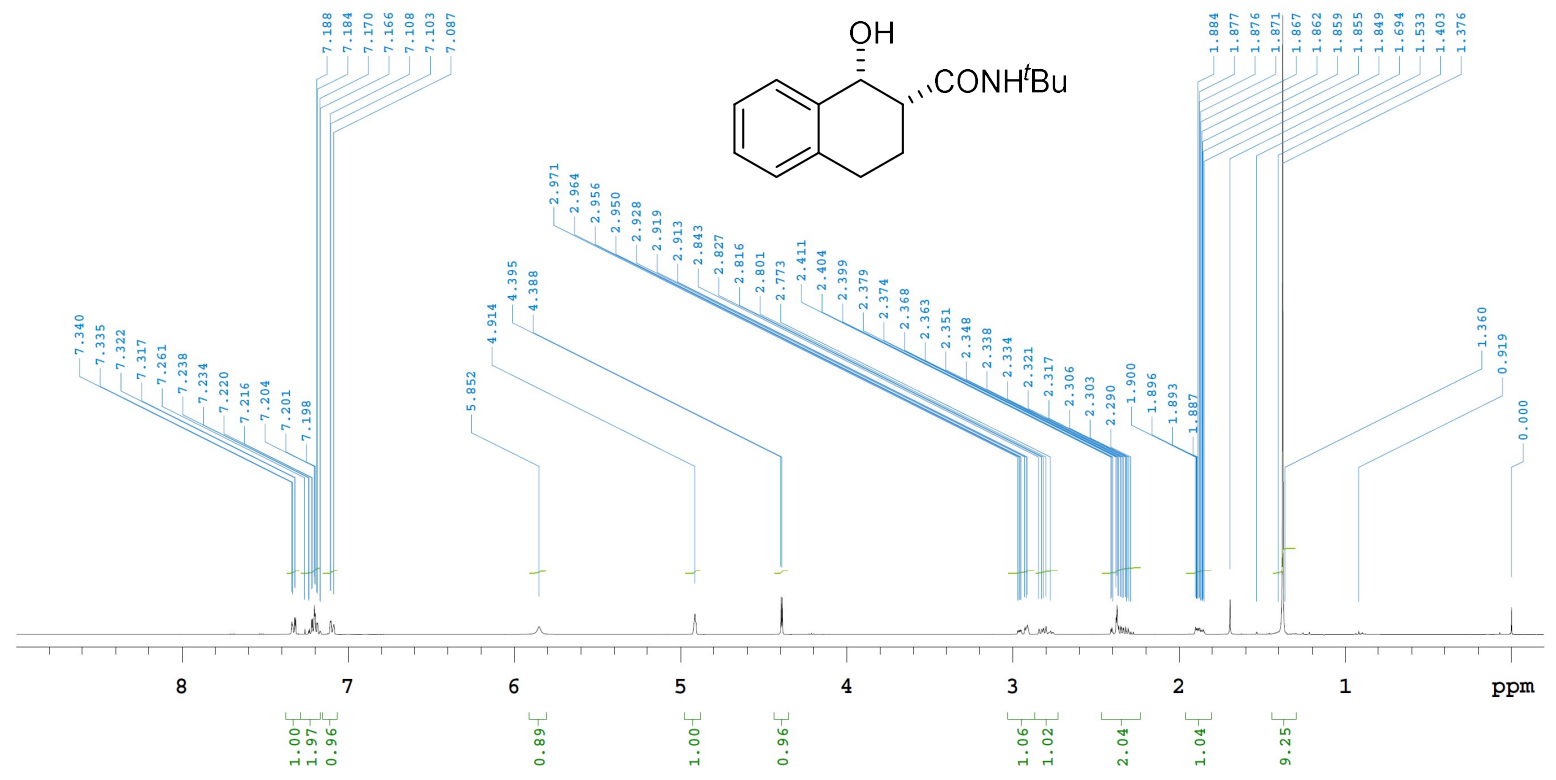

${ }^{13} \mathrm{C} \mathrm{NMR}\left(125 \mathrm{MHz}, \mathrm{CDCl}_{3}\right)$
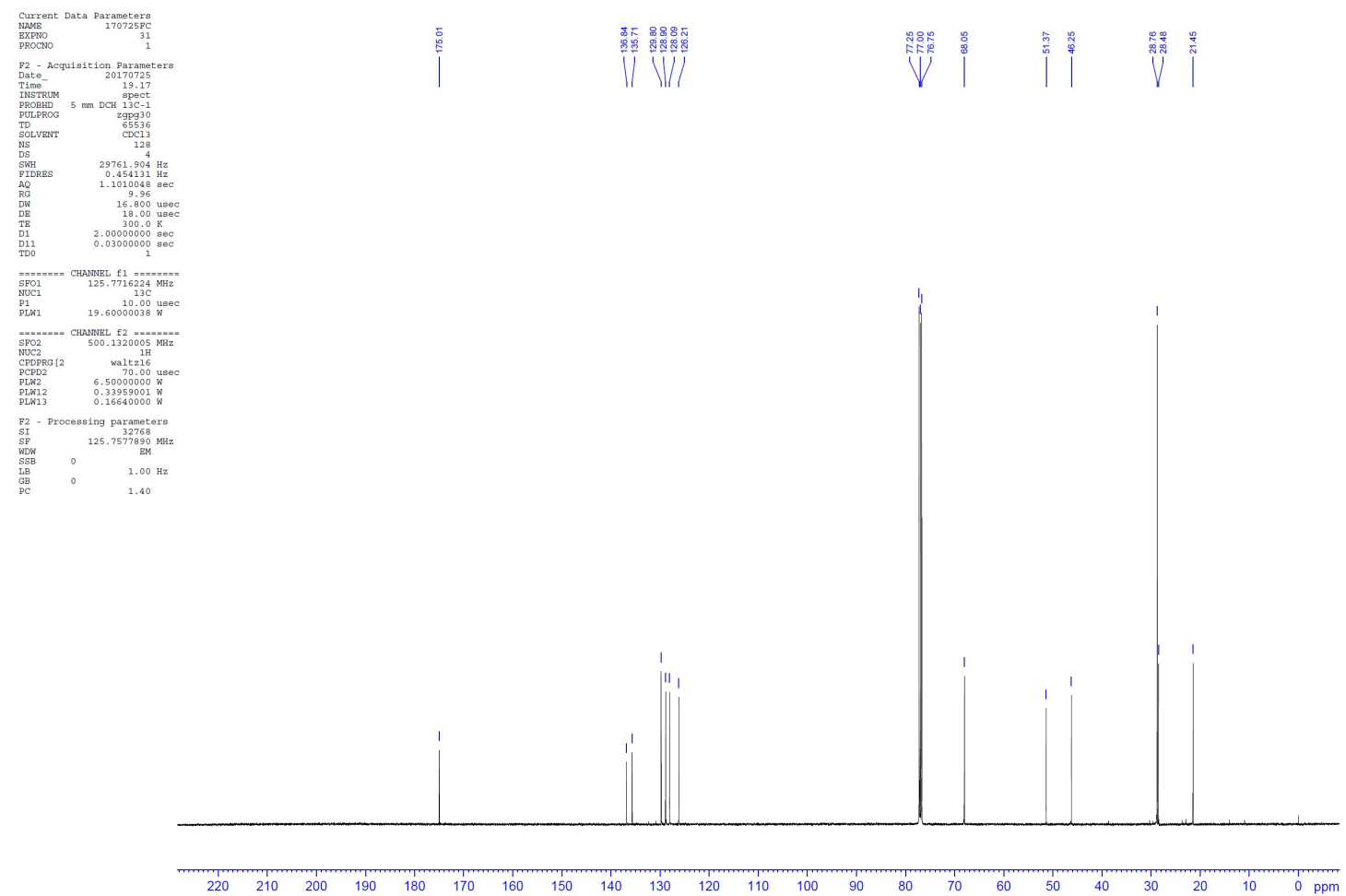
(5R,6R)- $N$-(tert-Butyl)-5-hydroxy-6,7,8,9-tetrahydro-5H-benzo[7]annulene-6-carboxa mide (cis-6e)

${ }^{1} \mathrm{H}-\mathrm{NMR}\left(400 \mathrm{MHz}, \mathrm{CDCl}_{3}\right)$

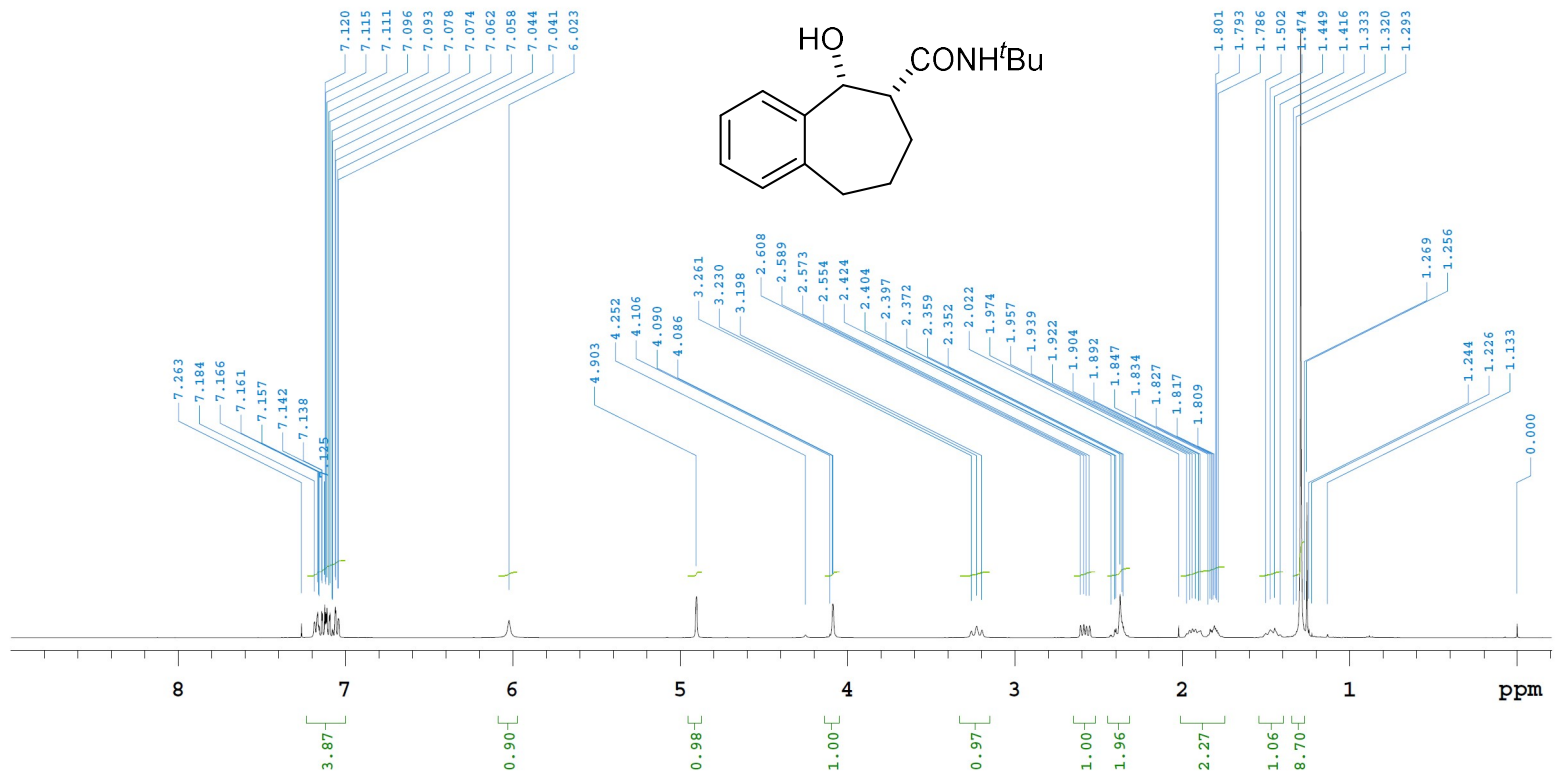

${ }^{13} \mathrm{C} \mathrm{NMR}\left(125 \mathrm{MHz}, \mathrm{CDCl}_{3}\right)$

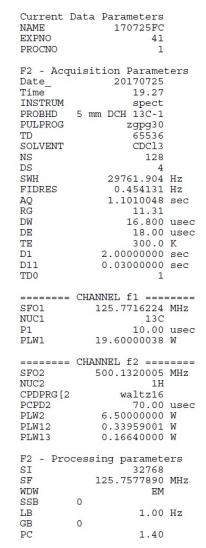

$|1|$

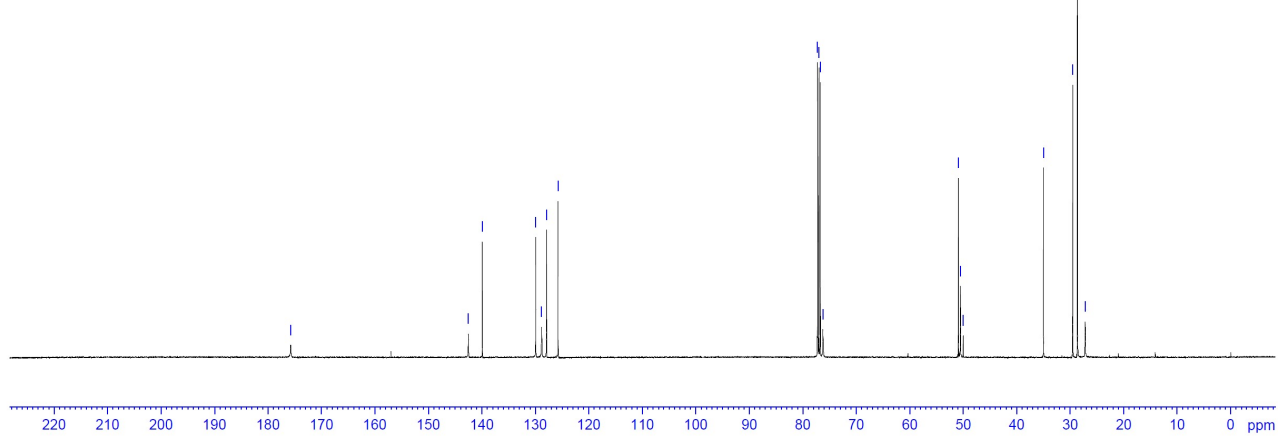


(1S,2R)-2-(Phenylsulfonyl)-2,3-dihydro-1H-inden-1-ol (cis-6f)

${ }^{1} \mathrm{H}-\mathrm{NMR}\left(400 \mathrm{MHz}, \mathrm{CDCl}_{3}\right)$

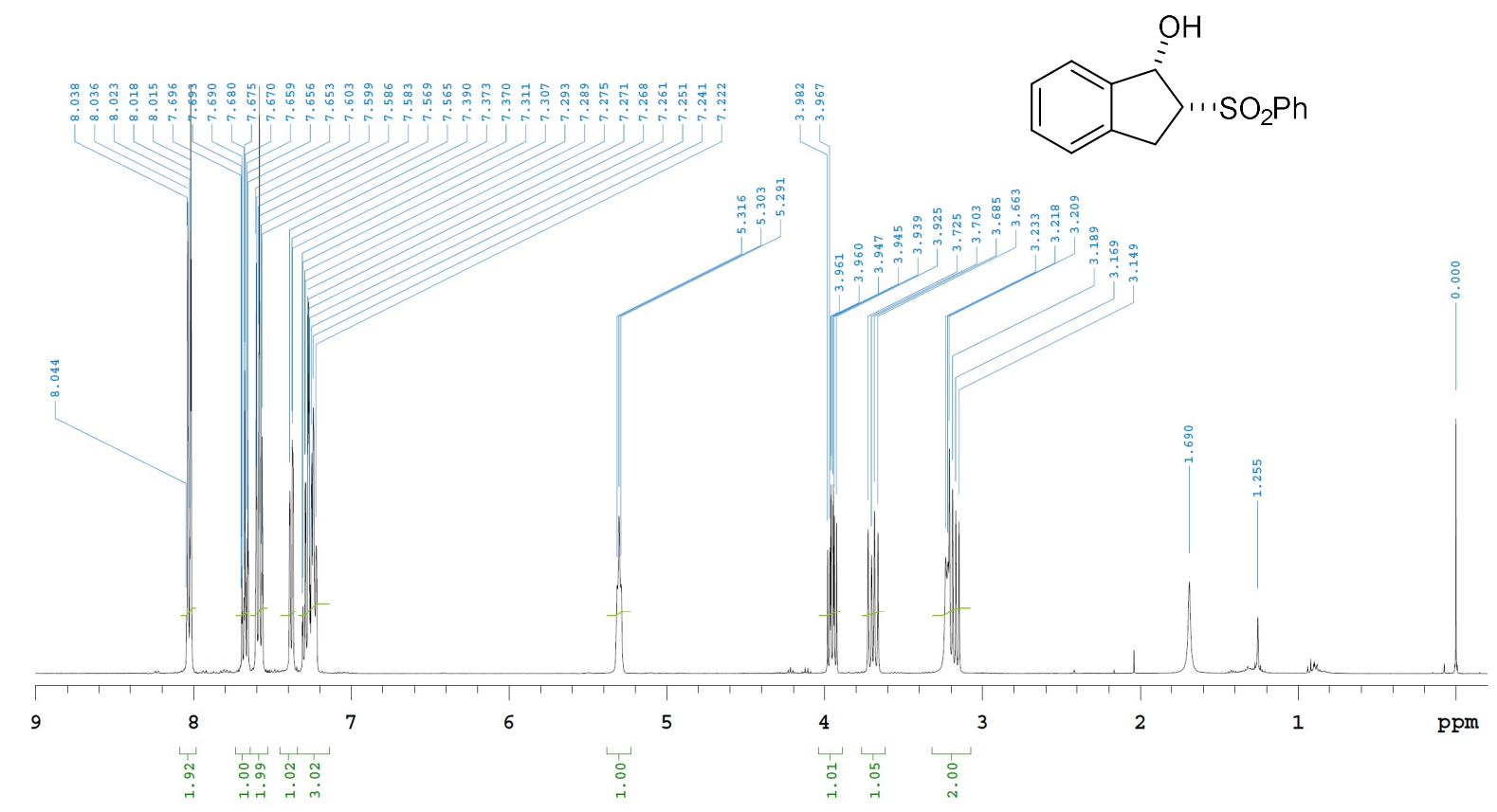

${ }^{13} \mathrm{C}$ NMR (125 MHz, $\left.\mathrm{CDCl}_{3}\right)$
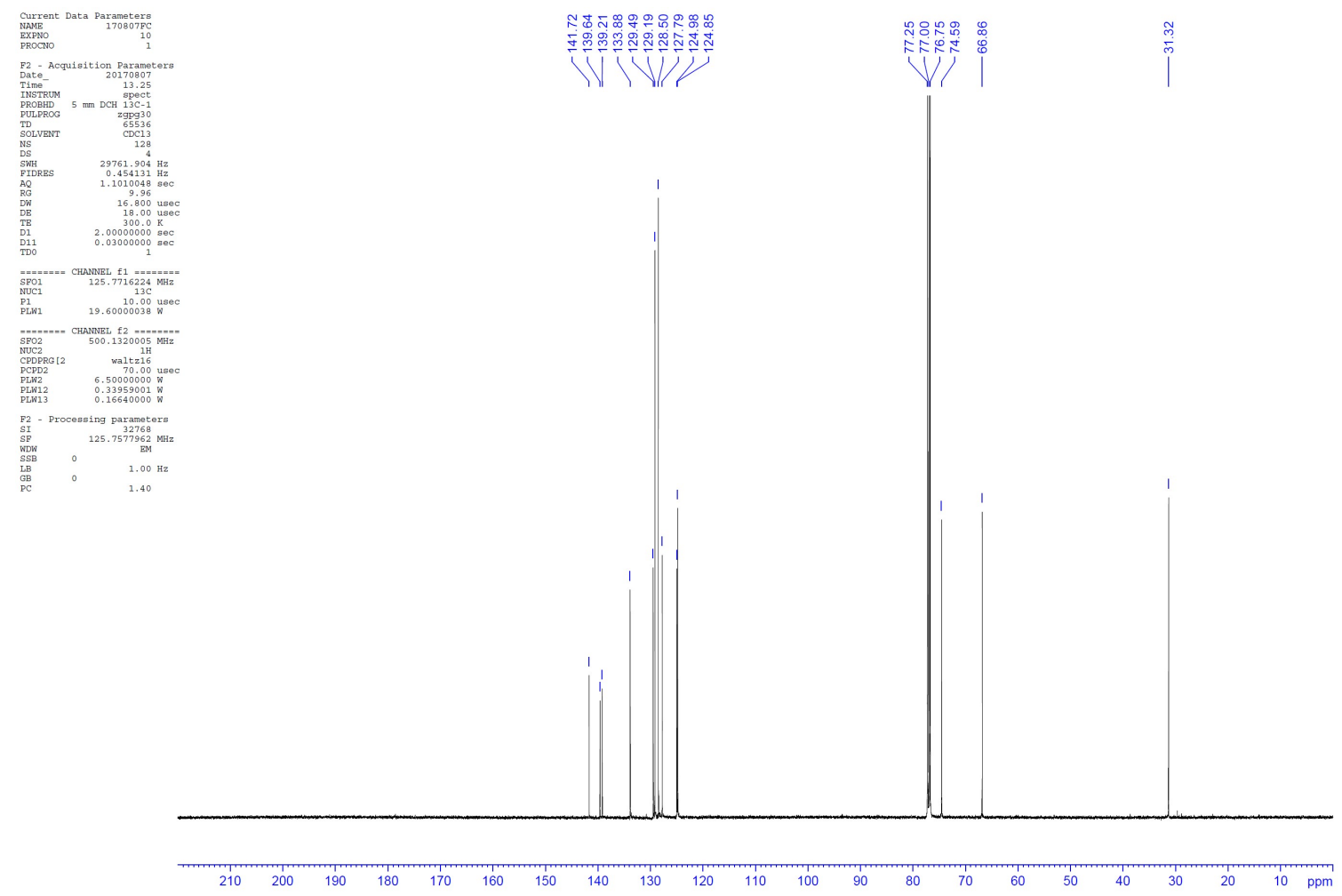
(1S,2R)-2-(Phenylsulfonyl)-1,2,3,4-tetrahydronaphthalen-1-ol (cis-6g)

${ }^{1} \mathrm{H}-\mathrm{NMR}\left(400 \mathrm{MHz}, \mathrm{CDCl}_{3}\right)$

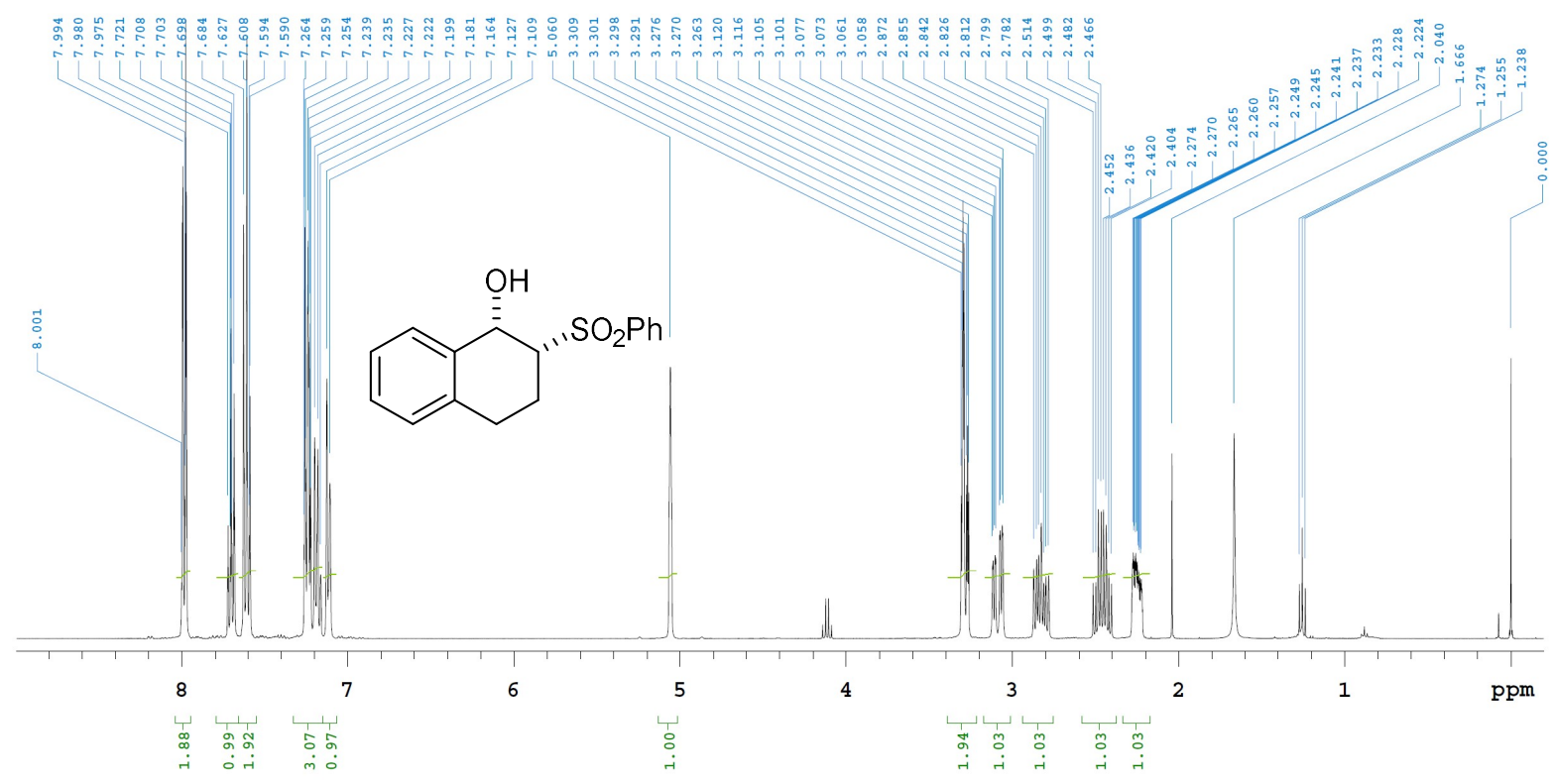

${ }^{13} \mathrm{C} \mathrm{NMR}\left(125 \mathrm{MHz}, \mathrm{CDCl}_{3}\right)$
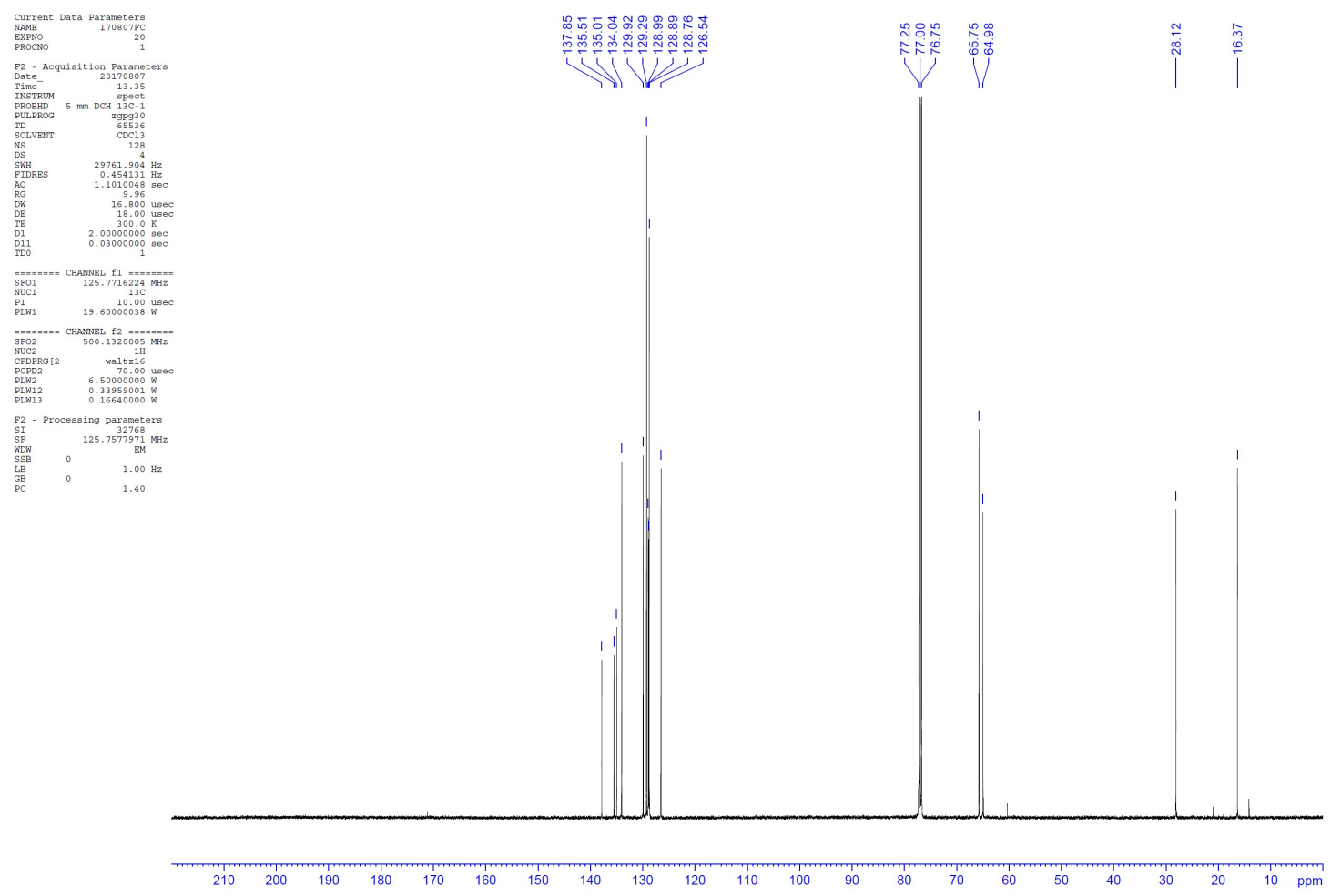
$(1 R, 2 R)-N$-(tert-Butyl)-2-hydroxy-1,2-dihydroacenaphthylene-1-carboxamide (cis-6h) ${ }^{1} \mathrm{H}-\mathrm{NMR}\left(400 \mathrm{MHz}, \mathrm{CDCl}_{3}\right)$

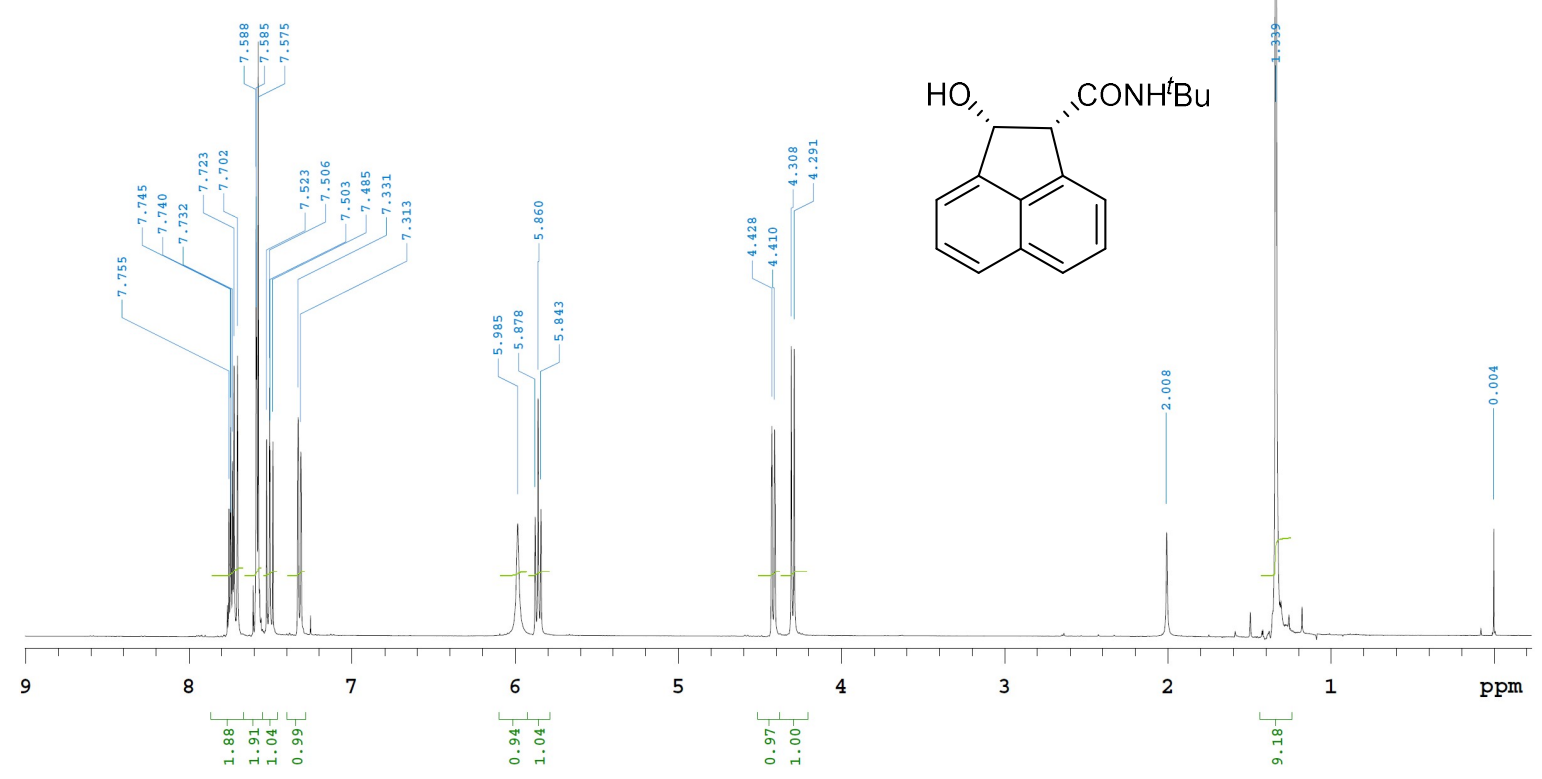

${ }^{13} \mathrm{C}$ NMR (125 MHz, $\left.\mathrm{CDCl}_{3}\right)$
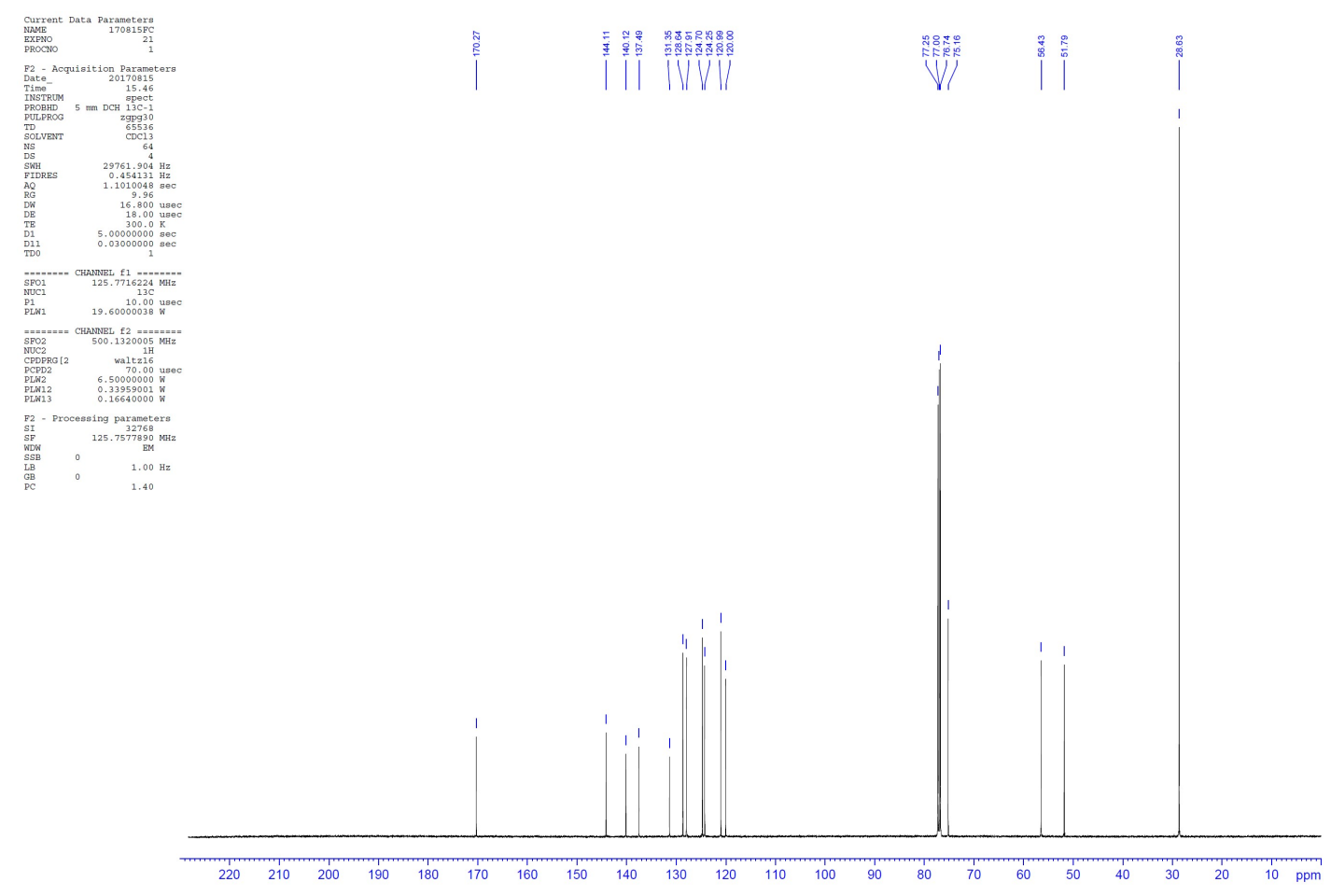
Methyl (1R,2R)-2-hydroxy-1,2-dihydroacenaphthylene-1-carboxylate (cis-6i)

${ }^{1} \mathrm{H}-\mathrm{NMR}\left(500 \mathrm{MHz}, \mathrm{CDCl}_{3}\right)$

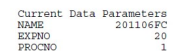

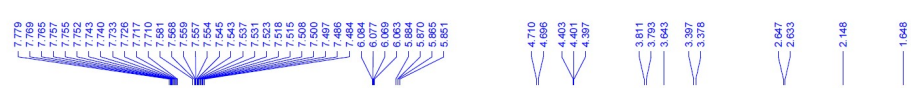
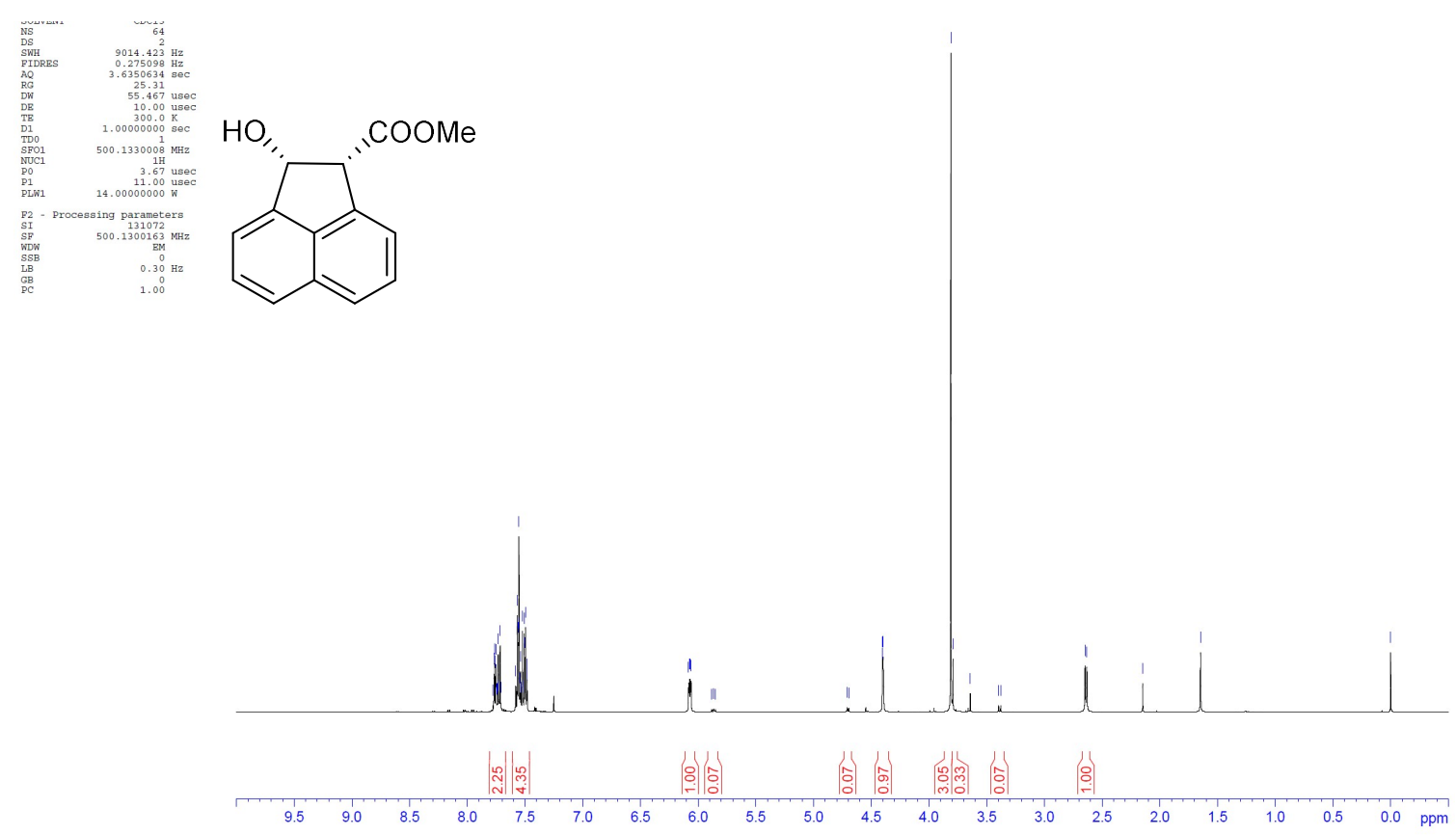

${ }^{13} \mathrm{C} \mathrm{NMR}\left(125 \mathrm{MHz}, \mathrm{CDCl}_{3}\right)$

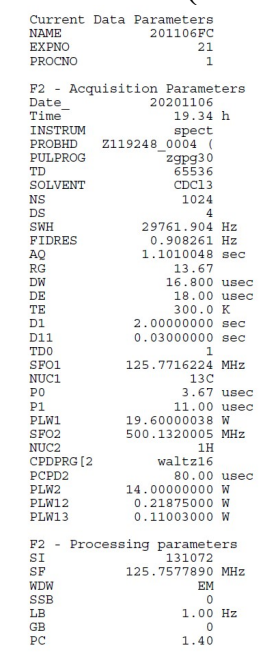


(1S,6R)-6-Chloro-3-methylcyclohex-2-en-1-ol (cis-8a)

${ }^{1} \mathrm{H}-\mathrm{NMR}\left(500 \mathrm{MHz}, \mathrm{CDCl}_{3}\right.$ )
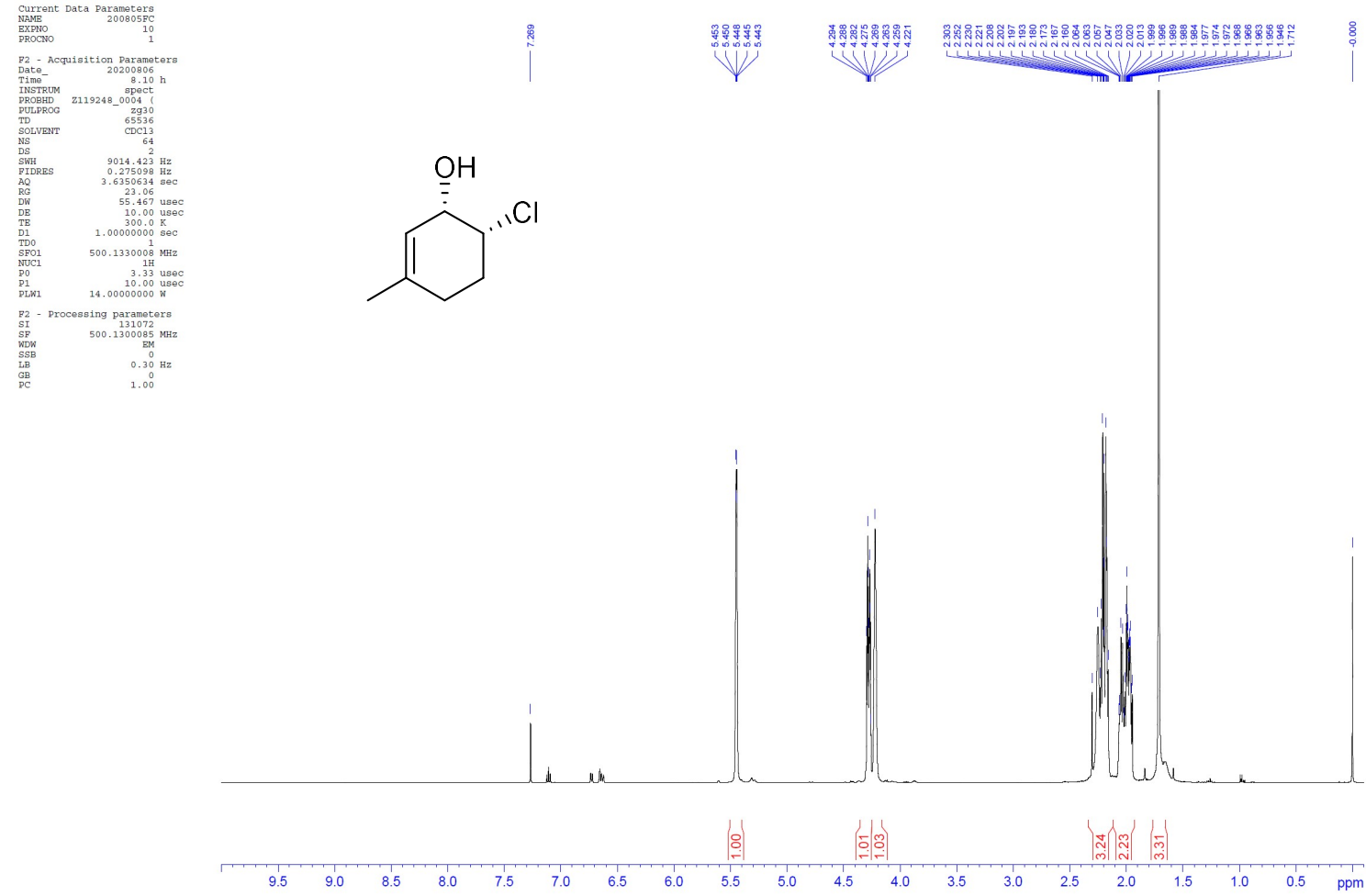

${ }^{13} \mathrm{C} \mathrm{NMR}\left(125 \mathrm{MHz}, \mathrm{CDCl}_{3}\right)$
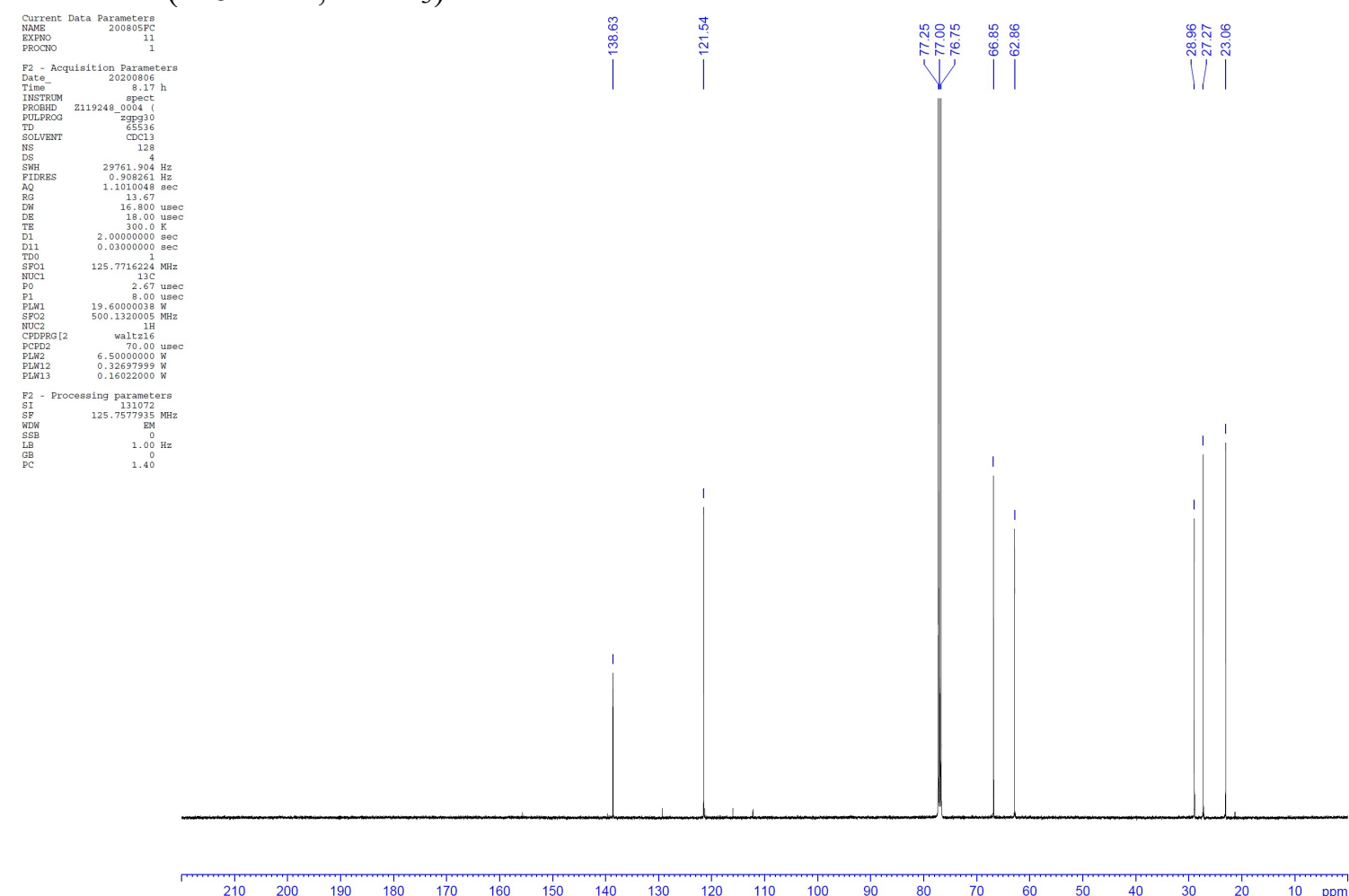
Ethyl (1R,2S)-2-hydroxy-4-methylcyclohex-3-ene-1-carboxylate (cis-8b)

${ }^{1} \mathrm{H}-\mathrm{NMR}\left(500 \mathrm{MHz}, \mathrm{CDCl}_{3}\right)$
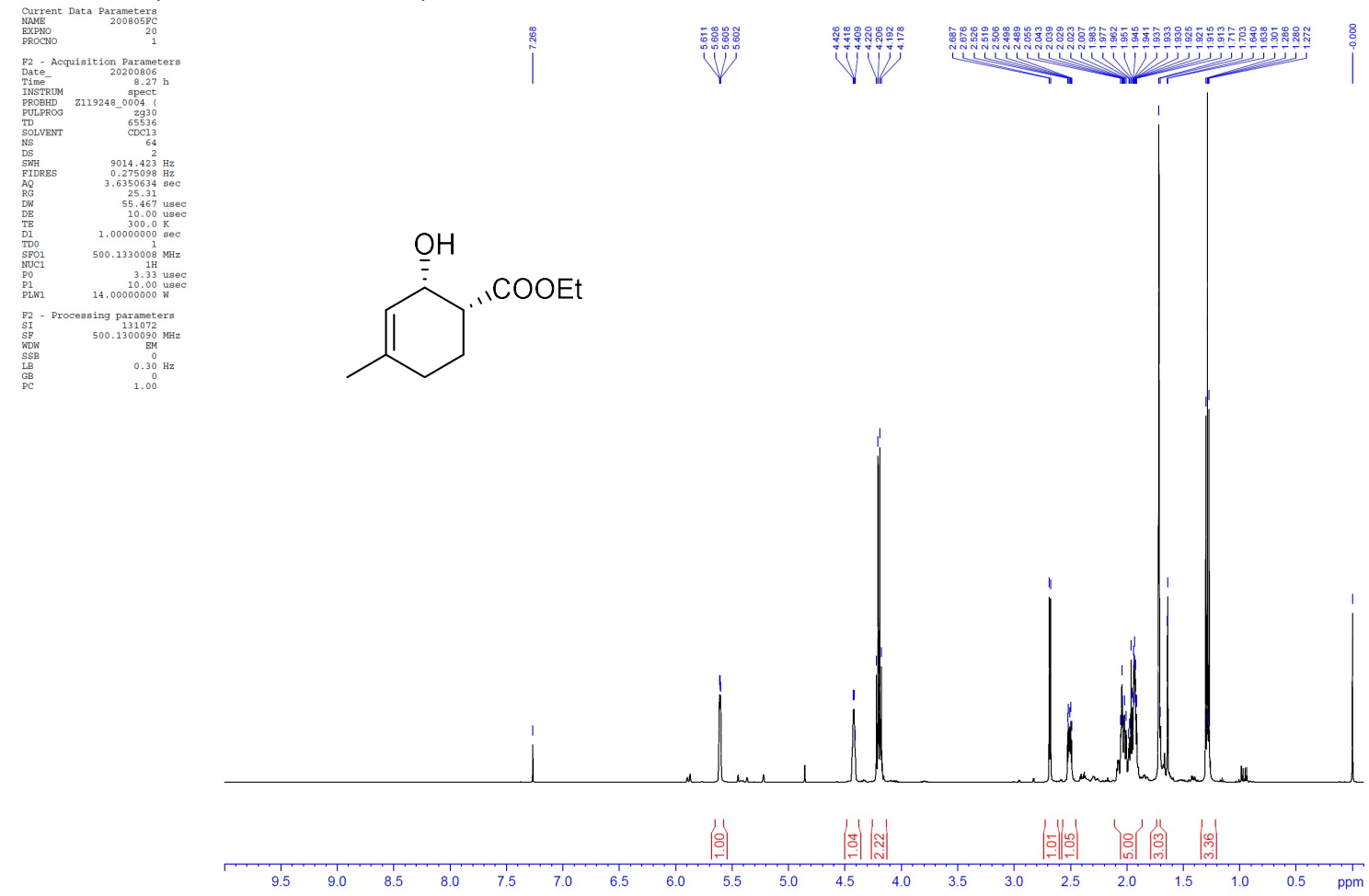

${ }^{13} \mathrm{C} \mathrm{NMR}\left(125 \mathrm{MHz}, \mathrm{CDCl}_{3}\right)$

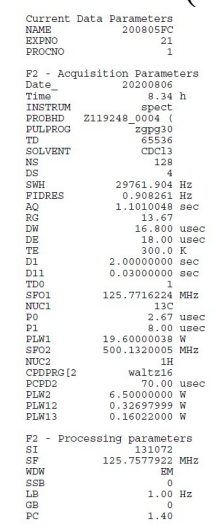


$(1 R, 2 S)-N$-(tert-Butyl)-2-hydroxy-4-methylcyclohex-3-ene-1-carboxamide (cis-8c)

${ }^{1} \mathrm{H}-\mathrm{NMR}\left(500 \mathrm{MHz}, \mathrm{CDCl}_{3}\right.$ )
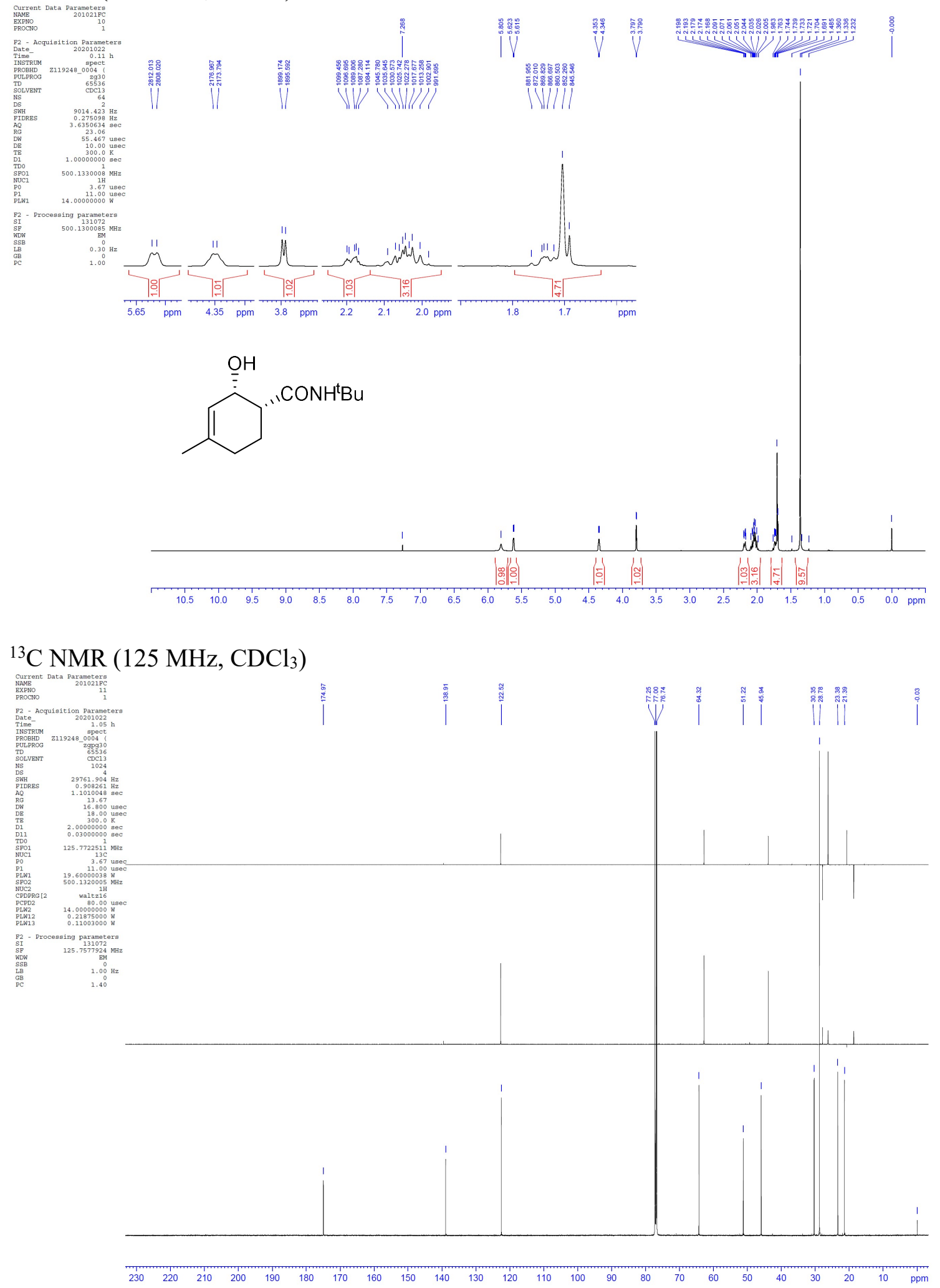
7-(Benzyloxy)-3,4-dihydronaphthalen-1(2H)-one (A)

${ }^{1} \mathrm{H}-\mathrm{NMR}\left(500 \mathrm{MHz}, \mathrm{CDCl}_{3}\right)$
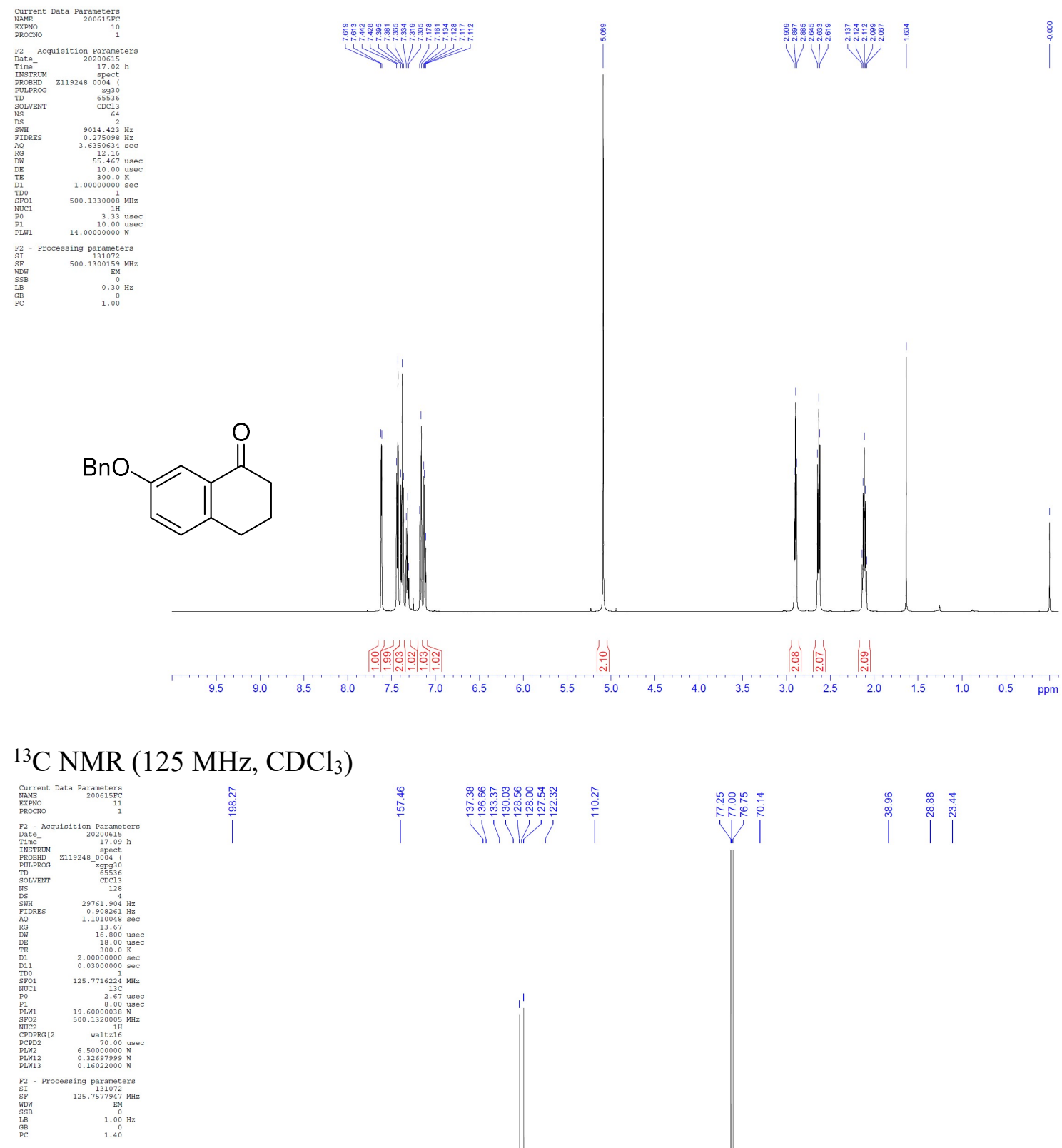
7-(Benzyloxy)-2-chloro-3,4-dihydronaphthalen-1(2H)-one (10)

${ }^{1} \mathrm{H}-\mathrm{NMR}\left(500 \mathrm{MHz}, \mathrm{CDCl}_{3}\right)$

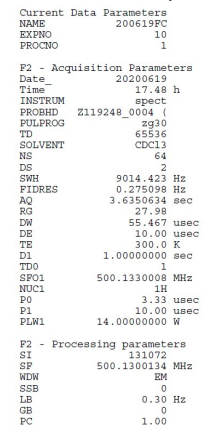<smiles>O=C1c2cc(OCc3ccccc3)ccc2CCC1Cl</smiles>

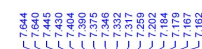
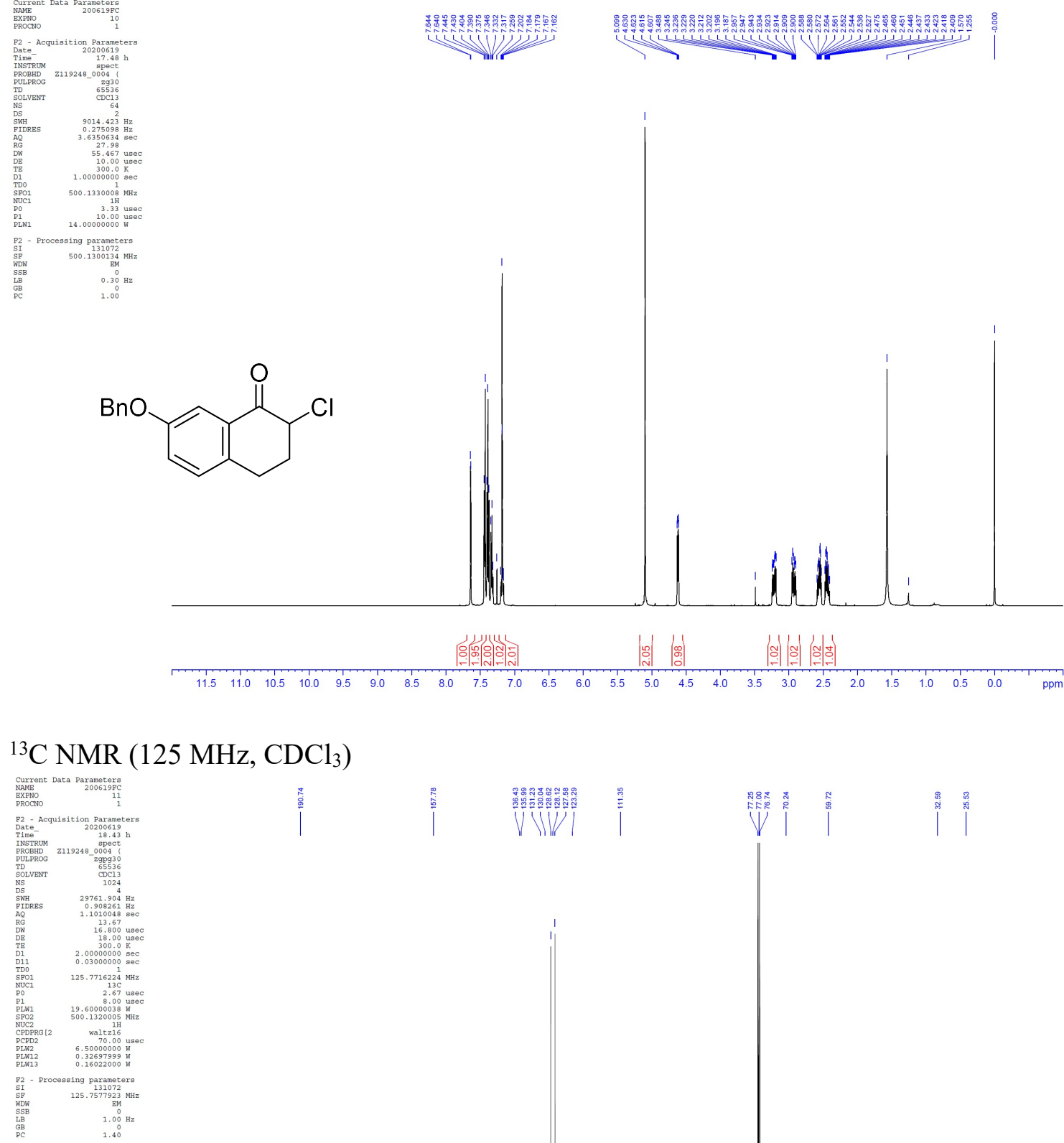


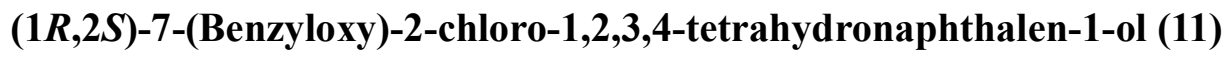

${ }^{1} \mathrm{H}-\mathrm{NMR}\left(500 \mathrm{MHz}, \mathrm{CDCl}_{3}\right)$

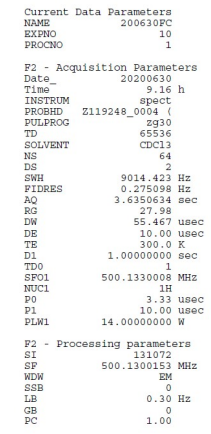

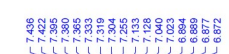

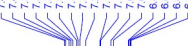

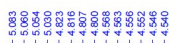

wiv V
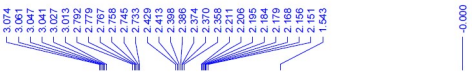<smiles>Oc1ccc2c(c1)[C@H](O)[C@H](Cl)CC2</smiles>

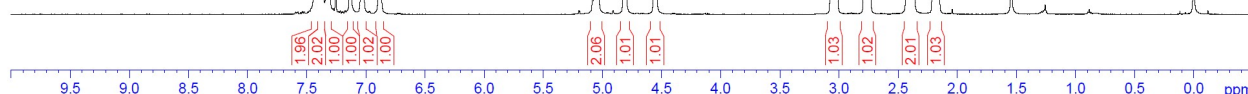

${ }^{13} \mathrm{C} \mathrm{NMR}\left(125 \mathrm{MHz}, \mathrm{CDCl}_{3}\right)$

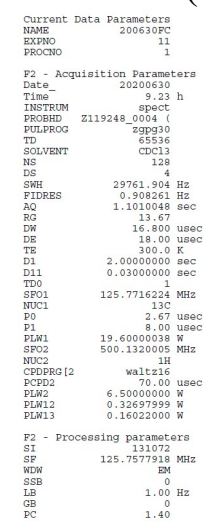


(1R,2R)-2-Azido-7-(benzyloxy)-1,2,3,4-tetrahydronaphthalen-1-ol (B)

${ }^{1} \mathrm{H}-\mathrm{NMR}\left(500 \mathrm{MHz}, \mathrm{CDCl}_{3}\right)$
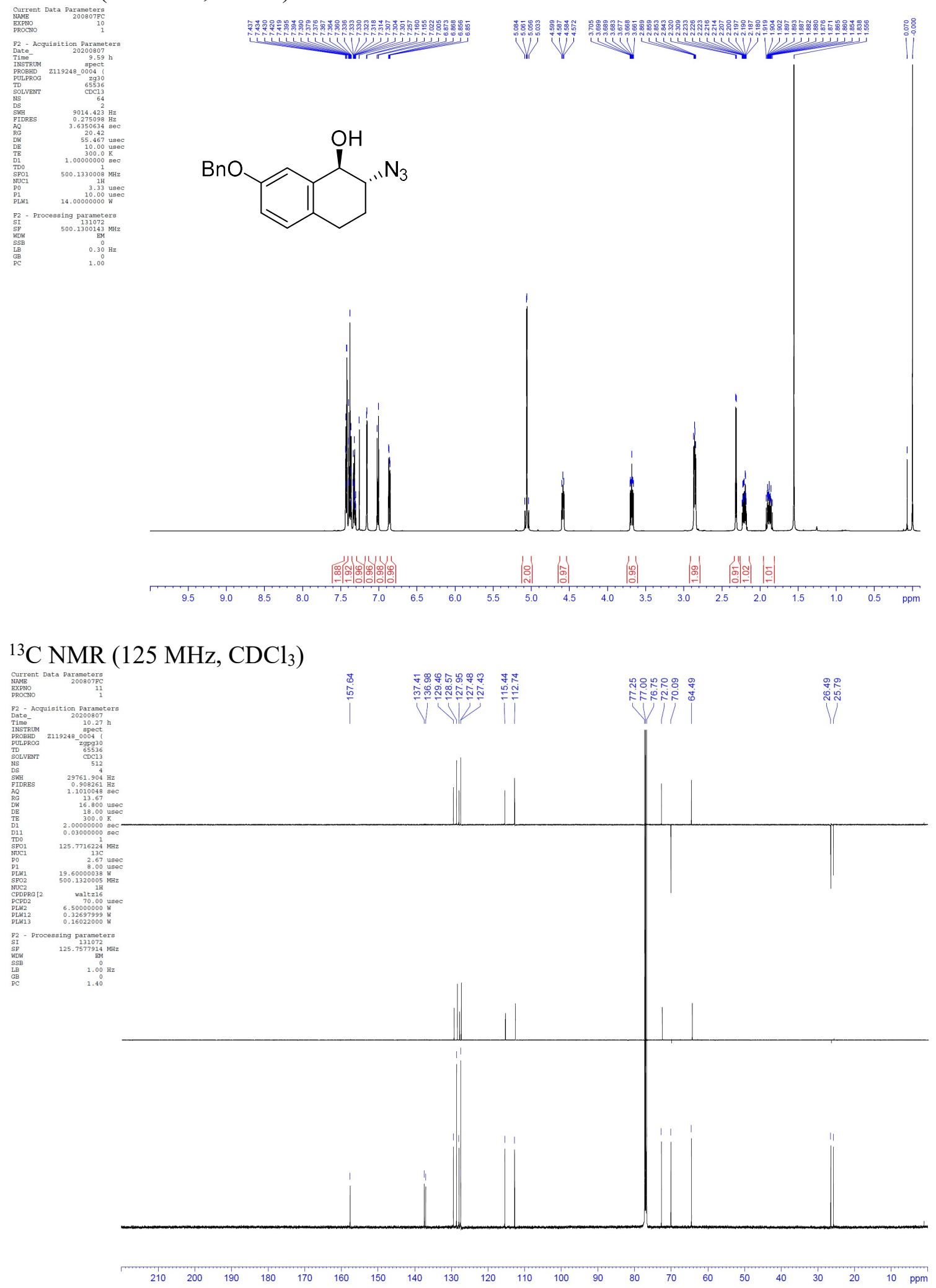
${ }^{1} \mathrm{H}-{ }^{1} \mathrm{H}-\mathrm{COSY}$

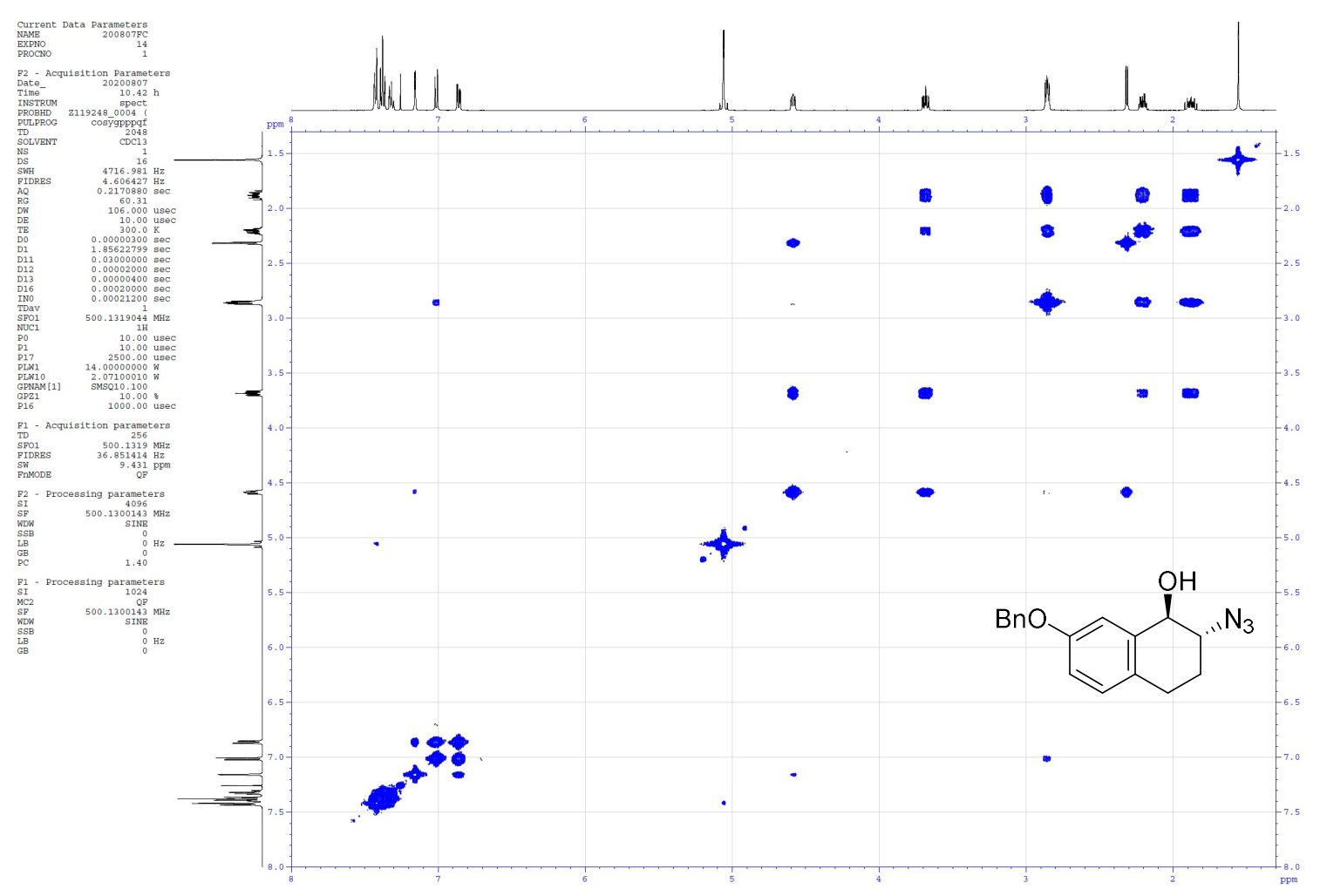


(1R,2R)-2-Amino-7-(benzyloxy)-1,2,3,4-tetrahydronaphthalen-1-ol (12)

${ }^{1} \mathrm{H}-\mathrm{NMR}\left(500 \mathrm{MHz}, \mathrm{CDCl}_{3}\right)$
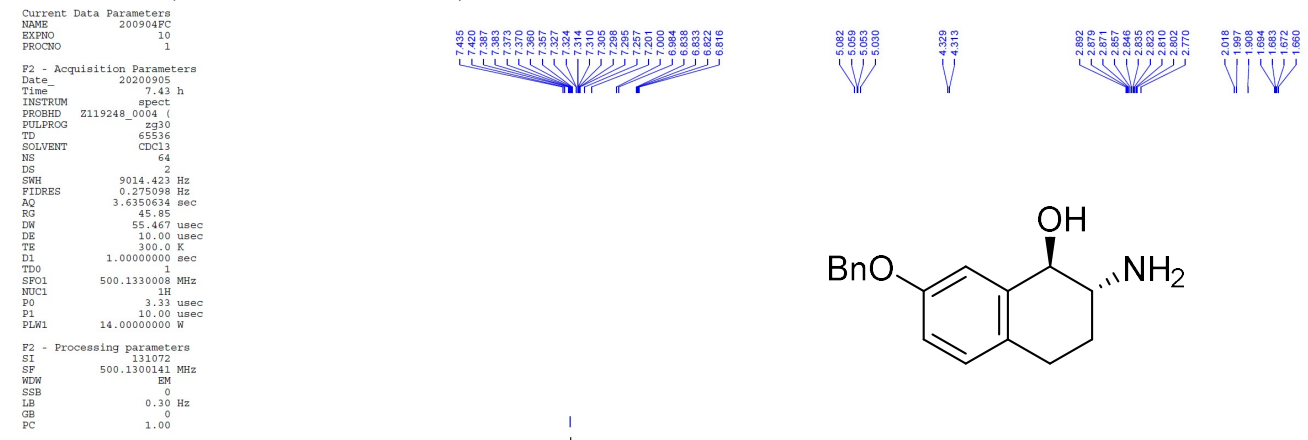<smiles>N[C@H]1CCc2ccc(OCc3ccccc3)cc2C1O</smiles>

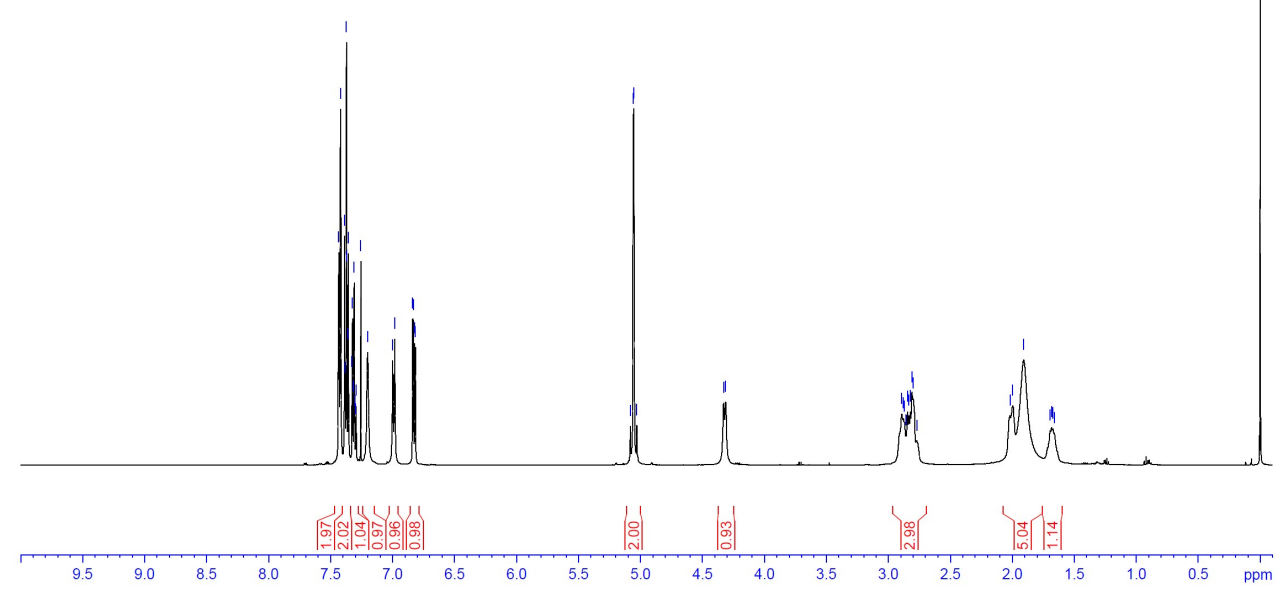

${ }^{13} \mathrm{C}$ NMR $\left(125 \mathrm{MHz}, \mathrm{CDCl}_{3}\right)$
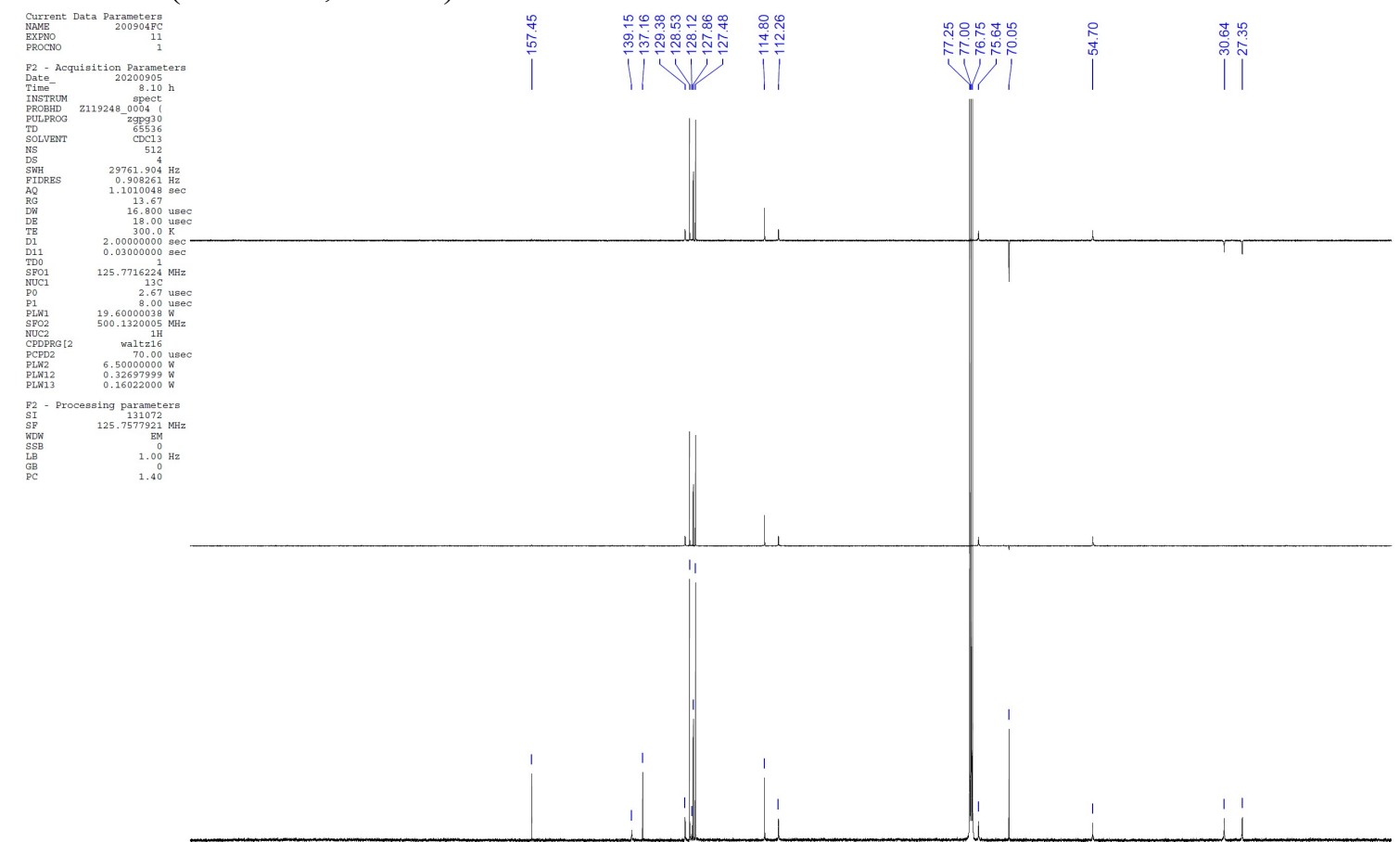

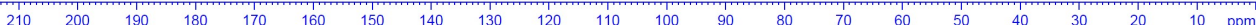


${ }^{1} \mathrm{H}-{ }^{1} \mathrm{H}-\mathrm{COSY}$

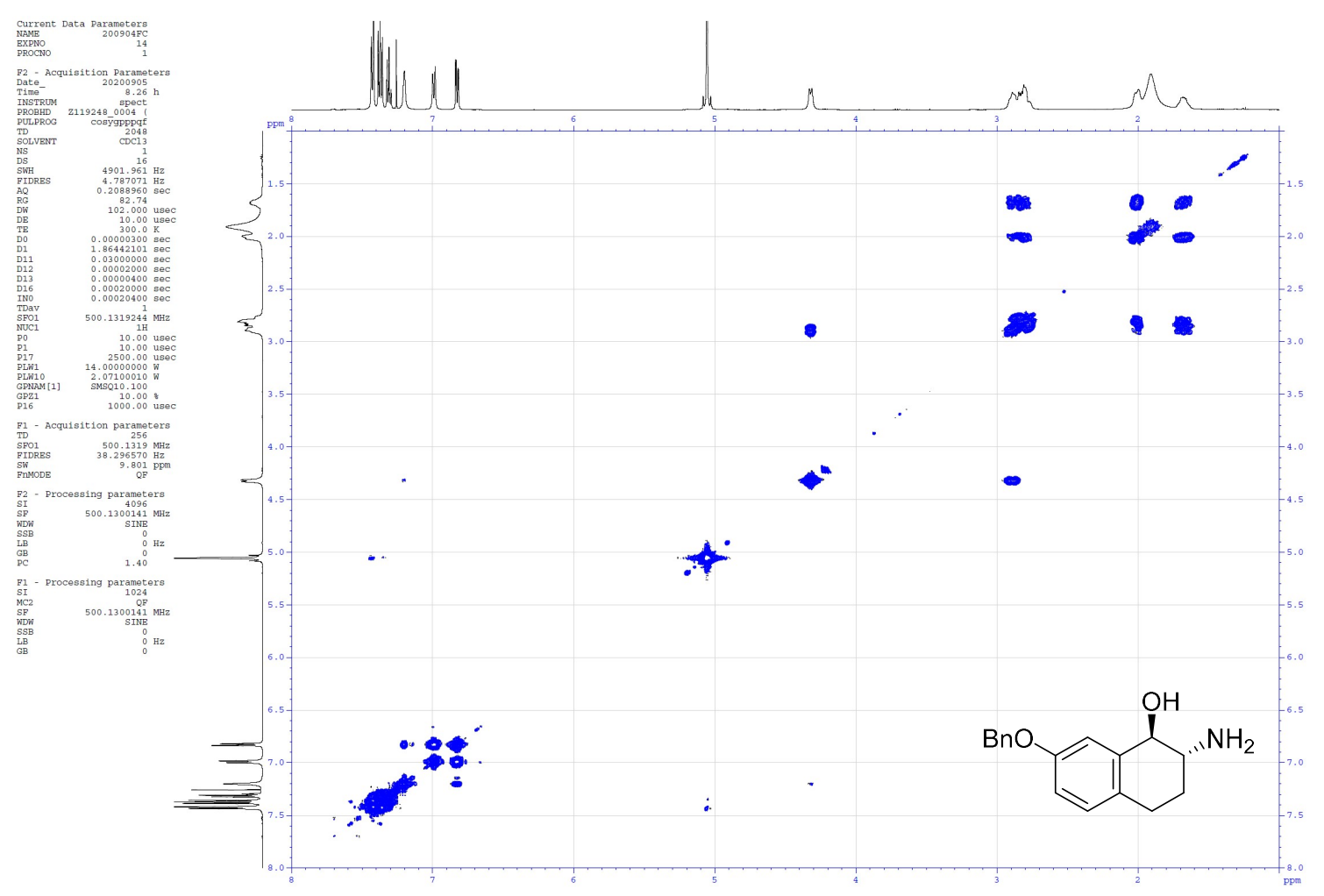


(4aR,10bR)-9-(Benzyloxy)-4a,5,6,10b-tetrahydro-2H-naphtho[1,2-b][1,4]oxazin-3(4H)one (13)
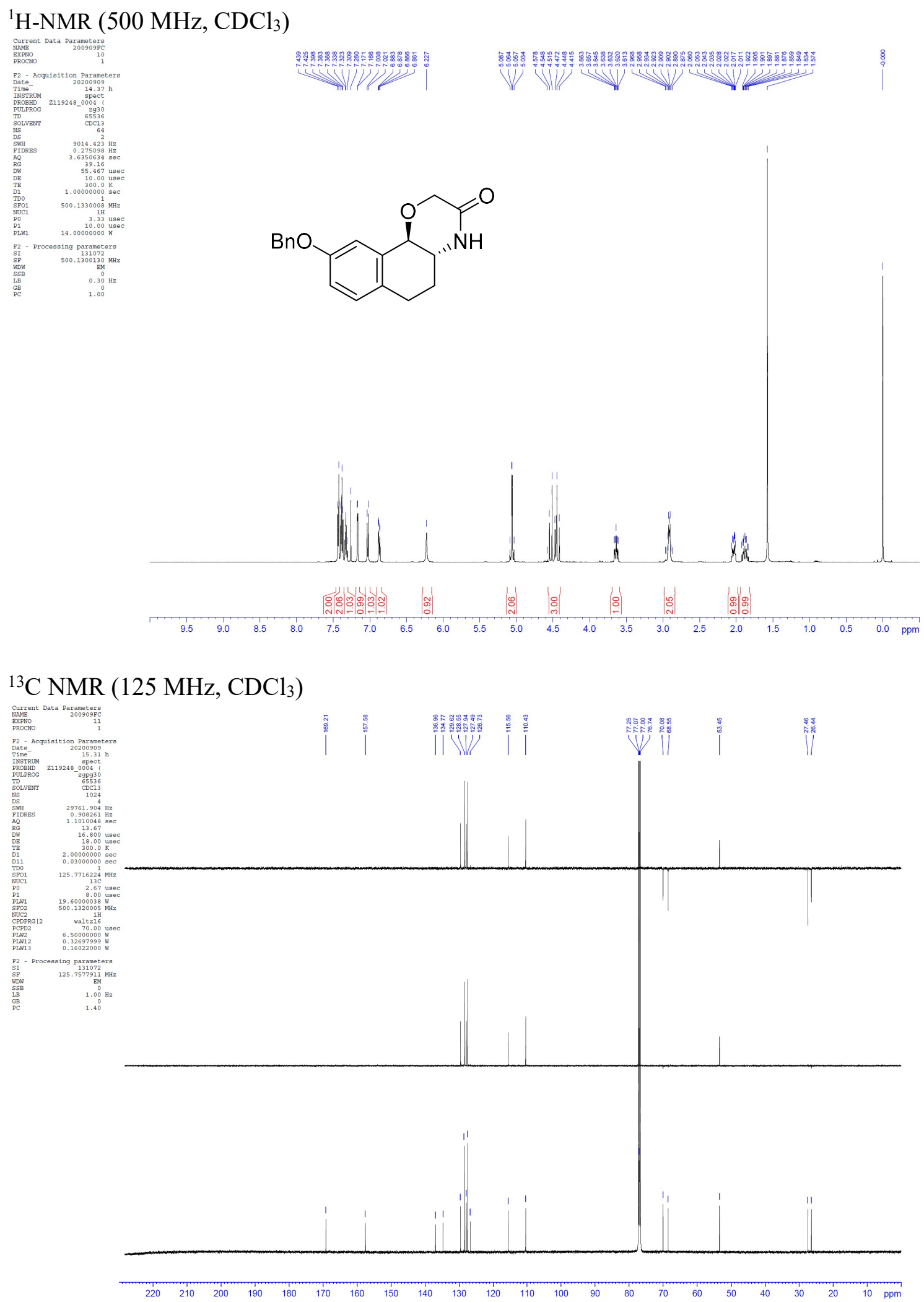
${ }^{1} \mathrm{H}-{ }^{1} \mathrm{H}-\mathrm{COSY}$

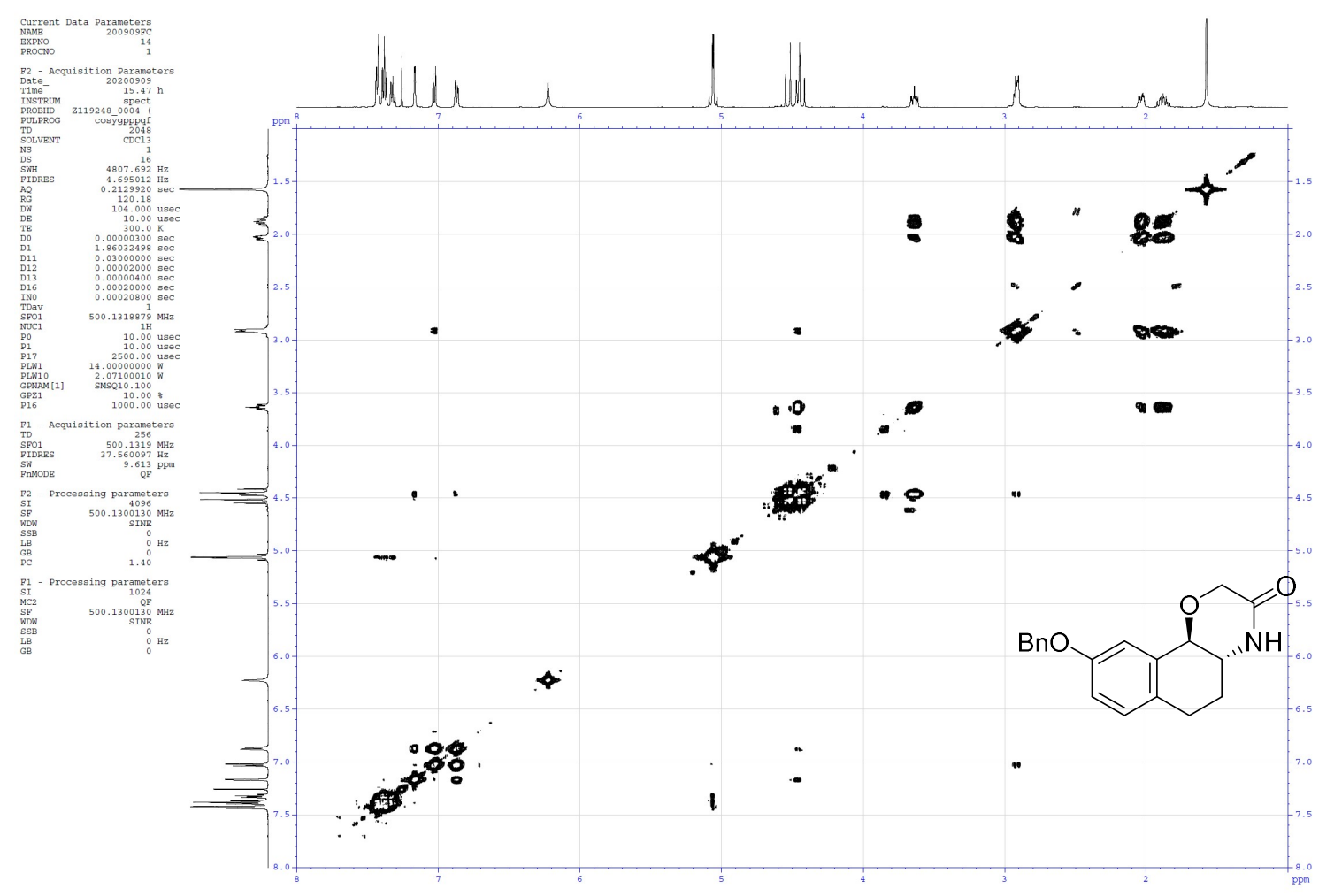


$(4 \mathrm{a} R, 10 \mathrm{~b} R)-9-(B e n z y l o x y)-4-p r o p i o n y l-4 a, 5,6,10 \mathrm{~b}-$ tetrahydro-2 $H$-naphtho[1,2-b][1,4]0 xazin-3(4H)-one (14)

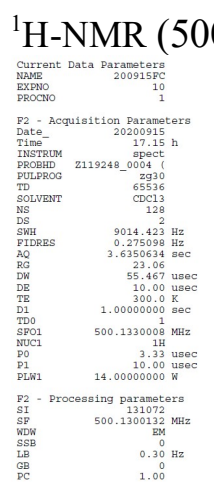<smiles>CCC(=O)N1C(=O)CO[C@H]2c3cc(OCc4ccccc4)ccc3CC[C@@H]21</smiles>

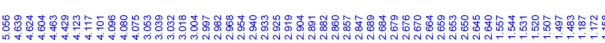
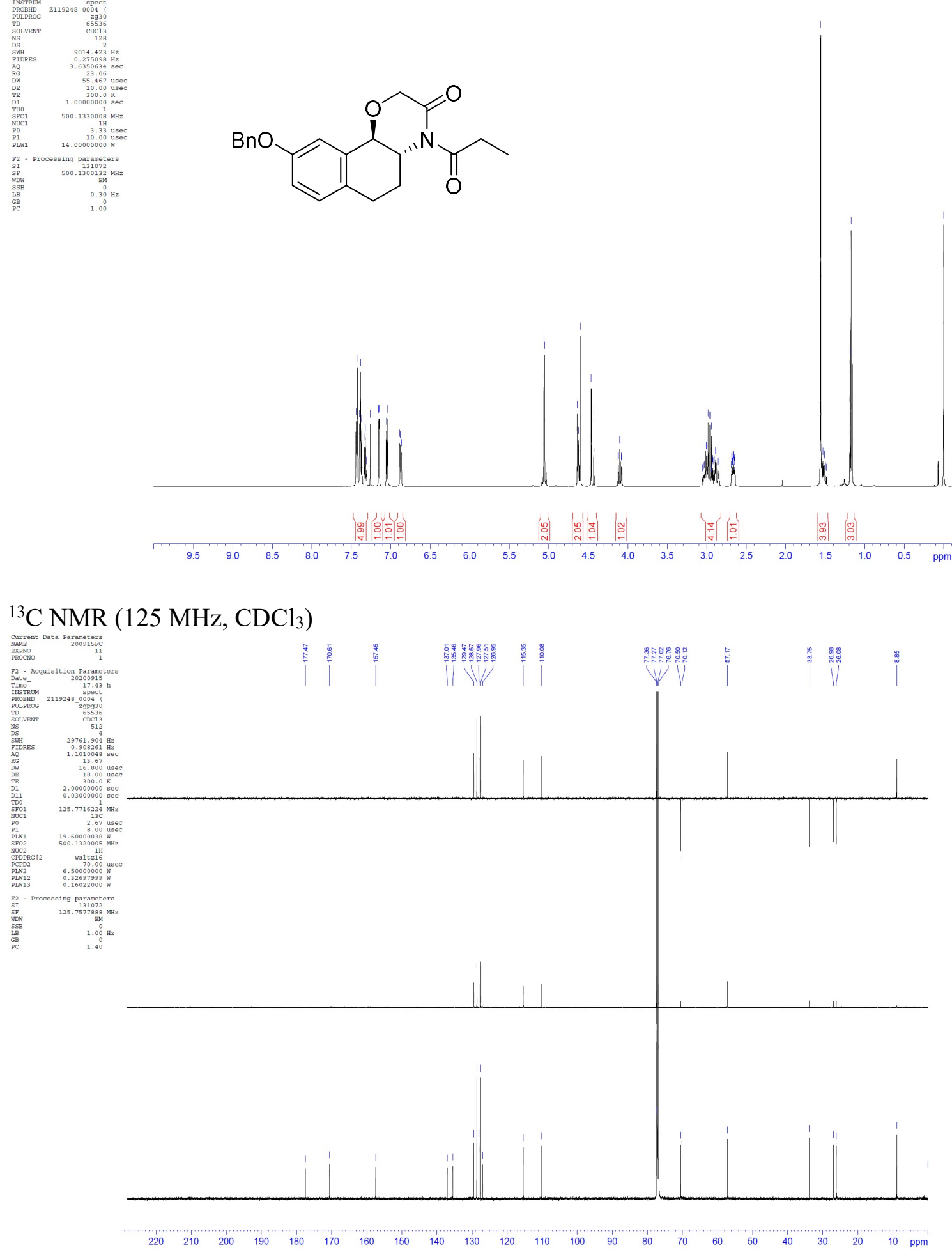
${ }^{1} \mathrm{H}-{ }^{1} \mathrm{H}-\mathrm{COSY}$

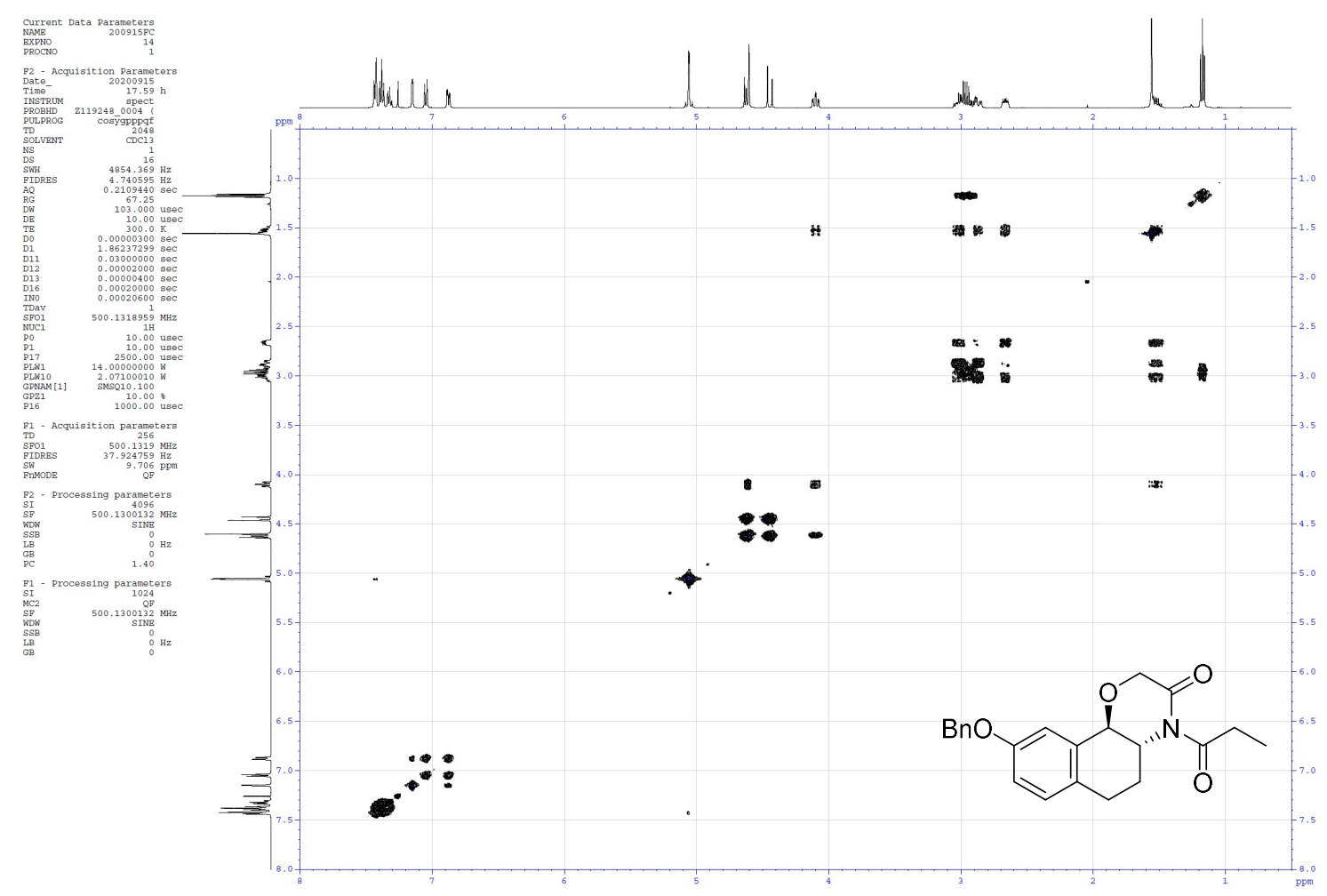


(4aR,10bR)-9-(Benzyloxy)-4-propyl-3,4,4a,5,6,10b-hexahydro-2H-naphtho[1,2-b][1,4]0 xazine (C)

${ }^{1} \mathrm{H}-\mathrm{NMR}\left(500 \mathrm{MHz}, \mathrm{CDCl}_{3}\right)$

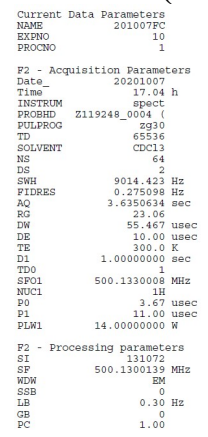

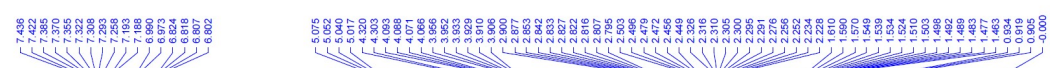<smiles>CCCN1CCO[C@H]2c3cc(OBr)ccc3CC[C@H]21</smiles>
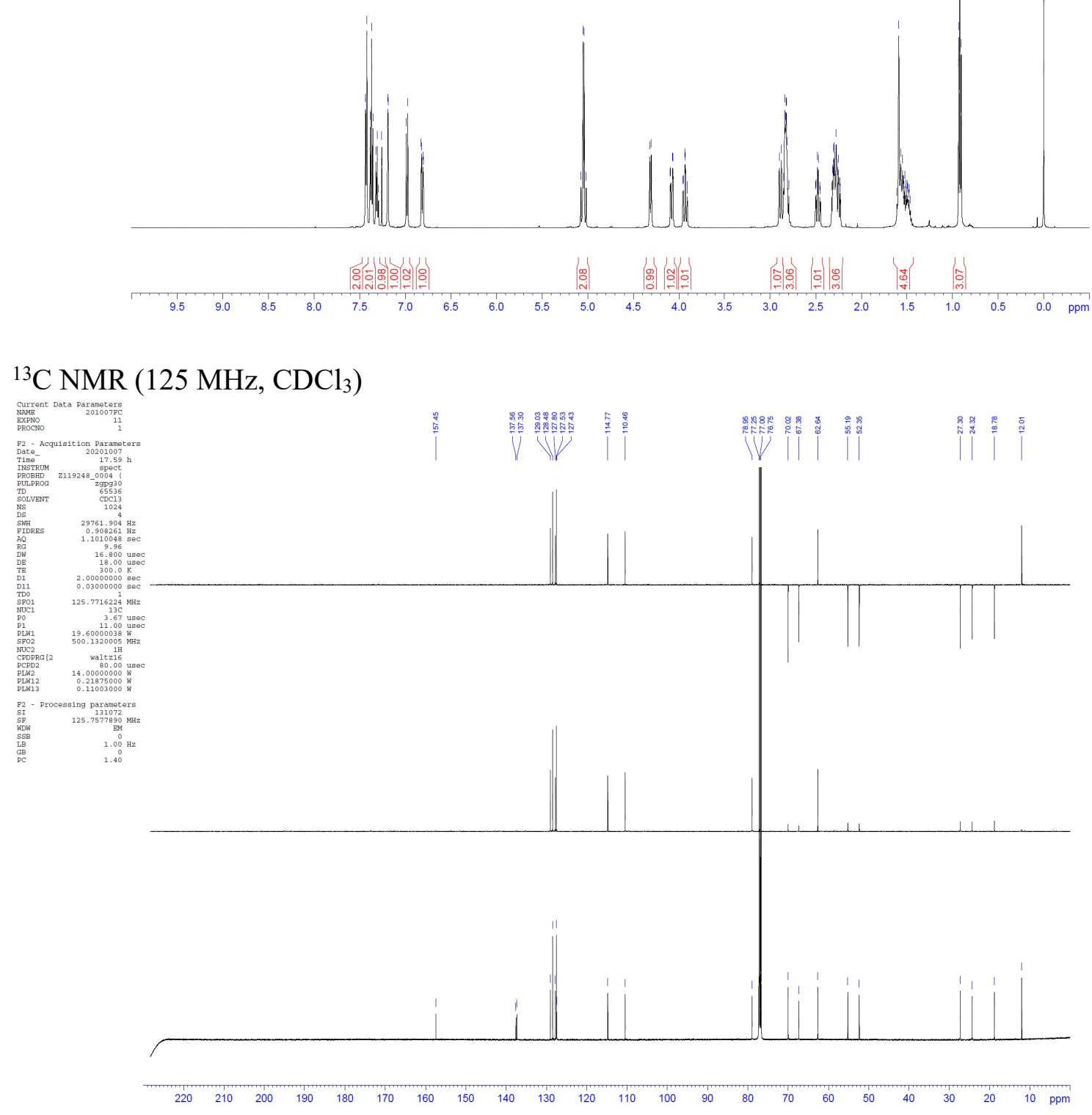
${ }^{1} \mathrm{H}-{ }^{1} \mathrm{H}-\mathrm{COSY}$

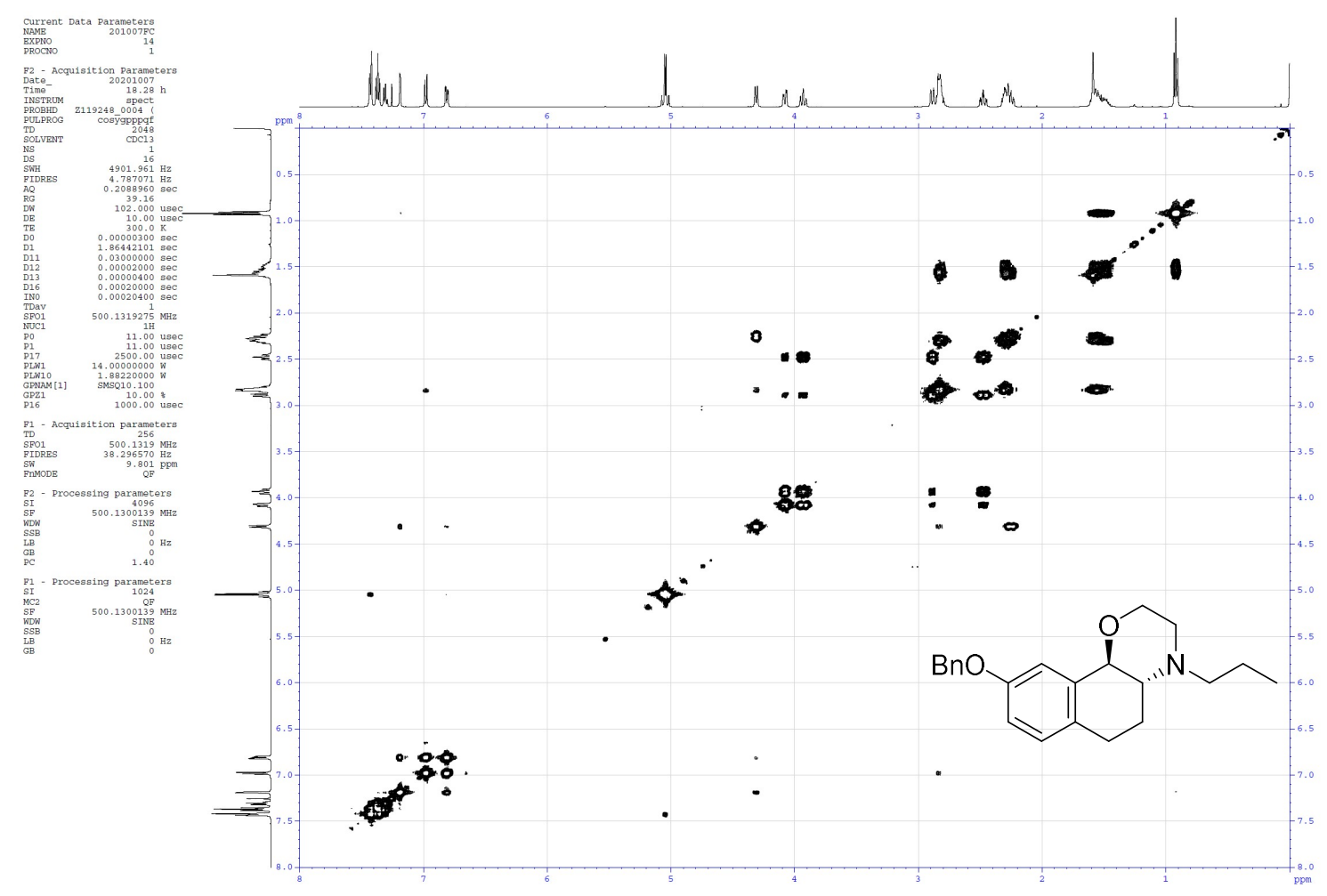


(+)-PHNO:

(4aR,10bR)-4-Propyl-3,4,4a,5,6,10b-hexahydro-2H-naphtho[1,2-b][1,4]oxazin-9-ol

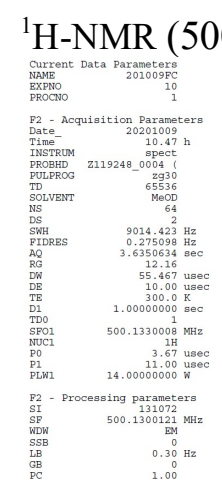
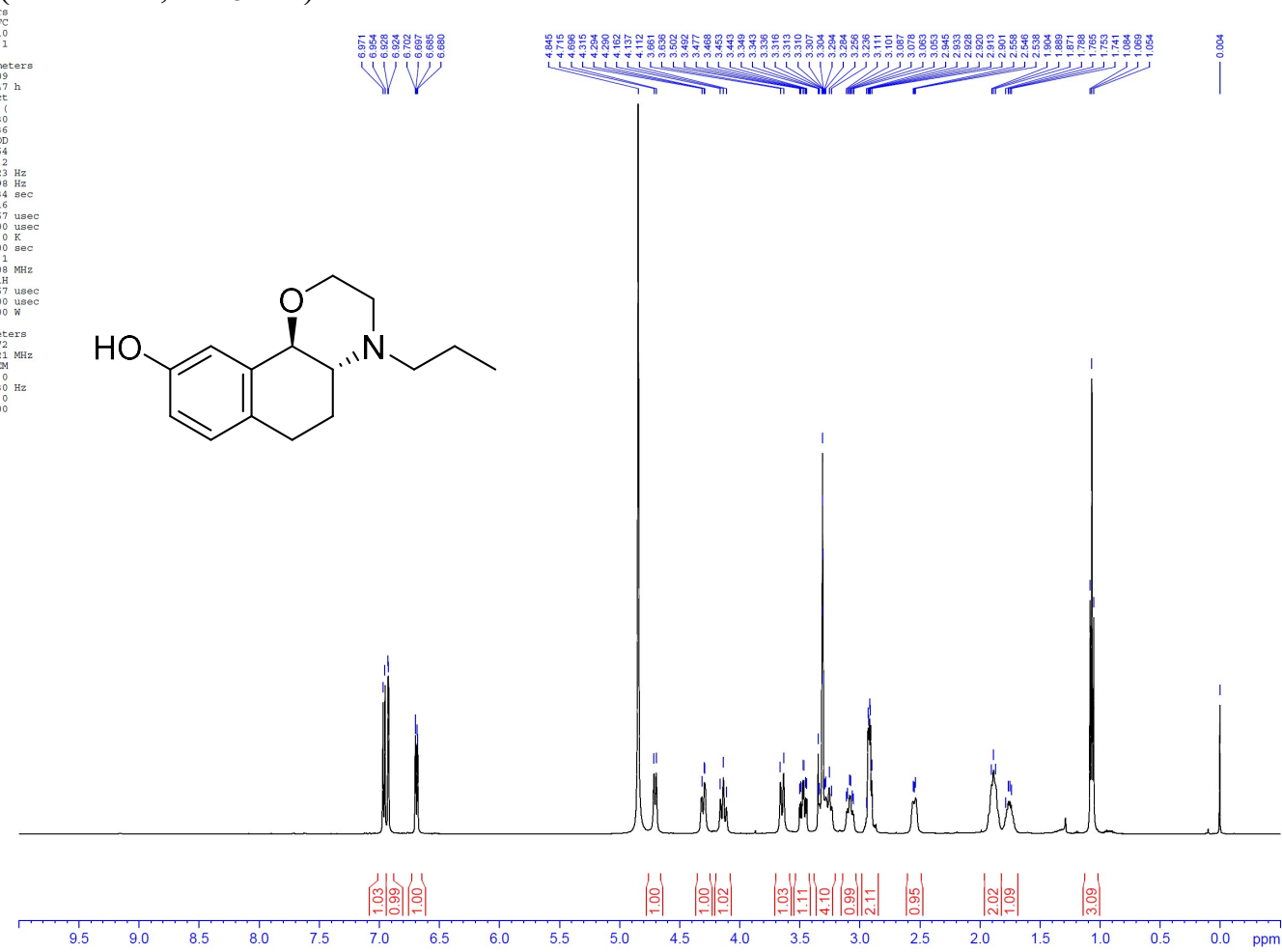

${ }^{13} \mathrm{C}$ NMR (125 MHz, $\left.\mathrm{CD}_{3} \mathrm{OD}\right)$

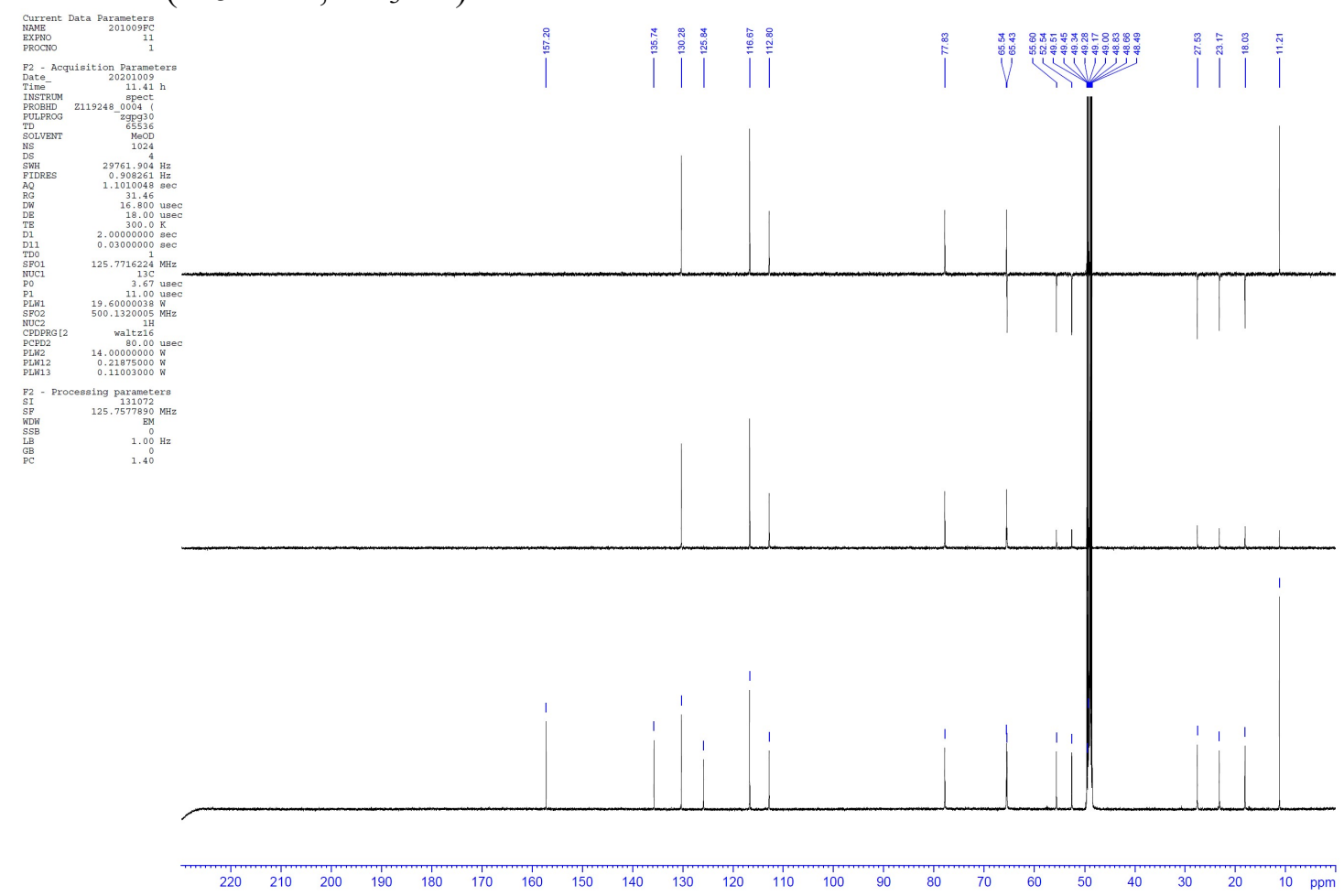


${ }^{1} \mathrm{H}-{ }^{1} \mathrm{H}-\mathrm{COSY}$

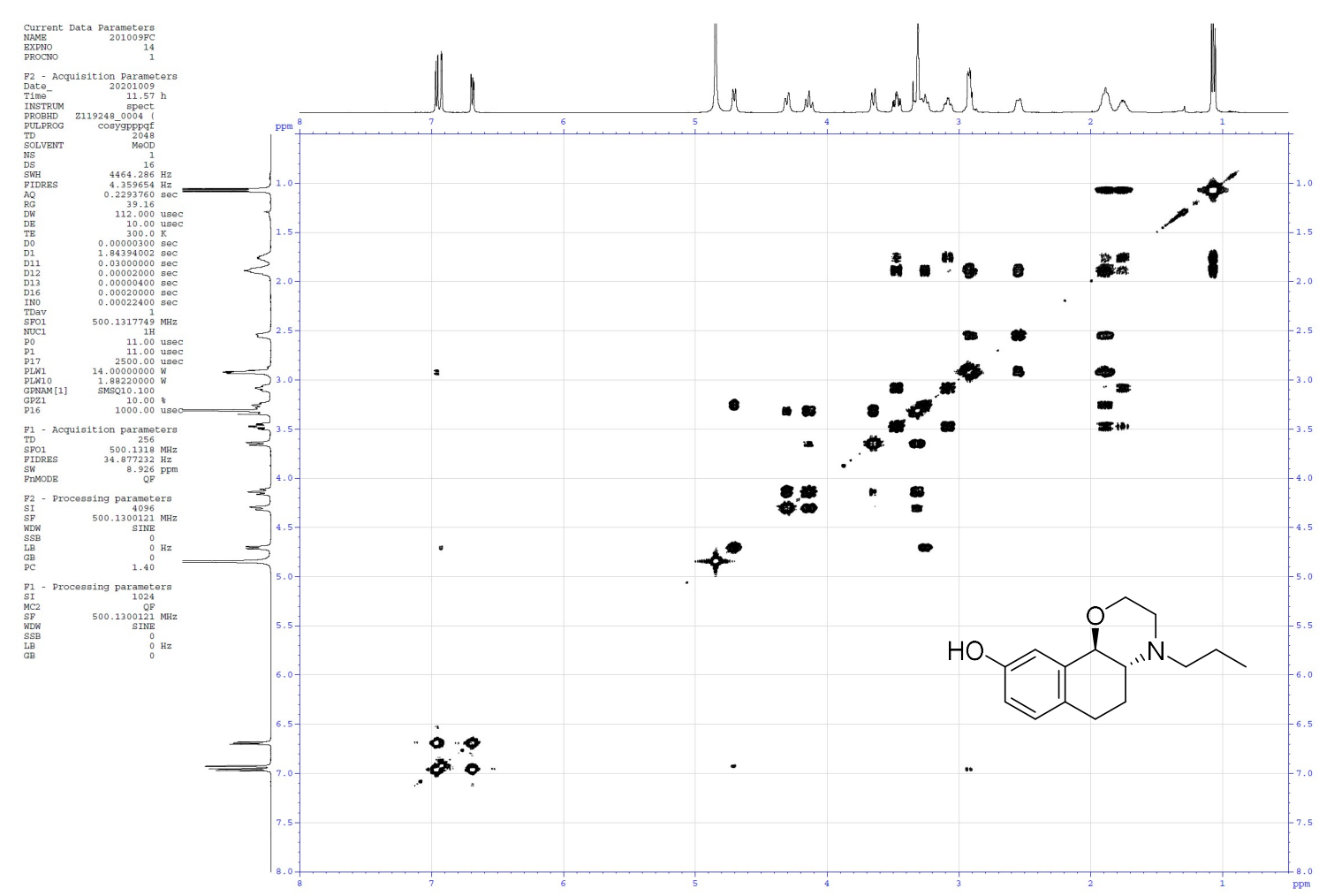

HSQC

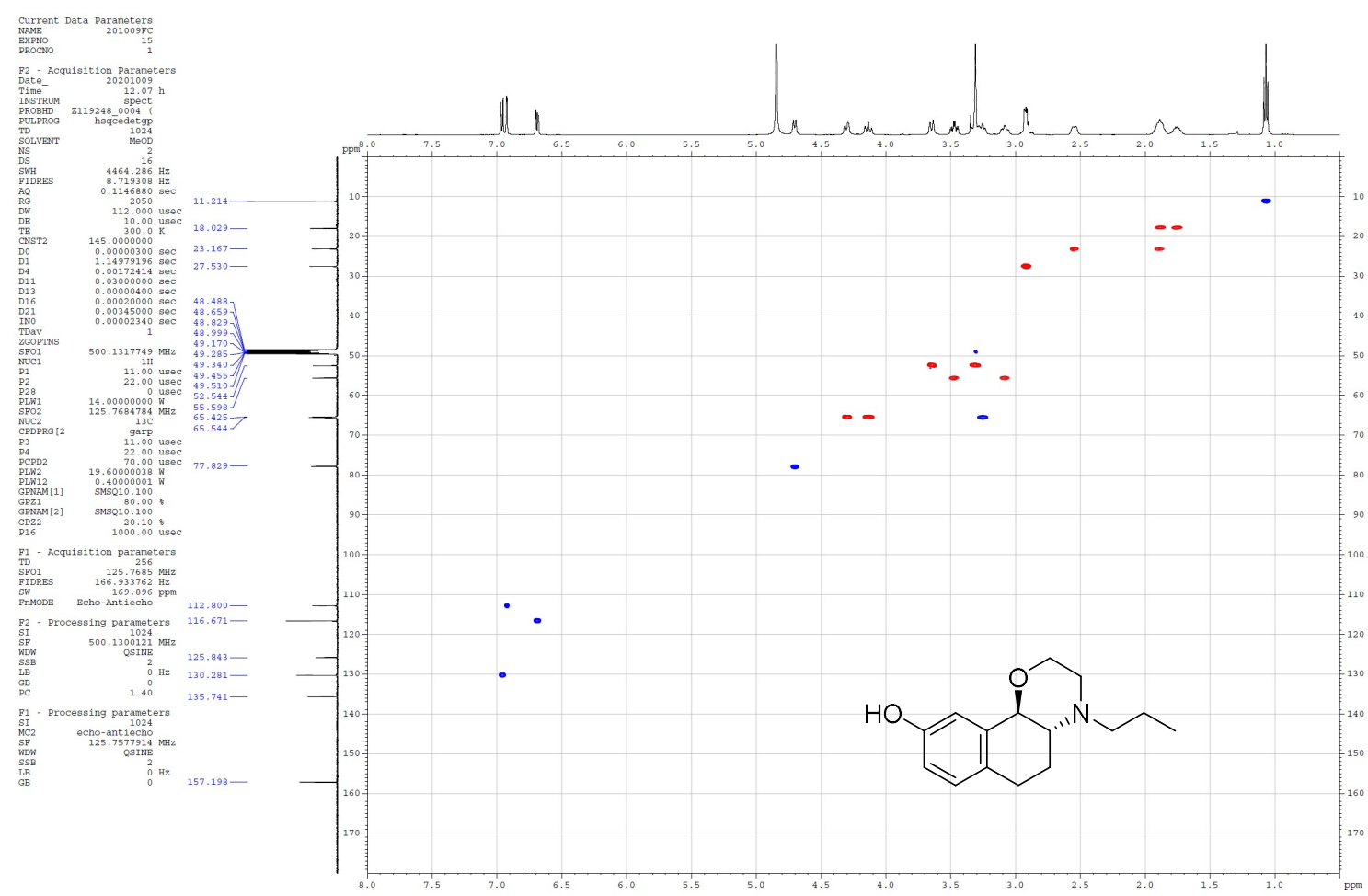


HMBC

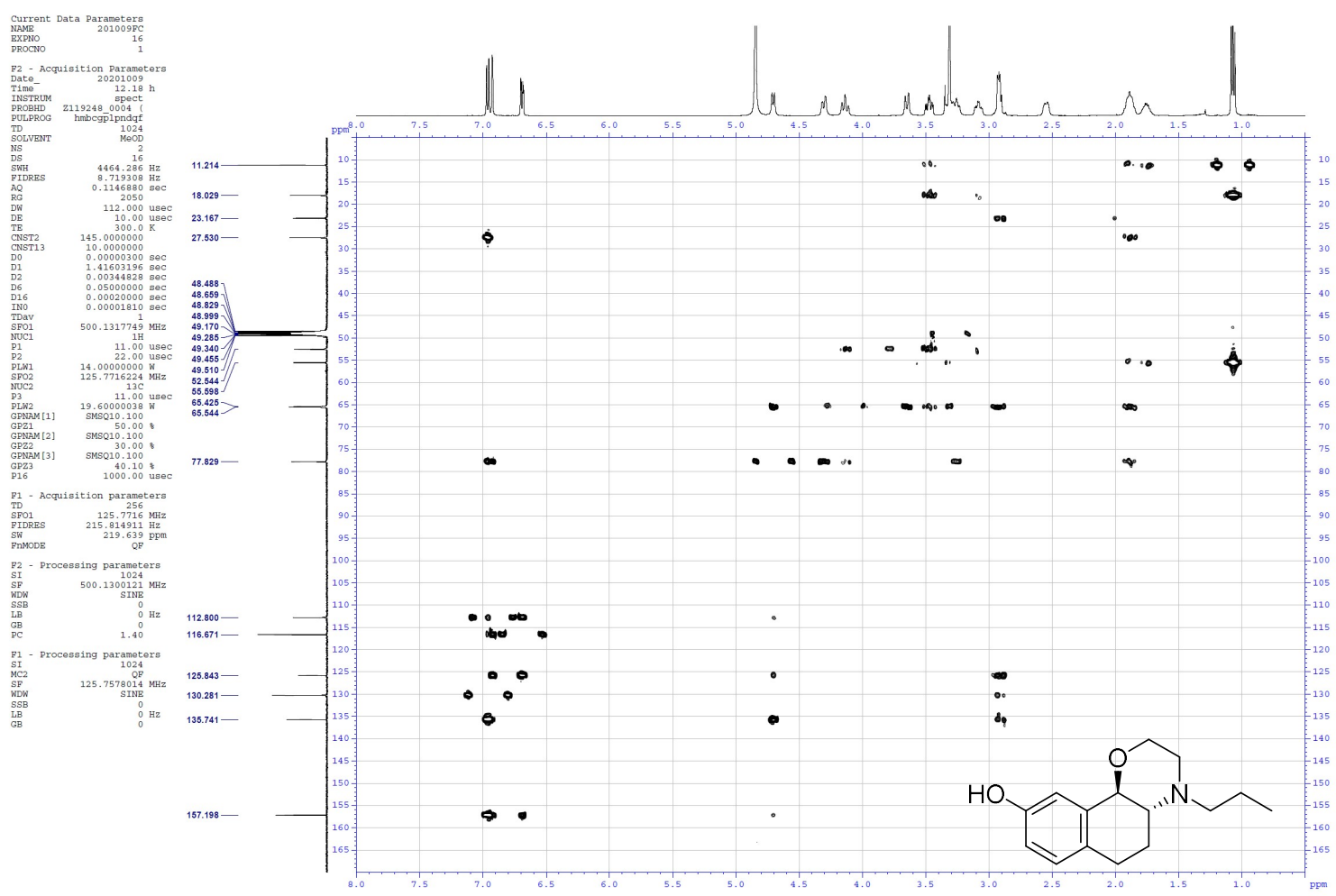


(1R,2R)-1-((4,5-Dichloro-2-((3aR,6R,7aR)-8,8-dimethyl-2,2-dioxidohexahydro-3H-3a,6 -methanobenzo[c]isothiazole-1-carbonyl)benzoyl)oxy)-2,3-dihydro-1H-indene-2-carbo xylate (D)
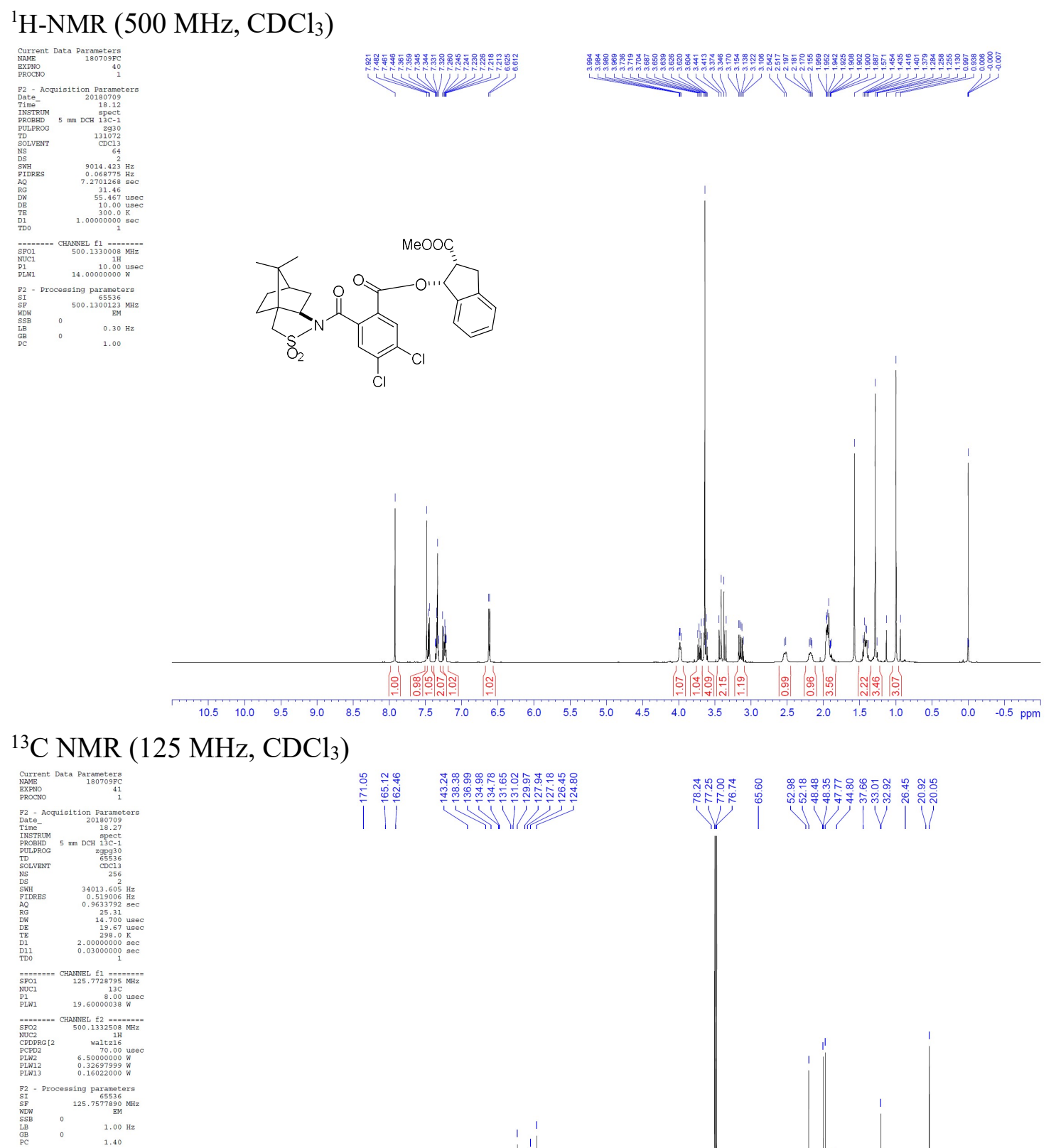


\section{Chiral HPLC Charts for Reduction Products.}

\section{(1S,2R)-2-Chloro-1,2,3,4-tetrahydronaphthalen-1-ol (cis-4a)}

Chiralcel OJ-H, $250 \times 4.6 \mathrm{~mm}$ column, hexane/2-propanol 97:3, $1.0 \mathrm{~mL} / \mathrm{min}, 220 \mathrm{~nm}, 35^{\circ} \mathrm{C}$

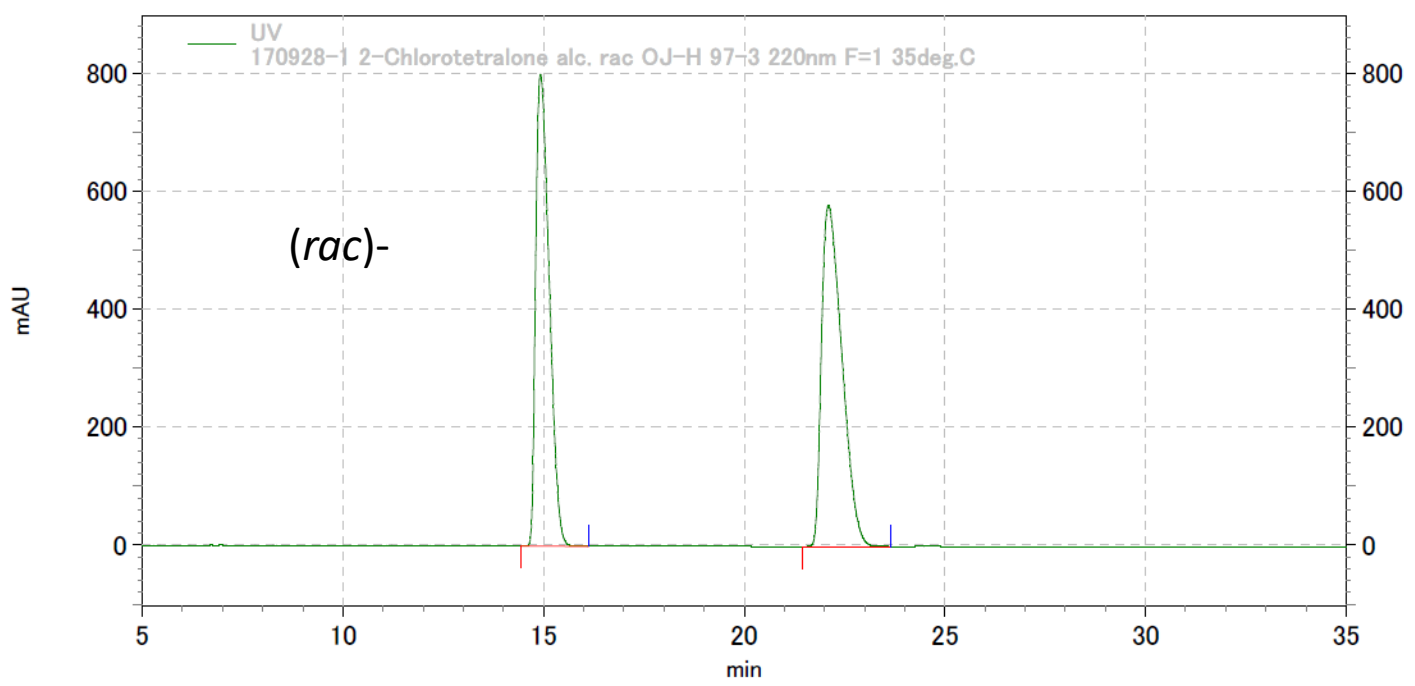

170928-1 2-Chlorotetralone alc. rac 0J-H 97-3 220nm F=1 35deg. C

\begin{tabular}{rlllll} 
UV & & & & \\
Pk & R. T. & Area & Area\% & B.C. & Symmetry \\
No. & & & & & \\
\hline 1 & 14.923 & 74747436 & 47.727 & MM & 0.00000 \\
2 & 22.100 & 81866362 & 52.273 & MM & 0.00000
\end{tabular}
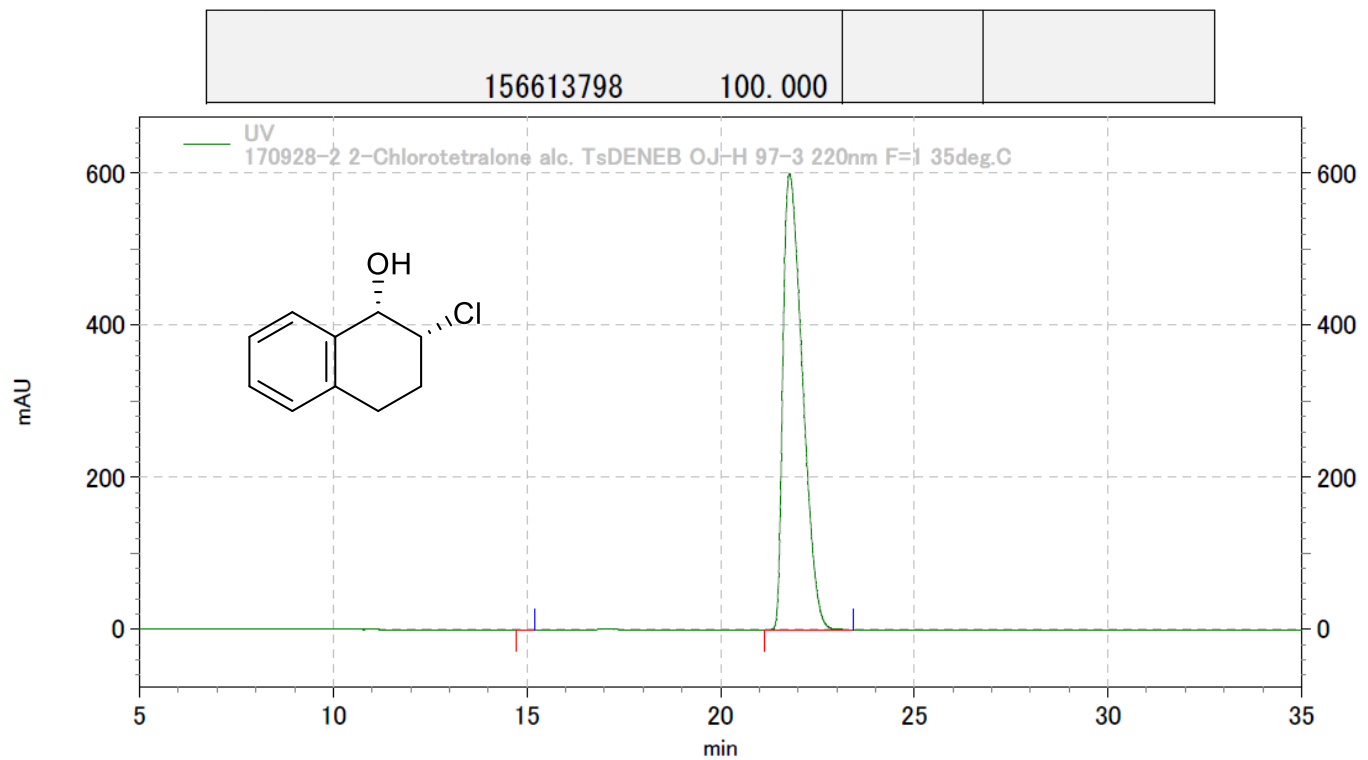

170928-2 2-Chlorotetralone alc. TsDENEB 0J-H 97-3 220nm F=1 35deg. C

\begin{tabular}{|c|c|c|c|c|c|}
\hline $\begin{array}{l}\text { UV } \\
\text { Pk } \\
\text { No. }\end{array}$ & R. T. & Area & Area\% & B. C. & Symmetry \\
\hline $\begin{array}{l}1 \\
2\end{array}$ & $\begin{array}{l}14.947 \\
21.783\end{array}$ & $\begin{array}{r}8140 \\
83725704\end{array}$ & $\begin{array}{r}0.010 \\
99.990\end{array}$ & $\begin{array}{l}\text { MM } \\
\text { MM }\end{array}$ & $\begin{array}{l}0.00000 \\
0.00000\end{array}$ \\
\hline & & 83733844 & 100.000 & & \\
\hline
\end{tabular}




\section{(1S,2R)-2-Chloro-7-methoxy-1,2,3,4-tetrahydronaphthalen-1-ol (cis-4b)}

Chiralpak AS-3, $250 \times 4.6 \mathrm{~mm}$ column, hexane/2-propanol 95:5, $1.0 \mathrm{~mL} / \mathrm{min}, 220 \mathrm{~nm}, 35^{\circ} \mathrm{C}$

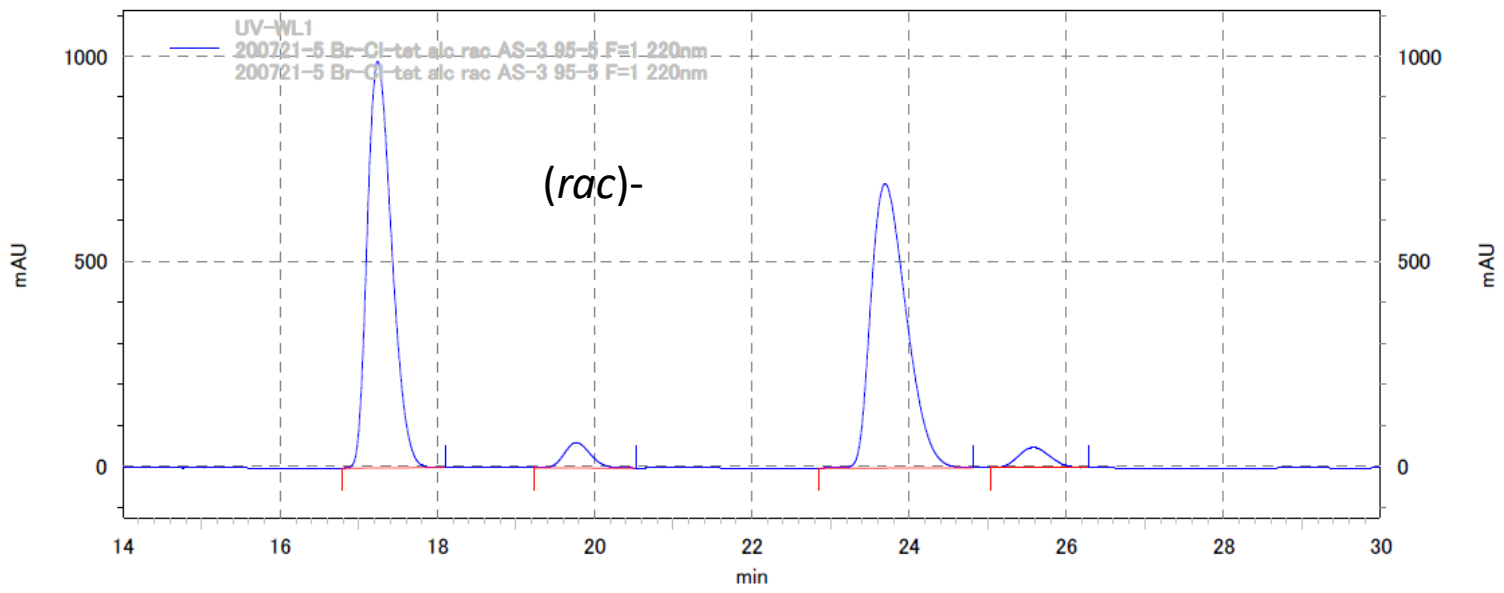

UV-WL1

\begin{tabular}{lrrr|}
\multicolumn{1}{c}{ Peak No. } & Area & Area(\%) \\
\hline & 17.240 & 85515366 & 46.680 \\
& 19.767 & 5755976 & 3.142 \\
& 23.693 & 86230771 & 47.071 \\
& 25.580 & 5690836 & 3.106 \\
\hline & & & 100.000 \\
\hline
\end{tabular}

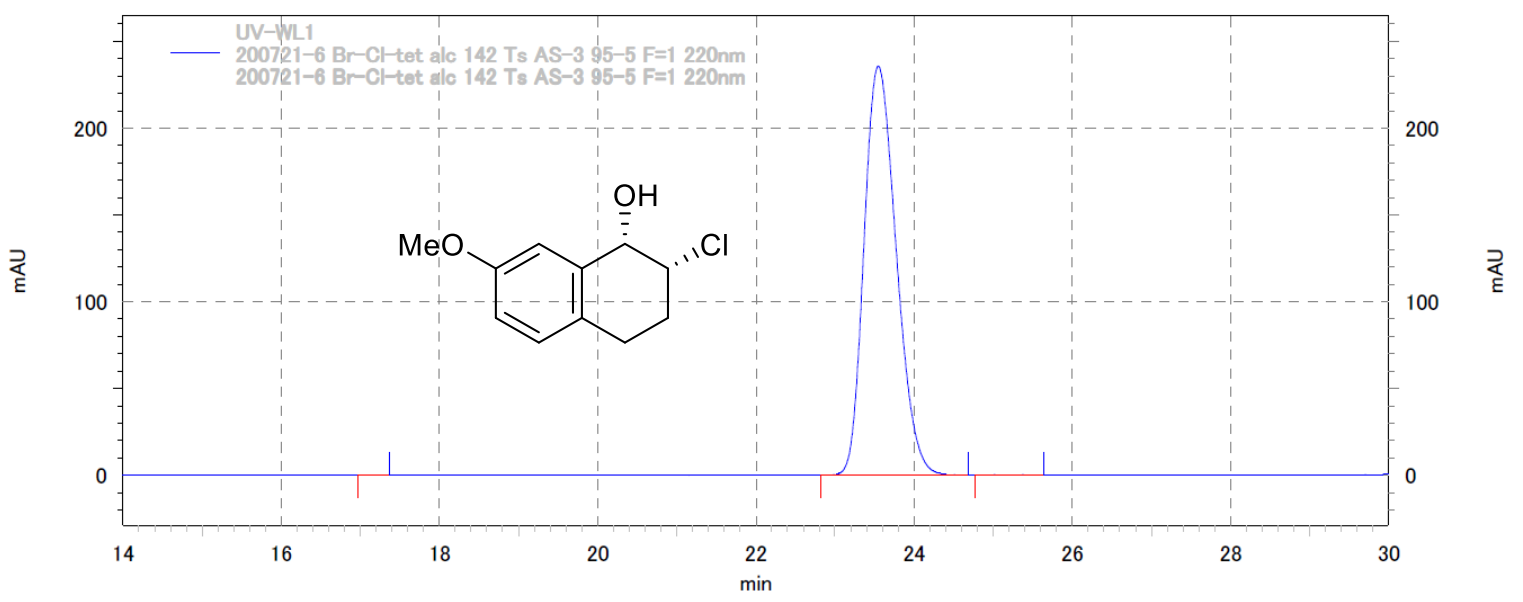

\begin{tabular}{|c|c|c|c|}
\hline Peak No. & $\mathrm{RT}$ (min) & Area & Area(\%) \\
\hline & 17.147 & 6404 & 0.024 \\
\hline & 23.547 & 26605318 & 99.886 \\
\hline & 25.187 & 23905 & 0.090 \\
\hline
\end{tabular}




\section{(1S,2R)-2-Chloro-7-bromo-1,2,3,4-tetrahydronaphthalen-1-ol (cis-4c)}

Chiralcel OD-3, $150 \times 4.6 \mathrm{~mm}$ column, hexane/2-propanol 98:2, $0.8 \mathrm{~mL} / \mathrm{min}, 220 \mathrm{~nm}, 35^{\circ} \mathrm{C}$

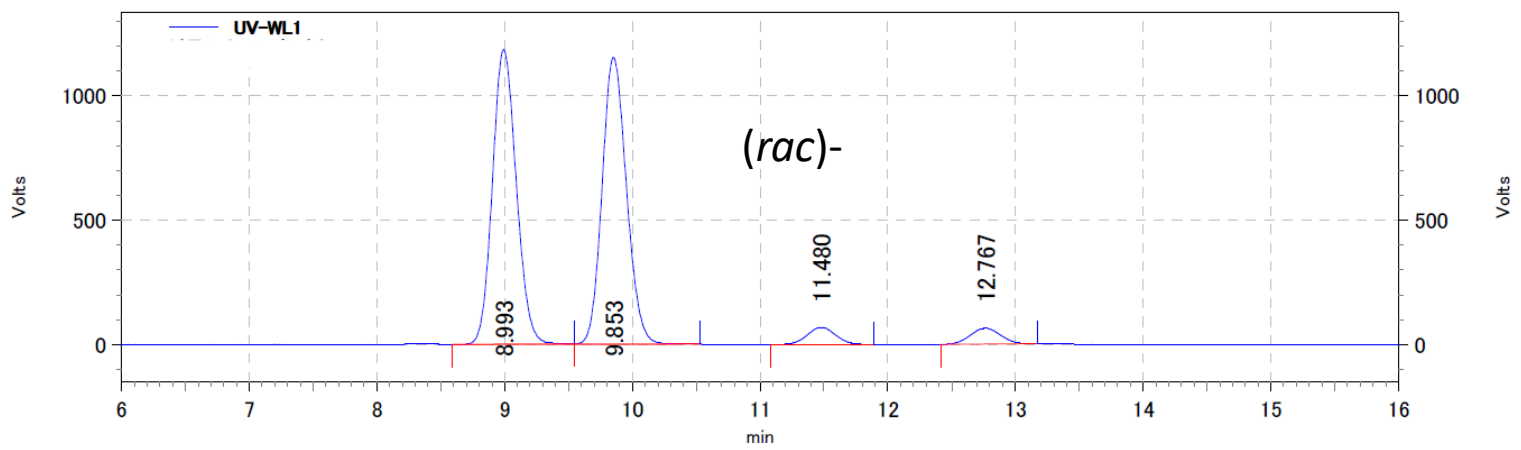

\begin{tabular}{|c|c|c|c|}
\hline Peak No. & $\mathrm{RT}(\min )$ & Area & Area(\%) \\
\hline & 8.993 & 61756314 & 46.899 \\
\hline & 9.853 & 61453398 & 46.669 \\
\hline & 11.480 & 4276521 & 3.248 \\
\hline & 12.767 & 4191807 & 3.183 \\
\hline & & 131678040 & 100.000 \\
\hline
\end{tabular}

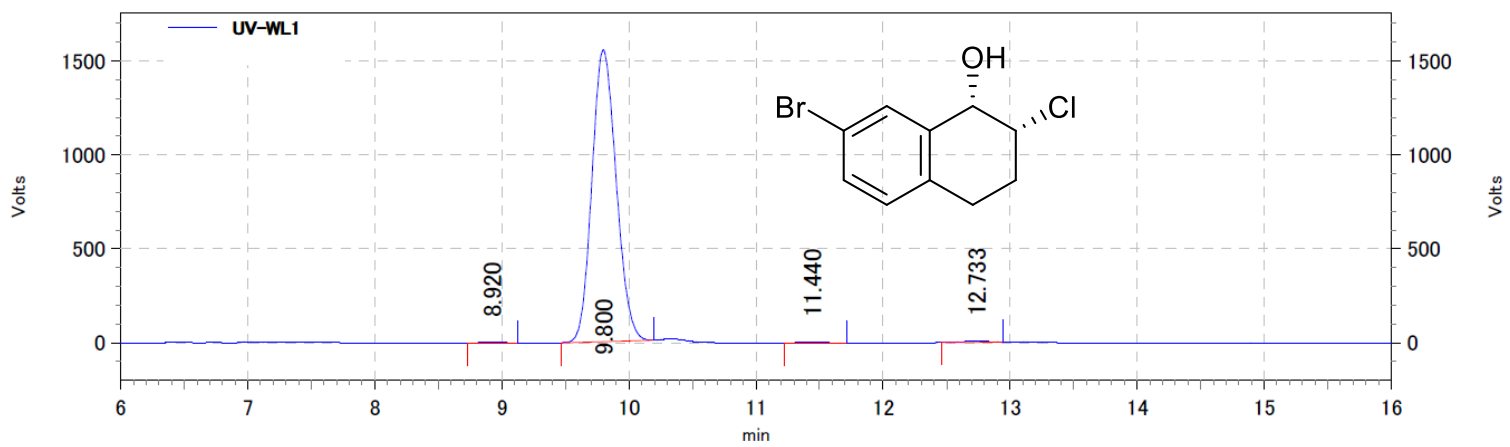

\begin{tabular}{|c|c|c|c|}
\hline Peak No. & $\mathrm{RT}(\min )$ & Area & Area(\%) \\
\hline & 8.920 & 50090 & 0.060 \\
\hline & 9.800 & 82548466 & 99.436 \\
\hline & 11.440 & 68446 & 0.082 \\
\hline & 12.733 & 350022 & 0.422 \\
\hline
\end{tabular}




\section{(1S,2R)-2-Chloro-2,3-dihydro-1H-inden-1-ol (cis-4d)}

Chiralcel OJ-H, $250 \times 4.6 \mathrm{~mm}$ column, hexane/2-propanol 97:3, $1.0 \mathrm{~mL} / \mathrm{min}, 220 \mathrm{~nm}, 30^{\circ} \mathrm{C}$

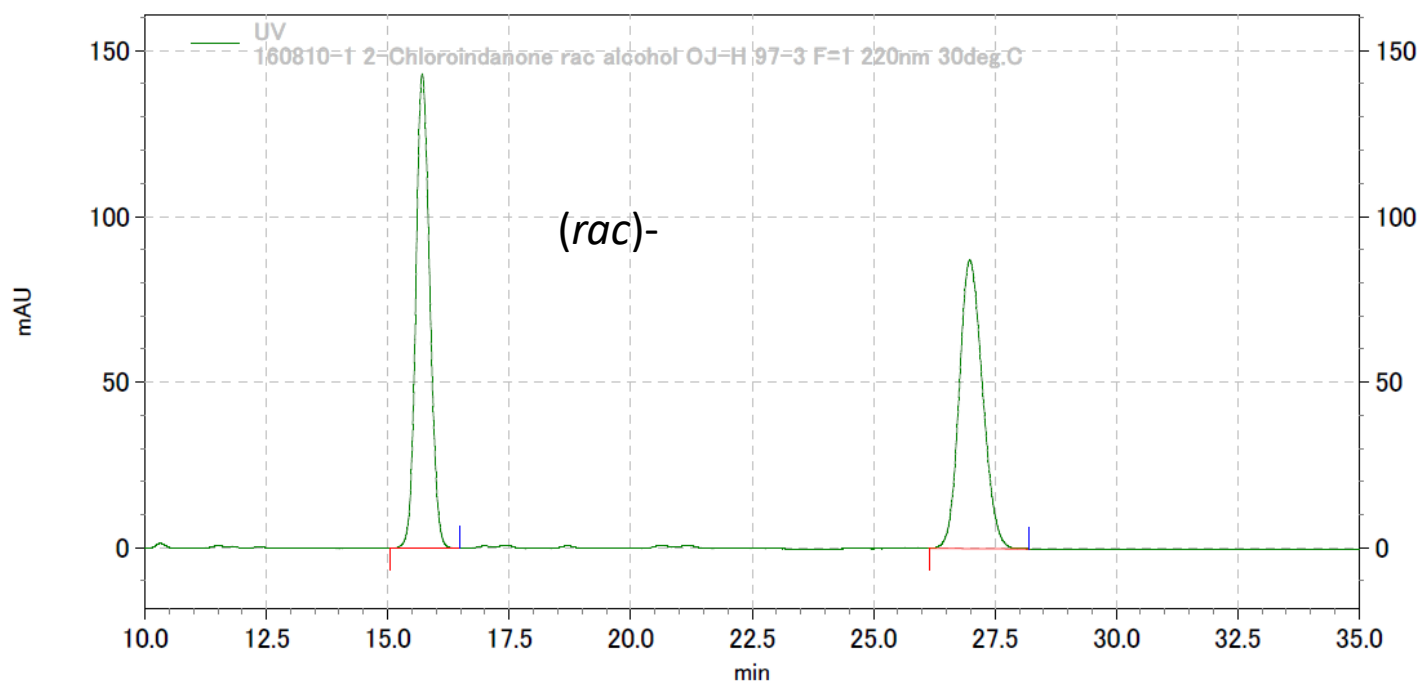

160810-1 2-Chloroindanone rac alcohol 0J-H 97-3 F=1 220nm 30deg. C

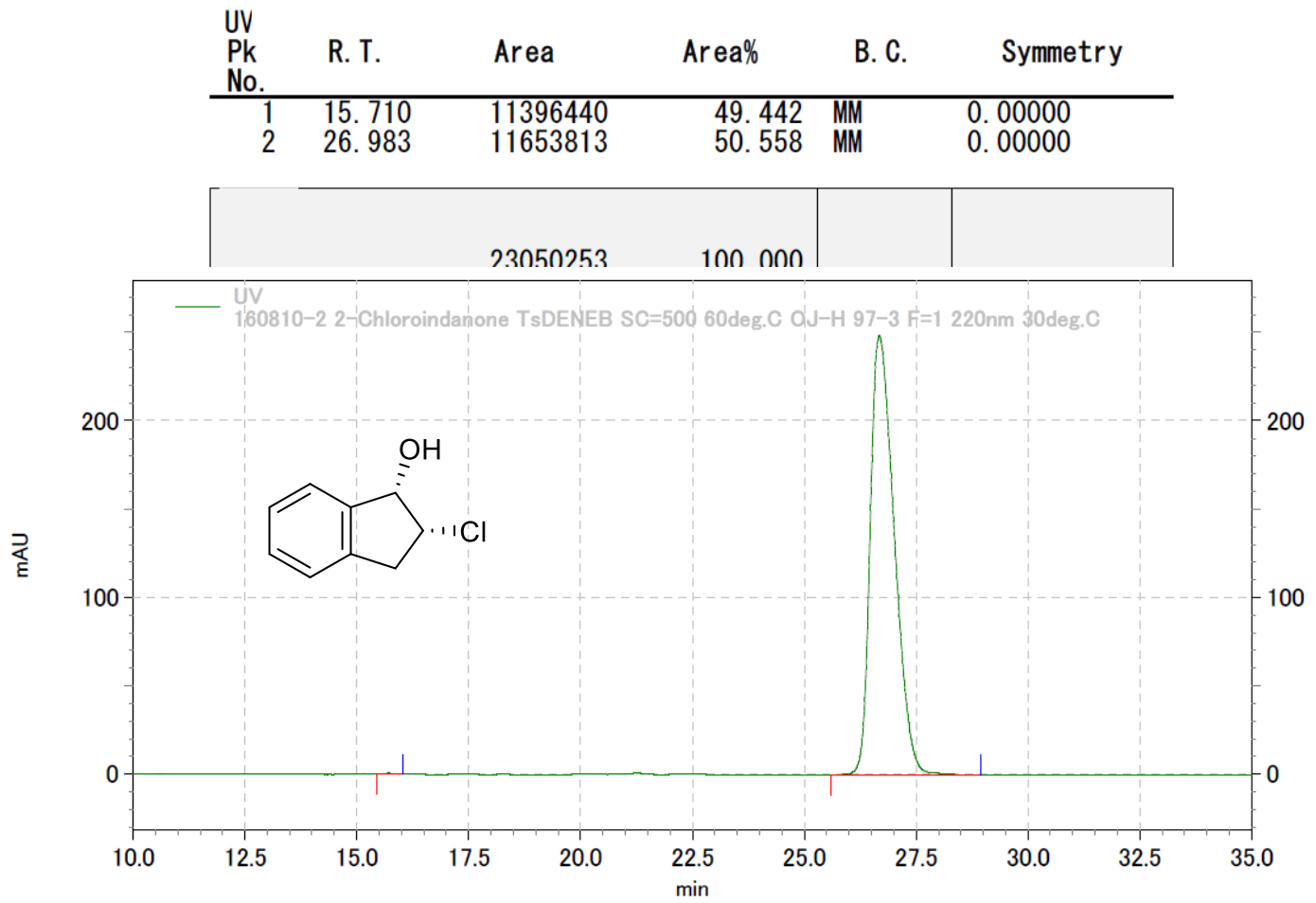

160810-2 2-Chloroindanone TsDENEB SC=500 60deg. C 0J-H 97-3 F=1 220nm 30deg. C

\begin{tabular}{|c|c|c|c|c|c|}
\hline UV & R. T. & Area & Area\% & B. C. & Symmetry \\
\hline $\begin{array}{l}1 \\
2\end{array}$ & $\begin{array}{l}15.710 \\
26.677\end{array}$ & $\begin{array}{r}44268 \\
37058781\end{array}$ & $\begin{array}{r}0.119 \\
99.881\end{array}$ & $\begin{array}{l}\text { MM } \\
\text { MM }\end{array}$ & $\begin{array}{l}0.00000 \\
0.00000\end{array}$ \\
\hline & & 37103049 & 100.000 & & \\
\hline
\end{tabular}




\section{(1S,2R)-2-Chloro-5-methoxy-2,3-dihydro-1H-inden-1-ol (cis-4e)}

Chiralcel OJ-3, $250 \times 4.6 \mathrm{~mm}$ column, hexane/2-propanol 90:10, $1.0 \mathrm{~mL} / \mathrm{min}, 220 \mathrm{~nm}, 35^{\circ} \mathrm{C}$

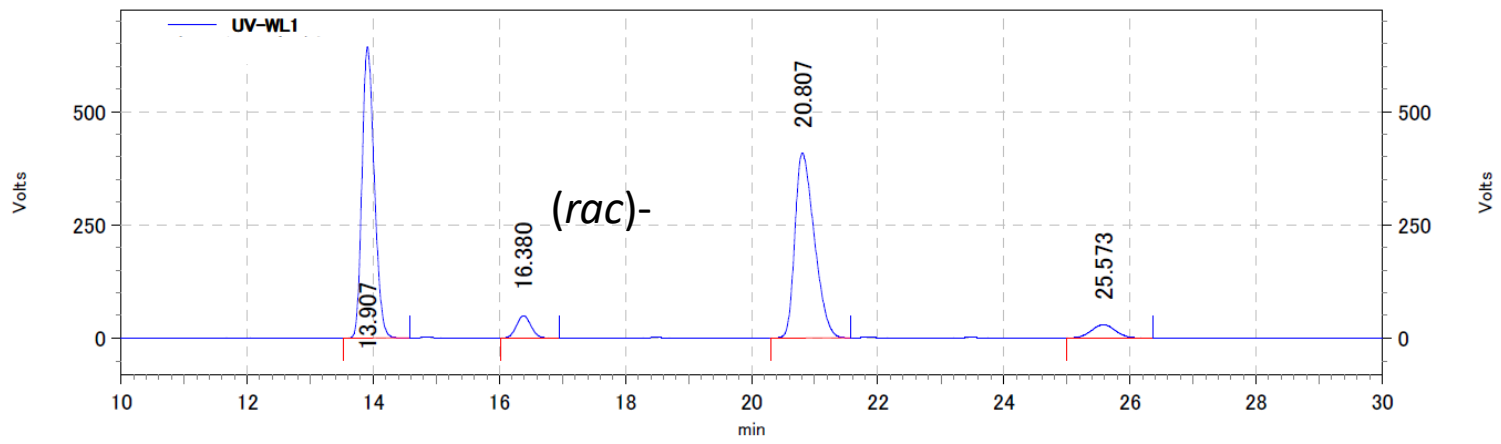

\begin{tabular}{|c|c|c|c|}
\hline Peak No. & $\mathrm{RT}$ (min) & Area & Area(\%) \\
\hline & 13.907 & 34911872 & 45.836 \\
\hline & 16.380 & 3168191 & 4.159 \\
\hline & 20.807 & 34925959 & 45.854 \\
\hline & 25.573 & 3161647 & 4.151 \\
\hline & & 76167669 & 100.000 \\
\hline
\end{tabular}

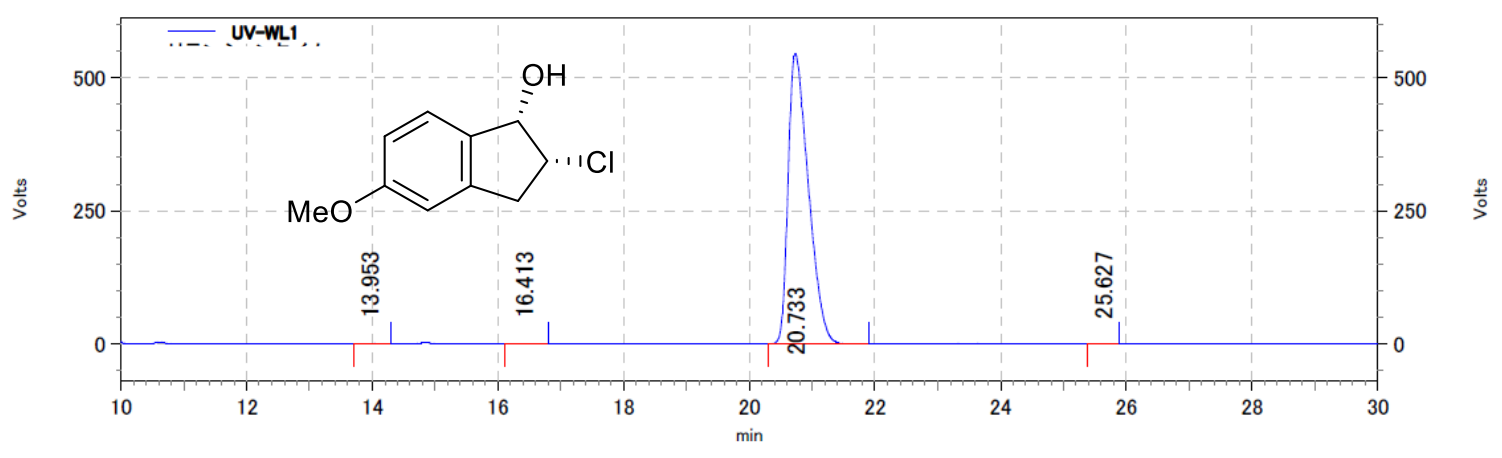

UV-WL1

Peak No.

$\mathrm{RT}(\min )$

13.953

16.413

20.733

25.627

Area

Area(\%)

0.151

0.099

488468

99.733

8460

0.017

\begin{tabular}{|r|r|rr|}
\hline & & 48949439 & 100.000 \\
\hline
\end{tabular}




\section{(1S,2R)-2,5-Dichloro-2,3-dihydro-1H-inden-1-ol (cis-4f)}

Chiralcel OZ-3, $250 \times 4.6 \mathrm{~mm}$ column, hexane/2-propanol 97:3, $1.0 \mathrm{~mL} / \mathrm{min}, 220 \mathrm{~nm}, 35^{\circ} \mathrm{C}$

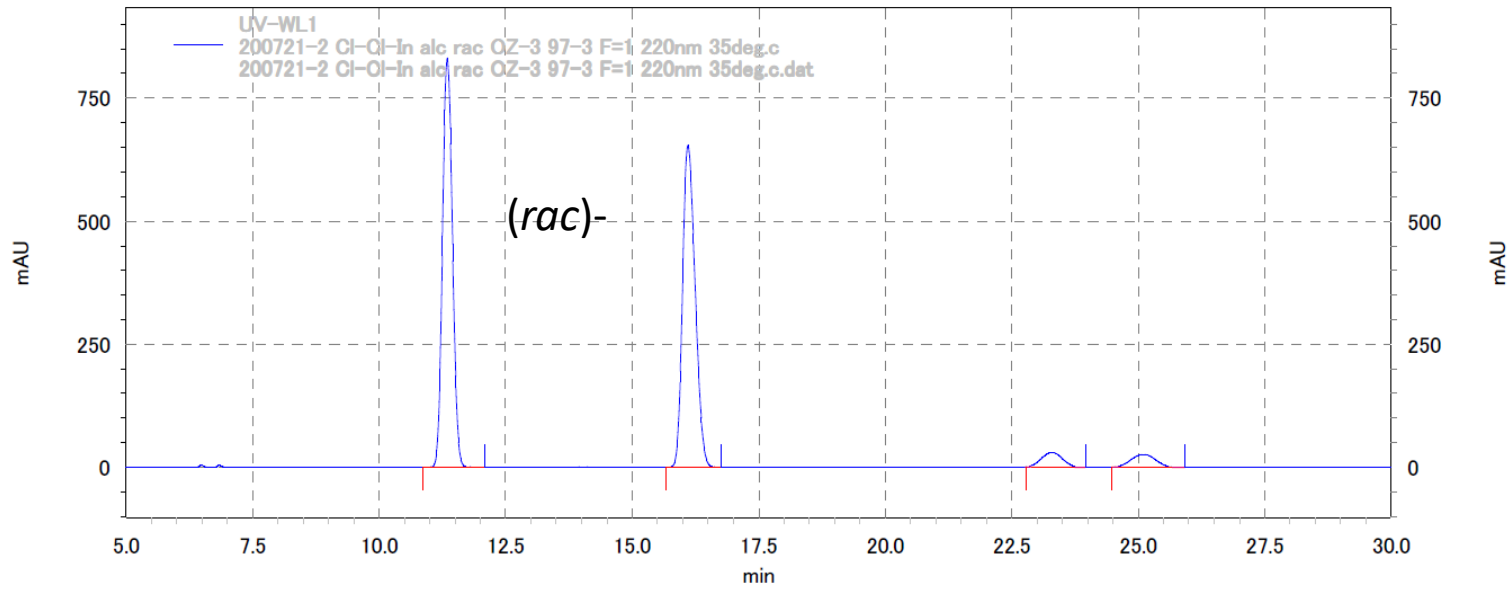

UV-WL1

Peak No.

$\mathrm{RT}(\min )$

11.347

16.107

23.293

25.107

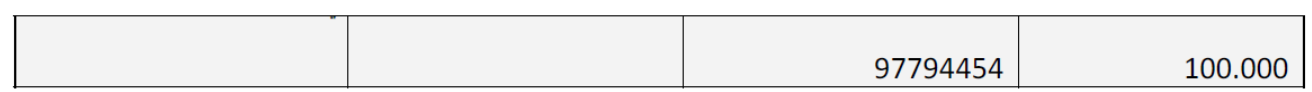

Area

45419668

45423171

3441136

3510479

UV-WL1

Peak No.

$\mathrm{RT}$ (min)

11.260

15.873

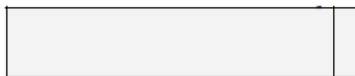

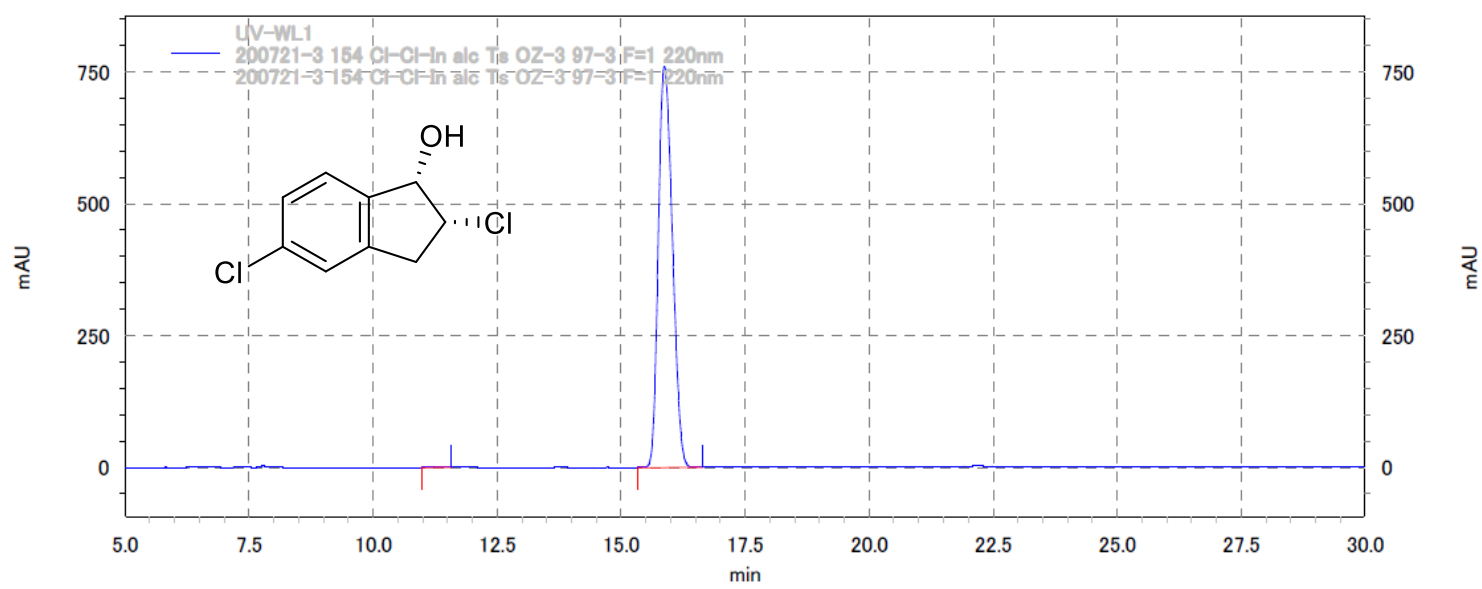

\begin{tabular}{|c|c|c|c|}
\hline Peak No. & $\mathrm{RT}(\mathrm{min})$ & Area & Area(\%) \\
\hline & 11.260 & 135993 & 0.226 \\
\hline & 15.873 & 60159464 & 99.774 \\
\hline & & 60295457 & 100.000 \\
\hline
\end{tabular}




\section{(1S,2R)-2-Chloro-2,3-dihydro-1H-indene-1,7-diol (cis-4g)}

Chiralcel OJ-3, $250 \times 4.6 \mathrm{~mm}$ column, hexane/2-propanol 90:10, $1.0 \mathrm{~mL} / \mathrm{min}, 220 \mathrm{~nm}, 35^{\circ} \mathrm{C}$

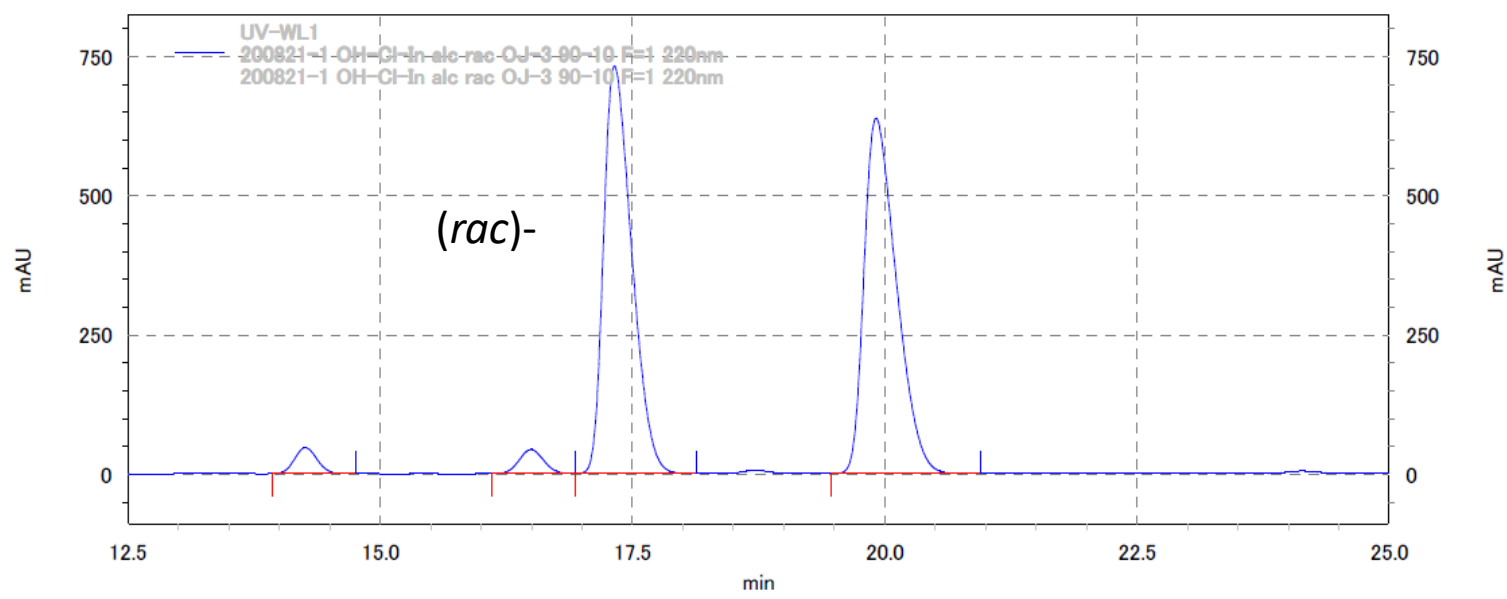

\begin{tabular}{rrrr} 
UV-WL1 & & & \\
& Peak No. & Area & Area(\%) \\
\hline & 14.260 & 2946350 & 2.463 \\
& 16.493 & 2914958 & 2.437 \\
& 17.320 & 56877116 & 47.553 \\
& 19.913 & 56868811 & 47.546 \\
\hline & & 119607235 & 100.000 \\
\hline
\end{tabular}

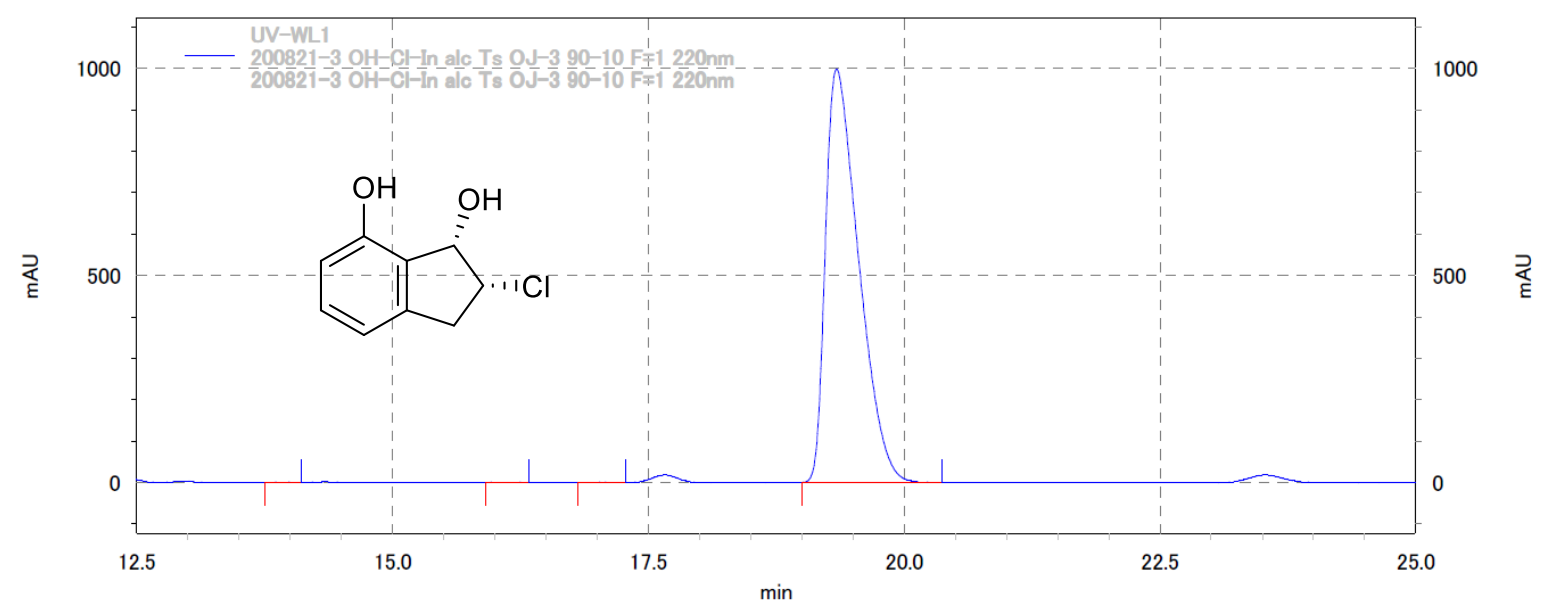

UV-WL1

\begin{tabular}{rrrr|} 
Peak No. & RT $(\min )$ & Area & Area(\%) \\
\hline & 13.927 & 28714 & 0.032 \\
& 16.100 & 75888 & 0.084 \\
& 17.053 & 38705 & 0.043 \\
& 19.340 & 89962946 & 99.841 \\
\hline & & 90106253 & 100.000 \\
\hline
\end{tabular}




\section{(1S,2R)-2-Chloro-3,3-dimethyl-2,3-dihydro-1H-inden-1-ol (cis-4h)}

Chiralcel OZ-3, $250 \times 4.6 \mathrm{~mm}$ column, hexane/2-propanol 97:3, $1.0 \mathrm{~mL} / \mathrm{min}, 220 \mathrm{~nm}, 35^{\circ} \mathrm{C}$

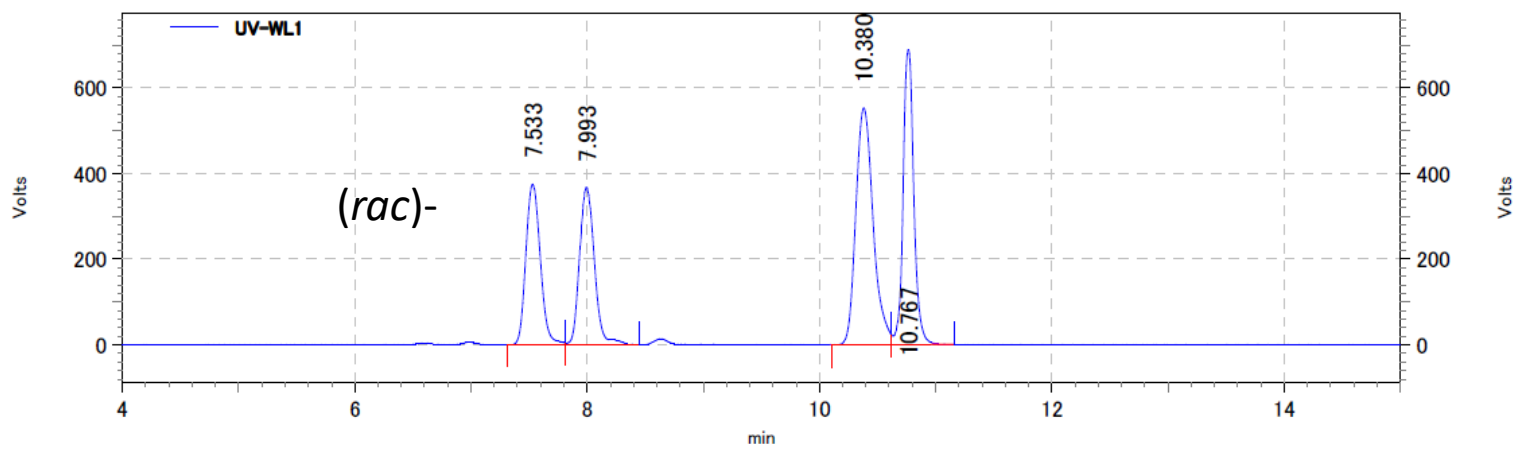

\begin{tabular}{|c|c|c|c|}
\hline Peak No. & $\mathrm{RT}(\mathrm{min})$ & Area & Area(\%) \\
\hline & 7.533 & 12712461 & 19.117 \\
\hline & 7.993 & 12936775 & 19.454 \\
\hline & 10.380 & 23074268 & 34.699 \\
\hline & 10.767 & 17774831 & 26.730 \\
\hline & & 66498335 & 100.000 \\
\hline
\end{tabular}

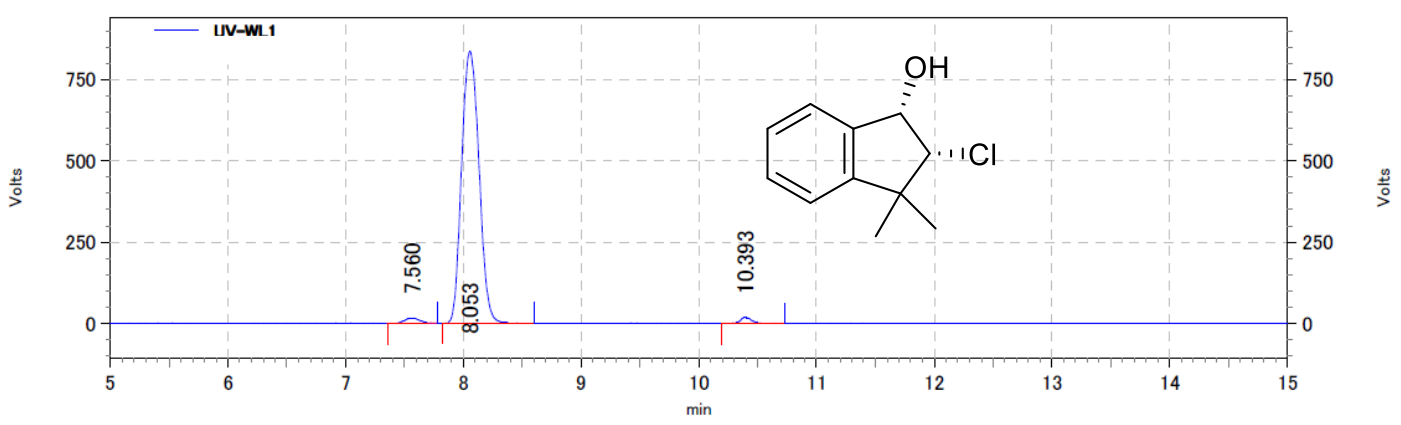

\begin{tabular}{|c|c|c|c|}
\hline Peak No. & RT (min) & Area & Area(\%) \\
\hline & 7.560 & 589243 & 1.658 \\
\hline & 8.053 & 34450576 & 96.914 \\
\hline & 10.393 & 507927 & 1.429 \\
\hline & & 35547746 & 100.000 \\
\hline
\end{tabular}




\section{(5S,6R)-6-Chloro-6,7,8,9-tetrahydro-5H-benzo[7]annulen-5-ol (cis-4i)}

Chiralcel OJ-H, $250 \times 4.6 \mathrm{~mm}$ column, hexane/2-propanol 97:3, $1.0 \mathrm{~mL} / \mathrm{min}, 220 \mathrm{~nm}, 30^{\circ} \mathrm{C}$

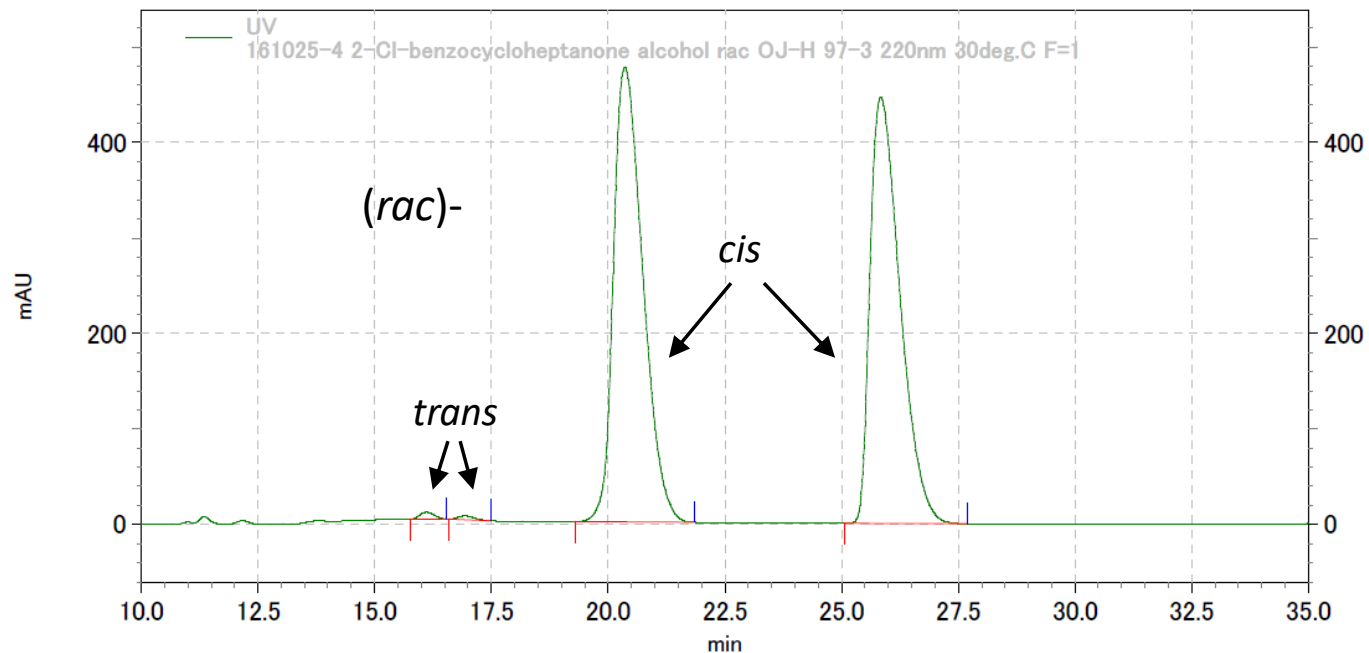

161025-4 2-Cl-benzocycloheptanone alcohol rac 0J-H 97-3 220nm 30deg. C F=1

\begin{tabular}{|c|c|c|c|c|c|}
\hline $\begin{array}{l}\text { UV } \\
\mathrm{Pk} \\
\mathrm{No}\end{array}$ & R. T. & Area & Area\% & B. C. & Symmetry \\
\hline $\begin{array}{l}1 \\
2 \\
3 \\
4\end{array}$ & $\begin{array}{l}16.107 \\
16.940 \\
20.360 \\
25.840\end{array}$ & $\begin{array}{r}642538 \\
395895 \\
83376870 \\
79687413\end{array}$ & $\begin{array}{r}0.392 \\
0.241 \\
50.808 \\
48.559\end{array}$ & $\begin{array}{l}\text { MM } \\
\text { MM } \\
\text { MM } \\
\text { MM }\end{array}$ & $\begin{array}{l}0.00000 \\
0.00000 \\
0.00000 \\
0.00000\end{array}$ \\
\hline & & 164102716 & 100.000 & & \\
\hline
\end{tabular}

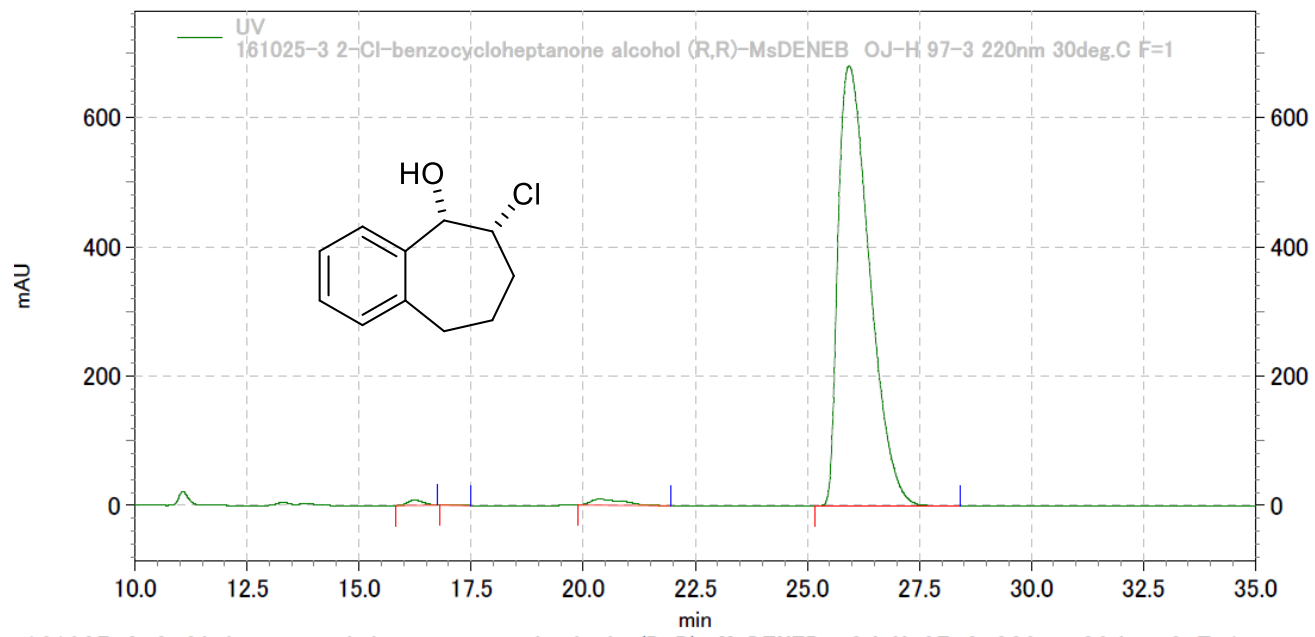

161025-3 2-Cl-benzocycloheptanone alcohol (R, R)-MsDENEB OJ-H 97-3 220nm 30deg. C F=1

\begin{tabular}{|c|c|c|c|c|c|}
\hline $\begin{array}{l}\text { UV } \\
\mathrm{Pk} \\
\text { No. }\end{array}$ & R. T. & Area & Area\% & B. C. & Symmetry \\
\hline $\begin{array}{l}1 \\
2 \\
3 \\
4\end{array}$ & $\begin{array}{l}16.243 \\
17.073 \\
20.367 \\
25.933\end{array}$ & $\begin{array}{r}778674 \\
62859 \\
1954138 \\
132640493\end{array}$ & $\begin{array}{r}0.575 \\
0.046 \\
1.443 \\
97.936\end{array}$ & $\begin{array}{l}\mathrm{MM} \\
\mathrm{MM} \\
\mathrm{MM} \\
\mathrm{MM}\end{array}$ & $\begin{array}{l}0.00000 \\
0.00000 \\
0.00000 \\
0.00000\end{array}$ \\
\hline & & 135436164 & 100.000 & & \\
\hline
\end{tabular}




\section{(1S,2R)-2-Chloro-1,2-dihydroacenaphthylen-1-ol (cis-4j)}

Chiralpak AS-3, $250 \times 4.6 \mathrm{~mm}$ column, hexane/2-propanol 95:5, $1.0 \mathrm{~mL} / \mathrm{min}, 220 \mathrm{~nm}, 35^{\circ} \mathrm{C}$

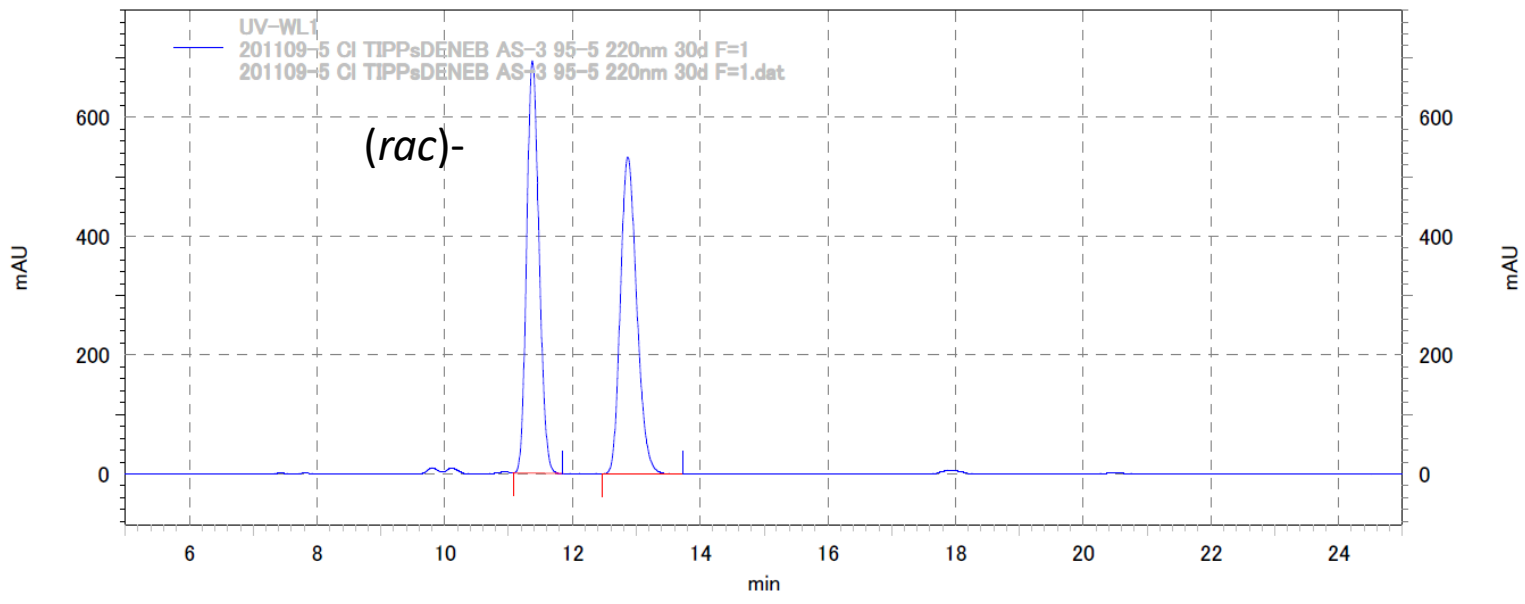

UV-WL1

\begin{tabular}{l|rrrr|}
\multicolumn{1}{c}{ Peak No. } & Area & Area(\%) \\
\hline \multicolumn{1}{|c}{11.373} & 37349001 & 49.605 \\
& 12.867 & 37943433 & 50.395 \\
\hline & & 75292434 & 100.000 \\
\hline
\end{tabular}

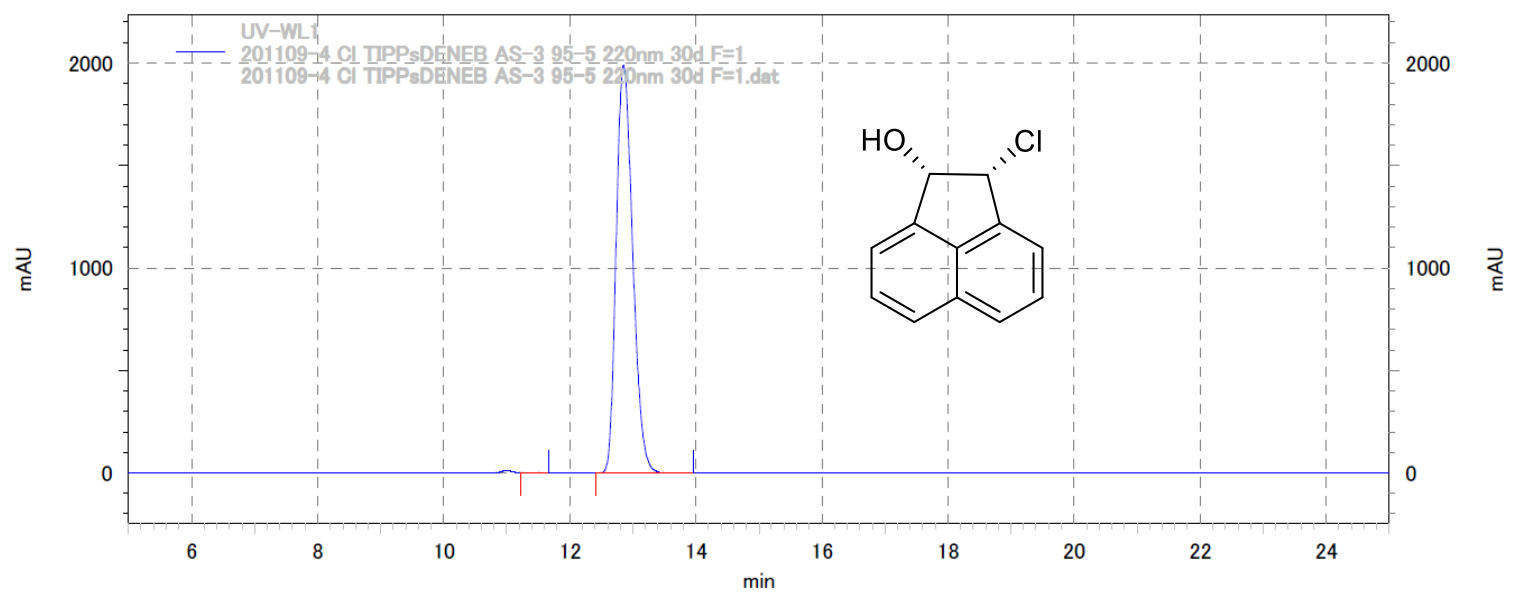

UV-WL1

\begin{tabular}{lrrrr|}
\multicolumn{1}{c}{ Peak No. } & Area & Area $(\%)$ \\
\hline \multicolumn{1}{l}{} & 11.380 & 129452 & 0.089 \\
12.853 & 144713733 & 99.911 \\
\hline & & 144843185 & 100.000 \\
\hline
\end{tabular}




\section{(1S,2R)-2-Bromo-2,3-dihydro-1H-inden-1-ol (cis-4k)}

Chiralpak AS-3, $250 \times 4.6 \mathrm{~mm}$ column, hexane/2-propanol 97:3, $1.0 \mathrm{~mL} / \mathrm{min}, 220 \mathrm{~nm}, 35^{\circ} \mathrm{C}$

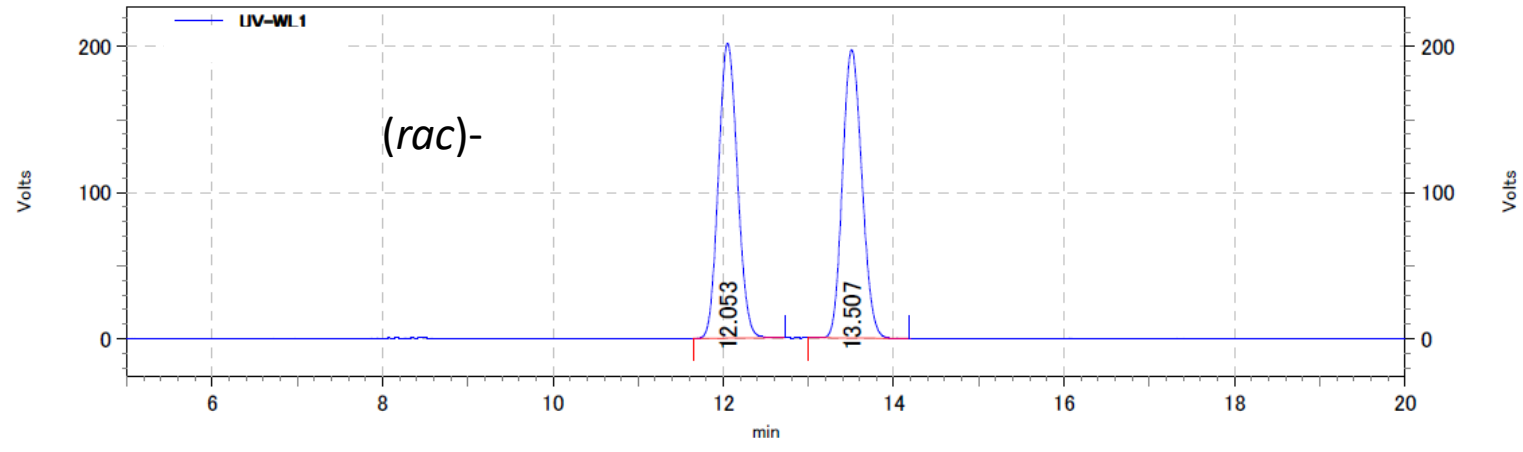

\begin{tabular}{rrrr} 
UV-WL1 & \multicolumn{1}{r}{ Areak $(\min )$} & Area & Area(\%) \\
\hline & 12.053 & 12523188 & 50.011 \\
& 13.507 & 12517532 & 49.989 \\
\hline & & & \\
\hline & & 25040720 & 100.000 \\
\hline
\end{tabular}

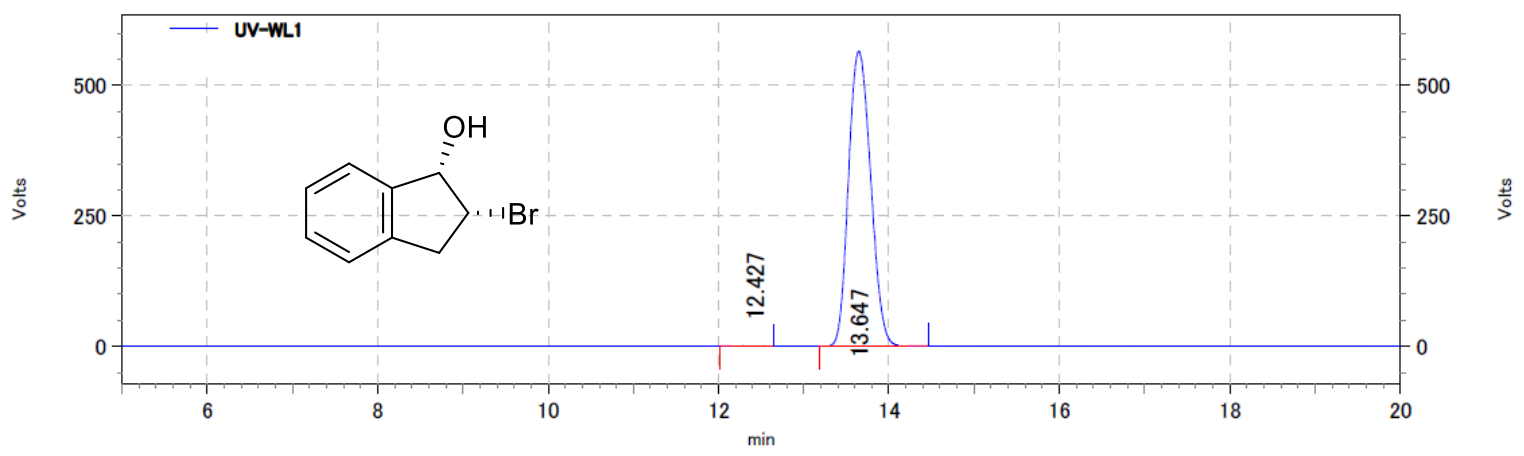

\begin{tabular}{rrrrr} 
UV-WL1 & \multicolumn{1}{c}{ Area } & Area(\%) \\
\hline & 12.427 & 98000 & 0.239 \\
& 13.647 & 40863490 & 99.761 \\
\hline & & & \\
\hline & & 40961490 & 100.000 \\
\hline
\end{tabular}




\section{(1S,2R)-2-Bromo-1,2,3,4-tetrahydronaphthalen-1-ol (cis-4l)}

Chiralpak AS-3, $250 \times 4.6 \mathrm{~mm}$ column, hexane/2-propanol 97:3, $1.0 \mathrm{~mL} / \mathrm{min}, 220 \mathrm{~nm}, 35^{\circ} \mathrm{C}$

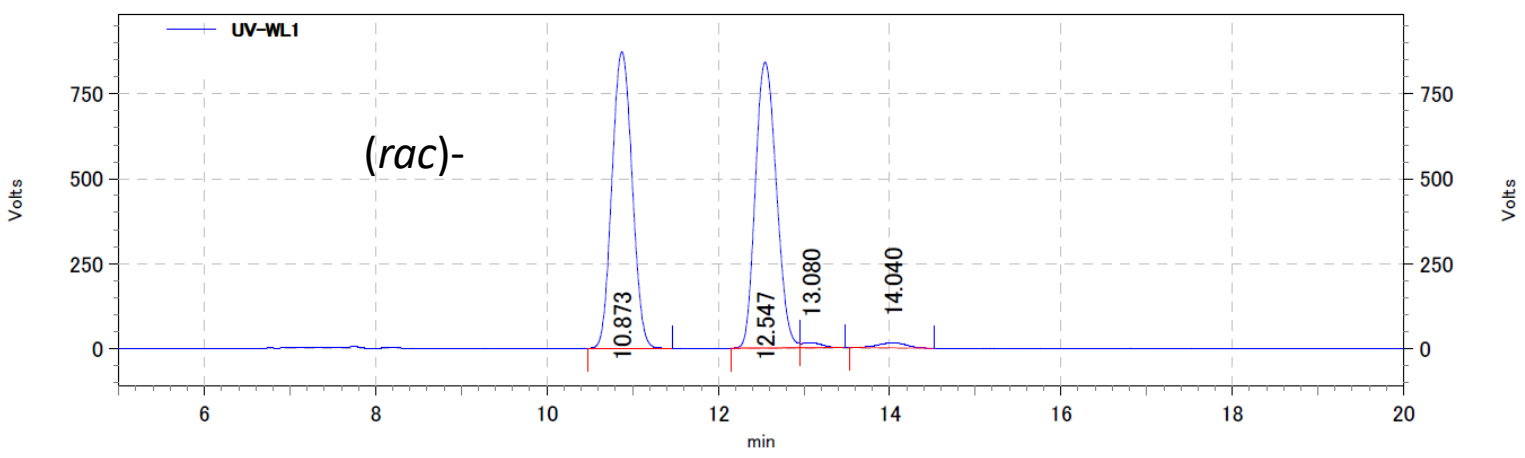

\begin{tabular}{|c|c|c|c|}
\hline Peak No. & $\mathrm{RT}(\mathrm{min})$ & Area & Area(\%) \\
\hline & 10.873 & 57284448 & 48.827 \\
\hline & 12.547 & 57651692 & 49.140 \\
\hline & 13.080 & 983889 & 0.839 \\
\hline & 14.040 & 1400110 & 1.193 \\
\hline & & 117320139 & 100.000 \\
\hline
\end{tabular}

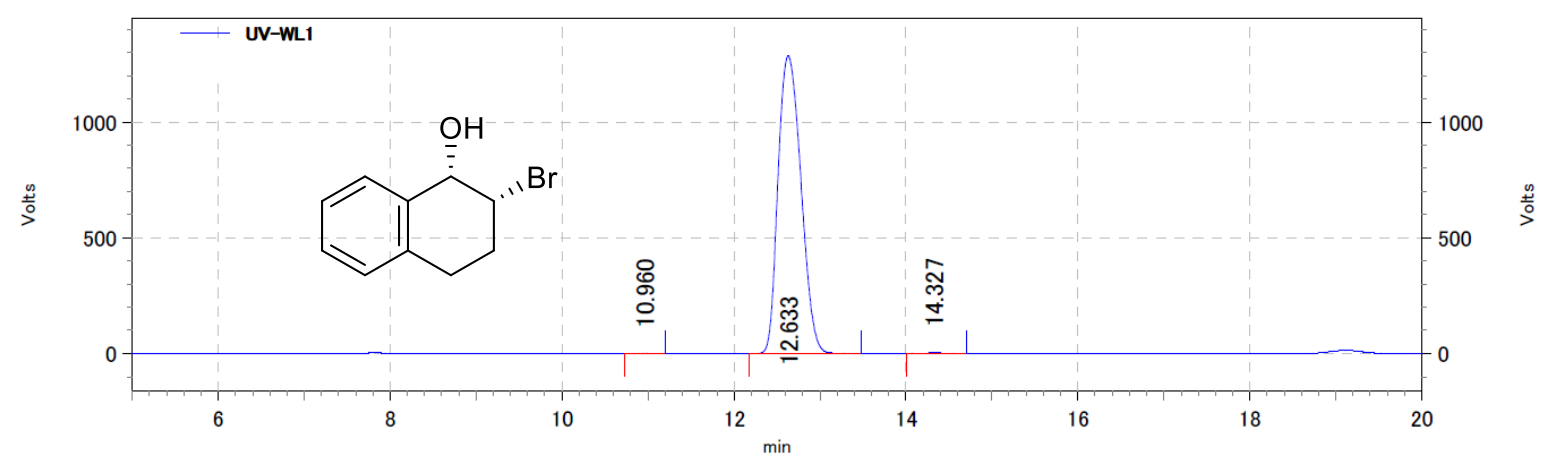

\begin{tabular}{rrrrr|} 
UV-WL1 & \multicolumn{3}{c}{ Area } & Area(\%) \\
\hline \multicolumn{1}{r}{ Peak No. } & 10.960 & 44289 & 0.046 \\
& 12.633 & 95254540 & 99.767 \\
& 14.327 & 178205 & 0.187 \\
\hline & & 95477034 & 100.000 \\
\hline
\end{tabular}


Methyl (1R,2R)-1-hydroxy-2,3-dihydro-1H-indene-2-carboxylate (cis-6a)

Chiralcel OJ-H, $250 \times 4.6 \mathrm{~mm}$ column, hexane/2-propanol 97:3, $1.0 \mathrm{~mL} / \mathrm{min}, 220 \mathrm{~nm}, 35^{\circ} \mathrm{C}$

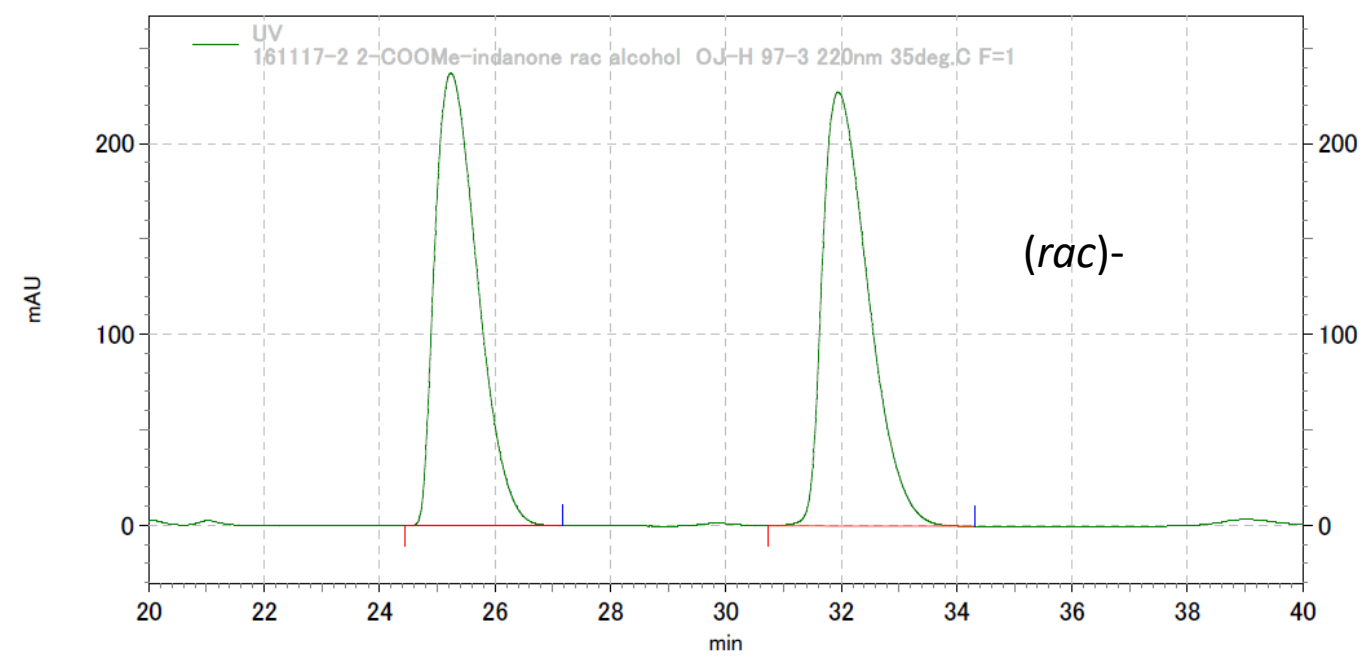

161117-2 2-CO0Me-indanone rac alcohol 0J-H 97-3 220nm 35deg. C F=1

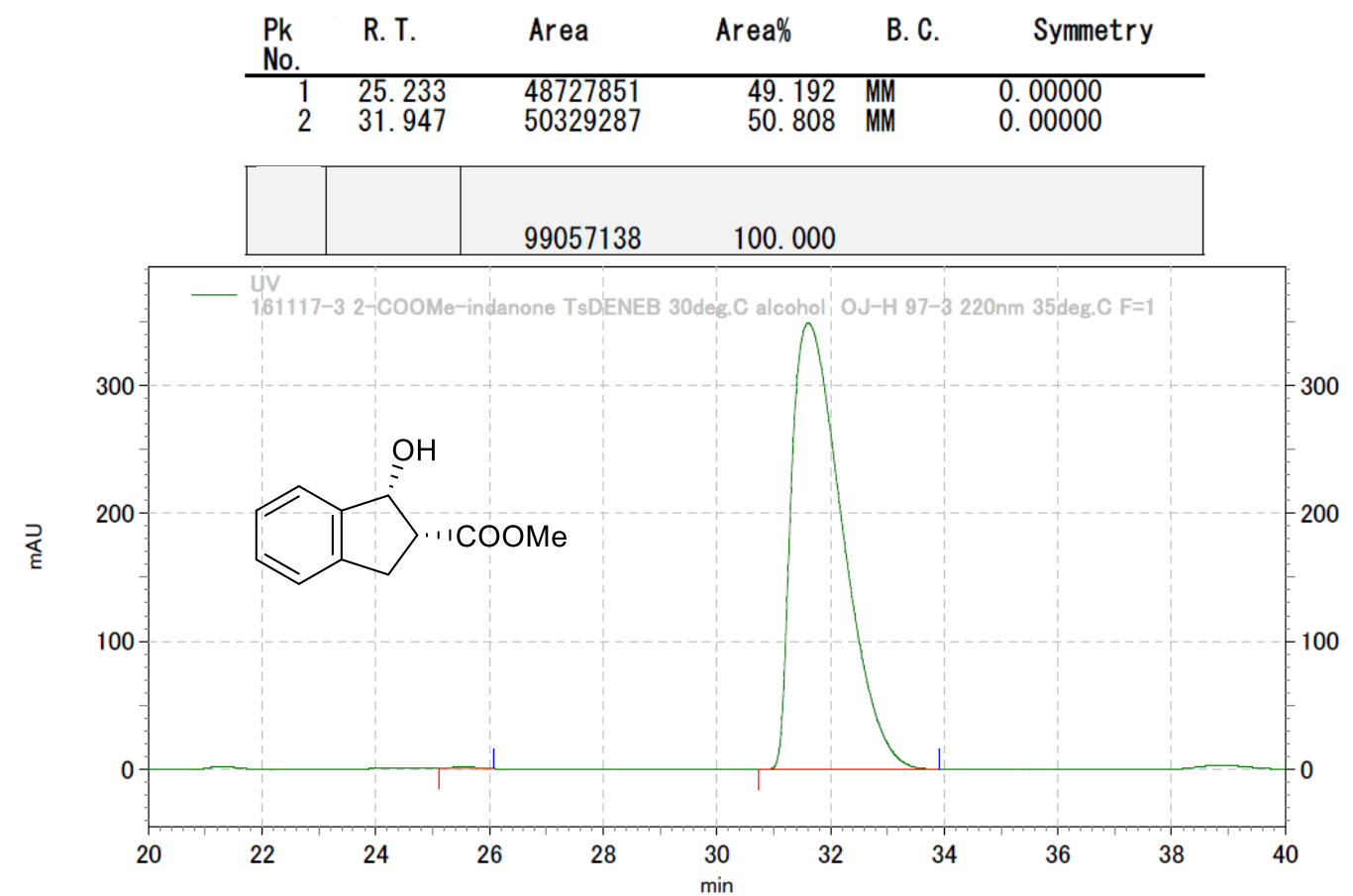

161117-3 2-CO0Me-indanone TsDENEB 30deg. C alcohol 0J-H 97-3 220nm 35deg. C F=1

\begin{tabular}{rlrrrr}
$\begin{array}{r}\text { Pk } \\
\text { No. }\end{array}$ & R. T. & \multicolumn{1}{c}{ Area } & Area\% & B. C. & Symmetry \\
\hline 1 & 25.537 & 181911 & 0.210 & MM & 0.00000 \\
2 & 31.607 & 86374325 & 99.790 & MM & 0.00000 \\
\hline & & & & & \\
\hline & & 86556236 & 100.000 & \\
\hline
\end{tabular}


Methyl (5R,6R)-5-hydroxy-6,7,8,9-tetrahydro-5H-benzo[7]annulene-6-carboxylate (cis-6b)

Chiralcel OD-H, $250 \times 4.6 \mathrm{~mm}$ column, hexane/2-propanol 98:2, $1.0 \mathrm{~mL} / \mathrm{min}, 220 \mathrm{~nm}, 40^{\circ} \mathrm{C}$

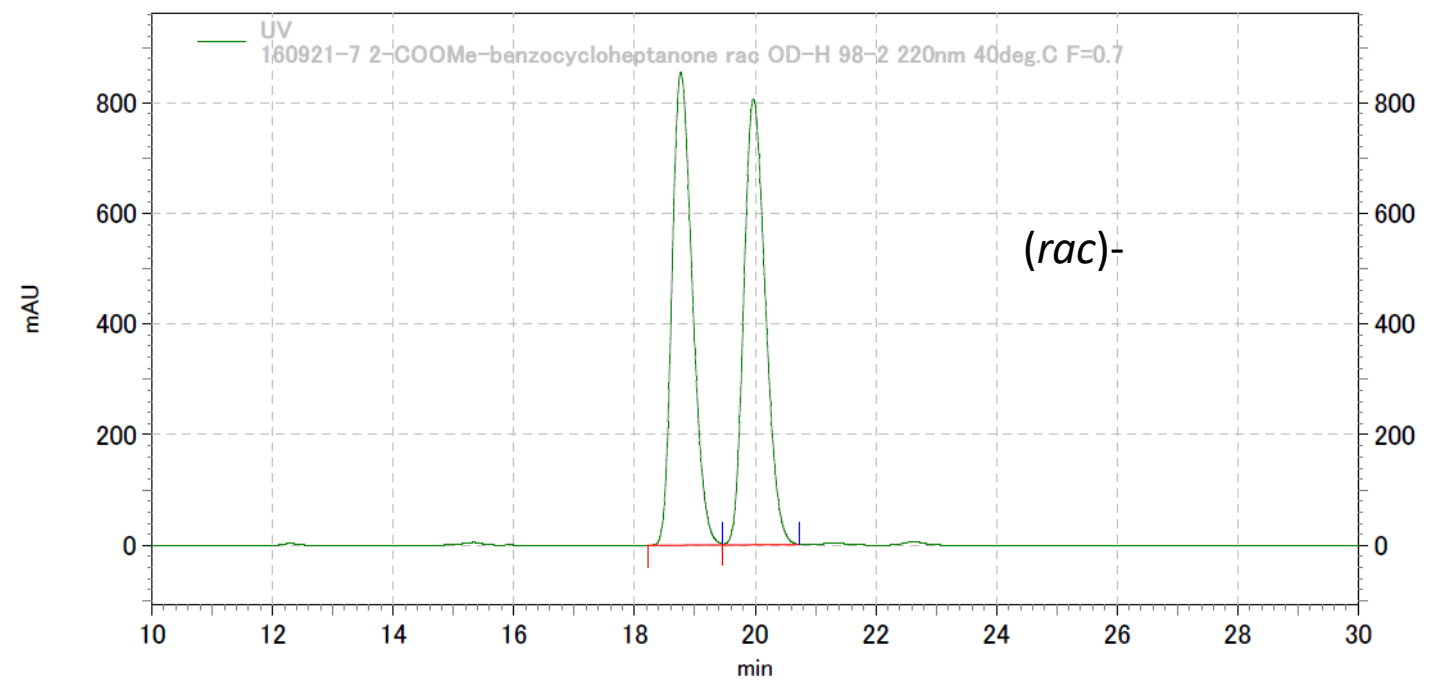

160921-7 2-C00Me-benzocycloheptanone rac OD-H 98-2 220nm 40deg. C F=0. 7

\begin{tabular}{rlllll}
$\begin{array}{r}\text { Pk } \\
\text { No. }\end{array}$ & R. T. & Area & Area\% & B. C. & Symmetry \\
\hline 1 & 18.770 & 79700620 & 49.868 & $\mathrm{Mx}$ & 0.00000 \\
2 & 19.973 & 80123545 & 50.132 & $\mathrm{xM}$ & 0.00000
\end{tabular}

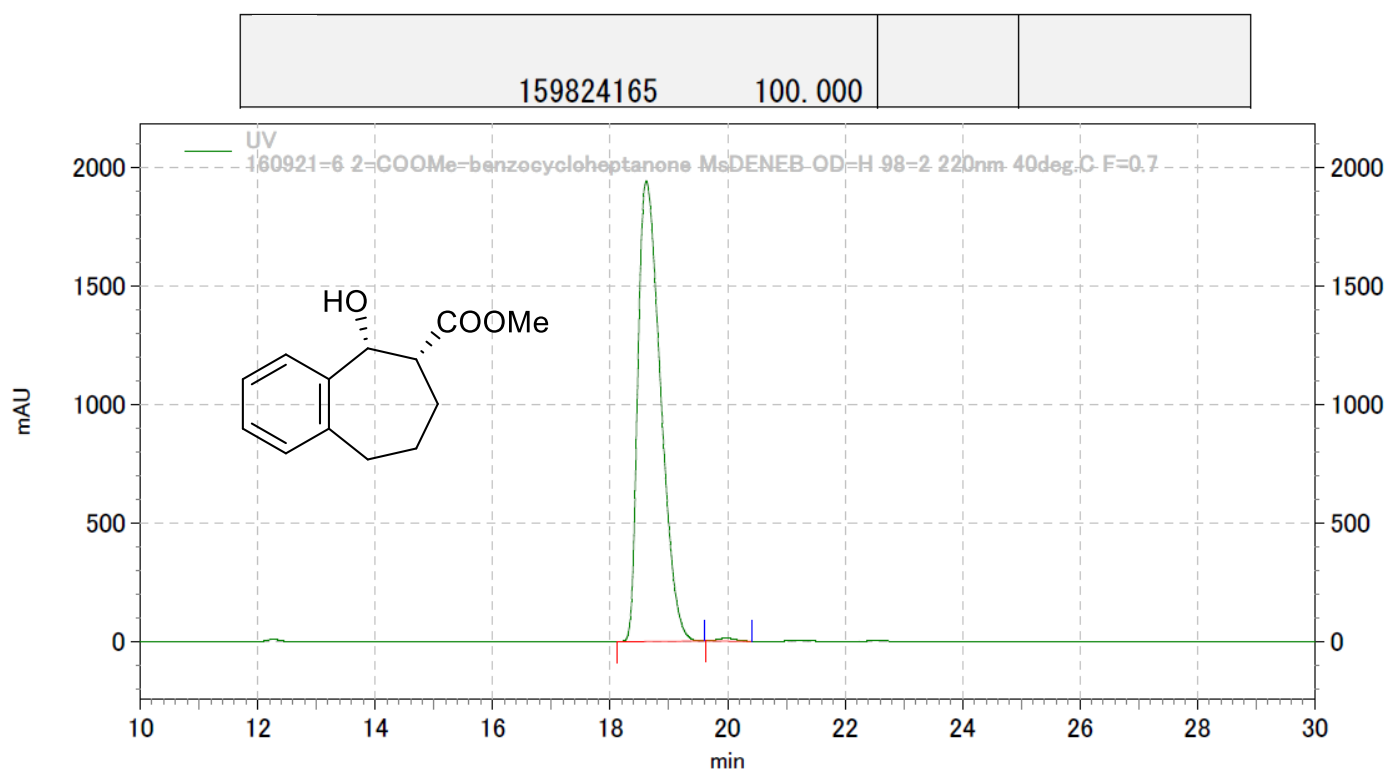

160921-6 2-CO0Me-benzocycloheptanone MsDENEB OD-H 98-2 220nm 40deg. C F=0.7

\begin{tabular}{rlrrrr}
$\begin{array}{r}\text { Pk } \\
\text { No. }\end{array}$ & R. T. & \multicolumn{1}{c}{ Area } & \multicolumn{1}{l}{ Area\% } & B. C. & Symmetry \\
\hline 1 & 18.617 & 208040895 & 99.466 & MM & 0.00000 \\
2 & 19.980 & 1117509 & 0.534 & MM & 0.00000 \\
\hline & & & & \\
\hline & & & & & \\
\hline
\end{tabular}


Chiralcel OJ-H, $250 \times 4.6 \mathrm{~mm}$ column, hexane/2-propanol 95:5, $1.0 \mathrm{~mL} / \mathrm{min}, 220 \mathrm{~nm}, 30^{\circ} \mathrm{C}$

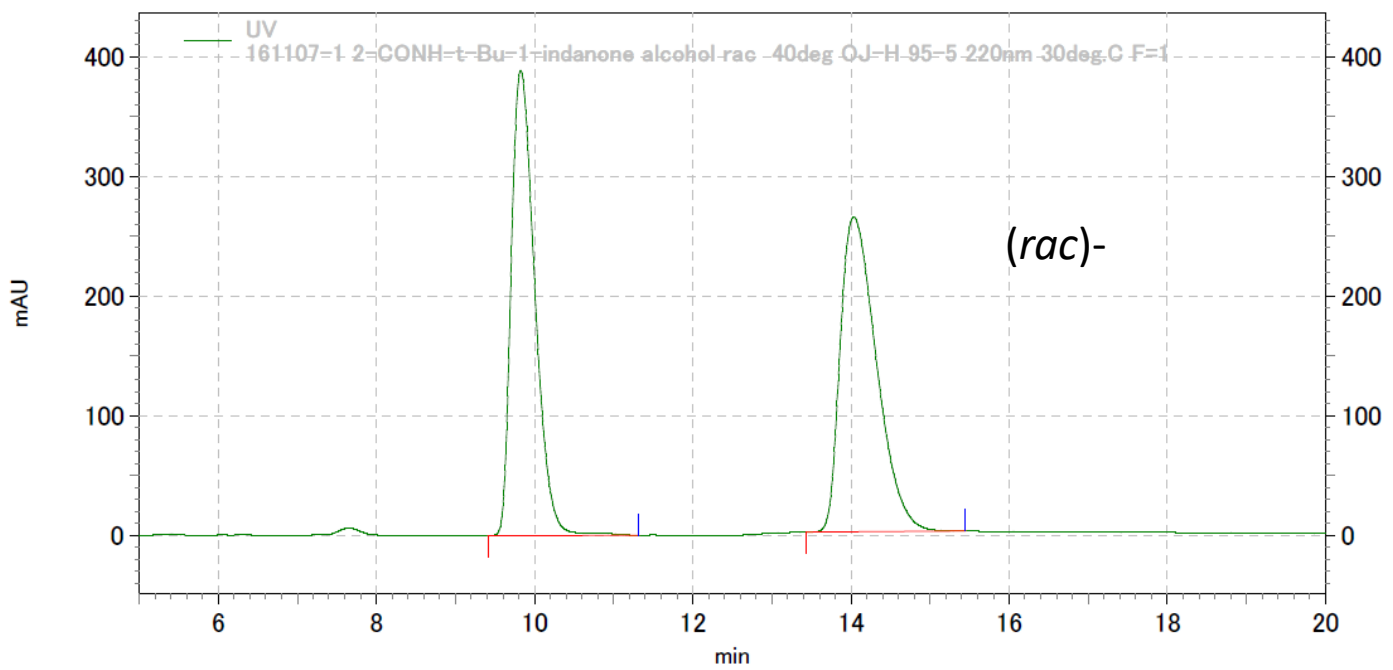

161107-1 2-CONH-t-Bu-1-indanone alcohol rac 40deg 0J-H 95-5 220nm 30deg. C F=1

\begin{tabular}{rrlrrl}
\multicolumn{1}{c}{$\begin{array}{l}\text { Pk } \\
\text { No. }\end{array}$} & \multicolumn{1}{c}{ R. T. } & Area & Area\% & B. C. & Symmetry \\
\hline 1 & 9.823 & 32142166 & 48.960 & MM & 0.00000 \\
2 & 14.037 & 33508072 & 51.040 & MM & 0.00000
\end{tabular}

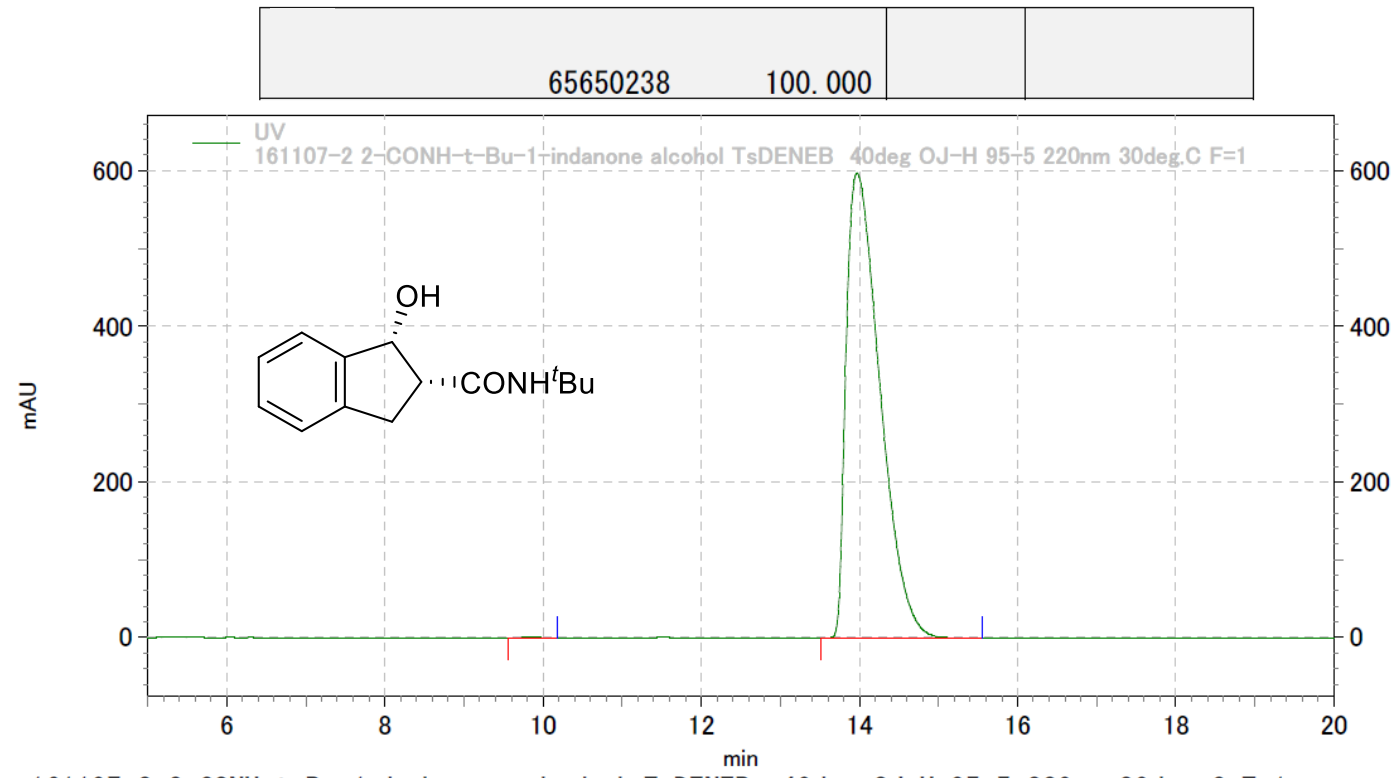

161107-2 2-CONH-t-Bu-1-indanone alcohol TsDENEB 40deg 0J-H 95-5 220nm 30deg. C F=1

\begin{tabular}{rrrrrr}
$\begin{array}{rlr}\text { Pk } \\
\text { No. }\end{array}$ & R. T. & \multicolumn{1}{l}{ Area } & \multicolumn{1}{l}{ Area\% } & \multicolumn{1}{l}{ B. C. } & \multicolumn{1}{c}{ Symmetry } \\
\hline 1 & 9.853 & 52883 & 0.075 & MM & 0.00000 \\
2 & 13.970 & 70913719 & 99.925 & MM & 0.00000 \\
\hline & & & & & \\
\hline & 70966602 & 100.000 & & \\
\hline
\end{tabular}


$(1 R, 2 R)-N$-(tert-Butyl)-1-hydroxy-1,2,3,4-tetrahydronaphthalene-2-carboxamide (cis-6d)

Chiralcel OJ-H, $250 \times 4.6 \mathrm{~mm}$ column, hexane/2-propanol 95:5, $1.0 \mathrm{~mL} / \mathrm{min}, 220 \mathrm{~nm}, 30^{\circ} \mathrm{C}$

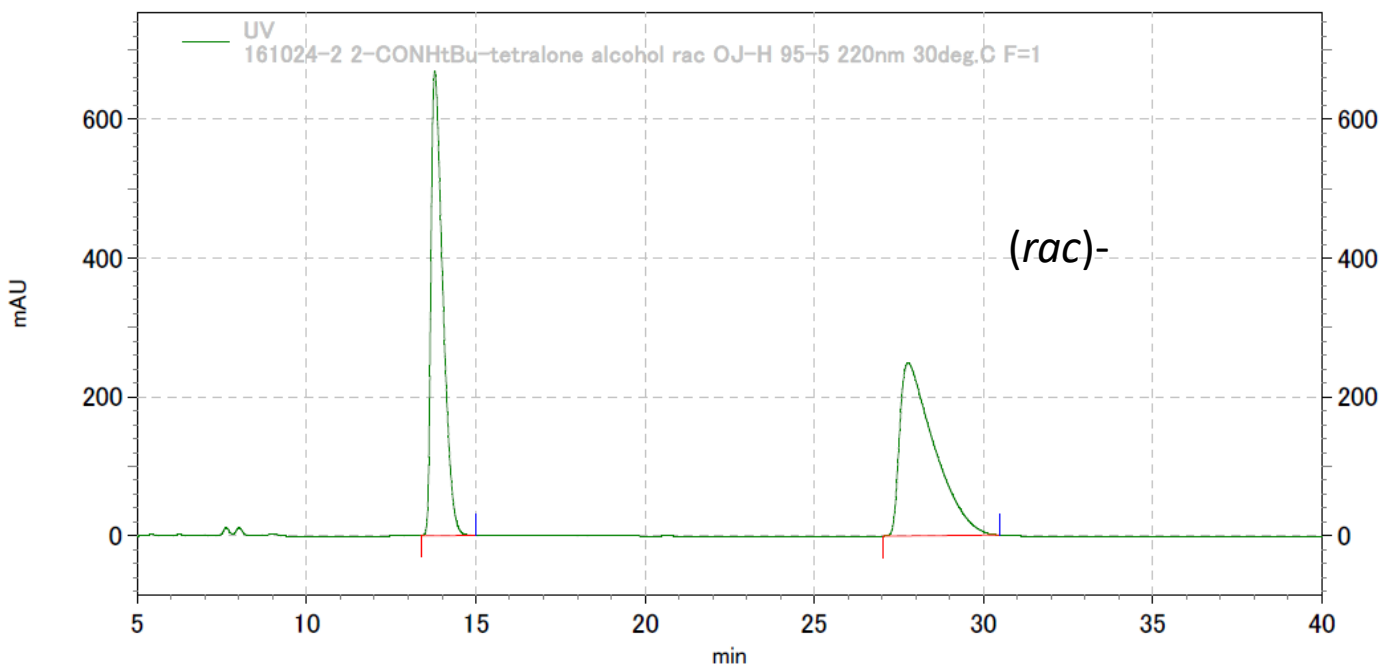

161024-2 2-CONHtBu-tetralone alcohol rac 0J-H 95-5 220nm 30deg. C F=1

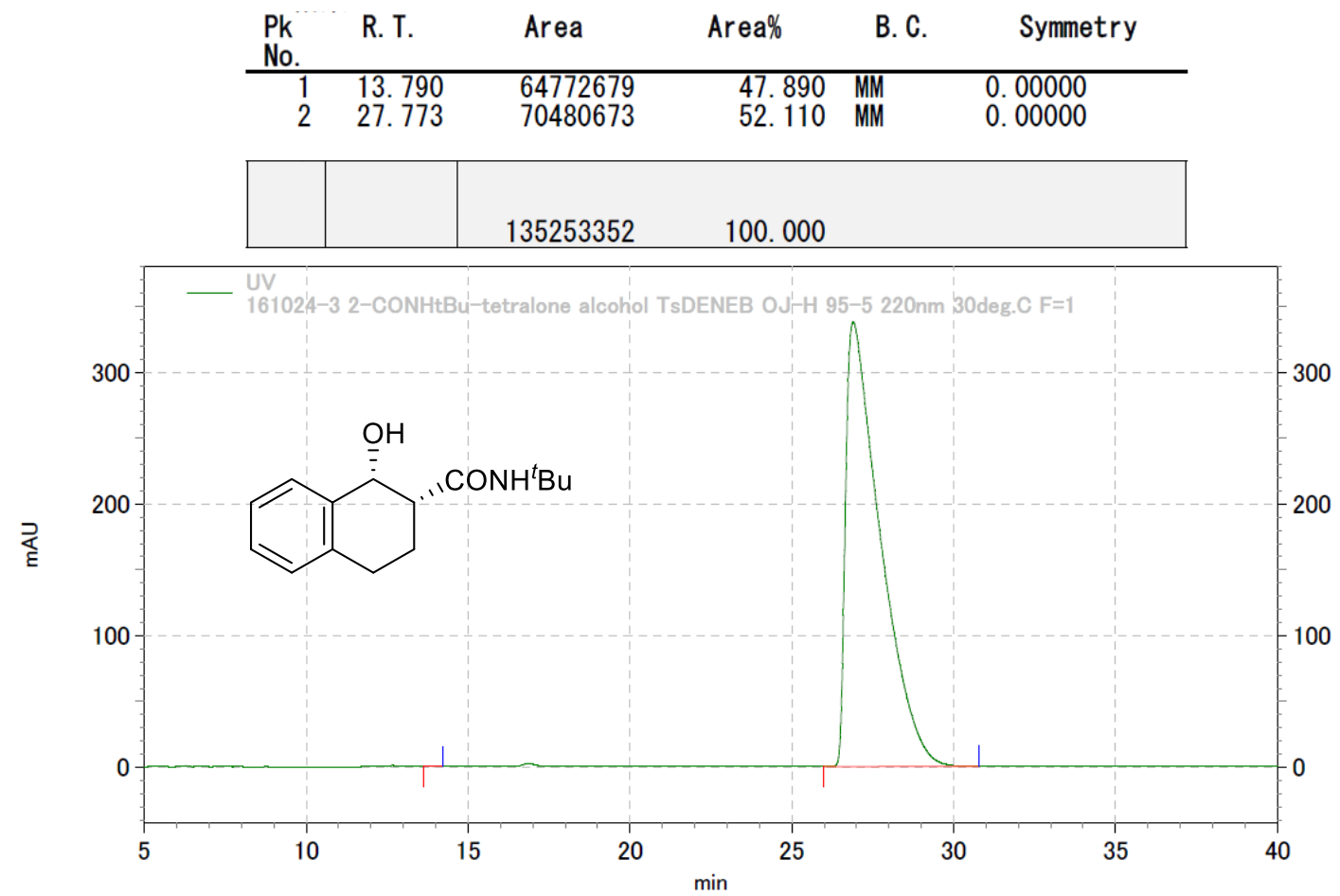

161024-3 2-CONHtBu-tetralone alcohol TsDENEB 0J-H 95-5 220nm 30deg. C F=1

\begin{tabular}{rlrrrr}
$\begin{array}{r}\text { Pk } \\
\text { No. }\end{array}$ & R. T. & Area & Area\% & \multicolumn{1}{c}{ B. C. } & \multicolumn{1}{c}{ Symmetry } \\
\hline 1 & 13.863 & 11017 & 0.011 & MM & 0.00000 \\
2 & 26.897 & 100377496 & 99.989 & MM & 0.00000 \\
\hline & & & & & \\
\hline & & & & & \\
\hline
\end{tabular}


$(5 R, 6 R)-N$-(tert-Butyl)-5-hydroxy-6,7,8,9-tetrahydro-5H-benzo[7]annulene-6-carboxa mide (cis-6e)

Chiralpak AS-H, $250 \times 4.6 \mathrm{~mm}$ column, hexane/2-propanol 95:5, $1.0 \mathrm{~mL} / \mathrm{min}, 220 \mathrm{~nm}, 30^{\circ} \mathrm{C}$

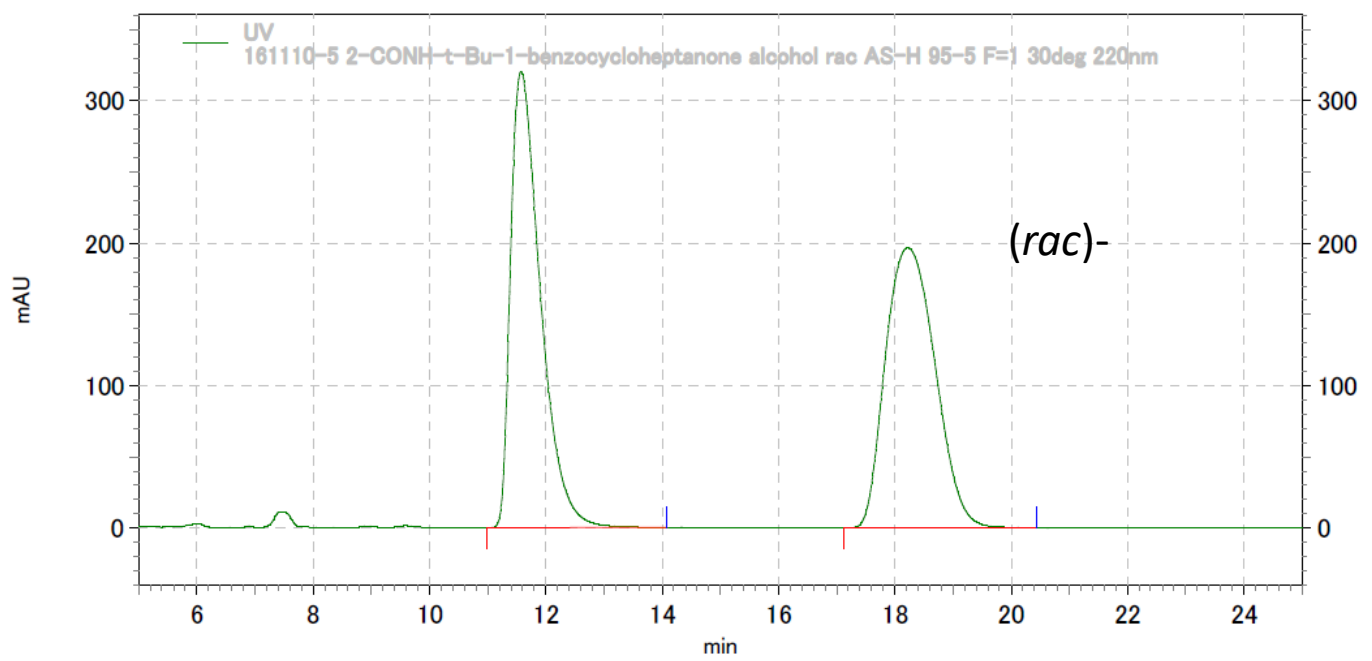

161110-5 2-CONH-t-Bu-1-benzocycloheptanone alcohol rac AS-H 95-5 F=1 30deg 220nm

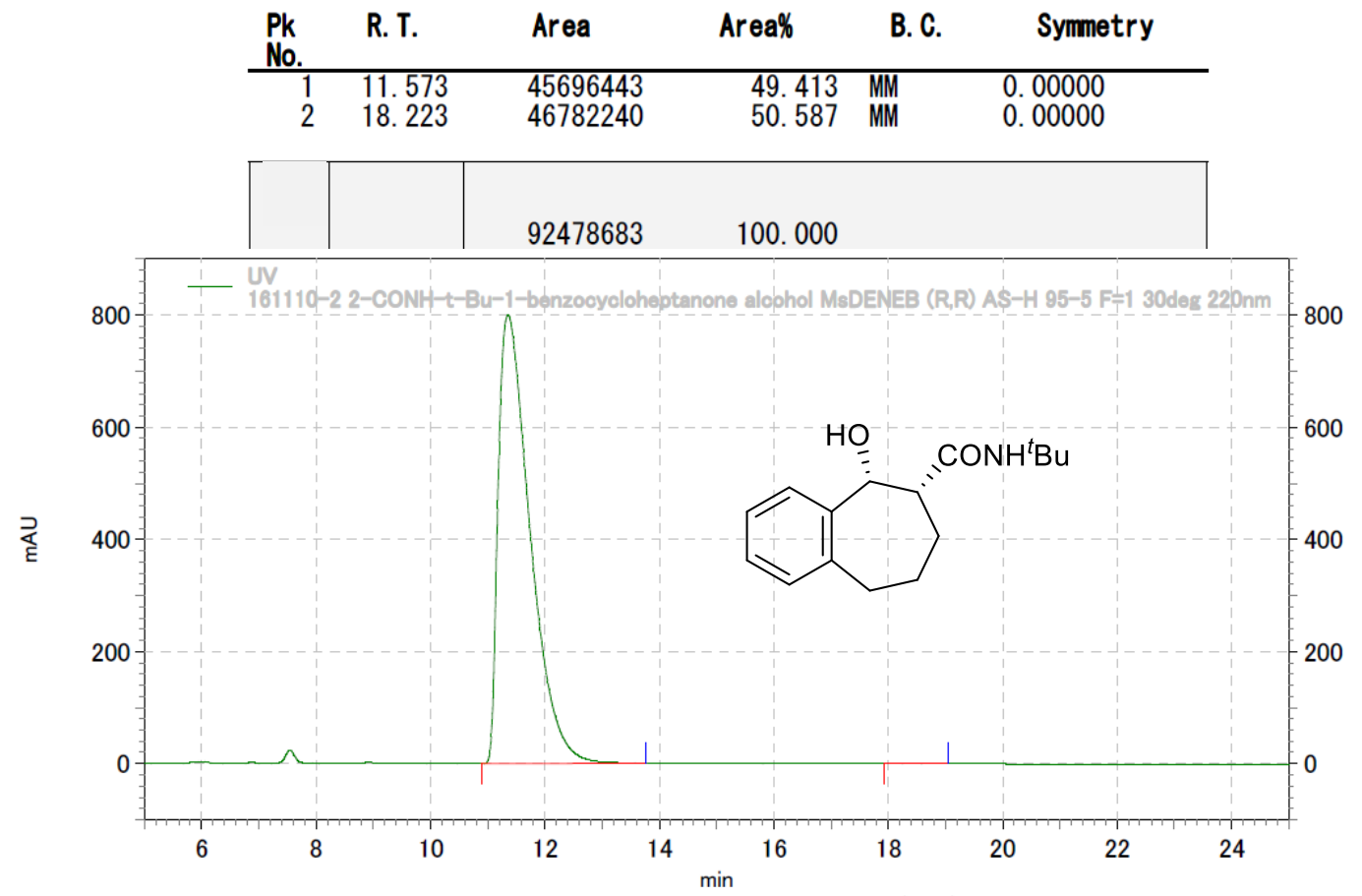

161110-2 2-CONH-t-Bu-1-benzocycloheptanone alcohol MsDENEB (R, R) AS-H 95-5 F=1 30deg 220nm

\begin{tabular}{rlrrrr}
$\begin{array}{r}\text { Pk } \\
\text { No. }\end{array}$ & R. T. & \multicolumn{1}{c}{ Area } & \multicolumn{1}{c}{ Area\% } & B.C. & Symmetry \\
\hline 1 & 11.357 & 123714744 & 99.953 & MM & 0.00000 \\
2 & 18.373 & 58123 & 0.047 & MM & 0.00000 \\
\hline & & & & & \\
\hline & & 123772867 & 100.000 & \\
\hline
\end{tabular}




\section{(1S,2R)-2-(Phenylsulfonyl)-2,3-dihydro-1H-inden-1-ol (cis-6f)}

Chiralpak AD-H, $250 \times 4.6 \mathrm{~mm}$ column, hexane/2-propanol 80:20, $1.0 \mathrm{~mL} / \mathrm{min}, 220 \mathrm{~nm}, 30^{\circ} \mathrm{C}$

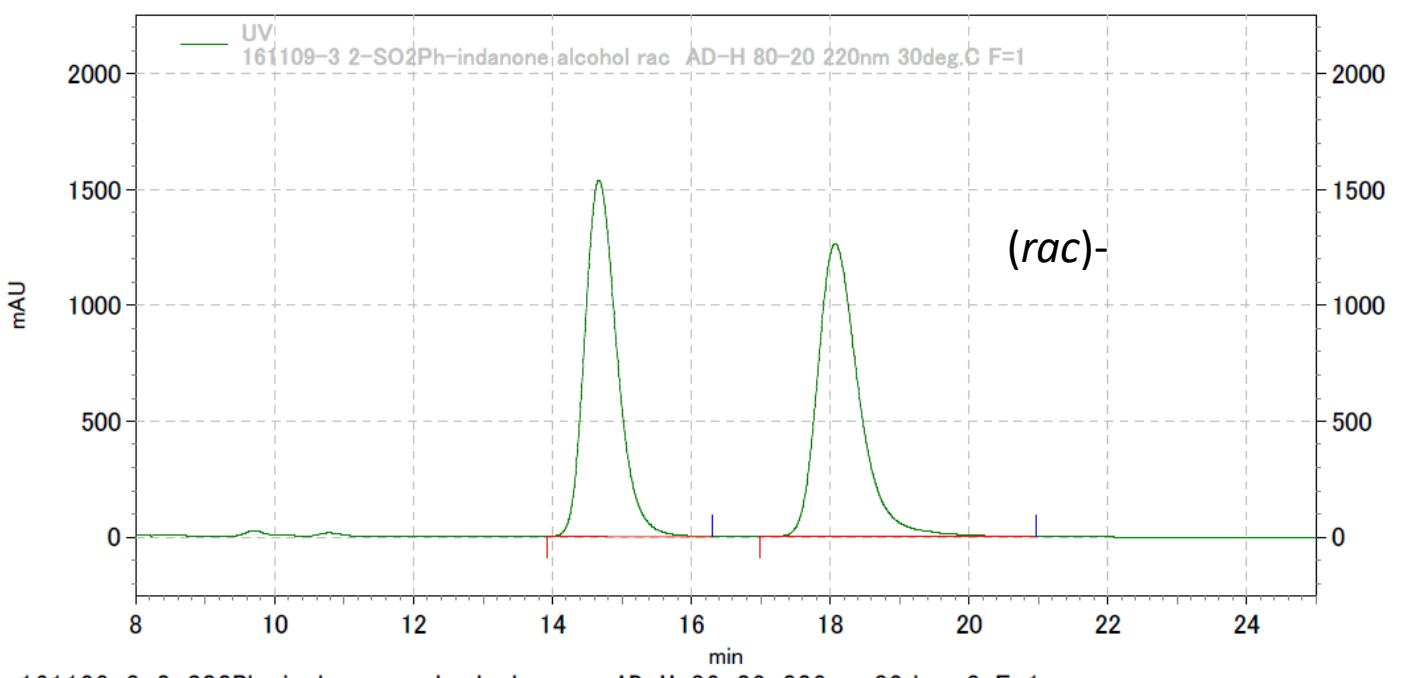

161109-3 2-S02Ph-indanone alcohol rac AD-H 80-20 220nm 30deg. C F=1

\begin{tabular}{|c|c|c|c|c|c|}
\hline $\begin{array}{l}\text { Pk } \\
\text { No. }\end{array}$ & R. T. & Area & Area\% & B. C. & Symmetry \\
\hline $\begin{array}{l}1 \\
2\end{array}$ & $\begin{array}{l}14.670 \\
18.073\end{array}$ & $\begin{array}{l}197747070 \\
208256916\end{array}$ & $\begin{array}{l}48.706 \\
51.294\end{array}$ & $\begin{array}{l}\text { MM } \\
\text { MM }\end{array}$ & $\begin{array}{l}0.00000 \\
0.00000\end{array}$ \\
\hline
\end{tabular}
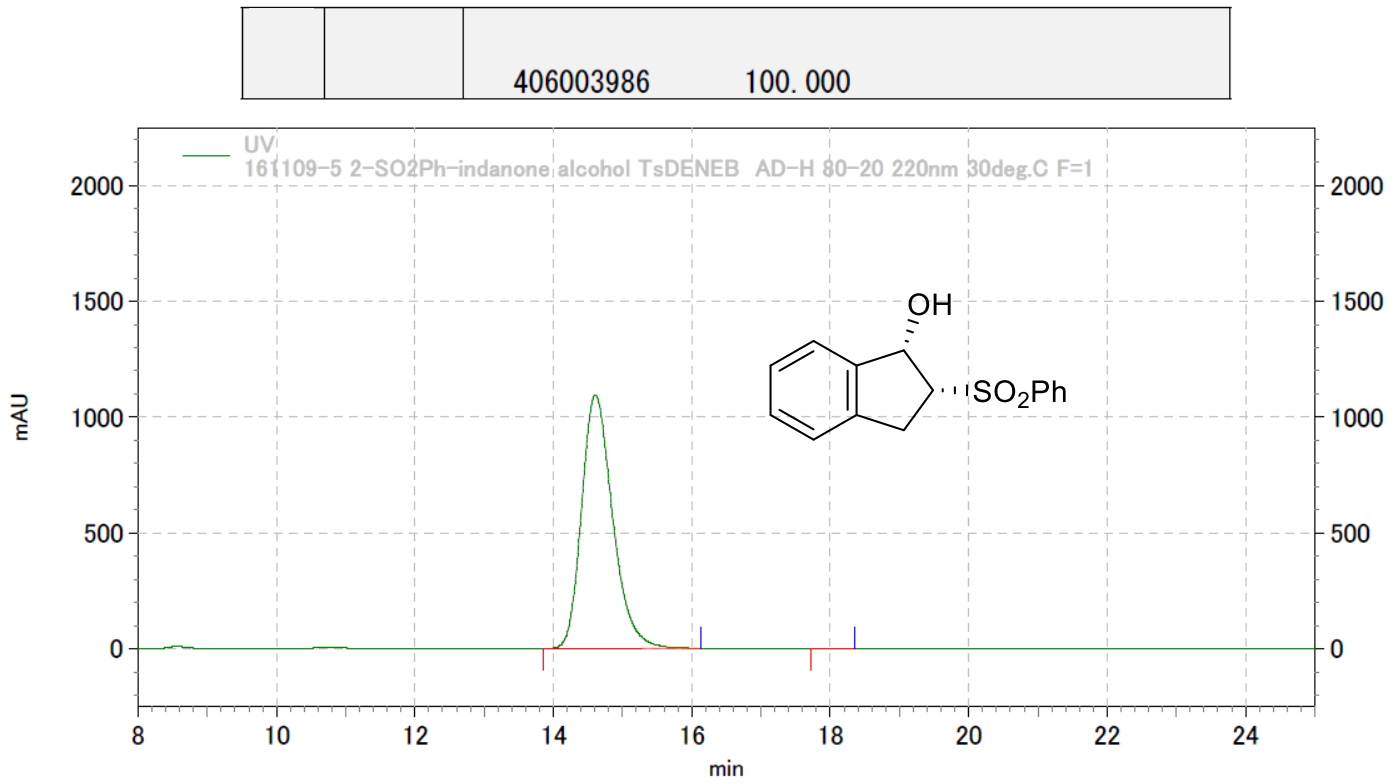

161109-5 2-S02Ph-indanone alcohol TsDENEB AD-H 80-20 220nm 30deg. C F=1

\begin{tabular}{|c|c|c|c|c|c|}
\hline $\begin{array}{l}\text { Pk } \\
\text { No }\end{array}$ & R. T. & Area & Area\% & B. C. & Symmetry \\
\hline $\begin{array}{l}1 \\
2\end{array}$ & $\begin{array}{l}14.607 \\
18.013\end{array}$ & $\begin{array}{r}136858087 \\
42030\end{array}$ & $\begin{array}{r}99.969 \\
0.031\end{array}$ & $\begin{array}{l}\text { MM } \\
\text { MM }\end{array}$ & $\begin{array}{l}0.00000 \\
0.00000\end{array}$ \\
\hline & & 136900117 & 100.000 & & \\
\hline
\end{tabular}




\section{(1S,2R)-2-(Phenylsulfonyl)-1,2,3,4-tetrahydronaphthalen-1-ol (cis-6g)}

Chiralpak AD-H, $250 \times 4.6 \mathrm{~mm}$ column, hexane/2-propanol 80:20, $1.0 \mathrm{~mL} / \mathrm{min}, 220 \mathrm{~nm}, 30^{\circ} \mathrm{C}$

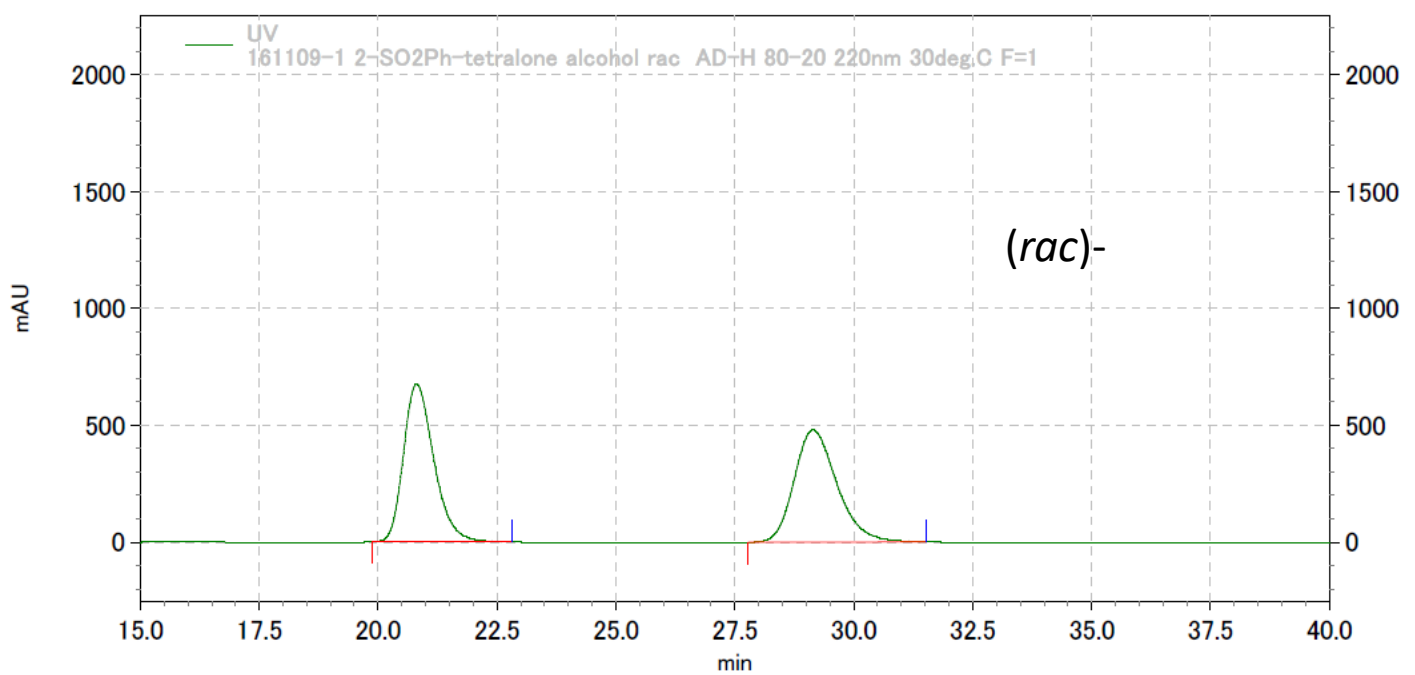

161109-1 2-S02Ph-tetralone alcohol rac AD-H 80-20 220nm 30deg. C F=1

\begin{tabular}{rlcccc}
$\begin{array}{c}\text { Pk } \\
\text { No. }\end{array}$ & R. T. & Area & Area\% & B. C. & Symmetry \\
\hline 1 & 20.807 & 120166934 & 50.111 & MM & 0.00000 \\
2 & 29.147 & 119635804 & 49.889 & MM & 0.00000
\end{tabular}
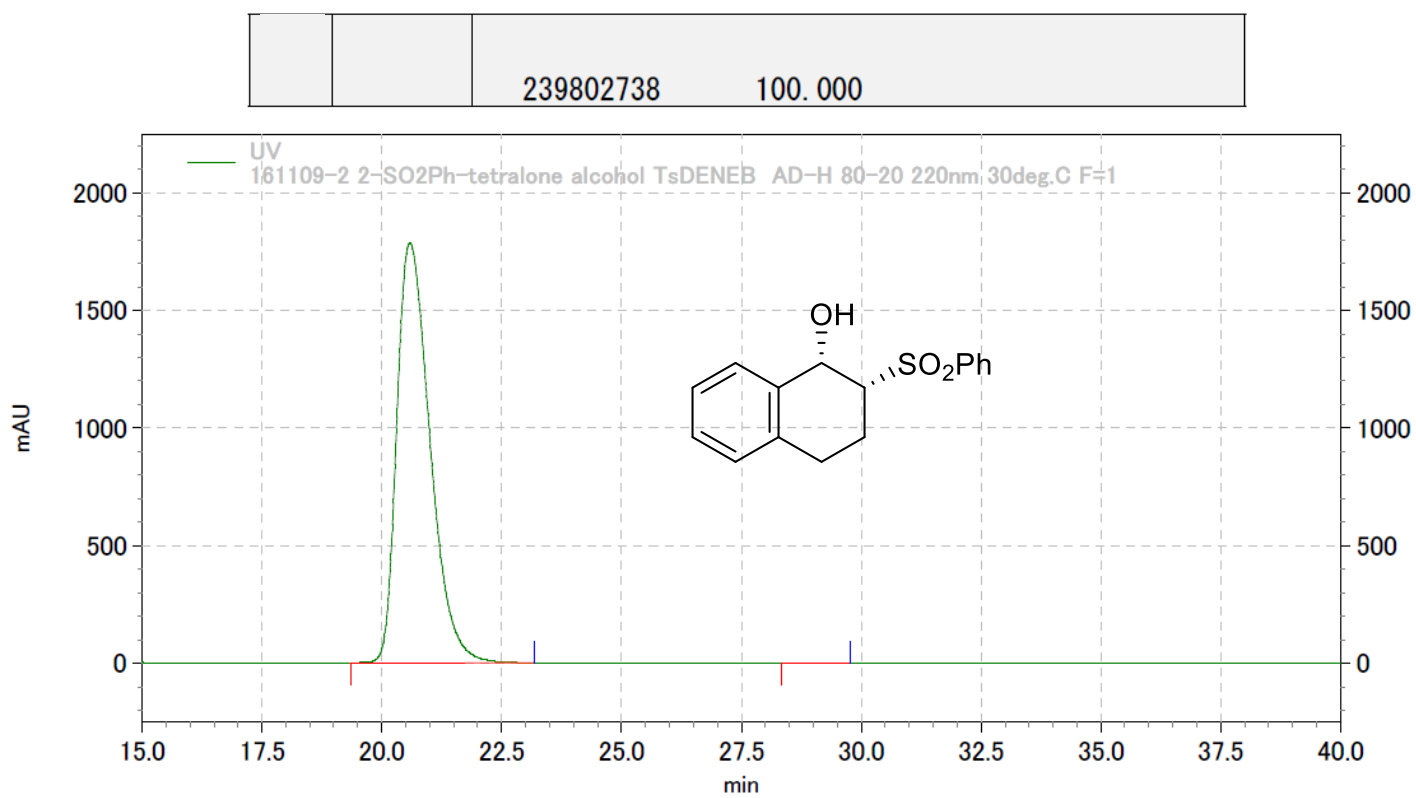

161109-2 2-S02Ph-tetralone alcohol TsDENEB AD-H 80-20 220nm 30deg. C F=1

\begin{tabular}{|c|c|c|c|c|c|}
\hline $\mathrm{Pk}$ & R. T. & Area & Area\% & B. $\mathrm{C}$ & Symmetry \\
\hline 2 & $\begin{array}{l}20.587 \\
29.020\end{array}$ & $\begin{array}{r}344181403 \\
65411\end{array}$ & $\begin{array}{r}99.981 \\
0.019\end{array}$ & $\begin{array}{l}\text { MM } \\
M M\end{array}$ & $\begin{array}{l}0.00000 \\
0.00000\end{array}$ \\
\hline & & 344246814 & 100.000 & & \\
\hline
\end{tabular}


Chiralpak AS-H, $250 \times 4.6 \mathrm{~mm}$ column, hexane/2-propanol 95:5, $1.0 \mathrm{~mL} / \mathrm{min}, 220 \mathrm{~nm}, 30{ }^{\circ} \mathrm{C}$

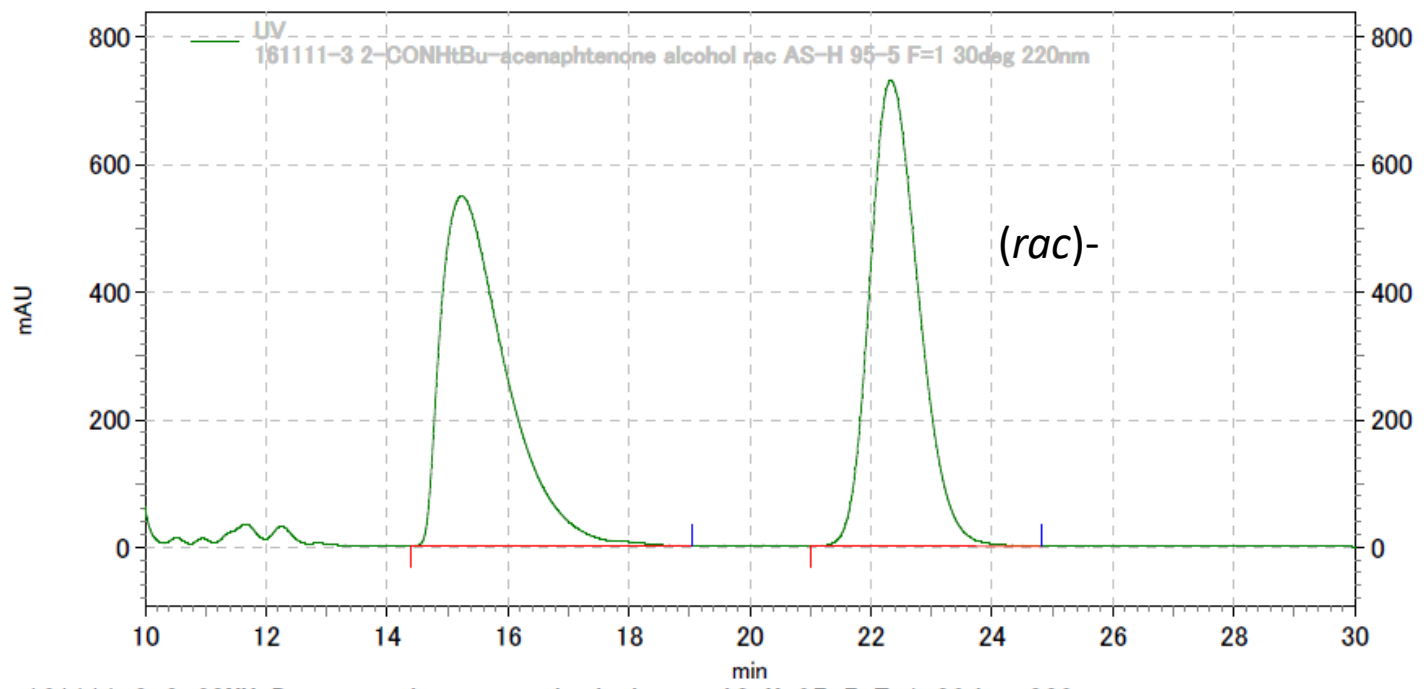

161111-3 2-CONHtBu-acenaphtenone alcohol rac AS-H 95-5 F=1 30deg 220nm

\begin{tabular}{rlrrrr}
$\begin{array}{r}\text { Pk } \\
\text { No. }\end{array}$ & R. T. & \multicolumn{1}{c}{ Area } & \multicolumn{1}{l}{ Area\% } & B. C. & Symmetry \\
\hline 1 & 15.233 & 163179682 & 49.878 & MM & 0.00000 \\
2 & 22.330 & 163975320 & 50.122 & MM & 0.00000 \\
\hline & & & & & \\
\hline & & 327155002 & 100.000 & \\
\hline
\end{tabular}

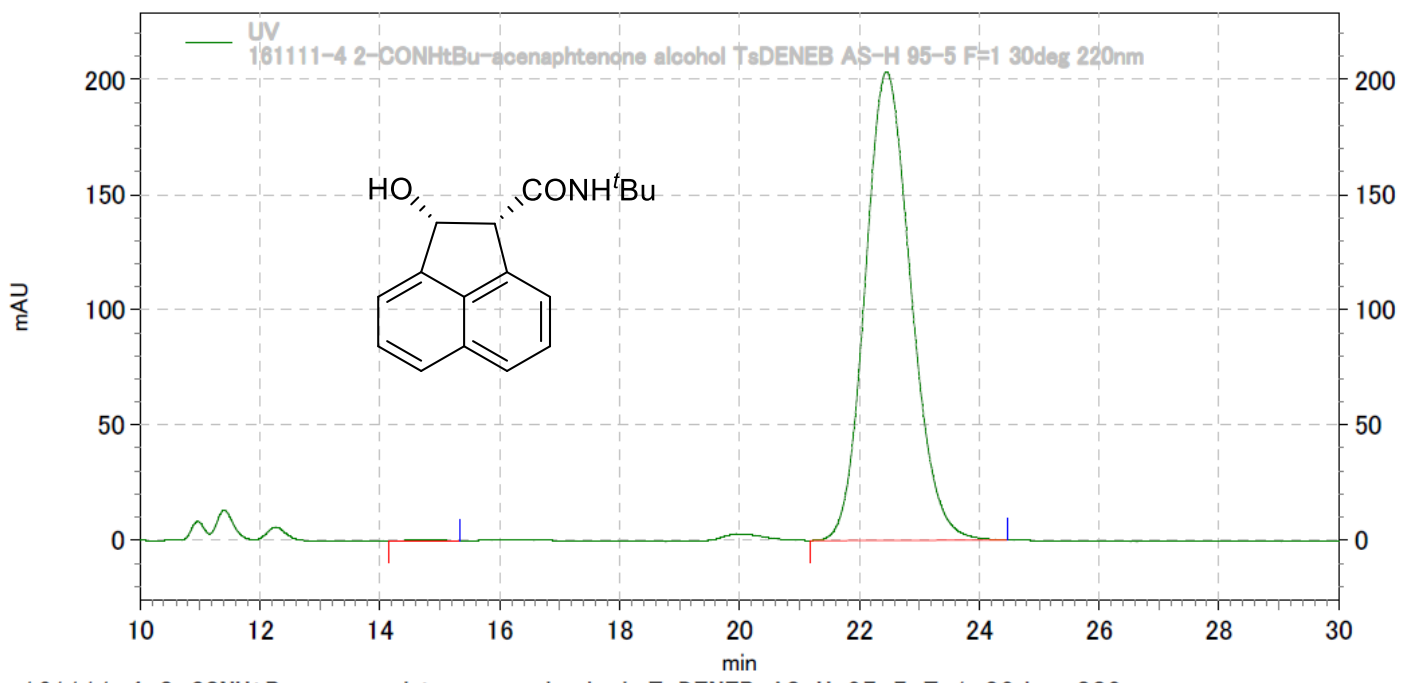

161111-4 2-CONHtBu-acenaphtenone alcohol TsDENEB AS-H 95-5 F=1 30deg 220nm

\begin{tabular}{rlrrrr}
$\begin{array}{c}\text { Pk } \\
\text { No. }\end{array}$ & R. T. & \multicolumn{1}{c}{ Area } & \multicolumn{1}{c}{ Area\% } & B. C. & Symmetry \\
\hline 1 & 14.700 & 32795 & 0.076 & MM & 0.00000 \\
2 & 22.453 & 43210198 & 99.924 & MM & 0.00000 \\
\hline & & & & & \\
\hline & & 43242993 & 100.000 & \\
\hline
\end{tabular}


Methyl (1R,2R)-2-hydroxy-1,2-dihydroacenaphthylene-1-carboxylate (cis-6i)

Chiralcel OJ-H, $250 \times 4.6 \mathrm{~mm}$ column, hexane/2-propanol 97:3, $1.0 \mathrm{~mL} / \mathrm{min}, 220 \mathrm{~nm}, 30^{\circ} \mathrm{C}$

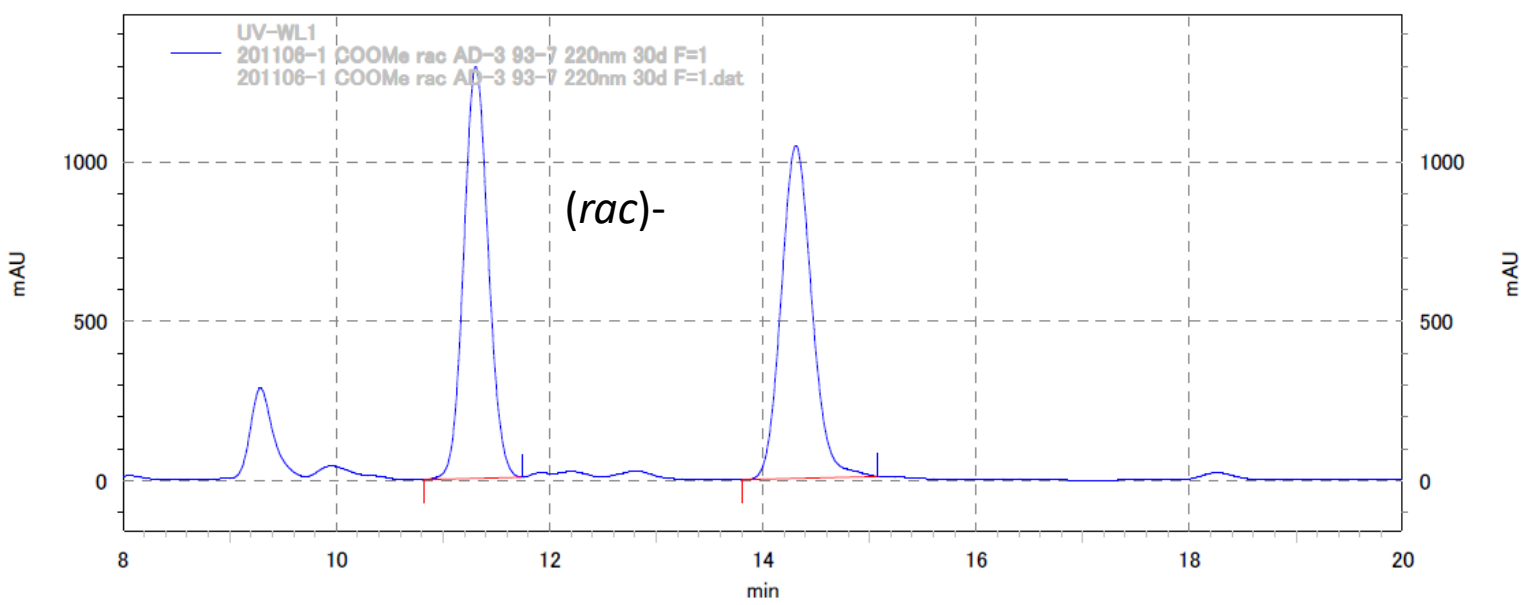

\begin{tabular}{|c|c|c|c|}
\hline Peak No. & $\mathrm{RT}(\mathrm{min})$ & Area & Area(\%) \\
\hline & 11.307 & 82668618 & 49.698 \\
\hline & 14.313 & 83674467 & 50.302 \\
\hline & & 166343085 & 100.000 \\
\hline
\end{tabular}

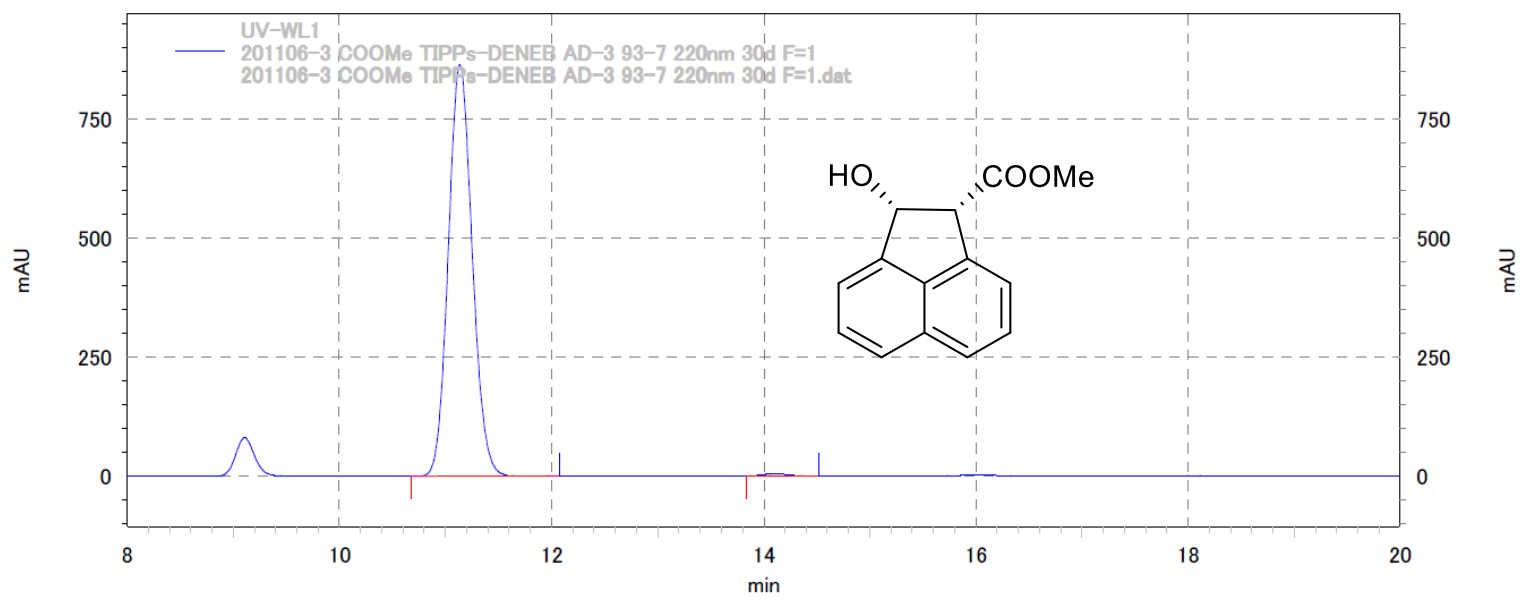

\begin{tabular}{rrrrr} 
UV-WL1 & \multicolumn{3}{c}{ Area(\%) } \\
\hline & RT $($ min) & Area & 99.482 \\
& 11.133 & 54124591 & 0.518 \\
\hline & 14.100 & 281879 & 100.000 \\
\hline
\end{tabular}




\section{(1S,6R)-6-Chloro-3-methylcyclohex-2-en-1-ol (cis-8a)}

Chiralpak AD-3, $150 \times 4.6 \mathrm{~mm}$ column, hexane/2-propanol 98:2, $1.0 \mathrm{~mL} / \mathrm{min}, 210 \mathrm{~nm}, 35^{\circ} \mathrm{C}$

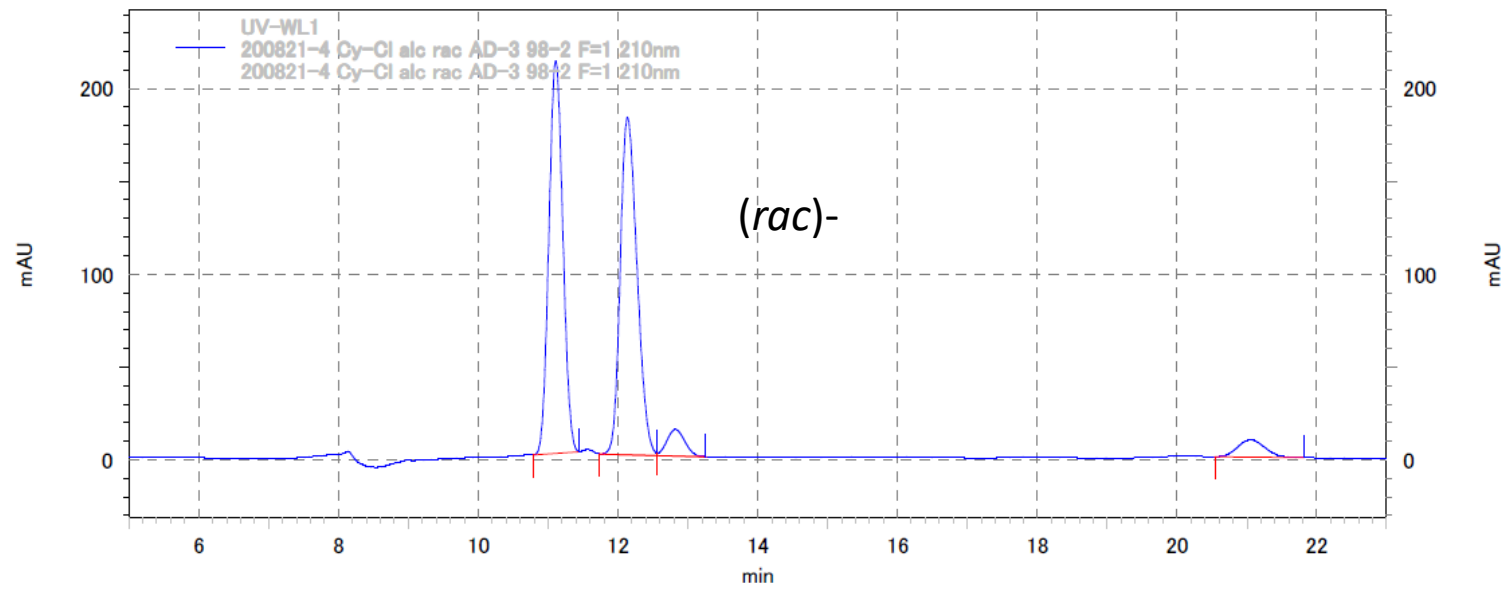

UV-WL1

\begin{tabular}{lrrr} 
Peak No. & RT(min) & Area & 45.324 \\
\hline & 11.107 & 11759791 & 46.827 \\
& 12.133 & 12149681 & 3.918 \\
& 12.820 & 1016629 & 3.930 \\
\hline & 21.053 & 1019741 & \\
\hline & & & \\
& & 25945842 & 100.000 \\
\hline
\end{tabular}

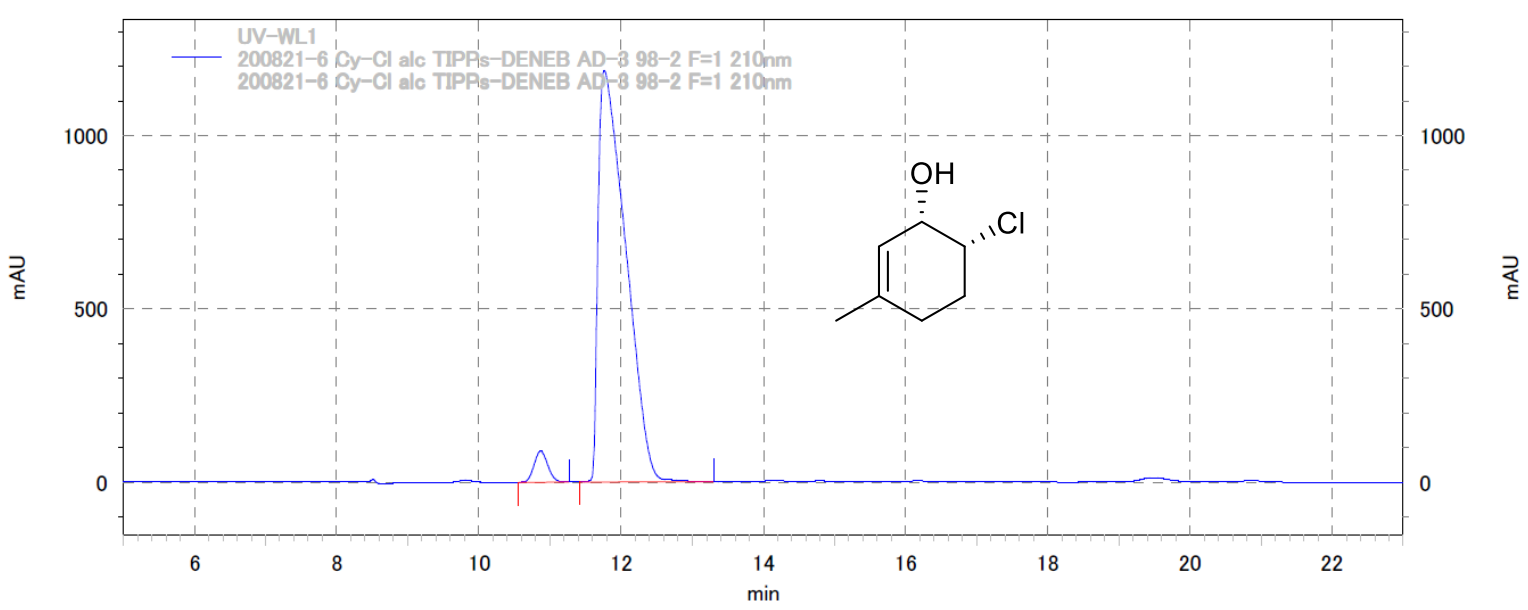

UV-WL1

Peak No.

$\mathrm{RT}$ (min)

10.867

Area

Area(\%)

11.767

127502629

3.616

96.384 
Ethyl (1R,2S)-2-hydroxy-4-methylcyclohex-3-ene-1-carboxylate (cis-8b)

Chiralcel OZ-3, $250 \times 4.6 \mathrm{~mm}$ column, hexane/2-propanol 95:5, $1.0 \mathrm{~mL} / \mathrm{min}, 210 \mathrm{~nm}, 30^{\circ} \mathrm{C}$
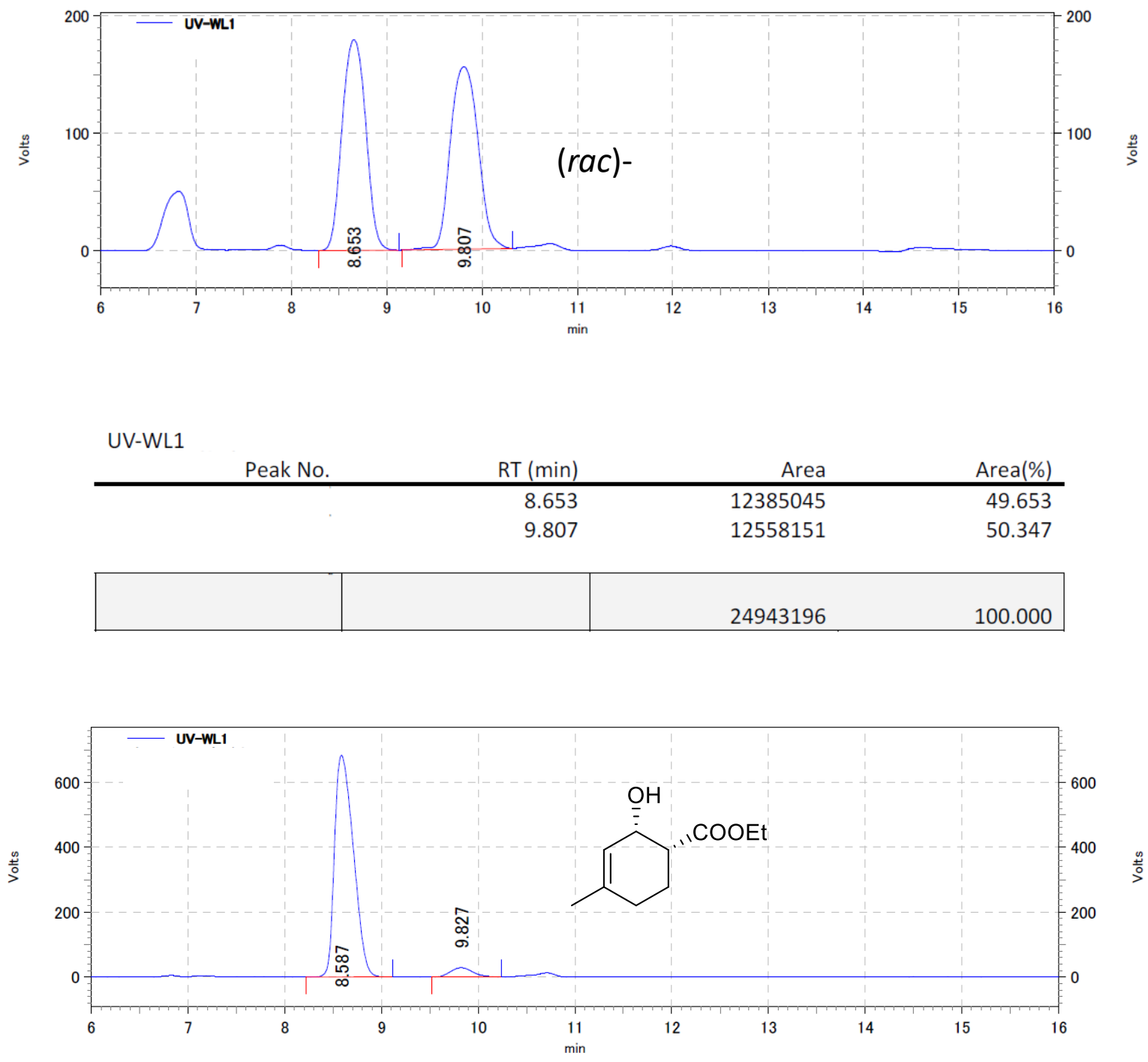

\begin{tabular}{rrrrr} 
UV-WL1 Peak No. & RT $(\min )$ & Area & Area(\%) \\
\hline & 8.587 & 36978958 & 95.237 \\
& 9.827 & 1849563 & 4.763 \\
& & 38828521 & 100.000 \\
\hline
\end{tabular}


$(1 R, 2 S)-N$-(tert-Butyl)-2-hydroxy-4-methylcyclohex-3-ene-1-carboxamide (cis-8c)

Chiralpak QN-AX, $150 \times 4.6 \mathrm{~mm}$ column, hexane/2-propanol 96:4, $1.0 \mathrm{~mL} / \mathrm{min}, 210 \mathrm{~nm}, 30^{\circ} \mathrm{C}$

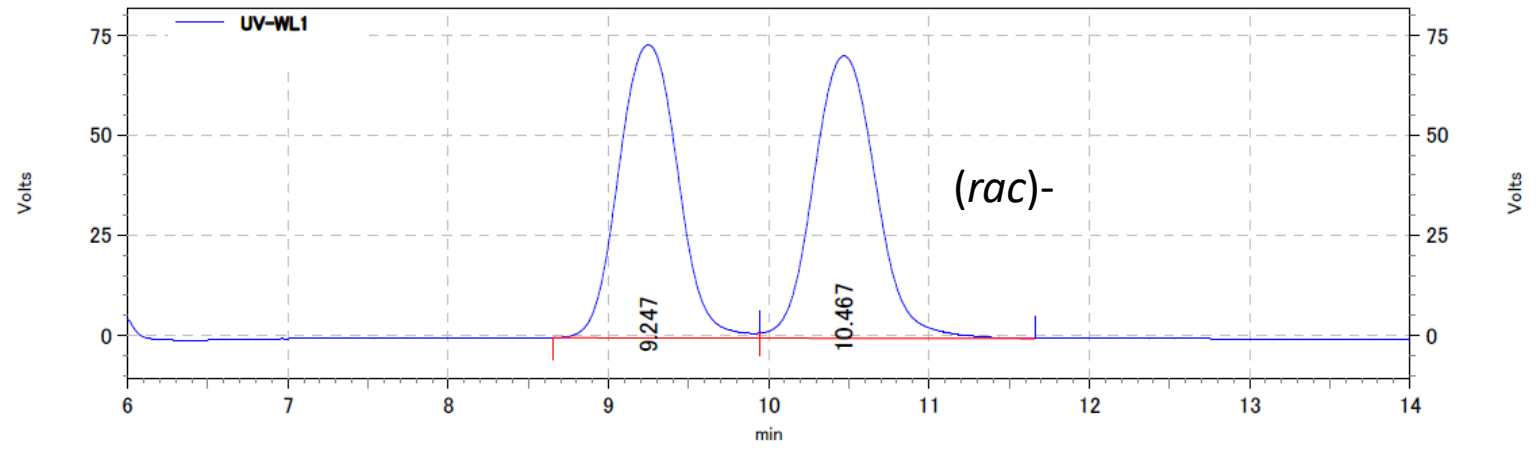

UV-WL1

Peak No.

$\mathrm{RT}(\min )$

9.247

Area

7480981

7618629

Area(\%)

10.467

50.456
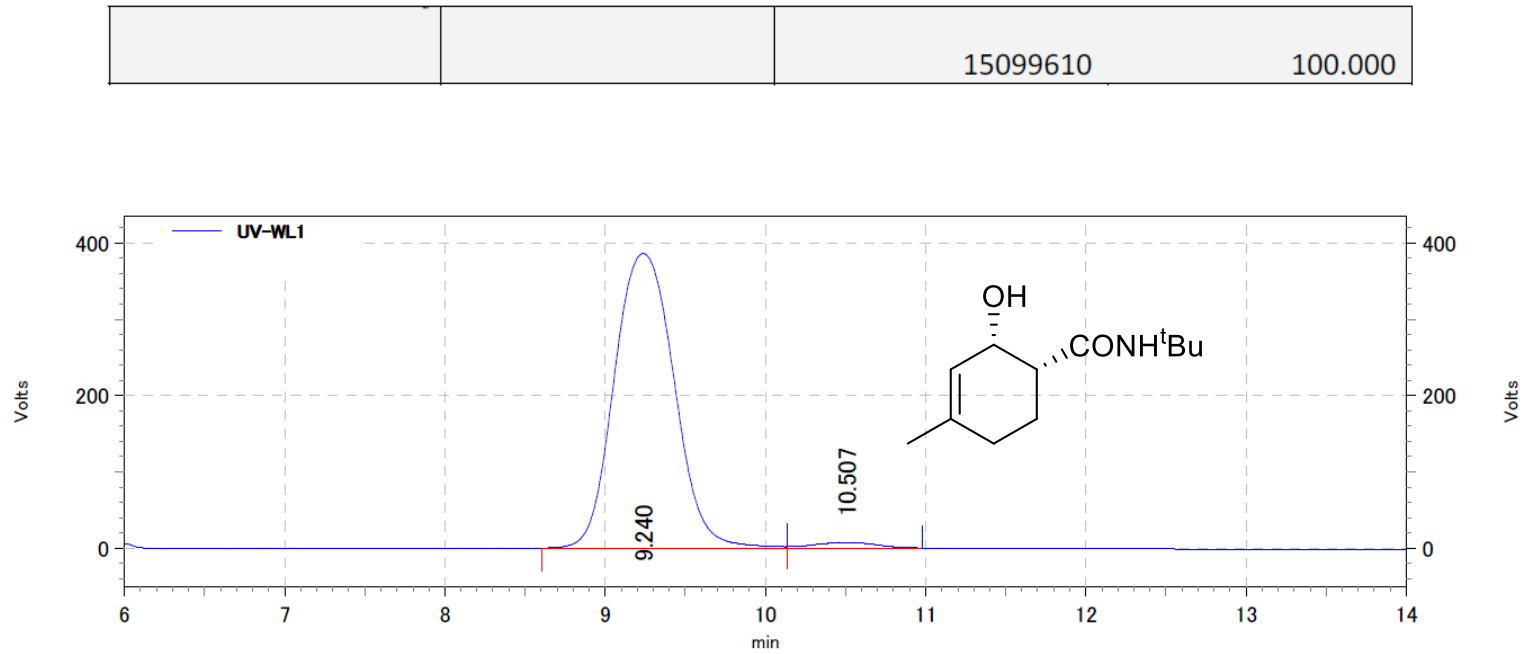

\begin{tabular}{rrrrr} 
UV-WL1 Peak No. & RT (min) & Area & Area(\%) \\
\hline & 9.240 & 40076731 & 98.058 \\
& 10.507 & 793887 & 1.942 \\
\hline & & & & 100.000 \\
\hline
\end{tabular}




\section{(1R,2S)-7-(Benzyloxy)-2-chloro-1,2,3,4-tetrahydronaphthalen-1-ol (11)}

Chiralcel OZ-3, $250 \times 4.6 \mathrm{~mm}$ column, hexane/2-propanol 95:5, $1.0 \mathrm{~mL} / \mathrm{min}, 210 \mathrm{~nm}, 35^{\circ} \mathrm{C}$

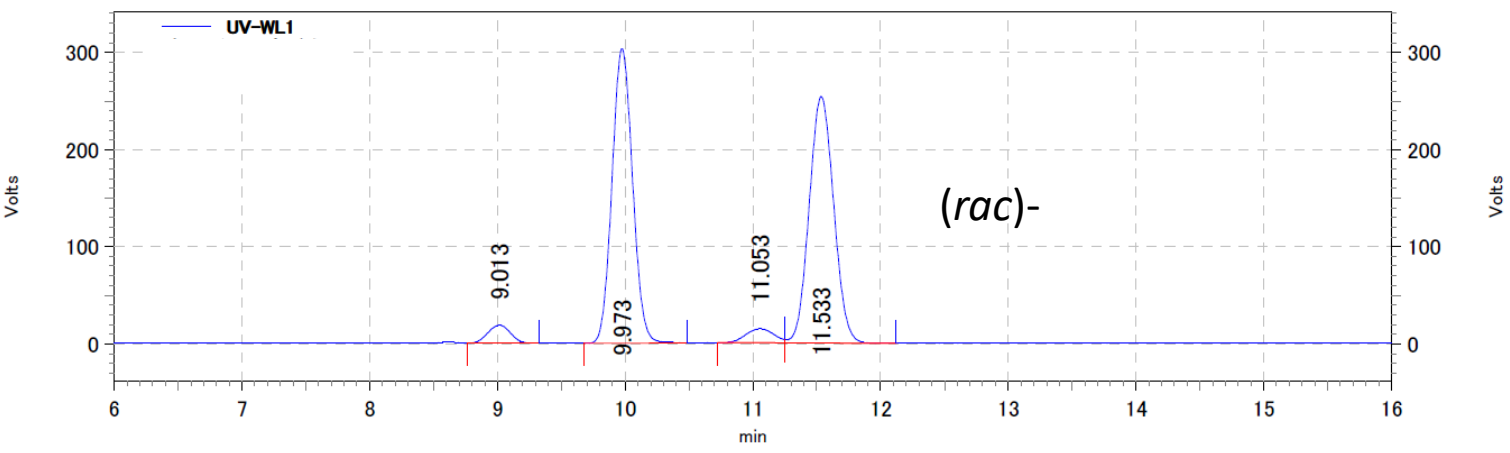

\begin{tabular}{|c|c|c|c|}
\hline Peak No. & $\mathrm{RT}(\min )$ & Area & Area(\%) \\
\hline & 9.013 & 914016 & 3.159 \\
\hline & 9.973 & 13582448 & 46.947 \\
\hline & 11.053 & 868570 & 3.002 \\
\hline & 11.533 & 13566505 & 46.892 \\
\hline & & 28931539 & 100.000 \\
\hline
\end{tabular}

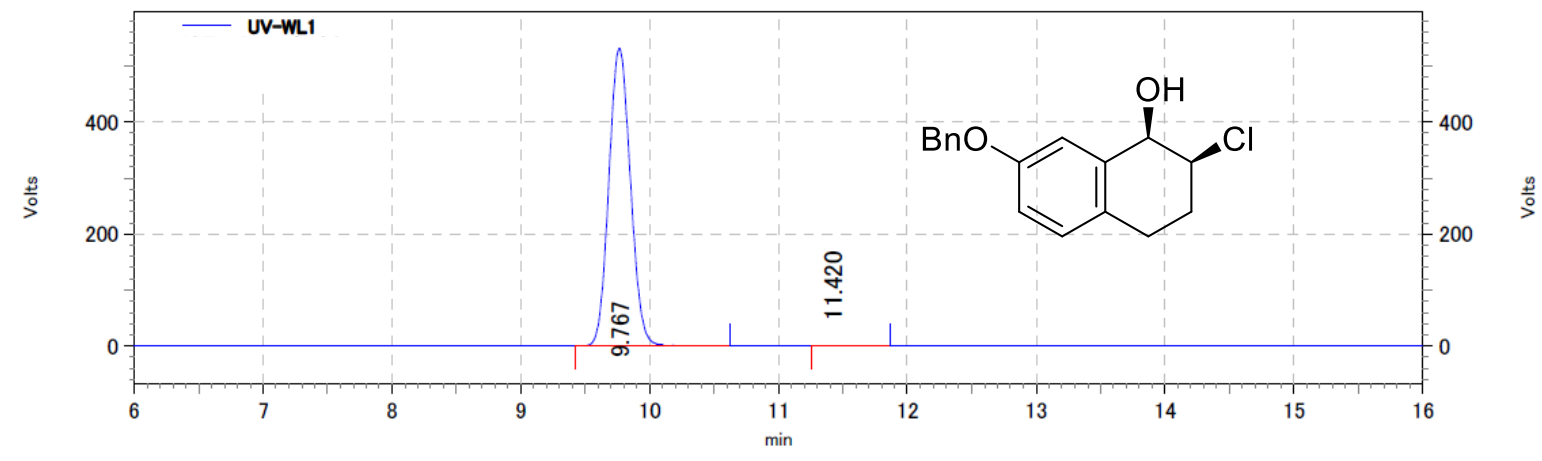

\begin{tabular}{|c|c|c|c|}
\hline Peak No. & $\mathrm{RT}(\min )$ & Area & Area(\%) \\
\hline & 9.767 & 24801566 & 99.969 \\
\hline & 11.420 & 7748 & 0.031 \\
\hline & & 24809314 & 100.000 \\
\hline
\end{tabular}




\section{(+)-PHNO:}

$(4 \mathrm{a} R, 10 \mathrm{~b} R)-4-P r o p y l-3,4,4 a, 5,6,10 b-h e x a h y d r o-2 H$-naphtho[1,2-b][1,4]oxazin-9-ol

Chiralcel OZ-3, $250 \times 4.6 \mathrm{~mm}$ column, hexane/2-propanol 90:10, $1.0 \mathrm{~mL} / \mathrm{min}, 220 \mathrm{~nm}, 30^{\circ} \mathrm{C}$

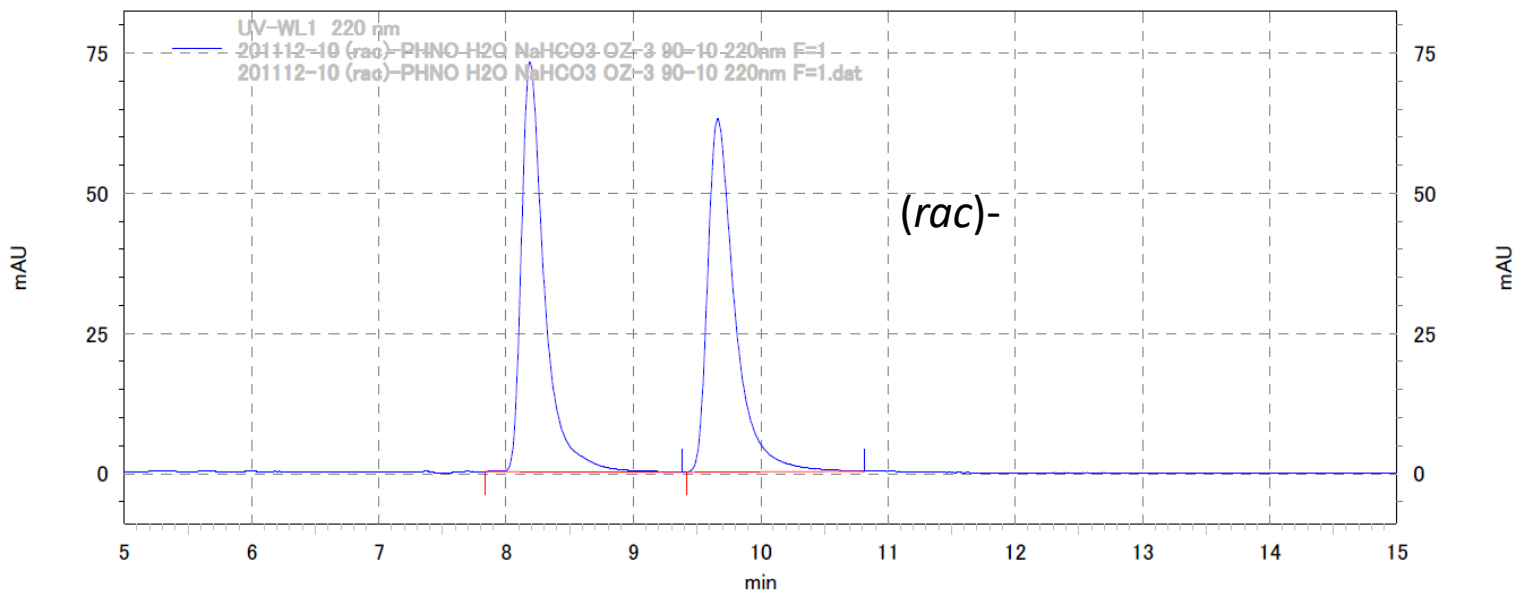

UV-WL1 $220 \mathrm{~nm}$

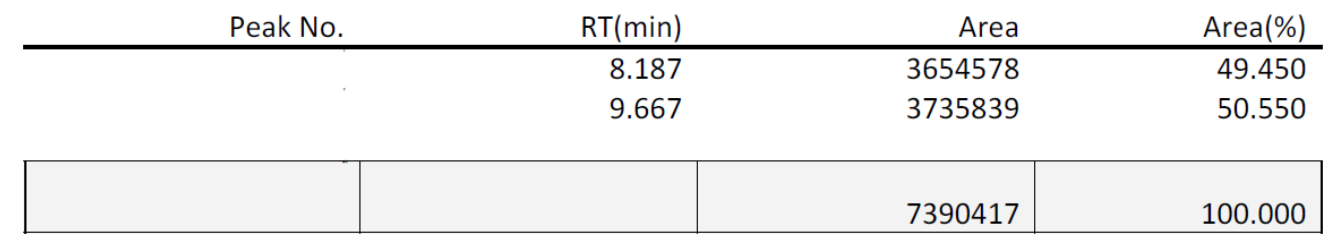

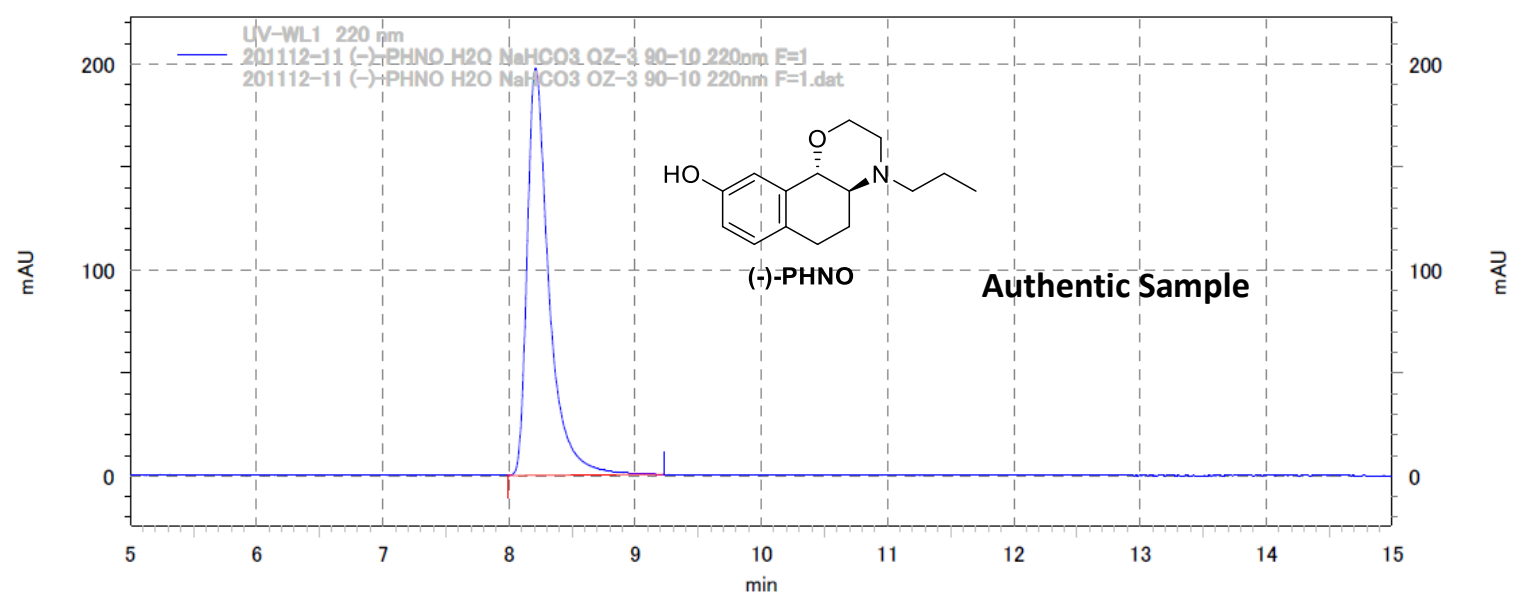

UV-WL1 $220 \mathrm{~nm}$

\begin{tabular}{|r|rrr|}
\multicolumn{1}{c}{ Peak No. } & RT $(\min )$ & Area & Area(\%) \\
\hline & 8.213 & 9486288 & 100.000 \\
& & & \\
\hline & & 9486288 & 100.000 \\
\hline
\end{tabular}




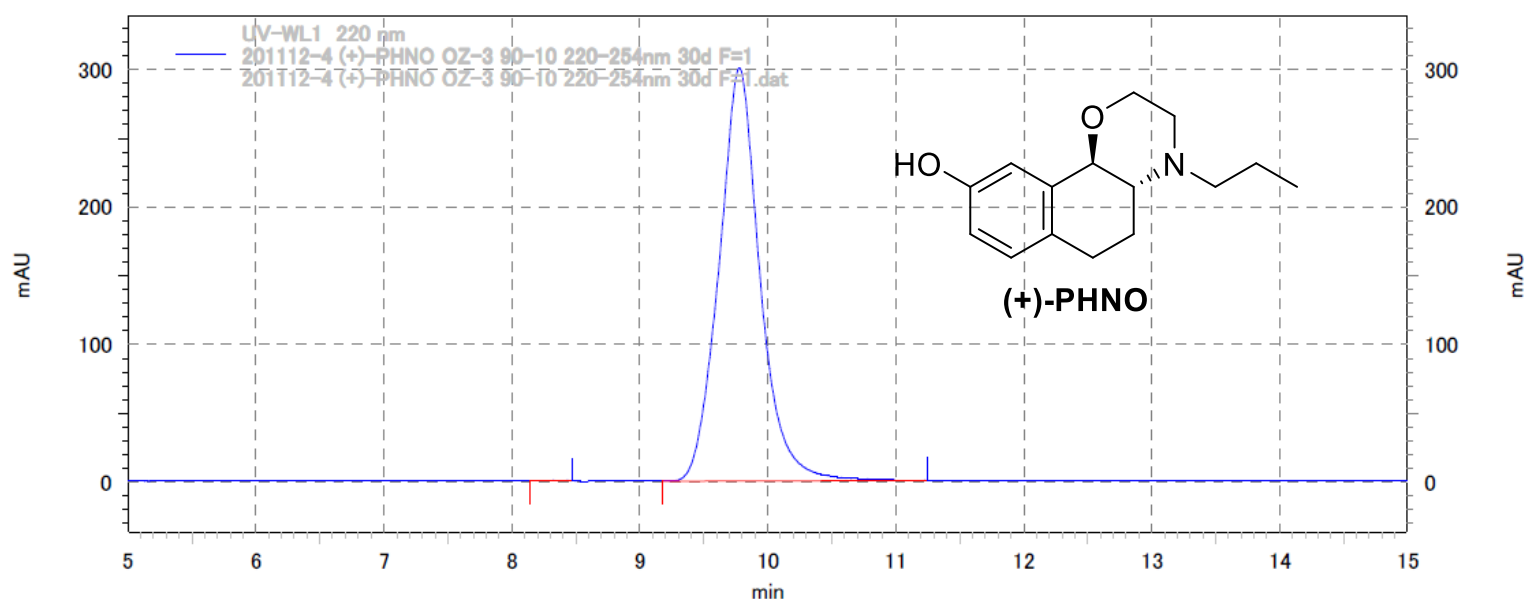

UV-WL1 220 nm

Peak No.

$\mathrm{RT}$ (min)

Area

Area(\%)

8.393

23370

0.087

9.780

26753512

99.913 


\section{J. HR-MS Spectra for Substrates and Products.}

\section{2-Chloro-3,4-dihydronaphthalen-1(2H)-one (3a)}

(APCI positive)

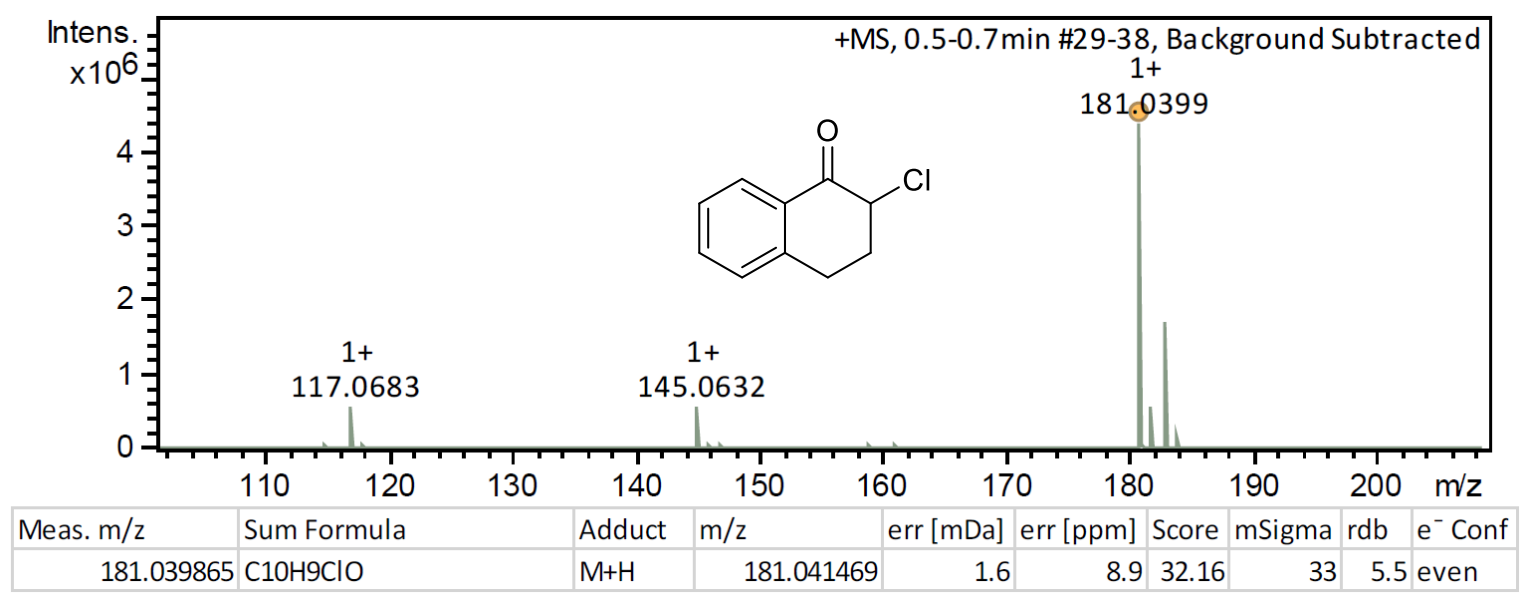

\section{2-Chloro-7-methoxy-3,4-dihydronaphthalen-1(2H)-one (3b)}

(APCI positive)

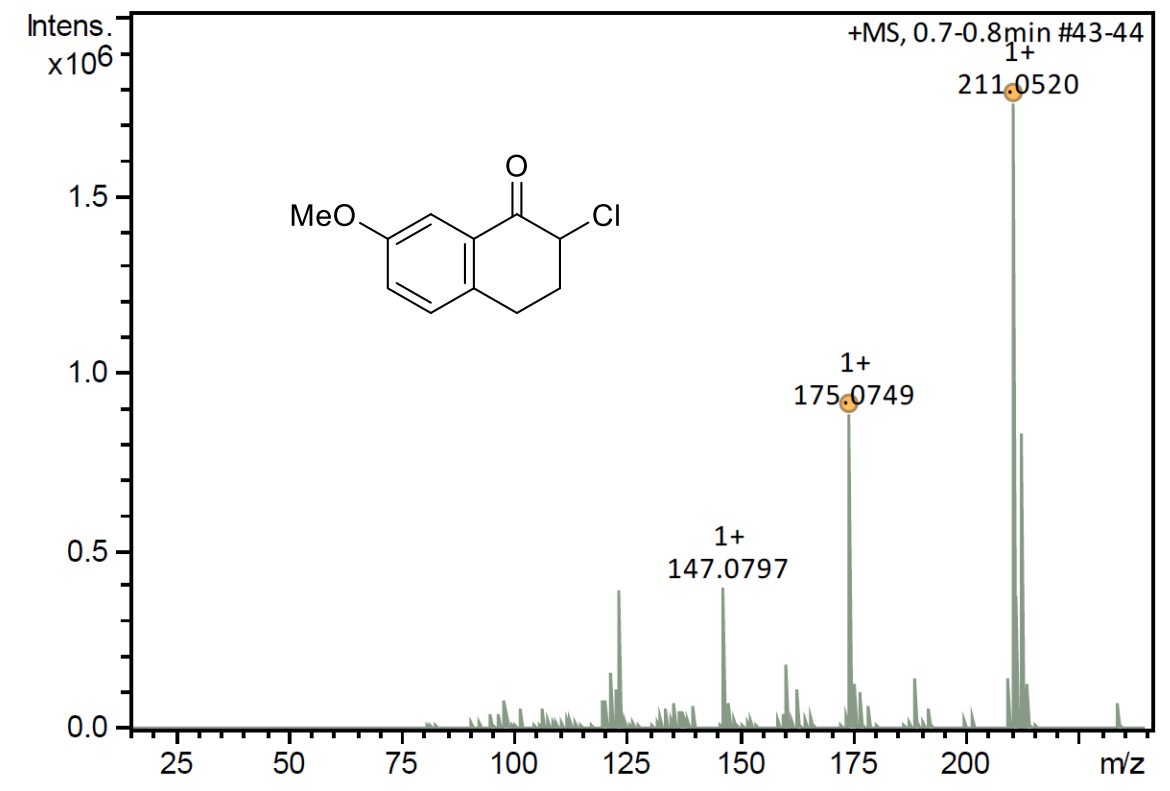

\begin{tabular}{|c|l|l|r|r|r|r|r|r|r|r|}
\hline Meas. $\mathrm{m} / \mathrm{z}$ & Sum Formula & Adduct & Score & $\mathrm{m} / \mathrm{z}$ & err $[\mathrm{mDa}]$ & err [ppm] $\mathrm{mSigma}$ & rdb $^{-} \mathrm{e}^{-}$Conf \\
\hline 147.079736 & $\mathrm{C} 10 \mathrm{H} 100$ & $\mathrm{M}+\mathrm{H}$ & 100 & 147.080441 & 0.7 & 4.8 & 58.6 & 5.5 even \\
\hline 175.074873 & $\mathrm{C} 11 \mathrm{H} 1002$ & $\mathrm{M}+\mathrm{H}$ & 100 & 175.075356 & 0.5 & 2.8 & 64.5 & 6.5 even \\
\hline 211.051954 & $\mathrm{C} 11 \mathrm{H} 11 \mathrm{ClO} 2$ & $\mathrm{M}+\mathrm{H}$ & 100 & 211.052034 & 0.1 & 0.4 & 87.3 & 5.5 even \\
\hline
\end{tabular}


2-Chloro-7-bromo-3,4-dihydronaphthalen-1(2H)-one (3c)

(APCI positive)

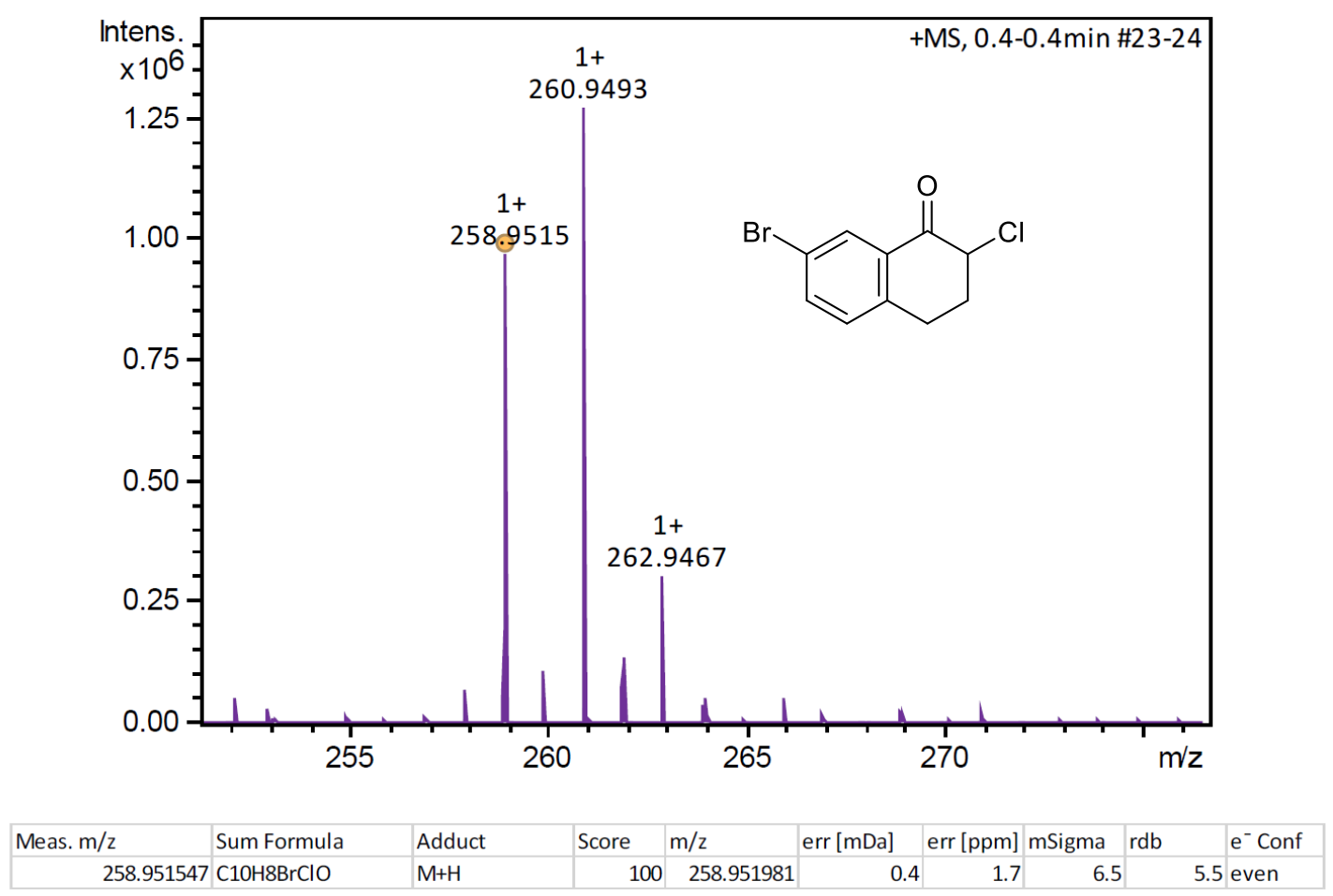

\section{2-Chloro-2,3-dihydro-1H-inden-1-one (3d)}

(APCI positive)

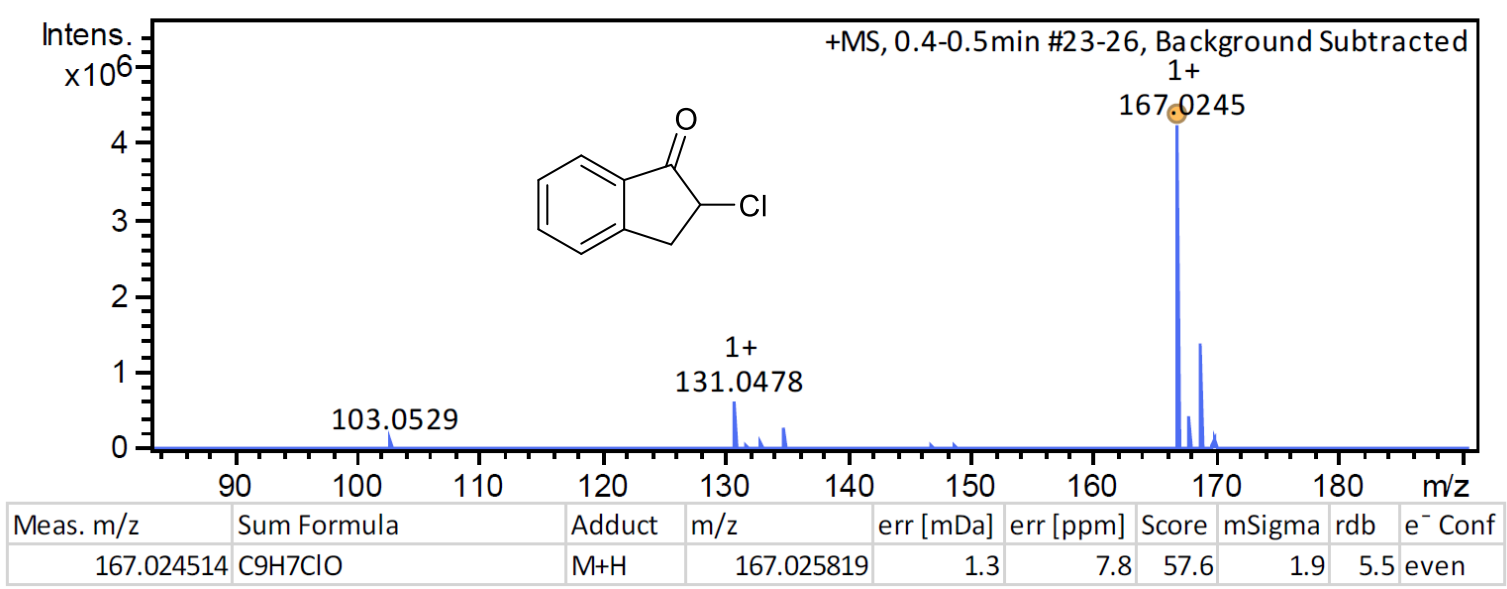


2-Chloro-5-methoxy-2,3-dihydro-1H-inden-1-one (3e)

(APCI positive)

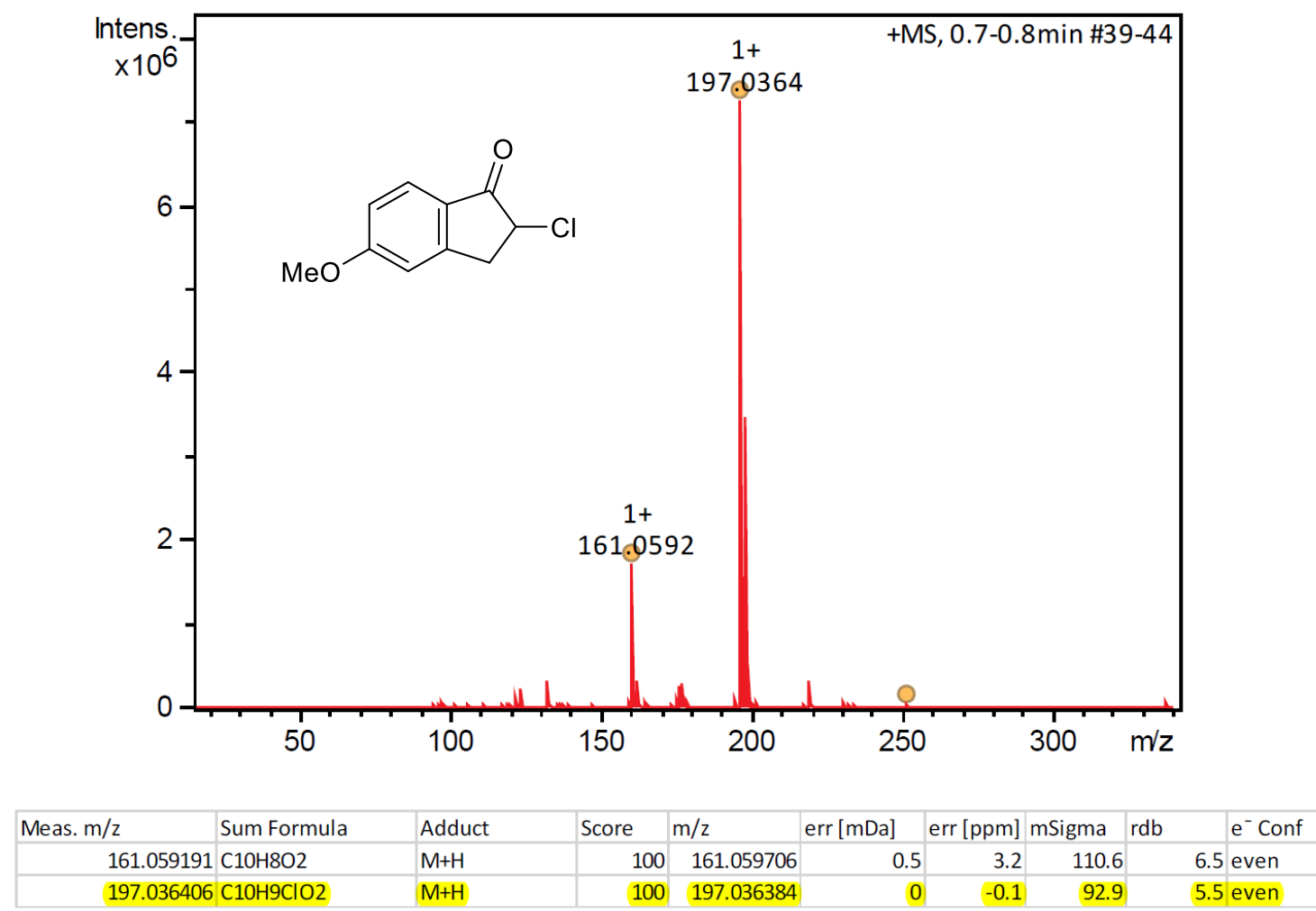

\section{2,5-Dichloro-2,3-dihydro-1 $H$-inden-1-one (3f)}

(APCI positive)

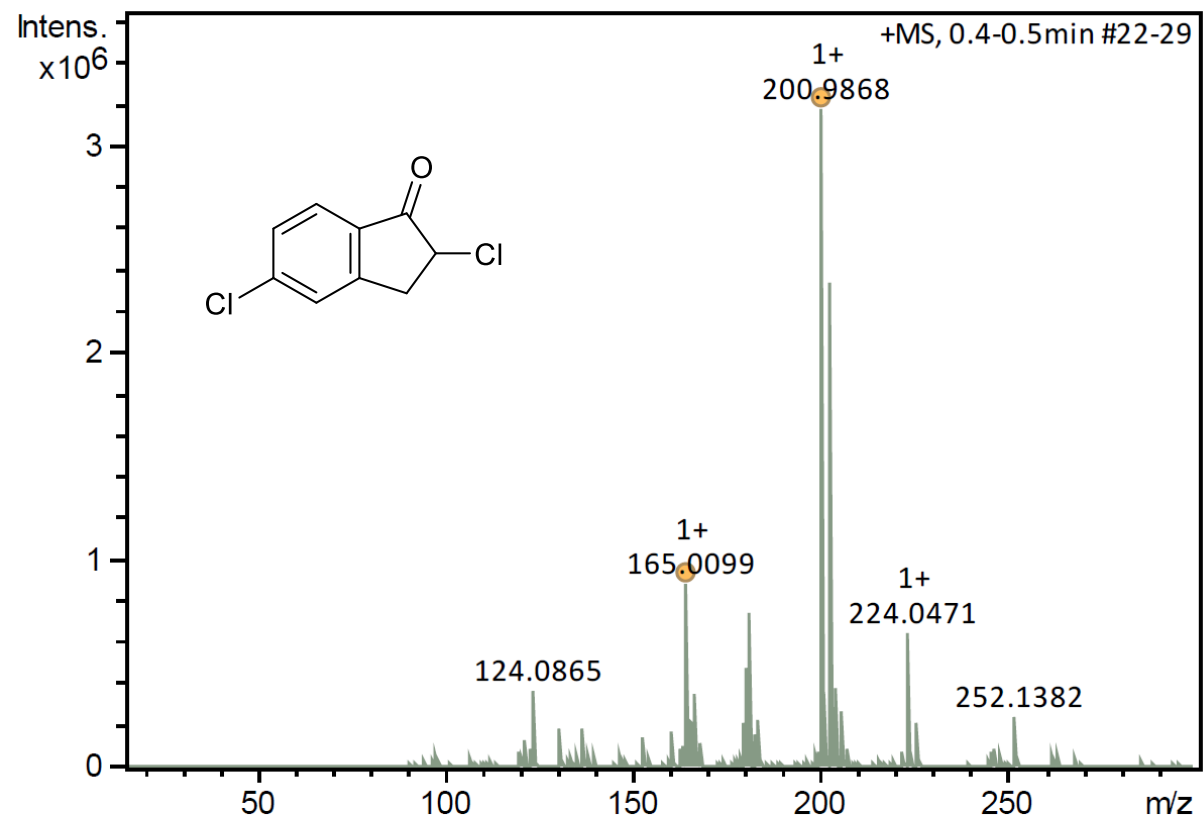

\begin{tabular}{|c|c|c|c|c|c|c|c|c|c|}
\hline Meas. $\mathrm{m} / \mathrm{z}$ & Sum Formula & Adduct & Score & $\mathrm{m} / \mathrm{z}$ & $\operatorname{err}[\mathrm{mDa}]$ & err [ppm] & mSigma & $\mathrm{rdb}$ & $\mathrm{e}^{-}$Conf \\
\hline 165.009895 & $\mathrm{C} 9 \mathrm{H} 5 \mathrm{ClO}$ & $\mathrm{M}+\mathrm{H}$ & 100 & 165.010169 & 0.3 & 1.7 & 87.2 & 6.5 & even \\
\hline 200.986772 & $\mathrm{C}_{2} \mathrm{H}_{6 \mathrm{Cl}} 2 \mathrm{O}$ & $\mathrm{M}+\mathrm{H}$ & 100 & 200.986847 & 0.1 & 0.4 & 38.4 & $5.5 \epsilon$ & even \\
\hline
\end{tabular}


2-Chloro-7-hydroxy-2,3-dihydro-1H-inden-1-one (3g)

(APCI positive)

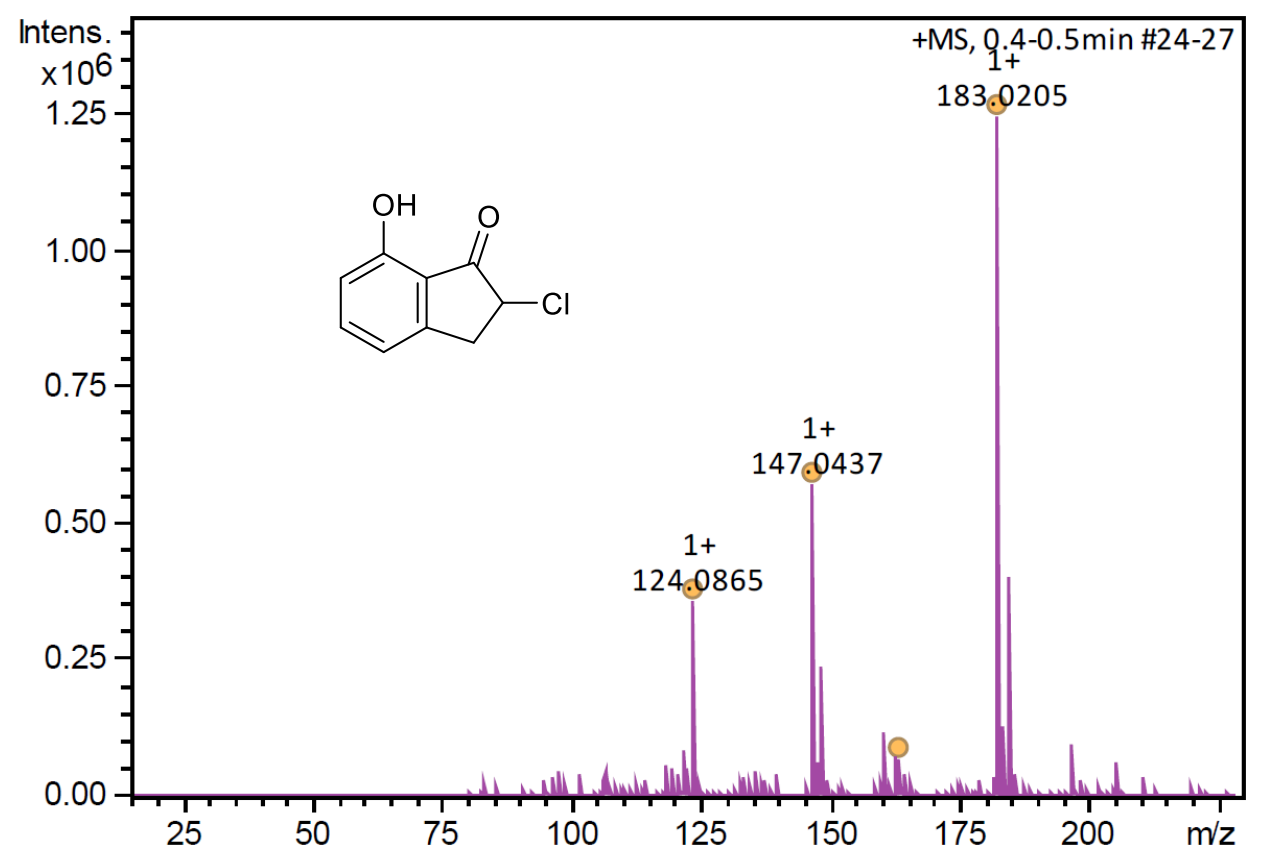

\begin{tabular}{|c|c|c|c|c|c|c|c|c|c|}
\hline Meas. $\mathrm{m} / \mathrm{z}$ & Sum Formula & Adduct & Score & $\mathrm{m} / \mathrm{z}$ & err [mDa] & err [ppm] & mSigma & $\mathrm{rdb}$ & $\mathrm{e}^{-}$Conf \\
\hline 147.043749 & $\mathrm{C} 9 \mathrm{H} 6 \mathrm{O} 2$ & $\mathrm{M}+\mathrm{H}$ & 100 & 147.044056 & 0.3 & 2.1 & 10.4 & 6.5 & even \\
\hline 183.020525 & $\mathrm{C} 9 \mathrm{H} 7 \mathrm{ClO} 2$ & $\mathrm{M}+\mathrm{H}$ & 100 & 183.020734 & 0.2 & 1.1 & 3.3 & 5.5 & even \\
\hline
\end{tabular}

\section{2-Chloro-3,3-dimethyl-2,3-dihydro-1H-inden-1-one (3h)}

(APCI positive)

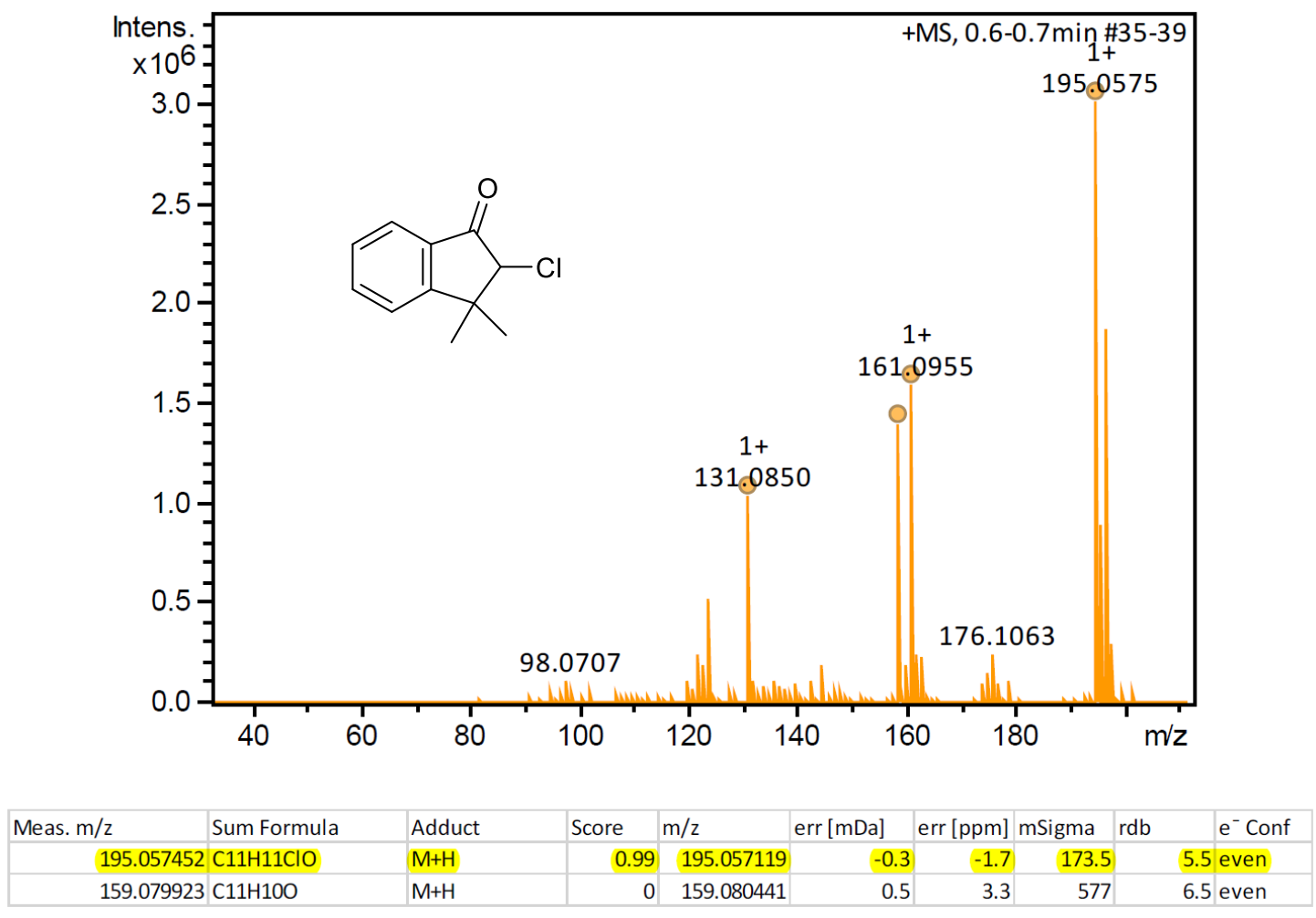




\section{6-Chloro-6,7,8,9-tetrahydro-5H-benzo[7]annulen-5-one (3i)}

(APCI positive)

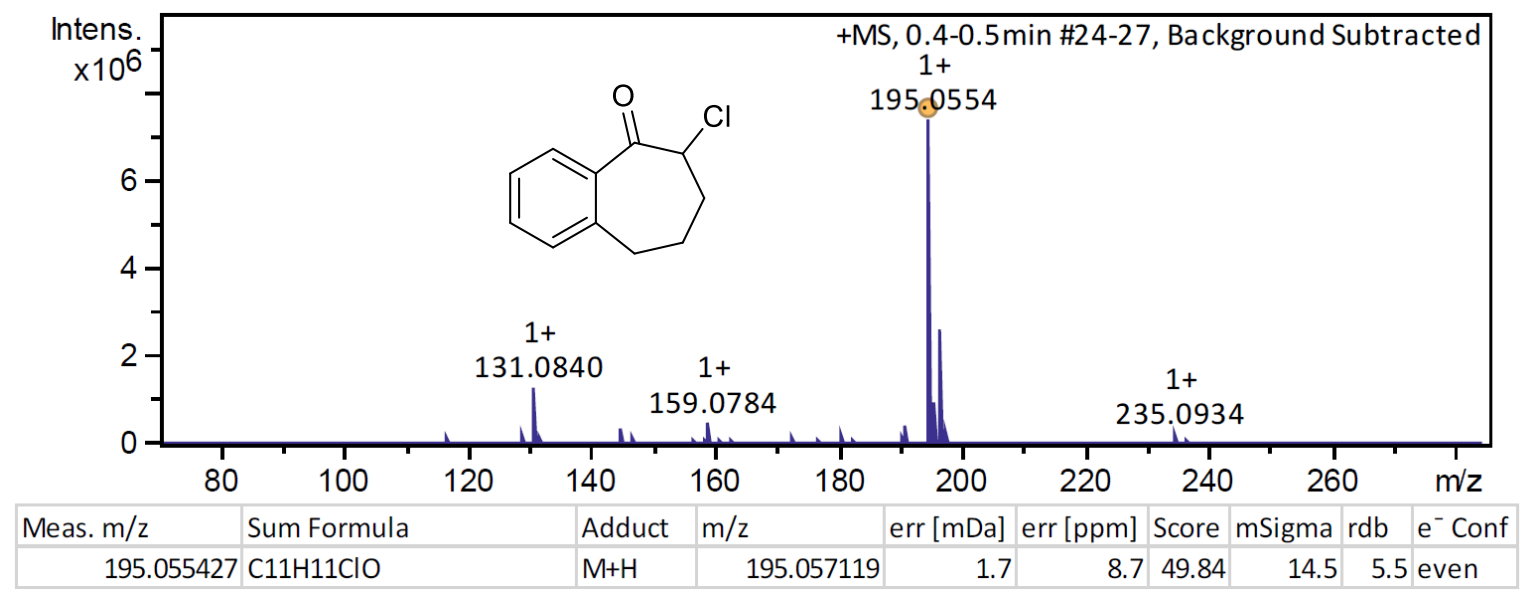

\section{2-Chloroacenaphthylen-1(2H)-one (3j)}

(ESI positive)

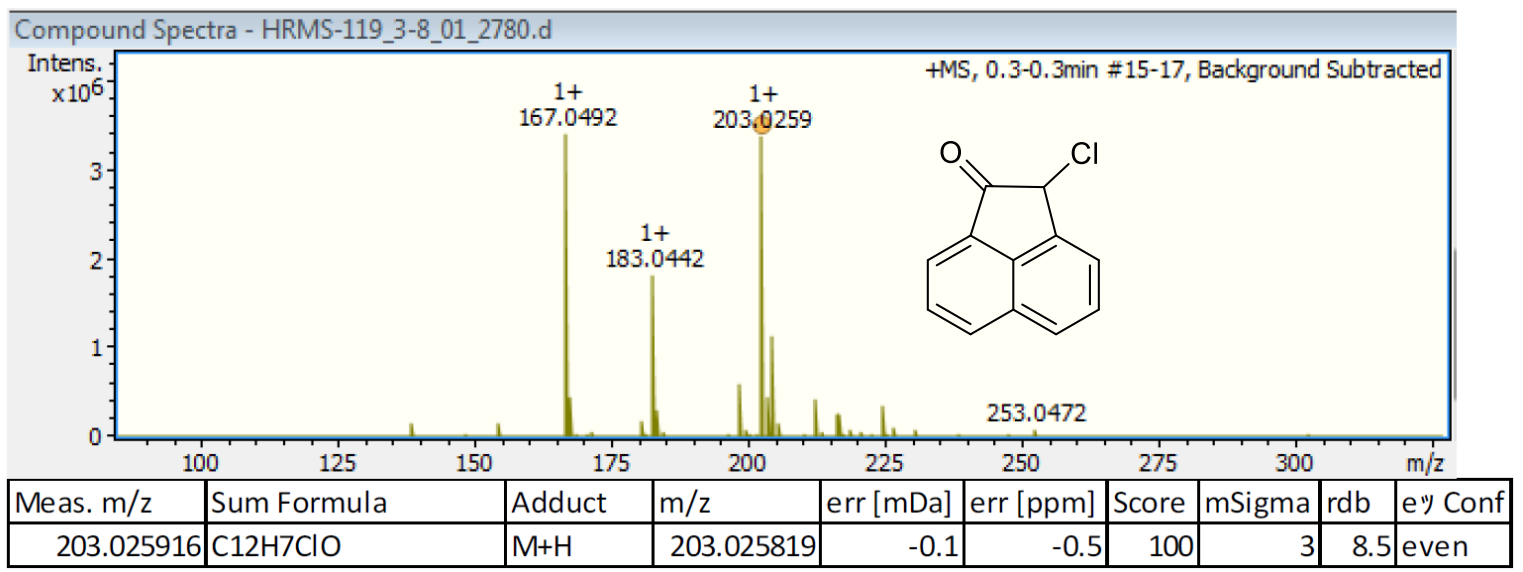


Methyl 1-oxo-2,3-dihydro-1H-indene-2-carboxylate (5a)

(ESI positive)

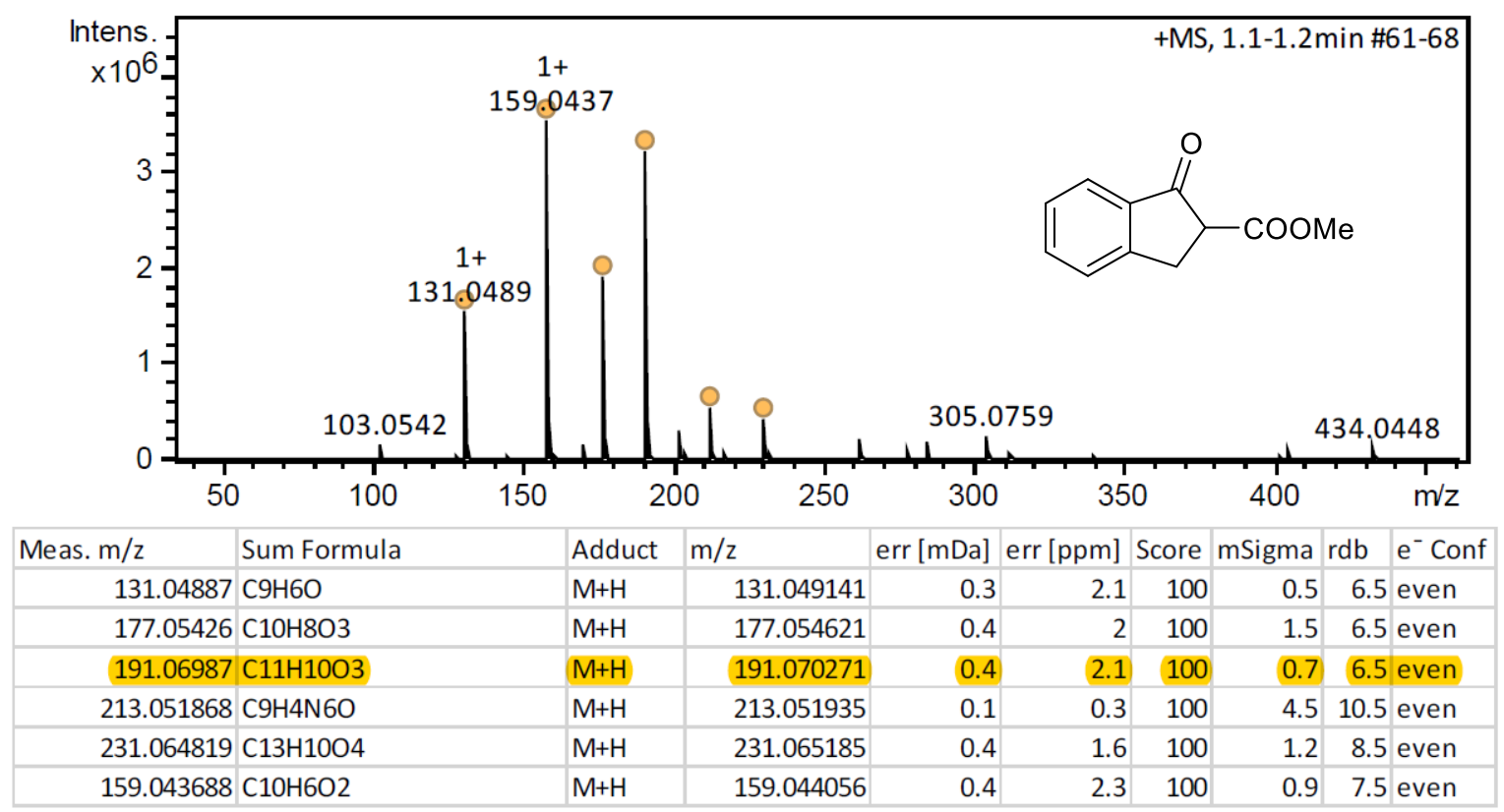

Methyl 5-oxo-6,7,8,9-tetrahydro-5 $H$-benzo[7]annulene-6-carboxylate (5b)

(ESI positive)

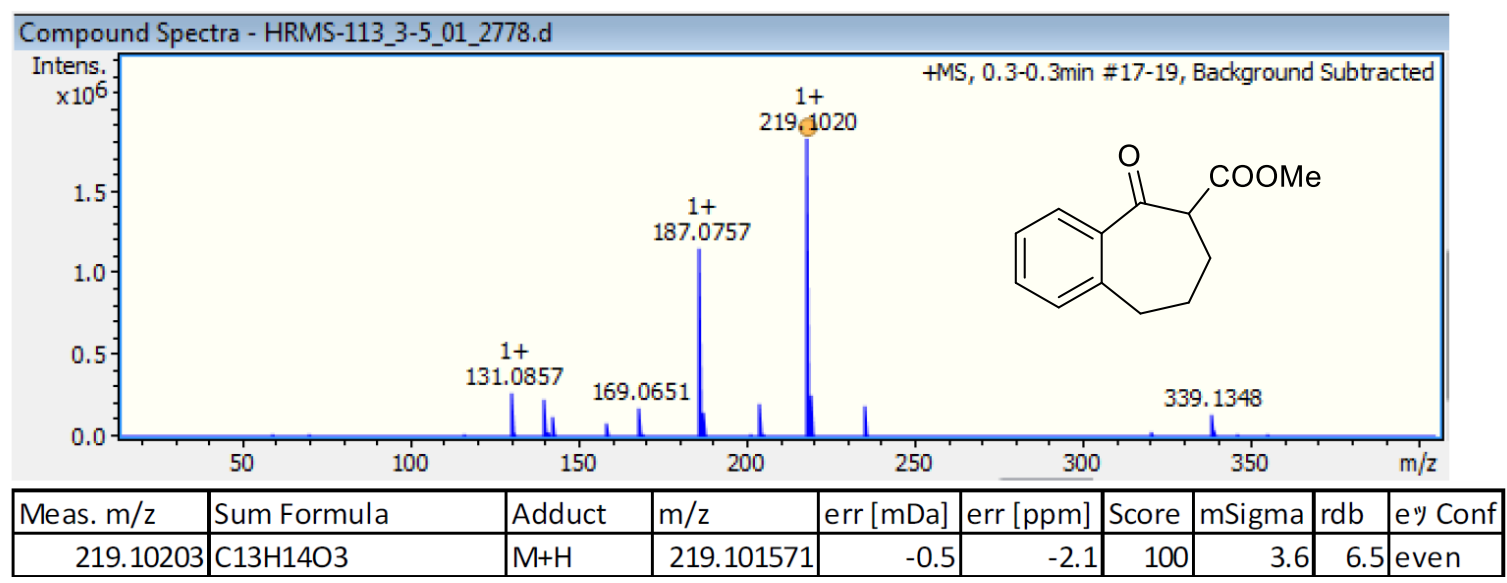


$N$-(tert-Butyl)-1-oxo-2,3-dihydro-1H-indene-2-carboxamide (5c)

(APCI positive)

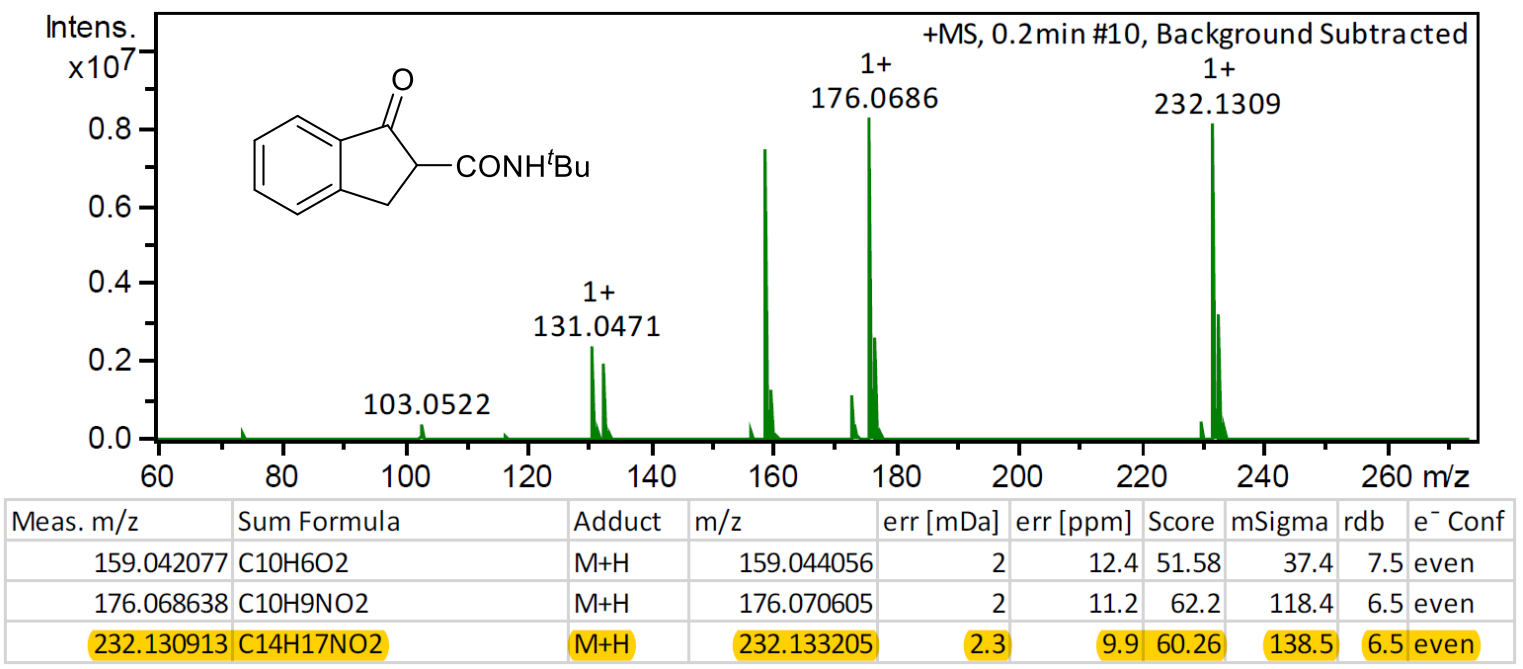

\section{$N$-(tert-Butyl)-1-oxo-1,2,3,4-tetrahydronaphthalene-2-carboxamide (5d)}

(ESI positive)

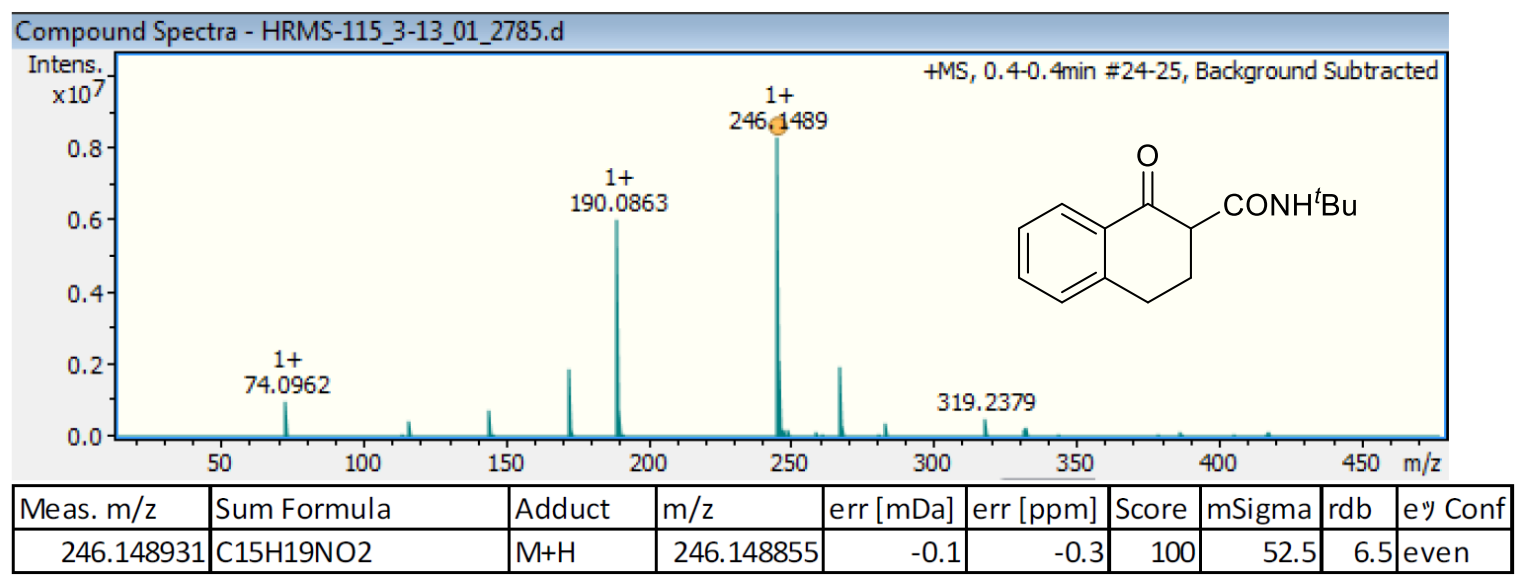


$N$-(tert-butyl)-5-oxo-6,7,8,9-tetrahydro-5H-benzo[7]annulene-6-carboxamide (5e)

(ESI positive)

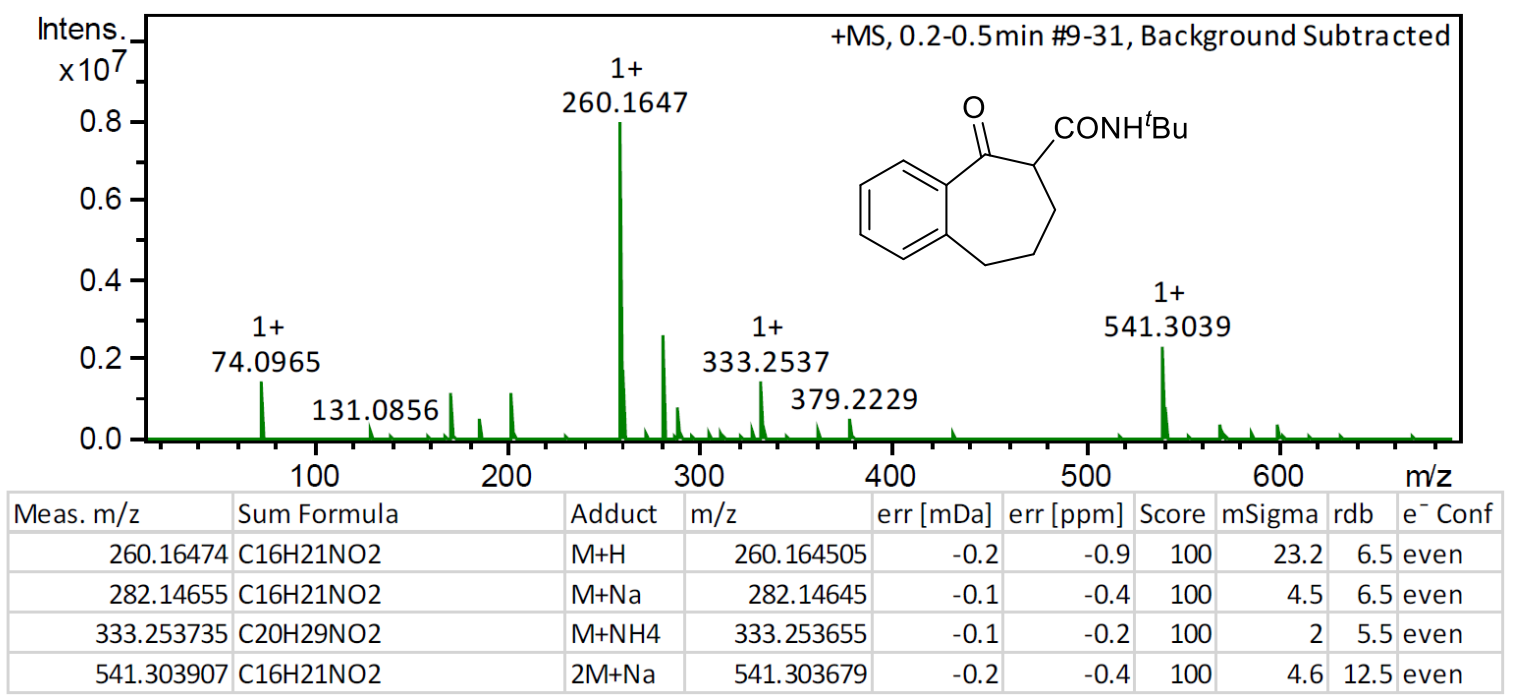

\section{2-(Phenylsulfonyl)-3,4-dihydronaphthalen-1(2H)-one (5f)}

(ESI positive)

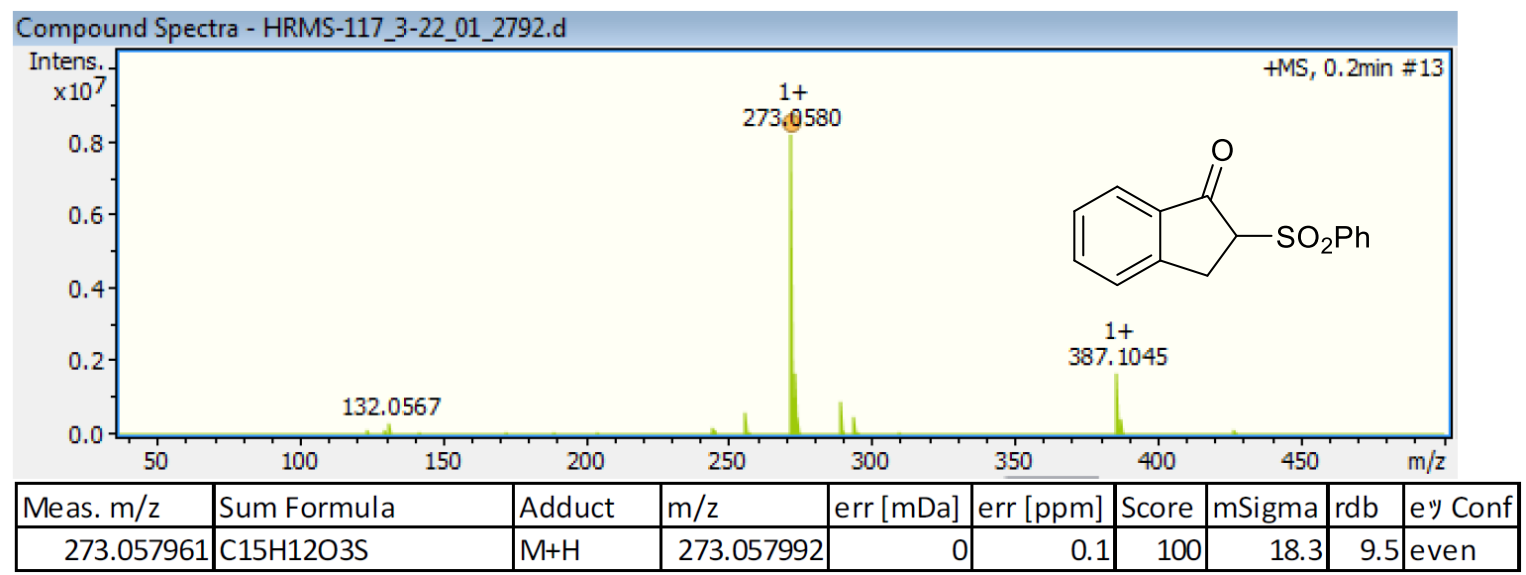




\section{2-(Phenylsulfonyl)-2,3-dihydro-1H-inden-1-one (5g)}

(ESI positive)

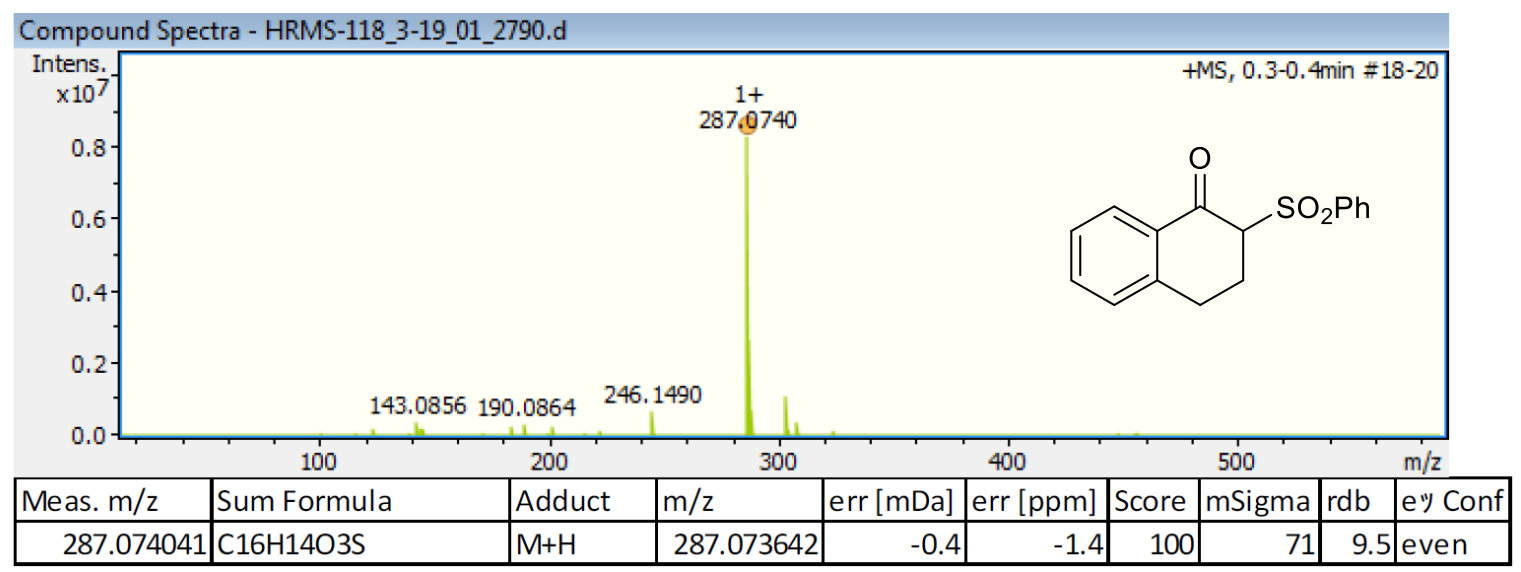

\section{$N$-(tert-Butyl)-2-oxo-1,2-dihydroacenaphthylene-1-carboxamide (5h)}

(ESI positive)

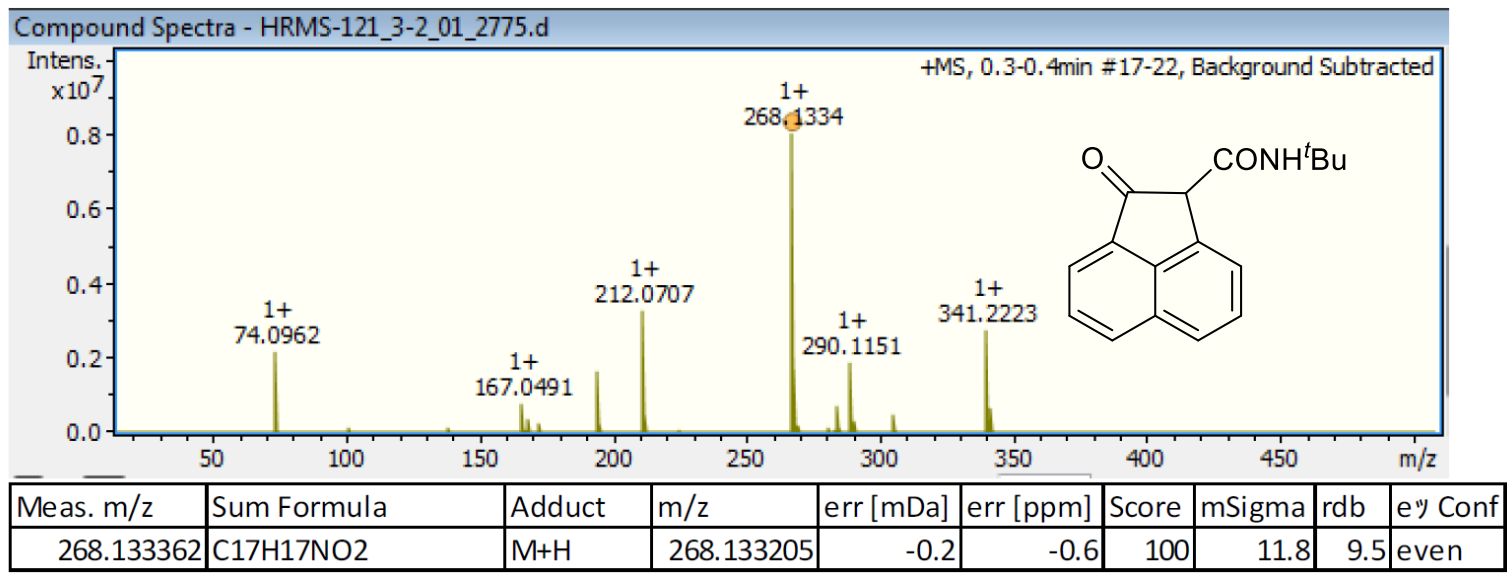


Methyl 2-oxo-1,2-dihydroacenaphthylene-1-carboxylate (5i)

(ESI positive)

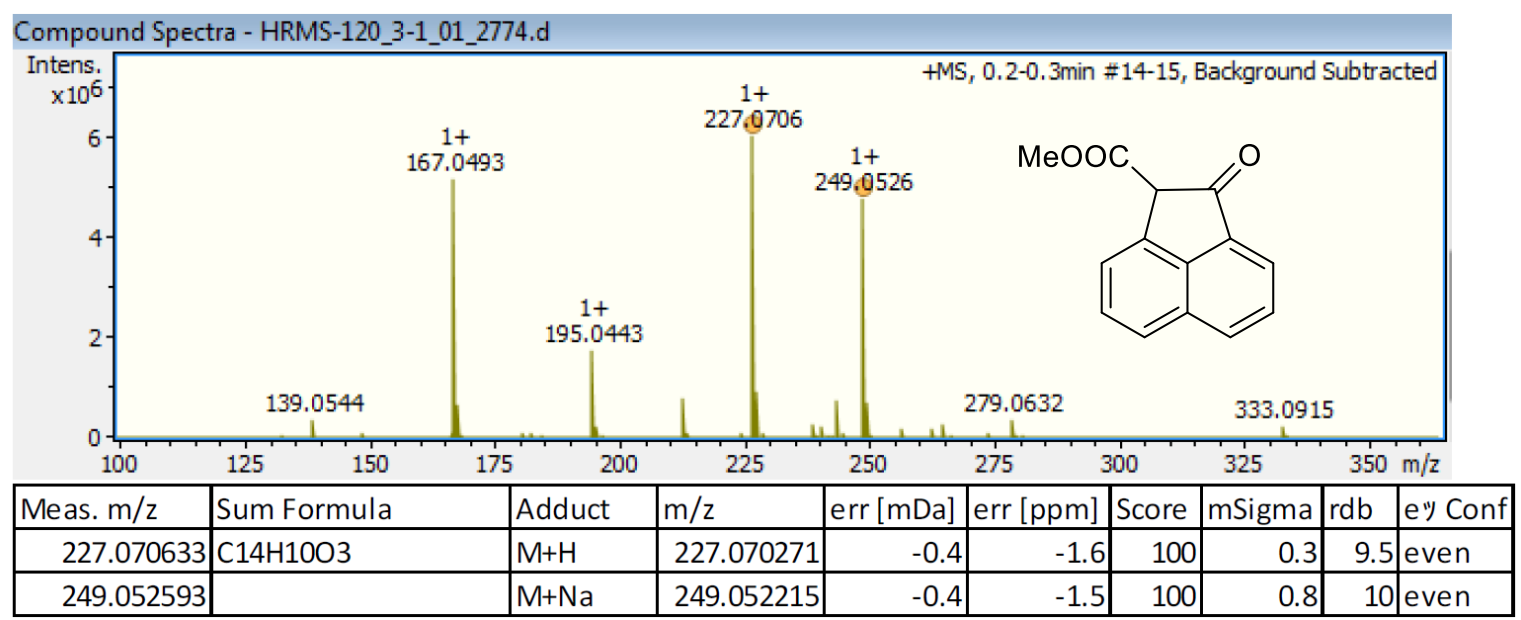

\section{6-Chloro-3-methylcyclohex-2-en-1-one (7a)}

(APCI positive)

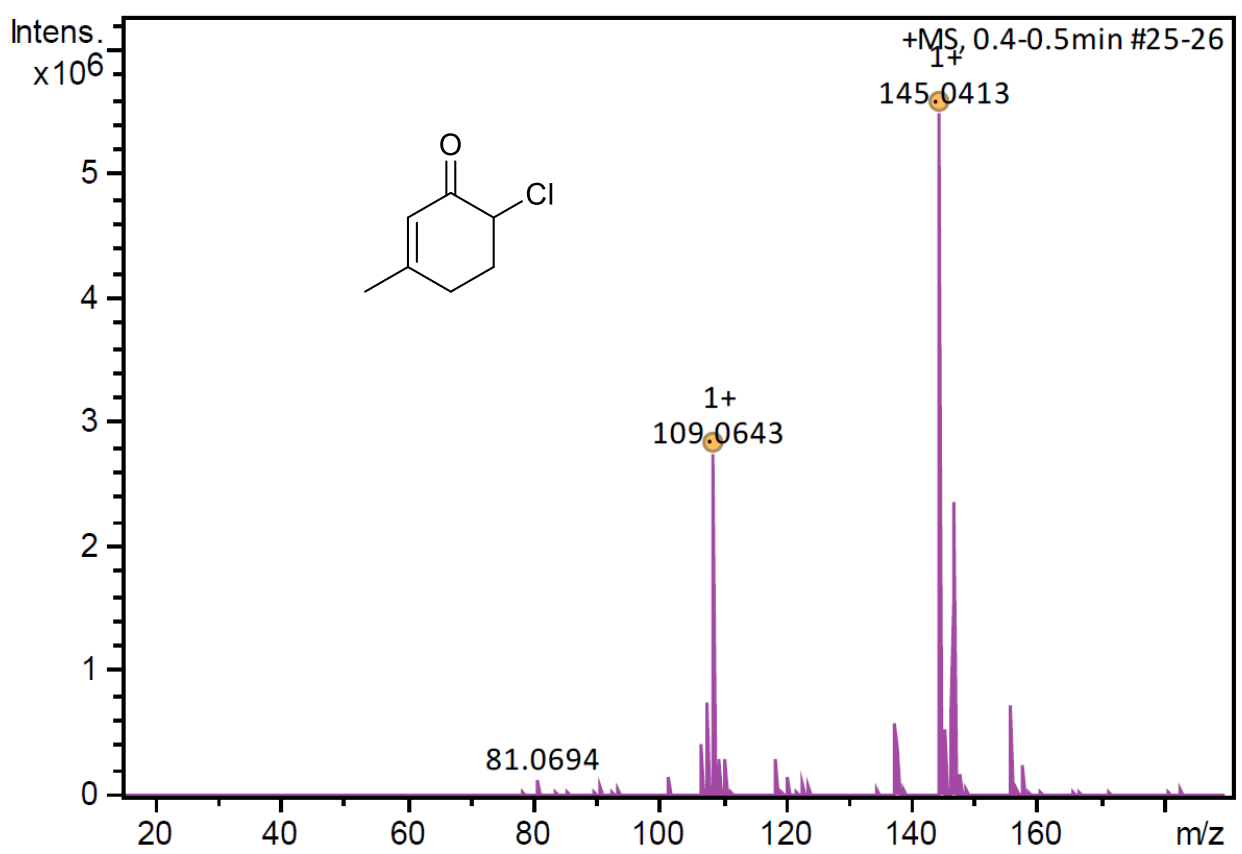

\begin{tabular}{|c|l|l|r|r|r|r|r|r|r|r|}
\hline Meas. $\mathrm{m} / \mathrm{z}$ & Sum Formula & Adduct & Score & $\mathrm{m} / \mathrm{z}$ & err [mDa] & err [ppm] & $\mathrm{mSigma}$ & rdb & $\mathrm{e}^{-}$Conf \\
\hline 145.041276 & $\mathrm{C7H9ClO}$ & $\mathrm{M}+\mathrm{H}$ & 100 & 145.041469 & 0.2 & 1.3 & 54 \\
\hline 109.064262 & $\mathrm{C7H} 80$ & $\mathrm{M}+\mathrm{H}$ & 37.78 & 109.064791 & 0.5 & 4.9 & 64 & 3.5 even \\
\hline
\end{tabular}


Ethyl 4-methyl-2-oxocyclohex-3-ene-1-carboxylate (7b)

(FI)

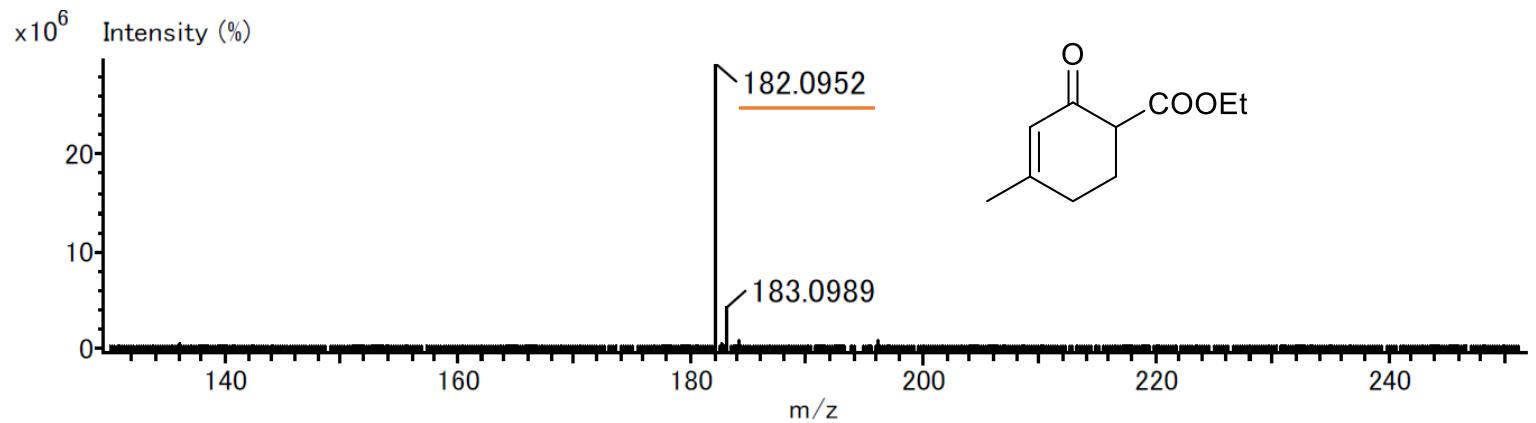

$N$-(tert-Butyl)-4-methyl-2-oxocyclohex-3-ene-1-carboxamide (7c)

(FI)

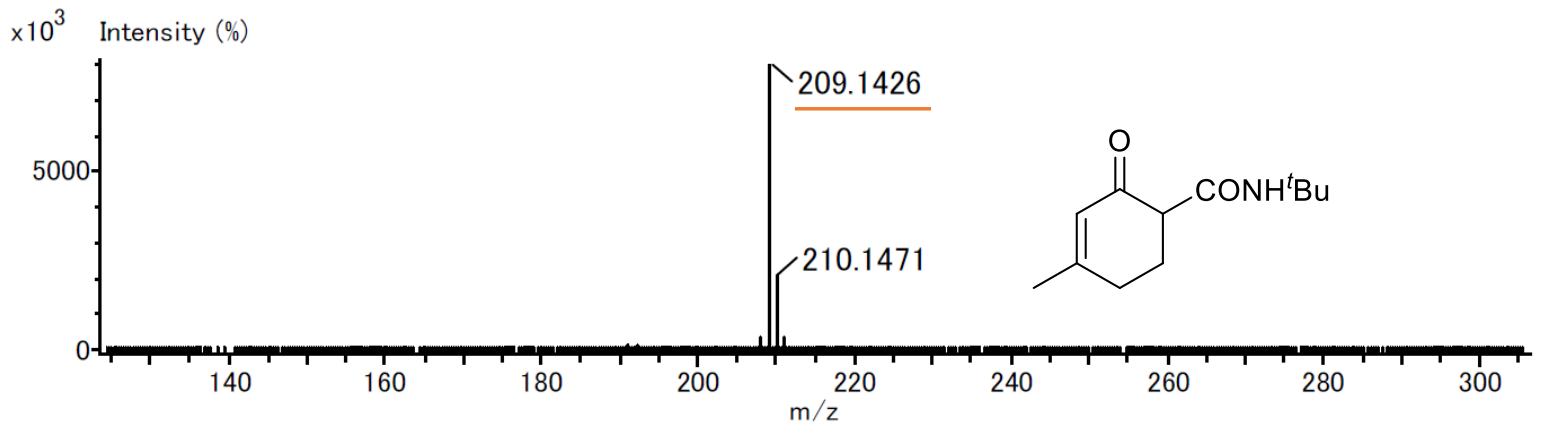


(1S,2R)-2-Chloro-1,2,3,4-tetrahydronaphthalen-1-ol (cis-4a)

(FI)

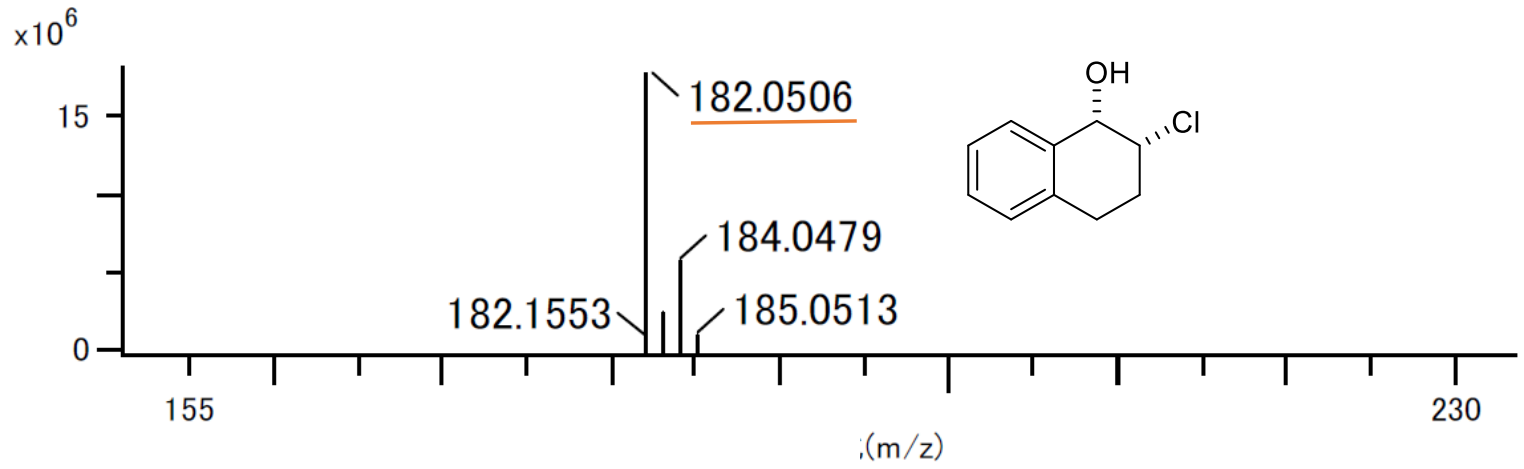

(1S,2R)-2-Chloro-7-methoxy-1,2,3,4-tetrahydronaphthalen-1-ol (cis-4b)

(APCI positive)

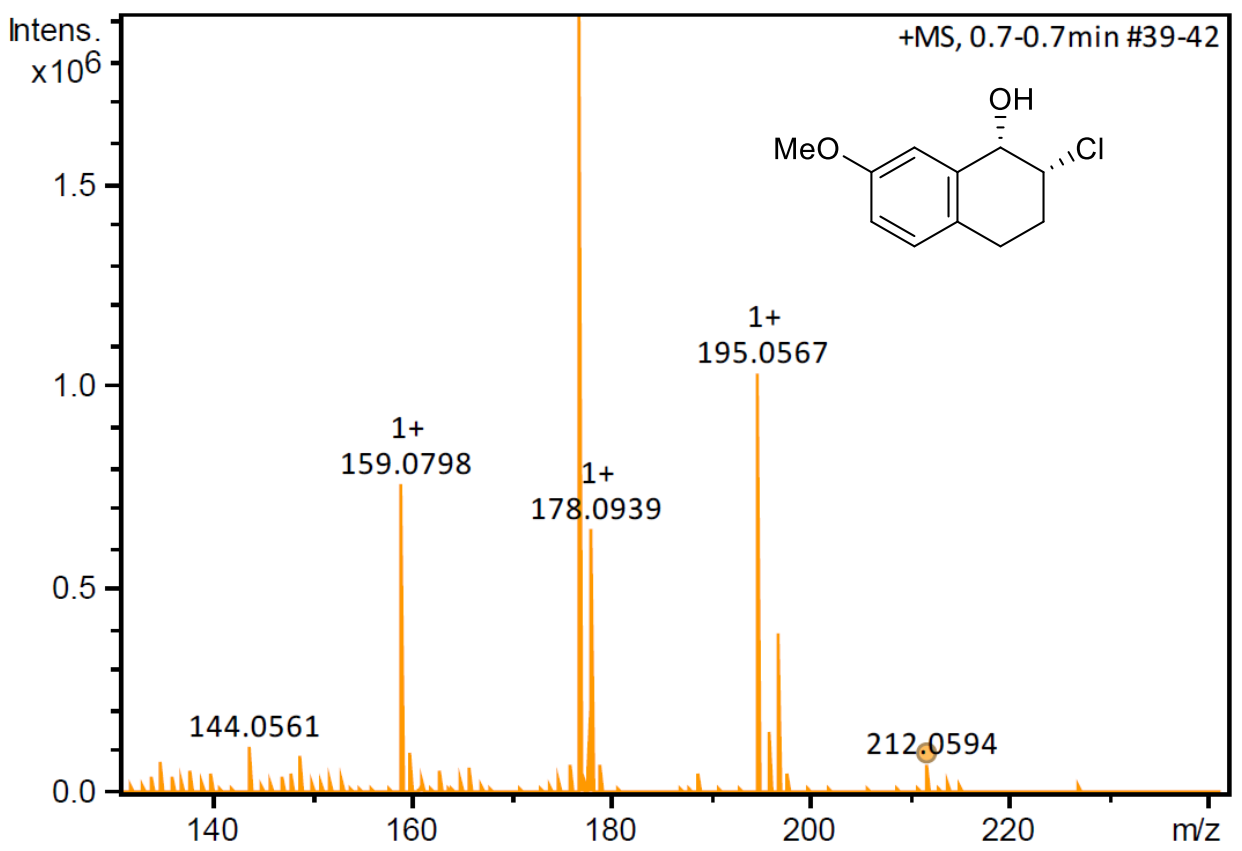

\begin{tabular}{|c|c|c|c|c|c|c|c|c|c|}
\hline Meas. $\mathrm{m} / \mathrm{z}$ & Sum Formula & Adduct & Score & $\mathrm{m} / \mathrm{z}$ & err [mDa] & err [ppm] & mSigma & $\mathrm{rdb}$ & $\mathrm{e}^{-}$Conf \\
\hline 159.079774 & $\mathrm{C} 11 \mathrm{H} 100$ & $\mathrm{M}+\mathrm{H}$ & 100 & 159.080441 & 0.7 & 4.2 & 19 & 6.5 & even \\
\hline 177.090768 & $\mathrm{C} 11 \mathrm{H} 12 \mathrm{O} 2$ & $\mathrm{M}+\mathrm{H}$ & 100 & 177.091006 & 0.2 & 1.3 & 42.7 & 5.5 & even \\
\hline 195.056745 & $\mathrm{C} 11 \mathrm{H} 11 \mathrm{ClO}$ & $\mathrm{M}+\mathrm{H}$ & 100 & 195.057119 & 0.4 & 1.9 & 27.9 & 5.5 & even \\
\hline 212.059407 & $\mathrm{C} 11 \mathrm{H} 13 \mathrm{ClO} 2$ & M & 100 & 212.059859 & 0.5 & 2.1 & 8.8 & & odd \\
\hline
\end{tabular}


(1S,2R)-2-Chloro-7-bromo-1,2,3,4-tetrahydronaphthalen-1-ol (cis-4c)

(CI)

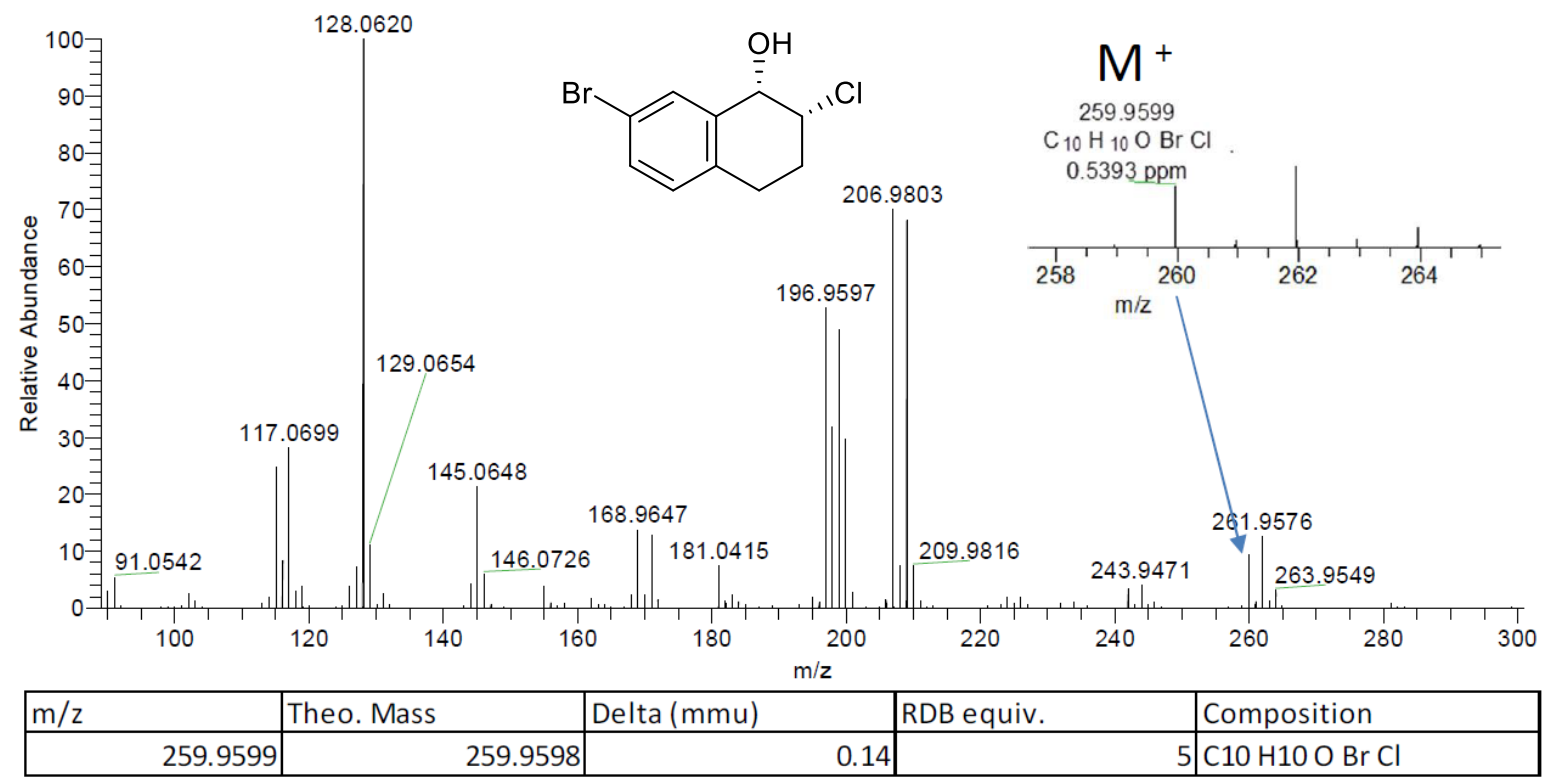

(1S,2R)-2-Chloro-2,3-dihydro-1H-inden-1-ol (cis-4d)

(FI)

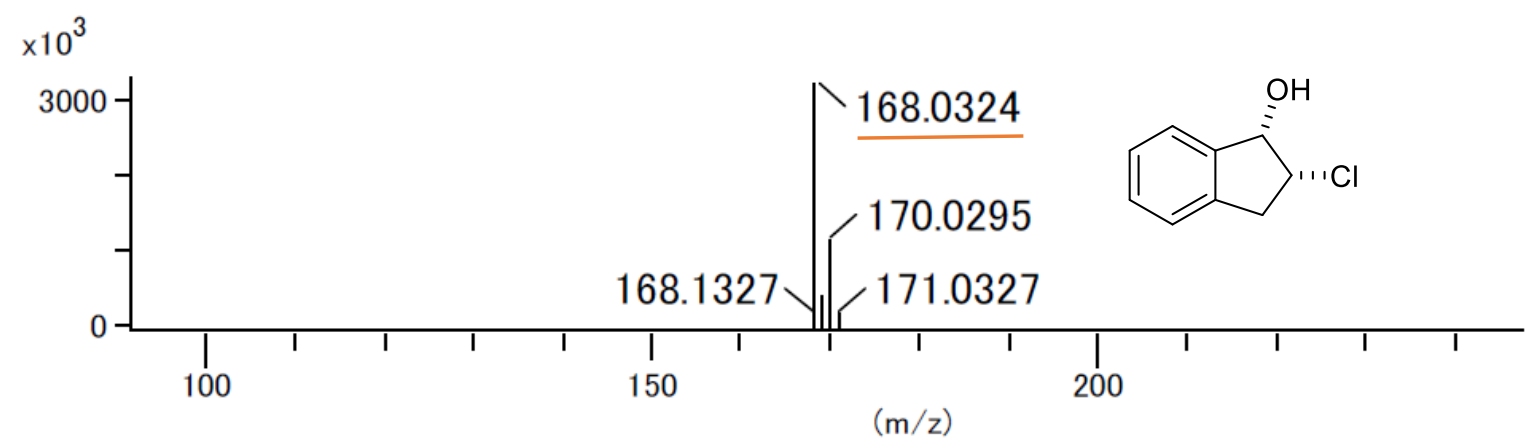


(CI)

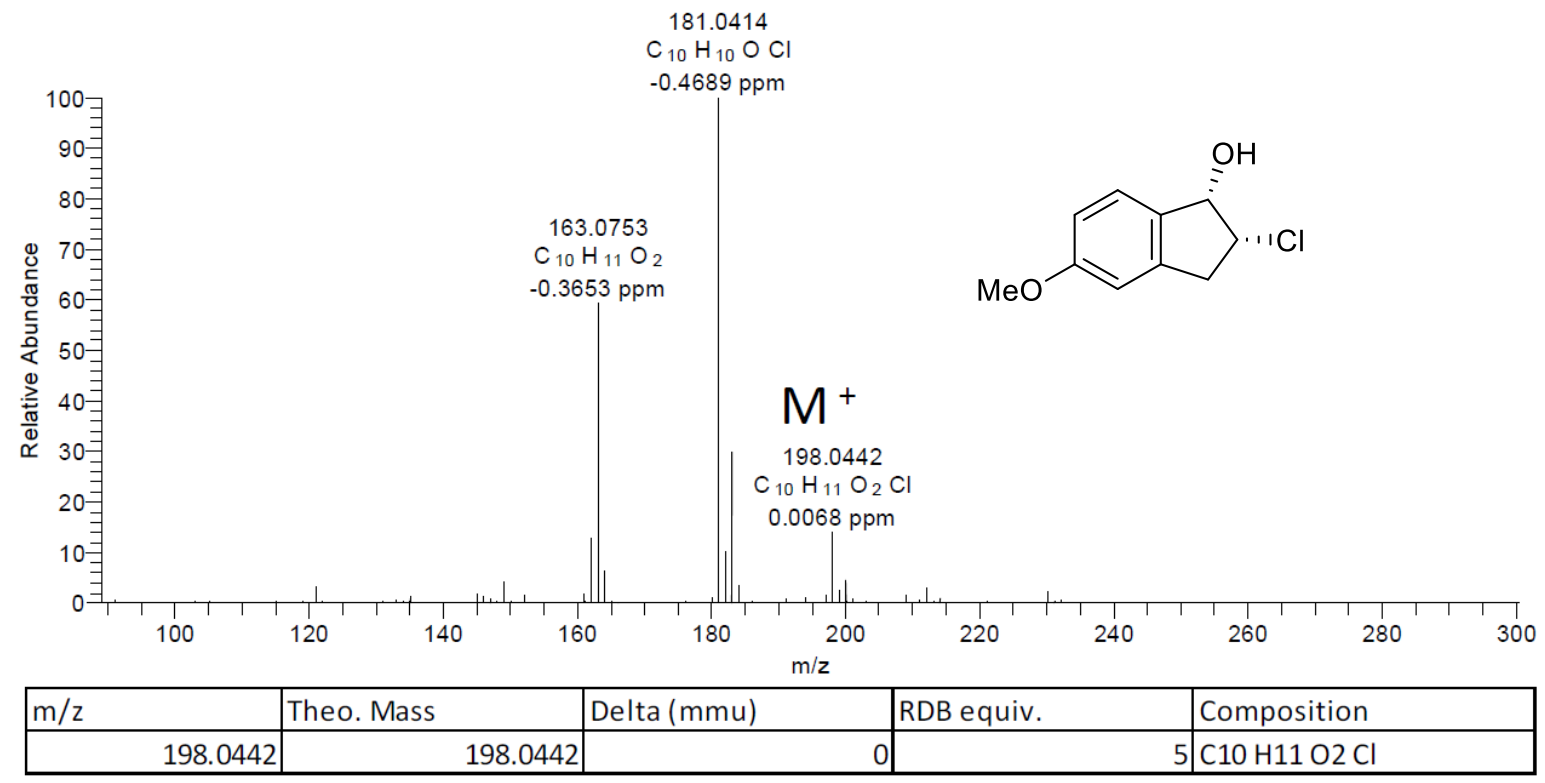

\section{(1S,2R)-2,5-Dichloro-2,3-dihydro-1H-inden-1-ol (cis-4f)}

(CI)

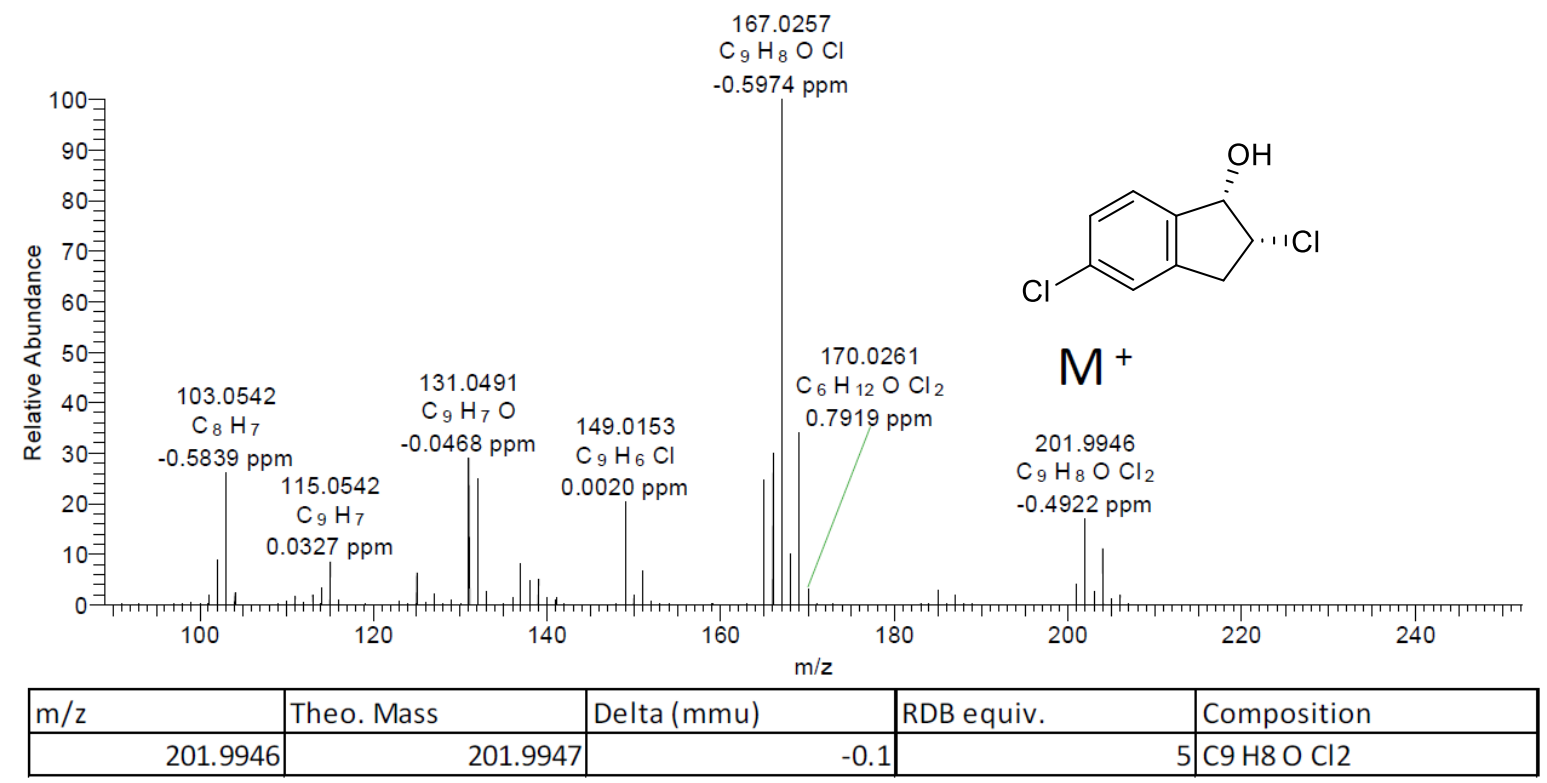


(1S,2R)-2,5-Dichloro-2,3-dihydro-1H-inden-1-ol (cis-4g)

(FI)

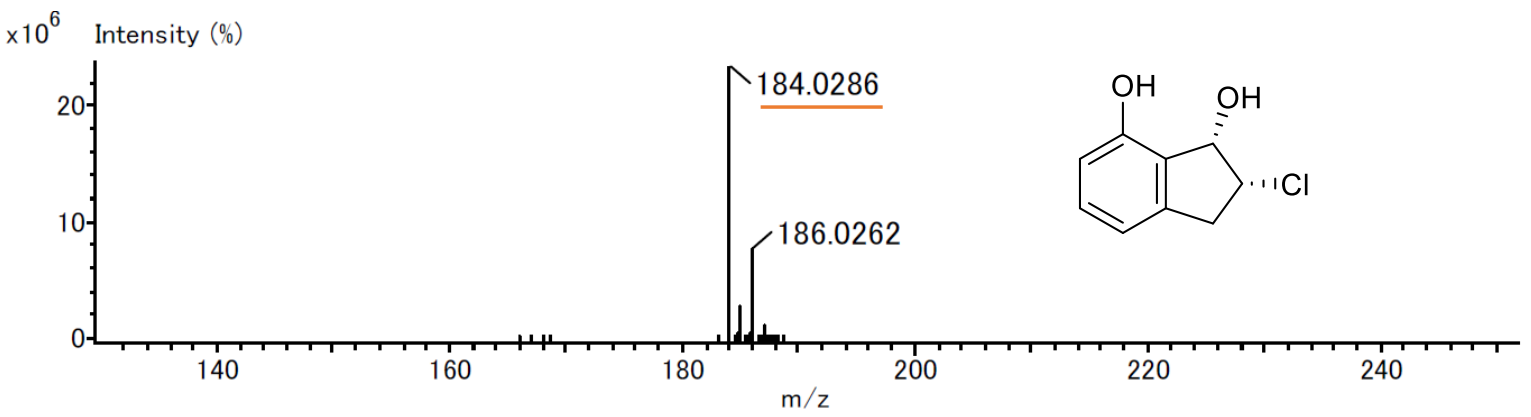

(1S,2R)-2,5-Dichloro-2,3-dihydro-1H-inden-1-ol (cis-4h)

(FI)

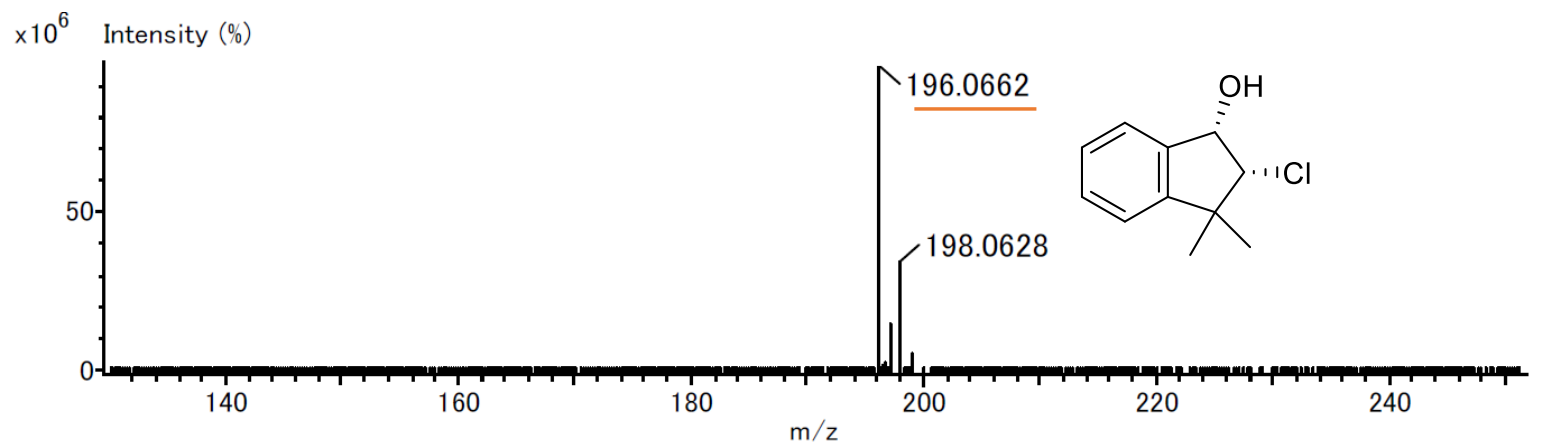


(5S,6R)-6-Chloro-6,7,8,9-tetrahydro-5H-benzo[7]annulen-5-ol (cis-4i)

(FI)

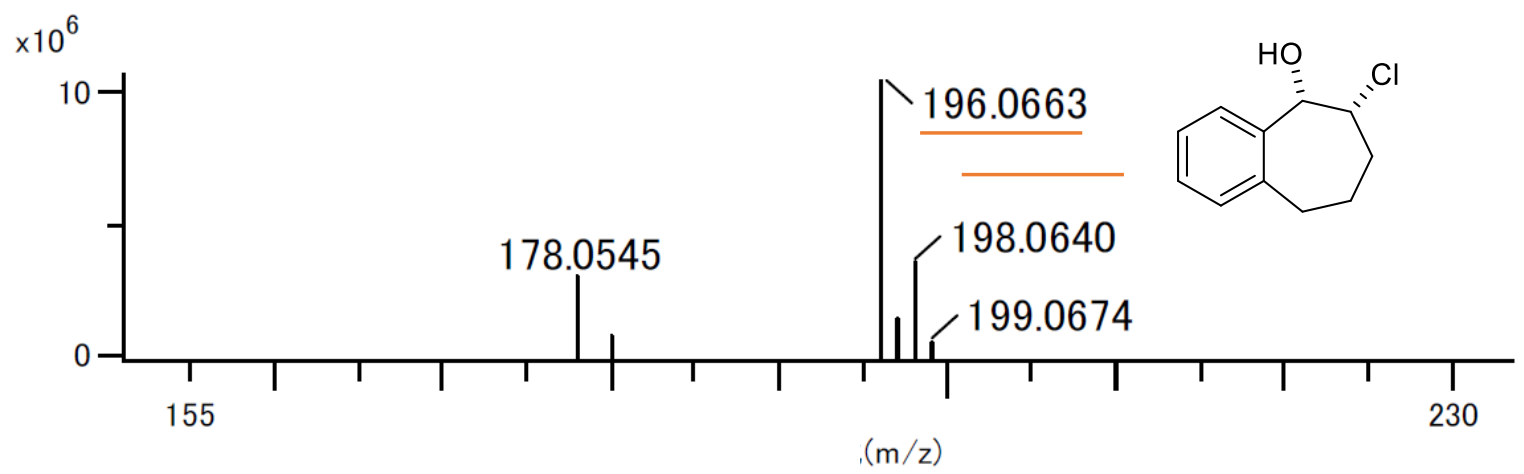

(1S,2R)-2-Chloro-1,2-dihydroacenaphthylen-1-ol (cis-4j)

(FI)

$\times 10^{6}$

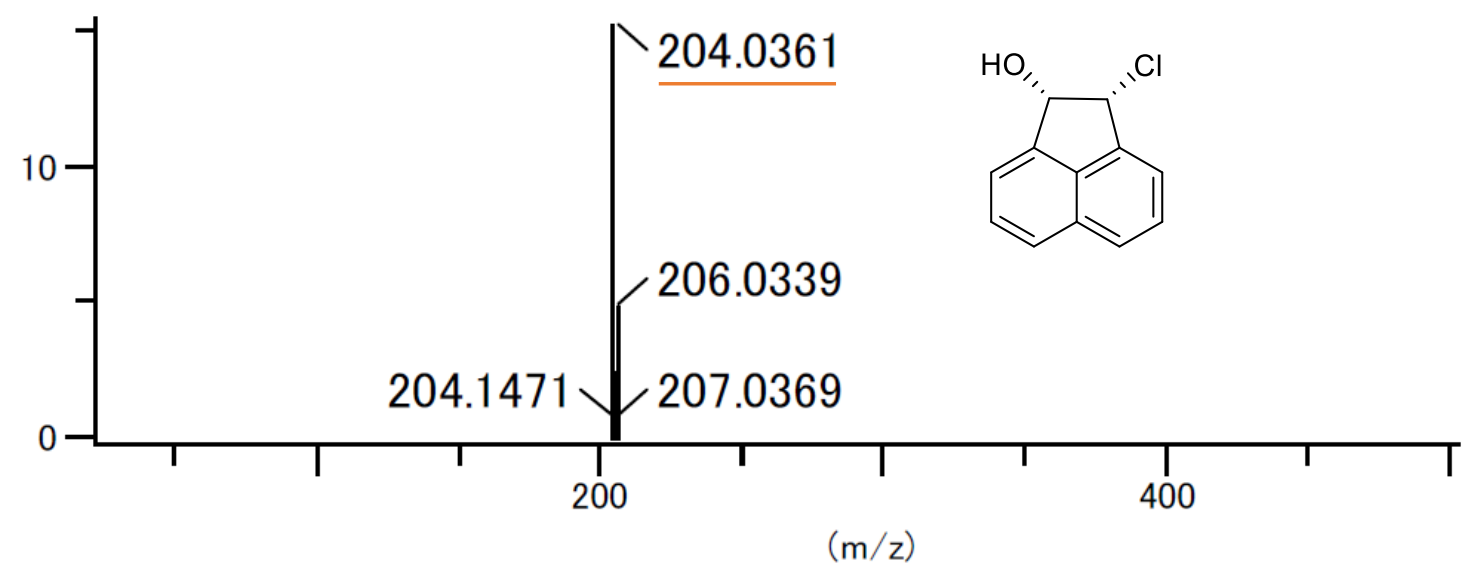


(1S,2R)-2-Bromo-2,3-dihydro-1H-inden-1-ol (cis-4k)

(FI)

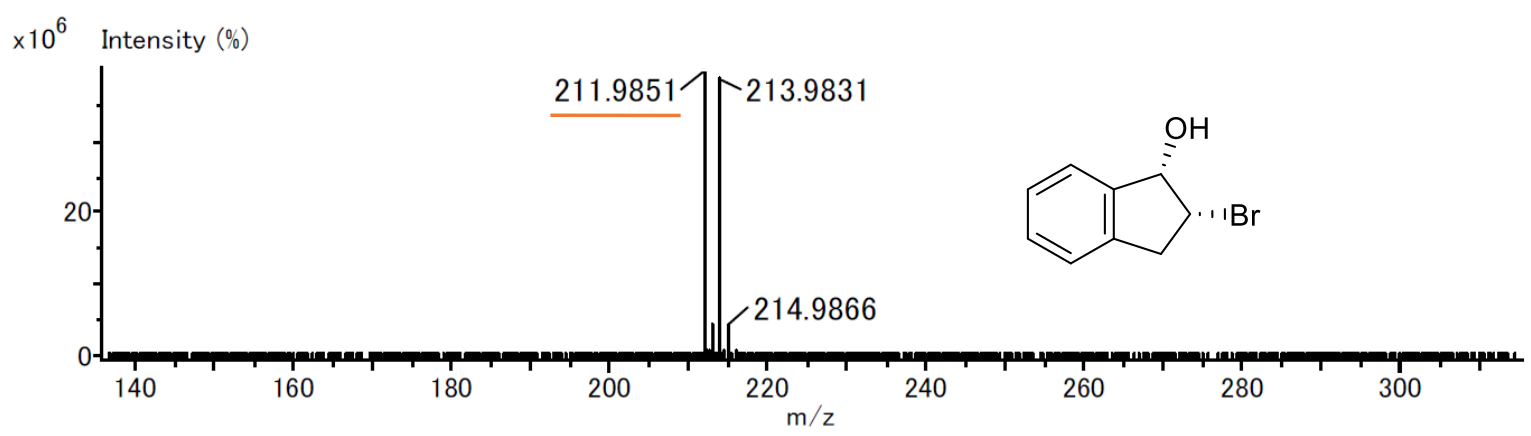

(1S,2R)-2-Bromo-1,2,3,4-tetrahydronaphthalen-1-ol (cis-4l)

(FI)

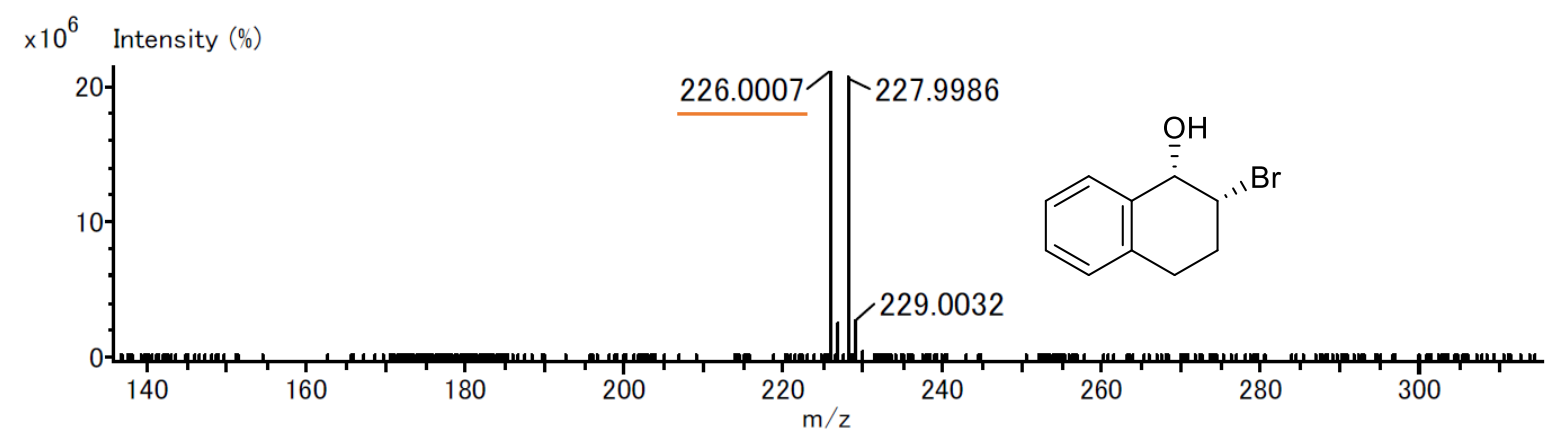


Methyl (1R,2R)-1-hydroxy-2,3-dihydro-1H-indene-2-carboxylate (cis-6a)

(FI)

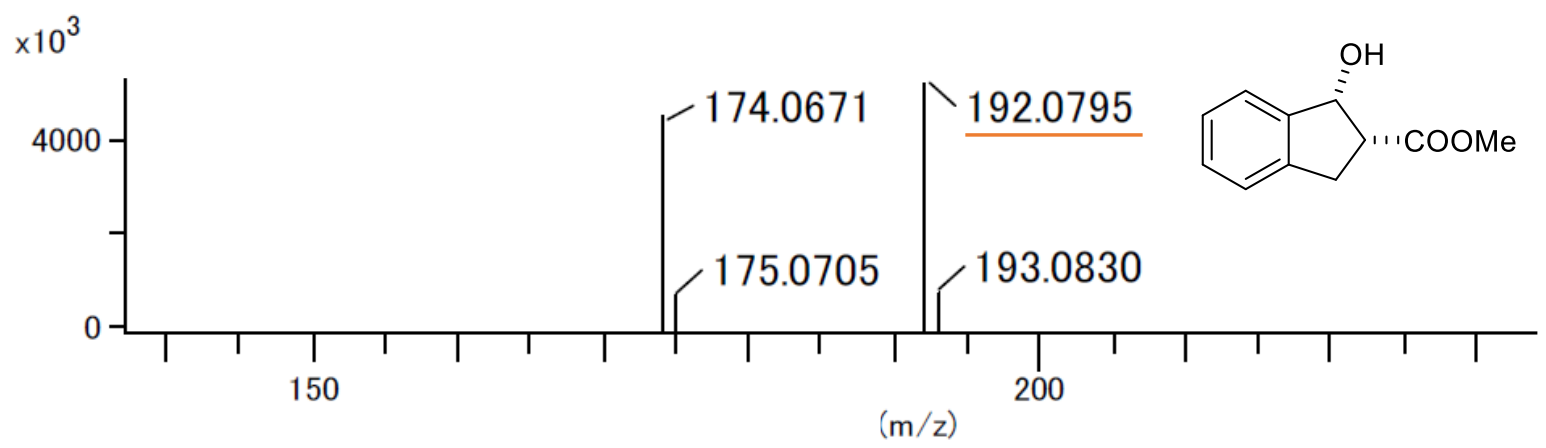

Methyl $(5 R, 6 R)-5$-hydroxy-6,7,8,9-tetrahydro-5H-benzo[7]annulene-6-carboxylate (cis-6b)

(FI)

$\times 10^{6}$

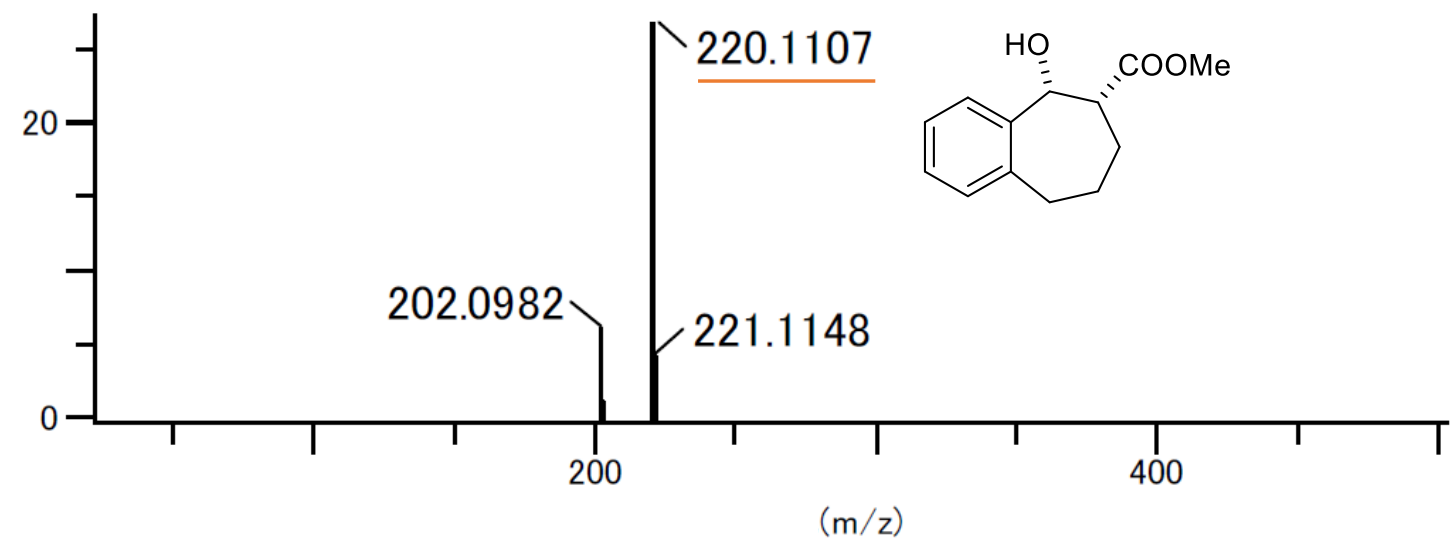


(1R,2R)-N-(tert-Butyl)-1-hydroxy-2,3-dihydro-1H-indene-2-carboxamide (cis-6c)

(FI)

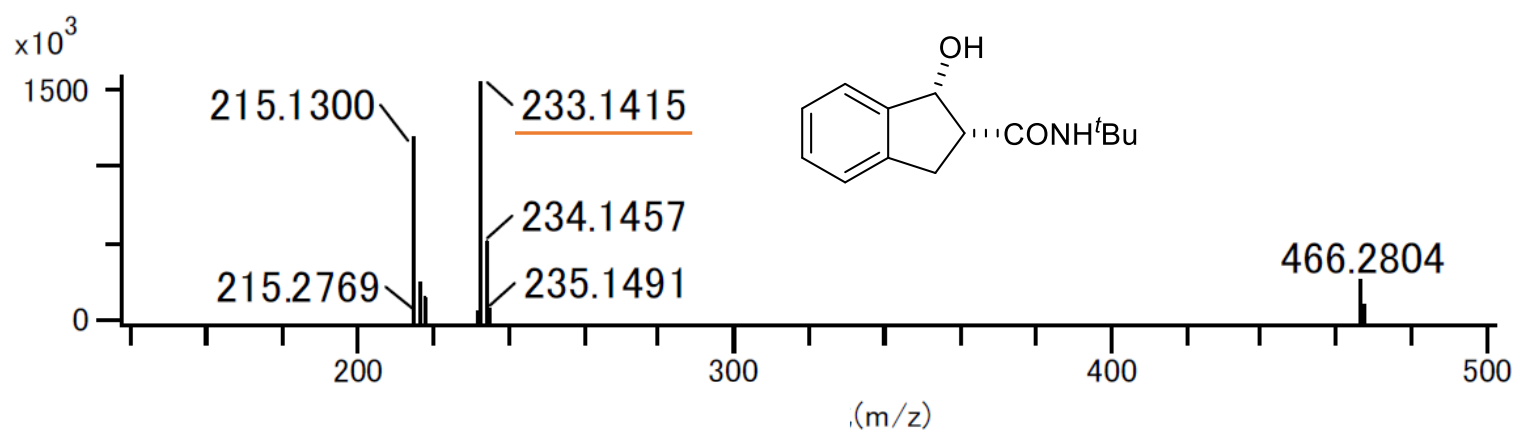

(1R,2R)- $N$-(tert-Butyl)-1-hydroxy-1,2,3,4-tetrahydronaphthalene-2-carboxamide (cis-6d)

(FI)

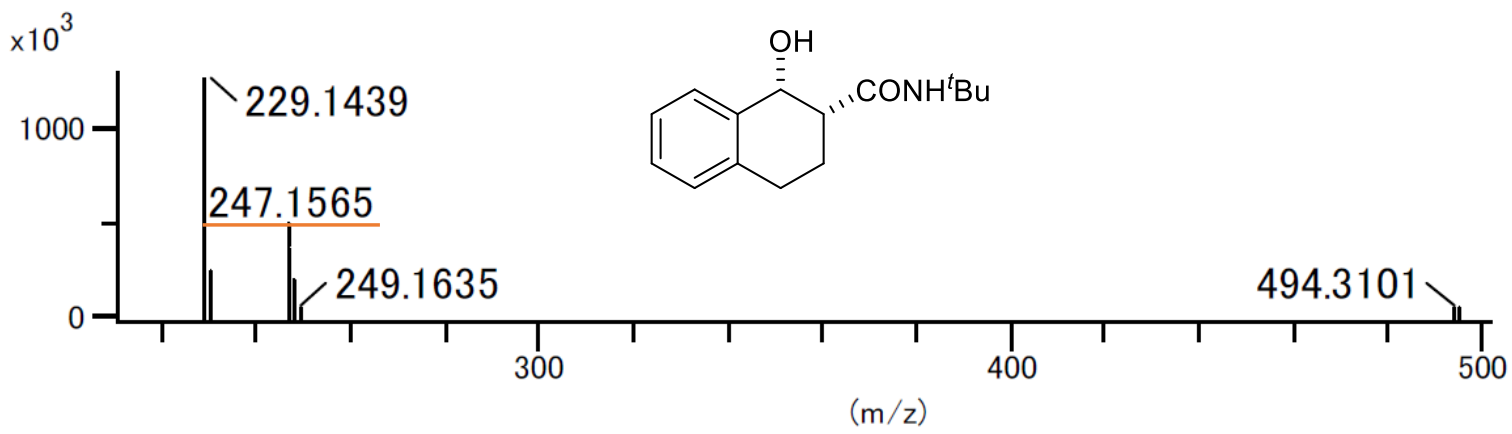


(5R,6R)- $N$-(tert-Butyl)-5-hydroxy-6,7,8,9-tetrahydro-5H-benzo[7]annulene-6-carboxa mide (cis-6e)

(FI)

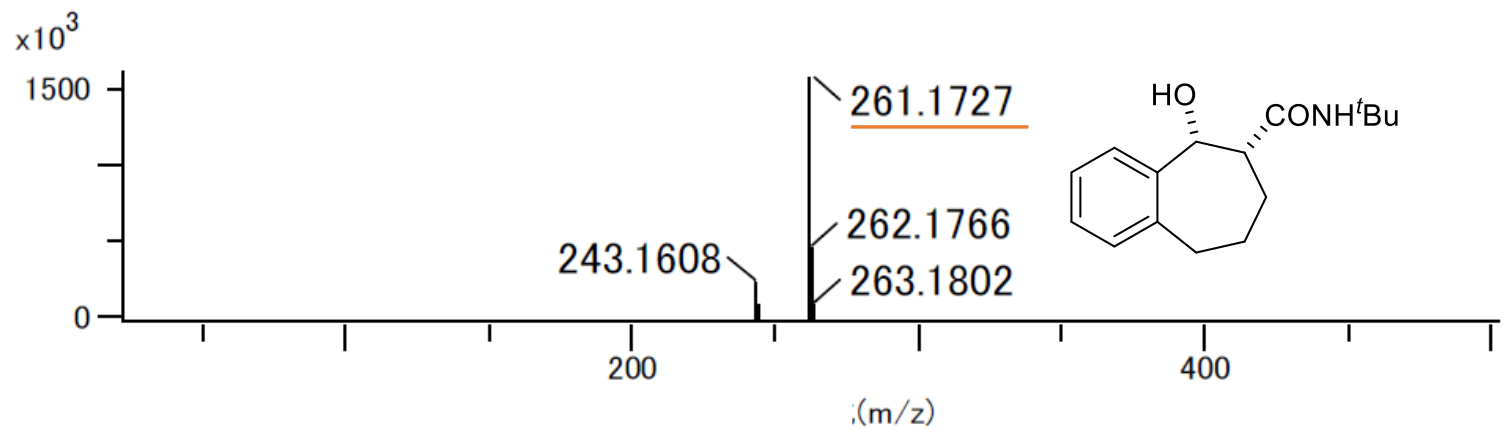

(1S,2R)-2-(Phenylsulfonyl)-2,3-dihydro-1H-inden-1-ol (cis-6f)

(FI)

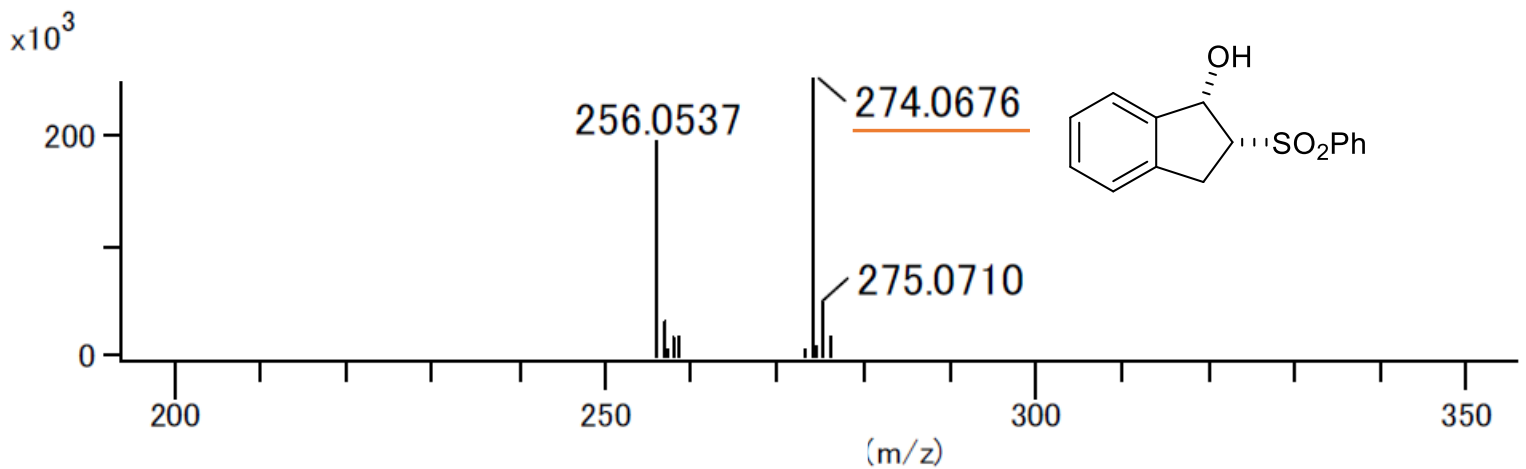




\section{(1S,2R)-2-(Phenylsulfonyl)-1,2,3,4-tetrahydronaphthalen-1-ol (cis-6g)}

(ESI positive)

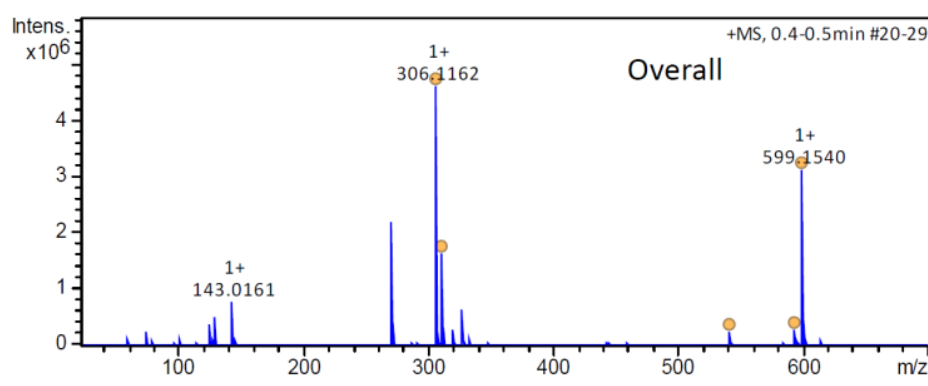<smiles>O[C@H]1c2ccccc2CC[C@@H]1Oc1ccccc1</smiles>
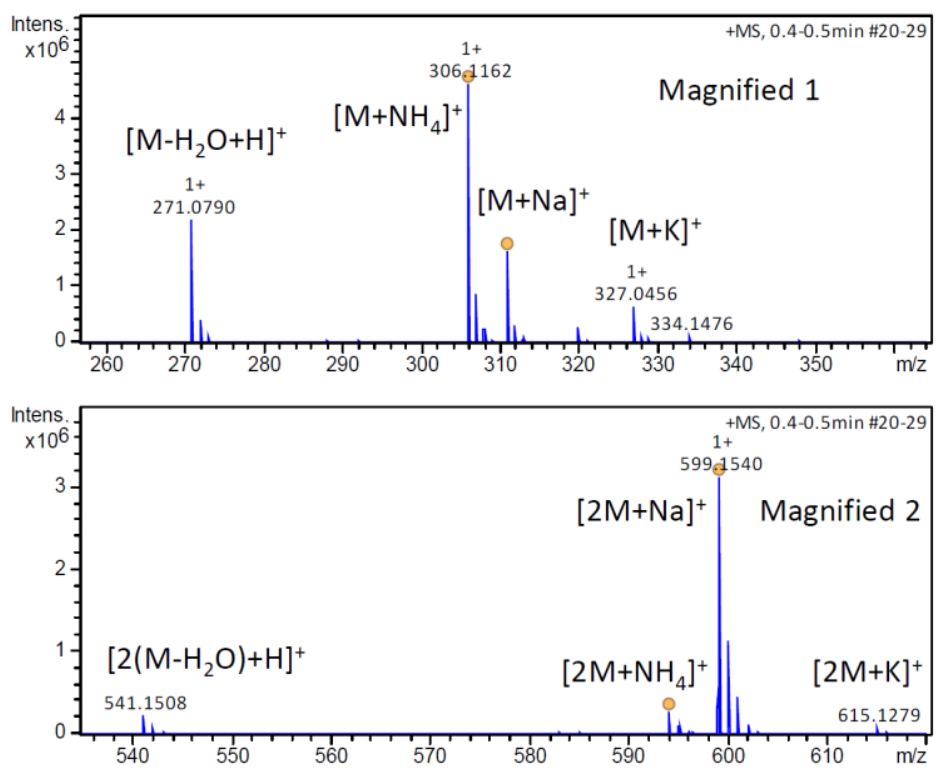

\begin{tabular}{|c|c|c|c|c|c|c|c|c|c|}
\hline Meas. $\mathrm{m} / \mathrm{z}$ & Sum Formula & Adduct & $\mathrm{m} / \mathrm{z}$ & err [mDa] & err [ppm] & Score & mSigma & $\mathrm{rdb}$ & $\mathrm{e}^{-}$Conf \\
\hline \multirow[t]{3}{*}{271.079008} & $\mathrm{C} 16 \mathrm{H} 14 \mathrm{O} 2 \mathrm{~S}$ & $\mathrm{M}+\mathrm{H}$ & 271.078727 & -0.3 & -1 & 100 & 8.6 & 9.5 & even \\
\hline & $\mathrm{C} 11 \mathrm{H} 20 \mathrm{O} 2 \mathrm{~S} 2$ & $\mathrm{M}+\mathrm{Na}$ & 271.079693 & 0.7 & 2.5 & 100 & 34.5 & 1.5 & even \\
\hline & $\mathrm{C} 16 \mathrm{H} 16 \mathrm{OSS}$ & $\mathrm{M}-\mathrm{H} 2 \mathrm{O}+\mathrm{H}$ & 271.078727 & -0.3 & -1 & 100 & 8.6 & 9.5 & even \\
\hline \multirow[t]{2}{*}{306.116233} & $\mathrm{C} 13 \mathrm{H} 2106 \mathrm{~S}$ & $\mathrm{M}+\mathrm{H}$ & 306.113161 & -3.1 & -10 & 100 & 18.5 & & odd \\
\hline & $\mathrm{C} 16 \mathrm{H} 16 \mathrm{OSS}$ & $\mathrm{M}+\mathrm{NH} 4$ & 306.115841 & -0.4 & -1.3 & 100 & 6.9 & 7.5 & even \\
\hline \multirow[t]{2}{*}{311.071586} & $\mathrm{C} 18 \mathrm{H} 14 \mathrm{OSS}$ & $\mathrm{M}+\mathrm{H}$ & 311.073642 & 2.1 & 6.6 & 100 & 15.1 & 11.5 & even \\
\hline & $\mathrm{C} 16 \mathrm{H} 16 \mathrm{OS} \mathrm{S}$ & $\mathrm{M}+\mathrm{Na}$ & 311.071236 & -0.3 & -1.1 & 100 & 7.9 & 8.5 & even \\
\hline \multirow[t]{4}{*}{327.045576} & $\mathrm{C} 21 \mathrm{H} 10 \mathrm{O} 2 \mathrm{~S}$ & $\mathrm{M}+\mathrm{H}$ & 327.047427 & 1.9 & 5.7 & 100 & 37.2 & 16.5 & even \\
\hline & $\mathrm{C} 10 \mathrm{H} 1307 \mathrm{~S} 2$ & $\mathrm{M}+\mathrm{NH} 4$ & 327.044095 & -1.5 & -4.5 & 100 & 26.8 & & odd \\
\hline & $\mathrm{C} 19 \mathrm{H} 12 \mathrm{O} 2 \mathrm{~S}$ & $\mathrm{M}+\mathrm{Na}$ & 327.045021 & -0.6 & -1.7 & 100 & 32.5 & 13.5 & even \\
\hline & $\mathrm{C} 16 \mathrm{H} 16 \mathrm{OS} \mathrm{S}$ & $M+K$ & 327.045173 & -0.4 & -1.2 & 100 & 6.4 & 8.5 & even \\
\hline \multirow[t]{3}{*}{541.150836} & $\mathrm{C} 32 \mathrm{H} 28 \mathrm{O} 4 \mathrm{~S} 2$ & $\mathrm{M}+\mathrm{H}$ & 541.150178 & -0.7 & -1.2 & 100 & 11.9 & 18.5 & even \\
\hline & $\mathrm{C} 26 \mathrm{H} 3009 \mathrm{~S}$ & $\mathrm{M}+\mathrm{Na}$ & 541.150274 & -0.6 & -1 & 100 & 39.5 & 11.5 & even \\
\hline & $\mathrm{C} 16 \mathrm{H} 14 \mathrm{O} 2 \mathrm{~S}$ & $2 \mathrm{M}+\mathrm{H}$ & 541.150178 & -0.7 & -1.2 & 100 & 11.9 & 18.5 & even \\
\hline \multirow[t]{3}{*}{594.19847} & $\mathrm{C} 32 \mathrm{H} 32 \mathrm{O} 6 \mathrm{~S} 2$ & $\mathrm{M}+\mathrm{NH} 4$ & 594.197856 & -0.6 & -1 & 100 & 12 & 15.5 & even \\
\hline & C41H31OS & $\mathrm{M}+\mathrm{Na}$ & 594.198782 & 0.3 & 0.5 & 100 & 41.6 & 26 & odd \\
\hline & $\mathrm{C} 16 \mathrm{H} 16 \mathrm{OSS}$ & $2 \mathrm{M}+\mathrm{NH} 4$ & 594.197856 & -0.6 & -1 & 100 & 12 & 15.5 & even \\
\hline \multirow[t]{4}{*}{599.153976} & $\mathrm{C} 34 \mathrm{H} 30 \mathrm{OSS} 2$ & $\mathrm{M}+\mathrm{H}$ & 599.155657 & 1.7 & 2.8 & 100 & 16.7 & 19.5 & even \\
\hline & $\mathrm{C} 32 \mathrm{H} 32 \mathrm{O} 6 \mathrm{~S} 2$ & $\mathrm{M}+\mathrm{Na}$ & 599.153251 & -0.7 & -1.2 & 100 & 9.1 & 16.5 & even \\
\hline & $\mathrm{C} 17 \mathrm{H} 15 \mathrm{OSS}$ & $2 \mathrm{M}+\mathrm{H}$ & 599.155657 & 1.7 & 2.8 & 100 & 16.7 & 19.5 & even \\
\hline & $\mathrm{C} 16 \mathrm{H} 16 \mathrm{OSS}$ & $2 \mathrm{M}+\mathrm{Na}$ & 599.153251 & -0.7 & -1.2 & 100 & 9.1 & 16.5 & even \\
\hline \multirow[t]{4}{*}{615.127885} & $\mathrm{C} 37 \mathrm{H} 26 \mathrm{O} 52$ & $\mathrm{M}+\mathrm{H}$ & 615.129442 & 1.6 & 2.5 & 100 & 32.1 & 24.5 & even \\
\hline & $\mathrm{C} 35 \mathrm{H} 28 \mathrm{O} 5 \mathrm{~S} 2$ & $\mathrm{M}+\mathrm{Na}$ & 615.127037 & -0.8 & -1.4 & 100 & 27 & 21.5 & even \\
\hline & $\mathrm{C} 32 \mathrm{H} 32 \mathrm{OSS} 2$ & $M+K$ & 615.127189 & -0.7 & -1.1 & 100 & 15.3 & 16.5 & even \\
\hline & $\mathrm{C} 16 \mathrm{H} 16 \mathrm{OSS}$ & $2 M+K$ & 615.127189 & -0.7 & -1.1 & 100 & 15.3 & 16.5 & even \\
\hline
\end{tabular}




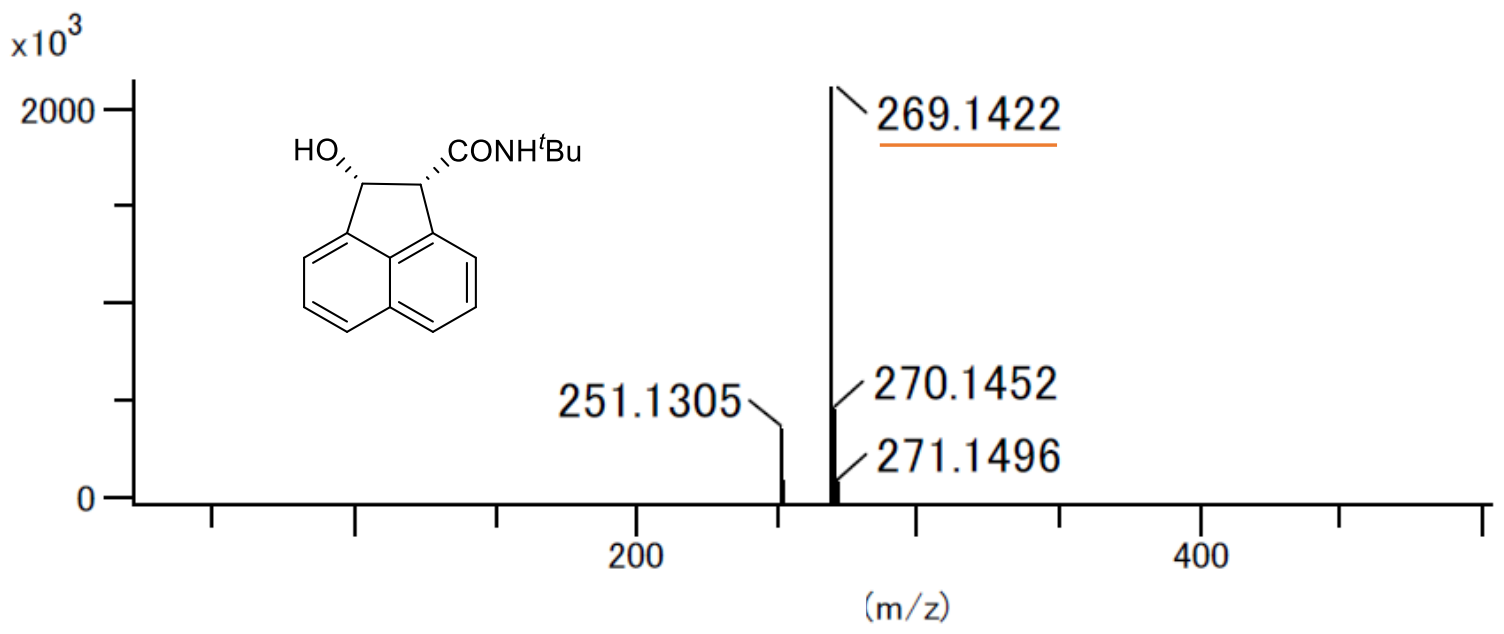

Methyl (1R,2R)-2-hydroxy-1,2-dihydroacenaphthylene-1-carboxylate (cis-6i)

(FI)

$\times 10^{6}$

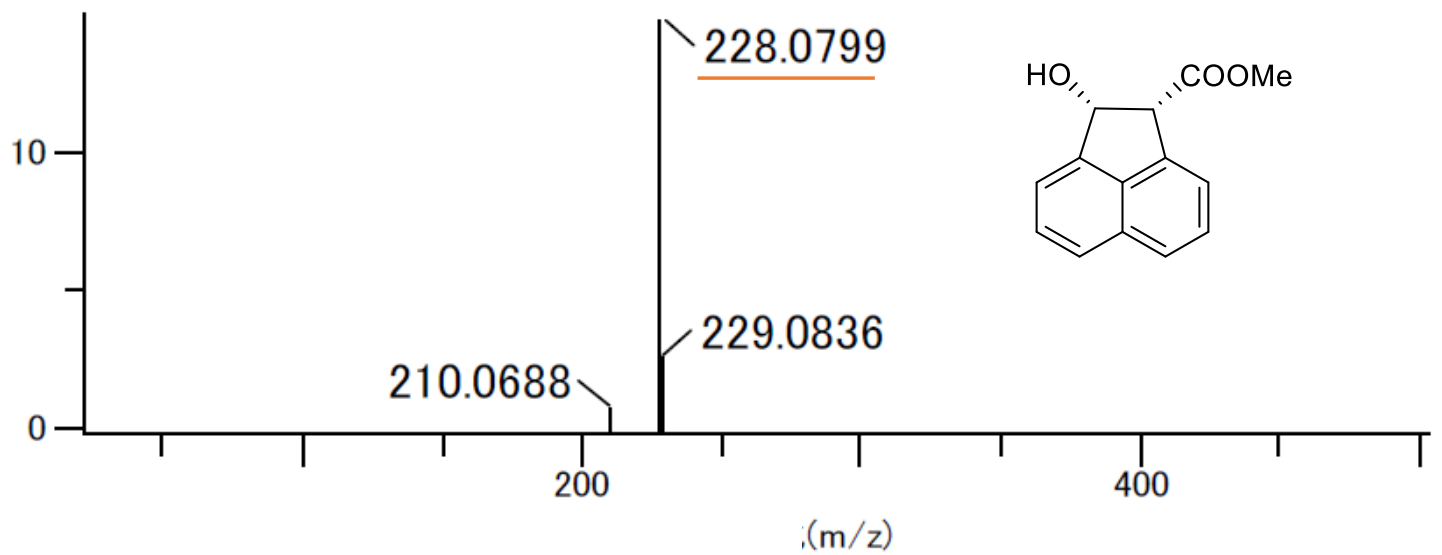


(1S,6R)-6-Chloro-3-methylcyclohex-2-en-1-ol (cis-8a)

(CI)

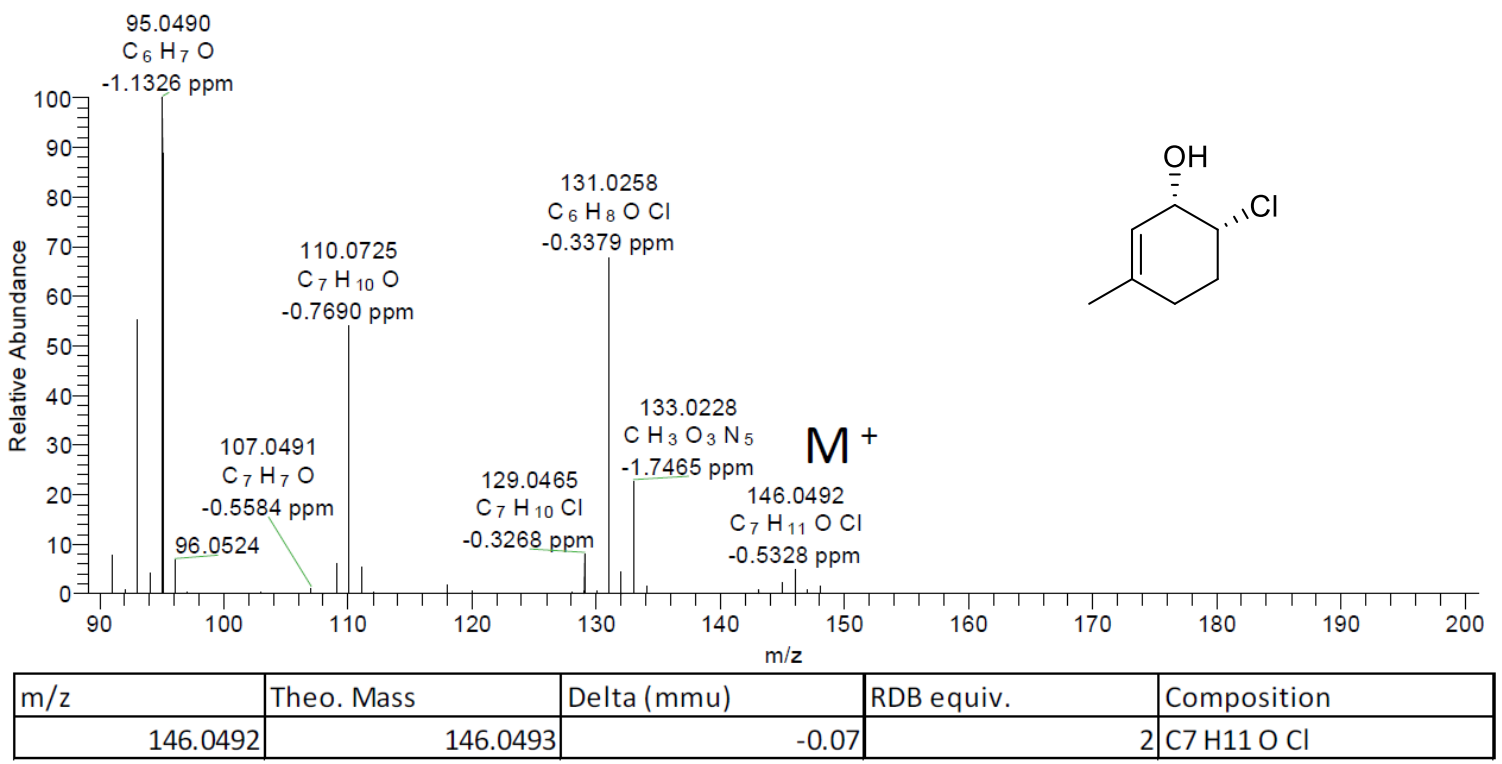

Ethyl (1R,2S)-2-hydroxy-4-methylcyclohex-3-ene-1-carboxylate (cis-8b)

(FI)

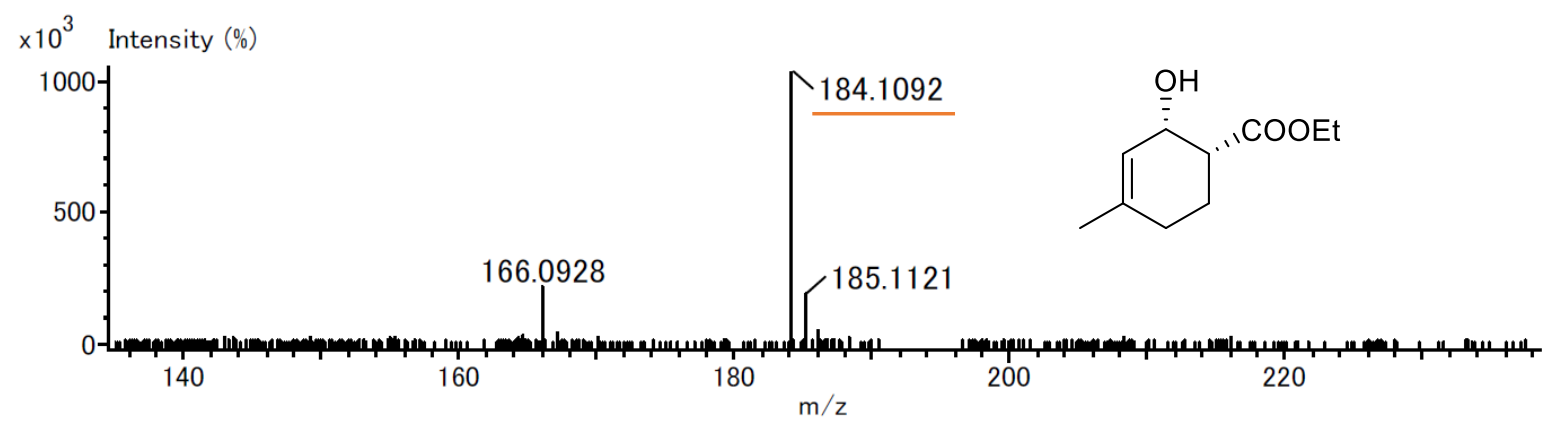


$(1 R, 2 S)-N$-(tert-Butyl)-2-hydroxy-4-methylcyclohex-3-ene-1-carboxamide (cis-8c)

(FI)

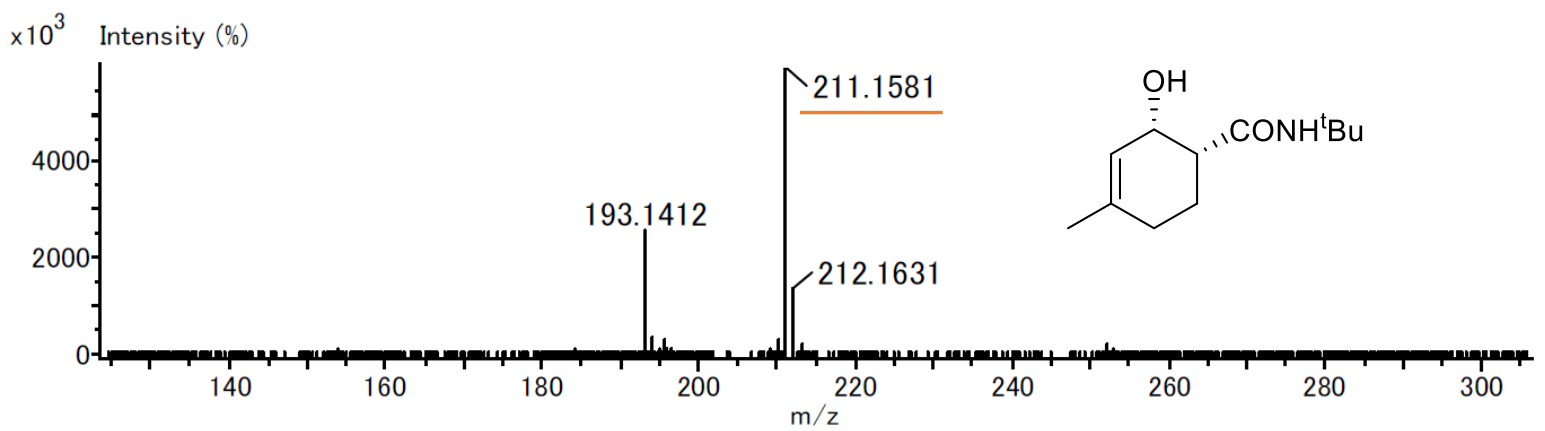

7-(Benzyloxy)-3,4-dihydronaphthalen-1(2H)-one (A)

(APCI positive)

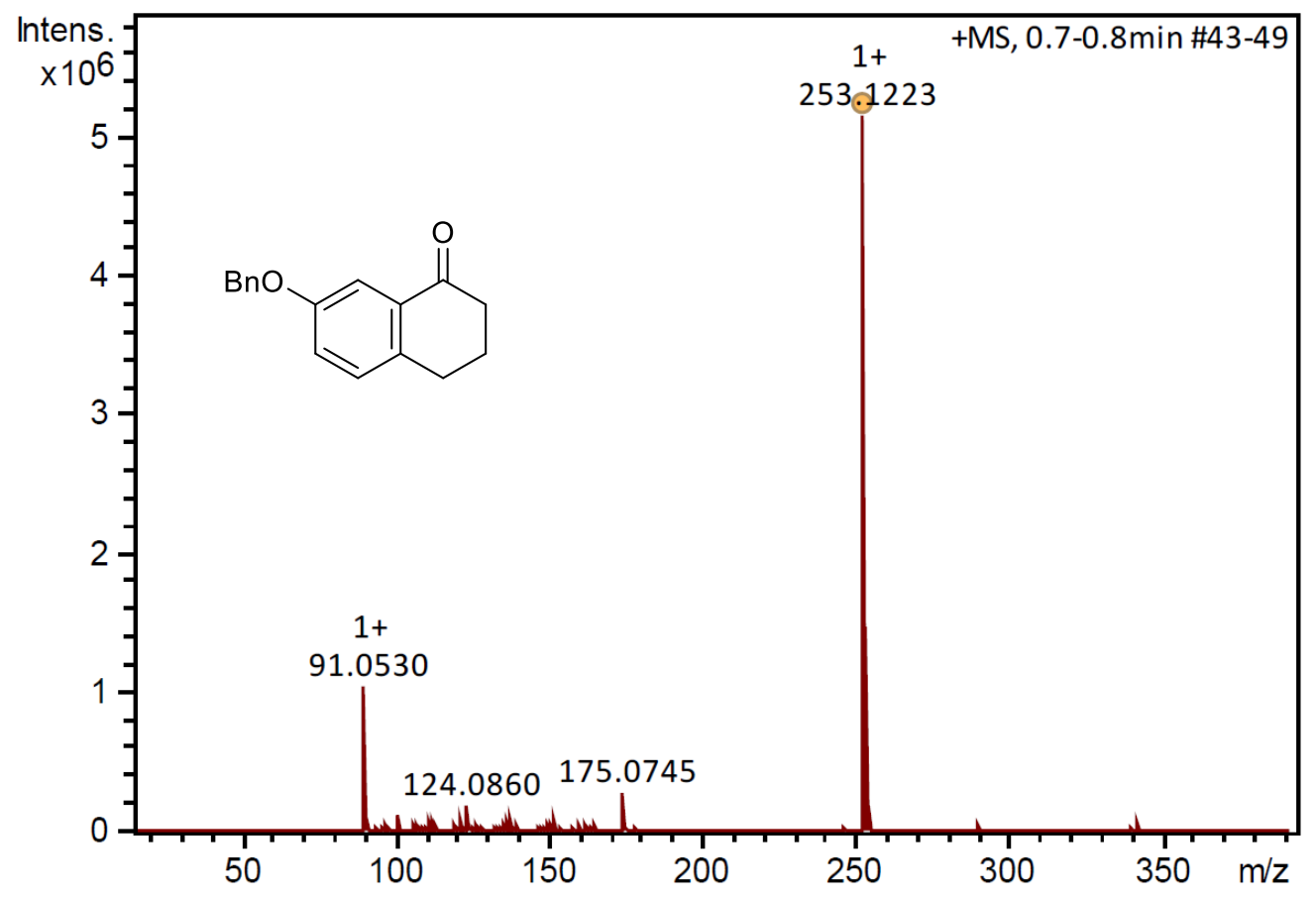

Meas. $\mathrm{m} / \mathrm{z}$

Sum Formula

Adduct Score $m / z$

err $[\mathrm{mD}$ 253.122329 $\mathrm{C} 17 \mathrm{H} 16 \mathrm{O} 2$

$\mathrm{M}+\mathrm{H}$

$100 \quad 253.122306$

\begin{tabular}{lr} 
& err [ppm] \\
\hline 0 & -0.1
\end{tabular}

\begin{tabular}{|l|l|l|}
\hline 59.8 & & 9.5 even
\end{tabular}


7-(Benzyloxy)-2-chloro-3,4-dihydronaphthalen-1(2H)-one (10)

(APCI positive)

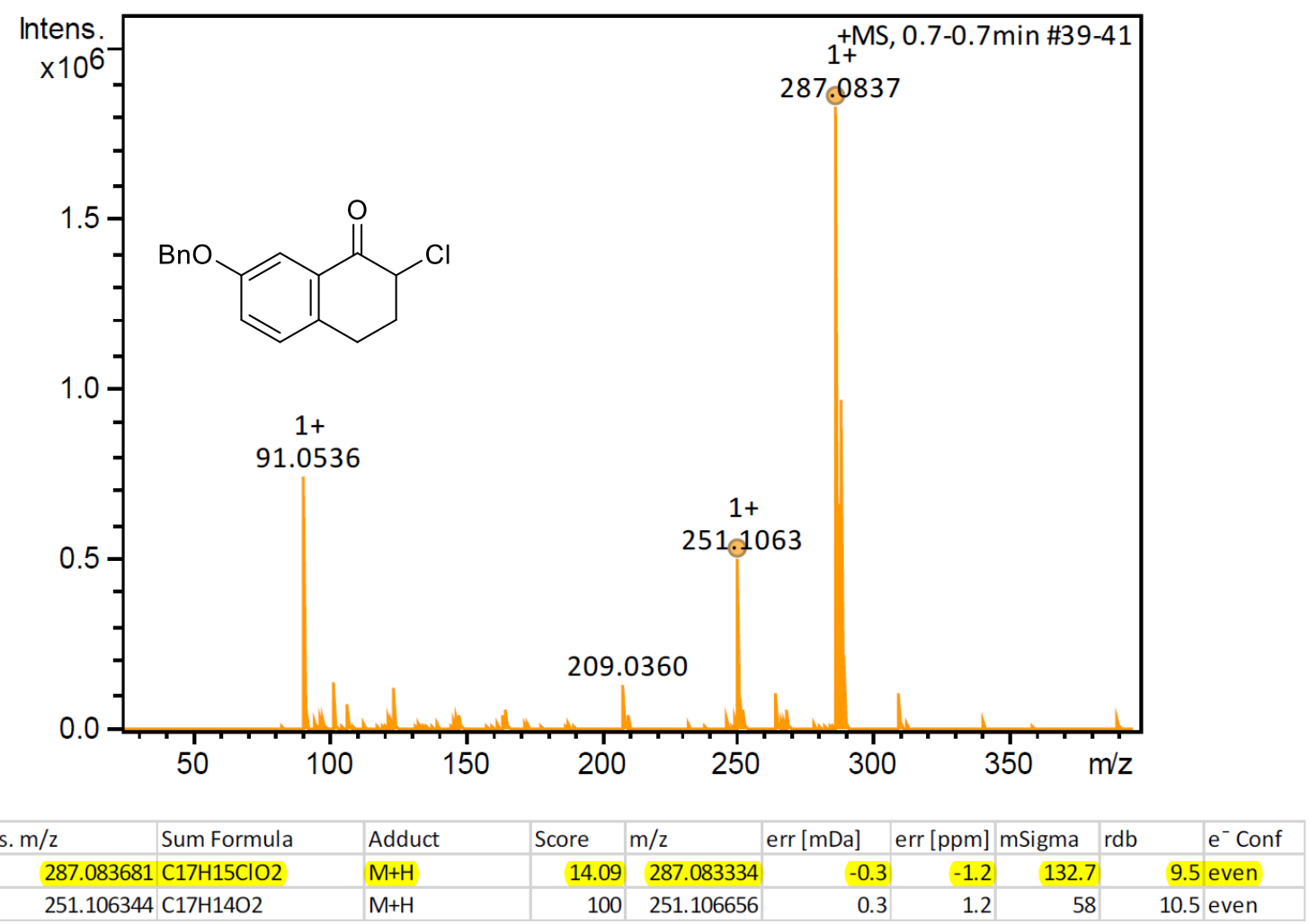

\section{(1R,2S)-7-(Benzyloxy)-2-chloro-1,2,3,4-tetrahydronaphthalen-1-ol (11)}

(CI)

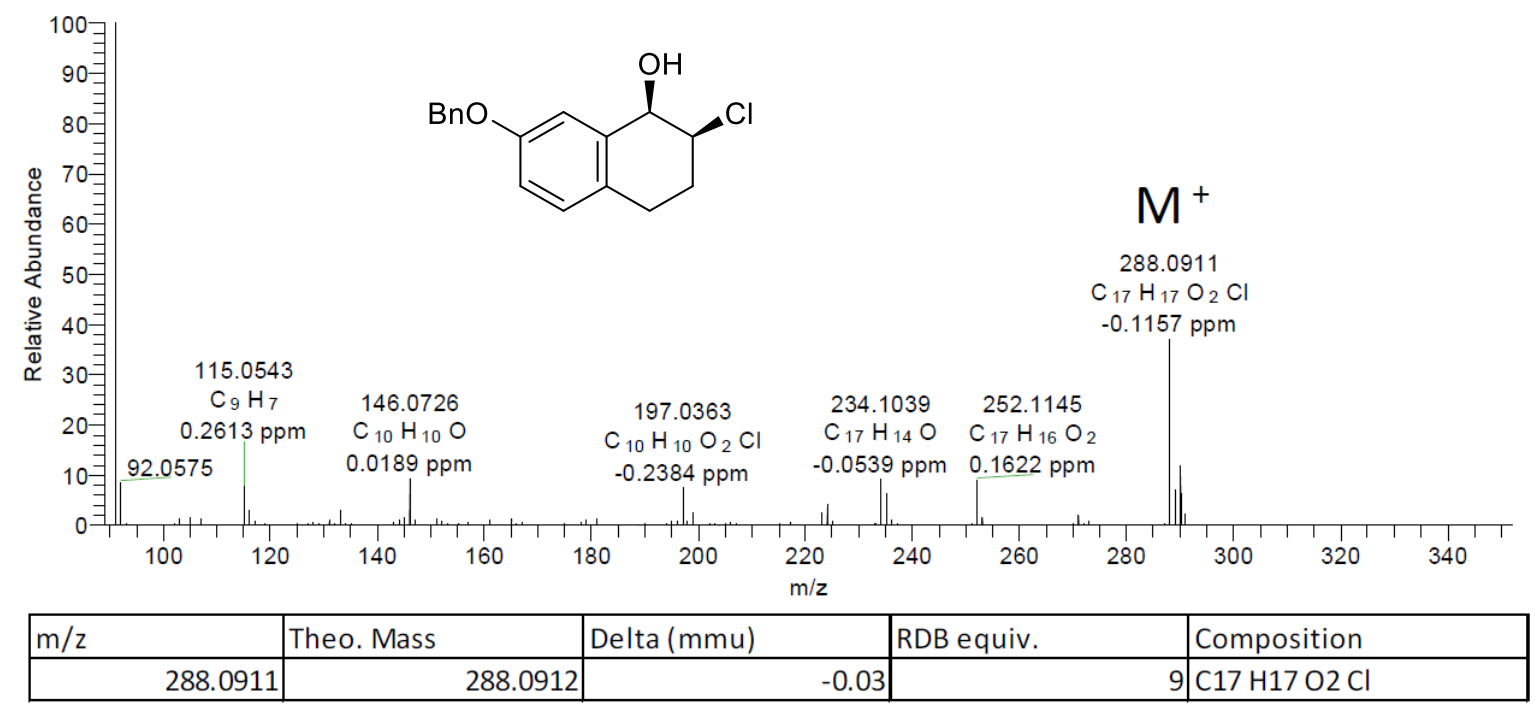


(1R,2R)-2-Azido-7-(benzyloxy)-1,2,3,4-tetrahydronaphthalen-1-ol (B)

(FI)

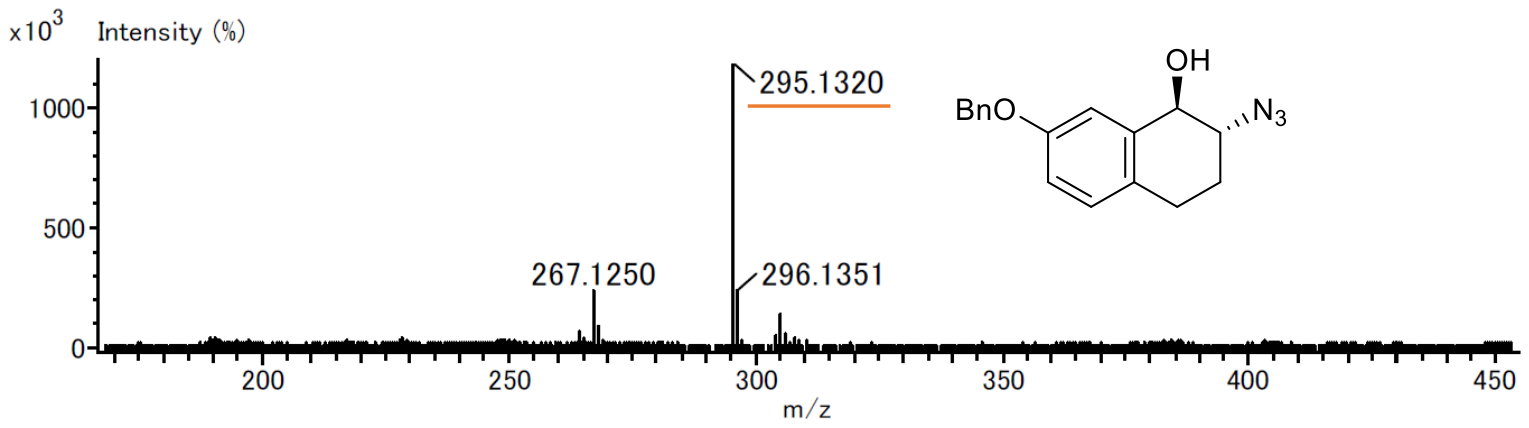

(1R,2R)-2-Amino-7-(benzyloxy)-1,2,3,4-tetrahydronaphthalen-1-ol (12)

(ESI positive)

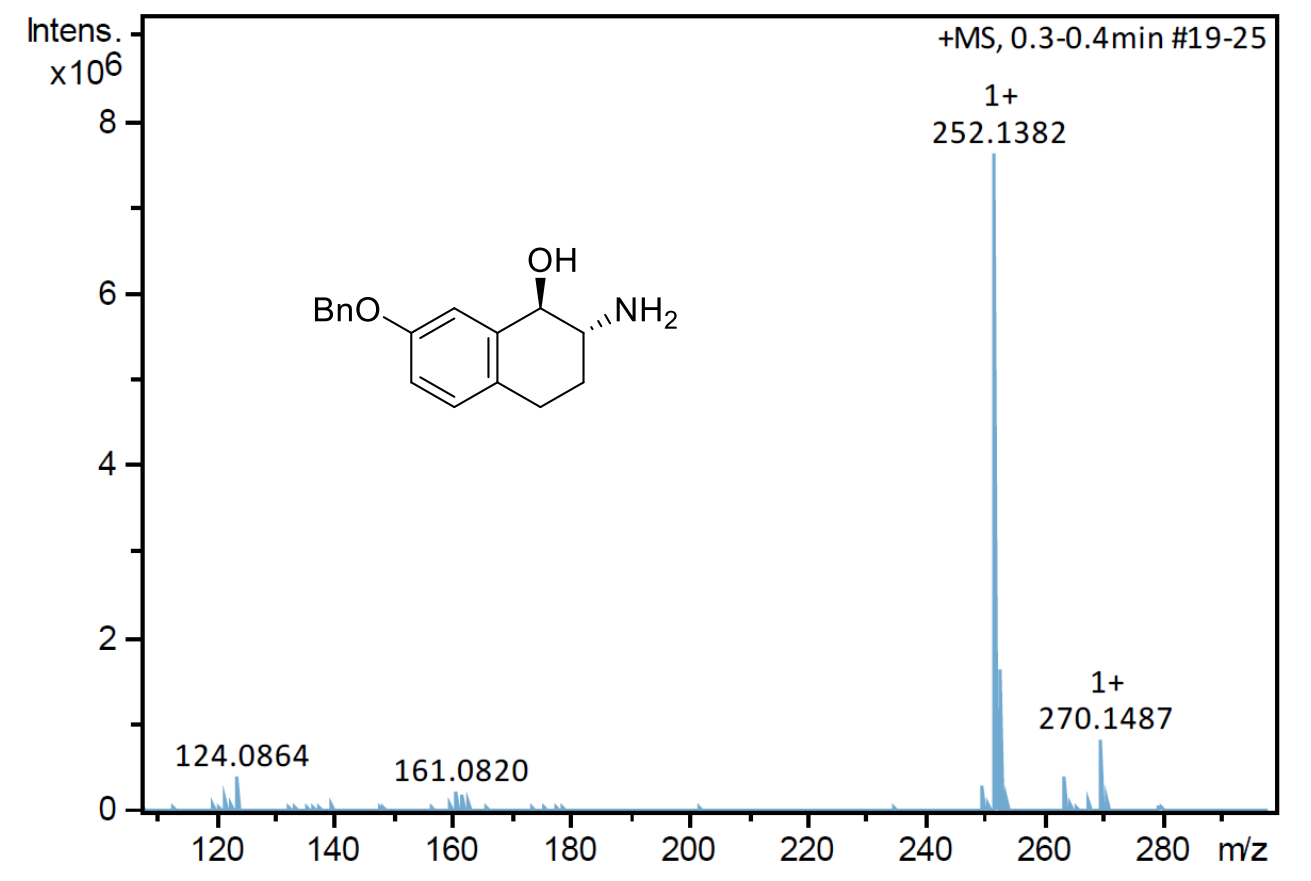

\begin{tabular}{|c|c|c|c|c|c|c|c|c|c|}
\hline Meas. $\mathrm{m} / \mathrm{z}$ & Sum Formula & Adduct & Score & $\mathrm{m} / \mathrm{z}$ & err [mDa] & err [ppm] & mSigma & $\mathrm{rdb}$ & $\mathrm{e}^{-}$Conf \\
\hline 252.138209 & $\mathrm{C} 17 \mathrm{H} 17 \mathrm{NO}$ & $\mathrm{M}+\mathrm{H}$ & 100 & 252.138291 & 0.1 & 0.3 & 13.9 & 9.5 & even \\
\hline 270.148655 & C17H19NO2 & $\mathrm{M}+\mathrm{H}$ & 100 & 270.148855 & 0.2 & 0.7 & 0.3 & 8.5 & even \\
\hline
\end{tabular}


$(4 \mathrm{a} R, 10 \mathrm{~b} R)-9-(B e n z y l o x y)-4 \mathrm{a}, 5,6,10 \mathrm{~b}-$ tetrahydro-2H-naphtho[1,2-b][1,4]oxazin-3(4H)one (13)

(APCI positive)

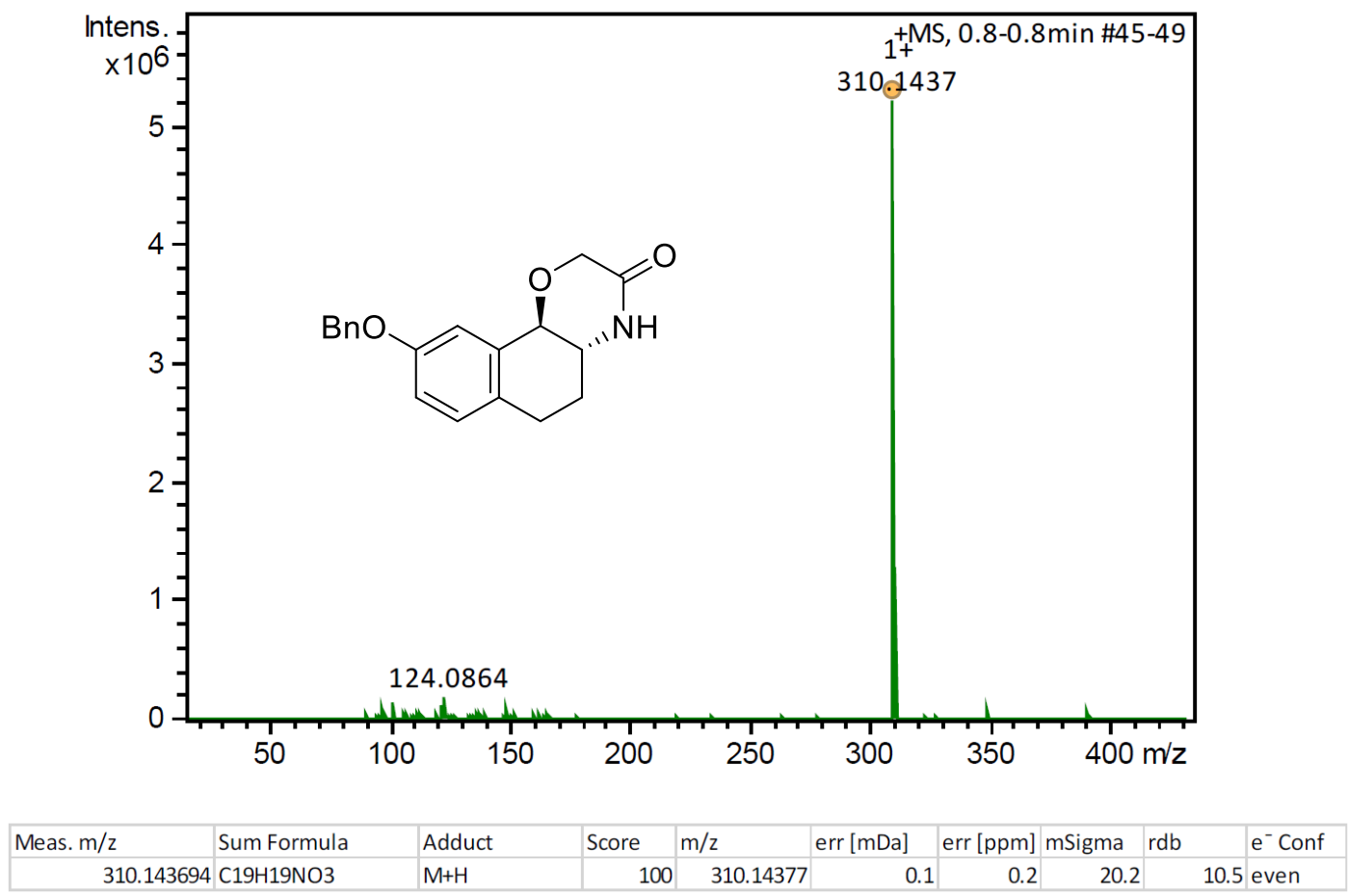

$(4 \mathrm{a} R, 10 \mathrm{~b} R)-9-(B e n z y l o x y)-4-p r o p i o n y l-4 a, 5,6,10 \mathrm{~b}-t$ trahydro-2H-naphtho[1,2-b][1,4]o xazin-3(4H)-one (14)

(APCI positive)

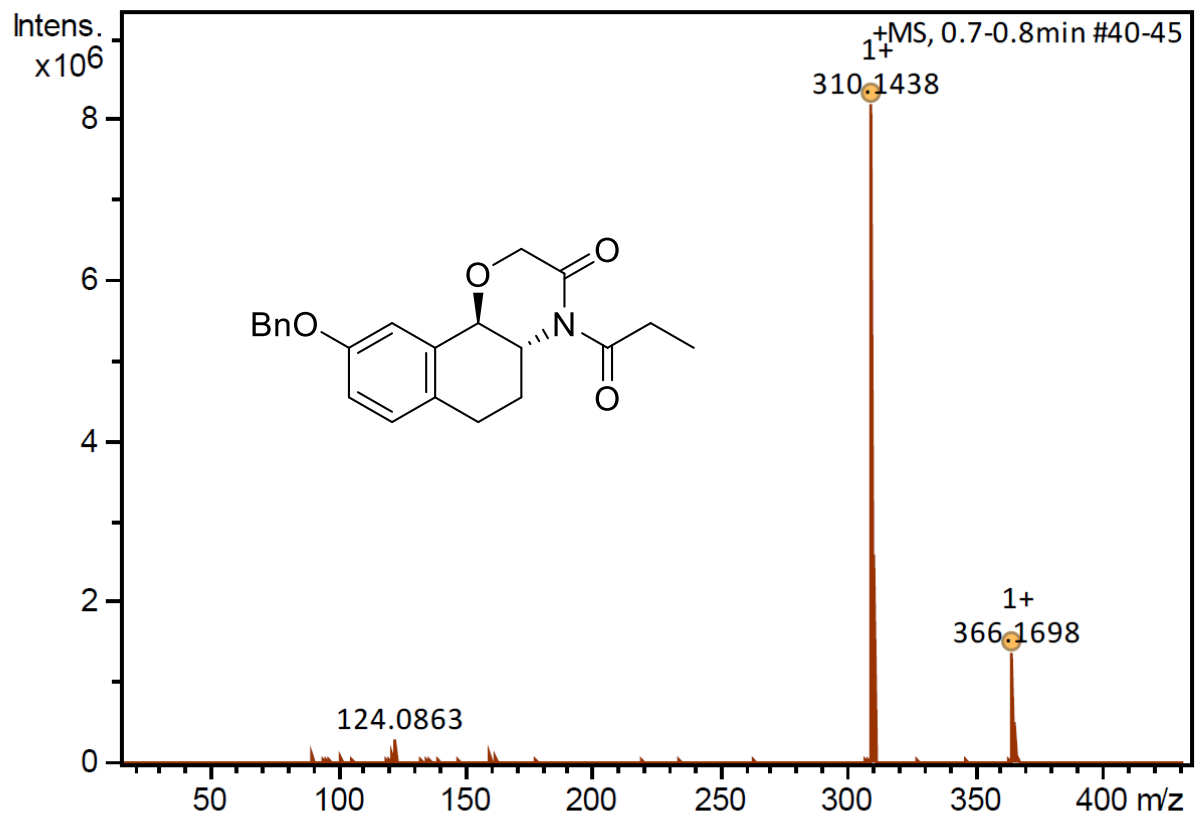


(4aR,10bR)-9-(Benzyloxy)-4-propyl-3,4,4a,5,6,10b-hexahydro-2H-naphtho[1,2-b][1,4]o xazine $(C)$

(ESI positive)

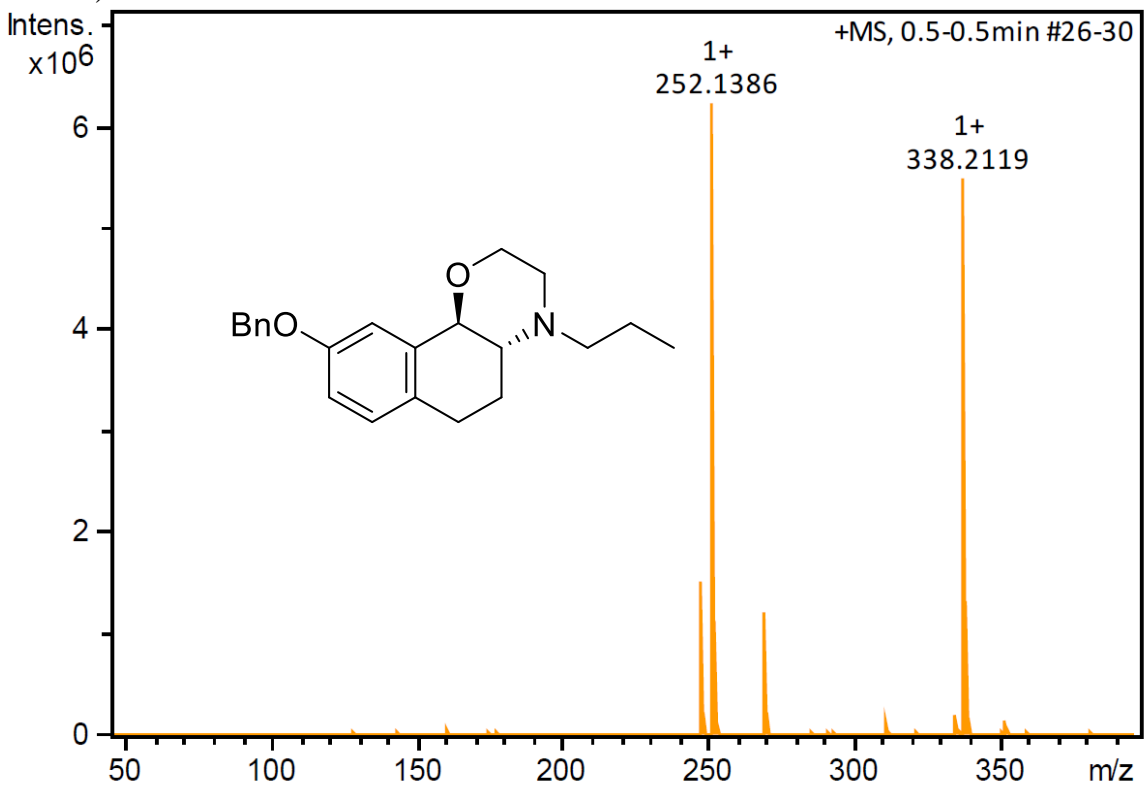

\begin{tabular}{|c|l|l|r|r|r|r|r|r|r|r|}
\hline Meas. $\mathrm{m} / \mathrm{z}$ & Sum Formula & Adduct & \multicolumn{1}{|c|}{ Score } & $\mathrm{m} / \mathrm{z}$ & err [mDa] & err [ppm] & $\mathrm{mSigma}$ & $\mathrm{rdb}^{-}$ & $\mathrm{e}^{-}$Conf \\
\hline 252.138637 & C17H17NO & $\mathrm{M}+\mathrm{H}$ & 100 & 252.138291 & -0.3 & -1.4 & 3.5 & 9.5 & even \\
\hline 338.211901 & $\mathrm{C} 22 \mathrm{H} 27 \mathrm{NO} 2$ & $\mathrm{M}+\mathrm{H}$ & 100 & 338.211456 & -0.4 & -1.3 & 1.6 & 9.5 even \\
\hline
\end{tabular}

\section{(+)-PHNO:}

$(4 \mathrm{a} R, 10 \mathrm{~b} R)$-4-Propyl-3,4,4a,5,6,10b-hexahydro-2H-naphtho[1,2-b][1,4]oxazin-9-ol (ESI positive)

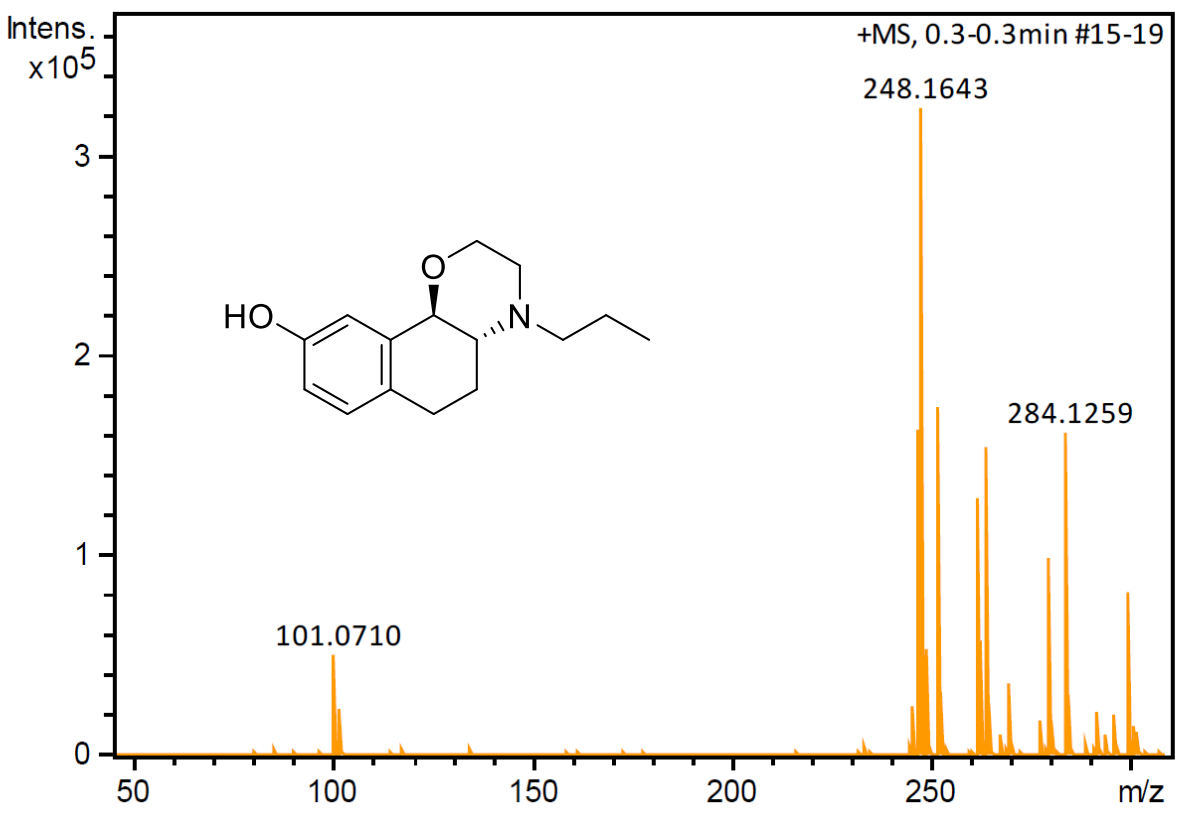


$(1 R, 2 R)$-1-((4,5-Dichloro-2-((3aR,6R,7aR)-8,8-dimethyl-2,2-dioxidohexahydro-3H-3a,6 -methanobenzo[ $[c]$ isothiazole-1-carbonyl)benzoyl)oxy)-2,3-dihydro- $1 H$-indene-2-carbo xylate (D)

(ESI positive)

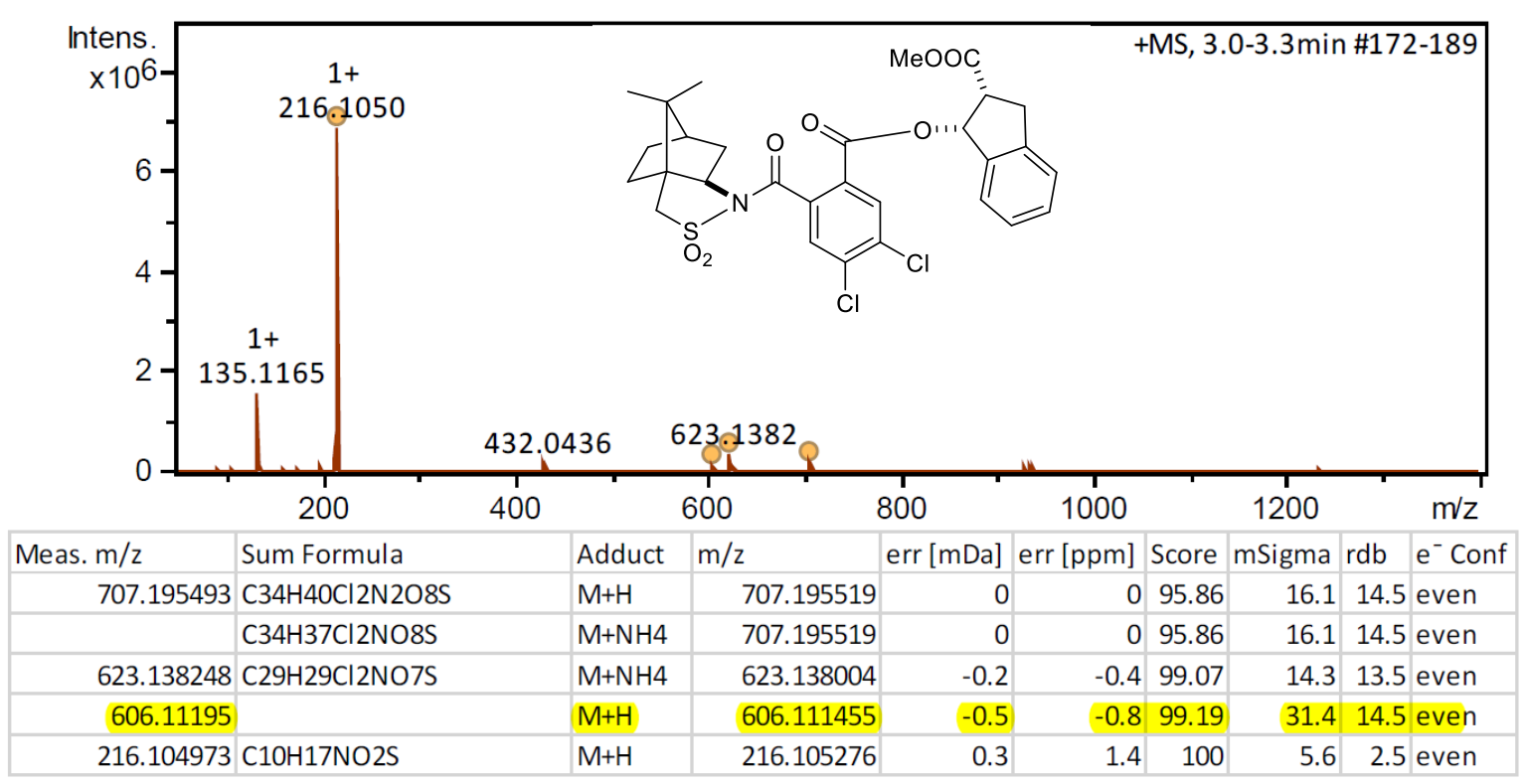

